\title{
Metformin and second- or third-generation sulphonylurea combination therapy for adults with type 2 diabetes mellitus
} (Review)

Madsen KS, Kähler P, Kähler LKA, Madsbad S, Gnesin F, Metzendorf MI, Richter B, Hemmingsen B

Madsen KS, Kähler P, Kähler LKA, Madsbad S, Gnesin F, Metzendorf MI, Richter B, Hemmingsen B.

Metformin and second- or third-generation sulphonylurea combination therapy for adults with type 2 diabetes mellitus.

Cochrane Database of Systematic Reviews 2019, Issue 4. Art. No.: CD012368.

DOI: 10.1002/14651858.CD012368.pub2.

www.cochranelibrary.com

Metformin and second- or third-generation sulphonylurea combination therapy for adults with type 2 diabetes 
TABLE OF CONTENTS

HEADER

ABSTRACT

PLAIN LANGUAGE SUMMARY

SUMMARY OF FINDINGS

BACKGROUND

OBJECTIVES

METHODS

RESULTS

Figure 1.

Figure 2.

Figure 3.

DISCUSSION

AUTHORS' CONCLUSIONS

ACKNOWLEDGEMENTS

REFERENCES

CHARACTERISTICS OF STUDIES

DATA AND ANALYSES

Analysis 1.1. Comparison 1 Metformin plus sulphonylurea vs metformin plus placebo, Outcome 1 All-cause mortality. ............

Analysis 1.2. Comparison 1 Metformin plus sulphonylurea vs metformin plus placebo, Outcome 2 Serious adverse events. ....

Analysis 1.3. Comparison 1 Metformin plus sulphonylurea vs metformin plus placebo, Outcome 3 Cardiovascular mortality. ...

Analysis 1.4. Comparison 1 Metformin plus sulphonylurea vs metformin plus placebo, Outcome 4 Non-fatal myocardial infarction.

Analysis 1.5. Comparison 1 Metformin plus sulphonylurea vs metformin plus placebo, Outcome 5 Heart failure.

Analysis 1.6. Comparison 1 Metformin plus sulphonylurea vs metformin plus placebo, Outcome 6 Non-serious adverse events.

Analysis 1.7. Comparison 1 Metformin plus sulphonylurea vs metformin plus placebo, Outcome 7 Mild/moderate hypoglycaemia.

Analysis 1.8. Comparison 1 Metformin plus sulphonylurea vs metformin plus placebo, Outcome 8 Serious hypoglycaemia. ....

Analysis 1.9. Comparison 1 Metformin plus sulphonylurea vs metformin plus placebo, Outcome 9 Weight change. .................

Analysis 1.10. Comparison 1 Metformin plus sulphonylurea vs metformin plus placebo, Outcome 10 Change in $\mathrm{HbA1c}$. ..........

Analysis 2.1. Comparison 2 Metformin plus sulphonylurea vs metformin plus GLP-1 analogue, Outcome 1 All-cause mortality. .

Analysis 2.2. Comparison 2 Metformin plus sulphonylurea vs metformin plus GLP-1 analogue, Outcome 2 Serious adverse events.

Analysis 2.3. Comparison 2 Metformin plus sulphonylurea vs metformin plus GLP-1 analogue, Outcome 3 Cardiovascular mortality.

Analysis 2.4. Comparison 2 Metformin plus sulphonylurea vs metformin plus GLP-1 analogue, Outcome 4 Non-fatal myocardial infarction.

Analysis 2.5. Comparison 2 Metformin plus sulphonylurea vs metformin plus GLP-1 analogue, Outcome 5 Heart failure. ......... Analysis 2.6. Comparison 2 Metformin plus sulphonylurea vs metformin plus GLP-1 analogue, Outcome 6 End-stage renal disease.

Analysis 2.7. Comparison 2 Metformin plus sulphonylurea vs metformin plus GLP-1 analogue, Outcome 7 Non-serious adverse events.

Analysis 2.8. Comparison 2 Metformin plus sulphonylurea vs metformin plus GLP-1 analogue, Outcome 8 Mild/moderate hypoglycaemia.

Analysis 2.9. Comparison 2 Metformin plus sulphonylurea vs metformin plus GLP-1 analogue, Outcome 9 Serious hypoglycaemia.

Analysis 2.10. Comparison 2 Metformin plus sulphonylurea vs metformin plus GLP-1 analogue, Outcome 10 Weight (change). Analysis 2.11. Comparison 2 Metformin plus sulphonylurea vs metformin plus GLP-1 analogue, Outcome 11 Change in $\mathrm{HbA1c}$. Analysis 3.1. Comparison 3 Metformin plus sulphonylurea vs metformin plus DPP-4 inhibitor, Outcome 1 All-cause mortality. ... Analysis 3.2. Comparison 3 Metformin plus sulphonylurea vs metformin plus DPP-4 inhibitor, Outcome 2 Serious adverse events.

Analysis 3.3. Comparison 3 Metformin plus sulphonylurea vs metformin plus DPP-4 inhibitor, Outcome 3 Cardiovascular mortality.

4 
Analysis 3.4. Comparison 3 Metformin plus sulphonylurea vs metformin plus DPP-4 inhibitor, Outcome 4 Non-fatal myocardial infarction.

Analysis 3.5. Comparison 3 Metformin plus sulphonylurea vs metformin plus DPP-4 inhibitor, Outcome 5 Heart failure. .......... Analysis 3.6. Comparison 3 Metformin plus sulphonylurea vs metformin plus DPP-4 inhibitor, Outcome 6 Non-fatal stroke. .... Analysis 3.7. Comparison 3 Metformin plus sulphonylurea vs metformin plus DPP-4 inhibitor, Outcome 7 Non-serious adverse events.

Analysis 3.8. Comparison 3 Metformin plus sulphonylurea vs metformin plus DPP-4 inhibitor, Outcome 8 Mild/moderate hypoglycaemia.

Analysis 3.9. Comparison 3 Metformin plus sulphonylurea vs metformin plus DPP-4 inhibitor, Outcome 9 Serious hypoglycaemia.

Analysis 3.10. Comparison 3 Metformin plus sulphonylurea vs metformin plus DPP-4 inhibitor, Outcome 10 Weight change (kg).

Analysis 3.11. Comparison 3 Metformin plus sulphonylurea vs metformin plus DPP-4 inhibitor, Outcome 11 Change in HbA1c. . Analysis 3.12. Comparison 3 Metformin plus sulphonylurea vs metformin plus DPP-4 inhibitor, Outcome 12 Fasting plasma glucose.

Analysis 3.13. Comparison 3 Metformin plus sulphonylurea vs metformin plus DPP-4 inhibitor, Outcome 13 BMI.

Analysis 4.1. Comparison 4 Metformin plus sulphonylurea vs metformin plus long-acting DPP-4 inhibitor, Outcome 1 All-cause mortality.

Analysis 4.2. Comparison 4 Metformin plus sulphonylurea vs metformin plus long-acting DPP-4 inhibitor, Outcome 2 Serious adverse events.

Analysis 4.3. Comparison 4 Metformin plus sulphonylurea vs metformin plus long-acting DPP-4 inhibitor, Outcome 3 Cardiovascular mortality.

Analysis 4.4. Comparison 4 Metformin plus sulphonylurea vs metformin plus long-acting DPP-4 inhibitor, Outcome 4 Non-fatal myocardial infarction.

Analysis 4.5. Comparison 4 Metformin plus sulphonylurea vs metformin plus long-acting DPP-4 inhibitor, Outcome 5 Nonserious adverse events.

Analysis 4.6. Comparison 4 Metformin plus sulphonylurea vs metformin plus long-acting DPP-4 inhibitor, Outcome 6 Mild/ moderate hypoglycaemia.

Analysis 4.7. Comparison 4 Metformin plus sulphonylurea vs metformin plus long-acting DPP-4 inhibitor, Outcome 7 Serious hypoglycaemia.

Analysis 4.8. Comparison 4 Metformin plus sulphonylurea vs metformin plus long-acting DPP-4 inhibitor, Outcome 8 Weight change (kg).

Analysis 4.9. Comparison 4 Metformin plus sulphonylurea vs metformin plus long-acting DPP-4 inhibitor, Outcome 9 Change in HbAlc (\%).

Analysis 5.1. Comparison 5 Metformin plus sulphonylurea vs metformin plus thiazolidinedione, Outcome 1 All-cause mortality.

Analysis 5.2. Comparison 5 Metformin plus sulphonylurea vs metformin plus thiazolidinedione, Outcome 2 Serious adverse events.

Analysis 5.3. Comparison 5 Metformin plus sulphonylurea vs metformin plus thiazolidinedione, Outcome 3 Cardiovascular mortality.

Analysis 5.4. Comparison 5 Metformin plus sulphonylurea vs metformin plus thiazolidinedione, Outcome 4 Non-fatal myocardial infarction.

Analysis 5.5. Comparison 5 Metformin plus sulphonylurea vs metformin plus thiazolidinedione, Outcome 5 Heart failure. ...... Analysis 5.6. Comparison 5 Metformin plus sulphonylurea vs metformin plus thiazolidinedione, Outcome 6 Non-fatal stroke. .. Analysis 5.7. Comparison 5 Metformin plus sulphonylurea vs metformin plus thiazolidinedione, Outcome 7 Amputation of lower extremity.

Analysis 5.8. Comparison 5 Metformin plus sulphonylurea vs metformin plus thiazolidinedione, Outcome 8 Blindness or severe vision loss.

Analysis 5.9. Comparison 5 Metformin plus sulphonylurea vs metformin plus thiazolidinedione, Outcome 9 End-stage renal disease.

Analysis 5.10. Comparison 5 Metformin plus sulphonylurea vs metformin plus thiazolidinedione, Outcome 10 Non-serious adverse events.

Analysis 5.11. Comparison 5 Metformin plus sulphonylurea vs metformin plus thiazolidinedione, Outcome 11 Mild/moderate hypoglycaemia.

Analysis 5.12. Comparison 5 Metformin plus sulphonylurea vs metformin plus thiazolidinedione, Outcome 12 Serious hypoglycaemia.

198

199

200

200

201

202

202

203

203

204

204

204

205

205

205

205 
Analysis 5.13. Comparison 5 Metformin plus sulphonylurea vs metformin plus thiazolidinedione, Outcome 13 Weight (change).

Analysis 5.14. Comparison 5 Metformin plus sulphonylurea vs metformin plus thiazolidinedione, Outcome 14 Change in HbA1c.

Analysis 6.1. Comparison 6 Metformin plus sulphonylurea vs metformin plus thiazolidinedione (subgroups duration of intervention), Outcome 1 All-cause mortality.

Analysis 6.2. Comparison 6 Metformin plus sulphonylurea vs metformin plus thiazolidinedione (subgroups duration of intervention), Outcome 2 Serious adverse events.

Analysis 6.3. Comparison 6 Metformin plus sulphonylurea vs metformin plus thiazolidinedione (subgroups duration of intervention), Outcome 3 Cardiovascular mortality.

Analysis 6.4. Comparison 6 Metformin plus sulphonylurea vs metformin plus thiazolidinedione (subgroups duration of intervention), Outcome 4 Non-fatal myocardial infarction.

Analysis 6.5. Comparison 6 Metformin plus sulphonylurea vs metformin plus thiazolidinedione (subgroups duration of intervention), Outcome 5 Heart failure.

Analysis 6.6. Comparison 6 Metformin plus sulphonylurea vs metformin plus thiazolidinedione (subgroups duration of intervention), Outcome 6 Non-fatal stroke.

Analysis 6.7. Comparison 6 Metformin plus sulphonylurea vs metformin plus thiazolidinedione (subgroups duration of intervention), Outcome 7 Non-serious adverse events.

Analysis 6.8. Comparison 6 Metformin plus sulphonylurea vs metformin plus thiazolidinedione (subgroups duration of intervention), Outcome 8 Mild/moderate hypoglycaemia.

Analysis 6.9. Comparison 6 Metformin plus sulphonylurea vs metformin plus thiazolidinedione (subgroups duration of intervention), Outcome 9 Serious hypoglycaemia.

Analysis 6.10. Comparison 6 Metformin plus sulphonylurea vs metformin plus thiazolidinedione (subgroups duration of intervention), Outcome 10 Weight change.

Analysis 6.11. Comparison 6 Metformin plus sulphonylurea vs metformin plus thiazolidinedione (subgroups duration of intervention), Outcome 11 Change in HbAlc.

Analysis 7.1. Comparison 7 Metformin plus sulphonylurea vs metformin plus glinide, Outcome 1 All-cause mortality. ............. Analysis 7.2. Comparison 7 Metformin plus sulphonylurea vs metformin plus glinide, Outcome 2 Serious adverse events. ....... Analysis 7.3. Comparison 7 Metformin plus sulphonylurea vs metformin plus glinide, Outcome 3 Cardiovascular mortality. ..... Analysis 7.4. Comparison 7 Metformin plus sulphonylurea vs metformin plus glinide, Outcome 4 Mild/moderate hypoglycaemia.

Analysis 7.5. Comparison 7 Metformin plus sulphonylurea vs metformin plus glinide, Outcome 5 Serious hypoglycaemia. ..... Analysis 7.6. Comparison 7 Metformin plus sulphonylurea vs metformin plus glinide, Outcome 6 Weight change. ................... Analysis 7.7. Comparison 7 Metformin plus sulphonylurea vs metformin plus glinide, Outcome 7 Change in HbA1c. ................ Analysis 8.1. Comparison 8 Metformin plus sulphonylurea vs metformin plus SGLT-2 inhibitor, Outcome 1 All-cause mortality. . Analysis 8.2. Comparison 8 Metformin plus sulphonylurea vs metformin plus SGLT-2 inhibitor, Outcome 2 Serious adverse events.

Analysis 8.3. Comparison 8 Metformin plus sulphonylurea vs metformin plus SGLT-2 inhibitor, Outcome 3 Cardiovascular mortality.

Analysis 8.4. Comparison 8 Metformin plus sulphonylurea vs metformin plus SGLT-2 inhibitor, Outcome 4 Non-fatal myocardial infarction.

Analysis 8.5. Comparison 8 Metformin plus sulphonylurea vs metformin plus SGLT-2 inhibitor, Outcome 5 Heart failure. ......... Analysis 8.6. Comparison 8 Metformin plus sulphonylurea vs metformin plus SGLT-2 inhibitor, Outcome 6 Non-fatal stroke. .... Analysis 8.7. Comparison 8 Metformin plus sulphonylurea vs metformin plus SGLT-2 inhibitor, Outcome 7 Amputation of lower extremity.

Analysis 8.8. Comparison 8 Metformin plus sulphonylurea vs metformin plus SGLT-2 inhibitor, Outcome 8 Non-serious adverse events.

Analysis 8.9. Comparison 8 Metformin plus sulphonylurea vs metformin plus SGLT-2 inhibitor, Outcome 9 Mild/moderate hypoglycaemia.

Analysis 8.10. Comparison 8 Metformin plus sulphonylurea vs metformin plus SGLT-2 inhibitor, Outcome 10 Serious hypoglycaemia.

Analysis 8.11. Comparison 8 Metformin plus sulphonylurea vs metformin plus SGLT-2 inhibitor, Outcome 11 Weight change. ... Analysis 8.12. Comparison 8 Metformin plus sulphonylurea vs metformin plus SGLT-2 inhibitor, Outcome 12 Change in HbA1c. . 
[Intervention Review]

\section{Metformin and second- or third-generation sulphonylurea combination therapy for adults with type 2 diabetes mellitus}

Kasper S Madsen¹, Pernille Kähler², Lise Katrine Aronsen Kähler ${ }^{1}$, Sten Madsbad³, Filip Gnesin, Maria-Inti Metzendorf ${ }^{5}$, Bernd Richter 5 , Bianca Hemmingsen 5

1Faculty of Health and Medical Sciences, University of Copenhagen, Copenhagen N, Denmark. ${ }^{2}$ Copenhagen Medical University, Faculty of Health and Medical Sciences, Copenhagen, Denmark. ${ }^{3}$ Department of Endocrinology, Hvidovre Hospital, University of Copenhagen, Hvidovre, Denmark. 4Department of Endocrinology, Diabetes and Metabolism, Department 7652, Rigshospitalet, Copenhagen, Denmark. ${ }^{5}$ Cochrane Metabolic and Endocrine Disorders Group, Institute of General Practice, Medical Faculty of the Heinrich-Heine-University Düsseldorf, Düsseldorf, Germany

Contact address: Kasper S Madsen, Faculty of Health and Medical Sciences, University of Copenhagen, Blegdamsvej 3B, Copenhagen N, 2200, Denmark. kasperstaberg@gmail.com.

Editorial group: Cochrane Metabolic and Endocrine Disorders Group.

Publication status and date: New, published in Issue 4, 2019.

Citation: Madsen KS, Kähler P, Kähler LKA, Madsbad S, Gnesin F, Metzendorf MI, Richter B, Hemmingsen B. Metformin and second- or third-generation sulphonylurea combination therapy for adults with type 2 diabetes mellitus. Cochrane Database of Systematic Reviews 2019, Issue 4. Art. No.: CD012368. DOI: 10.1002/14651858.CD012368.pub2.

Copyright @ 2019 The Cochrane Collaboration. Published by John Wiley \& Sons, Ltd.

\section{A B S T R A C T}

\section{Background}

The number of people with type 2 diabetes mellitus (T2DM) is increasing worldwide. The combination of metformin and sulphonylurea (M $+\mathrm{S}$ ) is a widely used treatment. Whether $\mathrm{M}+\mathrm{S}$ shows better or worse effects in comparison with other antidiabetic medications for people with T2DM is still controversial.

\section{Objectives}

To assess the effects of metformin and sulphonylurea (second- or third-generation) combination therapy for adults with type 2 diabetes mellitus.

\section{Search methods}

We updated the search of a recent systematic review from the Agency for Healthcare Research and Quality (AHRQ). The updated search included CENTRAL, MEDLINE, Embase, ClinicalTrials.gov and WHO ICTRP. The date of the last search was March 2018. We searched manufacturers' websites and reference lists of included trials, systematic reviews, meta-analyses and health technology assessment reports. We asked investigators of the included trials for information about additional trials.

\section{Selection criteria}

We included randomised controlled trials (RCTs) randomising participants 18 years old or more with T2DM to M+S compared with metformin plus another glucose-lowering intervention or metformin monotherapy with a treatment duration of 52 weeks or more.

\section{Data collection and analysis}

Two review authors read all abstracts and full-text articles and records, assessed risk of bias and extracted outcome data independently. We used a random-effects model to perform meta-analysis, and calculated risk ratios (RRs) for dichotomous outcomes and mean differences (MDs) for continuous outcomes, using 95\% confidence intervals ( $\mathrm{Cls}$ ) for effect estimates. We assessed the certainty of the evidence using the GRADE instrument. 


\section{Main results}

We included 32 RCTs randomising 28,746 people. Treatment duration ranged between one to four years. We judged none of these trials as low risk of bias for all 'Risk of bias' domains. Most important events per person were all-cause and cardiovascular mortality, serious adverse events (SAE), non-fatal stroke (NFS), non-fatal myocardial infarction (MI) and microvascular complications. Most important comparisons were as follows:

Five trials compared $M+S(N=1194)$ with metformin plus a glucagon-like peptide 1 analogue $(N=1675)$ : all-cause mortality was $11 / 1057$ (1\%) versus $11 / 1537$ (0.7\%), risk ratio (RR) 1.15 (95\% confidence interval (CI) 0.49 to 2.67); 3 trials; 2594 participants; low-certainty evidence; cardiovascular mortality $1 / 307(0.3 \%)$ versus $1 / 302$ (0.3\%), low-certainty evidence; serious adverse events (SAE) $128 / 1057$ (12.1\%) versus 194/1537 (12.6\%), RR 0.90 (95\% Cl 0.73 to 1.11); 3 trials; 2594 participants; very low-certainty evidence; non-fatal myocardial infarction (MI) 2/549 (0.4\%) versus 6/1026 (0.6\%), RR 0.57 (95\% Cl 0.12 to 2.82); 2 trials; 1575 participants; very low-certainty evidence.

Nine trials compared $\mathrm{M}+\mathrm{S}(\mathrm{N}=5414)$ with metformin plus a dipeptidyl-peptidase 4 inhibitor $(\mathrm{N}=6346)$ : all-cause mortality was $33 / 5387$ (0.6\%) versus $26 / 6307$ (0.4\%), RR 1.32 (95\% Cl 0.76 to 2.28); 9 trials; 11,694 participants; low-certainty evidence; cardiovascular mortality $11 / 2989$ ( $0.4 \%$ ) versus 9/3885 (0.2\%), RR 1.54 (95\% Cl 0.63 to 3.79); 6 trials; 6874 participants; low-certainty evidence; SAE 735/5387 (13.6\%) versus $779 / 6307$ (12.4\%), RR 1.07 (95\% Cl 0.97 to 1.18); 9 trials; 11,694 participants; very low-certainty evidence; NFS $14 / 2098$ (0.7\%) versus $8 / 2995$ (0.3\%), RR 2.21 (95\% Cl 0.74 to 6.58$)$; 4 trials; 5093 participants; very low-certainty evidence; non-fatal MI $15 / 2989$ (0.5\%) versus 13/3885 (0.3\%), RR 1.45 (95\% Cl 0.69 to 3.07); 6 trials; 6874 participants; very low-certainty evidence; one trial in 64 participants reported no microvascular complications were observed (very low-certainty evidence).

Eleven trials compared $\mathrm{M}+\mathrm{S}(\mathrm{N}=3626)$ with metformin plus a thiazolidinedione $(\mathrm{N}=3685)$ : all-cause mortality was $123 / 3300(3.7 \%)$ versus 114/3354 (3.4\%), RR 1.09 (95\% Cl 0.85 to 1.40); 6 trials; 6654 participants; low-certainty evidence; cardiovascular mortality $37 / 2946$ (1.3\%) versus $41 / 2994$ (1.4\%), RR 0.78 (95\% Cl 0.36 to 1.67); 4 trials; 5940 participants; low-certainty evidence; SAE 666/3300 (20.2\%) versus 671/3354 (20\%), RR 1.01 (95\% Cl 0.93 to 1.11); 6 trials; 6654 participants; very low-certainty evidence; NFS 20/1540 (1.3\%) versus $16 / 1583$ (1\%), RR 1.29 (95\% Cl 0.67 to 2.47); P = 0.45; 2 trials; 3123 participants; very low-certainty evidence; non-fatal MI 25/1841 (1.4\%) versus 21/1877 (1.1\%), RR 1.21 (95\% Cl 0.68 to 2.14$) ; \mathrm{P}=0.51$; 3 trials; 3718 participants; very low-certainty evidence; three trials (3123 participants) reported no microvascular complications (very low-certainty evidence).

Three trials compared $M+S(N=462)$ with metformin plus a glinide $(N=476)$ : one person died in each intervention group (3 trials; 874 participants; low-certainty evidence); no cardiovascular mortality (2 trials; 446 participants; low-certainty evidence); SAE 34/424 (8\%) versus $27 / 450$ (6\%), RR 1.68 (95\% Cl 0.54 to 5.21); P = 0.37; 3 trials; 874 participants; low-certainty evidence; no NFS (1 trial; 233 participants; very low-certainty evidence); non-fatal MI 2/215 (0.9\%) participants in the M+S group; 2 trials; 446 participants; low-certainty evidence; no microvascular complications (1 trial; 233 participants; low-certainty evidence).

Four trials compared $\mathrm{M}+\mathrm{S}(\mathrm{N}=2109)$ with metformin plus a sodium-glucose co-transporter 2 inhibitor $(\mathrm{N}=3032)$ : all-cause mortality was $13 / 2107$ (0.6\%) versus 19/3027 (0.6\%), RR 0.96 (95\% Cl 0.44 to 2.09); 4 trials; 5134 participants; very low-certainty evidence; cardiovascular mortality $4 / 1327(0.3 \%)$ versus $6 / 2262(0.3 \%)$, RR 1.22 (95\% Cl 0.33 to 4.41$)$; 3 trials; 3589 participants; very low-certainty evidence; SAE $315 / 2107$ (15.5\%) versus 375/3027 (12.4\%), RR 1.02 (95\% Cl 0.76 to 1.37); 4 trials; 5134 participants; very low-certainty evidence; NFS 3/919 $(0.3 \%)$ versus $7 / 1856(0.4 \%)$, RR 0.87 ( $95 \% \mathrm{Cl} 0.22$ to 3.34$) ; 2$ trials; 2775 participants; very low-certainty evidence; non-fatal MI $7 / 890$ ( $0.8 \%)$ versus 8/1374 (0.6\%), RR 1.43 (95\% Cl 0.49 to 4.18; 2 trials); 2264 participants; very low-certainty evidence; amputation of lower extremity $1 / 437(0.2 \%)$ versus $1 / 888(0.1 \%)$; very low-certainty evidence.

Trials reported more hypoglycaemic episodes with $\mathrm{M}+\mathrm{S}$ combination compared to all other metformin-antidiabetic agent combinations. Results for $\mathrm{M}+\mathrm{S}$ versus metformin monotherapy were inconclusive. There were no RCTs comparing M+S with metformin plus insulin. We identified nine ongoing trials and two trials are awaiting assessment. Together these trials will include approximately 16,631 participants.

\section{Authors' conclusions}

There is inconclusive evidence whether $\mathrm{M}+\mathrm{S}$ combination therapy compared with metformin plus another glucose-lowering intervention results in benefit or harm for most patient-important outcomes (mortality, SAEs, macrovascular and microvascular complications) with the exception of hypoglycaemia (more harm for M+S combination). No RCT reported on health-related quality of life.

\section{PLAIN LANGUAGE SUMMARY}

\section{Metformin and sulphonylurea combination therapy for adults with type 2 diabetes mellitus}

\section{Review question}

We wanted to investigate the effects of the combination of the antidiabetic medications metformin plus sulphonylurea compared with other antidiabetic interventions in people with type 2 diabetes.

\section{Background}


Many people with type 2 diabetes are treated with several types of glucose-lowering drugs such as 'sulphonylureas' (for example glibenclamide or glyburide, glipizide and gliclazide). These medications lower blood glucose by stimulating the secretion of insulin in the body, thereby increasing insulin levels in the blood. Another antidiabetic agent, metformin lowers blood glucose by improving the body's ability to make insulin work better (insulin sensitivity). The combination of metformin plus sulphonylurea is widely used. We wanted to investigate the effects of metformin plus sulphonylurea on patient-important outcomes such as complications of diabetes (for example kidney and eye disease, heart attacks, strokes), death from any cause, health-related quality of life and side effects of the medications.

\section{Study characteristics}

We found 32 randomised controlled studies (clinical trials where people are randomly put into one of two or more treatment groups), which allocated 28,746 people with type 2 diabetes to either metformin plus sulphonylurea or a comparator group. The comparator groups consisted of the following types of antidiabetic medications in addition to metformin: five studies with glucagon-like peptide 1 analogues, nine studies with dipeptidyl-peptidase 4 inhibitors, 11 studies with thiazolidinediones, three studies with glinides and four studies with sodium-glucose co-transporter 2 inhibitors.

Participants of the studies were treated for between one and four years. There were big differences between people taking part in the studies, especially with regard to age, how long people had diabetes and whether diabetes complications were present at the start of the study.

This evidence is up to date as of March 2018.

\section{Key results}

Data on patient-important outcomes were few, and data were sparse for all comparisons of metformin plus sulphonylurea with other antidiabetic medications. The available data did not show firm differences between metformin plus sulphonylurea and other combinations of metformin with antidiabetic drugs or metformin only for most patient-important outcomes. There were more events with low blood sugar (hypoglycaemic episodes) with metformin plus sulphonylurea combination treatment compared to all other combinations of metformin with another antidiabetic compound.

We did not identify studies reporting on health-related quality of life. We identified nine ongoing studies and two yet unpublished studies are awaiting assessment. Together these studies will include around 16,631 participants. Once results are published these studies could significantly influence the findings of our review.

\section{Certainty of the evidence}

All included studies had deficiencies in the way they were conducted or how study authors reported the results. For individual comparisons of the antidiabetic medications the number of participants was often small, resulting in a high risk of random error (play of chance). 


\section{S U M MARY OF F I N I NGS}

Summary of findings for the main comparison. Metformin-sulphonylurea (second- or third-generation) combination therapy compared with metformin plus another antidiabetic drug for adults with type 2 diabetes mellitus

Metformin-sulphonylurea (second- or third-generation) combination therapy compared with metformin plus another antidiabetic drug for adults with type 2 diabetes mellitus

Patient: people with type 2 diabetes mellitus

Settings: outpatients

Intervention: metformin + sulphonylurea

Comparison: metformin plus another antidiabetic drug

\begin{tabular}{|c|c|c|c|c|c|c|}
\hline Outcomes & $\begin{array}{l}\text { Metformin + } \\
\text { antidiabetic } \\
\text { drug }\end{array}$ & $\begin{array}{l}\text { Metformin + } \\
\text { sulphonylurea }\end{array}$ & $\begin{array}{l}\text { Relative effect } \\
(95 \% \mathrm{CI})\end{array}$ & $\begin{array}{l}\text { No. of partici- } \\
\text { pants } \\
\text { (trials) }\end{array}$ & $\begin{array}{l}\text { Certainty of } \\
\text { the evidence } \\
\text { (GRADE) }\end{array}$ & Comments \\
\hline \multicolumn{7}{|l|}{ All-cause mortality (N) } \\
\hline $\begin{array}{l}M+G L P 1-A \\
\text { Follow-up: } 2-3 \text { years }\end{array}$ & 7 per 1000 & $\begin{array}{l}8 \text { per } 1000 \text { ( } 4 \text { to } \\
19 \text { ) }\end{array}$ & $\begin{array}{l}\text { RR } 1.15 \text { (0.49 to } \\
2.67 \text { ) }\end{array}$ & $2594(3)$ & $\begin{array}{l}\oplus \oplus \ominus \ominus^{\mathrm{a} 1} \\
\text { Low }\end{array}$ & \\
\hline $\begin{array}{l}\text { M + DPP4-I } \\
\text { Follow-up: } 1-3 \text { years }\end{array}$ & 4 per 1000 & $\begin{array}{l}5 \text { per } 1000 \text { ( } 3 \text { to } \\
9)\end{array}$ & $\begin{array}{l}\text { RR } 1.32 \text { (0.76 to } \\
2.28 \text { ) }\end{array}$ & $11,694(9)$ & $\begin{array}{l}\oplus \oplus \ominus \ominus \\
\text { Low } b 1\end{array}$ & \\
\hline $\begin{array}{l}M+\text { thiazolidinedione } \\
\text { Follow-up: } 1-5.5 \text { years }\end{array}$ & 34 per 1000 & $\begin{array}{l}37 \text { per } 1000(29 \\
\text { to } 48)\end{array}$ & $\begin{array}{l}\text { RR } 1.09 \text { (0.85 to } \\
1.40)\end{array}$ & $6654(6)$ & $\begin{array}{l}\oplus \oplus \oplus \ominus \\
\text { Low } c 1\end{array}$ & \\
\hline $\begin{array}{l}M+\text { nateglinide } \\
\text { Follow-up: } 1-2 \text { years }\end{array}$ & See comment & & & $874(3)$ & $\begin{array}{l}\oplus \oplus \ominus \ominus \\
\text { Low }^{\mathrm{d} 1}\end{array}$ & $\begin{array}{l}1 \text { participant died in each inter- } \\
\text { vention group }\end{array}$ \\
\hline $\begin{array}{l}\text { M + SGLT2-I } \\
\text { Follow-up: 2-4 years }\end{array}$ & 6 per 1000 & $\begin{array}{l}6 \text { per } 1000 \text { (3 to } \\
13)\end{array}$ & $\begin{array}{l}\text { RR } 0.96 \text { (0.44 to } \\
2.09 \text { ) }\end{array}$ & $5134(4)$ & $\begin{array}{l}\oplus \ominus \ominus \ominus \\
\text { Very lowe1 }\end{array}$ & \\
\hline \multicolumn{7}{|c|}{ Cardiovascular mortality (N) } \\
\hline $\begin{array}{l}M+G L P 1-A \\
\text { Follow-up: } 2-3 \text { years }\end{array}$ & See comment & & & $609(1)$ & $\begin{array}{l}\oplus \oplus \oplus \ominus \\
\text { Low }^{\mathrm{a} 2}\end{array}$ & $\begin{array}{l}1 / 307(0.3 \%) \text { participants died } \\
\text { due to cardiovascular disease in } \\
\text { the } M+S \text { group compared with }\end{array}$ \\
\hline
\end{tabular}




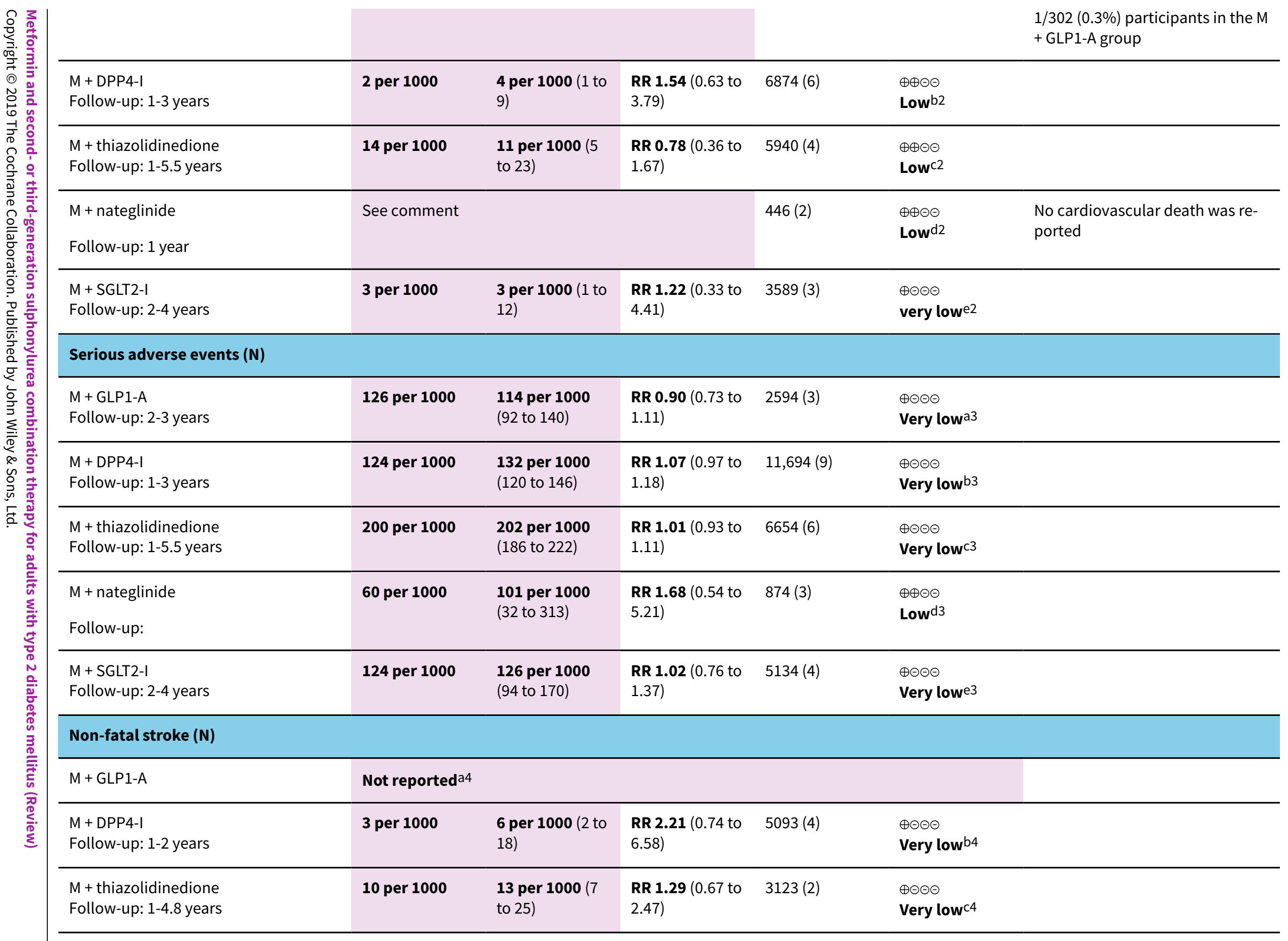




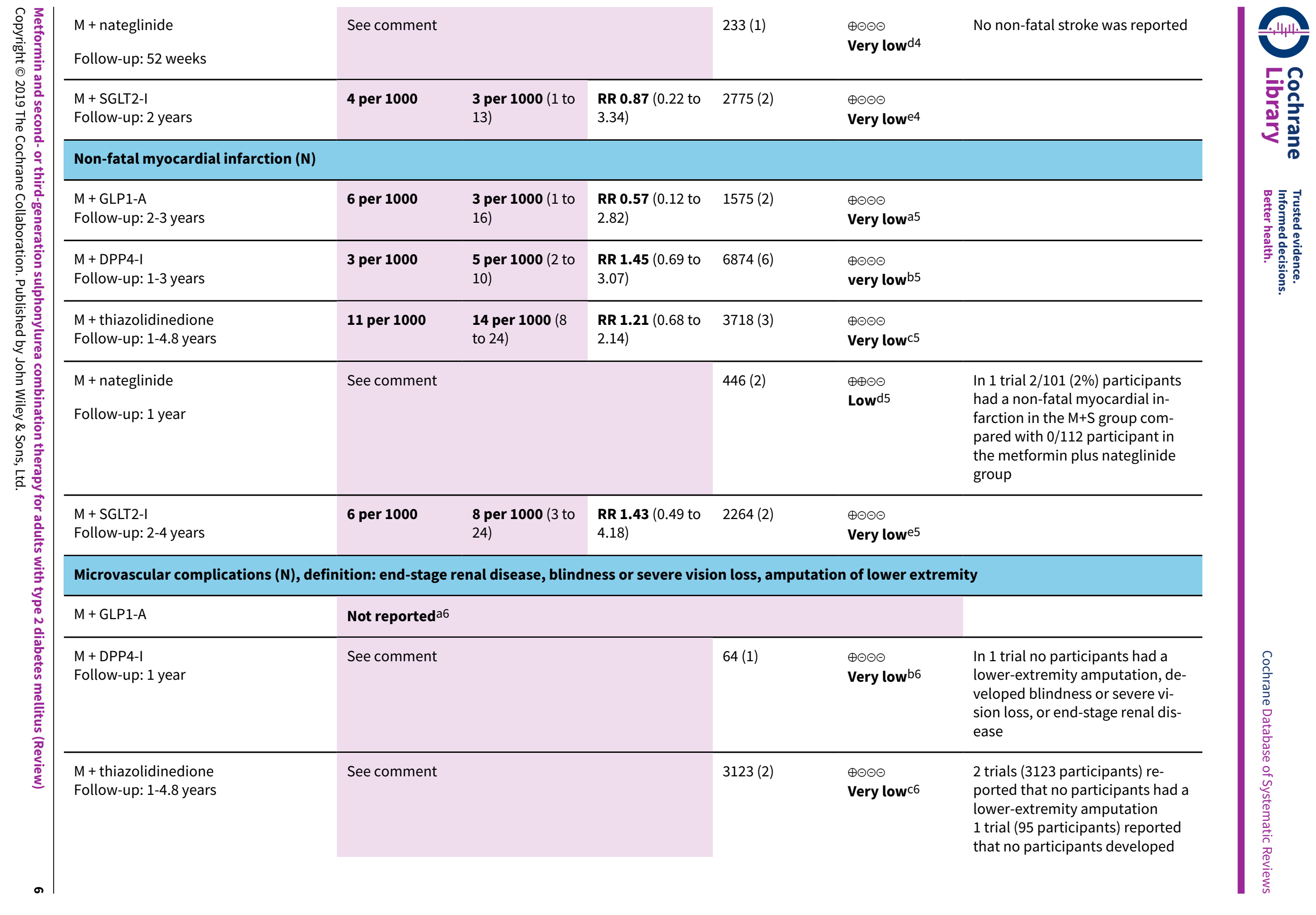




\begin{tabular}{|c|c|c|c|c|c|}
\hline \multirow[t]{4}{*}{ 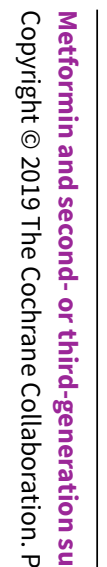 } & & & & & $\begin{array}{l}\text { blindness or severe vision loss, or } \\
\text { end-stage renal disease }\end{array}$ \\
\hline & $\begin{array}{l}M+\text { nateglinide } \\
\text { Follow-up: } 52 \text { weeks }\end{array}$ & See comment & $233(1)$ & $\begin{array}{l}\oplus \oplus \oplus \ominus \\
\text { Low }^{\mathrm{d} 6}\end{array}$ & $\begin{array}{l}\text { No microvascular complications } \\
\text { were reported }\end{array}$ \\
\hline & $\begin{array}{l}\text { M + SGLT2-I } \\
\text { Follow-up: } 2 \text { years }\end{array}$ & See comment & $1325(1)$ & $\begin{array}{l}\oplus \ominus \ominus \ominus \\
\text { Very lowe6 }\end{array}$ & $\begin{array}{l}\text { In } 1 \text { trial } 1 / 437(0.2 \%) \text { partici- } \\
\text { pants had an amputation of the } \\
\text { lower extremity in the } M+S \text { group } \\
\text { compared with } 1 / 888(0.1 \%) \text { in } \\
\text { the } M+\text { SGLT2-I group }\end{array}$ \\
\hline & Health-related quality of life & Not reported & & & \\
\hline
\end{tabular}

${ }^{*}$ The basis for the assumed risk (e.g. the median control group risk across trials) is provided in footnotes. The corresponding risk (and its $95 \%$ confidence interval) is based on the assumed risk in the comparison group and the relative effect of the intervention (and its $95 \% \mathrm{CI}$ )

CI: confidence interval; DPP4-I: dipeptidyl peptidase-4 inhibitor; GLP1-A: glucagon-like peptide 1 analogue; HbA1c: glycosylated haemoglobin A1c; M: metformin; M+S: metformin + sulphonylurea; N: number; N/R: not reported; RR: risk ratio; SGLT2-I: sodium-glucose co-transporter 2 inhibitor; T: thiazolidinedione

\section{GRADE Working Group grades of evidence}

High certainty: we are very confident that the true effect lies close to that of the estimate of the effect.

Moderate certainty: we are moderately confident in the effect estimate; the true effect is likely to be close to the estimate of the effect, but there is a possibility that it is Moderate certainty: we
substantially different.

Low certainty: our confidence in the effect estimate is limited; the true effect may be substantially different from the estimate of the effect.

Very low certainty: we have very little confidence in the effect estimate; the true effect is likely to be substantially different from the estimate of effect.

\section{All-cause mortality}

a1Downgraded by two levels because of serious imprecision ( $\mathrm{Cl}$ consistent with both benefit and harm, small number of trials) - see Appendix 17.

b1Downgraded by one level because of inconsistency (non-consistent direction of effect) and by one level because of imprecision (Cl consistent with both benefit and harm) see Appendix 18.

c1Downgraded by two levels because of serious imprecision ( $\mathrm{Cl}$ consistent with both benefit and harm, small number of trials) - see Appendix 20 .

d1Downgraded by two levels because of serious imprecision ( $\mathrm{Cl}$ consistent with both benefit and harm, small number of trials) - see Appendix 21.

e1Downgraded by one level because of inconsistency (point estimates varied widely) and by two levels because of serious imprecision (Cl consistent with both benefit and harm, small number of trials, low event rate) - see Appendix 22.

\section{Cardiovascular mortality}

a2Downgraded by two levels because of serious imprecision (small number of trials, Cl consistent with both benefit and harm) - see Appendix 17.

b2Downgraded by one level because of because of inconsistency (non-consistent direction of effect) and by one level because of imprecision (Cl consistent with both benefit and harm) - see Appendix 18.

c2Downgraded by two levels because of serious imprecision (small number of trials, Cl consistent with both benefit and harm) - see Appendix 20.

d2Downgraded by two levels because of serious imprecision (small number of trials, unknown event rate) - see Appendix 20.

e2Downgraded by one level because of inconsistency (point estimates varied widely) and by two levels because of serious imprecision (Cl consistent with both benefit and harm, small number of trials, low event rate) - see Appendix 22. 
a3Downgraded by one level because of attrition bias and by two levels because of serious imprecision (Cl consistent with both benefit and harm, small number of trials) - see Appendix 17.

b3Downgraded by one level because of attrition bias, by one level because of inconsistency (non-consistent direction of effect) and by one level because of imprecision ( $\mathrm{C}$ consistent with both benefit and harm) - see Appendix 18

c3Downgraded by one level because of attrition bias, by one level because of inconsistency (non-consistent direction of effect) and by one level because of serious imprecision (Cl consistent with both benefit and harm, small number of trials) - see Appendix 20.

d3Downgraded by two levels because of serious imprecision ( $\mathrm{Cl}$ consistent with both benefit and harm, small number of trials) - see Appendix 21 .

e3 Downgraded by one level because of attrition bias and inconsistency (point estimates varied widely) and by two levels because of serious imprecision (CI consistent with both benefit and harm, small number of trials) - see Appendix 22.

\section{Non-fatal stroke}

a4 No adequate data for analysis.

b4 Downgraded by one level because of attrition bias and by two levels because of serious imprecision (Cl consistent with both benefit and harm, small number of trials, low event rate) - see Appendix 18.

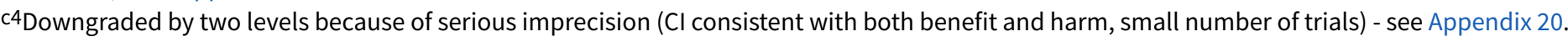

d4Downgraded by two levels of evidence because of serious imprecision (low number of trials, unknown event rate) - see Appendix 21.

e4Downgraded by one level because of attrition bias and by two levels because of serious imprecision ( $\mathrm{Cl}$ consistent with both benefit and harm, small number of trials, low event rate) - see Appendix 22.

\section{Non-fatal myocardial infarction}

a5Downgraded by one level because of attrition bias and by two levels because of serious imprecision (small number of trials, $\mathrm{Cl}$ consistent with both benefit and harm) - see Appendix 17.

b5Downgraded by one level because of attrition bias and by two levels because of serious imprecision ( $\mathrm{Cl}$ consistent with both benefit and harm, small number of trials, low event rate) - see Appendix 18.

c5Downgraded by one level because of attrition bias and by two levels because of serious imprecision (Cl consistent with both benefit and harm, small number of trials) - see Appendix 20.

d5Downgraded by two levels because of serious imprecision (low number of trials, low event rate) - see Appendix 21.

e15Downgraded by one level because of attrition bias and by two levels because of serious imprecision (Cl consistent with both benefit and harm, small number of trials, low event rate) - see Appendix 22.

\section{Microvascular complications}

a6 No adequate data for analysis.

b6Downgraded by three levels because of very serious imprecision (small number of participants, one trial only, unknown event rate) - see Appendix 18.

c6Downgraded by one level because of attrition bias and reporting bias and by two levels because of serious imprecision (Cl consistent with both benefit and harm, small number of trials, unknown event rate) - see Appendix 20.

d6Downgraded by two levels because of serious imprecision (low number of trials, unknown event rate) - see Appendix 21.

e6 Downgraded by one level because of attrition bias and by two levels because of serious imprecision ( $\mathrm{Cl}$ consistent with both benefit and harm, small number of trials, low event rate) - see Appendix 22 


\section{B A C K G R O U N D}

A number of medical organisations have developed guidelines or recommendations for treatment of type 2 diabetes mellitus (T2DM). Most people with T2DM are initially recommended to reduce calorie intake and increase physical activity in order to improve glycaemic control (ADA 2016). However, in order to achieve and maintain specific glycaemic targets, the majority of people with T2DM will require pharmacological glucose-lowering interventions. Metformin is currently the first-line glucose-lowering drug for people with T2DM because of its postulated benefits, including absence of weight gain, or even weight loss, and lack of hypoglycaemia (Inzucchi 2012; Nathan 2009). If behavioural interventions like diet and exercise and maximum tolerated doses of one oral glucose-lowering drug fail to achieve the glycaemic target, other glucose-lowering drugs are often added (ADA 2016). As T2DM is a progressive condition, a substantial proportion of people with T2DM will, with time, require insulin. Some guidelines recommend continuing metformin in this situation (ADA 2016).

As described below, people with T2DM have an elevated risk of developing macrovascular as well as microvascular complications (Almdal 2004). In the treatment of people with T2DM, researchers have considered which glycaemic target is appropriate in order to lower the risk of these complications. One hypothesis so far has been that lower glycosylated haemoglobin A1c ( $\mathrm{HbA1c}$ ) values are associated with reduced macrovascular and microvascular complications. However, this paradigm has been challenged by a Cochrane Review investigating intensive glycaemic control compared to conventional glycaemic control in people with T2DM (Hemmingsen 2011). In this review, authors found insufficient information to confirm or exclude a risk reduction in macrovascular as well as microvascular complications with intensive compared to conventional glycaemic control.

\section{Description of the condition}

Worldwide, the number of people with diabetes was estimated to be 177 million in 2000 and is foreseen to rise to 366 million in 2030 (Wild 2004). T2DM comprises 90\% of people with diabetes and is associated with excess bodyweight and physical inactivity (WHO 2015). T2DM is characterised by hyperglycaemia, insulin resistance and impaired insulin secretion (LeRoith 2002). Although the definition of T2DM relies on elevated blood glucose, T2DM occurs not in isolation, but as part of a complex metabolic-cardiovascular syndrome that includes dyslipidaemia, hypertension, obesity, clotting abnormalities, microalbuminuria and accelerated atherosclerosis, although not every one of these disorders occurs in every person with T2DM (DeFronzo 1999). People with T2DM have an elevated risk of developing macrovascular disease (such as cardiovascular death, myocardial infarction, stroke and peripheral ischaemia) as well as microvascular complications (such as retinopathy, nephropathy and neuropathy) (Almdal 2004).

\section{Description of the intervention}

Since the introduction of the sulphonylureas in the 1950s these glucose-lowering drugs have been the mainstay in the treatment of T2DM. The first to be introduced on the market were the first-generation sulphonylureas (acetohexamide, carbutamide, chlorpropamide, tolazamide and tolbutamide). Later, the second and third generations of sulphonylureas were introduced, and have now almost completely replaced the first-generation sulphonylureas (Harrower 2000). The secondgeneration sulphonylureas (e.g. glibenclamide (in the USA: glyburide), glipizide and gliclazide) and the third-generation sulphonylureas (gliclazide modified release (MR), glipizide gastrointestinal therapeutic system (GITS) and glimepiride) are thought to have a better safety profile (Harrower 2000). In the late 1950 s the biguanide metformin was introduced as another glucoselowering drug (Bailey 1996).

Metformin is usually the first choice of a glucose-lowering drug if diet and exercise are insufficient in controlling T2DM. However, in case of metformin intolerance or contraindications, sulphonylureas might be prescribed as monotherapy. Sulphonylureas are mostly prescribed as a part of combination therapy with other glucose-lowering drugs, especially metformin (ADA 2016). All sulphonylureas are administered orally. The daily dose recommended in people with T2DM depends on the specific sulphonylurea compound. The sulphonylureas have different pharmacokinetic profiles due to different bindings to the sulphonylurea receptor in the pancreatic $\beta$-cells. Chlorpropamide has a half-life of 36 hours, whereas glimepiride has a half-life of around 5 hours (McCall 2001). Metformin has an estimated plasma half-life of 1.5 to 4.9 hours (Bailey 1996). Because of variations in the half-life of the different sulphonylureas, some have to be taken once daily and others two or three times daily. For glimepiride, the recommended dose is up to $6 \mathrm{mg}$ per day (Langtry 1998). For gliclazide the daily dose is $30 \mathrm{mg}$ to $120 \mathrm{mg}$ (Deacon 2015; Harrower 2000a). Metformin is likewise administered orally. Titration of metformin begins with a low dose $(500 \mathrm{mg}$ ) taken once or twice per day with meals. The maximum recommended dose is up to $1000 \mathrm{mg}$ twice daily (Nathan 2009).

\section{Adverse effects of the intervention}

All sulphonylureas have the potential to cause hypoglycaemia. The risk of hypoglycaemia differs between the different types of sulphonylureas and some agents like glibenclamide are prone to cause prolonged hypoglycaemia. The risk of hypoglycaemia is more pronounced for the first-generation sulphonylureas than the newer generations of sulphonylureas (Harrower 2000). In people with T2DM receiving metformin, gastrointestinal adverse effects, including abdominal discomfort and diarrhoea, are the most common adverse events, occurring in $20 \%$ to $30 \%$ of patients. Because metformin does not increase insulin secretion, hypoglycaemia is uncommon in people with T2DM taking metformin monotherapy (DeFronzo 1999). Previously, metformin was considered to be contraindicated in many chronic conditions, due to an increased risk of lactic acidosis. However, a Cochrane Review has concluded that there is no evidence that metformin is associated with an increased risk of lactic acidosis and the list of contraindications for metformin use should be reassessed (Salpeter 2010).

The University Group Diabetes Program (UGDP) trial suggested that tolbutamide was associated with adverse cardiovascular effects compared with placebo and insulin in people with T2DM (UGDP 1976). Later, other randomised clinical trials did not demonstrate clear evidence of an increased risk of cardiovascular events with sulphonylurea use compared with other glucose-lowering drugs in people with T2DM (ADOPT 2006; UKPDS-33 1998). Several observational studies have indicated increased mortality and risk of cardiovascular disease with sulphonylurea monotherapy 
compared with metformin monotherapy in people with T2DM (Morgan 2014; Roumie 2012; Schramm 2011). However, the risk seems to depend on the type of sulphonylurea (Pantalone 2012; Schramm 2011). Moreover, because of uncontrolled, or undetected, or both confounding factors in observational studies, the results of these studies have to be verified by randomised controlled trials (RCTs; Deeks 2003).

A UKPDS substudy showed that the early addition of metformin in sulphonylurea-treated participants was associated with an increased risk of mortality compared with continuation of sulphonylurea alone (UKPDS-34 1998). Several observational studies have investigated the association between the combination of metformin and sulphonylureas and the risk of cardiovascular disease and mortality. Overall, these studies show conflicting results (Evans 2006; Gulliford 2004; Johnson 2002; Kahler 2007).

\section{How the intervention might work}

The primary mechanism of action for the sulphonylureas is to stimulate insulin release from the pancreatic $\beta$-cells. Sulphonylureas increase pancreatic insulin release by closing of potassium-sensitive adenosine triphosphate (P-ATP) channels in the $\beta$-cells (Harrower 2000; Scott 2012). Metformin is thought to increase insulin sensitivity, which may lead to a variety of metabolic effects. Inhibition of hepatic glucose production (through increased hepatic sensitivity to insulin) is regarded as the principal mechanism through which metformin lowers blood glucose (Krentz 2005). The enzyme adenosine 5'-monophosphateactivated protein kinase (AMPK) has been identified as a target of the drug. Through phosphorylation of key proteins affecting energy production, AMPK regulates and co-ordinates cellular glucose and lipid metabolism (Krentz 2005).

\section{Why it is important to do this review}

Several studies have investigated the combination therapy of metformin and sulphonylureas and the risk of cardiovascular disease and mortality (Evans 2006; Gulliford 2004; Johnson 2002; Kahler 2007; UKPDS-34 1998). However, the data are primarily based on observational studies and show conflicting results. Therefore, it is still unclear whether metformin and sulphonylurea in combination increase the risk of cardiovascular disease and mortality. Guidelines suggest flexibility in choosing the next drug after metformin monotherapy failure (ADA 2016). It therefore remains to be clarified which drug class is the most suitable second line, since most people with T2DM will need a combination therapy over time in order to achieve glycaemic targets. This systematic review aims to evaluate whether sulphonylureas are the best choice of combination therapy with metformin.

\section{OB JECTIVES}

To assess the effects of metformin and sulphonylurea (secondor third-generation) combination therapy for adults with type 2 diabetes mellitus.

\section{METHODS}

\section{Criteria for considering studies for this review}

\section{Types of studies}

We included randomised controlled trials (RCTs).

\section{Types of participants}

Adults aged 18 years or older with type 2 diabetes mellitus (T2DM).

\section{Diagnostic criteria for diabetes mellitus}

In order to be consistent with changes in the classification of and diagnostic criteria for diabetes mellitus over the years, the diagnosis should be established using the standard criteria valid at the time the trial commenced (e.g. ADA 2003; ADA 2008; WHO 1998). Ideally, the diagnostic criteria should have been described. We used the trial authors' definition of diabetes mellitus if necessary. We planned to subject diagnostic criteria to a sensitivity analysis.

\section{Types of interventions}

We planned to investigate the following comparisons of intervention versus control/comparator.

\section{Intervention}

- Metformin plus second- or third-generation sulphonylurea (M + S) combination therapy

\section{Comparator}

- Metformin plus another glucose-lowering intervention as a combination therapy (e.g. metformin plus dipeptidylpeptidase-4 inhibitor, metformin plus insulin)

- Metformin plus placebo

- Metformin monotherapy

Concomitant interventions would have to be the same in both the intervention and comparator groups to establish fair comparisons.

If a trial included multiple arms, we included any arm that met the review's inclusion criteria.

\section{Minimum duration of intervention}

We included trials with a minimum duration of intervention of 52 weeks. Because we primarily intended to investigate patientimportant outcomes, we focused on longer-term trials, since macrovascular and microvascular complications develop over time.

\section{Minimum duration of follow-up}

We included trials with a duration of the intervention of 52 weeks or more. Extended follow-up periods (also called open-label extension studies) defined as a follow-up of participants once the original trial was terminated, as specified in the power calculation for this trial, are frequently of an observational nature, and we have only evaluated them for adverse events (Buch 2011; Megan 2012).

\section{Summary of specific exclusion criteria}

- We excluded combinations of more than two glucose-lowering agents.

- We excluded studies investigated women diagnosed with gestational diabetes.

\section{Types of outcome measures}

We did not exclude a trial only on the basis that one or several of our primary or secondary outcome measures were not reported in the publication. In case none of our primary or secondary outcomes 
were reported we did not include this trial but provided some basic information in an additional table.

\section{Primary outcomes}

- All-cause mortality

- Health-related quality of life

- Serious adverse events

\section{Secondary outcomes}

- Cardiovascular mortality

- Non-fatal myocardial infarction

- Heart failure

- Non-fatal stroke

- Amputation of lower extremity

- Blindness or severe vision loss

- End-stage renal disease

- Non-serious adverse events

- Hypoglycaemia

- Socio-economic effects

\section{Additional explorative outcomes}

- Weight

- HbA1c (glycosylated haemoglobin A1c)

\section{Method of outcome measurement}

- All-cause mortality: defined as death from any cause

- Health-related quality of life: defined as mental and physical health-related quality of life, separate and combined, evaluated by a validated instrument such as Short-Form 36

- Serious adverse events: defined according to the International Conference on Harmonization Guidelines as any event that leads to death, that is life-threatening, required in-patient hospitalisation or prolongation of existing hospitalisation, resulted in persistent or significant disability, and any important medical event that may have jeopardised the participant or required intervention to prevent it (ICH 1997), or as reported in trials.

- Cardiovascular mortality: defined as death from myocardial infarction, heart failure or stroke

- Non-fatal myocardial infarction, heart failure, non-fatal stroke, amputation of lower extremity, blindness or severe vision loss, hypoglycaemia (mild/moderate, serious): defined as reported in trials. Measured at the end of the intervention and at the end of follow-up.

- End-stage renal disease: defined as dialysis, renal transplantation or death due to renal disease

- Non-serious adverse events: defined as number of participants with any untoward medical occurrence not necessarily having a causal relationship with the intervention.

- Weight and HbAlc: measured in $\mathrm{kg}$ and $\%$

- Socio-economic effects: for example costs of the intervention, absence from work, medication consumption

\section{Timing of outcome measurement}

- For all-cause mortality, serious adverse events and non-serious adverse events: any time after participants were randomised to intervention/comparator groups
- For all other outcomes measures: at the end of the intervention and at the end of follow-up.

Specification of key prognostic variables

- Ethnicity

- Obesity

- Hypertension

- Previous gestational diabetes

- Age

- Existing cardiovascular disease

- Kidney disease

\section{Search methods for identification of studies}

\section{Electronic searches}

In 2016 the Agency for Healthcare Research and Quality (AHRQ) published an updated systematic review with meta-analyses on the effectiveness and safety of glucose-lowering interventions for people with T2DM, including metformin-based combination therapies (Maruthur 2016). This report included search results from several databases up to April 2015 and a further update of MEDLINE up to December 2015.

We based our search on the results of this systematic AHRQ report and added new references identified by a revised search strategy from 2015 onwards, in the following literature databases.

- Cochrane Central Register of Controlled Trials (CENTRAL; 2018, Issue 3) via the Cochrane Register of Studies Online (CRSO)

- MEDLINE Ovid (Epub Ahead of Print, In-Process \& Other NonIndexed Citations, Ovid MEDLINE(R) Daily and Ovid MEDLINE(R); from 1946 to 5 March 2018)

- Embase Ovid (from 1974 to 13 July 2016)

Additionally we searched the following trials registers:

- ClinicalTrials.gov (5 March 2018)

- World Health Organization International Clinical Trials Registry Platform (ICTRP) (5 March 2018)

We continuously applied a MEDLINE (Ovid SP) email alert service to identify newly published trials using the same search strategy as described for MEDLINE (for details on search strategies, see Appendix 1).

\section{Searching other resources}

We searched the reference lists of included trials, systematic reviews, meta-analyses and health technology assessment reports for other potentially eligible trials or ancillary publications. In addition, we contacted authors of included trials to identify any additional information about the retrieved trials and to determine whether further trials existed that we had missed.

We also searched manufacturers' websites and the databases of regulatory agencies (European Medicines Agency (EMA), US Food and Drugs Administration (FDA); Hart 2012; Schroll 2015).

We did not use abstracts or conference proceedings for data extraction because this information source does not fulfil the CONSORT requirements which is "an evidence-based, minimum set 
of recommendations for reporting randomized trials" (CONSORT; Scherer 2007).

\section{Data collection and analysis}

\section{Selection of studies}

Two review authors ( $\mathrm{KM}$ and $\mathrm{PK}$, LK or FG) independently scanned the abstract, title, or both, of every record we retrieved in the literature searches, to determine which trials we should assess further. We obtained the full text of all potentially-relevant records. We resolved any disagreements through consensus or by recourse to an additional review author $(\mathrm{BH})$. If we could not resolve a disagreement, we categorised the trial as a 'study awaiting classification' and contacted the trial authors for clarification. We prepared a flow diagram of the number of trials identified and excluded at each stage in accordance with the PRISMA flow diagram of trial selection (Liberati 2009).

\section{Data extraction and management}

For trials that fulfilled our inclusion criteria, two review authors (KM and PK, LK or FG) independently extracted key participant and intervention characteristics. We reported data on efficacy outcomes and adverse events using standard data extraction sheets from the Cochrane Metabolic and Endocrine Disorders (CMED) Group. We resolved any disagreements by discussion or, if required, by consultation with an additional review author $(\mathrm{BH})$ (for details see Characteristics of included studies; Table 1; Table 2; Appendix 2; Appendix 3; Appendix 4; Appendix 5; Appendix 6; Appendix 7; Appendix 8; Appendix 9; Appendix 10; Appendix 11; Appendix 12; Appendix 13; Appendix 14). We tried to retrieve the protocol for each included trial.

We provided information about potentially relevant ongoing trials in the Characteristics of ongoing studies table and in Appendix 7 'Matrix of trial outcome (publications and trial endpoints)'.

We emailed all authors of included trials to enquire whether they were willing to answer questions regarding their trials. We presented the results of this survey in Appendix 15 'Survey of trial investigators providing information on studies'. We sought relevant missing information on the trial from the primary author(s) of the article, if possible.

\section{Dealing with duplicate and companion publications}

In the event of duplicate publications, companion documents or multiple reports of a primary trial, we maximised the information yield by collating all available data and used the most complete dataset aggregated across all known publications. We listed duplicate publications, companion documents, multiple reports of a primary trial and trial documents of included trials (such as trial registry information) as secondary references under the study identifier (ID) of the included trial. Furthermore, we also listed duplicate publications, companion documents, multiple reports of a trial and trial documents of excluded trials (such as trial registry information) as secondary references under the study ID of the excluded trial.

\section{Data from clinical trial registers}

In case data of included trials were available as study results in clinical trials registers such as ClinicalTrials.gov, we made full use of this information and extracted the data. If there was also a full publication of the trial, we collated and critically appraised all available data. If a trial fulfilled the inclusion criteria and was marked as a completed study in the clinical trials register but no additional information was available, we added this trial to the table of 'Studies awaiting classification'.

\section{Assessment of risk of bias in included studies}

Two review authors (KM and PK, LK or FG) independently assessed the risk of bias of each included trial. We resolved disagreements by consensus, or by consultation with an additional review author $(\mathrm{BH})$. In cases of disagreement, we consulted the remainder of the review author team and made a judgement based on consensus. If adequate information was not available from the publications, trial protocols, or other sources, we contacted the trial authors for more detail to request missing data on 'Risk of bias' items.

We used the Cochrane 'Risk of bias' assessment tool (Higgins 2011a; Higgins 2017) assigning assessments of low, high, or unclear risk of bias (for details, see Appendix 2; Appendix 3). We evaluated individual bias items as described in the Cochrane Handbook for Systematic Reviews of Interventions, according to the criteria and associated categorisations contained therein (Higgins 2017).

\section{Summary assessment of risk of bias}

We presented a 'Risk of bias' graph and a 'Risk of bias' summary figure.

We distinguished between self-reported, investigator-assessed and adjudicated outcome measures.

We considered the following outcomes as self-reported.

- Health-related quality of life

- Non-serious adverse events

- Hypoglycaemia

- Weight

We considered the following outcomes as investigator-assessed.

- All-cause mortality

- Serious adverse events

- Cardiovascular mortality

- Non-fatal myocardial infarction

- Heart failure

- Non-fatal stroke

- Amputation of lower extremity

- Blindness or severe vision loss

- End-stage renal disease

- Hypoglycaemia

- Socio-economic effects

- Weight

- HbA1c

\section{Risk of bias for a trial across outcomes}

Some risk of bias domains, such as selection bias (sequence generation and allocation sequence concealment), affected the risk of bias across all outcome measures in a trial. In case of high risk of selection bias, we marked all outcomes investigated in the associated trial as high risk. Otherwise, we did not perform a 
summary assessment of the risk of bias across all outcomes for a trial.

\section{Risk of bias for an outcome within a trial and across domains}

We assessed the risk of bias for an outcome measure by including all entries relevant to that outcome (i.e. both trial-level entries and outcome-specific entries). We considered low risk of bias to denote a low risk of bias for all key domains, unclear risk to denote an unclear risk of bias for one or more key domains and high risk to denote a high risk of bias for one or more key domains.

\section{Risk of bias for an outcome across trials and across domains}

These were our main summary assessments that we incorporated into our judgements about the certainty of the evidence in Summary of findings for the main comparison. We defined outcomes as at low risk of bias when most information came from trials at low risk of bias, unclear risk when most information came from trials at low or unclear risk of bias, and high risk when a sufficient proportion of information came from trials at high risk of bias.

\section{Measures of treatment effect}

When at least two trials were available for a comparison of a given outcome, we expressed dichotomous data as risk ratio (RR) or an odds ratio (OR) with $95 \%$ confidence interval $(\mathrm{Cl})$. For continuous outcomes measured on the same scale (e.g. weight loss in $\mathrm{kg}$ ) we estimated the intervention effect using the mean difference (MD) with $95 \% \mathrm{Cl}$. For continuous outcomes measuring the same underlying concept (e.g. health-related quality of life) but applying different measurement scales, we calculated the standardised mean difference (SMD). We planned to calculate time-to-event data as hazard ratio $(\mathrm{HR})$ with $95 \% \mathrm{Cl}$ with the generic inverse variance method.

The scales measuring health-related quality of life could go in different directions. Some scales increase in values with improved health-related quality of life, whereas other scales decrease in values with improved health-related quality of life. To adjust for the different directions of the scales, we planned to multiply the scales that reported better health-related quality of life with decreasing values by -1 .

\section{Unit of analysis issues}

We took into account the level at which randomisation occurred, for example in cross-over trials, cluster-randomised trials and multiple observations for the same outcome. If more than one comparison from the same trial was eligible for inclusion in the same meta-analysis, we either combined groups to create a single pair-wise comparison or we appropriately reduced the sample size so that the same participants did not contribute multiply (splitting the 'shared' group into two or more groups). Although the latter approach offers some solution for adjusting the precision of the comparison, it does not account for correlation arising from inclusion of the same set of participants in multiple comparisons (Higgins 2011b).

We planned to re-analyse cluster-RCTs that did not appropriately adjust for potential clustering of participants within clusters in their analyses. Variance of the intervention effects was planned to be inflated by a design effect (DEFF). Calculation of a DEFF involves estimation of an intra-cluster correlation (ICC). We planned to obtain estimates of ICCs by contacting trial authors, or by imputing
ICC values using either estimates from other included trials that reported ICCs or external estimates from empirical research (e.g. Bell 2013). We planned to examine the impact of clustering by performing sensitivity analyses.

\section{Dealing with missing data}

We tried to obtain missing data from trial authors and carefully evaluate important numerical data such as screened, randomlyassigned participants as well as intention-to-treat (ITT), and astreated and per-protocol populations. We investigated attrition rates (e.g. dropouts, losses to follow-up, withdrawals), and we critically appraised issues concerning missing data and imputation methods (e.g. last observation carried forward (LOCF)).

In trials where the standard deviation of the outcome was not available at follow-up or could not be recreated, we standardised by the average of the pooled baseline standard deviation (SD) from those trials in which this information was reported.

When included trials did not report means and SDs for outcomes and we did not receive the necessary information from trial authors, we imputed these values by estimating the mean and variance from the median, range, and the size of the sample (Hozo 2005).

We planned to investigate the impact of imputation on metaanalyses by performing sensitivity analyses.

\section{Assessment of heterogeneity}

In the event of substantial clinical or methodological heterogeneity, we planned not to report trial results as the pooled effect estimate in a meta-analysis.

We identified heterogeneity (inconsistency) by visually inspecting the forest plots and by using a standard $\mathrm{Chi}^{2}$ test with a significance level of $\alpha=0.1$ (Deeks 2017). In view of the low power of this test, we also considered the $\mathrm{I}^{2}$ statistic, which quantifies inconsistency across trials to assess the impact of heterogeneity on the metaanalysis (Higgins 2002; Higgins 2003); an $1^{2}$ statistic of $75 \%$ or more indicates a considerable level of heterogeneity (Higgins 2011b).

When heterogeneity was present, we attempted to determine possible reasons for it by examining individual trial and subgroup characteristics.

\section{Assessment of reporting biases}

If we included 10 or more trials investigating a particular outcome, we planned to use funnel plots to assess small-trial effects. Several explanations may account for funnel plot asymmetry, including true heterogeneity of effect with respect to trial size, poor methodological design (and hence bias of small trials) and publication bias (Sterne 2017). Therefore, we planned to interpret the results carefully (Sterne 2011).

\section{Data synthesis}

We undertook meta-analysis only if we judged participants, interventions, comparisons and outcomes to be sufficiently similar to ensure an answer that was clinically meaningful. Unless good evidence showed homogeneous effects across trials, we primarily summarised data at low risk of bias using a random-effects model (Wood 2008). We interpreted random-effects meta-analyses with consideration to the whole distribution of effects, ideally by 
presenting a prediction interval (Higgins 2009). A prediction interval specifies a predicted range for the true treatment effect in an individual trial (Riley 2011). In addition, we performed statistical analyses according to the statistical guidelines presented in the Cochrane Handbook for Systematic Reviews of Interventions (Deeks 2017).

\section{Trial sequential analyses}

In a single trial, sparse data and interim analyses increase the risk of type I and type II errors. To avoid type I errors, group sequential monitoring boundaries are applied to decide whether a trial could be terminated early because of a sufficiently small $P$ value, that is the cumulative Z-curve crosses the monitoring boundaries (Lan 1983). Likewise, before reaching the planned sample size of a trial, the trial may be stopped due to futility if the cumulative Zscore crosses the futility monitoring boundaries (Higgins 2011b). Sequential monitoring boundaries for benefit, harm, or futility can be applied to meta-analyses as well, called trial sequential monitoring boundaries (Higgins 2011; Wetterslev 2008). In a trial sequential analysis (TSA), the addition of each trial in a cumulative meta-analysis is regarded as an interim meta-analysis and helps to clarify if significance is reached or futility is reached or whether additional trials are needed (Wetterslev 2008).

TSA combines a calculation of the diversity-adjusted required information size (cumulated meta-analysis sample size to detect or reject a specific relative intervention effect) for meta-analysis with the threshold of data associated with statistics (Pogue 1997; Wetterslev 2008).

The idea in TSA is that if the cumulative Z-curve crosses the boundary for benefit or harm before a diversity-adjusted required information size is reached, a sufficient level of evidence for the anticipated intervention effect has been reached with the assumed type I error and no further trials may be needed. If the cumulative Z-curve crosses the boundary for futility before a diversity-adjusted required information size is reached, the assumed intervention effect can be rejected with the assumed type II error and no further trials may be needed. If the Z-curve does not cross any boundary, then there is insufficient evidence to reach a conclusion. To construct the trial sequential monitoring boundaries, the required information size is needed and is calculated as the least number of participants needed in a well-powered single trial and subsequently adjusted for diversity among the included trials in the meta-analysis (Wetterslev 2008). We applied TSA as it decreases the risk of type I and II errors due to sparse data and multiple updating in a cumulative meta-analysis, and it provides us with important information in order to estimate the risks of imprecision when the required information size is not reached. Additionally, TSA provides important information regarding the need for additional trials and the required information size of such trials (Wetterslev 2008).

We applied trial sequential monitoring boundaries according to an estimated clinical important effect. We based the required information size on an a priori effect corresponding to a $10 \%$ relative risk reduction ( $R R R$ ).

We performed TSA for continuous outcomes with mean differences, by using the trials applying the same scale to calculate the required sample size. For the continuous outcomes we tested the evidence for the achieved differences in the cumulative meta-analyses.
For the heterogeneity adjustment of the required information size we used the diversity $\left(D^{2}\right)$ estimated in the meta-analyses of included trials.

We performed TSA on primary and secondary outcomes.

\section{Subgroup analysis and investigation of heterogeneity}

We expected the following characteristics to introduce clinical heterogeneity and carried out the following subgroup analyses (on our primary and secondary outcomes) including investigation of interactions (Altman 2003).

- Trials with a long duration ( $\geq 2$ years) versus trials with a short duration ( $<2$ years).

- Trials including obese participants versus trials including nonobese participants.

\section{Sensitivity analysis}

We performed sensitivity analyses on our primary and secondary outcomes to explore the influence of the following factors (when applicable) on effect sizes, by restricting the analysis to the following.

- Published trials

- Effect of risk of bias, as specified in the Assessment of risk of bias in included studies section

- Very long or large trials, to establish the extent to which they dominate the results

- Use of the following filters: diagnostic criteria, imputation, language of publication, source of funding (industry versus other), or country

We also tested the robustness of results by repeating analyses using different measures of effect size (i.e. RR, OR, etc.) and different statistical models (fixed-effect and random-effects models).

\section{Certainty of the evidence}

We presented the overall certainty of the evidence for each outcome specified below, according to the GRADE approach, which takes into account issues relating not only to internal validity (risk of bias, inconsistency, imprecision, publication bias) but also to external validity, such as directness of results. Two review authors (KM and PK, LK or FG) independently rated the certainty of the evidence for each outcome.

We included an appendix titled 'Checklist to aid consistency and reproducibility of GRADE assessments' to help with the standardisation of the 'Summary of findings' tables (Meader 2014). Alternatively, we would have used the GRADEpro Guideline Development Tool (GDT) software and presented evidence profile tables as an appendix (GRADEproGDT 2015). We presented results for outcomes as described in the Types of outcome measures section. If meta-analysis was not possible, we presented the results in a narrative format in the 'Summary of findings' table. We justified all decisions to downgrade the certainty of the evidence using footnotes, and we made comments to aid the reader's understanding of the Cochrane Review when necessary.

\section{'Summary of findings' table}

We presented a summary of the evidence in Summary of findings for the main comparison. This provides key information about 
the best estimate of the magnitude of effect, in relative terms and as absolute differences for each relevant comparison of alternative management strategies, numbers of participants and trials addressing each important outcome, and rates the overall confidence in effect estimates for each outcome. We created the 'Summary of findings' table using the methods described in the Cochrane Handbook for Systematic Reviews of Interventions (Schünemann 2017), along with Review Manager 5 (RevMan 5) table editor (Review Manager 2014). We reported the following outcomes, listed according to priority.

- All-cause mortality

- Cardiovascular mortality

- Serious adverse events

- Non-fatal stroke

- Non-fatal myocardial infarction

- Microvascular complications (end-stage renal disease, blindness or severe vision loss, amputation of lower extremity)

- Health-related quality of life

Figure 1. Trial flow diagram

\section{RES U L T S}

\section{Description of studies}

For a detailed description of trials, see Table 1, Table 2, 'Characteristics of included studies', 'Characteristics of excluded studies, and 'Characteristics of ongoing studies'.

\section{Results of the search}

Our databases search identified 2754 records to be screened. We excluded most of the records on the basis of their titles and abstracts because they clearly did not meet the inclusion criteria. We assessed a total of 296 full-text articles/records for eligibility. After screening, 32 trials with 41 trial arms, published in 164 publications/records finally met our inclusion criteria.

Handsearching of systematic reviews and reference lists identified two trials described in seven publications (Gerich 2005; Ristic 2007). Handsearching of manufacturers' websites identified eight records.

We excluded a total of 118 full-text articles/records, 80 of these publications/records described 62 trials and 38 of these publications/records did not describe trials. We identified nine ongoing trials described in 11 publications/records (see 'Ongoing studies'). We identified two trials awaiting assessment (see 'Characteristics of studies awaiting classification'). For an overview of trial selection, please see Figure 1. 
AHRQ: Agency for Healthcare Research and Quality; DPP4-I: dipeptidyl-peptidase 4 inhibitor; GLP1-A: glucagon-like peptide 1 analogue; M+S: metformin + sulphonylurea; SGLT2-I: sodium-glucose co-transporter 2 inhibitor

2358 records identified through database
searching
Cochrane Library: 473
MEDLINE: 765
Embase: 461
WHO International Clinical Trials Registry
Platform: 307
ClinicalTrials.gov: 352

969 additional records identified through non-database sources (contacts with experts, manufacturers, handsearching of literature)

AHRQ-report: 268

Handsearching of systematic reviews and reference lists: 2 trials (7 records)

Handsearching of manufacturers' websites: 8

Other: 686

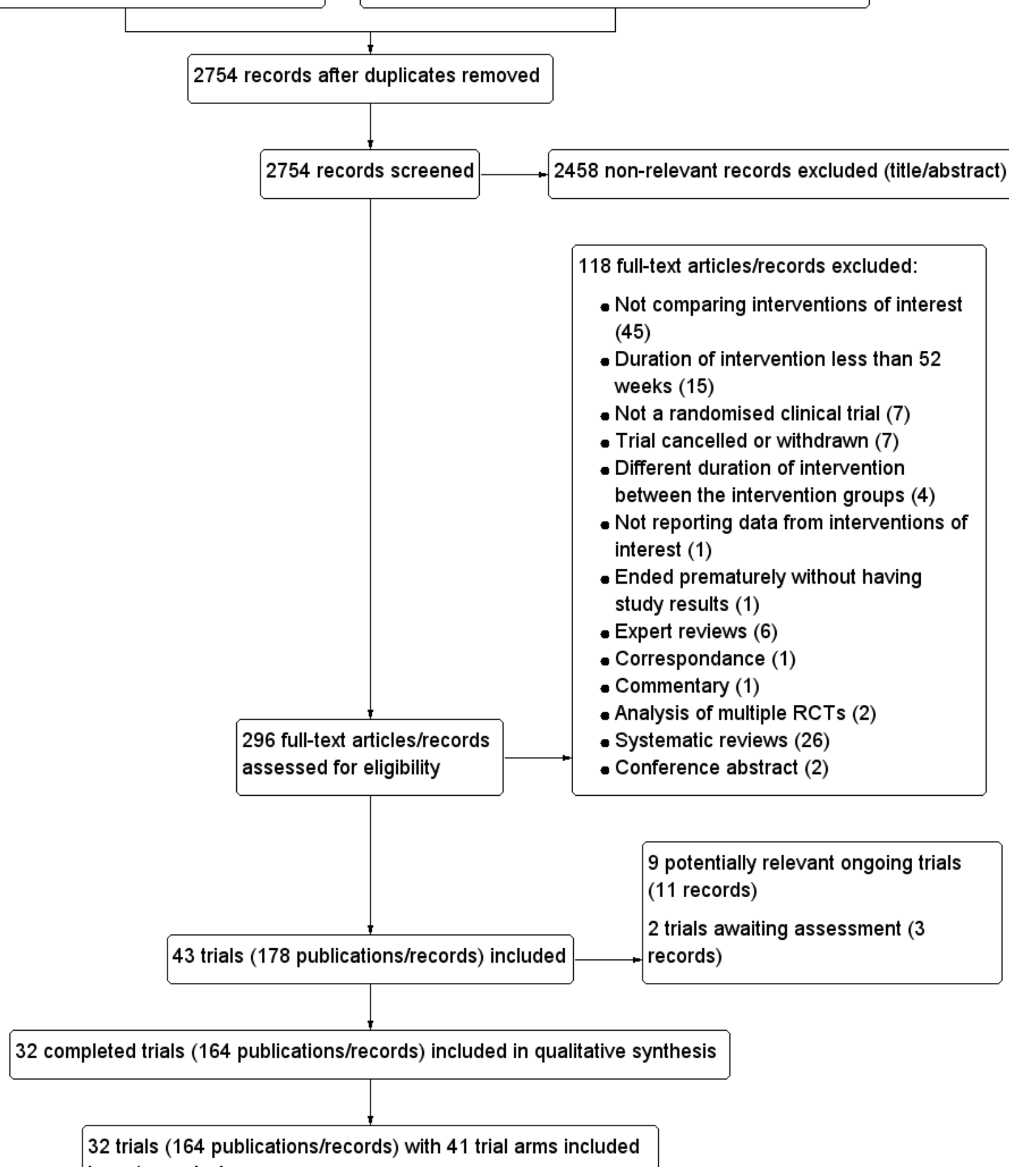


Figure 1. (Continued)

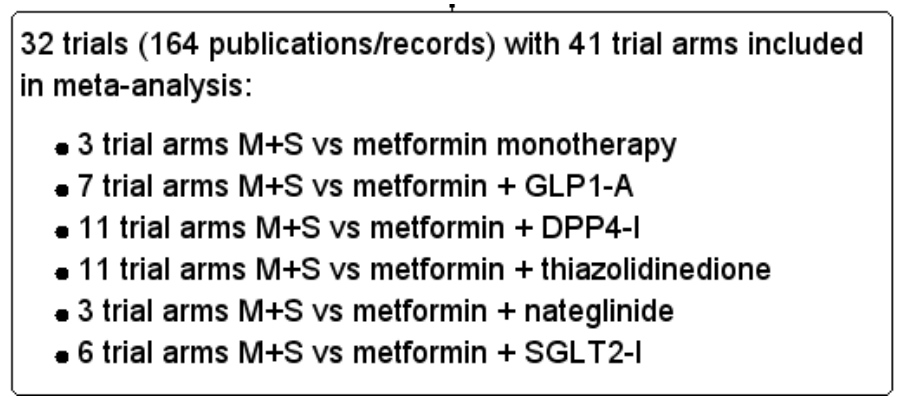

\section{Included studies}

A detailed description of the characteristics of included trials is presented elsewhere (see Characteristics of included studies and Appendix 4; Appendix 5; Appendix 6; Appendix 7; Appendix 8; Appendix 9; Appendix 10; Appendix 11; Appendix 12; Appendix 13; Appendix 14). The following is a succinct overview.

\section{Source of data}

Twenty-six trials reported data with relevance for this review, published in medical journals (Charbonnel 2005; Del Prato 2014; Del Prato 2015; Derosa 2005; Derosa 2009b; Derosa 2010; Derosa 2011a; Derosa 2011b; Filozof 2010; Gallwitz 2012a; Gallwitz 2012b; Gerich 2005; Göke 2013; Hamann 2008; Handelsman 2017; Hollander 2017; Leiter 2015; Maffioli 2013; Matthews 2010; Nauck 2013; Petrica 2009; Petrica 2011; Ridderstråle 2014; Schernthaner 2015; Seck 2010; Vaccaro 2017).

Fourteen trials reported data in trials registers (Ahrén 2014; Del Prato 2014; Del Prato 2015; Gallwitz 2012a; Gallwitz 2012b; Göke 2013; Handelsman 2017; Home 2009; Leiter 2015; Nauck 2013; NCT00367055; Ridderstråle 2014; Schernthaner 2015; Seck 2010).

Six trials reported data on the manufacturer's website (Ahrén 2014; Gerich 2005; Hamann 2008; Matthews 2010; NCT00367055; Ristic 2007).

We contacted all trial authors or investigators by email (see Appendix 15). When important information was lacking on ongoing studies and excluded trials, we contacted investigators for clarification (see Appendix 15). We only received additional data on four included trials through correspondence with authors (Dei Cas 2017; Derosa 2005; Home 2009; Vaccaro 2017).

\section{Comparisons}

One trial compared $\mathrm{M}+\mathrm{S}$ with metformin monotherapy (Derosa 2009a). Two trials compared M+S with metformin plus placebo (Ahrén 2014; Nauck 2013). Five trials compared M+S with metformin plus a GLP-1 analogue (Ahrén 2014; Derosa 2010; Derosa 2011a; Gallwitz 2012a; Nauck 2013). Nine trials compared M+S with metformin plus a DPP-4 inhibitor (Ahrén 2014; Dei Cas 2017; Del Prato 2014; Filozof 2010; Gallwitz 2012b; Göke 2013; Matthews 2010; Schernthaner 2015; Seck 2010). One trial compared $\mathrm{M}+\mathrm{S}$ with metformin plus long-acting DPP-4 inhibitor (Handelsman 2017). Eleven trials compared M+S with metformin plus a thiazolidinedione (Charbonnel 2005; Derosa 2005; Derosa 2009a; Derosa 2011b; Hamann 2008; Home 2009; Maffioli 2013; NCT00367055; Petrica 2009; Petrica 2011; Vaccaro 2017). Three trials compared $\mathrm{M}+\mathrm{S}$ with metformin plus a glinide (Derosa 2009b; Gerich 2005; Ristic 2007). Four trials compared M+S with metformin plus a SGLT-2 inhibitor (Del Prato 2015; Hollander 2017; Leiter 2015; Ridderstråle 2014).

Several trials implemented more than two comparison groups within their trial designs. For an overview of trials, trial arms, comparators, interventions and number of randomised participants see Table 2 .

\section{Overview of trial populations}

All, but seven trials provided information on sample size calculations (Derosa 2009b; Derosa 2010; Gerich 2005; NCT00367055; Petrica 2009; Petrica 2011; Seck 2010). All, but 10 trials reported the total number of participants screened (Derosa 2005; Derosa 2009a; Derosa 2009b; Derosa 2010; Derosa 2011a; Derosa 2011b; Filozof 2010; Maffioli 2013; NCT00367055; Ristic 2007). A total of 12,863 participants were randomised to $M+S$ and 15,833 participants were randomised to a comparator. The percentage of participants finishing the trial was approximately $69 \%$ in the $M+S$ group and $69 \%$ in the comparator groups. Number of randomised participants ranged from 22 to 1556 in the M+S groups and from 22 to 1765 in the comparator groups (Table 1).

\section{Trial design}

All of the 32 trials were randomised controlled trials with a parallel design. In 15 trials the primary trial structure had a noninferiority design (Ahrén 2014; Del Prato 2014; Del Prato 2015; Filozof 2010; Gallwitz 2012a; Gallwitz 2012b; Göke 2013; Hamann 2008; Handelsman 2017; Hollander 2017; Home 2009; Leiter 2015; Matthews 2010; Ridderstråle 2014; Seck 2010).

Two trials had both a placebo group and an active comparator group (Ahrén 2014; Nauck 2013), the rest of the included trials had an active comparator group. Two trials were single-centre trials (Dei Cas 2017; Maffioli 2013). Two trials did not report number of centres (Petrica 2009; Petrica 2011). The remaining trials were multicentre trials, defined as two or more centres. Seven trials were openlabelled for participants and personnel (Dei Cas 2017; Gallwitz 2012a; Home 2009; NCT00367055; Petrica 2009; Petrica 2011; Vaccaro 2017). Two trials were single-blind for investigators but not for the participants (Derosa 2010; Derosa 2011a). The remaining trials were double-blinded for investigators and participants. Three trials did not report blinding of outcome assessors for any outcome (Dei Cas 2017; Gallwitz 2012a; NCT00367055). The remaining trials reported blinding of outcome assessors for one or more outcomes. Trials were performed between the years 
2001 to 2017. The duration of the intervention ranged from 52 weeks to 208 weeks. Twenty trials had a run-in period (Ahrén 2014; Del Prato 2014; Del Prato 2015; Derosa 2005; Derosa 2009b; Derosa 2010; Filozof 2010; Gallwitz 2012a; Gerich 2005; Göke 2013; Hamann 2008; Handelsman 2017; Hollander 2017; Home 2009; Leiter 2015; Maffioli 2013; Nauck 2013; Ridderstråle 2014; Schernthaner 2015; Seck 2010). The remaining trials did not report any run-in period. Two trials were terminated early (Matthews 2010; Vaccaro 2017). Matthews 2010 had a higher than expected discontinuation rate and fewer participants than expected reached the primary endpoint. Vaccaro 2017 had a lower than expected rate of primary endpoint events during follow-up.

Eleven trials had an extended follow-up period (Ahrén 2014; Charbonnel 2005; Del Prato 2015; Göke 2013; Hollander 2017; Home 2009; Leiter 2015; Nauck 2013; Ridderstråle 2014; Ristic 2007; Seck 2010). With the exception of Ristic 2007 all had high attrition rates between the original time of follow-up and the extended follow-up. Four trials reported that they were able to keep the double-blinding conditions (Del Prato 2015; Göke 2013; Leiter 2015; Ristic 2007).

\section{Settings}

All trials were conducted in outpatient clinics.

\section{Participants}

All the trials included people with T2DM and with $\mathrm{HbA1c}$ ranging from 7\% to 9\% (Dei Cas 2017; Hollander 2017; Home 2009; Schernthaner 2015; Vaccaro 2017); 7\% to 9.5\% (Leiter 2015); $6.5 \%$ to 10\% (Del Prato 2015; Gallwitz 2012b; Göke 2013; Seck 2010); $7 \%$ to 10\% (Ahrén 2014; Del Prato 2014; Hamann 2008; Ridderstråle 2014); $7 \%$ to $11 \%$ (Gerich 2005; Nauck 2013); $6.8 \%$ to $9 \%$ (Ristic 2007); $7.5 \%$ to $11 \%$ (Charbonnel 2005; Filozof 2010); $6.5 \%$ to $8.5 \%$ (Matthews 2010; NCT00367055); 6.5\% to 9\% (Gallwitz 2012a; Handelsman 2017); more than 6.5\% (Derosa 2009a); more than 7\% (Derosa 2005; Derosa 2009b; Derosa 2011b; Petrica 2009; Petrica 2011) and more than 8\% (Derosa 2010; Derosa 2011a; Maffioli 2013).

One trial did not report the duration of T2DM at baseline (Derosa 2009a). For the rest of the trials the mean duration of T2DM ranged from newly diagnosed to more than 10 years.

All trials included both genders. The percentage of women ranged from $29 \%$ to $65 \%$.

The mean age of the participants ranged from 52 years to 73 years. One trial only included participants aged 65 years and more (Schernthaner 2015).

Mean $\mathrm{HbAlc}$ at baseline ranged from $7.3 \%$ to $9.3 \%$.

Ten trials did not report any comorbidities, cointerventions or comedications (Ahrén 2014; Del Prato 2014; Filozof 2010; Gerich 2005; Handelsman 2017; Hollander 2017; Home 2009; Nauck 2013; NCT00367055; Ristic 2007).

Major exclusion criteria were type 1 diabetes mellitus or secondary forms of diabetes, diabetic complications (nephropathy, retinopathy, neuropathy), history of ketoacidosis, organ failure (renal, hepatic, heart), history of pancreatitis, new cardiovascular event or pregnancy.

\section{Diagnosis}

Five trials applied the diagnostic criteria of T2DM as defined by the European Association for the Study of Diabetes (EASD) 2007 guideline (Derosa 2009a; Derosa 2010; Derosa 2011a; Derosa 2011b; Maffioli 2013), four trials used the definition of the World Health Organization (WHO) 1999/2006 criteria (Gallwitz 2012a; Hamann 2008; Home 2009; NCT00367055), two trials used the American Diabetes Association (ADA) 2001 criteria (Derosa 2005; Derosa 2009b), one trial applied the ADA 1997 criteria (Dei Cas 2017), and one trial applied the ADA 2017 criteria (Hollander 2017). The remaining trials did not report diagnostic criteria for T2DM.

\section{Interventions}

Metformin was administered in all intervention and comparator arms and was mostly given in doses of $500 \mathrm{mg} /$ day to $3000 \mathrm{mg} /$ day (Appendix 4).

Second-generation sulphonylurea was administered either as glibenclamide, in doses of $5 \mathrm{mg}$ /day to $15 \mathrm{mg} /$ day (Dei Cas 2017; Derosa 2009b; Derosa 2010; Derosa 2011b; Gerich 2005; Maffioli 2013); gliclazide, in doses of 30 to $320 \mathrm{mg}$ /day (Charbonnel 2005; Filozof 2010; NCT00367055; Ristic 2007); or glipizide, in doses of 5 to 20 mg/day (Del Prato 2014; Del Prato 2015; Göke 2013; Seck 2010). Third-generation sulphonylurea was administered as glimepiride, in doses of $1 \mathrm{mg} /$ day to $8 \mathrm{mg} /$ day (Ahrén 2014; Derosa 2005; Derosa 2009a; Derosa 2011a; Gallwitz 2012a; Gallwitz 2012b; Handelsman 2017; Hollander 2017; Home 2009; Leiter 2015; Matthews 2010; Nauck 2013; Petrica 2009; Petrica 2011; Ridderstråle 2014; Schernthaner 2015). Two trials administered more than one kind of sulphonylurea, that is, glibenclamide or gliclazide (Hamann 2008), or glibenclamide, gliclazide or glimepiride (Vaccaro 2017).

GLP-1 analogues were administered as albiglutide, in doses of 30 $\mathrm{mg}$ to $50 \mathrm{mg}$ once weekly (Ahrén 2014); exenatide, in doses of 10 $\mu \mathrm{g} /$ day to $20 \mu \mathrm{g} /$ day (Derosa 2010; Derosa 2011a; Gallwitz 2012a); or liraglutide, in doses of $0.6 \mathrm{mg} /$ day to $1.8 \mathrm{mg} /$ day (Nauck 2013).

DPP-4 inhibitors were administered as either sitagliptin, in doses of 100 mg/day (Ahrén 2014; Seck 2010); vildagliptin (in doses of 100 mg/day (Dei Cas 2017; Filozof 2010; Matthews 2010); alogliptin, in doses of $12.5 \mathrm{mg} /$ day to $25 \mathrm{mg} /$ day (Del Prato 2014); linagliptin (in doses of $5 \mathrm{mg} /$ day (Gallwitz 2012b); or saxagliptin, in doses of $5 \mathrm{mg} /$ day (Göke 2013; Schernthaner 2015).

Thiazolidinediones were administered as either rosiglitazone, in doses of $4 \mathrm{mg} /$ day to $8 \mathrm{mg} /$ day (Derosa 2005; Hamann 2008; Home 2009; NCT00367055; Petrica 2009); or pioglitazone, in doses of 15 $\mathrm{mg} /$ day to $45 \mathrm{mg}$ /day (Derosa 2011b; Maffioli 2013; Vaccaro 2017).

Glinides were administered as nateglinide, in doses of $180 \mathrm{mg} /$ day to $540 \mathrm{mg}$ /day (Derosa 2009b; Gerich 2005; Ristic 2007).

SGLT-2 inhibitors were administered as either dapagliflozin, in doses of $2.5 \mathrm{mg} /$ day to $10 \mathrm{mg} /$ day (Del Prato 2015); canagliflozin, in doses of $100 \mathrm{mg} /$ day to $300 \mathrm{mg} /$ day (Leiter 2015); empagliflozin, in doses of $25 \mathrm{mg} /$ day (Ridderstråle 2014); or ertugliflozin, in doses of $5 \mathrm{mg} /$ day to $15 \mathrm{mg} /$ day (Hollander 2017).

Long-acting DPP-4 inhibitors were administered as omarigliptin, in doses of $25 \mathrm{mg} /$ week (Handelsman 2017). 
Two trials compared M+S with metformin plus placebo (Ahrén 2014; Nauck 2013).

Twelve trials reported counselling for diet and exercise (Del Prato 2015; Derosa 2005; Derosa 2009a; Derosa 2009b; Derosa 2010; Derosa 2011a; Derosa 2011b; Göke 2013; Maffioli 2013; Ridderstråle 2014; Schernthaner 2015; Seck 2010). One trial counselled on diet only (Charbonnel 2005). The remaining trials did not provide information about counselling.

\section{Outcomes}

Six trials did not define a primary outcome (Derosa 2009a; Derosa 2009b; Derosa 2010; Maffioli 2013; Petrica 2009; Petrica 2011). None of these trials was registered in ClinicalTrials.gov or had a design paper published.

Twenty trials were registered at ClinicalTrials.gov (Ahrén 2014; Dei Cas 2017; Del Prato 2014; Del Prato 2015; Filozof 2010; Gallwitz 2012a; Gallwitz 2012b; Göke 2013; Hamann 2008; Handelsman 2017; Hollander 2017; Home 2009; Leiter 2015; Matthews 2010; Nauck 2013; NCT00367055; Ridderstråle 2014; Schernthaner 2015; Seck 2010; Vaccaro 2017).

Most trials used $\mathrm{HbA1C}$ as the primary outcome measure.

We included 32 trials. All the included trials but eight reported one or more of the primary outcomes of relevance for this review (Derosa 2009a; Derosa 2009b; Derosa 2010; Derosa 2011a; Derosa 2011b; Maffioli 2013; Petrica 2009; Petrica 2011). All the included trials but nine reported on all-cause mortality (Dei Cas 2017; Derosa 2009a; Derosa 2009b; Derosa 2010; Derosa 2011a; Derosa 2011b; Maffioli 2013; Petrica 2009; Petrica 2011). All the included trials but nine reported on serious adverse events and non-serious adverse events (Derosa 2009a; Derosa 2009b; Derosa 2010; Derosa 2011a; Derosa 2011b; Maffioli 2013; Petrica 2009; Petrica 2011; Ristic 2007), see Appendix 11. Three trials only reported adverse events leading to discontinuation (Derosa 2009a; Derosa 2010; Maffioli 2013). In three trials, the authors provided safety-data without the number of participants included in the safety-analysis (Derosa 2009b; Derosa 2011a; Derosa 2011b). One trial reported on adverse events for up to six months and 6 to 12 months separately (Ristic 2007).

One trial reported on health-related quality of life (Nauck 2013). This trial measured the outcome 'impact of weight on quality of life' (IWQOL). Authors did not report results per intervention groups.
Four trials reported on 'amputation of lower extremity' (Dei Cas 2017; Derosa 2005; Hollander 2017; Vaccaro 2017). Three trials reported on 'end-stage renal disease' (Nauck 2013; Dei Cas 2017; Derosa 2005). Two trials reported on 'blindness or severe vision loss' (Dei Cas 2017; Derosa 2005).

Three trials reported cost effectiveness data (Del Prato 2014; Del Prato 2015; Göke 2013).

For definitions of outcomes see Appendix 9 and Appendix 10.

\section{Excluded studies}

We excluded 118 publications/records after full-text evaluation (see Characteristics of excluded studies) mainly for the following reasons. The trial did not compare interventions of interest ( $\mathrm{N}$ $=45$ ). Two of these trials investigated an investigational drug (EUCTR2004-002549-11-FI (tesaglitazar); NCT01481116 (fasiglifam)) and one trial investigated a drug no longer approved for use (Rubin 2008 (muraglitazar)). Other reasons for exclusion included: duration of intervention less than 52 weeks $(\mathrm{N}=15)$, not an RCT ( $N$ $=7$ ), trial cancelled or withdrawn $(\mathrm{N}=7)$ and different duration of the intervention between the intervention groups $(\mathrm{N}=4)$. One trial compared interventions of interest (metformin compared with $M$ $+S$ ), but the trial only reported on metformin compared with usual care (Cryer 2005). We contacted the trial authors to request data, but did not receive a reply. One trial ended prematurely and had no study results (EUCTR2009-014727-23-IT). Six publications were expert reviews (Albarran 2013; Fleming 2015; Nishio 2015; Odawara 2015; Scheen 2016; Seufert 2014), one was a correspondence (Kannan 2015), one was a commentary on a systematic review (Bellary 2011) and two were analysing pooled data from multiple RCTs (Reid 2016; Rosenstock 2015). We identified 26 systematic reviews (Amate 2015; Aylsworth 2014; Belsey 2008; Chan 2015; Dai 2014; Foroutan 2016; Geng 2015; Goring 2014; Gu 2015; Guthrie 2015; Hershon 2016; Hou 2015; Kuecker 2016; Lim 2015; Liu 2014; Maruthur 2016; Mearns 2015; Mishriky 2015; Monami 2008; Phung 2010; Phung 2014; Rosenstock 2013; Varvaki 2016; Whalen 2015; Zhou 2015; Zintzaras 2014), and one conference abstract (Alvares 2015). This publication is listed in Additional references.

\section{Risk of bias in included studies}

For details on the risk of bias of the included trials see Characteristics of included studies. For an overview of review authors' judgements about each risk of bias item for individual trials and across all trials see Figure 2 and Figure 3. 
Figure 2. 'Risk of bias' graph: review authors' judgements about each 'Risk of bias' item presented as percentages across all included trials (blank cells indicate that the particular outcome was not measured in some trials).

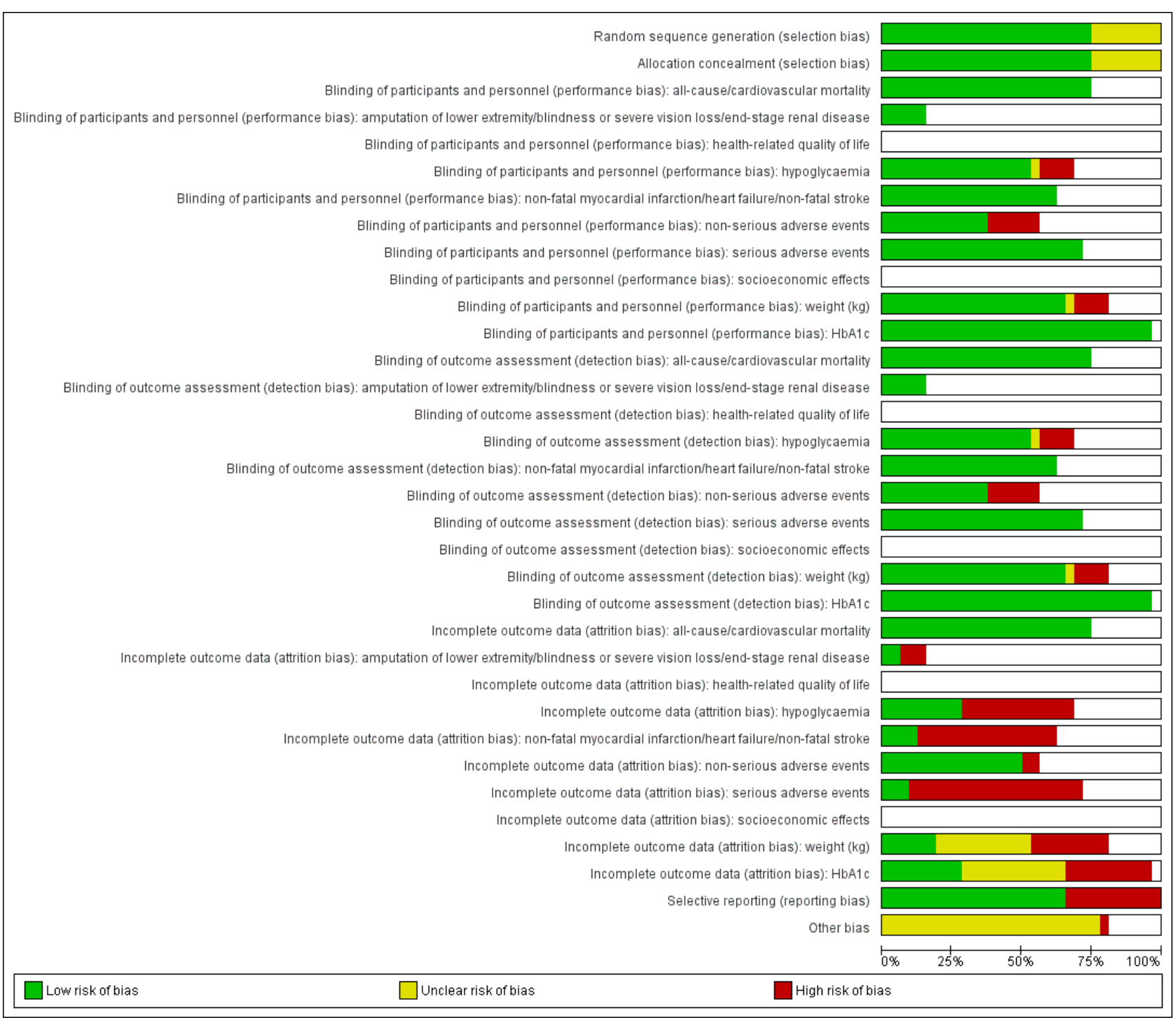


Figure 3. 'Risk of bias' summary: review authors' judgements about each 'Risk of bias' item for each included trial ((blank cells indicate that the particular outcome was not measured in some trials)

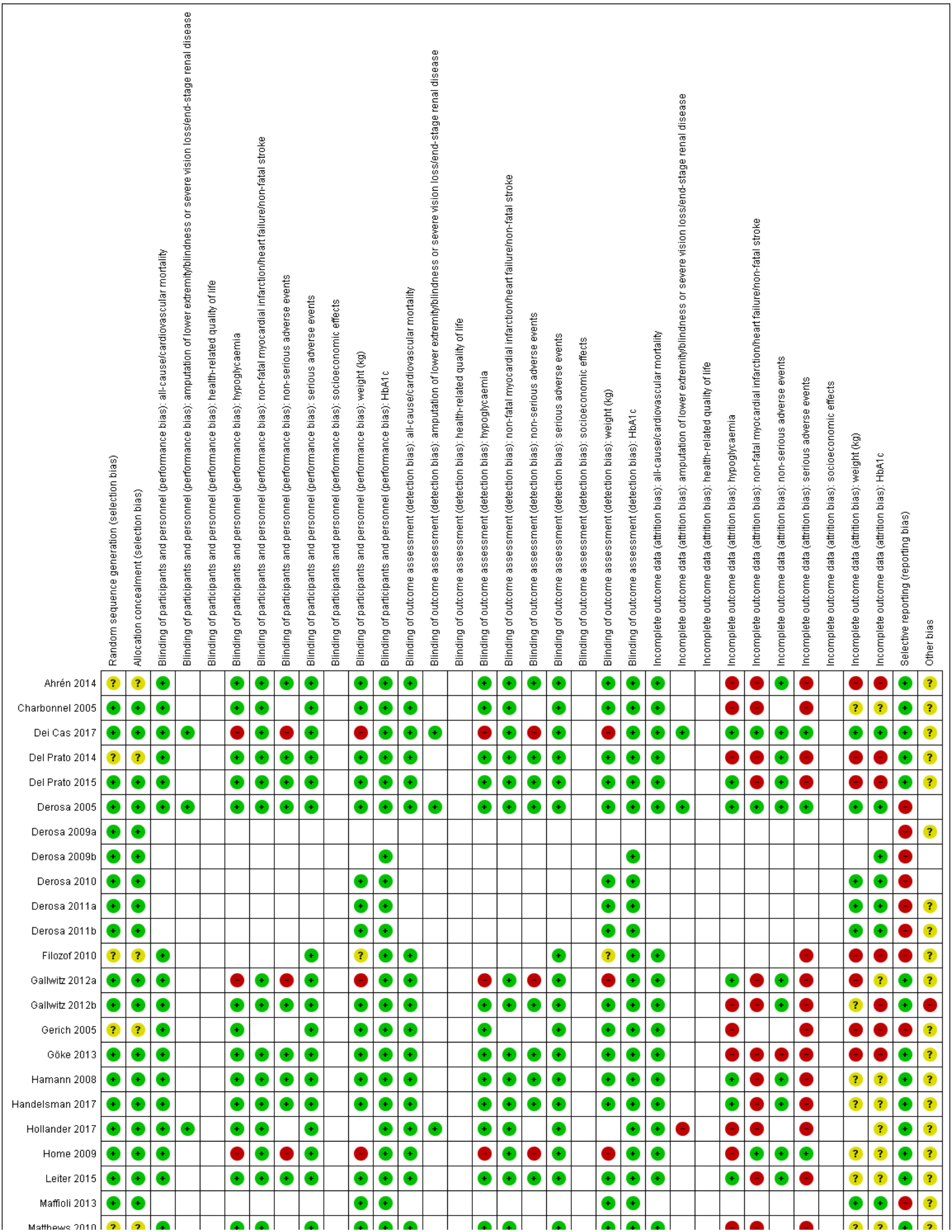


Figure 3. (Continued)

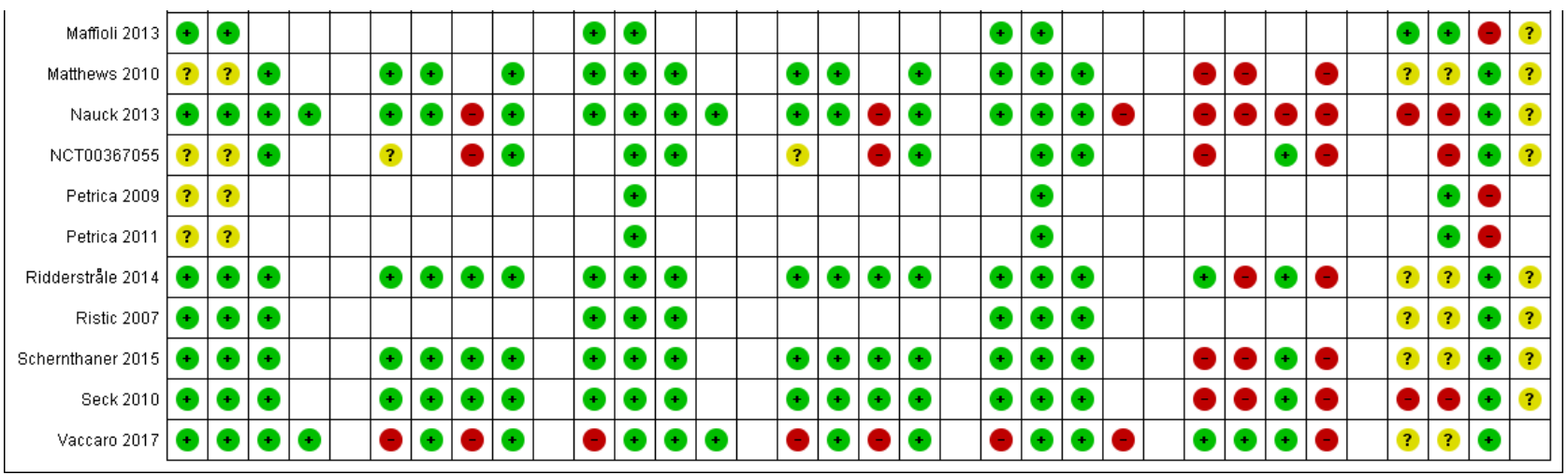

\section{Allocation}

We judged 24 trials at low risk of selection bias regarding the method of randomisation and allocation concealment (Charbonnel 2005; Dei Cas 2017; Del Prato 2015; Derosa 2005; Derosa 2009a; Derosa 2009b; Derosa 2010; Derosa 2011a; Derosa 2011b; Gallwitz 2012a; Gallwitz 2012b; Göke 2013; Hamann 2008; Handelsman 2017; Hollander 2017; Home 2009; Leiter 2015; Maffioli 2013; Nauck 2013; Ridderstråle 2014; Ristic 2007; Schernthaner 2015; Seck 2010; Vaccaro 2017). The remaining trials only reported that the participants were randomised but did not provide any further description (Ahrén 2014; Del Prato 2014; Filozof 2010; Gerich 2005; Matthews 2010; NCT00367055; Petrica 2009; Petrica 2011). Therefore, we judged these trials at unclear risk of bias regarding randomisation and allocation concealment.

We evaluated trial baseline data for our predefined prognostic baseline variables. None of the included trials reported data on all our key prognostic variables. However, all trials reported some variables of interest. None of the trials reporting prognostic variables showed important differences between the intervention groups.

\section{Blinding}

Blinding of participants and investigators for all outcomes was adequate in 21 trials (Ahrén 2014; Charbonnel 2005; Del Prato 2014; Del Prato 2015; Derosa 2005; Derosa 2009b; Derosa 2011b; Gallwitz 2012b; Gerich 2005; Göke 2013; Hamann 2008; Handelsman 2017; Hollander 2017; Leiter 2015; Maffioli 2013; Matthews 2010; Nauck 2013; Ridderstråle 2014; Ristic 2007; Schernthaner 2015; Seck 2010). Trials ensured blinding of participants and investigators by using identical placebo tablets or injections. Seven trials were open-label (Dei Cas 2017; Gallwitz 2012a; Home 2009; NCT00367055; Petrica 2009; Petrica 2011; Vaccaro 2017). Two trials were single-blinded (Derosa 2010; Derosa 2011a).

In one trial the participants and investigators were blinded in the first 26 weeks of the intervention period followed by a 78-week open-label extension phase (Nauck 2013).

Eight trials described a blinded outcome committee evaluating cardiovascular and cerebrovascular events (Ahrén 2014; Del Prato 2014; Filozof 2010; Gallwitz 2012b; Home 2009; Matthews 2010; Ridderstråle 2014; Vaccaro 2017).
Where measured, all primary outcomes of this review were investigator-assessed and we judged these at low risk of performance and detection bias. The trials reporting blood glucose measurements were all performed by the investigators and we judged these outcomes measures at low risk of performance and detection bias. Overall, the risk of performance bias and detection bias was low or unclear for our secondary outcomes.

\section{Incomplete outcome data}

All trials reported the complete number of participants randomised and finishing the trial. Two trials adequately addressed incomplete data for all outcomes (Dei Cas 2017; Derosa 2005). We judged the following outcomes to be at unclear or high risk of attrition bias in one or more trials: amputation of lower extremity, blindness or severe vision loss, end-stage renal disease (Hollander 2017; Nauck 2013; Vaccaro 2017); hypoglycaemia (Ahrén 2014; Charbonnel 2005; Del Prato 2014; Gallwitz 2012b; Gerich 2005; Göke 2013; Hollander 2017; Home 2009; Matthews 2010; Nauck 2013; NCT00367055; Schernthaner 2015; Seck 2010); non-fatal myocardial infarction, heart failure, non-fatal stroke (Ahrén 2014; Charbonnel 2005; Del Prato 2014; Del Prato 2015; Gallwitz 2012a; Gallwitz 2012b; Göke 2013; Hamann 2008; Handelsman 2017; Hollander 2017; Leiter 2015; Matthews 2010; Nauck 2013; Ridderstråle 2014; Schernthaner 2015; Seck 2010); non-serious adverse events (Filozof 2010; Göke 2013; Nauck 2013); serious adverse events (Ahrén 2014; Charbonnel 2005; Del Prato 2014; Del Prato 2015; Filozof 2010; Gallwitz 2012a; Gallwitz 2012b; Gerich 2005; Göke 2013; Hamann 2008; Handelsman 2017; Hollander 2017; Leiter 2015; Matthews 2010; Nauck 2013; NCT00367055; Ridderstråle 2014; Schernthaner 2015; Seck 2010; Vaccaro 2017); weight (Ahrén 2014; Charbonnel 2005; Del Prato 2014; Del Prato 2015; Filozof 2010; Gallwitz 2012a; Gallwitz 2012b; Gerich 2005; Göke 2013; Hamann 2008; Handelsman 2017; Home 2009; Leiter 2015; Matthews 2010; Nauck 2013; NCT00367055; Ridderstråle 2014; Ristic 2007; Schernthaner 2015; Seck 2010; Vaccaro 2017) and HbA1c (Ahrén 2014; Charbonnel 2005; Del Prato 2014; Del Prato 2015; Filozof 2010; Gallwitz 2012a; Gallwitz 2012b; Gerich 2005; Göke 2013; Hamann 2008; Handelsman 2017; Hollander 2017; Home 2009; Leiter 2015; Matthews 2010; Nauck 2013; NCT00367055; Ridderstråle 2014; Ristic 2007; Schernthaner 2015; Seck 2010; Vaccaro 2017).

The reasons for a high risk of attrition bias for dichotomous outcomes were: high dropout rate; dropout rate not balanced between intervention groups; reason for dropout not balanced 
between intervention groups; no information on imputation method; missing data imputed with 'last observation carried forward' technique; proportion of missing outcomes compared with the observed event risk was substantial enough to result in potentially clinically relevant bias for the intervention effect estimate.

The reasons for an unclear or high risk of attrition bias for continuous outcomes were: high dropout rate; dropout rate not balanced between intervention groups; reason for dropout not balanced between intervention groups; no information on imputation method; missing data imputed with 'last observation carried forward' technique; only participants with a value at baseline and at a specified visit were analysed; only participants who completed the study (excluding participants receiving rescue therapy) were included in the analysis; analyses based on per protocol population; and substantial amount of missing outcomes to potentially result in clinically relevant bias for the observed effect size.

\section{Selective reporting}

Twenty-two of the included trials had a published protocol (Ahrén 2014; Dei Cas 2017; Del Prato 2014; Del Prato 2015; Filozof 2010; Gallwitz 2012a; Gallwitz 2012b; Gerich 2005; Göke 2013; Hamann 2008; Handelsman 2017; Hollander 2017; Home 2009; Leiter 2015; Matthews 2010; Nauck 2013; NCT00367055; Ridderstråle 2014; Ristic 2007; Schernthaner 2015; Seck 2010; Vaccaro 2017).

We judged 11 of the included trials at high risk of reporting bias for one or more of our outcomes (Derosa 2005; Derosa 2009a; Derosa 2009b; Derosa 2010; Derosa 2011a; Derosa 2011b; Filozof 2010; Gerich 2005; Maffioli 2013; Petrica 2009; Petrica 2011). For more details, see Appendix 8.

We judged nine trials as at high risk of selective outcome reporting regarding one or more of the primary outcomes with relevance to this review (Derosa 2005; Derosa 2009a; Derosa 2009b; Derosa 2010; Derosa 2011a; Derosa 2011b; Maffioli 2013; Petrica 2009; Petrica 2011); we judged 10 trials as high risk of selective outcome reporting regarding one or more of the secondary outcomes with relevance to this review (Derosa 2005; Derosa 2009a; Derosa 2009b; Derosa 2010; Derosa 2011a; Derosa 2011b; Gerich 2005; Maffioli 2013; Petrica 2009; Petrica 2011).

We judged two trials as high risk of selective outcome reporting regarding one or more of the additional explorative outcomes of relevance to this review (Derosa 2009a; Filozof 2010).

\section{Other potential sources of bias}

Twenty-two trials received support or funding from a pharmaceutical company (Ahrén 2014; Charbonnel 2005; Dei Cas 2017; Del Prato 2014; Del Prato 2015; Filozof 2010; Gallwitz 2012a; Gallwitz 2012b; Gerich 2005; Göke 2013; Hamann 2008; Handelsman 2017; Hollander 2017; Home 2009; Leiter 2015; Matthews 2010; Nauck 2013; NCT00367055; Ridderstråle 2014; Ristic 2007; Schernthaner 2015; Seck 2010). For some comparisons, the same author had performed similar trials (Derosa 2005; Derosa 2009a; Derosa 2010; Derosa 2011a; Derosa 2011b; Maffioli 2013).

\section{Effects of interventions}

See: Summary of findings for the main comparison Metforminsulphonylurea (second- or third-generation) combination therapy compared with metformin plus another antidiabetic drug for adults with type 2 diabetes mellitus

See Summary of findings for the main comparison for details on the most important comparisons of this review $(M+S$ compared with metformin plus GLP-1 analogue, metformin plus DPP-4 inhibitor, metformin plus thiazolidinedione, metformin plus glinide and metformin plus SGLt-2 inhibitor).

\section{Baseline characteristics}

For details on baseline characteristics, see Appendix 5 and Appendix 6.

\section{Metformin-sulfonylurea combination therapy versus metformin plus insulin}

We identified no trials comparing $M+S$ with metformin plus insulin.

\section{Metformin-sulfonylurea combination therapy versus metformin monotherapy}

One trial compared $\mathrm{M}+\mathrm{S}$ combination therapy with metformin monotherapy (Derosa 2009a). The intervention group received metformin in doses of $850 \mathrm{mg} /$ day and glimepiride in doses of 2 $\mathrm{mg} /$ day to $6 \mathrm{mg} /$ day. The comparator group received metformin in doses of $1000 \mathrm{mg} /$ day to $3000 \mathrm{mg} /$ day.

\section{Primary outcomes}

The included trial did not report on all-cause mortality, healthrelated quality of life or serious adverse events.

\section{Secondary outcomes}

The included trial did not report on cardiovascular mortality, non-fatal myocardial infarction, heart failure, non-fatal stroke, amputation of lower extremity, blindness or severe vision loss, endstage renal disease, non-serious adverse events, hypoglycaemia or socioeconomic effects.

\section{Additional explorative outcomes \\ Weight}

The included trial did not report on weight.

\section{HbA1c}

The included trial reported final HbAlc measurements of $7.8 \%$ (SD 0.4) and 7.9\% (SD 0.5) in the M+S group compared with the metformin monotherapy, respectively. They did not report the number of participants included in the analysis.

\section{Metformin-sulfonylurea combination therapy versus metformin plus placebo}

Two trials compared $\mathrm{M}+\mathrm{S}$ combination therapy with metformin plus placebo (Ahrén 2014; Nauck 2013). Both trials administered glimepiride, given in doses of $1 \mathrm{mg} /$ day to $4 \mathrm{mg} /$ day; and metformin in doses of $\geq 1500 \mathrm{mg} /$ day. Both trials had multiple intervention arms with glimepiride, GLP-1 analogue, DPP-4 inhibitor or placebo in combination with metformin (Ahrén 2014), as well as glimepiride, GLP-1 analogue or placebo in combination with metformin (Nauck 2013). For details of the certainty of the evidence see Appendix 16. 


\section{Primary outcomes}

\section{All-cause mortality}

Both included trials reported data on all-cause mortality (lowcertainty evidence because of serious imprecision; Analysis 1.1). One trial reported 6 deaths out of 307 participants (2\%) and 1 death out of 101 participants $(1 \%)$ in the $M+S$ combination group compared with the metformin plus placebo group, respectively (Ahrén 2014). However, the number of deaths was unclear due to varied reporting. We contacted the trial authors for clarification but did not receive a reply. To be sure to account for all deaths, we extracted data from the publication that reported the highest number. One trial reported data after an 18-month, open-label extension: there were no deaths in 242 participants and no deaths in 121 participants in the $\mathrm{M}+\mathrm{S}$ combination group compared with the metformin plus placebo group, respectively (Nauck 2013).

\section{Health-related quality of life}

None of the included trials reported on this outcome.

\section{Serious adverse effects}

Both included trials reported that a total of 84 participants experienced a serious adverse event; in the $M+S$ group 60/549 $(10.9 \%)$ participants had a serious adverse event compared with $24 / 222(10.8 \%)$ participants in the metformin plus placebo group (RR $0.97,95 \% \mathrm{Cl} 0.59$ to $1.61 ; \mathrm{P}=0.91 ; 2$ trials; 771 participants; very low-certainty evidence because of attrition bias and serious imprecision; Analysis 1.2). One trial reported data after an 18month, open-label extension phase (Nauck 2013).

TSA showed that $2.45 \%$ of the diversity-adjusted required information size to detect or reject a $10 \%$ relative risk reduction (RRR) had been accrued. Diversity was $21 \%$. As only a minor fraction of the diversity-adjusted required information size to detect or reject a $10 \%$ RRR had been accrued, we could not calculate the TSAadjusted $95 \% \mathrm{Cl}$.

\section{Secondary outcomes}

\section{Cardiovascular mortality}

Both included trials reported data on cardiovascular mortality (lowcertainty evidence because of serious imprecision; Analysis 1.3). One trial reported 1 cardiovascular death in $307(0.3 \%)$ participants in the $\mathrm{M}+\mathrm{S}$ group compared with 1 cardiovascular death in $101(1 \%)$ participants in the metformin plus placebo group (Ahrén 2014). One trial reported that no participants died in either intervention group after an 18-month, open-label extension phase (Nauck 2013).

\section{Non-fatal myocardial infarction}

Both included trials reported that a total of three participants experienced a non-fatal myocardial infarction; in the $\mathrm{M}+\mathrm{S}$ group $2 / 549(0.4 \%)$ participants had a myocardial infarction compared with $1 / 222(0.5 \%)$ participants in the metformin plus placebo group (RR $0.63,95 \% \mathrm{Cl} 0.08$ to $5.10 ; \mathrm{P}=0.67 ; 2$ trials; 771 participants; very low-certainty evidence because of attrition bias and serious imprecision; Analysis 1.4). One trial reported data after an 18month, open-label extension phase (Nauck 2013).

TSA showed that $0.13 \%$ of the diversity-adjusted required information size to detect or reject a $10 \%$ RRR had been accrued. Diversity was zero. As only a minor fraction of the diversity-adjusted required information size to detect or reject a 10\% RRR had been accrued, we could not calculate the TSA-adjusted $95 \% \mathrm{Cl}$.

\section{Heart failure}

Both included trials reported data on heart failure (Analysis 1.5). One trial reported one heart failure in 307 participants $(0.3 \%)$ and no heart failure in 101 participants in the $\mathrm{M}+\mathrm{S}$ group compared with the metformin plus placebo group, respectively (Ahrén 2014). One trial reported that no participants experienced a heart failure in either intervention group after an 18-month, open-label extension phase (Nauck 2013).

\section{Non-fatal stroke, amputation of lower extremity, blindness or severe vision loss}

None of the included trials reported on this outcome.

\section{End-stage renal disease}

One trial reported that no participants had end-stage renal disease in either intervention group after an 18-month, open-label extension phase (very low-certainty evidence because of attrition bias and serious imprecision; Nauck 2013).

\section{Non-serious adverse events}

Both included trials reported that a total of 505 participants experienced a non-serious adverse event: in the $M+S$ group 386/549 $(70.3 \%)$ participants had a non-serious adverse event compared with 119/222 (53.6\%) participants in the metformin plus placebo group (random RR 1.25, 95\% Cl 0.96 to $1.64 ; \mathrm{P}=0.10$; fixed RR 1.24, $95 \% \mathrm{Cl} 1.10$ to $1.41 ; \mathrm{P}<0.001 ; 2$ trials; 771 participants; Analysis 1.6). None of the trials provided a detailed definition of the outcome. One trial reported data after an 18-month, open-label extension phase (Nauck 2013).

TSA showed that $5.8 \%$ of the diversity-adjusted required information size to detect or reject a 10\% RRR had been accrued. Diversity was $78 \%$. The TSA-adjusted $95 \% \mathrm{CI}$ was 0.47 to 3.32 .

\section{Hypoglycaemia}

Both included trials reported that a total of 181 participants experienced a mild or moderate hypoglycaemic episode; in the M+S group there were $160 / 549$ (29.1\%) participants with hypoglycaemic episodes compared with $21 / 222$ (9.5\%) participants in the metformin plus placebo group (random RR 3.93, 95\% Cl 0.71 to $21.88 ; \mathrm{P}=0.12$; fixed RR $2.87,95 \% \mathrm{Cl} 1.90$ to $4.34 ; \mathrm{P}<0.001$; 2 trials; 771 participants; Analysis 1.7 in favour of metformin plus placebo). Ahrén 2014 defined mild or moderate hypoglycaemia by blood glucose levels $3.9 \mathrm{mmol} / \mathrm{L}$ or lower and Nauck 2013 used blood glucose values $3.1 \mathrm{mmol} / \mathrm{L}$ or lower. One trial reported data after an 18-month, open-label extension phase (Nauck 2013).

TSA showed that $0.18 \%$ of the diversity-adjusted required information size to detect or reject a $10 \%$ RRR had been accrued. Diversity was $93 \%$. As only a minor fraction of the diversity-adjusted required information size to detect or reject a 10\% RRR had been accrued, we could not calculate the TSA-adjusted $95 \% \mathrm{Cl}$.

Both included trials reported data on serious hypoglycaemia (Analysis 1.8). One trial reported one participant with serious hypoglycaemia out of 307 participants $(0.3 \%)$ and no participants with serious hypoglycaemia out of 101 participants in the $M$ $+S$ group compared with the metformin plus placebo group, 
respectively (Ahrén 2014). One trial reported that no participants had serious hypoglycaemia in either intervention group after an 18month, open-label extension phase (Nauck 2013).

\section{Socioeconomic effects}

None of the included trials reported on this outcome.

\section{Additional explorative outcomes}

\section{Weight}

Both included trials reported weight change in favour of metformin plus placebo (mean difference (MD) $3.4 \mathrm{~kg}, 95 \% \mathrm{Cl} 1.4$ to 5.4 ; $\mathrm{P}=$ $0.001 ; 2$ trials; 476 participants; Analysis 1.9). Nauck 2013 reported data after an 18-month, open-label extension phase.

\section{HbA1c}

Both included trials reported change in $\mathrm{HbAlc}$ in favour of $\mathrm{M}+\mathrm{S}$ (random MD - 0.5\%, 95\% Cl-1.1 to 0.1; $\mathrm{P}=0.13$; fixed MD -0.6\%, 95\% $\mathrm{Cl}-0.8$ to $-0.4 ; \mathrm{P}<0.001 ; 2$ trials; 472 participants; Analysis 1.10). One trial reported data after an 18-month, open-label extension phase (Nauck 2013).

\section{Metformin-sulfonylurea combination therapy versus metformin plus GLP-1 analogue}

Five trials compared $\mathrm{M}+\mathrm{S}$ with metformin plus a GLP-1 analogue (Ahrén 2014; Derosa 2010; Derosa 2011a; Gallwitz 2012a; Nauck 2013). Four trials administered glimepiride in doses of $2 \mathrm{mg} /$ day to 6 mg/day (Ahrén 2014; Derosa 2011a; Gallwitz 2012a; Nauck 2013), and one trial administered glibenclamide in doses of $15 \mathrm{mg} /$ day (Derosa 2010). Three trials administered exenatide in doses of 10 $\mu \mathrm{g} /$ day to $20 \mu \mathrm{g} /$ day (Derosa 2010; Derosa 2011a; Gallwitz 2012a), one trial administered albiglutide in doses of $30 \mathrm{mg} /$ week to $50 \mathrm{mg} /$ week (Ahrén 2014), and one trial administered liraglutide in doses of $0.6 \mathrm{mg} /$ day to $1.8 \mathrm{mg} /$ day (Nauck 2013). Metformin was given in doses of $1000 \mathrm{mg} /$ day to $1500 \mathrm{mg} /$ day or more.

\section{Primary outcomes}

\section{All-cause mortality}

Three trials reported that a total of 22 participants died: in the $M$ +S group 11/1057 participants (1.0\%) died compared with 11/1537 participants $(0.7 \%)$ in the metformin plus GLP-1 analogue group (RR $1.15,95 \% \mathrm{Cl} 0.49$ to $2.67 ; \mathrm{P}=0.75 ; 3$ trials; 2594 participants; lowcertainty evidence; Analysis 2.1). Calculation of the $95 \%$ prediction interval did not provide a meaningful estimate. One trial reported data after an 18-month, open-label extension phase (Nauck 2013).

Derosa 2011 a reported that no participants died but did not provide the number of participants included in the analysis.

Because all trials were published in English, we could not perform sensitivity analyses according to publication status or language of publication. Sensitivity analysis restricted to trials with low risk of selection bias did not change the direction of the effect estimate (RR $0.93,95 \% \mathrm{Cl} 0.30$ to $2.93 ; \mathrm{P}=0.91 ; 2$ trials; 1985 participants). We could not perform sensitivity analysis excluding large trials because none of the included trials for this comparison had more than 1000 participants randomised to each intervention group. We could not perform sensitivity analysis excluding trials funded by a pharmaceutical company since all trials received funding from a pharmaceutical company.
TSA showed that $0.61 \%$ of the diversity-adjusted information size had been accrued to detect or reject a 10\% RRR. Diversity was zero. As only a minor fraction of the diversity-adjusted required information size to detect or reject a 10\% RRR had been accrued, we could not calculate the TSA-adjusted $95 \% \mathrm{Cl}$.

\section{Health-related quality of life}

We did not identify trials with data on health-related quality of life for this comparison. One trial reported on 'impact of weight on quality of life' (IWQOL; Nauck 2013). They reported the result separately for each intervention group.

\section{Serious adverse effects}

Three trials reported that a total of 322 participants experienced a serious adverse event: in the $\mathrm{M}+\mathrm{S}$ group $128 / 1057$ participants (12.1\%) had a serious adverse event compared with 194/1537 participants (12.6\%) in the metformin plus GLP-1 analogue group ( $R R 0.90,95 \% \mathrm{Cl} 0.73$ to $1.11 ; \mathrm{P}=0.32 ; 3$ trials; 2594 participants; very low-certainty evidence; Analysis 2.2 ). The $95 \%$ prediction interval ranged between 0.23 and 3.51. One of the trials reported data after an 18-month, open-label extension (Nauck 2013). Derosa 2011a reported that no participants experienced serious adverse events but did not provide the number of participants included in the analysis.

Because all trials were published in English, we could not perform sensitivity analyses according to publication status or language of publication. Sensitivity analysis according to trials with low risk of selection bias and duration of intervention (excluding trials with duration of intervention longer than 104 weeks) contained the same two trials (Gallwitz 2012a; Nauck 2013). The effect estimate did not change substantially (RR $0.94,95 \% \mathrm{Cl} 0.73$ to $1.20 ; \mathrm{P}=$ 0.60; 2 trials; 1985 participants). We could not perform sensitivity analysis excluding large trials because no trials had more than 1000 participants randomised to each intervention group. We could not perform sensitivity analysis excluding trials funded by a pharmaceutical company because all trials received funding from a pharmaceutical company.

TSA showed that $12.4 \%$ of the diversity-adjusted information size had been accrued to detect or reject a 10\% RRR. Diversity was zero. The TSA-adjusted $95 \% \mathrm{Cl}$ was 0.38 to 2.15 .

\section{Secondary outcomes}

\section{Cardiovascular mortality}

Three trials reported on cardiovascular disease (Ahrén 2014; Derosa 2011a; Nauck 2013). Derosa 2011a reported that no participants died due to cardiovascular disease but did not provide the number of participants included in the analysis. Nauck 2013 reported that no participants died due to cardiovascular disease in any of the intervention groups referring to the 18-month, open-label extension phase.

Ahrén 2014 reported that in the $M+S$ group $1 / 307(0.3 \%)$ participants died of cardiovascular disease compared with $1 / 302$ $(0.3 \%)$ participants in the metformin plus GLP-1 analogue group (Analysis 2.3; low-certainty evidence).

\section{Non-fatal myocardial infarction}

Two trials reported that a total of eight participants experienced a non-fatal myocardial infarction: in the $\mathrm{M}+\mathrm{S}$ group $2 / 549(0.4 \%)$ 
participants had a non-fatal myocardial infarction compared with $6 / 1026(0.6 \%)$ participants in the metformin plus GLP-1 analogue group (RR $0.57,95 \% \mathrm{Cl} 0.12$ to $2.82 ; \mathrm{P}=0.49 ; 2$ trials; 1575 participants; very low-certainty evidence; Analysis 2.4).

Nauck 2013 reported data after an 18-month, open-label extension phase. Derosa 2011a reported that no participants experienced a non-fatal myocardial infarction but did not provide the number of participants included in the analysis.

We could not perform sensitivity analyses due to lack of data.

TSA showed that $0.4 \%$ of the diversity-adjusted information size had been accrued to detect or reject a 10\% RRR. Diversity was zero. As only a minor fraction of the diversity-adjusted required information size to detect or reject a 10\% RRR had been accrued, we could not calculate the TSA-adjusted $95 \% \mathrm{Cl}$.

\section{Heart failure}

Four trials reported that a total of five participants developed heart failure: in the $\mathrm{M}+\mathrm{S}$ group $1 / 1057(0.1 \%)$ participants developed heart failure compared with $4 / 1537(0.3 \%)$ participants in the metformin plus GLP-1 analogue group (RR $0.54,95 \% \mathrm{Cl} 0.10$ to 2.77 ; $\mathrm{P}=0.46 ; 3$ trials; 2594 participants; Analysis 2.5). Calculation of the 95\% prediction interval did not provide a meaningful estimate.

Nauck 2013 reported data after an 18-month, open-label extension phase. Derosa 2011a reported that no participants developed heart failure but did not provide the number of participants included in the analysis.

Because all trials were published in English, we could not perform sensitivity analyses according to publication status or language of publication. Sensitivity analysis restricted to trials with low risk of selection bias and duration of intervention (excluding trials with duration of intervention more than 104 weeks) contained the same two trials (Gallwitz 2012a; Nauck 2013). The effect estimate did not change substantially (RR $0.58,95 \% \mathrm{Cl} 0.06$ to $5.54 ; \mathrm{P}=$ 0.63; 2 trials; 1985 participants). We could not perform sensitivity analysis excluding large trials because no trials had more than 1000 participants randomised to each intervention group. We could not perform sensitivity analysis excluding trials funded by a pharmaceutical company because all trials received funding from a pharmaceutical company.

TSA showed that $0.26 \%$ of the diversity-adjusted information size had been accrued to detect or reject a $10 \%$ RRR. Diversity was zero. As only a minor fraction of the diversity-adjusted required information size to detect or reject a $10 \%$ RRR had been accrued, we could not calculate the TSA-adjusted $95 \% \mathrm{Cl}$.

\section{Non-fatal stroke}

Derosa 2011a reported that no participants experienced a stroke during the intervention period but did not provide the number of participants included in the analysis.

\section{Amputation of lower extremity}

Derosa 2011a reported that no participants had amputation of lower extremity during the intervention period but did not provide the number of participants included in the analysis.

\section{Blindness or severe vision loss}

Derosa 2011a reported that no participants had blindness or severe vision loss during the intervention period but did not provide the number of participants included in the analysis.

\section{End-stage renal disease}

One trial provided data on end-stage renal disease, however only after the 18-month, open-label extension phase (Nauck 2013). In the $M+S$ group no participants out of 242 participants had endstage renal disease compared with 1 participant out of $724(0.1 \%)$ participants in the metformin plus GLP-1 analogue group (Analysis 2.6).

Derosa 2011a reported that no participants had end-stage renal disease during the intervention period but did not provide the number of participants included in the analysis.

\section{Non-serious adverse events}

Three trials reported that a total of 1621 participants experienced a non-serious adverse event: in the $\mathrm{M}+\mathrm{S}$ group 634/1057 (60.0\%) participants had a non-serious adverse event compared with 987/1537 (64.2\%) participants in the metformin plus GLP-1 analogue group (Analysis 2.7). Due to substantial heterogeneity, aggregating the trials in a meta-analysis was not appropriate. With regard to the $\mathrm{M}+\mathrm{S}$ groups, the trial with most of the non-serious adverse events had two placebo interventions (GLP-1 analogue as subcutaneous injection and placebo DPP-4 inhibitor as tablet) in addition to M+S (Ahrén 2014).

One trial was open-label and did not apply placebo (Gallwitz 2012a), and one trial (Nauck 2013), reported data after an 18month, open-label extension. With regard to the metformin plus GLP-1 analogue treatment arms, each trial used a different GLP-1 analogue: one administered albiglutide (Ahrén 2014), and one administered exenatide (Gallwitz 2012a), or liraglutide (Nauck 2013).

Derosa 2011a reported that no participants experienced nonserious adverse events but did not provide the number of participants included in the analysis.

Because all trials were published in English, we could not perform sensitivity analyses according to publication status or language of publication. Sensitivity analyses restricted to trials with low risk of selection bias and duration of intervention (excluding trials with duration of intervention longer than 104 weeks) contained the same two trials (Gallwitz 2012a; Nauck 2013). The effect estimate was in favour of $\mathrm{M}+\mathrm{S}$ combination therapy (RR $0.84,95 \% \mathrm{Cl} 0.76$ to 0.92; $\mathrm{P}=0.0001 ; 2$ trials; 1985 participants). We could not perform sensitivity analysis excluding large trials because no trials had more than 1000 participants randomised to each intervention group. We could not perform sensitivity analysis excluding trials funded by a pharmaceutical company because all trials received funding from a pharmaceutical company.

\section{Hypoglycaemia}

Three trials reported that a total of 569 participants experienced a mild or moderate hypoglycaemic episode: in the $\mathrm{M}+\mathrm{S}$ group 400/1057 (37.8\%) participants had a hypoglycaemic episode compared with 169/1537 (11.0\%) participants in the metformin plus GLP-1 analogue group (RR 3.24, 95\% Cl 2.05 to 5.13; P < 0.001; 3 trials; 2594 participants; Analysis 2.8; in favour of metformin 
plus GLP-1 analogue). Calculation of the $95 \%$ prediction interval did not provide a meaningful estimate. The trial with mostly mild or moderate hypoglycaemic events defined hypoglycaemia as any sign or symptom of hypoglycaemia or blood glucose $3.9 \mathrm{mmol} / \mathrm{L}$ or less (Gallwitz 2012a), whereas the other trials defined hypoglycaemia as symptoms of hypoglycaemia plus blood glucose values $3.9 \mathrm{mmol} / \mathrm{L}$ or less, or blood glucose values 3.9 $\mathrm{mmol} / \mathrm{L}$ or less without symptoms (Ahrén 2014), or symptoms of hypoglycaemia or blood glucose values $3.1 \mathrm{mmol} / \mathrm{L}$ or less (Nauck 2013). Nauck 2013 reported data after an 18-month, open-label extension phase.

Derosa 2011a reported two events of mild or moderate hypoglycaemia in the glimepiride group and one in the exenatide group but did not provide the number of participants included in the analysis.

Because all trials were published in English, we could not perform sensitivity analyses according to publication status or language of publication. Sensitivity analyses restricted to trials with low risk of selection bias and duration of intervention (excluding trials with duration of intervention longer than 104 weeks) contained the same two trials (Gallwitz 2012a; Nauck 2013). We could not perform sensitivity analysis excluding large trials because no trials had more than 1000 participants randomised to each intervention group. We could not perform sensitivity analysis excluding trials funded by a pharmaceutical company because all trials received funding from a pharmaceutical company.

Only three participants reported serious hypoglycaemia: in the $M$ $+S$ group $1 / 1057(0.1 \%)$ participants had a serious hypoglycaemic event compared with $2 / 1537(0.1 \%)$ participants in the metformin plus GLP-1 analogue group (RR 1.00, 95\% Cl 0.16 to $6.30 ; \mathrm{P}=1.00 ; 3$ trials; 2594 participants; Analysis 2.9).

Nauck 2013 reported data after an 18-month, open-label extension phase. Derosa 2011a reported that no event of serious hypoglycaemia was observed but did not provide the number of participants included in the analysis.

Because all trials were published in English, we could not perform sensitivity analyses according to publication status or language of publication. Sensitivity analyses restricted to trials with low risk of selection bias and duration of intervention (excluding trials with duration of intervention longer than 104 weeks) contained the same two trials (Gallwitz 2012a; Nauck 2013). The effect estimate did not change substantially ( $\mathrm{RR} 0.58,95 \% \mathrm{Cl} 0.06$ to $5.54 ; \mathrm{P}=$ 0.63; 2 trials; 1985 participants). We could not perform sensitivity analysis excluding large trials because no trial had more than 1000 participants randomised to each intervention group. We could not perform sensitivity analysis excluding trials funded by a pharmaceutical company because all trials received funding from a pharmaceutical company.

TSA for severe hypoglycaemia showed that $0.09 \%$ of the diversityadjusted information size had been accrued to detect or reject a $10 \%$ RRR. Diversity was zero. As only a minor fraction of the diversity-adjusted required information size to detect or reject a $10 \%$ RRR had been accrued, we could not calculate the TSAadjusted $95 \% \mathrm{Cl}$.

\section{Socioeconomic effects}

We did not identify trials with data on socioeconomic effects for this comparison.

\section{Additional explorative outcomes Weight}

Five trials reported weight change in favour of metformin plus GLP-1 analogue (MD $5.5 \mathrm{~kg}, 95 \% \mathrm{Cl} 3.6$ to 7.5 ; P < 0.001; 5 trials; 1777 participants; Analysis 2.10$)$. Calculation of the $95 \%$ prediction interval did not provide a meaningful estimate. Heterogeneity in the findings could have been caused by the various durations of follow-up, ranging from 52 weeks to 156 weeks, and various doses of glimepiride ranging from $1 \mathrm{mg} /$ day to $6 \mathrm{mg} /$ day. Hollander 2017 did not report the number of participants included in the analysis.

Nauck 2013 reported data after an 18-month, open-label extension phase.

\section{HbA1c}

Five trials reported change in $\mathrm{HbA} 1 \mathrm{c}$ (MD $0.01 \%, 95 \% \mathrm{Cl}-0.2$ to $0.2 ; \mathrm{P}$ $=0.91 ; 5$ trials; 2346 participants; Analysis 2.11$)$. The $95 \%$ prediction interval ranged between $0 \%$ and $1.0 \%$

Nauck 2013 reported data after an 18-month, open-label extension phase. Trials with 52 weeks of treatment reported at greater decrease in Hbalc with sulphonylurea treatment, whereas trials with 104 weeks of treatment or more reported a greater decrease in $\mathrm{HbA} 1 \mathrm{c}$ with GLP-1 analogue treatment.

TSA showed that $0.61 \%$ of the diversity-adjusted information size had been accrued to detect or reject a $10 \%$ RRR. Diversity was $91 \%$. As only a minor fraction of the diversity-adjusted required information size to detect or reject a 10\% RRR had been accrued, we could not calculate the TSA-adjusted $95 \% \mathrm{Cl}$.

\section{Metformin-sulfonylurea combination therapy versus metformin plus DPP-4 inhibitor}

Nine trials compared $M+S$ combination therapy with metformin plus a DPP-4 inhibitor (Ahrén 2014; Dei Cas 2017; Del Prato 2014; Filozof 2010; Gallwitz 2012b; Göke 2013; Matthews 2010; Schernthaner 2015; Seck 2010). Four trials administered glimepiride in doses of $1 \mathrm{mg} /$ day to $6 \mathrm{mg}$ /day (Ahrén 2014; Gallwitz 2012b; Matthews 2010; Schernthaner 2015), three trials administered glipizide in doses of $5 \mathrm{mg} /$ day to $20 \mathrm{mg}$ /day (Del Prato 2014; Göke 2013; Seck 2010), one trial administered glibenclamide in doses of $10 \mathrm{mg} /$ day (Dei Cas 2017), and one trial administered gliclazide in doses of $80 \mathrm{mg} /$ day to $320 \mathrm{mg} /$ day (Filozof 2010). Three trials administered vildagliptin in doses of $100 \mathrm{mg} /$ day (Dei Cas 2017; Filozof 2010; Matthews 2010), two trials administered sitagliptin in doses of $100 \mathrm{mg} /$ day (Ahrén 2014; Seck 2010), two trials administered saxagliptin in doses of $5 \mathrm{mg} /$ day ( Göke 2013; Schernthaner 2015), one trial administered alogliptin (in doses of $12.5 \mathrm{mg} /$ day to $25 \mathrm{mg} /$ day (Del Prato 2014), and one trial administered linagliptin in doses of $5 \mathrm{mg} /$ day (Gallwitz 2012b). One trial administered metformin at any dose (Schernthaner 2015), the remaining trials administered metformin in doses of $1500 \mathrm{mg} /$ day or more. 


\section{Primary outcomes}

\section{All-cause mortality}

Nine trials reported that a total of 59 participants died: in the $\mathrm{M}+\mathrm{S}$ group 33 participants died out of 5387 (0.6\%) participants, compared with 26 deaths in $6307(0.4 \%)$ participants in the metformin plus DPP-4 inhibitor group (RR $1.32,95 \% \mathrm{Cl} 0.76$ to 2.28; $P=0.32 ; 9$ trials; 11,694 participants; low-certainty evidence; Analysis 3.1). The $95 \%$ prediction interval ranged between 0.68 and 2.55 .

A test for subgroup differences according to duration of follow-up did not indicate interaction $(P=0.77)$.

Because all trials were published in English, we could not perform sensitivity analyses according to publication status or language of publication. Sensitivity analysis restricted to trials with low risk of selection bias did not substantially change the effect estimate (RR 1.29, 95\% Cl 0.41 to $4.10 ; \mathrm{P}=0.66 ; 5$ trials; 4363 participants). Sensitivity analysis excluding large trials did not substantially change the effect estimate (RR $1.52,95 \% \mathrm{Cl} 0.81$ to 2.88 ; $\mathrm{P}=0.19$; 8 trials; 8595 participants). We could not perform sensitivity analysis excluding trials funded by a pharmaceutical company because all trials had received funding from a pharmaceutical company.

TSA showed that $2.4 \%$ of the diversity-adjusted information size had been accrued to detect or reject a 10\% RRR. Diversity was zero. As only a minor fraction of the diversity-adjusted required information size to detect or reject a 10\% RRR had been accrued, we could not calculate the TSA-adjusted $95 \% \mathrm{Cl}$.

\section{Health-related quality of life}

We did not identify trials reporting data on health-related quality of life for this comparison.

\section{Serious adverse effects}

Nine trials reported that a total of 1514 participants experienced a serious adverse event: in the $M+S$ group $735 / 5387(13.6 \%)$ participants had a serious adverse event compared with $779 / 6307$ (12.4\%) participants in the metformin plus DPP-4 inhibitor group (RR $1.07,95 \% \mathrm{Cl} 0.97$ to $1.18 ; \mathrm{P}=0.17 ; 9$ trials; 11,694 participants; very low-certainty evidence; Analysis 3.2). The 95\% prediction interval ranged between 0.95 and 1.20 .

A test for subgroup differences according to duration of follow-up did not indicate interaction $(P=0.82)$.

Because all trials were published in English, we could not perform sensitivity analyses according to publication status or language of publication. Sensitivity analysis restricted to only trials with low risk of selection bias did not substantially change the effect estimate (RR $1.09,95 \% \mathrm{Cl} 0.93$ to $1.28 ; \mathrm{P}=0.27 ; 5$ trials; 4363 participants). Sensitivity analysis excluding large trials did not substantially change the effect estimate (RR $1.06,95 \% \mathrm{Cl} 0.94$ to 1.20; $P=0.33 ; 8$ trials; 8595 participants). We could not perform sensitivity analysis excluding trials funded by a pharmaceutical company because all trials received funding from a pharmaceutical company.

TSA showed that $61 \%$ of the diversity-adjusted required information size to detect or reject a $10 \%$ RRR had been accrued. Diversity was zero. The TSA-adjusted $95 \% \mathrm{Cl}$ was 0.93 to 1.23 .

\section{Secondary outcomes}

\section{Cardiovascular mortality}

Six trials reported that a total of 20 participants died due to cardiovascular disease: in the $\mathrm{M}+\mathrm{S}$ group 11 participants died out of $2989(0.4 \%)$ participants compared with 9 out of 3885 $(0.2 \%)$ participants in the metformin plus DPP-4 inhibitor group (RR 1.54, 95\% Cl 0.63 to 3.79; $\mathrm{P}=0.34 ; 6$ trials; 6874 participants; low-certainty evidence; Analysis 3.3). The $95 \%$ prediction interval ranged between 0.43 and 5.52. Two trials reported a composite outcome of cardiovascular and cerebrovascular events (Filozof 2010: $M+S$ 12/493 (2.4\%) participants, metformin plus DPP-4 inhibitor 7/510 (1.4\%) participants; Matthews 2010: M+S 60/1546 (3.9\%) participants, metformin plus DPP-4 inhibitor 59/1553 (3.8\%) participants).

We could not perform a test for subgroup differences according to duration of follow-up due to lack of data.

Because all trials were published in English, we could not perform sensitivity analyses according to publication status or language of publication. Sensitivity analysis restricted to only trials with low risk of selection bias did not substantially change the effect estimate (RR $1.72,95 \% \mathrm{Cl} 0.44$ to $6.69 ; \mathrm{P}=0.43 ; 4$ trials; 3645 participants).

We could not perform sensitivity analysis excluding large trials because no trials had more than 1000 participants randomised to each intervention group. We could not perform sensitivity analysis excluding trials funded by a pharmaceutical company because all trials received funding from a pharmaceutical company.

TSA showed that $0.92 \%$ of the diversity-adjusted information size had been accrued to detect or reject a 10\% RRR. Diversity was zero. As only a minor fraction of the diversity-adjusted required information size to detect or reject a $10 \%$ RRR had been accrued, we could not calculate the TSA-adjusted $95 \% \mathrm{Cl}$.

\section{Non-fatal myocardial infarction}

Six trials reported that a total of 28 participants experienced a non-fatal myocardial infarction: in the M+S group $15 / 2989(0.5 \%)$ participants had a myocardial infarction compared with $13 / 3885$ (0.3\%) participants in the metformin plus DPP-4 inhibitor group (RR $1.45,95 \% \mathrm{Cl} 0.69$ to $3.07 ; \mathrm{P}=0.33 ; 6$ trials; 6874 participants; very low-certainty evidence; Analysis 3.4). The $95 \%$ prediction interval ranged between 0.50 and 4.20 .

We could not perform a test for subgroup differences according to duration of follow-up due to lack of data.

Because all trials were published in English, we could not perform sensitivity analyses according to publication status or language of publication. Sensitivity analysis restricted to trials with low risk of selection bias did not substantially change the effect estimate (RR $1.44,95 \% \mathrm{Cl} 0.55$ to $3.77 ; \mathrm{P}=0.45 ; 4$ trials; 3645 participants). We could not perform sensitivity analysis excluding large trials because no trials had more than 1000 participants randomised to each intervention group. We could not perform sensitivity analysis excluding trials funded by a pharmaceutical company because all trials received funding from a pharmaceutical company.

TSA showed that $0.95 \%$ of the diversity-adjusted information size had been accrued to detect or reject a 10\% RRR. Diversity was 
zero. As only a minor fraction of the diversity-adjusted required information size to detect or reject a 10\% RRR had been accrued, we could not calculate the TSA-adjusted $95 \% \mathrm{Cl}$.

\section{Heart failure}

Eight trials reported that a total of 29 participants developed heart failure: in the $\mathrm{M}+\mathrm{S}$ group 15/4894 (0.3\%) participants developed heart failure compared with $14 / 5797(0.2 \%)$ participants in the metformin plus DPP-4 inhibitor group (RR $1.05,95 \% \mathrm{Cl} 0.47$ to 2.34; $\mathrm{P}=0.90 ; 8$ trials; 10,691 participants; Analysis 3.5). The $95 \%$ prediction interval ranged between 0.39 and 2.86 .

A test for subgroup differences according to duration of follow-up did not indicate interaction $(P=0.08)$.

Because all trials were published in English, we could not perform sensitivity analyses according to publication status or language of publication. Sensitivity analysis restricted to only trials with low risk of selection bias did not substantially change the direction of the effect estimate (RR $1.21,95 \% \mathrm{Cl} 0.38$ to $3.78 ; \mathrm{P}=0.75 ; 5$ trials; 4363 participants). Sensitivity analysis excluding large trials did not substantially change the direction of the effect estimate (RR $1.07,95 \% \mathrm{Cl} 0.42$ to $2.69 ; \mathrm{P}=0.89 ; 7$ trials; 7592 participants). We could not perform sensitivity analysis excluding trials funded by a pharmaceutical company because all trials received funding from a pharmaceutical company.

\section{Non-fatal stroke}

Four trials reported that a total of 22 participants experienced a non-fatal stroke: in the M+S group 14/2098 (0.7\%) participants had a non-fatal stroke compared with $8 / 2995(0.3 \%)$ participants in the metformin plus DPP-4 inhibitor group (RR $2.21,95 \% \mathrm{Cl} 0.74$ to 6.58 ; $\mathrm{P}=0.15 ; 4$ trials; 5093 participants; very low-certainty evidence; Analysis 3.6). The $95 \%$ prediction interval ranged between 0.12 and 40.89 .

We could not perform subgroup analysis due to lack of data.

Because all trials were published in English, we could not perform sensitivity analyses according to publication status or language of publication. We could not perform sensitivity analysis restricted to trials with low risk of selection bias due to lack of data. We could not perform sensitivity analysis excluding long trials because all trials had a follow-up of 104 weeks or less. We could not perform sensitivity analysis excluding large trials because no trials had more than 1000 participants randomised to each intervention group. We could not perform sensitivity analysis excluding trials funded by a pharmaceutical company because all trials received funding from a pharmaceutical company.

TSA showed that $0.76 \%$ of the diversity-adjusted information size had been accrued to detect or reject a 10\% RRR. Diversity was $23 \%$. As only a minor fraction of the diversity-adjusted required information size to detect or reject a 10\% RRR had been accrued, we could not calculate the TSA-adjusted $95 \% \mathrm{Cl}$.

\section{Amputation of lower extremity}

Dei Cas 2017 did not observe amputation of the lower extremity in either intervention group (64 participants; very low-certainty evidence).

\section{Blindness or severe vision loss}

Dei Cas 2017 did not observe blindness or severe vision loss in either intervention group (64 participants; very low-certainty evidence).

\section{End-stage renal disease}

Dei Cas 2017 did not observe end-stage renal disease in either intervention group (64 participants; very low-certainty evidence).

\section{Non-serious adverse events}

Seven trials reported that a total of 4751 participants experienced a non-serious adverse event: in the $\mathrm{M}+\mathrm{S}$ group $2156 / 3348$ (64.4\%) participants had a non-serious adverse event compared with $2595 / 4244(61.1 \%)$ participants in the metformin plus DPP-4 inhibitor group (RR 1.18, 95\% Cl 1.03 to $1.35 ; \mathrm{P}=0.02 ; 7$ trials; 7592 participants; Analysis 3.7 in favour of metformin plus DPP-4 inhibitor). The 95\% prediction interval ranged between 0.73 and 1.91. There was substantial heterogeneity, which could be caused by various definitions of the outcome (most trials did not adequately specify this outcome measure).

A test for subgroup differences according to duration of follow-up did not indicate interaction $(P=0.11)$.

Because all trials were published in English, we could not perform sensitivity analyses according to publication status or language of publication. Sensitivity analysis restricted to trials with low risk of selection bias did not substantially change the effect estimate (RR 1.27, 95\% Cl 1.06 to 1.52; $\mathrm{P}=0.01 ; 5$ trials; 4363 participants). We could not perform sensitivity analysis excluding large trials because no trials had more than 1000 participants randomised to each intervention group. We could not perform sensitivity analysis excluding trials funded by a pharmaceutical company because all trials received funding from a pharmaceutical company.

TSA showed that $21.5 \%$ of the diversity-adjusted required information size to detect or reject a $10 \%$ RRR had been accrued. Diversity was $94 \%$. The TSA-adjusted $95 \% \mathrm{Cl}$ was 0.88 to 1.58 .

\section{Hypoglycaemia}

Seven trials reported that a total of 1544 participants experienced a mild or moderate hypoglycaemic event: in the M+S group 1359/4535 (30.0\%) participants had a mild or moderate hypoglycaemic episode compared with $185 / 5438$ (3.4\%) participants in the metformin plus DPP-4 inhibitor group (RR $7.42,95 \% \mathrm{Cl} 4.77$ to 11.53 ; $\mathrm{P}<0.001 ; 7$ trials; 9973 participants; Analysis 3.8 in favour of metformin plus DPP-4 inhibitor). The $95 \%$ prediction interval ranged between 1.86 and 29.66. There was substantial heterogeneity, probably caused by various definitions of the outcome. All trials that stated a definition of outcome defined mild/moderate hypoglycaemia in different ways (Ahrén 2014; Del Prato 2014; Göke 2013; Matthews 2010).

The test for subgroup differences analysing trials according to duration of intervention showed a statistically significant difference between subgroups ( $P=0.04$; Analysis 3.8). However, Cls overlap indicating that in fact there was no true interaction.

Because all trials were published in English, we could not perform sensitivity analyses according to publication status or language of publication. Sensitivity analysis restricted to trials with low risk of selection bias did not substantially change the effect estimate (RR $6.71,95 \% \mathrm{Cl} 3.94$ to $11.44 ; \mathrm{P}<0.001 ; 4$ trials; 3645 participants). 
Sensitivity analysis excluding large trials did not substantially change the effect estimate (RR 6.00, 95\% Cl 4.33 to $8.32 ; \mathrm{P}<0.001 ; 6$ trials; 6874 participants). We could not perform sensitivity analysis excluding trials funded by a pharmaceutical company because all trials received funding from a pharmaceutical company.

TSA showed that $1.4 \%$ of the diversity-adjusted information size had been accrued to detect or reject a 10\% RRR. Diversity was $88 \%$. As only a minor fraction of the diversity-adjusted required information size to detect or reject a 10\% RRR had been accrued, we could not calculate the TSA-adjusted $95 \% \mathrm{Cl}$.

Eight trials reported that a total of 55 participants experienced a serious hypoglycaemic event: in the $\mathrm{M}+\mathrm{S}$ group $51 / 4894(1.0 \%)$ participants had a serious hypoglycaemic episode compared with $4 / 5797(0.1 \%)$ participants in the metformin plus DPP-4 inhibitor group (RR 8.04, 95\% Cl 3.31 to 19.53; $\mathrm{P}<0.01$; 8 trials; 10,691 participants; Analysis 3.9 in favour of metformin plus DPP-4 inhibitor). The 95\% prediction interval ranged between 2.66 and 24.35 .

A test for subgroup differences according to duration of follow-up did not indicate interaction $(P=0.78)$.

Because all trials were published in English, we could not perform sensitivity analyses according to publication status or language of publication. Sensitivity analysis restricted to trials with low risk of selection bias did not substantially change the effect estimate (RR $8.62,95 \% \mathrm{Cl} 2.81$ to $26.43 ; \mathrm{P}=0.0002 ; 5$ trials; 4363 participants). Sensitivity analysis excluding large trials did not substantially change the effect estimate (RR 6.93, 95\% Cl 2.72 to $17.65 ; \mathrm{P}<0.001 ; 7$ trials; 7592 participants). We could not perform sensitivity analysis excluding trials funded by a pharmaceutical company because all trials received funding from a pharmaceutical company.

TSA showed that $0.62 \%$ of the diversity-adjusted information size had been accrued to detect or reject a $10 \%$ RRR. Diversity was $0 \%$. As only a minor fraction of the diversity-adjusted required information size to detect or reject a 10\% RRR had been accrued, we could not calculate the TSA-adjusted $95 \% \mathrm{Cl}$.

\section{Socioeconomic effects}

Two trials performed economic analyses of trial data (Del Prato 2014; Göke 2013).

One trial presented an economic analysis using the IMS Core Diabetes Model (Del Prato 2014). Treatment with alogliptin $12.5 \mathrm{mg}$ or $25 \mathrm{mg}$ compared with sulphonylurea was associated with an incremental cost-effectiveness ratio of GBP 10,959 or GBP 7217 per quality adjusted life year, respectively.

One trial presented an economic analysis using the Cardiff Stochastic Simulation Cost-utility Model (Göke 2013). The overall mean cost per quality adjusted life year gained with saxagliptin plus metformin compared with M+S was GBP 7888 .

\section{Additional explorative outcomes}

Weight

Nine trials reported weight change. Metformin plus DPP-4 inhibitor compared with $\mathrm{M}+\mathrm{S}$ combination therapy resulted in a weight loss of $2.2 \mathrm{~kg}(95 \% \mathrm{Cl} 1.7$ to 2.6 ; $\mathrm{P}<0.001 ; 9$ trials; 10,228 participants;
Analysis 3.10). The 95\% prediction interval ranged between $0.8 \mathrm{~kg}$ and $3.5 \mathrm{~kg}$.

\section{HbA1c}

Nine trials reported $\mathrm{HbA} 1 \mathrm{c}$ (random MD $-0.1 \%, 95 \% \mathrm{Cl}-0.1$ to 0.03 ; $\mathrm{P}=0.25$; 9 trials; 9320 participants; fixed MD $-0.1 \%, 95 \% \mathrm{Cl}-0.1$ to -0.04 ; $\mathrm{P}<0.001$; Analysis 3.11 in favour of $\mathrm{M}+\mathrm{S}$ ). The $95 \%$ prediction interval ranged between $-0.3 \%$ and $0.2 \%$.

\section{Metformin-sulfonylurea combination therapy versus metformin plus a long-acting DPP-4 inhibitor}

One trial compared $\mathrm{M}+\mathrm{S}$ with metformin plus a long-acting DPP-4 inhibitor (Handelsman 2017). Glimepiride was given in doses of $1 \mathrm{mg} /$ day to $6 \mathrm{mg} /$ day, omarigliptin in doses of $25 \mathrm{mg} /$ week and metformin in doses of $1500 \mathrm{mg} /$ day or more. For details of the certainty of the evidence see Appendix 19.

\section{Primary outcomes}

\section{All-cause mortality}

In the $M+S$ group no participants died out of 375 participants, compared with 2 deaths in $375(0.5 \%)$ participants in the metformin plus long-acting DPP-4 inhibitor group (RR $0.20,95 \% \mathrm{Cl} 0.01$ to 4.15; $\mathrm{P}=0.30$; very low-certainty evidence because of indirectness and very serious imprecision; Analysis 4.1).

\section{Health-related quality of life}

Handelsman 2017 did not report on this outcome.

\section{Serious adverse effects}

In the $\mathrm{M}+\mathrm{S}$ group $18 / 375$ (4.8\%) participants experienced a serious adverse event compared with $24 / 375(6.4 \%)$ participants in the metformin plus long-acting DPP-4 inhibitor group (RR $0.75,95 \% \mathrm{Cl}$ 0.41 to $1.36 ; P=0.34$; very low-certainty evidence because of very serious imprecision, Analysis 4.2).

\section{Secondary outcomes}

\section{Cardiovascular mortality}

In the $\mathrm{M}+\mathrm{S}$ group no participants out of 375 died compared with 1 death in $375(0.3 \%)$ participants in the metformin plus longacting DPP-4 inhibitor (RR $0.33,95 \% \mathrm{Cl} 0.01$ to 8.16 ; $\mathrm{P}=0.50$; very low-certainty evidence because of indirectness and very serious imprecision; Analysis 4.3).

\section{Non-fatal myocardial infarction}

In the $\mathrm{M}+\mathrm{S}$ group $1 / 375(0.3 \%)$ participants experienced a nonfatal myocardial infarction compared with $0 / 375$ participants in the metformin plus long-acting DPP-4 inhibitor (RR 3.00, 95\% Cl 0.12 to $73.41 ; \mathrm{P}=0.50$; very low-certainty evidence because of indirectness and very serious imprecision, Analysis 4.4).

Heart failure, non-fatal stroke, amputation of lower extremity, blindness or severe vision loss, end-stage renal disease

Handelsman 2017 did not report on these outcomes.

\section{Non-serious adverse events}

In the $\mathrm{M}+\mathrm{S}$ group $125 / 375$ (33.3\%) participants experienced a nonserious adverse event compared with $43 / 375$ (11.5\%) participants in the metformin plus long-acting DPP-4 inhibitor (RR 2.91, 95\% Cl 
2.12 to $3.99 ; P<0.001$; Analysis 4.5), favouring metformin plus longacting DPP-4 inhibitor.

\section{Hypoglycaemia}

In the $\mathrm{M}+\mathrm{S}$ group $110 / 375$ (29.3\%) participants experienced a mild or moderate hypoglycaemic episode compared with $21 / 375$ (5.6\%) participants in the metformin plus long-acting DPP-4 inhibitor (RR $5.24,95 \% \mathrm{Cl} 3.36$ to 8.17 ; $\mathrm{P}<0.001$; Analysis 4.6 in favour of metformin plus long-acting DPP-4 inhibitor).

In the $\mathrm{M}+\mathrm{S}$ group $6 / 375$ (1.6\%) participants experienced a serious hypoglycaemic episode compared with $1 / 375(0.3 \%)$ participant in the metformin plus long-acting DPP-4 inhibitor (RR 6.00, 95\% Cl 0.73 to $49.60 ; P=0.10$; Analysis 4.7 ).

\section{Socioeconomic effects}

Handelsman 2017 did not report on this outcome.

\section{Additional explorative outcomes}

\section{Weight}

Change from baseline in body weight increased by $1.5 \mathrm{~kg}$ (SD 4.0) in 375 participants in the $M+S$ group compared with a weight reduction of $0.4 \mathrm{~kg}$ in 375 participants in the metformin plus longacting DPP-4 inhibitor group (MD 1.9 kg, 95\% Cl 1.3 to 2.5; P < 0.001; Analysis 4.8).

\section{HbA1c}

In the $\mathrm{M}+\mathrm{S}$ group there was a MD in HbAlc change of $-0.5 \%$ in 375 participants compared with $-0.3 \%$ in 375 participants in the metformin plus long-acting DPP-4 inhibitor group (MD of $-0.2 \%$, $95 \% \mathrm{Cl}-0.3$ to $-0.1 ; \mathrm{P}=0.006$; Analysis 4.9 ).

\section{Metformin-sulfonylurea combination therapy versus metformin plus thiazolidinedione}

Eleven trials compared $\mathrm{M}+\mathrm{S}$ combination therapy with metformin plus a thiazolidinedione (Charbonnel 2005; Derosa 2005; Derosa 2009a; Derosa 2011b; Hamann 2008; Home 2009; Maffioli 2013; NCT00367055; Petrica 2009; Petrica 2011; Vaccaro 2017). Four trials administered glimepiride in doses of $2 \mathrm{mg} /$ day to $4 \mathrm{mg} /$ day (Derosa 2005; Derosa 2009a; Petrica 2009; Petrica 2011), two trials administered glibenclamide in doses of $5 \mathrm{mg} /$ day to $15 \mathrm{mg} /$ day (Derosa 2011b; Maffioli 2013), and two trials administered gliclazide in doses of $80 \mathrm{mg} /$ day to $320 \mathrm{mg} /$ day (Charbonnel 2005; NCT00367055). Three trials treated participants with various sulphonylureas: glibenclamide and gliclazide in doses of $5 \mathrm{mg} /$ day to $15 \mathrm{mg} /$ day and $80 \mathrm{mg} /$ day to $320 \mathrm{mg} /$ day, respectively (Hamann 2008); glibenclamide, gliclazide and glimepiride in doses up to $15 \mathrm{mg} /$ day, $240 \mathrm{mg} /$ day and $4 \mathrm{mg} /$ day, respectively (Home 2009); glibenclamide, gliclazide and glimepiride in doses of $5 \mathrm{mg} /$ day to $15 \mathrm{mg} /$ day, $30 \mathrm{mg} /$ day to $120 \mathrm{mg} /$ day and $2 \mathrm{mg} /$ day to $6 \mathrm{mg} /$ day, respectively (Vaccaro 2017). Five trials administered rosiglitazone in doses of $4 \mathrm{mg} /$ day to $8 \mathrm{mg} /$ day (Derosa 2005; Hamann 2008; Home 2009; NCT00367055; Petrica 2009), and six trials administered pioglitazone in doses of $15 \mathrm{mg} /$ day to $45 \mathrm{mg} /$ day (Charbonnel 2005; Derosa 2009a; Derosa 2011b; Maffioli 2013; Petrica 2011; Vaccaro 2017). Metformin was given in doses of 850 $\mathrm{mg} /$ day to $2550 \mathrm{mg} /$ day.

\section{Primary outcomes}

\section{All-cause mortality}

Six trials reported that a total of 237 participants died: in the $M+S$ group 123/3300 (3.7\%) participants died compared with 114/3354 (3.4\%) participants in the metformin plus thiazolidinedione group (RR 1.09, 95\% Cl 0.85 to 1.40; $\mathrm{P}=0.51 ; 6$ trials; 6654 participants; low-certainty evidence; Analysis 5.1). The $95 \%$ prediction interval ranged between 0.75 and 1.55 . One of the trials provided data from a time-to-event analysis on all-cause mortality: the HR for metformin plus pioglitazone versus $\mathrm{M}+\mathrm{S}$ was 1.10 (95\% $\mathrm{Cl} 0.75$ to $1.61, \mathrm{P}=0.63$; Vaccaro 2017).

In one trial, comparing $\mathrm{M}+\mathrm{S}$ with metformin plus pioglitazone, the investigators reported that no participants died but did not provide the number of participants included in the analysis (Derosa 2011b).

A test for subgroup differences comparing rosiglitazone with pioglitazone did not indicate interaction ( $P=0.84$; Analysis 5.1). A test for subgroup differences according to duration of follow-up did not indicate interaction ( $P=0.91$; Analysis 6.1).

Because all trials were published in English, we could not perform sensitivity analyses according to publication status or language of publication. Sensitivity analysis according to trials with low risk of selection bias did not substantially change the effect estimate (RR $1.08,95 \% \mathrm{Cl} 0.84$ to $1.38 ; \mathrm{P}=0.57$; 5 trials; 6570 participants). Sensitivity analysis excluding large trials did not substantially change the effect estimate (RR 2.08, 95\% $\mathrm{Cl} 0.49$ to $8.80 ; \mathrm{P}=0.32 ; 4$ trials; 1404 participants). We could not perform sensitivity analysis excluding trials funded by a pharmaceutical company due to lack of data.

TSA showed that $7.7 \%$ of the diversity-adjusted required information size to detect or reject a 10\% RRR had been accrued. Diversity was $0 \%$. The TSA-adjusted $95 \% \mathrm{Cl}$ was 0.39 to 3.01 .

\section{Health-related quality of life}

None of the included trials reported on this outcome.

\section{Serious adverse effects}

Six trials reported that a total of 1337 participants experienced a serious adverse event: in the M+S group 666/3300 (20.2\%) participants had a serious adverse event compared with 671/3354 (20.0\%) participants in the metformin plus thiazolidinedione group ( $R R$ 1.01, $95 \% \mathrm{Cl} 0.93$ to $1.11 ; \mathrm{P}=0.80 ; 6$ trials; 6654 participants; very low-certainty evidence; Analysis 5.2). The $95 \%$ prediction interval ranged between 0.88 and 1.16 .

Derosa 2011 b compared $M+S$ with metformin plus pioglitazone and reported that no participants experienced a serious adverse event but did not provide the number of participants included in the analysis.

A test for subgroup differences comparing rosiglitazone with pioglitazone did not indicate interaction ( $P=0.84$; Analysis 5.2). A test for subgroup differences according to duration of follow-up did not indicate interaction ( $P=0.28$; Analysis 6.2$)$.

Because all trials were published in English, we could not perform sensitivity analyses according to publication status or language of publication. Sensitivity analysis according to trials with low risk of selection bias did not substantially change the effect estimate 
(RR 1.01, 95\% Cl 0.92 to 1.10; $\mathrm{P}=0.90 ; 5$ trials; 6570 participants) Sensitivity analysis excluding large trials did not substantially change the effect estimate (RR $1.13,95 \% \mathrm{Cl} 0.69$ to 1.86 ; $\mathrm{P}=0.63 ; 4$ trials; 1404 participants). We could not perform sensitivity analysis excluding trials funded by a pharmaceutical company due to lack of data.

TSA showed that $10.6 \%$ of the diversity-adjusted required information size to detect or reject a 10\% RRR had been accrued. Diversity was $81 \%$. The TSA-adjusted $95 \% \mathrm{Cl}$ was 0.41 to 2.22 .

\section{Secondary outcomes}

\section{Cardiovascular mortality}

Four trials reported that a total of 78 participants died due to cardiovascular disease: in the $\mathrm{M}+\mathrm{S}$ group $37 / 2946(1.3 \%)$ participants died compared with $41 / 2994$ (1.4\%) participants in the metformin plus thiazolidinedione group (RR $0.78,95 \% \mathrm{Cl} 0.36$ to 1.67; $\mathrm{P}=0.52 ; 4$ trials; 5940 participants; low-certainty evidence; Analysis 5.3). The $95 \%$ prediction interval ranged between 0.07 and 8.92. Vaccaro 2017 provided data from a time-to-event analysis on cardiovascular mortality: metformin plus pioglitazone versus $\mathrm{M}+\mathrm{S}$ had a HR of $2.24(95 \% \mathrm{Cl} 0.69$ to $7.28, \mathrm{P}=0.18)$. Derosa 2011b, compared $\mathrm{M}+\mathrm{S}$ with metformin plus pioglitazone and reported that no participants died due to cardiovascular disease but did not provide the number of participants included in the analysis.

A test for subgroup differences comparing rosiglitazone with pioglitazone did not indicate interaction ( $P=0.40$; Analysis 5.3). A test for subgroup differences according to duration of follow-up did not indicate interaction ( $P=0.36$; Analysis 6.3 ).

Because all trials were published in English, we could not perform sensitivity analyses according to publication status or language of publication. We could not perform sensitivity analysis according to trials with low risk of selection bias since all trials were evaluated as low risk of selection bias. We could not perform sensitivity analyses excluding large trials and trials funded by a pharmaceutical company due to lack of data.

TSA showed that $1.3 \%$ of the diversity-adjusted required information size to detect or reject a 10\% RRR had been accrued. Diversity was $66 \%$. As only a minor fraction of the diversity-adjusted required information size to detect or reject a 10\% RRR had been accrued, we could not calculate the TSA-adjusted $95 \% \mathrm{Cl}$.

\section{Non-fatal myocardial infarction}

Three trials reported that a total of 46 participants experienced a non-fatal myocardial infarction: in the $M+S$ group 25/1841 (1.4\%) participants had a non-fatal myocardial infarction compared with $21 / 1877$ (1.1\%) participants in the metformin plus thiazolidinedione group ( $R R 1.21,95 \% \mathrm{Cl} 0.68$ to $2.14 ; \mathrm{P}=0.51 ; 3$ trials; 3718 participants; very low-certainty evidence; Analysis 5.4). The $95 \%$ prediction interval ranged between 0.03 and 48.76 .

Vaccaro 2017 provided data from a time-to-event analysis on non-fatal myocardial infarction: $M+S$ versus metformin plus pioglitazone had a HR of $0.87(95 \% \mathrm{Cl} 0.48$ to $1.55 ; \mathrm{P}=0.63)$.

Derosa $2011 \mathrm{~b}$ compared $\mathrm{M}+\mathrm{S}$ with metformin plus pioglitazone and reported that no participants experienced a non-fatal myocardial infarction but did not provide the number of participants included in the analysis.
A test for subgroup differences comparing rosiglitazone with pioglitazone did not indicate interaction ( $P=0.58$; Analysis 5.4). A test for subgroup differences according to duration of follow-up did not indicate interaction ( $P=0.58$; Analysis 6.4).

Because all trials were published in English, we could not perform sensitivity analyses according to publication status or language of publication. We could not perform sensitivity analysis according to trials with low risk of selection bias because all trials were evaluated as low risk of selection bias. Sensitivity analyses excluding large trials and trials funded by a pharmaceutical company could not be performed due to lack of data.

TSA showed that $1.72 \%$ of the diversity-adjusted required information size to detect or reject a 10\% RRR had been accrued. Diversity was $0 \%$. As only a minor fraction of the diversity-adjusted required information size to detect or reject a 10\% RRR had been accrued, we could not calculate the TSA-adjusted $95 \% \mathrm{Cl}$.

\section{Heart failure}

Five trials reported that a total of 83 participants developed heart failure: in the $\mathrm{M}+\mathrm{S}$ group 33/3259 (1.0\%) participants developed heart failure compared with 50/3311 (1.5\%) participants in the metformin plus thiazolidinedione group (RR $0.67,95 \% \mathrm{Cl} 0.43$ to 1.04; $\mathrm{P}=0.08 ; 5$ trials; 6570 participants; Analysis 5.5). The $95 \%$ prediction interval ranged between 0.33 and 1.37 .

Vaccaro 2017 provided data from a time-to-event analysis on heart failure: the HR comparing participants in the sulphonylureas group with participants in the pioglitazone group was $1.57(95 \% \mathrm{Cl} 0.76$ to $3.24 ; \mathrm{P}=0.22$.

Derosa 2011 b compared $\mathrm{M}+\mathrm{S}$ with metformin plus pioglitazone and reported that no participants developed heart failure but did not report the number of participants included in the analysis.

A test for subgroup differences comparing rosiglitazone with pioglitazone did not indicate interaction ( $P=0.65$; Analysis 5.5). A test for subgroup differences according to duration of follow-up did not indicate interaction ( $P=0.79$; Analysis 6.5$)$.

Because all trials were published in English, we could not perform sensitivity analyses according to publication status or language of publication. We could not perform sensitivity analysis according to trials with low risk of selection bias because all trials were evaluated as low risk of selection bias. Sensitivity analysis excluding large trials did not substantially change the effect estimate (RR 0.51 , $95 \% \mathrm{Cl} 0.12$ to $2.07 ; \mathrm{P}=0.35 ; 3$ trials; 1320 participants). We could not perform sensitivity analysis excluding trials funded by a pharmaceutical company due to lack of data.

TSA showed that $3.3 \%$ of the diversity-adjusted required information size to detect or reject a 10\% RRR had been accrued. Diversity was $0 \%$. As only a minor fraction of the diversity-adjusted required information size to detect or reject a 10\% RRR had been accrued, we could not calculate the TSA-adjusted $95 \% \mathrm{Cl}$.

\section{Non-fatal stroke}

Two trials compared $\mathrm{M}+\mathrm{S}$ with metformin plus thiazolidinedione: in the $\mathrm{M}+\mathrm{S}$ group $20 / 1540(1.3 \%)$ participants had a non-fatal stroke compared with $16 / 1583(1 \%)$ participants in the metformin plus thiazolidinedione group (RR $1.29,95 \% \mathrm{Cl} 0.67$ to $2.47 ; \mathrm{P}=0.45 ; 2$ 
trials; 3123 participants; very low-certainty evidence; Analysis 5.6). Vaccaro 2017 administered pioglitazone combined with metformin and also provided data from a time-to-event analysis on non-fatal stroke: $\mathrm{M}+\mathrm{S}$ compared with metformin plus pioglitazone showed a HR of 0.79 (95\% Cl 0.41 to $1.53 ; \mathrm{P}=0.49 ; 3028$ participants). Only Vaccaro 2017, in his trial of long duration, observed non-fatal strokes (Analysis 6.6).

Derosa 2011b administered pioglitazone combined with metformin and reported that no participants experienced a non-fatal stroke but did not provide the number of participants included in the analysis.

\section{Amputation of lower extremity}

Two trials with 3123 participants provided data on amputation of lower extremity (very low-certainty evidence; Analysis 5.7). Neither of the trials reported any events.

\section{Blindness or severe vision loss}

One trial with 95 participants provided data on blindness or severe vision loss (very low-certainty evidence; Analysis 5.8). They did not report any events.

\section{End-stage renal disease}

One trial with 95 participants provided data on end-stage renal disease (very low-certainty evidence; Analysis 5.9). They did not report any events.

\section{Non-serious adverse events}

Five trials reported that a total of 3072 participants experienced a non-serious adverse event: in the M+S group 1510/2987 (50.6\%) participants had a non-serious adverse event compared with $1562 / 3037(51.4 \%)$ participants in the metformin plus thiazolidinedione group (RR $0.94,95 \% \mathrm{Cl} 0.44$ to $2.01 ; \mathrm{P}=0.87 ; 5$ trials; 6024 participants; Analysis 5.10). The $95 \%$ prediction interval ranged between 0.05 and 16.49 .

Derosa 2011b compared M+S with metformin plus pioglitazone and reported that two and three participants experienced non-serious adverse events, respectively. However, they did not provide the number of participants included in the analysis.

A test for subgroup differences comparing rosiglitazone with pioglitazone did not indicate interaction $(P=0.36$; Analysis 5.10). A test for subgroup differences according to duration of follow-up did not indicate interaction ( $P=0.94$; Analysis 6.7).

Because all trials were published in English, we could not perform sensitivity analyses according to publication status or language of publication. Sensitivity analysis according to trials with low risk of selection bias did not substantially change the effect estimate (RR $0.92,95 \% \mathrm{Cl} 0.36$ to $2.35 ; \mathrm{P}=0.87 ; 4$ trials; 5940 participants). Sensitivity analysis excluding large trials did not substantially change the effect estimate (RR $0.97,95 \% \mathrm{Cl} 0.76$ to $1.25 ; \mathrm{P}=0.83 ; 3$ trials; 774 participants). Sensitivity analysis excluding trials funded by a pharmaceutical company did not substantially change the effect estimate (RR $0.93,95 \% \mathrm{Cl} 0.82$ to $1.05 ; \mathrm{P}=0.24 ; 2$ trials; 3123 participants).

TSA showed that the cumulative z-curve crossed the futility boundaries suggesting that a $10 \%$ or greater RRR could be rejected at this point. Diversity was $0 \%$. The TSA-adjusted $95 \% \mathrm{Cl}$ was 0.74 to 1.19 .

\section{Hypoglycaemia}

Five trials reported that a total of 926 participants had a mild or moderate hypoglycaemic episode: in the M+S group 721/2999 $(24.0 \%)$ participants had a mild or moderate hypoglycaemic episode compared with 205/3060 (6.7\%) participants in the metformin plus thiazolidinedione group (RR $3.63,95 \% \mathrm{Cl} 2.98$ to 4.44; $\mathrm{P}<0.001 ; 5$ trials; 6059 participants; Analysis 5.11 in favour of metformin plus thiazolidinedione). The $95 \%$ prediction interval ranged between 2.30 and 5.73. Derosa 2011b compared M+S with metformin plus pioglitazone and reported two and one events of mild/moderate hypoglycaemia, respectively. However, they did not provide the number of participants included in the analysis.

A test for subgroup differences comparing rosiglitazone with pioglitazone did not indicate interaction ( $P=0.62$; Analysis 5.11). A test for subgroup differences according to duration of follow-up did not indicate interaction ( $P=0.48$; Analysis 6.8).

Because all trials were published in English, we could not perform sensitivity analyses according to publication status or language of publication. Sensitivity analysis according to trials with low risk of selection bias did not substantially change the effect estimate (RR $3.63,95 \% \mathrm{Cl} 2.92$ to $4.52 ; \mathrm{P}<0.00001 ; 4$ trials; 5975 participants). Sensitivity analysis excluding large trials did not substantially change the effect estimate (RR 5.99, 95\% Cl 2.43 to 14.76; $\mathrm{P}=$ $0.0001 ; 3$ trials; 809 participants). Sensitivity analysis excluding trials funded by a pharmaceutical company did not substantially change the effect estimate (RR 3.37, 95\% Cl 2.84 to 3.99; $\mathrm{P}<0.00001$; 2 trials; 3123 participants).

TSA showed that $8.0 \%$ of the diversity-adjusted required information size to detect or reject a $10 \%$ RRR had been accrued. Diversity was $45 \%$. The TSA-adjusted $95 \% \mathrm{Cl}$ was 1.63 to 8.09 .

Five trials reported that 36 participants experienced serious hypoglycaemia: in the M+S group 30/3259 (0.9\%) participants had a serious hypoglycaemic episode compared with 6/3311 $(0.2 \%)$ participants in the metformin plus thiazolidinedione group (random RR 3.98, 95\% Cl 0.34 to 46.01; $\mathrm{P}=0.27$; fixed RR 4.77, $95 \% \mathrm{Cl} 2.05$ to 11.09 ; $\mathrm{P}<0.001 ; 5$ trials; 6570 participants; Analysis 5.12; in favour of metformin plus thiazolidinedione). However, there was substantial heterogeneity, probably caused by various definitions of serious hypoglycaemia. Only one trial provided a clear description of serious hypoglycaemia (Vaccaro 2017).

Derosa 2011b compared M+S with metformin plus pioglitazone and reported no serious hypoglycaemic events. However, they did not provide the number of participants included in the analysis.

A test for subgroup differences comparing rosiglitazone with pioglitazone showed a statistically significant difference between subgroups ( $P=0.009$; Analysis 5.12 ). However, Cls overlap slightly indicating that in fact there was no true interaction. A test for subgroup differences according to duration of follow-up did not indicate interaction $(P=0.85$; Analysis 6.9).

Because all trials were published in English, we could not perform sensitivity analyses according to publication status or language of publication. We could not perform sensitivity analysis according to trials with low risk of selection bias because all trials were evaluated 
as low risk of selection bias. We could not perform sensitivity analysis of large trials and trials funded by a pharmaceutical company due to lack of data.

TSA showed that $0.08 \%$ of the diversity-adjusted required information size to detect or reject a 10\% RRR had been accrued. Diversity was $79 \%$. As only a minor fraction of the diversity-adjusted required information size to detect or reject a 10\% RRR had been accrued, we could not calculate the TSA-adjusted $95 \% \mathrm{Cl}$.

\section{Socioeconomic effects}

None of the included trials reported on this outcome.

\section{Additional explorative outcomes}

\section{Weight}

Seven trials reported weight change (random MD $-0.6 \mathrm{~kg}, 95 \%$ $\mathrm{Cl}-2.8$ to $1.6 ; \mathrm{P}=0.62$; fixed $\mathrm{MD}-2.0 \mathrm{~kg}, 95 \% \mathrm{Cl}-2.4$ to -1.6 ; $\mathrm{P}<0.001 ; 7$ trials; 6877 participants; Analysis 5.13; in favour of $\mathrm{M}+\mathrm{S}$ ). The $95 \%$ prediction interval ranged between $-8.3 \mathrm{~kg}$ and $7.2 \mathrm{~kg}$.There was substantial heterogeneity probably caused by two of the rosiglitazone trials (Derosa 2005; Home 2009), and by duration of follow-up (ranging from one year to 5.5 years), various sulphonylureas (glimepiride, glibenclamide, gliclazide) and various doses of rosiglitazone ranging from $4 \mathrm{mg} /$ day to $8 \mathrm{mg} /$ day.

\section{HbA1c}

Ten trials reported change in $\mathrm{HbAlc}$ (random MD 0.2\%, 95\% Cl 0.04 to $0.3 ; \mathrm{P}=0.01$; fixed $\mathrm{MD} 0.2 \%, 95 \% \mathrm{Cl} 0.1$ to $0.2 ; \mathrm{P}<0.001 ; 10$ trials; 7020 participants; Analysis 5.14; in favour of metformin plus thiazolidinedione). The 95\% prediction interval ranged between $-0.2 \%$ and $0.5 \%$. There was substantial heterogeneity probably caused by duration of follow-up (ranging from 1 year to 5.5 years), various sulphonylureas (glimepiride, glibenclamide, gliclazide) and various doses of sulphonylureas and thiazolidinediones.

Derosa 2009a reported $\mathrm{HbA1c}$ final values of $7.8 \%$ (SD 0.4) in the $\mathrm{M}+\mathrm{S}$ group and $7.2 \%$ (SD 0.3) in the metformin plus pioglitazone group. However, they did not provide the number of participants included in the analysis.

\section{Metformin-sulfonylurea combination therapy versus metformin plus glinide}

Three trials compared $\mathrm{M}+\mathrm{S}$ combination therapy with metformin plus a glinide (Derosa 2009b; Gerich 2005; Ristic 2007). Two trials administered glibenclamide in doses of $1.25 \mathrm{mg} /$ day to $15 \mathrm{mg} /$ day and one trial administered gliclazide in doses of $80 \mathrm{mg} /$ day to 240 $\mathrm{mg} /$ day. All trials administered nateglinide in doses of $180 \mathrm{mg} /$ day to $540 \mathrm{mg} /$ day. The trials administered metformin in doses of 500 $\mathrm{mg} /$ day to $3000 \mathrm{mg} /$ day.

\section{Primary outcomes}

\section{All-cause mortality}

Three trials with 874 participants reported data on all-cause mortality and one person died in each intervention group (lowcertainty evidence; Analysis 7.1)

\section{Health-related quality of life}

None of the included trials reported on this outcome.

\section{Serious adverse effects}

Three trials reported that a total of 61 participants experienced a serious adverse event: in the $\mathrm{M}+\mathrm{S}$ group 34/424 (8\%) participants had a serious adverse event compared with 27/450 (6\%) participants in the metformin plus thiazolidinedione group (RR $1.68,95 \% \mathrm{Cl} 0.54$ to $5.21 ; \mathrm{P}=0.37 ; 3$ trials; 874 participants; lowcertainty evidence; Analysis 7.2).

In one trial serious adverse events were reported for up to six months and six to 12 months separately. For up to six months 5/126 (4.0\%) participants in the $\mathrm{M}+\mathrm{S}$ group had a serious adverse event compared with $7 / 130(5.4 \%)$ participants in the metformin plus glinide group. For six to 12 months, 7/101 (6.9\%) participants and $2 / 112(1.8 \%)$ participants experienced a serious adverse event in the $\mathrm{M}+\mathrm{S}$ group compared with the metformin plus glinide group, respectively (Ristic 2007).

\section{Secondary outcomes}

\section{Cardiovascular mortality}

Two trials with 446 participants provided data on cardiovascular mortality (Derosa 2009b; Ristic 2007). No cardiovascular death was observed in either trial (low-certainty evidence; Analysis 7.3).

\section{Non-fatal myocardial infarction}

Two trials provided data on non-fatal myocardial infarction in 446 participants. In total two non-fatal myocardial infarctions were reported in $2 / 215(0.9 \%)$ participants in the $\mathrm{M}+\mathrm{S}$ group compared with $0 / 231$ participants in the metformin plus thiazolidinedione group (2 trials; 446 participants; low-certainty evidence). Derosa $2009 \mathrm{~b}$ stated that no participants experienced a non-fatal myocardial infarction. One trial reported non-fatal myocardial infarction for up to six months and six to 12 months separately (Ristic 2007). For up to six months no event occurred in either intervention group. For six to 12 months, 2/101 (2\%) participants had non-fatal myocardial infarction in the $\mathrm{M}+\mathrm{S}$ group compared to $0 / 112$ participant in the metformin plus glinide group (RR 5.54, 95\% $\mathrm{Cl} 0.27$ to $114.02 ; \mathrm{P}=0.27$ ).

\section{Heart failure}

Two trials provided data on heart failure. Derosa 2009b stated that no heart failure occurred. One trial reported data on heart failure for up to six months and six to 12 months separately. For up to six months no event occurred in either intervention group. For six to 12 months, 0/101 participants and 1/112 (0.9\%) participants developed heart failure in the $\mathrm{M}+\mathrm{S}$ group compared to the metformin plus glinide group, respectively (Ristic 2007).

\section{Non-fatal stroke}

Derosa 2009b stated that no non-fatal stroke occurred (233 participants; very low-certainty evidence).

\section{Amputation of lower extremity}

Derosa 2009b stated that no amputation of lower extremity occurred (233 participants; low-certainty evidence).

\section{Blindness or severe vision loss}

Derosa 2009b stated that no blindness or severe vision loss occurred (233 participants; low-certainty evidence). 


\section{End-stage renal disease}

Derosa 2009b stated that no end-stage renal disease occurred (233 participants; low-certainty evidence).

\section{Non-serious adverse events}

Derosa 2009b stated that no non-serious adverse events occurred.

\section{Hypoglycaemia}

Two trials provided data on mild or moderate hypoglycaemia. Gerich 2005 reported data from a subgroup analysis of participants 65 years and older: in the $\mathrm{M}+\mathrm{S}$ group $7 / 40(17.5 \%)$ participants had mild or moderate hypoglycaemia compared with 1/35 (2.9\%) participants in the metformin plus glinide group (Analysis 7.4). Derosa 2009b reported two mild or moderate hypoglycaemic episodes in the $\mathrm{M}+\mathrm{S}$ group compared with three events in the metformin plus glinide group.

Two trials provided data on serious hypoglycaemia. Gerich 2005 reported that $2 / 209(1 \%)$ participants in the $M+S$ group compared with 0/219 participants in the metformin plus glinide group experienced a serious hypoglycaemic episode (Analysis 7.5). Derosa 2009 b stated that no serious hypoglycaemia occurred.

\section{Socioeconomic effects}

Neither of the included trials reported on this outcome.

\section{Additional explorative outcomes}

\section{Weight}

Two trials reported weight change (MD $1.1 \mathrm{~kg}, 95 \% \mathrm{Cl}-0.1$ to 2.3; $\mathrm{P}$ = 0.06; 2 trials; 619 participants; Analysis 7.6).

\section{HbAlc}

Three trials reported change in $\mathrm{HbAlc}$ (random MD 0.2\%, 95\% $\mathrm{Cl}-0.6$ to $1.0 ; \mathrm{P}=0.69$; fixed $\mathrm{MD} 0.4 \%, 95 \% \mathrm{Cl} 0.3$ to $0.5 ; \mathrm{P}$ $<0.00001$; 3 trials; 852 participants; Analysis 7.7; in favour of metformin plus glinide). Calculation of the $95 \%$ prediction interval did not provide a meaningful estimate. There was substantial heterogeneity probably caused by various durations of follow-up (ranging from 52 weeks to 104 weeks), various sulphonylureas (glibenclamide and gliclazide) and various doses of nateglinide (ranging from $180 \mathrm{mg} /$ day to $540 \mathrm{mg} /$ day).

\section{Metformin-sulfonylurea combination therapy versus metformin plus sodium-glucose co-transporter 2 (SGLT-2) inhibitor}

Four trials compared $\mathrm{M}+\mathrm{S}$ combination therapy with metformin plus a SGLT-2 inhibitor (Del Prato 2015; Hollander 2017; Leiter 2015; Ridderstråle 2014). Three trials administered glimepiride in doses of $1 \mathrm{mg} /$ day to $8 \mathrm{mg} /$ day (Hollander 2017; Leiter 2015; Ridderstråle 2014), and one trial administered glipizide in doses of $5 \mathrm{mg} / \mathrm{day}$ to $20 \mathrm{mg} /$ day (Del Prato 2015). All trials administered various SGLT-2 inhibitors: dapagliflozin in doses of $2.5 \mathrm{mg} /$ day to $10 \mathrm{mg} /$ day (Del Prato 2015), ertugliflozin in doses of $15 \mathrm{mg} /$ day (Hollander 2017), canagliflozin in doses of $100 \mathrm{mg} /$ day to $300 \mathrm{mg} /$ day (Leiter 2015), and empagliflozin in doses of 25 mg/day (Ridderstråle 2014). Metformin was given in doses of $1000 \mathrm{mg} /$ day to $1500 \mathrm{mg} /$ day or more.

\section{Primary outcomes}

\section{All-cause mortality}

Four trials reported that a total of 32 participants died: in the $M$ +S group 13/2107 (0.6\%) participants died compared with 19/3027 $(0.6 \%)$ participants in the metformin plus SGLT-2 inhibitor group (RR 0.96, $95 \% \mathrm{Cl} 0.44$ to $2.09 ; \mathrm{P}=0.91 ; 4$ trials; 5134 participants; very low-certainty evidence; Analysis 8.1 ). The $95 \%$ prediction interval ranged between 0.17 and 5.30 .

Because all trials were published in English, we could not perform sensitivity analyses according to publication status or language of publication. We could not perform sensitivity analysis according to trials with low risk of selection bias because all trials were evaluated as low risk of selection bias. Sensitivity analysis excluding long trials did not substantially change the effect estimate (RR 0.72 , $95 \% \mathrm{Cl} 0.30$ to $1.76 ; \mathrm{P}=0.47 ; 3$ trials; 4320 participants). One trial reported data for 104 weeks (Ridderstråle 2014). We could not perform sensitivity analysis excluding large trials because all trials randomised fewer than 1000 participants to each intervention group. We could not perform sensitivity analysis excluding trials funded by a pharmaceutical company because all trials received funding from a pharmaceutical company.

TSA showed that $1.04 \%$ of the diversity-adjusted required information size to detect or reject a $10 \%$ RRR had been accrued. Diversity was $0 \%$. As only a minor fraction of the diversity-adjusted required information size to detect or reject a 10\% RRR had been accrued, we could not calculate the TSA-adjusted $95 \% \mathrm{Cl}$.

\section{Health-related quality of life}

None of the included trials reported on this outcome.

\section{Serious adverse effects}

Four trials reported that a total of 690 participants experienced a serious adverse event: in the $M+S$ group 315/2107 (15.5\%) participants had a serious adverse event compared with 375/3027 (12.4\%) participants in the metformin plus SGLT-2 inhibitor group (RR $1.02,95 \% \mathrm{Cl} 0.76$ to $1.37 ; \mathrm{P}=0.90 ; 4$ trials; 5134 participants; very low-certainty evidence; Analysis 8.2 ). The $95 \%$ prediction interval ranged between 0.30 and 3.51 .

Because all trials were published in English, we could not perform sensitivity analyses according to publication status or language of publication. We could not perform sensitivity analysis according to trials with low risk of selection bias because all trials were evaluated as low risk of selection bias. Sensitivity analysis excluding long trials did not substantially change the effect estimate (RR 0.93 , $95 \% \mathrm{Cl} 0.35$ to $2.49 ; \mathrm{P}=0.88 ; 2$ trials; 2775 participants). We could not perform sensitivity analysis excluding large trials because all trials randomised fewer than 1000 participants to each intervention group. We could not perform sensitivity analysis excluding trials funded by a pharmaceutical company because all trials received funding from a pharmaceutical company.

TSA showed that $12.4 \%$ of the diversity-adjusted required information size to detect or reject a $10 \%$ RRR had been accrued. Diversity was $78 \%$. The TSA-adjusted $95 \% \mathrm{Cl}$ was 0.31 to 3.36 . 


\section{Secondary outcomes}

\section{Cardiovascular mortality}

Three trials reported that a total of 10 participants died due to cardiovascular disease: in the $\mathrm{M}+\mathrm{S}$ group $4 / 1327$ (0.3\%) participants died compared with $6 / 2262(0.3 \%)$ participants in the metformin plus SGLT-2 inhibitor group (RR $1.22,95 \% \mathrm{Cl} 0.33$ to 4.41; $\mathrm{P}=0.77$; 3 trials; 3589 participants; very low-certainty evidence; Analysis 8.3). Calculation of the $95 \%$ prediction interval did not provide a meaningful estimate.

Because all trials were published in English, we could not perform sensitivity analyses according to publication status or language of publication. We could not perform sensitivity analysis according to trials with low risk of selection bias because all trials were evaluated as low risk of selection bias. Sensitivity analysis excluding long trials did not substantially change the effect estimate (RR 1.02, $95 \% \mathrm{Cl} 0.25$ to $4.18 ; \mathrm{P}=0.98 ; 2$ trials; 2775 participants). We could not perform sensitivity analysis excluding large trials because all trials randomised fewer than 1000 participants to each intervention group. We could not perform sensitivity analysis excluding trials funded by a pharmaceutical company because all trials received funding from a pharmaceutical company.

TSA showed that $0.36 \%$ of the diversity-adjusted required information size to detect or reject a 10\% RRR had been accrued. Diversity was $0 \%$. As only a minor fraction of the diversity-adjusted required information size to detect or reject a 10\% RRR had been accrued, we could not calculate the TSA-adjusted $95 \% \mathrm{Cl}$.

\section{Non-fatal myocardial infarction}

Two trials reported that a total of 15 participants experienced a non-fatal myocardial infarction: in the $M+S$ group $7 / 890(0.8 \%)$ participants had a non-fatal myocardial infarction compared with $8 / 1374(0.6 \%)$ participants in the metformin plus SGLT-2 inhibitor group (RR 1.43, 95\% Cl 0.49 to $4.18 ; \mathrm{P}=0.52 ; 2$ trials; 2264 participants; very low-certainty evidence; Analysis 8.4).

We could not perform sensitivity analyses due to lack of data.

TSA showed that $0.46 \%$ of the diversity-adjusted required information size to detect or reject a $10 \%$ RRR had been accrued. Diversity was $0 \%$. As only a minor fraction of the diversity-adjusted required information size to detect or reject a 10\% RRR had been accrued, we could not calculate the TSA-adjusted $95 \% \mathrm{Cl}$.

\section{Heart failure}

Three trials reported that a total of four participants developed heart failure: in the $M+S$ group $4 / 1670$ (0.2\%) participants developed heart failure compared with $0 / 2139$ participant in the metformin plus SGLT-2 inhibitor group (Peto OR 9.21, 95\% Cl 1.26 to 67.24; $\mathrm{P}=0.03 ; 3$ trials; 3809 participants; Analysis 8.5).

Because all trials were published in English, we could not perform sensitivity analyses according to publication status or language of publication. We could not perform sensitivity analysis according to trials with low risk of selection bias because all trials were evaluated as low risk of selection bias. Sensitivity analysis excluding long trials did not substantially change the effect estimate but widened the $\mathrm{Cl}$ (Peto OR 11.84, 95\% Cl 0.68 to 205.34; $\mathrm{P}=0.09 ; 2$ trials; 2264 participants). We could not perform sensitivity analysis excluding large trials because all trials randomised fewer than 1000 participants to each intervention group. We could not perform sensitivity analysis excluding trials funded by a pharmaceutical company because all trials received funding from a pharmaceutical company.

None of the participants in the comparator group (metformin plus SGLT-2 inhibitor) developed heart failure. TSA could therefore not be performed as it was not possible to calculate an event rate in the comparator group. However, applying 0.5 events in each of the trials in the comparator group showed that $10.9 \%$ of the diversityadjusted required information size to detect or reject a 10\% RRR had been accrued. Diversity was 0\%. The TSA-adjusted 95\% $\mathrm{Cl}$ did not provide a meaningful estimate.

\section{Non-fatal stroke}

Two trials reported that a total of 10 participants experienced a non-fatal stroke: in the M+S group 3/919 $(0.3 \%)$ participants had a non-fatal stroke compared with $7 / 1856(0.4 \%)$ participants in the metformin plus SGLT-2 inhibitor group (RR $0.87,95 \% \mathrm{Cl} 0.22$ to 3.34; $\mathrm{P}=0.83 ; 2$ trials; 2775 participants; very low-certainty evidence; Analysis 8.6).

We could not perform sensitivity analyses due to lack of data.

TSA showed that $0.37 \%$ of the diversity-adjusted required information size to detect or reject a $10 \%$ RRR had been accrued. Diversity was $0 \%$. As only a minor fraction of the diversity-adjusted required information size to detect or reject a 10\% RRR had been accrued, we could not calculate the TSA-adjusted $95 \% \mathrm{Cl}$.

\section{Amputation of lower extremity}

Hollander 2017 reported one amputation of lower extremity in $437(0.2 \%)$ participants in the $M+S$ group compared with one amputation in $888(0.1 \%)$ participants in the metformin plus SGLT-2 inhibitor group (very low-certainty evidence, Analysis 8.7).

\section{Blindness or severe vision loss}

None of the included trials reported on this outcome.

\section{End-stage renal disease}

None of the included trials reported on this outcome.

\section{Non-serious adverse events}

Three trials reported that a total of 2284 participants had nonserious adverse events: in the $M+S$ group $1139 / 1670$ (68.2\%) participants experienced a non-serious adverse event compared with $1145 / 2139$ (53.5\%) participants in the metformin plus SGLT-2 inhibitor group (RR $1.27,95 \% \mathrm{Cl} 1.01$ to $1.59 ; \mathrm{P}=0.04 ; 3$ trials; 3809 participants; Analysis 8.8 ; in favour of metformin plus SGLT-2 inhibitor). The $95 \%$ prediction interval ranged between 0.07 and 23.77. None of the trials provided a detailed definition of this outcome measure.

Because all trials were published in English, we could not perform sensitivity analyses according to publication status or language of publication. We could not perform sensitivity analysis according to trials with low risk of selection bias because all trials were evaluated as low risk of selection bias. Sensitivity analysis excluding long trials did not substantially change the direction of the effect estimate (RR $1.38,95 \% \mathrm{Cl} 1.06$ to $1.80 ; \mathrm{P}=0.02 ; 2$ trials; 2264 participants). We included one trial with a duration of intervention longer than 104 weeks of treatment in the analysis because they reported data for 
non-serious adverse events after 52 weeks of treatment (Del Prato 2015). We could not perform sensitivity analysis excluding large trials because all trials randomised fewer than 1000 participants to each intervention group. We could not perform sensitivity analysis excluding trials funded by a pharmaceutical company because all trials received funding from a pharmaceutical company.

TSA showed that $6.2 \%$ of the diversity-adjusted required information size to detect or reject a 10\% RRR had been accrued. Diversity was $96 \%$. The TSA-adjusted $95 \% \mathrm{Cl}$ was 0.53 to 3.01 .

\section{Hypoglycaemia}

Three trials reported that a total of 603 participants experienced mild or moderate hypoglycaemia: in the M+S group 514/1670 $(30.8 \%)$ participants had a mild or moderate hypoglycaemic episode compared with $89 / 1639$ (5.4\%) participants in the metformin plus SGLT-2 inhibitor group (RR $5.60,95 \% \mathrm{Cl} 2.38$ to 13.14; $\mathrm{P}<0.001 ; 3$ trials; 3309 participants; Analysis 8.9; in favour of metformin plus SGLT-2 inhibitor). Calculation of the $95 \%$ prediction interval did not provide a meaningful estimate.

Because all trials were published in English, we could not perform sensitivity analyses according to publication status or language of publication. We could not perform sensitivity analysis according to trials with low risk of selection bias because all trials were evaluated as low risk of selection bias. We could not perform sensitivity analysis excluding long trials due to lack of data. We could not perform sensitivity analysis excluding large trials because all trials randomised fewer than 1000 participants to each intervention group. We could not perform sensitivity analysis excluding trials funded by a pharmaceutical company because all trials received funding from a pharmaceutical company.

TSA showed that $0.44 \%$ of the diversity-adjusted required information size to detect or reject a 10\% RRR had been accrued. Diversity was $93 \%$. As only a minor fraction of the diversity-adjusted required information size to detect or reject a 10\% RRR had been accrued, we could not calculate the TSA-adjusted $95 \% \mathrm{CI}$.

Four trials reported that a total of 38 participants had serious hypoglycaemia: in the M+S group 30/2107 (1.4\%) participants had a serious hypoglycaemic episode compared with $8 / 3027(0.3 \%)$ participants in the metformin plus SGLT-2 inhibitor group (RR $6.16,95 \% \mathrm{Cl} 2.92$ to $12.97 ; \mathrm{P}<0.001 ; 4$ trials; 5134 participants; Analysis 8.10; in favour of metformin plus SGLT-2 inhibitor). The $95 \%$ prediction interval ranged between 1.20 and 31.58 . For one of the trials included in the meta-analysis, the number of serious hypoglycaemic events was unclear due to varied reporting (Leiter 2015). We contacted the trial authors for clarification but did not receive a reply. To be sure to have included all serious hypoglycaemic events, we extracted the highest number of serious hypoglycaemic events reported.

Because all trials were published in English, we could not perform sensitivity analyses according to publication status or language of publication. We could not perform sensitivity analysis according to trials with low risk of selection bias because all trials were evaluated as low risk of selection bias. Sensitivity analysis excluding long trials did not substantially change the effect estimate (RR 6.39, 95\% Cl 2.89 to 14.12 ; $\mathrm{P}<0.001 ; 2$ trials; 2775 participants). We could not perform sensitivity analysis excluding large trials because all trials randomised fewer than 1000 participants to each intervention group. We could not perform sensitivity analysis excluding trials funded by a pharmaceutical company because all trials received funding from a pharmaceutical company.

TSA showed that $0.56 \%$ of the diversity-adjusted required information size to detect or reject a 10\% RRR had been accrued. Diversity was $0 \%$. As only a minor fraction of the diversity-adjusted required information size to detect or reject a 10\% RRR had been accrued, we could not calculate the TSA-adjusted $95 \% \mathrm{Cl}$.

\section{Socioeconomic effects}

One trial performed three economic analyses of trial data using the Cardiff Diabetes Model (Del Prato 2015). One economic analysis aimed to assess the cost-effectiveness of SGLT-2 inhibitor compared with sulphonylurea when added to metformin for treatment of people in the UK with diabetes mellitus inadequately controlled on metformin alone. There was a mean incremental benefit of 0.47 quality-adjusted life years $(95 \% \mathrm{Cl} 0.42$ to 0.67$)$, calculated for dapagliflozin plus metformin, the incremental cost-effectiveness ratio point estimate was GBP 2671 per quality-adjusted life year.

Another economic analysis aimed to assess the cost-effectiveness of SGLT-2 inhibitor compared with sulphonylurea when added to metformin for treatment of Nordic people with diabetes mellitus inadequately controlled on metformin alone. The mean lifetime gain in quality-adjusted life years for metformin plus dapagliflozin compared to $\mathrm{M}+\mathrm{S}$ was 0.25 in Denmark, 0.27 in Finland, 0.24 in Norway and 0.28 in Sweden. The cost per quality-adjusted life year gained was EUR 7944 in Denmark, EUR 5424 in Finland, EUR 4769 in Norway and EUR 6093 in Sweden.

The third economic analysis aimed to assess the cost-effectiveness of SGLT-2 inhibitor compared with sulphonylurea when added to metformin for treatment of Spanish people with diabetes mellitus inadequately controlled on metformin alone. Dapagliflozin was a cost-effective option compared with sulphonylureas and resulted in a cost per quality-adjusted life year gained of EUR 3560.

\section{Additional explorative outcomes \\ Weight}

Three trials reported weight change (MD $4.4 \mathrm{~kg}, 95 \% \mathrm{Cl} 4.1$ to 4.8; $\mathrm{P}<0.001$; 3 trials; 3294 participants; Analysis 8.11; in favour of metformin plus SGLT-2 inhibitor). The $95 \%$ prediction interval ranged between $2.7 \mathrm{~kg}$ and $7.3 \mathrm{~kg}$.

\section{HbA1c}

Four trials reported $\mathrm{HbAlc}$ (random MD $0.1 \%, 95 \% \mathrm{Cl}-0.1$ to 0.2 ; $\mathrm{P}=0.26$; fixed $\mathrm{MD} 0.1 \%, 95 \% \mathrm{Cl} 0.02$ to $0.1 ; \mathrm{P}=0.005 ; 4$ trials; 4182 participants; Analysis 8.12; in favour of metformin plus SGLT-2 inhibitor). There was substantial heterogeneity, probably caused by various durations of intervention. For one trial (Hollander 2017), we extracted data after 52 weeks of treatment showing benefit of sulphonylurea treatment, whereas for the remaining trials we extracted data after 104 weeks or more showing benefit of SGLT-2 inhibitor treatment. Furthermore, heterogeneity could have been caused by various sulphonylureas (glipizide and glimepiride) administered in various doses ( $1 \mathrm{mg} /$ day to $8 \mathrm{mg} /$ day) and various SGLT-2 inhibitors (dapagliflozin, ertugliflozin, canagliflozin, empagliflozin). 


\section{Subgroup analyses}

We only performed subgroup analyses on $\mathrm{M}+\mathrm{S}$ combination therapy versus the combination of metformin plus DPP-4 inhibitor and metformin plus thiazolidinediones (see above). The remaining combination comparators did not include enough trials to perform subgroup analyses.

We performed subgroup analyses for the comparison of $\mathrm{M}+\mathrm{S}$ versus metformin plus thiazolidinediones dividing trials into studies investigating rosiglitazone or pioglitazone; see Analysis 5.1 to Analysis 5.14.

We performed subgroup analyses on trials with a long duration (two years and more) versus trials with a short duration (shorter than two years); see Analysis 6.1 to Analysis 6.11.

We did not perform a subgroup analysis on trials including obese participants $(\mathrm{BMI} \geq 30$ ) versus trials including non-obese participants $(\mathrm{BMI}<30)$ due to similar BMIs among the included trials.

\section{Sensitivity analyses}

We planned to perform sensitivity analyses for the following factors.

- Published trials

- Language of publication

- Analysis restricted to trials with low risk of selection bias

- Long trials (trials with duration of intervention longer than 104 weeks excluded)

- Large trials (trials with more than 1000 participants randomised to each intervention group excluded).

- Source of funding (trials funded by a pharmaceutical company excluded).

We did not perform sensitivity analysis on diagnostic criteria (often not reported), country (mostly performed in multiple countries) and imputation.

\section{Assessment of reporting bias}

We did not draw funnel plots due to limited number of trials for a particular outcome (maximum $\mathrm{N}=8$ ).

\section{Ongoing trials}

We identified nine ongoing RCTs, which potentially will provide data of interest for this review ( EUCTR2011-003335-63-IT; EUCTR2012-000152-34-IT; JPRN-UMIN000008815; NCT01243424; NCT01794143; NCT02142309; NCT02730377; NCT02769481; NCT03332771). The ongoing trials will include about 15,147 participants. All ongoing trials assessed one or more outcomes of interest for our review.

\section{Studies awaiting classification}

We classified two trials as 'studies awaiting classification'. One trial (Müller-Wieland 2018), was only published shortly before the publication of this review and one trial (NCT02564926), was submitted in January 2019 but results are not yet publicly available. Both trials compared $\mathrm{M}+\mathrm{S}$ with metformin plus SGLT-2 inhibitors. The trials included 1484 participants. Neither trial reports outcomes relevant for Summary of findings for the main comparison.

\section{DISCUSSION}

\section{Summary of main results}

This Cochrane Review investigated the effects of $M+S$ combination therapy compared with metformin plus another pharmacological glucose-lowering intervention, placebo or metformin monotherapy in people with T2DM. We included 32 trials with a total of 28,746 randomised participants. We judged all trials to have unclear or high risk of bias in one or more 'Risk of bias' domains. The amount of evidence on patient-important outcomes was limited. The use of $\mathrm{M}+\mathrm{S}$ neither revealed a clear advantage nor a disadvantage for the outcome measures specified in the 'Summary of findings' table of this review. However, there were fewer hypoglycaemic episodes when comparing $\mathrm{M}+\mathrm{S}$ with all other metformin plus another glucose-lowering combination therapies. In two cases $(M+S$ versus metformin plus placebo, $M+S$ versus metformin plus DPP-4 inhibitor), hypoglycaemia was reduced in the comparator groups and at the same time $\mathrm{HbAlc}$ was improved in the $M+S$ group (Table 3$)$. In three cases $(M+S$ versus metformin plus thiazolidinedione, $M+S$ versus metformin plus glinide, $M+S$ versus metformin plus SGLT-2 inhibitor), there were both improved $\mathrm{HbA1c}$ values and fewer hypoglycaemic episodes in the comparator groups (Table 3). One has to take into account that the risk of hypoglycaemia increases with low glucose level targets which may not apply to the majority of elderly people with diabetes (ADA 2019).

\section{Overall completeness and applicability of evidence}

We conducted an extensive search for trials, including publications in all languages, and tried to obtain additional data on all trials. Handsearching of systematic reviews and reference lists identified two additional trials to be included (Gerich 2005; Ristic 2007). Handsearching of manufacturers' websites identified eight additional references to be included. For all trials, we contacted one or more trial authors to obtain supplemental information on 'Risk of bias' domains and outcomes. In addition, we asked trial authors about any additional information about the retrieved trial(s) and to specify whether further trials existed that we may had missed. Six trial investigators of 10 trials either just confirmed a question or provided additional data that could be implemented for the 'Risk of bias' assessment or the meta-analyses of outcomes (Dei Cas 2017; Derosa 2005; Derosa 2009b; Derosa 2011a; Derosa 2011b; Gallwitz 2012a; Gallwitz 2012b; Göke 2013; Home 2009; Vaccaro 2017). For all trials, we identified contact information for one or more authors.

The diagnosis of T2DM was primary established using the European Association for the Study of Diabetes (EASD), World Health Organization (WHO) and American Diabetes Association (ADA) definitions. Nineteen trials did not specify how they established diagnosis of T2DM (Ahrén 2014; Charbonnel 2005; Del Prato 2014; Del Prato 2015; Filozof 2010; Gallwitz 2012b; Gerich 2005; Göke 2013; Handelsman 2017; Leiter 2015; Matthews 2010; Nauck 2013; Petrica 2009; Petrica 2011; Ridderstråle 2014; Ristic 2007; Schernthaner 2015; Seck 2010; Vaccaro 2017). The included trials used different types of sulphonylureas; 16 trials administered a second-generation sulphonylurea and 16 trials administered a third-generation sulphonylurea. 
A potential selection bias within the trials may exist as more healthy and motivated people are expected to participate in a clinical trial. However, one Cochrane systematic review observed that clinical outcomes in people participating in RCTs are comparable to outcomes in comparable individuals outside RCTs (Vist 2008).

\section{Quality of the evidence}

None of the 32 included trials in our review was classified as having low risk of bias in all 'Risk of bias' domains. The description of randomisation and allocation in the included trials was insufficient in eight trials (Ahrén 2014; Del Prato 2014; Filozof 2010; Gerich 2005; Matthews 2010; NCT00367055; Petrica 2009; Petrica 2011). Eleven trials had insufficient reporting of one or more outcomes of relevance for our review and, therefore, we classified them as having high risk of bias for selective outcome reporting bias (Derosa 2005; Derosa 2009a; Derosa 2009b; Derosa 2010; Derosa 2011a; Derosa 2011b; Filozof 2010; Gerich 2005; Maffioli 2013; Petrica 2009; Petrica 2011). We were able to assess one or more of our predefined outcomes in all the included trials.

For all the comparisons, we judged the certainty of the evidence to be low or very low mainly because of very limited data, various risk of bias and imprecision.

Most trials received financial funding from the pharmaceutical industry. It is known that trials receiving funding or provision of free drugs or devices from a pharmaceutical company show more favourable results and conclusions compared to trials sponsored by other sources (Lundh 2017).

\section{Potential biases in the review process}

We were unable to draw funnel plots to assess small-study bias due to lack of data. If more data had been available and more metaanalyses could have been performed, we would have been able to investigate heterogeneity in more detail.

We were dealing with a substantially heterogeneous group of trials. Our meta-analyses, when performed, were limited by the inability to use individual participant data to assess whether distinct clinical characteristics may have influenced the effect estimates of the interventions. We explored heterogeneity by performing sensitivity and subgroup analyses. However, due to a limited number of trials we only performed subgroup analyses on $\mathrm{M}+\mathrm{S}$ versus metformin plus DPP- 4 inhibitor and $\mathrm{M}+\mathrm{S}$ versus metformin plus thiazolidinedione. Many of the included trials were not designed or powered to detect our predefined patientimportant outcomes.

Most of the included trials had a relatively small number of participants and the information sizes in the meta-analyses were equally small. This increases the risk of unrealistic estimates of the intervention effects due to bias (systematic errors) and chance (random errors) (Wetterslev 2008; Wood 2008). We have attempted to address systematic errors. We contacted all trial authors for clarification if one of the bias domains was not adequately reported. To reduce the risk of random errors, we conducted TSA on all predefined outcomes, whenever possible.

Several trials were published in more than one publication, which made it difficult for us to separate the primary publication from companion papers for some trials (for details, see Included studies).
We included trials with a minimum duration of 52 weeks to detect clinically relevant differences for the predefined outcomes. Unfortunately, the reporting of patient-relevant outcomes in the included trials was poor.

Two review authors carried out data extraction. However, the review authors extracting the data were not blinded as to which trial they were extracting data from.

\section{Agreements and disagreements with other studies or reviews}

In the search for additional trials we checked other reviews, systematic reviews and meta-analyses (Amate 2015; Andersen 2016; Aylsworth 2014; Belsey 2008; Chan 2015; Dai 2014; Foroutan 2016; Geng 2015; Goring 2014; Gu 2015; Guthrie 2015; Hershon 2016; Hou 2015; Kuecker 2016; Lim 2015; Liu 2014; Maruthur 2016; Mearns 2015; Mishriky 2015; Monami 2008; Phung 2010; Phung 2014; Rosenstock 2013; Sharma 2017; Varvaki 2016; Wang 2017; Whalen 2015; Zhou 2015; Zintzaras 2014). Because we primarily intended to investigate patient-important outcomes we only included trials with a minimum duration of intervention of 52 weeks. However, all but three reviews (Goring 2014; Rosenstock 2013; Varvaki 2016), included trials with a duration of intervention less than 52 weeks or did not describe the duration of intervention in the included trials. Furthermore, all but seven reviews (Amate 2015; Foroutan 2016; Hershon 2016; Maruthur 2016; Rosenstock 2013; Varvaki 2016; Wang 2017), focused on outcomes such as glycaemic control, weight, lipids etc. One review (Rosenstock 2013), and one systematic review (Varvaki 2016), only included trials with a duration of intervention of 52 weeks and more, and focused on patient-important outcomes. These reviews did not suggest an increased risk of cardiovascular events, allcause mortality or cardiovascular mortality, comparing $\mathrm{M}+\mathrm{S}$ with metformin plus another glucose-lowering drug. Several of the reviews and systematic reviews investigated exclusively $M+S$ vs metformin plus a DPP-4 inhibitor (Amate 2015; Foroutan 2016; Gu 2015; Hou 2015; Mishriky 2015; Sharma 2017; Wang 2017; Zhou 2015). One systematic review and meta-analysis compared $M+S$ with other metformin combination therapies as one group (Varvaki 2016). To our knowledge, our review is the first to investigate patient-important outcomes in long-term trials (defined as 52 weeks and more) looking at $\mathrm{M}+\mathrm{S}$ compared to metformin plus another glucose-lowering interventions separately.

\section{AUTHORS' CONCLUSIONS}

\section{Implications for practice}

There is no firm evidence whether metformin plus sulphonylurea combination compared with metformin plus another glucoselowering agent or metformin monotherapy increases benefit or harm for most patient-important outcomes (all-cause mortality, serious adverse events, macrovascular complications (cardiovascular mortality, non-fatal myocardial infarction, nonfatal stroke) and microvascular complications (amputation of lower extremity, blindness or severe vision loss, end-stage renal disease)). There were more reported hypoglycaemic episodes with metformin plus sulphonylurea combination in comparison to all other metformin-antidiabetic agent combinations. The risk of hypoglycaemia increases with low glucose level targets which may not apply to the majority of elderly people with diabetes. There were no trials comparing metformin plus sulphonylurea with 
metformin plus insulin. We identified nine ongoing trials and two trials are awaiting assessment. Together these trials will include around 16,631 participants and could have an impact on the findings of our review.

\section{Implications for research}

It remains to be clarified whether there are any substantial beneficial or harmful effects of metformin plus sulphonylurea in people with type 2 diabetes mellitus. Several ongoing trials with around 15,147 participants are investigating this topic and each trial will provide data on one or more outcomes of interest for our review. Trial completion dates are estimated between 2018 and 2021. Furthermore two trials with 1484 participants investigating metformin plus sulphonylurea compared with metformin plus SGLT-2 inhibitors are awaiting assessment. Future long-term randomised controlled trials should focus on patient-important outcomes (especially mortality, health- related quality of life, serious adverse events, macrovascular and microvascular complications).

\section{ACKNOWLEDGEMENTS}

We would like to thank Olga Kurbatova for screening a trial published in Russian for inclusion/exclusion (Onuchin 2010). We would like to thank Alessandra Dei Cas for providing data on the Dei Cas 2017 trial, Pamela Maffioli for providing data on the Derosa 2005 trial, Philip Home for providing data on the Home 2009 trial, Olga Vaccaro for providing data on the Vaccaro 2017 trial, Baptist Gallwitz for answering questions regarding the Gallwitz 2012a and Gallwitz 2012b trials and Ingrid Gause-Nilsson for answering questions regarding the Göke 2013 trial.We thank peer reviewer Peter T. Sawicki for his valuable comments and contribution to our review. We thank Denise Mitchell for her excellent copyediting of our review. Kasper Madsen thanks the Department of Endocrinology, Diabetes and Bone-Metabolic Research Unit, Rigshospitalet, for providing office facilities and great colleagues. 


\section{R E F E R E N C E S}

\section{References to studies included in this review}

Ahrén 2014 \{published and unpublished data\}

Ahrén B, Carr MC, Murphy K, Perkins C, Rendell M, Mallory J, et al. Albiglutide for the treatment of type 2 diabetes mellitus: an integrated safety analysis of the HARMONY phase 3 trials. Diabetes Research and Clinical Practice 2017;126:230-9. [DOI: 10.1016/j.diabres.2017.02.017]

* Ahrén B, Johnson SL, Stewart M, Cirkel DT, Yang F, Perry C, et al. HARMONY 3: 104-week randomized, double-blind, placeboand active-controlled trial assessing the efficacy and safety of albiglutide compared with placebo, sitagliptin, and glimepiride in patients with type 2 diabetes taking metformin. Diabetes Care 2014;37(8):2141-8. [DOI: $10.2337 / \mathrm{dc} 14-0024]$

Ahrén B, Stewart M, Cirkel D, Yang F, Perry C, Johnson S. HARMONY 3: 104 week (wk) efficacy of albiglutide (albi) compared to sitagliptin (sita) and glimepiride (SU) in patients (pts) with type 2 diabetes mellitus (T2DM) on metformin (met). 73rd Scientific Sessions. American Diabetes Assosciation. 2013 June 21-25; Chicago (IL). American Diabetes Assosciation, 2013. [52-LB]

Doggrell SA. Comparator clinical trials of surrogate endpoints with albiglutide are in HARMONY. Expert Review of Endocrinology and Metabolism 2015;10(3):273-6. [DOI: 10.1586/17446651.2015.995629]

Fisher M, Petrie MC, Ambery PD, Donaldson J, Ye J, McMurray JJ. Cardiovascular safety of albiglutide in the HARMONY Programme: a meta-analysis. Lancet Diabetes \& Endocrinology 2015;3(9):697-703. [DOI: 10.1016/S2213-8587(15)00233-8]

GSK Study ID 112753. Efficacy and safety of albiglutide in treatment of type 2 diabetes. gsk-studyregister.com/study/3736 (accessed 16 November 2016).

Home PD, Ahrén B, Reusch JE, Rendell M, Weissman PN, Cirkel DT, et al. Three-year data from 5 HARMONY phase 3 clinical trials of albiglutide in type 2 diabetes mellitus: long-term efficacy with or without rescue therapy. Diabetes Research and Clinical Practice 2017;131:49-60. [DOI: 10.1016/ j.diabres.2017.06.013]

Johnson S, Ahrén B, Stewart M, Cirkel D, Yang F, Perry C. HARMONY 3: 104 week efficacy of albiglutide compared to sitagliptin and glimepiride in patients with type 2 diabetes mellitus on metformin. Diabetologia 2013;56:S8-9.

Leiter LA, Mallory JM, Wilson TH, Reinhardt RR. Gastrointestinal safety across the albiglutide development programme. Diabetes, Obesity \& Metabolism 2016;18(9):930-5. [DOI: 10.1111/ dom.12679]

Matthews JE, Ahren B, Ye J, Carr MC, Stewart MW. HARMONY 3 year 3 results: albiglutide vs sitagliptin, glimepiride, and placebo in patients with T2DM on metformin. 50th Annual Meeting of the European Association for the Study of Diabetes. 15-19 September 2014. [abstract no 831]
NCT00838903. Efficacy and safety of albiglutide in treatment of type 2 diabetes. clinicaltrials.gov/ct2/show/study/NCT00838903 (accessed 16 November 2016).

\section{Charbonnel 2005 \{published data only\}}

Belcher G, Schernthaner G. Changes in liver tests during 1-year treatment of patients with type 2 diabetes with pioglitazone, metformin or gliclazide. Diabetic Medicine 2005;22(8):973-9. [DOI: 10.1111/j.1464-5491.2005.01595.x]

Charbonnel B, Roden M, Urquhart R, Mariz S, Johns D, Mihm M, et al. Pioglitazone elicits long-term improvements in insulin sensitivity in patients with type 2 diabetes: comparisons with gliclazide-based regimens. Diabetologia 2005;48(3):553-60. [DOI: 10.1007/s00125-004-1651-9]

* Charbonnel B, Schernthaner G, Brunetti P, Matthews DR, Urquhart R, Tan $\mathrm{MH}$, et al. Long-term efficacy and tolerability of add-on pioglitazone therapy to failing monotherapy compared with addition of gliclazide or metformin in patients with type 2 diabetes. Diabetologia 2005;48(6):1093-104. [DOI: 10.1007/ s00125-005-1751-1]

Matthews DR, Charbonnel BH, Hanefeld M, Brunetti P, Schernthaner $\mathrm{G}$. Long-term therapy with addition of pioglitazone to metformin compared with the addition of gliclazide to metformin in patients with type 2 diabetes: a randomized, comparative study. Diabetes/Metabolism Research and Reviews 2005;21(2):167-74. [DOI: 10.1002/dmrr.478]

Roden M, Mariz S, Brazzale AR, Pacini G. Free fatty acid kinetics during long-term treatment with pioglitazone added to sulfonylurea or metformin in type 2 diabetes. Journal of Internal Medicine 2009;265(4):476-87. [DOI: 10.1111/ j.1365-2796.2008.02040.x]

\section{Dei Cas 2017 \{published data only\}}

* Dei Cas A, Spigoni V, Cito M, Aldigeri R, Ridolfi V, Marchesi E, et al. Vildagliptin, but not glibenclamide, increases circulating endothelial progenitor cell number: a 12-month randomized controlled trial in patients with type 2 diabetes. Cardiovascular Diabetology 2017;16(1):27. [DOI: 10.1186/s12933-017-0503-0]

NCT01822548. Effect of vildagliptin vs. glibenclamide on circulating endothelial progenitor cell number type 2 diabetes. clinicaltrials.gov/ct2/show/NCT01822548 (accessed 26 June 2017).

Del Prato 2014 \{published data only\}

* Del Prato S, Camisasca R, Wilson C, Fleck P. Durability of the efficacy and safety of alogliptin compared with glipizide in type 2 diabetes mellitus: a 2-year study. Diabetes Obesity Metabolism 2014;16(12):1239-46. [DOI: doi.org/10.1111/dom.12377]

Del Prato S, Fleck P, Wilson C, Chaudhari P. Comparison of alogliptin and glipizide for composite endpoint of glycated haemoglobin reduction, no hypoglycaemia and no weight gain in type 2 diabetes mellitus. Diabetes, Obesity \& Metabolism 2016;18(6):623-7. [DOI: doi.org/10.1111/dom.12643] 
Gordon J, McEwan P, Hurst M, Puelles J. The cost-effectiveness of alogliptin versus sulfonylurea as add-on therapy to metformin in patients with uncontrolled type 2 diabetes mellitus. Diabetes Therapy 2016;7(4):825-45. [DOI: 10.1007/ s13300-016-0206-7]

NCT00856284. Efficacy and safety of alogliptin plus metformin compared to glipizide plus metformin in patients with type 2 diabetes mellitus (ENDURE). clinicaltrials.gov/ct2/show/ NCT00856284 (accessed 12 December 2016).

\section{Del Prato 2015 \{published data only\}}

Abad Paniagua EJ, Casado Escribano P, Fernández Rodriguez JM, Morales Escobar FJ, Betegón Nicolás L, SánchezCovisa J, et al. Cost-effectiveness analysis of dapagliflozin compared to DPP4 inhibitors and other oral antidiabetic drugs in the treatment of type-2 diabetes mellitus in Spain. Atencion Primaria 2015;47(8):505-13. [DOI: 10.1016/j.aprim.2014.11.002]

Charokopou M, McEwan P, Lister S, Callan L, Bergenheim K, Tolley K, et al. The cost-effectiveness of dapagliflozin versus sulfonylurea as an add-on to metformin in the treatment of Type 2 diabetes mellitus. Diabetic Medicine 2015;32(7):890-8. [DOI: 10.1111/dme.12772]

* Del Prato S, Nauck M, Durán-Garcia S, Maffei L, Rohwedder K, Theuerkauf $A$, et al. Long-term glycaemic response and tolerability of dapagliflozin versus a sulphonylurea as add-on therapy to metformin in patients with type 2 diabetes: 4-year data. Diabetes Obesity Metabolism 2015;17(6):581-90.

Fenici P, Sternhufvud C, Cain V, Mukherjee J, Rohwedder K. Dapagliflozin added to metformin is effective in achieving combined improvements in $\mathrm{HbAlc}$ and weight without hypoglycaemia over 4 years. Diabetologia 2015;58:S355.

Forst T, Rohwedder K, Sugg J, Johnsson E. Dapagliflozin decreases post-prandial glucose without an increase in Cpeptide or insulin. Diabetes 2015;64:A324.

Katz A, Yeh H. Dapagliflozin and insulin resistance in patients with type 2 diabetes. Diabetes 2015;64:A304-5.

NCT00660907. Efficacy and safety of dapagliflozin in combination with metformin in type 2 diabetes patients. clinicaltrials.gov/ct2/show/NCT00660907 (accessed 3 August 2017).

Nauck MA, Del Prato S, Duran-Garcia S, Rohwedder K, Langkilde AM, Sugg J, et al. Durability of glycaemic efficacy over 2 years with dapagliflozin versus glipizide as add-on therapies in patients whose type 2 diabetes mellitus is inadequately controlled with metformin. Diabetes, Obesity \& Metabolism 2014;16(11):1111-20.

Nauck MA, Del Prato S, Meier JJ, Duran-Garcia S, Rohwedder K, Elze $M$, et al. Dapagliflozin versus glipizide as add-on therapy in patients with type 2 diabetes who have inadequate glycemic control with metformin: a randomized, 52-week, doubleblind, active-controlled noninferiority trial. Diabetes Care 2011;34(9):2015-22.

Parikh S, Wilding J, Jabbour S, Hardy E. Dapagliflozin in type 2 diabetes: effectiveness across the spectrum of disease and over time. International Journal of Clinical Practice 2015;69(2):186-98.

Rohwedder K, Johnsson E. Baseline fasting plasma glucose may predict response to dapagliflozin when added to metformin. Diabetologia 2015;58:S349-50.

Sabale U, Ekman M, Granstrom O, Bergenheim K, McEwan P. Cost-effectiveness of dapagliflozin (Forxiga) added to metformin compared with sulfonylurea added to metformin in type 2 diabetes in the Nordic countries. Primary Care Diabetes 2015;9(1):39-47.

Stenlöf K, Cain V, Rohwedder K, Johnsson E. Maintenance of weight loss with dapagliflozin vs. glipizide as add-on to metformin over 4 years. Diabetes 2015;64:A27.

\section{Derosa 2005 \{published data only\}}

* Derosa G, Cicero AF, Gaddi AV, Ciccarelli L, Piccinni MN, Salvadeo S, et al. Long-term effects of glimepiride or rosiglitazone in combination with metformin on blood pressure control in type 2 diabetic patients affected by the metabolic syndrome: a 12-month, double-blind, randomized clinical trial. Clinical Therapeutics 2005;27(9):1383-91.

Derosa G, Gaddi A, Ciccarelli L, Fogari E, Ghelfi M, Ferrari I, et al. Long-term effect of glimepiride and rosiglitazone on nonconventional cardiovascular risk factors in metformin-treated patients affected by metabolic syndrome: a randomized, double-blind clinical trial. Journal of International Medical Research 2005;33(3):284-94.

Derosa G, Gaddi AV, Piccinni MN, Ciccarelli L, Salvadeo S, Peros E, et al. Antithrombotic effects of rosiglitazone-metformin versus glimepiride-metformin combination therapy in patients with type 2 diabetes mellitus and metabolic syndrome. Pharmacotherapy 2005;25(5):637-45.

Derosa G, Gaddi AV, Piccinni MN, Salvadeo S, Ciccarelli L, Fogari E, et al. Differential effect of glimepiride and rosiglitazone on metabolic control of type 2 diabetic patients treated with metformin: a randomized, double-blind, clinical trial. Diabetes, Obesity \& Metabolism 2006;8(2):197-205.

\section{Derosa 2009a \{published data only\}}

Derosa G, Maffioli P, Salvadeo SA, Ferrari I, Gravina A, Mereu R, et al. Direct comparison among oral hypoglycemic agents and their association with insulin resistance evaluated by euglycemic hyperinsulinemic clamp: the 60's study. Metabolism 2009;58(8):1059-66.

\section{Derosa 2009b \{published data only\}}

Derosa G, D'Angelo A, Fogari E, Salvadeo S, Gravina A, Ferrari I, et al. Effects of nateglinide and glibenclamide on prothrombotic factors in naive type 2 diabetic patients treated with metformin: a 1-year, double-blind, randomized clinical trial. Internal Medicine (Tokyo, Japan) 2007;46(22):1837-46.

* Derosa G, D'Angelo A, Fogari E, Salvadeo S, Gravina A, Ferrari I, et al. Nateglinide and glibenclamide metabolic effects in naive type 2 diabetic patients treated with metformin. Journal of Clinical Pharmacy and Therapeutics 2009;34(1):13-23. 


\section{Derosa 2010 \{published data only\}}

Derosa G, Maffioli P, Salvadeo SA, Ferrari I, Ragonesi PD, Querci $F$, et al. Exenatide versus glibenclamide in patients with diabetes. Diabetes Technology \& Therapeutics 2010;12(3):233-40.

\section{Derosa 2011a \{published data only\}}

Derosa G, Putignano P, Bossi AC, Bonaventura A, Querci F, Franzetti IG, et al. Exenatide or glimepiride added to metformin on metabolic control and on insulin resistance in type 2 diabetic patients. European Journal of Pharmacology 2011;666(1-3):251-6.

\section{Derosa 2011b \{published data only\}}

Derosa G, Cicero AF, Fogari E, D'Angelo A, Bianchi L, Maffioli P. Pioglitazone compared to glibenclamide on lipid profile and inflammation markers in type 2 diabetic patients during an oral fat load. Hormone and Metabolic Research 2011;43(7):505-12.

\section{Filozof 2010 \{published data only\}}

Filozof C, Gautier JF. A comparison of efficacy and safety of vildagliptin and gliclazide in combination with metformin in patients with type 2 diabetes inadequately controlled with metformin alone: a 52-week, randomized study. Diabetic Medicine 2010;27(3):318-26.

\section{Gallwitz 2012a \{published data only\}}

* Gallwitz B, Guzman J, Dotta F, Guerci B, Simó R, Basson BR, et al. Exenatide twice daily versus glimepiride for prevention of glycaemic deterioration in patients with type 2 diabetes with metformin failure (EUREXA): an open-label, randomised controlled trial. Lancet 2012;379(9833):2270-8.

Kazda C, Gallwitz B, Simó R, Guzmán JR, Kraus P, Nicolay C, et al. The European exenatide study of long-term exenatide vs glimepiride for type 2 diabetes: rationale and patient characteristics. Diabetes, Obesity \& Metabolism 2009;11(12):1131-7.

NCT00359762. Exenatide versus glimepiride in patients with type 2 diabetes. clinicaltrials.gov/ct2/show/NCT00359762 (accessed 6 July 2017).

Schernthaner G, Rosas-Guzman J, Dotta F, Guerci B, Simo R, Festa A, et al. Treatment escalation options for patients with type 2 diabetes after failure of exenatide twice daily or glimepiride added to metformin: results from the prospective European Exenatide (EUREXA) study. Diabetes, Obesity \& Metabolism 2015;17(7):689-98.

Simo R, Guerci B, Schernthaner G, Gallwitz B, Rosas-Guzman J, Dotta $\mathrm{F}$, et al. Long-term changes in cardiovascular risk markers during administration of exenatide twice daily or glimepiride: results from the European exenatide study. Cardiovascular Diabetology 2015;14(116):1-13.

\section{Gallwitz 2012b \{published data only\}}

Gallwitz B, Rosenstock J, Emser A, von Eynatten M, Woerle HJ. Linagliptin is more effective than glimepiride at achieving a composite outcome of target $\mathrm{HbA}(1)$ c. International Journal of Clinical Practice 2013;67(4):317-21.
Gallwitz B, Rosenstock J, Patel S, von Eynatten M, Hehnke U, Mehlburger L, et al. Regardless of the degree of glycaemic control, linagliptin has lower hypoglycaemia risk than all doses of glimepiride, at all time points, over the course of a 2-year trial. Diabetes, Obesity \& Metabolism 2015;17(3):276-84.

* Gallwitz B, Rosenstock J, Rauch T, Bhattacharya S, Patel S, von Eynatten M, et al. 2-year efficacy and safety of linagliptin compared with glimepiride in patients with type 2 diabetes inadequately controlled on metformin: a randomised, doubleblind, non-inferiority trial. Lancet 2012;380(9840):475-83.

Johansen OE, Boehm BO, Grill V, Torjesen PA, Bhattacharya S, Patel S, et al. C-peptide levels in latent autoimmune diabetes in adults treated with linagliptin versus glimepiride: exploratory results from a 2-year double-blind, randomized, controlled study. Diabetes Care 2014;37(1):e11-2.

Johansen OE, Neubacher D, von Eynatten M, Patel S, Woerle HJ. Cardiovascular safety with linagliptin in patients with type 2 diabetes mellitus: a pre-specified, prospective, and adjudicated meta-analysis of a phase 3 programme. Cardiovascular Diabetology 2012;11:3.

NCT00622284. Efficacy and safety of BI 1356 in combination with metformin in patients with type 2 diabetes. clinicaltrials.gov/ct2/show/study/NCT00622284 (accessed 7 July 2017).

Rosenstock J, Marx N, Neubacher D, Seck T, Patel S, Woerle HJ, et al. Cardiovascular safety of linagliptin in type 2 diabetes: a comprehensive patient-level pooled analysis of prospectively adjudicated cardiovascular events. Cardiovascular Diabetology 2015;14:57.

\section{Gerich 2005 \{published data only\}}

CDJN608A US07. Multicenter, randomized, double-blind, active controlled trial to compare the efficacy and safety of 104 weeks of nateglinide plus metformin vs glyburide plus metformin in drug naive subjects with type 2 diabetes mellitus who have inadequate control with diet and exercise. www.novctrd.com/ctrdWebApp/clinicaltrialrepository/ displayFile.do?trialResult=1497 (accessed 16 March 2017).

* Gerich J, Raskin P, Jean-Louis L, Purkayastha D, Baron MA. PRESERVE-beta: two-year efficacy and safety of initial combination therapy with nateglinide or glyburide plus metformin. Diabetes Care 2005;28(9):2093-9.

Schwarz SL, Gerich JE, Marcellari A, Jean-Louis L, Purkayastha D, Baron MA. Nateglinide, alone or in combination with metformin, is effective and well tolerated in treatmentnaive elderly patients with type 2 diabetes. Diabetes, Obesity \& Metabolism 2008;10(8):652-60.

\section{Göke 2013 \{published data only\}}

Cook W, Minervini G, Bryzinski B, Hirshberg B. Saxagliptin efficacy and safety in patients with type 2 diabetes mellitus stratified by cardiovascular disease history and cardiovascular risk factors: analysis of 3 clinical trials. Postgraduate Medicine 2014;126(6):19-32. 
Goke B, Gallwitz B, Eriksson J, Hellqvist A, Gause-Nilsson I. Saxagliptin is non-inferior to glipizide in patients with type 2 diabetes mellitus inadequately controlled on metformin alone: a 52-week randomised controlled trial. International Journal of Clinical Practice 2010;64(12):1619-31.

Granstrom O, Bergenheim K, McEwan P, Sennfalt K, Henriksson M. Cost-effectiveness of saxagliptin (Onglyza) in type 2 diabetes in Sweden. Primary Care Diabetes 2012;6(2):127-36.

* Göke B, Gallwitz B, Eriksson J G, Hellqvist Å, Gause-Nilsson I. Saxagliptin vs glipizide as add-on therapy in patients with type 2 diabetes mellitus inadequately controlled on metformin alone: long-term (52-week) extension of a 52-week randomised controlled trial. International Journal of Clinical Practice 2013;67(4):307-16.

Mintz ML, Minervini G. Saxagliptin versus glipizide as add-on therapy to metformin: assessment of hypoglycemia. Current Medical Research \& Opinion 2014;30(5):761-70.

NCT00575588. 52-week add-on to metformin comparison of saxagliptin and sulphonylurea, with a 52-week extension period. clinicaltrials.gov/ct2/show/NCT00575588 (accessed 10 July 2017).

\section{Hamann 2008 \{published data only\}}

GSK Study ID AVM100264. AVANDAMET versus metformin and sulphonylurea in people with poorly controlled type 2 diabetes. gsk-studyregister.com/study/7254 (accessed 12 July 2017).

* Hamann A, Garcia-Puig J, Paul G, Donaldson J, Stewart M. Comparison of fixed-dose rosiglitazone/metformin combination therapy with sulphonylurea plus metformin in overweight Individuals with type 2 diabetes inadequately controlled on metformin alone. Experimental and Clinical Endocrinology \& Diabetes 2008;116(1):6-13.

NCT00359112. AVANDAMET versus metformin and sulphonylurea in people with poorly controlled type 2 diabetes. clinicaltrials.gov/ct2/show/study/NCT00359112 (accessed 12 July 2017).

\section{Handelsman 2017 \{published data only\}}

* Handelsman Y, Lauring B, Gantz I, Iredale C, O'Neill EA, Wei $Z$, et al. A randomized, double-blind, non-inferiority trial evaluating the efficacy and safety of omarigliptin, a onceweekly DPP-4 inhibitor, or glimepiride in patients with type 2 diabetes inadequately controlled on metformin monotherapy. Current Medical Research and Opinion 2017;33(10):1861-8.

MK-3102-016. Clinical study report P016. A study of the safety and efficacy of omarigliptin (MK-3102) compared with glimepiride in participants with type 2 diabetes mellitus with inadequate glycemic control on metformin (MK-3102-016). merck.com/clinical-trials/study.html?id=3102-016\&tab=access (accessed 6 March 2018).

NCT01682759. A study of the safety and efficacy of omarigliptin (MK-3102) compared with glimepiride in participants with type 2 diabetes mellitus with inadequate glycemic control on metformin (MK-3102-016). clinicaltrials.gov/ct2/show/study/ NCT01682759 (accessed 6 March 2018).

\section{Hollander 2017 \{published data only\}}

* Hollander P, Liu J, Hill J, Johnson J, Jiang Z, Golm G, et al. Safety and efficacy of ertugliflozin compared to glimepiride in patients with type 2 diabetes inadequately controlled on metformin: the VERTIS SU trial. Diabetologia 2017;60:S19-S20.

Hollander P, Liu J, Hill J, Johnson J, Jiang ZW, Golm G, et al. Ertugliflozin compared with glimepiride in patients with type 2 diabetes mellitus inadequately controlled on metformin: The VERTIS SU randomized study. Diabetes Therapy 2018;9(1):193-207.

NCT01999218. Ertugliflozin vs. glimepiride in type 2 diabetes mellitus (T2DM) participants on metformin (MK-8835-002). clinicaltrials.gov/ct2/show/NCT01999218 (accessed 1 March 2018).

\section{Home 2009 \{published data only\}}

GSK Study ID BRL-049653/231. BRL-049653/231-RECORD: rosiglitazone evaluated for cardiac outcomes and regulation of glycaemia in diabetes. gsk-studyregister.com/study/7631 (accessed 1 April 2017).

Home PD, Jones NP, Pocock SJ, Beck-Nielsen H, Gomis R, Hanefeld M, et al. Rosiglitazone RECORD study: glucose control outcomes at 18 months. Diabetic Medicine 2007;24(6):626-34.

Home PD, Kahn SE, Jones NP, Noronha D, Beck-Nielsen H, Viberti G. Experience of malignancies with oral glucoselowering drugs in the randomised controlled ADOPT (A Diabetes Outcome Progression Trial) and RECORD (Rosiglitazone Evaluated for Cardiovascular Outcomes and Regulation of Glycaemia in Diabetes) clinical trials. Diabetologia 2010;53(9):1838-45.

* Home PD, Pocock SJ, Beck-Nielsen H, Curtis PS, Gomis R, Hanefeld M, et al. Rosiglitazone evaluated for cardiovascular outcomes in oral agent combination therapy for type 2 diabetes (RECORD): a multicentre, randomised, open-label trial. Lancet 2009;373(9681):2125-35.

Home PD, Pocock SJ, Beck-Nielsen H, Gomis R, Hanefeld M, Dargie $\mathrm{H}$, et al. Rosiglitazone evaluated for cardiac outcomes and regulation of glycaemia in diabetes (RECORD): study design and protocol. Diabetologia 2005;48(9):1726-35.

Home PD, Pocock SJ, Beck-Nielsen H, Gomis R, Hanefeld M, Jones NP, et al. Rosiglitazone evaluated for cardiovascular outcomes--an interim analysis. New England Journal of Medicine 2007;357(1):28-38.

Jones NP, Curtis PS, Home PD. Cancer and bone fractures in observational follow-up of the RECORD study. Acta Diabetologica 2015;52(3):539-46.

Komajda M, Curtis P, Hanefeld M, Beck-Nielsen H, Pocock SJ, Zambanini A, et al. Effect of the addition of rosiglitazone to metformin or sulfonylureas versus metformin/sulfonylurea combination therapy on ambulatory blood pressure in people with type 2 diabetes: a randomized controlled trial (the RECORD study). Cardiovascular Diabetology 2008;7:10. 
Komajda M, McMurray JJ, Beck-Nielsen H, Gomis R, Hanefeld M, Pocock SJ, et al. Heart failure events with rosiglitazone in type 2 diabetes: data from the RECORD clinical trial. European Heart Journal 2010;31(7):824-31.

Lopes RD, Dickerson S, Hafley G, Burns S, Tourt-Uhlig S, White J, et al. Methodology of a reevaluation of cardiovascular outcomes in the RECORD trial: study design and conduct. American Heart Journal 2013;166(2):208-16.

MacDonald MR, Petrie MC, Home PD, Komajda M, Jones NP, Beck-Nielsen $\mathrm{H}$, et al. Incidence and prevalence of unrecognized myocardial infarction in people with diabetes: a substudy of the Rosiglitazone Evaluated for Cardiac Outcomes and Regulation of Glycemia in Diabetes (RECORD) study. Diabetes Care 2011;34(6):1394-6.

Mahaffey KW, Hafley G, Dickerson S, Burns S, Tourt-Uhlig S, White $J$, et al. Results of a reevaluation of cardiovascular outcomes in the RECORD trial. American Heart Journal 2013;166(2):240-9.

NCT00379769. Rosiglitazone evaluated for cardiac outcomes and regulation of glycaemia in diabetes (RECORD). clinicaltrials.gov/ct2/show/NCT00379769 (accessed 1 April 2017).

\section{Leiter 2015 \{published data only\}}

Blonde L, Stenlof K, Fung A, Xie J, Canovatchel W, Meininger G. Effects of canagliflozin on body weight and body composition in patients with type 2 diabetes over 104 weeks. Postgraduate Medicine 2016;128(4):371-80.

Cefalu WT, Leiter LA, Yoon K-H, Arias P, Niskanen L, Xie J, et al. Efficacy and safety of canagliflozin versus glimepiride in patients with type 2 diabetes inadequately controlled with metformin (CANTATA-SU): 52 week results from a randomised, double-blind, phase 3 non-inferiority trial. Lancet 2013;382(9896):941-50.

Desai M, Merton K, Davies MJ, Vijapurkar U, Balis D. Canagliflozin provides greater improvement in risk factors of metabolic syndrome (MetS) versus glimepiride in patients with type 2 diabetes and MetS on background metformin Diabetologia 2016;59:S340-1.

Heerspink HJ, Desai M, Jardine M, Balis D, Meininger G, Perkovic V. Canagliflozin slows progression of renal function decline independently of glycemic effects. Journal of the American Society of Nephrology: JASN 2017;28(1):368-75.

John M, Cerdas S, Violante R, Deerochanawong C, Hassanein M, Slee A, et al. Efficacy and safety of canagliflozin in patients with type 2 diabetes mellitus living in hot climates. International Journal of Clinical Practice 2016;70(9):775-85.

Lavalle-Gonzalez FJ, Eliaschewitz FG, Cerdas S, Chacon Mdel P, Tong C, Alba M. Efficacy and safety of canagliflozin in patients with type 2 diabetes mellitus from Latin America. Current Medical Research \& Opinion 2016;32(3):427-39.

Leiter LA, Langslet G, Vijapurkar U, Davies MJ, Canovatchel W. Simultaneous reduction in both $\mathrm{HbAlc}$ and body weight with canagliflozin versus glimepiride in patients with type 2 diabetes on metformin. Diabetes Therapy 2016;7(2):269-78.

* Leiter LA, Yoon KH, Arias P, Langslet G, Xie J, Balis DA, et al. Canagliflozin provides durable glycemic improvements and body weight reduction over 104 weeks versus glimepiride in patients with type 2 diabetes on metformin: a randomized, double-blind, phase 3 study. Diabetes Care 2015;38(3):355-64.

NCT00968812. Canagliflozin treatment and trial analysissulfonylurea (CANTATA-SU) SGLT2 add-on to metformin versus glimepiride. clinicaltrials.gov/ct2/show/NCT00968812 (accessed 2 February 2017).

Nyirjesy P, Sobel JD, Fung A, Mayer C, Capuano G, Ways K, et al. Genital mycotic infections with canagliflozin, a sodium glucose co-transporter 2 inhibitor, in patients with type 2 diabetes mellitus: a pooled analysis of clinical studies. Current Medical Research and Opinion 2014;30(6):1109-19.

Patel CA, Bailey RA, Vijapurkar U, Meininger G, Blonde L. A posthoc analysis of the comparative efficacy of canagliflozin and glimepiride in the attainment of type 2 diabetes-related quality measures. BMC Health Services Research 2016;16(1):356.

Stenlof K, Blonde L, Fung A, Xie J, Canovatchel W, Meininger G. Distribution of weight loss with canagliflozin in patients with type 2 diabetes mellitus over 104 weeks. Diabetologia 2015;58:S354-5.

Van Gaal L, Garvey WT, Leiter LA, Vijapurkar U, List J, Cuddihy R, et al. Effects of canagliflozin versus glimepiride on adipokines, inflammatory biomarkers, and chemokines in patients with type 2 diabetes. Diabetologia 2017;60(Suppl. 1):S413-414.

Vercruysse F, Davies MJ, Merton K, Vijapurkar U, Simples J, Carroll A, et al. Achievement of glycaemic goals without hypoglycaemia with canagliflozin versus glimepiride in patients with type 2 diabetes. Diabetologia 2016;59:S339.

Watts NB, Bilezikian JP, Usiskin K, Edwards R, Desai M, Law G, et al. Effects of canagliflozin on fracture risk in patients with type 2 diabetes mellitus. Journal of Clinical Endocrinology and Metabolism 2016;101(1):157-66.

Maffioli 2013 \{published data only\}

Maffioli P, Fogari E, D’Angelo A, Perrone T, Derosa G. Ultrasonography modifications of visceral and subcutaneous adipose tissue after pioglitazone or glibenclamide therapy combined with rosuvastatin in type 2 diabetic patients not well controlled by metformin. European Journal of Gastroenterology \& Hepatology 2013;25(9):1113-22.

\section{Matthews 2010 \{published data only\}}

Ahren B, Foley JE, Dejager S, Akacha M, Shao Q, Heimann G, et al. Higher risk of hypoglycemia with glimepiride versus vildagliptin in patients with type 2 diabetes is not driven by high doses of glimepiride: divergent patient susceptibilities? Diabetes Therapy 2014;5(2):459-69.

Ahren B, Foley JE, Ferrannini E, Matthews DR, Zinman B, Dejager $S$, et al. Changes in prandial glucagon levels after a 2-year treatment with vildagliptin or glimepiride in patients 
with type 2 diabetes inadequately controlled with metformin monotherapy. Diabetes Care 2010;33(4):730-2.

Ahren B, Mathieu C, Bader G, Schweizer A, Foley JE. Efficacy of vildagliptin versus sulfonylureas as add-on therapy to metformin: comparison of results from randomised controlled and observational studies. Diabetologia 2014;57(7):1304-7.

Bader G, Geransar P, Schweizer A. Vildagliptin more effectively achieves a composite endpoint of $\mathrm{HbAlc}<7 \%$ without hypoglycaemia and weight gain compared with glimepiride after 2 years of treatment. Diabetes Research and Clinical Practice 2013;100(3):e78-81.

EudraCT 2004-004559-21. A multicenter, randomized, doubleblind, active controlled study to compare the long-term effect (up to 5 years) of treatment with LAF237 $50 \mathrm{mg}$ bid to glimepiride up to $6 \mathrm{mg}$ daily as add-on therapy in patients with type 2 diabetes inadequately controlled with metformin monotherapy. www.clinicaltrialsregister.eu/ctr-search/ trial/2004-004559-21/IT (accessed 11 March 2017).

Ferrannini E, Fonseca V, Zinman B, Matthews D, Ahrén B, Byiers S, et al. Fifty-two-week efficacy and safety of vildagliptin vs glimepiride in patients with type 2 diabetes mellitus inadequately controlled on metformin monotherapy. Diabetes, Obesity \& Metabolism 2009;11(2):157-66.

* Matthews DR, Dejager S, Ahren B, Fonseca V, Ferrannini E, Couturier A, et al. Vildagliptin add-on to metformin produces similar efficacy and reduced hypoglycaemic risk compared with glimepiride, with no weight gain: results from a 2-year study. Diabetes Obesesity \& Metabolism 2010;12(9):780-9.

NCT00106340. Vildagliptin compared to glimepiride in combination with metformin in patients with type 2 diabetes. clinicaltrials.gov/ct2/show/NCT00106340 (accessed 11 March 2017).

\section{Nauck 2013 \{published data only\}}

Blonde L, Russell-Jones D. The safety and efficacy of liraglutide with or without oral antidiabetic drug therapy in type 2 diabetes: an overview of the LEAD 1-5 studies. Diabetes, Obesity \& Metabolism 2009;11 Suppl 3:26-34.

Bode BW, Brett J, Falahati A, Pratley RE. Comparison of the efficacy and tolerability profile of liraglutide, a oncedaily human GLP-1 analog, in patients with type 2 diabetes $\geq 65$ and $<65$ years of age: a pooled analysis from phase III studies. American Journal of Geriatric Pharmacotherapy 2011;9(6):423-33.

Buse JB, Garber A, Rosenstock J, Schmidt WE, Brett JH, Videbaek N, et al. Liraglutide treatment is associated with a low frequency and magnitude of antibody formation with no apparent impact on glycemic response or increased frequency of adverse events: results from the Liraglutide Effect and Action in Diabetes (LEAD) trials. Journal of Clinical Endocrinology and Metabolism 2011;96(6):1695-702.

Davies MJ, Chubb BD, Smith IC, Valentine WJ. Cost-utility analysis of liraglutide compared with sulphonylurea or sitagliptin, all as add-on to metformin monotherapy in type 2 diabetes mellitus. Diabetic Medicine 2012;29(3):313-20.
Fonseca VA, Devries JH, Henry RR, Donsmark M, Thomsen HF, Plutzky J. Reductions in systolic blood pressure with liraglutide in patients with type 2 diabetes: insights from a patient-level pooled analysis of six randomized clinical trials. Journal of Diabetes and its Complications 2014;28(3):399-405.

Hegedus L, Moses AC, Zdravkovic M, Le Thi T, Daniels GH. GLP-1 and calcitonin concentration in humans: lack of evidence of calcitonin release from sequential screening in over 5000 subjects with type 2 diabetes or nondiabetic obese subjects treated with the human GLP-1 analog, liraglutide. Journal of Clinical Endocrinology and Metabolism 2011;96(3):853-60.

Henry RR, Buse JB, Sesti G, Davies MJ, Jensen KH, Brett J, et al. Efficacy of antihyperglycemic therapies and the influence of baseline hemoglobin $\mathrm{A}(1 \mathrm{C})$ : a meta-analysis of the liraglutide development program. Endocrine Practice 2011;17(6):906-13.

Hermansen K, Kolotkin RL, Hammer M, Zdravkovic M, Matthews D. Patient-reported outcomes in patients with type 2 diabetes treated with liraglutide or glimepiride, both as add-on to metformin. Primary Care Diabetes 2010;4(2):113-7.

Jendle J, Nauck MA, Matthews DR, Frid A, Hermansen K, During $M$, et al. Weight loss with liraglutide, a once-daily human glucagon-like peptide-1 analogue for type 2 diabetes treatment as monotherapy or added to metformin, is primarily as a result of a reduction in fat tissue. Diabetes, Obesity \& Metabolism 2009;11(12):1163-72.

McGill JB. Insights from the Liraglutide Clinical Development Program--the liraglutide effect and action in diabetes (LEAD) studies. Postgraduate Medicine 2009;121(3):16-25.

NCT00318461. To compare the effect of liraglutide when given together with metformin with the effect of metformin given alone and with the effect of glimepiride and metformin given together (LEAD-2). clinicaltrials.gov/ct2/show/NCT00318461 (accessed 28 March 2017).

Nauck M, Frid A, Hermansen K, Shah NS, Tankova T, Mitha IH, et al. Efficacy and safety comparison of liraglutide, glimepiride, and placebo, all in combination with metformin, in type 2 diabetes: the LEAD (liraglutide effect and action in diabetes)-2 study. Diabetes Care 2009;32(1):84-90.

* Nauck M, Frid A, Hermansen K, Thomsen AB, During M, Shah $\mathrm{N}$, et al. Long-term efficacy and safety comparison of liraglutide, glimepiride and placebo, all in combination with metformin in type 2 diabetes: 2-year results from the LEAD-2 study. Diabetes, Obesity \& Metabolism 2013;15(3):204-12.

Nauck M, Marre M. Adding liraglutide to oral antidiabetic drug monotherapy: efficacy and weight benefits. Postgraduate Medicine 2009;121(3):5-15.

Niswender K, Pi-Sunyer X, Buse J, Jensen KH, Toft AD, RussellJones $D$, et al. Weight change with liraglutide and comparator therapies: an analysis of seven phase 3 trials from the liraglutide diabetes development programme. Diabetes, Obesity \& Metabolism 2013;15(1):42-54

Roussel R, Martinez L, Vandebrouck T, Douik H, Emiel P, Guery M, et al. Evaluation of the long-term cost-effectiveness of 
liraglutide therapy for patients with type 2 diabetes in France. Journal of Medical Economics 2016;19(2):121-34.

Troels J, Kishore S, Steinberg W. Is there a link between liraglutide and pancreatitis? A post hoc review of pooled and patient-level data from completed liraglutide type 2 diabetes clinical trials. Diabetes Care 2015;38(6):1058-66.

Zinman B, Schmidt WE, Moses A, Lund N, Gough S. Achieving a clinically relevant composite outcome of an $\mathrm{HbA1c}$ of. Diabetes, Obesity \& Metabolism 2012;14(1):77-82.

\section{NCT00367055 \{published data only\}}

GSK Study ID 101765. Rosiglitazone-metformin combination versus metformin-sulfonylurea combination on beta-cell function in type 2 diabetes. gsk-studyregister.com/study/2645 (accessed 28 June 2017).

* NCT00367055. Rosiglitazone-metformin combination versus metformin-sulfonylurea combination on beta-cell function in type 2 diabetes. clinicaltrials.gov/ct2/show/study/NCT00367055 (accessed 28 June 2017).

\section{Petrica 2009 \{published data only\}}

Petrica L, Petrica M, Vlad A, Jianu CD, Gluhovschi G, lanculescu C, et al. Nephro- and neuroprotective effects of rosiglitazone versus glimepiride in normoalbuminuric patients with type 2 diabetes mellitus: a randomized controlled trial. Wiener Klinische Wochenschrift 2009;121(23-24):765-75.

\section{Petrica 2011 \{published data only\}}

Petrica L, Vlad A, Petrica M, Jianu CD, Gluhovschi G, Gadalean F, et al. Pioglitazone delays proximal tubule dysfunction and improves cerebral vessel endothelial dysfunction in normoalbuminuric people with type 2 diabetes mellitus. Diabetes Research and Clinical Practice 2011;94(1):22-32.

\section{Ridderstråle 2014 \{published data only\}}

Brice R, Spencer W, Asaro-Harris A, Zeller C, Hach T, Salsali A, et al. Analysis of empagliflozin vs glimepiride by Quality and Outcomes Framework targets: post hoc analysis of a head-tohead study. Diabetic Medicine 2015;32:95-6.

Chirila C, Zheng Q, Davenport E, Kaschinski D, Pfarr E, Hach T, et al. Treatment satisfaction in type 2 diabetes patients taking empagliflozin compared with patients taking glimepiride. Quality of Life Research 2016;25(5):1199-207.

Khunti K, Bingham-Gardiner P, Hassan SW, Zeller C, Naderali E, Salsali A, et al. Efficacy and safety of empagliflozin compared with glimepiride in South Asian patients with type 2 diabetes in a head-to-head study. Diabetic Medicine 2015;32:96.

Kohler S, Kaspers S, Salsali A, Zeller C, Woerle HJ. Effect of empagliflozin (EMPA) on bone fractures in patients with type 2 diabetes (T2DM). Diabetologia 2016;59:S26.

NCT01167881. Efficacy and safety of empagliflozin (BI 10773) with metformin in patients with type 2 diabetes. clinicaltrials.gov/ct2/show/NCT01167881 (accessed 18 July 2017).
Neeland IJ, McGuire DK, Eliasson B, Ridderstrale M, Zeller C, Woerle $\mathrm{HJ}$, et al. Comparison of adipose distribution indices with gold standard body composition assessments in the EMPAREG H2H SU Trial: a body composition sub-study. Diabetes Therapy 2015;6(4):635-42.

Ridderstrale M, Svaerd R, Zeller C, Kim G, Woerle HJ, Broedl UC. Rationale, design and baseline characteristics of a 4-year (208-week) phase III trial of empagliflozin, an SGLT2 inhibitor, versus glimepiride as add-on to metformin in patients with type 2 diabetes mellitus with insufficient glycemic control. Cardiovascular Diabetology 2013;12:129.

* Ridderstråle M, Andersen KR, Zeller C, Kim G, Woerle HJ, BroedI UC. Comparison of empagliflozin and glimepiride as add-on to metformin in patients with type 2 diabetes: a 104week randomised, active-controlled, double-blind, phase 3 trial. Lancet Diabetes \& Endocrinology 2014;2(9):691-700.

\section{Ristic 2007 \{published data only\}}

CDJN608A2308. A multicenter, double-blind, randomized, parallel-group study to evaluate the efficacy and safety of nateglinide and gliclazide in combination with metformin, in type 2 diabetes patients inadequately controlled on maximally tolerated doses of metformin alone. www.novctrd.com/ CtrdWeb/displaypdf.nov?trialresultid=1852 (accessed 17 July 2017).

CDJN608A2308. Six month extension to a multicenter, doubleblind, randomized, parallel-group study to evaluate the efficacy and safety of nateglinide and gliclazide in combination with metformin, in type 2 diabetes patients inadequately controlled on maximally tolerated doses of metformin alone. www.novctrd.com/CtrdWeb/displaypdf.nov?trialresultid=1850 (accessed 17 July 2017).

* Ristic S, Collober-Maugeais C, Cressier F, Tang P, Pecher E. Nateglinide or gliclazide in combination with metformin for treatment of patients with type 2 diabetes mellitus inadequately controlled on maximum doses of metformin alone: 1-year trial results. Diabetes, Obesity \& Metabolism 2007;9(4):506-11.

Ristic S, Collober-Maugeais C, Pecher E, Cressier F. Comparison of nateglinide and gliclazide in combination with metformin, for treatment of patients with type 2 diabetes mellitus inadequately controlled on maximum doses of metformin alone. Diabetic Medicine 2006;23(7):757-62.

\section{Schernthaner 2015 \{published data only\}}

NCT01006603. Saxagliptin compared to glimepiride in elderly type 2 diabetes patients, with inadequate glycemic control on metformin (GENERATION). clinicaltrials.gov/ct2/show/ NCT01006603 (accessed 2 August 2017).

Perl S, Cook W, Wei C, Ohman P, Hirshberg B. Effects of glimepiride versus saxagliptin on beta-cell function and hypoglycemia: a post hoc analysis in older patients with type 2 diabetes inadequately controlled with metformin. Clinical Therapeutics 2016;38(12):2578-88.

Perl S, Cook W, Wei C, Ohman P, Hirshberg B. Low betacell function at baseline is associated with higher rates of 
hypoglycemia in response to treatment with glimepiride in elderly patients with type 2 diabetes inadequately controlled with metformin. Diabetes 2015;64:A319.

* Schernthaner G, Durán-Garcia S, Hanefeld M, Langslet G, Niskanen L, Östgren CJ, et al. Efficacy and tolerability of saxagliptin compared with glimepiride in elderly patients with type 2 diabetes: a randomized, controlled study (GENERATION). Diabetes, Obesity \& Metabolism 2015;17(7):630-8.

\section{Seck 2010 \{published data only\}}

Krobot KJ, Ferrante SA, Davies MJ, Seck T, Meininger GE, Williams-Herman $D$, et al. Lower risk of hypoglycemia with sitagliptin compared to glipizide when either is added to metformin therapy: a pre-specified analysis adjusting for the most recently measured $\mathrm{HbA}(1 \mathrm{c})$ value. Current Medical Research and Opinion 2012;28(8):1281-7.

NCT00094770. An investigational drug study in patients with type 2 diabetes mellitus (0431-024). clinicaltrials.gov/ct2/show/ study/NCT00094770 (accessed 14 July 2017).

Nauck MA, Meininger G, Sheng D, Terranella L, Stein PP. Efficacy and safety of the dipeptidyl peptidase- 4 inhibitor, sitagliptin, compared with the sulfonylurea, glipizide, in patients with type 2 diabetes inadequately controlled on metformin alone: a randomized, double-blind, non-inferiority trial. Diabetes, Obesity \& Metabolism 2007;9(2):194-205.

Ommen ES, Xu L, O'Neill EA, Goldstein BJ, Kaufman KD, Engel SS. Comparison of treatment with sitagliptin or sulfonylurea in patients with type 2 diabetes mellitus and mild renal impairment: a post hoc analysis of clinical trials. Diabetes Therapy 2015;6(1):29-40.

* Seck T, Nauck M, Sheng D, Sunga S, Davies MJ, Stein PP, et al. Safety and efficacy of treatment with sitagliptin or glipizide in patients with type 2 diabetes inadequately controlled on metformin: a 2-year study. International Journal of Clinical Practice 2010;64(5):562-76.

Seck TL, Engel SS, Williams-Herman DE, Sisk CM, Golm GT, Wang $\mathrm{H}$, et al. Sitagliptin more effectively achieves a composite endpoint for $\mathrm{A} 1 \mathrm{C}$ reduction, lack of hypoglycemia and no body weight gain compared with glipizide. Diabetes Research and Clinical Practice 2011;93(1):e15-7.

Shankar RR, Xu L, Golm GT, O'Neill EA, Goldstein BJ, Kaufman KD, et al. A comparison of glycaemic effects of sitagliptin and sulfonylureas in elderly patients with type 2 diabetes mellitus. International Journal of Clinical Practice 2015;69(6):626-31.

Williams-Herman D, Round E, Swern AS, Musser B, Davies MJ, Stein PP, et al. Safety and tolerability of sitagliptin in patients with type 2 diabetes: a pooled analysis. BMC Endocrine Disorders 2008;8:14.

\section{Vaccaro 2017 \{published data only\}}

Chilelli. Long-term effect of pioglitazone vs glimepiride on lipoprotein oxidation in patients with type 2 diabetes: a prospective randomized study. Manuscript draft (accessed 19 March 2018).
NCT00700856. Thiazolidinediones or sulphonylureas and cardiovascular accidents intervention trial (TOSCA IT). clinicaltrials.gov/ct2/show/NCT00700856 (accessed 9 October 2017).

Vaccaro O, Masulli M, Bonora E, Del Prato S, Giorda CB, Maggioni AP, et al. Addition of either pioglitazone or a sulfonylurea in type 2 diabetic patients inadequately controlled with metformin alone: impact on cardiovascular events. A randomized controlled trial. Nutrition, Metabolism, and Cardiovascular Diseases 2012;22(11):997-1006.

Vaccaro O, Masulli M, Bonora E, Del Prato S, Nicolucci A, Rivellese AA, et al. The TOSCA.IT trial: a study designed to evaluate the effect of pioglitazone versus sulfonylureas on cardiovascular disease in type 2 diabetes. Diabetes Care 2012; Vol. 35, issue 12:e82. [PUBMED: 23173143]

* Vaccaro O, Masulli M, Nicolucci A, Bonora E, Del Prato S, Maggioni AP, et al. Effects on the incidence of cardiovascular events of the addition of pioglitazone versus sulfonylureas in patients with type 2 diabetes inadequately controlled with metformin (TOSCA.IT): a randomised, multicentre trial. Lancet Diabetes Endocrinology 2017;5(11):887-97.

\section{References to studies excluded from this review}

\section{ACCORD 2007 \{published data only\}}

Buse JB, Bigger JT, Byington RP, Cooper LS, Cushman WC, Friedewald WT, et al. Action to Control Cardiovascular Risk in Diabetes (ACCORD) trial: design and methods. American Journal of Cardiology 2007;99(12a):21i-33i.

NCT00000620. Action to control cardiovascular risk in diabetes (ACCORD). clinicaltrials.gov/ct2/show/NCT00000620 (accessed 3 March 2018).

\section{Alsharidah 2018 \{published data only\}}

Alsharidah M, Algeffari M, Abdel-Moneim AH, Lutfi MF, Alshelowi $\mathrm{H}$. Effect of combined gliclazide/metformin treatment on oxidative stress, lipid profile, and hepatorenal functions in type 2 diabetic patients. Saudi Pharmaceutical Journal 2018;26(1):1-6.

\section{Araki 2015 \{published data only\}}

Araki E, Tanizawa Y, Tanaka Y, Taniguchi A, Koiwai K, Kim G, et al. Long-term treatment with empagliflozin as add-on to oral antidiabetes therapy in Japanese patients with type 2 diabetes mellitus. Diabetes, Obesity \& Metabolism 2015;17(7):665-74.

NCT01368081. Empagliflozin (BI 10773) comprehensive addon study in Japanese subjects with type 2 diabetes mellitus. clinicaltrials.gov/ct2/show/NCT01368081 (accessed 3 March 2018).

\section{Bermudez-Pirela 2007 \{published data only\}}

Bermudez-Pirela VJ, Cano C, Medina MT, Souki A, Lemus MA, Leal EM, et al. Metformin plus low-dose glimeperide significantly improves Homeostasis Model Assessment for insulin resistance (HOMA(IR)) and beta-cell function (HOMA(beta-cell)) without hyperinsulinemia in patients with 
type 2 diabetes mellitus. American Journal of Therapeutics 2007;14(2):194-202.

\section{Berndt-Zipfel 2013 \{published data only\}}

Berndt-Zipfel C, Michelson G, Dworak M, Mitry M, Loffler A, Pfutzner A, et al. Vildagliptin in addition to metformin improves retinal blood flow and erythrocyte deformability in patients with type 2 diabetes mellitus - results from an exploratory study. Cardiovascular Diabetology 2013;12:59.

NCT01565096. Effect of adding vildagliptin on beta cell function and cardiovascular risk markers in patients with moderate metabolic control during metformin monotherapy. clinicaltrials.gov/ct2/show/NCT01565096 (accessed 3 March 2018).

\section{Bode 2013 \{published data only\}}

Bode B, Stenlof K, Sullivan D, Fung A, Usiskin K. Efficacy and safety of canagliflozin treatment in older subjects with type 2 diabetes mellitus: a randomized trial. Hospital Practice (1995) 2013;41(2):72-84.

NCT01106651. A safety and efficacy study of canagliflozin in older patients ( 55 to 80 years of age) with type 2 diabetes mellitus. clinicaltrials.gov/ct2/show/NCT01106651 (accessed 3 March 2018).

\section{Bruce 2006 \{published data only\}}

Bruce S, Park JS, Fiedorek FT, Howlett HC. Beta-cell response to metformin-glibenclamide combination tablets (Glucovance) in patients with type 2 diabetes. International Journal of Clinical Practice 2006;60(7):783-90.

NCT00035568. A research study to assess the mechanism by which glucovance, metformin, and glyburide work to control glucose levels In patients with type 2 diabetes. clinicaltrials.gov/ ct2/show/NCT00035568 (accessed 3 March 2018).

\section{Charbonnel 2006 \{published data only\}}

Charbonnel B, Karasik A, Liu J, Wu M, Meininger G. Efficacy and safety of the dipeptidyl peptidase-4 inhibitor sitagliptin added to ongoing metformin therapy in patients with type 2 diabetes inadequately controlled with metformin alone. Diabetes Care 2006;29(12):2638-43.

NCT00086515. Metformin add-on study in patients with type 2 diabetes mellitus (0431-020)(COMPLETED). clinicaltrials.gov/ ct2/show/NCT00086515 (accessed 3 March 2018).

\section{Cryer 2005 \{published data only\}}

Cryer DR, Nicholas SP, Henry DH, Mills DJ, Stadel BV. Comparative outcomes study of metformin intervention versus conventional approach the COSMIC Approach Study. Diabetes Care 2005;28(3):539-43.

\section{CTRI/2013/02/003417 \{published data only\}}

CTRI/2013/02/003417. Restudy of the PURSE HIS Population for the prevalence of risk factors causing blood vessel damage to heart muscle, brain tissue or peripheral tissue and to give advice on diet, exercise and if necessary small dose of drug to prevent conversion of pre-diabetes to diabetes. www.ctri.nic.in/Clinicaltrials/pdf_generate.php? trialid $=6005 \&$ EncHid $=\&$ modid $=\&$ compid $=\% 27, \% 276005 \operatorname{det} \% 27$ (accessed 9 March 2018).

\section{Derosa 2015 \{published data only\}}

Derosa G, D'Angelo A, Maffioli P. Sitagliptin in type 2 diabetes mellitus: efficacy after five years of therapy. Pharmacological Research 2015;100:127-34.

\section{EUCTR2004-002549-11-FI \{published data only\}}

Eudract: 2004-002549-11. An open, multi-centre and longterm extension study to evaluate the safety and tolerability of oral tesaglitazar therapy in patients with type 2 diabetes - GALLEX 1. www.clinicaltrialsregister.eu/ctr-search/search? query=eudract_number:2004-002549-11 (accessed 3 March 2018).

\section{EUCTR2006-001240-30-BE \{published data only\}}

Eudract: 2006-001240-30. Long term double blind comparison of gliclazide MR ( 30 to $120 \mathrm{mg}$ daily per os) and rosiglitazone ( 4 to $8 \mathrm{mg}$ daily per os) given in combination with metformin in type 2 diabetic patients. A 2-year international, multicentre, randomised, double-blind, parallelgroup study followed by a 2-year double blind extension ENDORSE. www.clinicaltrialsregister.eu/ctr-search/search? query=eudract_number:2006-001240-30 (accessed 3 March 2018).

\section{EUCTR2009-014727-23-IT \{published data only\}}

Eudract: 2009-014727-23. Multi-center, randomized, openlabel, two-parallel arm, intervention trial comparing DPPIV inhibitor vildagliptin with glibenclamide (glyburide) in achieving and maintaining good blood glucose control in type 2 diabetic patients in treatment failure with metformin alone - Long-term clinical effectiveness of DPP-IV inhibitors. www.clinicaltrialsregister.eu/ctr-search/search? query=eudract_number:2009-014727-23 (accessed 3 March 2018).

\section{EUCTR2009-017524-36-HU \{published data only\}}

Eudract: 2009-017524-36. A phase III, multicenter, doubleblind, active-controlled, 52-week extension study to evaluate the safety and efficacy of dutogliptin in patients with type 2 diabetes mellitus receiving background treatment with glimepiride alone or in combination with metformin or with pioglitazone alone. www.clinicaltrialsregister.eu/ctr-search/ search?query=eudract_number:2009-017524-36 (accessed 3 March 2018).

\section{Gregorio 1989 \{published data only\}}

Gregorio F, Ambrosi F, Angelici F, Cristallini S, Dini FL, Vespasiani G, et al. Body mass index, blood lactate and therapeutic effectiveness of metformin in type II diabetes mellitus. Medicina (Florence, Italy) 1989;9(2):200-4.

\section{Haering 2015 \{published data only\}}

* Haering HU, Merker L, Christiansen AV, Roux F, Salsali A, Kim G, et al. Empagliflozin as add-on to metformin plus sulphonylurea in patients with type 2 diabetes. Diabetes Research and Clinical Practice 2015;110(1):82-90. 
NCT01289990. Safety and efficacy of empagliflozin (BI 10773) and sitagliptin versus placebo over 76 weeks in patients with type 2 diabetes. clinicaltrials.gov/ct2/show/NCT01289990 (accessed 3 March 2018).

Roden M, Merker L, Christiansen AV, Roux F, Salsali A, Kim G, et al. Safety, tolerability and effects on cardiometabolic risk factors of empagliflozin monotherapy in drug-naive patients with type 2 diabetes: a double-blind extension of a phase III randomized controlled trial. Cardiovascular Diabetology 2015;14:154.

\section{Hassanein 2014 \{published data only\}}

Hassanein M, Abdallah K, Schweizer A. A double-blind, randomized trial, including frequent patient-physician contacts and Ramadan-focused advice, assessing vildagliptin and gliclazide in patients with type 2 diabetes fasting during Ramadan: the STEADFAST study. Vascular Health and Risk Management 2014;10:319-26.

\section{Heller 2018 \{published data only\}}

Heller SR, Pratley RE, Sinclair A, Festa A, Kiljanski J, Brusko CS, et al. Glycaemic outcomes of an individualized treatment approach for older vulnerable patients: a randomized, controlled study in type 2 diabetes mellitus (IMPERIUM). Diabetes, Obesity \& Metabolism 2018;20(1):148-56.

NCT02072096. A comparison of two treatment strategies in older participants with type 2 diabetes mellitus (T2DM) (IMPERIUM). clinicaltrials.gov/ct2/show/NCT02072096 (accessed 3 March 2018).

\section{Hermann 2001 \{published data only\}}

Hermann LS, Kalen J, Katzman P, Lager I, Nilsson A, Norrhamn $\mathrm{O}$, et al. Long-term glycaemic improvement after addition of metformin to insulin in insulin-treated obese type 2 diabetes patients. Diabetes, Obesity \& Metabolism 2001;3(6):428-34.

\section{Inagaki 2013 \{published data only\}}

* Inagaki N, Watada H, Murai M, Kagimura T, Gong Y, Patel S, et al. Linagliptin provides effective, well-tolerated add-on therapy to pre-existing oral antidiabetic therapy over 1 year in Japanese patients with type 2 diabetes. Diabetes, Obesity \& Metabolism 2013;15(9):833-43

NCT01204294. Comprehensive add on study in Japan. clinicaltrials.gov/ct2/show/NCT01204294 (accessed 3 March 2018).

\section{Iqbal 2014 \{published data only\}}

Iqbal N, Allen E, Ohman P. Long-term safety and tolerability of saxagliptin add-on therapy in older patients (aged $>/=65$ years) with type 2 diabetes. Clinical Interventions in Aging 2014;9:1479-87.

\section{ISRCTN19750520 \{published data only\}}

ISRCTN19750520. Reduced urine albumin excretion in community based collaborative care in elderly Chinese with type 2 diabetes. www.isrctn.com/ISRCTN19750520 (accessed 9 March 2018).

\section{ISRCTN41840459 \{published data only\}}

ISRCTN41840459. A study to assess the release profiles from fixed combination tablets (gliclazide MR/metformin). www.isrctn.com/ISRCTN41840459 (accessed 3 March 2018).

\section{Jackson 1987 \{published data only\}}

Jackson RA, Hawa MI, Jaspan JB, Sim BM, Disilvio L, Featherbe $D$, et al. Mechanism of metformin action in noninsulin-dependent diabetes. Diabetes 1987;36(5):632-40.

\section{Javaid 2007 \{published data only\}}

Javaid A, Hasan R, Zaib A, Mansoor S. A comparative study of the effects of hypoglycemic agents on serum electrolytes in the diabetic patients. Pakistan Journal of Pharmaceutical Sciences 2007;20(1):67-71.

\section{Johansen 2007 \{published data only\}}

Johansen OE, Gullestad L, Blaasaas KG, Orvik E, Birkeland KI. Effects of structured hospital-based care compared with standard care for type 2 diabetes-The Asker and Baerum Cardiovascular Diabetes Study, a randomized trial. Diabetic Medicine 2007;24(9):1019-27.

NCT00133718. A 2 year trial of patients with type 2 diabetes focusing on cardiovascular diagnostics and metabolic control. clinicaltrials.gov/ct2/show/NCT00133718? term=NCT00133718\&rank=1 (accessed 3 March 2018).

\section{JPRN-UMIN000005327 \{published data only\}}

JPRN-UMIN000005327. Comparisons of oral agents to standardize treatment for diabetes in Japan. upload.umin.ac.jp/cgi-open-bin/ctr_e/ctr_view.cgi? recptno=R000006064 (accessed 3 March 2018).

\section{Kala 2017 \{published data only\}}

Kala 2017. A comparative study of efficacy and safety among metformin with sitagliptin, metformin with voglibose, and metformin with glimepiride in patients with type 2 diabetes mellitus. Asian Journal of Pharmaceutical and Clinical Research 2017; Vol 10(Issue 12):313-16.

\section{Malha 2014 \{published data only\}}

Malha LP, Taan G, Zantout MS, Azar ST. Glycemic effects of vildagliptin in patients with type 2 diabetes before, during and after the period of fasting in Ramadan. Therapeutic Advances in Endocrinology and Metabolism 2014;5(1):3-9.

\section{Marre 2002 \{published data only\}}

Marre M, Howlett H, Lehert P, Allavoine T. Improved glycaemic control with metformin-glibenclamide combined tablet therapy (Glucovance) in type 2 diabetic patients inadequately controlled on metformin. Diabetic Medicine 2002;19(8):673-80.

\section{Meneghini 2010 \{published data only\}}

Meneghini LF, Traylor L, Schwartz SL. Improved glycemic control with insulin glargine versus pioglitazone as add-on therapy to sulfonylurea or metformin in patients with uncontrolled type 2 diabetes mellitus. Endocrine Practice 2010;16(4):588-99. 
Moon 2014 \{published data only\}

* Moon JS, Ha KS, Yoon JS, Lee HW, Lee HC, Won KC. The effect of glargine versus glimepiride on pancreatic beta-cell function in patients with type 2 diabetes uncontrolled on metformin monotherapy: open-label, randomized, controlled study. Acta Diabetologica 2014;51(2):277-85.

NCT00562172. Insulin glargine (lantus) vs sulfonylurea (SU) for BETA cell function (BETA study). clinicaltrials.gov/ct2/show/ NCT00562172 (accessed 3 March 2018).

Morikawa 2011 \{published data only\}

Morikawa A, Ishizeki K, Iwashima Y, Yokoyama H, Muto E, Oshima E, et al. Pioglitazone reduces urinary albumin excretion in renin-angiotensin system inhibitor-treated type 2 diabetic patients with hypertension and microalbuminuria: the APRIME study. Clinical and Experimental Nephrology 2011;15(6):848-53.

\section{Nauck 2006 \{published data only\}}

NCT01511172. Effect of liraglutide as add-on to metformin compared to either liraglutide or metformin alone, or to a combination of metformin and a SU (sulphonylurea) agent in subjects with type 2 diabetes. clinicaltrials.gov/ct2/show/ NCT01511172 (accessed 3 March 2018).

Nauck MA, Hompesch M, Filipczak R, Le TD, Zdravkovic M, Gumprecht J. Five weeks of treatment with the GLP-1 analogue liraglutide improves glycaemic control and lowers body weight in subjects with type 2 diabetes. Experimental and Clinical Endocrinology \& Diabetes 2006;114(8):417-23.

\section{NCT00269061 \{published data only\}}

NCT00269061. An exploratory MRI study in type 2 diabetic subjects: a randomized, double-blinded, placebo-controlled trial to evaluate the measurement of fluid volumes by MRI in the lower extremities of subjects receiving pioglitazone. clinicaltrials.gov/ct2/show/NCT00269061 (accessed 3 March 2018).

\section{NCT00449605 \{published data only\}}

NCT00449605. A glycemic control evaluation of glimepiride versus rimonabant on top of metformin in type 2 diabetes (ALLEGRO). clinicaltrials.gov/ct2/show/NCT00449605? term=NCT00449605\&rank=1 (accessed 3 March 2018).

\section{NCT00518882 \{published data only\}}

NCT00518882. Effect of liraglutide or exenatide added to an ongoing treatment on blood glucose control in subjects with type 2 diabetes (LEAD-6). clinicaltrials.gov/ct2/show/ NCT00518882 (accessed 9 March 2018).

\section{NCT00543751 \{published data only\}}

NCT00543751. Placebo controlled metformin and sulfonylurea combination study in patients with type 2 diabetes (0767-025). clinicaltrials.gov/ct2/show/NCT00543751 (accessed 9 March 2018).

\section{NCT00839527 \{published data only\}}

NCT00839527. A randomized, double-blind, placebo and activecontrolled, parallel-group, multicenter study to determine the efficacy and safety of albiglutide administered in combination with metformin and glimepiride compared with metformin plus glimepiride and placebo and with metformin plus glimepiride and pioglitazone in subjects with type 2 diabetes mellitus. clinicaltrials.gov/ct2/show/NCT00839527 (accessed 3 March 2018).

NCT00909597 \{published data only\}

NCT00909597. A study of taspoglutide versus pioglitazone in patients with type 2 diabetes. clinicaltrials.gov/ct2/show/ NCT00909597 (accessed 3 March 2018).

\section{NCT00947557 \{published data only\}}

NCT00947557. A phase III, randomized, double-blind, placebocontrolled, multicenter study to evaluate the safety and efficacy of dutogliptin in patients with type 2 diabetes mellitus on background treatment with glimepiride with or without metformin. clinicaltrials.gov/ct2/show/NCT00947557 (accessed 9 March 2018).

\section{NCT01087567 \{published data only\}}

NCT01087567. INSPIRE diabetes study: basal bolus insulin as primary treatment of type 2 diabetes. clinicaltrials.gov/ct2/ show/NCT01087567 (accessed 9 March 2018).

\section{NCT01106625 \{published data only\}}

NCT01106625. The CANTATA-MSU Trial (canagliflozin treatment and trial analysis - metformin and sulphonylurea). clinicaltrials.gov/ct2/show/NCT01106625 (accessed 3 March 2018).

\section{NCT01426737 \{published data only\}}

NCT01426737. The Swiss glucose variability study. clinicaltrials.gov/ct2/show/NCT01426737 (accessed 26 June 2017).

\section{NCT01455883 \{published data only\}}

NCT01455883. A study to evaluate ITCA 650 compared to glimepiride for the treatment of type 2 diabetes. clinicaltrials.gov/ct2/show/NCT01455883 (accessed 3 March 2018).

\section{NCT01481116 \{published data only\}}

NCT01481116. Efficacy and safety of TAK-875 compared to glimepiride when used with metformin in participants with type 2 diabetes. clinicaltrials.gov/ct2/show/results/NCT01481116 (accessed 26 June 2017).

\section{NCT01593137 \{published data only\}}

NCT01593137. A long-term, randomized, open-labeled, parallel-group trial to compare the effects of liraglutide and sulphonylurea (glimepiride) both in combination with metformin on clinical, endothelial and image markers of cardiovascular risk in patients with type 2 diabetes. clinicaltrials.gov/ct2/show/NCT01593137 (accessed 3 March 2018).

\section{NCT02244164 \{published data only\}}

NCT02244164. Pathophysiological study of the increase in pancreatic volume in type 2 diabetes treatments. clinicaltrials.gov/ct2/show/NCT02244164 (accessed 3 March 2018). 


\section{NCT02462369 \{published data only\}}

NCT02462369. Saxagliptin's effects on microalbuminuria improvement in type 2 diabetic patients. clinicaltrials.gov/ct2/ show/NCT02462369 (accessed 28 February 2018).

\section{NCT02587741 \{published data only\}}

NCT02587741. Comparison of diabetes retinopathy among type 2 diabetic patients treated with different regimens (CORRECT). clinicaltrials.gov/ct2/show/NCT02587741 (accessed 3 March 2018).

\section{NCT02616666 \{published data only\}}

NCT02616666. A pragmatic trial to evaluate the comparative effectiveness between dapagliflozin and standard of care in type 2 diabetes patients (DECIDE Study). clinicaltrials.gov/ct2/ show/NCT02616666 (accessed 9 March 2018).

\section{NCT03060980 \{published data only\}}

NCT03060980. Comparison of efficacy, safety, and tolerability of ITCA 650 to empagliflozin and glimepiride as add-on metformin clinicaltrials.gov/ct2/show/NCT03060980 (accessed 9 March 2018).

\section{Onuchin 2010 \{published data only\}}

Onuchin SG, Elsukova OS, Solov'ev OV, Onuchina EL. Capabilities of hypoglycemic therapy in women with decompensated type 2 diabetes mellitus. Terapevticheskii Arkhiv 2010;82(8):34-41.

\section{Rosenstock 2006 \{published data only\}}

Rosenstock J, Sugimoto D, Strange P, Stewart JA, SoltesRak E, Dailey G. Triple therapy in type 2 diabetes: insulin glargine or rosiglitazone added to combination therapy of sulfonylurea plus metformin in insulin-naive patients. Diabetes Care 2006;29(3):554-9.

\section{Rosenstock 2018 \{published data only\}}

NCT02033889. A study to evaluate the efficacy and safety of ertugliflozin in participants with type 2 diabetes mellitus and inadequate glycemic control on metformin monotherapy (MK-8835-007). clinicaltrials.gov/ct2/show/NCT02033889 (accessed 28 February 2018).

* Rosenstock J, Frias J, Pall D, Charbonnel B, Pascu R, Saur D, et al. Effect of ertugliflozin on glucose control, body weight, blood pressure and bone density in type 2 diabetes mellitus inadequately controlled on metformin monotherapy (VERTIS MET). Diabetes, Obesity \& Metabolism 2018;20(3):520-9.

\section{Rubin 2008 \{published data only\}}

NCT00095030. Study comparing muraglitazar with glimepiride in type 2 diabetics who are not controlled with metformin alone. clinicaltrials.gov/ct2/show/NCT00095030 (accessed 26 June 2017).

* Rubin CJ, Ledeine JM, Fiedorek FT. Improvement of glycaemic and lipid profiles with muraglitazar plus metformin in patients with type 2 diabetes: an active-control trial with glimepiride. Diabetes \& Vascular Disease Research 2008;5(3):168-76.

\section{Shankar 2017 \{published data only\}}

NCT01755156. A study to evaluate the safety, tolerability, and efficacy of the addition of omarigliptin (MK-3102) to participants with type 2 diabetes mellitus who have inadequate glycemic control on metformin therapy (MK-3102-024). clinicaltrials.gov/ ct2/show/NCT01755156 (accessed 28 February 2018).

* Shankar RR, Inzucchi SE, Scarabello V, Gantz I, Kaufman KD, Lai E, et al. A randomized clinical trial evaluating the efficacy and safety of the once-weekly dipeptidyl peptidase- 4 inhibitor omarigliptin in patients with type 2 diabetes inadequately controlled on metformin monotherapy. Current Medical Research and Opinion 2017;33(10):1853-60.

Tolman 2009 \{published data only\}

NCT00494312. Safety study of pioglitazone compared to glyburide on liver function. clinicaltrials.gov/ct2/show/ NCT00494312?term=NCT00494312\&rank=1 (accessed 3 March 2018).

Tolman KG, Freston JW, Kupfer S, Perez A. Liver safety in patients with type 2 diabetes treated with pioglitazone: results from a 3-year, randomized, comparator-controlled study in the US. Drug Safety 2009;32(9):787-800.

\section{UKPDS 1998 \{published data only\}}

ISRCTN75451837. UK prospective diabetes study - post study monitoring (PSM) and cohort follow-up (CFU). www.isrctn.com/ ISRCTN75451837 (accessed 9 March 2018).

UK Prospective Diabetes Study (UKPDS) Group. Intensive bloodglucose control with sulphonylureas or insulin compared with conventional treatment and risk of complications in patients with type 2 diabetes (UKPDS 33). Lancet 1998;352(9131):837-53.

\section{Weissman 2014 \{published data only\}}

Weissman PN, Carr MC, Ye J, Cirkel DT, Stewart M, Perry C, et al. HARMONY 4: randomised clinical trial comparing onceweekly albiglutide and insulin glargine in patients with type 2 diabetes inadequately controlled with metformin with or without sulfonylurea. Diabetologia 2014;57(12):2475-84.

\section{Yki-Järvinen 1999 \{published data only\}}

Yki-Järvinen H, Ryysy L, Nikkilä K, Tulokas T, Vanamo R, Heikkilä M. Comparison of bedtime insulin regimens in patients with type 2 diabetes mellitus. A randomized, controlled trial. Annals of Internal Medicine 1999;130(5):389-96.

\section{References to studies awaiting assessment}

Müller-Wieland 2018 \{published data only\}

* Müller-Wieland D, Kellerer M, Cypryk K, Skripova D, Rohwedder K, Johnsson E, et al. Efficacy and safety of dapagliflozin or dapagliflozin plus saxagliptin versus glimepiride as add-on to metformin in patients with type 2 diabetes. Diabetes, Obesity \& Metabolism 2018;20(11):2598-607.

NCT02471404. Efficacy and safety of dapagliflozin and dapagliflozin plus saxagliptin in combination with metformin in type 2 diabetes patients compared with sulphonylurea. 
clinicaltrials.gov/ct2/show/NCT02471404 (accessed 28 February 2018).

\section{NCT02564926 \{published data only\}}

NCT02564926. Foxiga Korea local phase 4 study (BEYOND). clinicaltrials.gov/ct2/show/NCT02564926 (accessed 28 February 2018).

\section{References to ongoing studies}

EUCTR2011-003335-63-IT \{published data only\}

EUCTR2011-003335-63-IT. Effects of liraglutide on ß-cell function in type 2 diabetic patients with secondary failure to oral hypoglycemic agents. A randomized, controlled, parallel groups, open-label, phase II study. apps.who.int/trialsearch/ Trial2.aspx?TrialID=EUCTR2011-003335-63-IT (accessed 28 February 2018).

\section{EUCTR2012-000152-34-IT \{published data only\}}

EUCTR2012-000152-34-IT. Evaluation of the effect of treatment with DPP-4 inhibitor on endothelial function versus sulphonylurea on markers of oxidative stress and inflammation and platelet function in patients with diabetes mellitus type 2 in primary failure with metformin and with $\mathrm{HbA1c}$ values below $8,5 \%$. apps.who.int/trialsearch/Trial2.aspx? TrialID=EUCTR2012-000152-34-IT (accessed 28 February 2018).

\section{JPRN-UMIN000008815 \{published data only\}}

JPRN-UMIN000008815. The effect of DPP-4 inhibitor on pancreatic beta cell function and renal function in type 2 diabetic patients. upload.umin.ac.jp/cgi-open-bin/ctr_e/ ctr_view.cgi?recptno=R000010271 (accessed 28 February 2018).

\section{NCT01243424 \{published data only\}}

Marx N, Rosenstock J, Kahn SE, Zinman B, Kastelein JJ, Lachin JM, et al. Design and baseline characteristics of the cardiovascular outcome trial of linagliptin versus glimepiride in type 2 diabetes (CAROLINA(R)). Diabetes \& Vascular Disease Research 2015;12(3):164-74.

* NCT01243424. CAROLINA: Cardiovascular outcome study of linagliptin versus glimepiride in patients with type 2 diabetes. clinicaltrials.gov/ct2/show/NCT01243424 (accessed 28 February 2018).

\section{NCT01794143 \{published data only\}}

* NCT01794143. A comparative effectiveness study of major glycemia-lowering medications for treatment of type 2 diabetes (GRADE). clinicaltrials.gov/ct2/show/NCT01794143 (accessed 28 February 2018).

Nathan DM, Buse JB, Kahn SE, Krause-Steinrauf H, Larkin ME, Staten $\mathrm{M}$, et al. Rationale and design of the glycemia reduction approaches in diabetes: a comparative effectiveness study (GRADE). Diabetes Care 2013;36(8):2254-61.

\section{NCT02142309 \{published data only\}}

NCT02142309. Glycemic durability after metformin failure (AMAZING). clinicaltrials.gov/ct2/show/NCT02142309 (accessed 28 February 2018)

\section{NCT02730377 \{published data only\}}

NCT02730377. Efficacy in controlling glycaemia with Victoza ${ }^{\circledR}$ (liraglutide) as add-on to metformin vs. OAD's as add-on to metformin after up to 104 weeks of treatment in subjects with type 2 diabetes (LIRA-PRIME). clinicaltrials.gov/ct2/show/ NCT02730377 (accessed 28 February 2018).

\section{NCT02769481 \{published data only\}}

NCT02769481. Safety and efficacy of bexagliflozin compared to glimepiride as add-on therapy to metformin in type 2 diabetes subjects. clinicaltrials.gov/ct2/show/NCT02769481 (accessed 28 February 2018)

\section{NCT03332771 \{published data only\}}

NCT03332771. Efficacy and safety of sotagliflozin versus glimepiride and placebo in subjects with type 2 diabetes mellitus that are taking metformin monotherapy (SOTA-GLIM). clinicaltrials.gov/ct2/show/NCT03332771 (accessed 9 March 2018).

\section{Additional references}

\section{ADA 2003}

Expert Committee on the Diagnosis and Classification of Diabetes Mellitus. Report of the expert committee on the diagnosis and classification of diabetes mellitus. Diabetes Care 2003;26(Suppl 1):S5-20.

\section{ADA 2008}

American Diabetes Association. Standards of medical care in diabetes - 2008. Diabetes Care 2008;31(Suppl 1):S12-54. [PUBMED: 18165335]

\section{ADA 2016}

American Diabetes Association. 7. Approaches to glycemic treatment. Diabetes Care 2016;39(Suppl 1):S52-9. [PUBMED: 26696682]

\section{ADA 2019}

American Diabetes Association. Standards of medical care in diabetes - 2019. care.diabetesjournals.org/content/ diacare/suppl/2018/12/17/42.Supplement_1.DC1/ DC_42_S1_Combined_FINAL.pdf (accessed 16 February 2019).

\section{ADOPT 2006}

Kahn SE, Haffner SM, Heise MA, Herman WH, Holman RR, Jones NP, et al. Glycemic durability of rosiglitazone, metformin, or glyburide monotherapy. New England Journal of Medicine 2006;355(23):2427-43. [PUBMED: 17145742]

\section{Albarran 2013}

Albarran OG, Ampudia-Blasco FJ. Dapagliflozin, the first SGLT-2 inhibitor in the treatment of type 2 diabetes. Medicina Clinica 2013;141 Suppl 2:36-43.

\section{Almdal 2004}

Almdal T, Scharling H, Jensen JS, Vestergaard H. The independent effect of type 2 diabetes mellitus on ischemic heart disease, stroke, and death: a population-based study of 
13,000 men and women with 20 years of follow-up. Archives of Internal Medicine 2004;164(13):1422-6. [PUBMED: 15249351]

\section{Altman 2003}

Altman DG, Bland JM. Interaction revisited: the difference between two estimates. BMJ 2003;326(7382):219. [PUBMED: 12543843]

\section{Alvares 2015}

Alvares J, Araujo VE, Izidoro JB, Diniz LM, Nascimento RC, Silva MR, et al. Efficacy and safety of antidiabetic drugs available on Brazilian public health system (Sus) - regular insulin, NPH insulin, metformin, glibenclamide and gliclazide In treatment of type 2 diabetes (T2DM) - systematic review and meta-analysis. Value in Health 2015;18(7):A862.

\section{Amate 2015}

Amate JM, Lopez-Cuadrado T, Almendro N, Bouza C, SazParkinson Z, Rivas-Ruiz R, et al. Effectiveness and safety of glimepiride and iDPP4, associated with metformin in second line pharmacotherapy of type 2 diabetes mellitus: systematic review and meta-analysis. International Journal of Clinical Practice 2015;69(3):292-304.

\section{Andersen 2016}

Andersen SE, Christensen M. Hypoglycaemia when adding sulphonylurea to metformin: a systematic review and network meta-analysis. British Journal of Clinical Pharmacology 2016;82(5):1291-302.

\section{Aylsworth 2014}

Aylsworth A, Dean Z, VanNorman C, Nkemdirim Okere A. Dapagliflozin for the treatment of type 2 diabetes mellitus. Annals of Pharmacotherapy 2014;48(9):1202-8.

\section{Bailey 1996}

Bailey CJ, Turner RC. Metformin. New England Journal of Medicine 1996;334(9):574-9. [PUBMED: 8569826]

\section{Bell 2013}

Bell ML, McKenzie JE. Designing psycho-oncology randomised trials and cluster randomised trials: variance components and intra-cluster correlation of commonly used psychosocial measures. Psycho-oncology 2013;22:1738-47.

\section{Bellary 2011}

Bellary S. For type 2 diabetes poorly controlled by metformin monotherapy, the addition of any non-insulin antidiabetic drug reduces $\mathrm{HbAlc}$ to a similar extent, but with differing effects on weight and hypoglycaemic risk. Evidence-based Medicine 2011;16(2):39-40.

\section{Belsey 2008}

Belsey J, Krishnarajah G. Glycaemic control and adverse events in patients with type 2 diabetes treated with metformin + sulphonylurea: a meta-analysis. Diabetes, Obesity \& Metabolism 2008;10 Suppl 1:1-7.

\section{Boutron 2014}

Boutron I, Altman DG, Hopewell S, Vera-Badillo F, Tannock I, Ravaud P. Impact of spin in the abstracts of articles reporting results of randomized controlled trials in the field of cancer: the SPIIN randomized controlled trial. Journal of Clinical Oncology 2014;32:4120-6.

\section{Buch 2011}

Buch MH, Aletaha D, Emery P, Smolen JS. Reporting of longterm extension studies: lack of consistency calls for consensus. Annals of the Rheumatic Diseases 2011;70(6):886-90.

\section{Chan 2015}

Chan SP, Colagiuri S. Systematic review and meta-analysis of the efficacy and hypoglycemic safety of gliclazide versus other insulinotropic agents. Diabetes Research and Clinical Practice 2015;110(1):75-81.

\section{CONSORT}

The CONSORT statement. www.consort-statement.org (accessed 19 may 2016).

\section{Corbett 2014}

Corbett MS, Higgins JP, Woolacott NF. Assessing baseline imbalance in randomised trials: implications for the Cochrane risk of bias tool. Research Synthesis Methods 2014;5:79-85.

\section{Dai 2014}

Dai X, Wang H, Jing Z, Fu P. The effect of a dual combination of noninsulin antidiabetic drugs on lipids: a systematic review and network meta-analysis. Current Medical Research and Opinion 2014;30(9):1777-86.

\section{Deacon 2015}

Deacon CF, Lebovitz HE. A comparative review of DPP-4 inhibitors and sulphonylureas. Diabetes, Obesity \& Metabolism 2015;18(4):333-47. [PUBMED: 26597596]

\section{Deeks 2003}

Deeks JJ, Dinnes J, D'Amico R, Sowden AJ, Sakarovitch C, Song $F$, et al. Evaluating non-randomised intervention studies. Health Technology Assessment (Winchester, England) 2003;7(27):iii-x, 1-173. [PUBMED: 14499048]

\section{Deeks 2017}

Deeks JJ, Higgins JP, Altman DG (editors): on behalf of the Cochrane Statistical Methods Group. Chapter 9: Analysing data and undertaking meta-analyses. In: Higgins JPT, Churchill R, Chandler J, Cumpston MS (editors). Cochrane Handbook for Systematic Reviews of Interventions version 5.2.0 (updated June 2017), Cochrane, 2017. Available from www.training.cochrane.org/handbook.

\section{DeFronzo 1999}

DeFronzo RA. Pharmacologic therapy for type 2 diabetes mellitus. Annals of Internal Medicine 1999;131(4):281-303. [PUBMED: 10454950]

\section{Evans 2006}

Evans JM, Ogston SA, Emslie-Smith A, Morris AD. Risk of mortality and adverse cardiovascular outcomes in type 2 diabetes: a comparison of patients treated with sulfonylureas and metformin. Diabetologia 2006;49(5):930-6. [PUBMED: 16525843] 


\section{Fleming 2015}

Fleming JW, Fleming LW, Davis CS. Fixed-dose combinations in type 2 diabetes - role of the canagliflozin metformin combination. Diabetes, Metabolic Syndrome and Obesity 2015;8:287-94.

\section{Foroutan 2016}

Foroutan N, Muratov S, Levine M. Safety and efficacy of dipeptidyl peptidase-4 inhibitors vs sulfonylurea in metforminbased combination therapy for type 2 diabetes mellitus: systematic review and meta-analysis. Clinical and Investigative Medicine, Medecine Clinique et Experimentale 2016;39(2):E48-62.

\section{Geng 2015}

Geng J, Yu H, Mao Y, Zhang P, Chen Y. Cost effectiveness of dipeptidyl peptidase- 4 inhibitors for type 2 diabetes. Pharmaco Economics 2015;33(6):581-97.

\section{Goring 2014}

Goring S, Hawkins N, Wygant G, Roudaut M, Townsend R, Wood I, et al. Dapagliflozin compared with other oral antidiabetes treatments when added to metformin monotherapy: a systematic review and network meta-analysis. Diabetes, Obesity \& Metabolism 2014;16(5):433-42.

\section{GRADEproGDT 2015 [Computer program]}

McMaster University (developed by Evidence Prime). GRADEproGDT. Hamilton (ON): McMaster University (developed by Evidence Prime), 2015 (accessed 28 February 2018).

\section{Gu 2015}

Gu S, Deng J, Shi L, Mu Y, Dong H. Cost-effectiveness of saxagliptin vs glimepiride as a second-line therapy added to metformin in type 2 diabetes in China. Journal of Medical Economics 2015;18(10):808-20.

\section{Gulliford 2004}

Gulliford M, Latinovic R. Mortality in type 2 diabetic subjects prescribed metformin and sulphonylurea drugs in combination: cohort study. Diabetes/metabolism Research and Reviews 2004;20(3):239-45. [PUBMED: 15133756]

\section{Guthrie 2015}

Guthrie RM. Clinical use of dipeptidyl peptidase-4 and sodium-glucose cotransporter 2 inhibitors in combination therapy for type 2 diabetes mellitus. Postgraduate Medicine 2015;127(5):463-79.

\section{Harrower 2000}

Harrower AD. Comparative tolerability of sulphonylureas in diabetes mellitus. Drug Safety 2000;22(4):313-20. [PUBMED: 10789825]

\section{Harrower 2000a}

Harrower A. Gliclazide modified release: from once-daily administration to 24-hour blood glucose control. Metabolism: Clinical and Experimental 2000;49(10 Suppl 2):7-11. [PUBMED: 11078469]

\section{Hart 2012}

Hart B, Lundh A, Bero L. Effect of reporting bias on metaanalyses of drug trials: reanalysis of meta-analyses. $B M J$ 2012;344:d7202. [DOI: 10.1136/bmj.d7202]

\section{Hemmingsen 2011}

Hemmingsen B, Lund SS, Gluud C, Vaag A, Almdal T, Hemmingsen $C$, et al. Intensive glycaemic control for patients with type 2 diabetes: systematic review with meta-analysis and trial sequential analysis of randomised clinical trials. $B M J$ (Clinical Research Ed.) 2011;343:d6898. [PUBMED: 22115901]

\section{Hershon 2016}

Hershon KS. Options for empagliflozin in combination therapy in type 2 diabetes mellitus. International Journal of General Medicine 2016;9:155-72.

\section{Higgins 2002}

Higgins JP, Thompson SG. Quantifying heterogeneity in a metaanalysis. Statistics in Medicine 2002;21:1539-58.

\section{Higgins 2003}

Higgins JP, Thompson SG, Deeks JJ, Altman DG. Measuring inconsistency in meta-analysis. BMJ 2003;327(7414):557-60.

\section{Higgins 2009}

Higgins JP, Thompson SG, Spiegelhalter DJ. A re-evaluation of random-effects meta-analysis. Journal of the Royal Statistical Society: Series A (Statistics in Society) 2009;172(1):137-59.

\section{Higgins 2011}

Higgins JP, Whitehead A, Simmonds M. Sequential methods for random-effects meta-analysis. Statistics in Medicine 2011;30(9):903-21. [PUBMED: 21472757]

\section{Higgins 2011a}

Higgins JP, Altman DG, Gøtzsche PC, Jüni P, Moher D, Oxman AD, et al. The Cochrane Collaboration's tool for assessing risk of bias in randomised trials. BMJ 2011;343:d5928.

\section{Higgins 2011b}

Higgins JP, Deeks JJ, Altman DG (editors). Chapter 16: Special topics in statistics. In: Higgins JPT, Green S (editors). Cochrane Handbook for Systematic Reviews of Interventions. Version 5.1.0 (updated March 2011). The Cochrane Collaboration, 2011. Available from www.cochrane-handbook.cochrane.org.

\section{Higgins 2017}

Higgins JP, Altman DG, Sterne JA (editors). Chapter 8: Assessing risk of bias in included studies. In: Higgins JPT, Churchill R, Chandler J, Cumpston MS (editors), Cochrane Handbook for Systematic Reviews of Interventions Version 5.2.0 (updated June 2017), Cochrane, 2017. Available from www.training.cochrane.org/handbook.

\section{Hou 2015}

Hou L, Zhao T, Liu Y, Zhang Y. Efficacy and safety of sitagliptin compared with sulfonylurea therapy in patients with type 2 diabetes showing inadequately controlled glycosylated hemoglobin with metformin monotherapy: a meta-analysis. Experimental and Therapeutic Medicine 2015;9(4):1528-36. 


\section{Hozo 2005}

Hozo SP, Djulbegovic B, Hozo I. Estimating the mean and variance from the median, range, and the size of a sample. BMC Medical Research Methodology 2005;5:13. [DOI: 10.1186/1471-2288-5-13]

\section{Hróbjartsson 2013}

Hróbjartsson A, Thomsen AS, Emanuelsson F, Tendal B, Hilden J, Boutron I, et al. Observer bias in randomized clinical trials with measurement scale outcomes: a systematic review of trials with both blinded and nonblinded assessors. Canadian Medical Association Journal 2013;185(4):E201-11.

\section{ICH 1997}

International Conference on Harmonisation Expert Working Group. International conference on harmonisation of technical requirements for registration of pharmaceuticals for human use. ICH harmonised tripartite guideline. Guideline for good clinical practice //1997 CFR \& ICH Guidelines. PA 19063-2043. USA: Barnett International/PAREXEL, 1997.

\section{Inzucchi 2012}

Inzucchi SE, Bergenstal RM, Buse JB, Diamant M, Ferrannini E, Nauck M, et al. Management of hyperglycemia in type 2 diabetes: a patient-centered approach: position statement of the American Diabetes Association (ADA) and the European Association for the Study of Diabetes (EASD). Diabetes Care 2012;35(6):1364-79. [PUBMED: 22517736]

\section{Johnson 2002}

Johnson JA, Majumdar SR, Simpson SH, Toth EL. Decreased mortality associated with the use of metformin compared with sulfonylurea monotherapy in type 2 diabetes. Diabetes Care 2002;25(12):2244-8. [PUBMED: 12453968]

\section{Jones 2015}

Jones CW, Keil LG, Holland WC, Caughey MC, Platts-Mills TF. Comparison of registered and published outcomes in randomized controlled trials: a systematic review. BMC Medicine 2015;13:282. [DOI: 10.1186/s12916-015-0520-3]

\section{Kahler 2007}

Kahler KH, Rajan M, Rhoads GG, Safford MM, Demissie K, Lu SE, et al. Impact of oral antihyperglycemic therapy on all-cause mortality among patients with diabetes in the Veterans Health Administration. Diabetes Care 2007;30(7):1689-93. [PUBMED: 17440170]

\section{Kannan 2015}

Kannan S, Mahadevan S, Ramakrishnan A. Fixed dose combinations for type 2 diabetes. Lancet Diabetes \& Endocrinology 2015;3(6):408.

\section{Kirkham 2010}

Kirkham JJ, Dwan KM, Altman DG, Gamble C, Dodd S, Smyth R, et al. The impact of outcome reporting bias in randomised controlled trials on a cohort of systematic reviews. BMJ 2010;340:c365. [DOI: 10.1136/bmj.c365]

\section{Krentz 2005}

Krentz AJ, Bailey CJ. Oral antidiabetic agents: current role in type 2 diabetes mellitus. Drugs 2005;65(3):385-411. [PUBMED: 15669880]

\section{Kuecker 2016}

Kuecker CM, Vivian EM. Patient considerations in type 2 diabetes - role of combination dapagliflozin-metformin XR. Diabetes, Metabolic Syndrome and Obesity: targets and therapy 2016;9:25-35

\section{Lan 1983}

Lan KK, DeMets DL. Discrete sequential boundaries for clinical trials. Biometrika 1983;70:659-63.

\section{Langtry 1998}

Langtry HD, Balfour JA. Glimepiride. A review of its use in the management of type 2 diabetes mellitus. Drugs 1998;55(4):563-84. [PUBMED: 9561345]

\section{LeRoith 2002}

LeRoith D. Beta-cell dysfunction and insulin resistance in type 2 diabetes: role of metabolic and genetic abnormalities. American Journal of Medicine 2002;113 Suppl 6A:3S-11S. [PUBMED: 12431757]

\section{Liberati 2009}

Liberati A, Altman DG, Tetzlaff J, Mulrow C, Gøtzsche PC, Ioannidis JPA, et al. The PRISMA statement for reporting systematic and meta-analyses of studies that evaluate interventions: explanation and elaboration. PLoS Medicine 2009;6(7):1-28. [DOI: 10.1371/journal.pmed.1000100]

\section{$\operatorname{Lim} 2015$}

Lim 2015. What's next after metformin? Focus on sulphonylurea: add-on or combination therapy. Pharmacy Practice 2015;13(3):606.

\section{Liu 2014}

Liu X, Xiao Q, Zhang L, Yang Q, Liu X, Xu L, et al. The long-term efficacy and safety of DPP-IV inhibitors monotherapy and in combination with metformin in 18,980 patients with type-2 diabetes mellitus--a meta-analysis. Pharmacoepidemiology and Drug Safety 2014;23(7):687-98.

\section{Lundh 2017}

Lundh A, Lexchin J, Mintzes B, Schroll JB, Bero L. Industry sponsorship and research outcome. Cochrane Database of Systematic Reviews 2017, Issue 2. [DOI: 10.1002/14651858.MR000033.pub3]

\section{Maruthur 2016}

Maruthur NM, Tseng E, Hutfless S, Wilson LM, Suarez-Cuervo C, Berger Z, et al. Diabetes medications as monotherapy or metformin-based combination therapy for type 2 diabetes: a systematic review and meta-analysis. Annals of Internal Medicine 2016;164(11):740-51. [DOI: 10.7326/M15-2650] 


\section{Mathieu 2009}

Mathieu S, Boutron I, Moher D, Altman DG, Ravaud P. Comparison of registered and published primary outcomes in randomized controlled trials. JAMA 2009;302:977-84.

\section{McCall 2001}

McCall AL. Clinical review of glimepiride. Expert Opinion on Pharmacotherapy 2001;2(4):699-713. [PUBMED: 11336617]

\section{Meader 2014}

Meader N, King K, Llewellyn A, Norman G, Brown J, Rodgers M, et al. A checklist designed to aid consistency and reproducibility of GRADE assessments: development and pilot validation. Systematic Reviews 2014;3:82.

\section{Mearns 2015}

Mearns ES, Sobieraj DM, White CM, Saulsberry WJ, Kohn CG, Doleh Y, et al. Comparative efficacy and safety of antidiabetic drug regimens added to metformin monotherapy in patients with type 2 diabetes: a network meta-analysis. PloS one 2015;10(4):e0125879.

\section{Megan 2012}

Megan B, Pickering RM, Weatherall M. Design, objectives, execution and reporting of published open-label extension studies. Journal of Evaluation in Clinical Practice 2012;18(2):209-15.

\section{Mishriky 2015}

Mishriky BM, Cummings DM, Tanenberg RJ. The efficacy and safety of DPP4 inhibitors compared to sulfonylureas as addon therapy to metformin in patients with type 2 diabetes: a systematic review and meta-analysis. Diabetes Research and Clinical Practice 2015;109(2):378-88.

\section{Monami 2008}

Monami M, Lamanna C, Marchionni N, Mannucci E. Comparison of different drugs as add-on treatments to metformin in type 2 diabetes: a meta-analysis. Diabetes Research and Clinical Practice 2008;79(2):196-203.

\section{Morgan 2014}

Morgan CL, Mukherjee J, Jenkins-Jones S, Holden SE, Currie CJ. Association between first-line monotherapy with sulphonylurea versus metformin and risk of all-cause mortality and cardiovascular events: a retrospective, observational study. Diabetes, Obesity \& Metabolism 2014;16(10):957-62. [PUBMED: 24720708]

\section{Nathan 2009}

Nathan DM, Buse JB, Davidson MB, Ferrannini E, Holman RR, Sherwin R, et al. Medical management of hyperglycaemia in type 2 diabetes mellitus: a consensus algorithm for the initiation and adjustment of therapy: a consensus statement from the American Diabetes Association and the European Association for the Study of Diabetes. Diabetologia 2009;52(1):17-30. [PUBMED: 18941734]

\section{Nishio 2015}

Nishio S, Abe M, Ito H. Anagliptin in the treatment of type 2 diabetes: safety, efficacy, and patient acceptability. Diabetes,
Metabolic Syndrome and Obesity: targets and therapy 2015;8:163-71.

\section{Odawara 2015}

Odawara M, Sagara R. Effects of vildagliptin as add-on treatment in patients with type 2 diabetes mellitus: insights from long-term clinical studies in Japan. Journal of Diabetes and Metabolic Disorders 2015;15:21.

\section{Pantalone 2012}

Pantalone KM, Kattan MW, Yu C, Wells BJ, Arrigain S, Jain A, et al. Increase in overall mortality risk in patients with type 2 diabetes receiving glipizide, glyburide or glimepiride monotherapy versus metformin: a retrospective analysis. Diabetes, Obesity \& Metabolism 2012;14(9):803-9. [PUBMED: 22486923]

\section{Phung 2010}

Phung OJ, Scholle JM, Talwar M, Coleman Cl. Effect of noninsulin antidiabetic drugs added to metformin therapy on glycemic control, weight gain, and hypoglycemia in type 2 diabetes. JAMA 2010;303(14):1410-8.

\section{Phung 2014}

Phung OJ, Sobieraj DM, Engel SS, Rajpathak SN. Early combination therapy for the treatment of type 2 diabetes mellitus: systematic review and meta-analysis. Diabetes, Obesity \& Metabolism 2014;16(5):410-7.

\section{Pogue 1997}

Pogue JM, Yusuf S. Cumulating evidence from randomized trials: utilizing sequential monitoring boundaries for cumulative meta-analysis. Controlled Clinical Trials 1997;18(6):580-93; discussion 661-6. [PUBMED: 9408720]

\section{Reid 2016}

Reid 2016. How much is too much? Outcomes in patients using high-dose insulin glargine. International Journal of Clinical Practice 2016;70(1):56-65.

\section{Review Manager 2014 [Computer program]}

Nordic Cochrane Centre, The Cochrane Collaboration. Review Manager 5 (RevMan 5). Version 5.3. Copenhagen: Nordic Cochrane Centre, The Cochrane Collaboration, 2014.

\section{Riley 2011}

Riley RD, Higgins JP, Deeks JJ. Interpretation of random effects meta-analyses. BMJ 2011;342:d549.

\section{Rosenstock 2013}

Rosenstock J, Marx N, Kahn SE, Zinman B, Kastelein JJ, Lachin JM, et al. Cardiovascular outcome trials in type 2 diabetes and the sulphonylurea controversy: rationale for the active-comparator CAROLINA trial. Diabetes \& Vascular Disease Research 2013;10(4):289-301.

\section{Rosenstock 2015}

Rosenstock J, Marx N, Neubacher D, Seck T, Patel S, Woerle HJ, et al. Cardiovascular safety of linagliptin in type 2 diabetes: a comprehensive patient-level pooled analysis of prospectively 
adjudicated cardiovascular events. Cardiovascular Diabetology 2015;14:57.

\section{Roumie 2012}

Roumie CL, Hung AM, Greevy RA, Grijalva CG, Liu X, Murff HJ, et al. Comparative effectiveness of sulfonylurea and metformin monotherapy on cardiovascular events in type 2 diabetes mellitus: a cohort study. Annals of Internal Medicine 2012;157(9):601-10. [PUBMED: 23128859]

\section{Salpeter 2010}

Salpeter SR, Greyber E, Pasternak GA, Salpeter EE. Risk of fatal and nonfatal lactic acidosis with metformin use in type 2 diabetes mellitus. Cochrane Database of Systematic Reviews 2010, Issue 4. [DOI: 10.1002/14651858.CD002967.pub4]

\section{Scheen 2016}

Scheen AJ. Dulaglutide (Trulicity(R)), a new once-weekly agonist of glucagon-like peptide-1 receptors for type 2 diabetes. Revue Medicale de Liege 2016;71(3):154-60.

\section{Scherer 2007}

Scherer RW, Langenberg P, von Elm E. Full publication of results initially presented in abstracts. Cochrane Database of Systematic Reviews 2007, Issue 2. [DOI: 10.1002/14651858.MR000005.pub3]

\section{Schramm 2011}

Schramm TK, Gislason GH, Vaag A, Rasmussen JN, Folke F, Hansen ML, et al. Mortality and cardiovascular risk associated with different insulin secretagogues compared with metformin in type 2 diabetes, with or without a previous myocardial infarction: a nationwide study. European Heart Journal 2011;32(15):1900-8. [PUBMED: 21471135]

\section{Schroll 2015}

Schroll JB, Bero L. Regulatory agencies hold the key to improving Cochrane Reviews of drugs [editorial]. Cochrane Database of Systematic Reviews 2015; Vol. 4:10.1002/14651858.ED000098.

\section{Schünemann 2017}

Schünemann HJ, Oxman AD, Higgins JP, Vist GE, Glasziou P, Akl E, et al. on behalf of the Cochrane GRADEing Methods Group and the Cochrane Statistical Methods Group. Chapter 11: Completing 'Summary of findings' tables and grading the confidence in or quality of the evidence. In: Higgins JPT, Churchill R, Chandler J, Cumpston MS (editors), Cochrane Handbook for Systematic Reviews of Interventions version 5.2.0 (updated June 2017). Cochrane, 2017. Available from www.training.cochrane.org/handbook.

\section{Scott 2012}

Scott LJ. Repaglinide: a review of its use in type 2 diabetes mellitus. Drugs 2012;72(2):249-72. [PUBMED: 22268393]

\section{Seufert 2014}

Seufert J. Oral add-on therapy to metformin in type 2 diabetes mellitus: a direct comparison between canagliflozin and glimepiride. Deutsche Medizinische Wochenschrift (1946) 2014;139 Suppl 2:S65-9.

\section{Sharma 2017}

Sharma M, Beckley N, Nazareth I, Petersen I. Effectiveness of sitagliptin compared to sulfonylureas for type 2 diabetes mellitus inadequately controlled on metformin: a systematic review and meta-analysis. BMJ Open 2017;7(10):e017260.

\section{Sterne 2011}

Sterne JA, Sutton AJ, Ioannidis JP, Terrin N, Jones DR, Lau J, et al. Recommendations for examining and interpreting funnel plot asymmetry in meta-analyses of randomised controlled trials. BMJ 2011;343:d4002.

\section{Sterne 2017}

Sterne JA, Egger M, Moher D, Boutron I (editors). Chapter 10: Addressing reporting biases. In: Higgins JPT, Churchill R, Chandler J, Cumpston MS (editors), Cochrane Handbook for Systematic Reviews of Interventions version 5.2.0 (updated June 2017), Cochrane, 2017. Available from www.training.cochrane.org/handbook.

\section{UGDP 1976}

University Group Diabetes Program. A study of the effects of hypoglycemia agents on vascular complications in patients with adult-onset diabetes. VI. Supplementary report on nonfatal events in patients treated with tolbutamide. Diabetes 1976;25(12):1129-53. [PUBMED: 992232]

\section{UKPDS-33 1998}

UK Prospective Diabetes Study (UKPDS) Group. Intensive bloodglucose control with sulphonylureas or insulin compared with conventional treatment and risk of complications in patients with type 2 diabetes (UKPDS 33). Lancet 1998;352(9131):837-53. [PUBMED: 9742976]

\section{UKPDS-34 1998}

UK Prospective Diabetes Study (UKPDS) Group. Effect of intensive blood-glucose control with metformin on complications in overweight patients with type 2 diabetes (UKPDS 34). Lancet 1998;352(9131):854-65. [PUBMED: 9742977]

\section{Varvaki 2016}

Varvaki Rados D, Catani Pinto L, Reck Remonti L, Bauermann Leitao C, Gross JL. The association between sulfonylurea use and all-cause and cardiovascular mortality: a meta-analysis with trial sequential analysis of randomized clinical trials. PLOS Medicine 2016;13(4):e1001992.

\section{Vist 2008}

Vist GE, Bryant D, Somerville L, Birminghem T, Oxman AD. Outcomes of patients who participate in randomized controlled trials compared to similar patients receiving similar interventions who do not participate. Cochrane Database of Systematic Reviews 2008, Issue 2. [DOI: 10.1002/14651858.MR000009.pub4]

\section{Wang 2017}

Wang F, He Y, Zhang R, Zeng Q, Zhao X. Combination therapy of metformin plus dipeptidyl peptidase-4 inhibitor versus metformin plus sulfonylurea and their association with a decreased risk of cardiovascular disease in type 2 diabetes mellitus patients. Medicine (Baltimore) 2017;96(36):e7638. 


\section{Wetterslev 2008}

Wetterslev J, Thorlund K, Brok J, Gluud C. Trial sequential analysis may establish when firm evidence is reached in cumulative meta-analysis. Journal of Clinical Epidemiology 2008;61(1):64-75. [PUBMED: 18083463]

\section{Whalen 2015}

Whalen K, Miller S, Onge ES. The role of sodium-glucose cotransporter 2 inhibitors in the treatment of type 2 diabetes. Clinical Therapeutics 2015;37(6):1150-66.

\section{WHO 1998}

Alberti KM, Zimmet PZ. Definition, diagnosis and classification of diabetes mellitus and its complications. Part I: diagnosis and classification of diabetes mellitus. Provisional report of a WHO consultation. Diabetic Medicine 1998;15(7):539-53.

\section{WHO 2015}

World Health Organization. Diabetes. www.who.int/ mediacentre/factsheets/fs312/en/ (accessed 29 February 2016).

\section{Wild 2004}

Wild S, Roglic G, Green A, Sicree R, King H. Global prevalence of diabetes: estimates for the year 2000 and projections for 2030. Diabetes Care 2004;27(5):1047-53. [PUBMED: 15111519]

\section{Wong 2006a}

Wong SS, Wilczynski NL, Haynes RB. Comparison of topperforming search strategies for detecting clinically sound treatment studies and systematic reviews in MEDLINE and Embase. Journal of the Medical Library Association 2006;94(4):451-5.

\section{Wong 2006b}

Wong SSL, Wilczynski NL, Haynes RB. Developing optimal search strategies for detecting clinically sound treatment studies in Embase. Journal of the Medical Library Association 2006;94(1):41-7.

\section{Wood 2008}

Wood L, Egger M, Gluud LL, Schulz KF, Jüni P, Altman DG, et al. Empirical evidence of bias in treatment effect estimates in controlled trials with different interventions and outcomes: meta-epidemiological study. BMJ 2008;336(7644):601-5.

\section{Zhou 2015}

Zhou 2015. The benefits and risks of DPP4-inhibitors vs sulfonylureas for patients with type 2 diabetes: accumulated evidence from randomised controlled trial. International Journal of Clinical Practice 2016;70(2):132-41.

\section{Zintzaras 2014}

Zintzaras E, Miligkos M, Ziakas P, Balk EM, Mademtzoglou D, Doxani $C$, et al. Assessment of the relative effectiveness and tolerability of treatments of type 2 diabetes mellitus: a network meta-analysis. Clinical Therapeutics 2014;36(10):1443-53.

\section{References to other published versions of this review Madsen 2016 \\ Madsen KS, Kähler P, Kähler LK, Madsbad S, Metzendorf M-I, Richter B, et al. Metformin and sulphonylurea (second- or third- generation) combination therapy for adults with type 2 diabetes mellitus. Cochrane Database of Systematic Reviews 2016, Issue 9. [DOI: 10.1002/14651858.CD012368]}

* Indicates the major publication for the study

\section{CHARACTERISTICS OF STUDIES}

Characteristics of included studies [ordered by study ID]

\section{Ahrén 2014}

Methods

Study design: randomised, double-blind, placebo- and active-controlled parallel-group study

Randomisation ratio: eligible participants were stratified by $\mathrm{HbA1c}$ level $(<8.0 \%$ versus $\geq 8.0 \%)$, history of $\mathrm{Ml}$, and age ( $<65$ versus $\geq 65$ years) and were randomly assigned (3:3:3:1) to receive, in addition to metformin, 1 of 4 treatments at baseline: albiglutide $30 \mathrm{mg}$, sitagliptin $100 \mathrm{mg}$, glimepiride $2 \mathrm{mg}$ or placebo

Non-inferiority and superiority design: the planned sample size provided $>90 \%$ power to demonstrate superiority versus placebo and non-inferiority versus sitagliptin and glimepiride (non-inferiority margin $=0.3 \%$ ). Superiority of albiglutide versus sitagliptin and glimepiride was tested if non-inferiority was established

Participants

Inclusion criteria: $\geq 18$ years of age, T2DM, inadequate glycaemic control while taking background metformin ( $\geq 1500 \mathrm{mg}$ or MTD) $\geq 3$ months before screening. Baseline $\mathrm{HbA1c}$ of $7.0 \%(53.0 \mathrm{mmol} /$ $\mathrm{mol})-10.0 \%$ ( $85.8 \mathrm{mmol} / \mathrm{mol}$ ); BMI $20-45 \mathrm{~kg} / \mathrm{m}^{2}$; creatinine clearance $>60 \mathrm{~mL} / \mathrm{min}$; normal TSH or were clinically euthyroid

Exclusion criteria: current ongoing symptomatic biliary disease or history of pancreatitis, recent clinically significant cardiovascular and/or cerebrovascular disease ( $\leq 2$ months before screening), treat- 
Ahrén 2014 (Continued)

ed gastroparesis, history of gastrointestinal surgery thought to significantly affect upper gastrointestinal function, history of most cancers not in remission for at least 3 years, personal or family history of medullary thyroid carcinoma or multiple endocrine neoplasia type 2, resting SBP > $160 \mathrm{mmHg}$ and/or $\mathrm{DBP}>100 \mathrm{mmHg}$, lipase above the ULN, haemoglobinopathy that could affect $\mathrm{HbA1c}$, and ALT or AST more than $2.5 \times$ ULN

Diagnostic criteria: not reported

Interventions

\section{Number of study centres: 289}

Run-in period: 4 weeks

Extension period: yes; all participants were assessed 8 weeks after the end of the intervention

\begin{tabular}{ll}
\hline Outcomes & Composite outcome measures reported: no \\
\hline Study details & Trial terminated early: no \\
& Trial ID: NCT00838903 \\
\hline Publication details & Language of publication: English \\
& Funding: commercial funding, GlaxoSmithKline (specify product): albiglutide \\
& Publication status: peer-reviewed journal \\
\hline Stated aim for study & Quote from publication: "To compare the efficacy and safety of weekly albiglutide with daily sitagliptin, \\
daily glimepiride, and placebo"
\end{tabular}

\section{Risk of bias}

\begin{tabular}{lll}
\hline Bias & Authors' judgement & Support for judgement \\
\hline $\begin{array}{ll}\text { Random sequence genera- } \\
\text { tion (selection bias) }\end{array}$ & Unclear risk & $\begin{array}{l}\text { Quote from publication: "Eligible patients were ... randomly assigned..." "De- } \\
\text { mographics and baseline characteristics were similar among the ... treatment } \\
\text { groups." }\end{array}$
\end{tabular}

Comment: insufficient information about the sequence generation process to permit judgement of 'low risk or 'high risk'

\begin{tabular}{|c|c|c|}
\hline $\begin{array}{l}\text { Allocation concealment } \\
\text { (selection bias) }\end{array}$ & Unclear risk & $\begin{array}{l}\text { Quote from publication: "Eligible patients were ... randomly assigned..." "De- } \\
\text { mographics and baseline characteristics were similar among the ... treatment } \\
\text { groups." }\end{array}$ \\
\hline
\end{tabular}

Comment: insufficient information about the allocation concealment to permit judgement of 'low risk' or 'high risk'

Blinding of participants Low risk and personnel (performance bias) all-cause/cardiovascular mortality

\begin{abstract}
Quote from publication: "Double blind (subject, investigator)". "Matching placebos for albiglutide, sitagliptin, and glimepiride were used to maintain blinding to treatment." "Possible cardiovascular events were prospectively recorded and adjudicated by an independent clinical endpoint committee masked to treatment allocation"
\end{abstract}

Comment: adjudicated/investigator-assessed outcome measurement. Blinding of key study personnel ensured

Quote from publication: "Double blind (subject, investigator)". "Matching placebos for albiglutide, sitagliptin, and glimepiride were used to maintain blinding to treatment".

\begin{tabular}{|c|c|c|}
\hline $\begin{array}{l}\text { Blinding of participants } \\
\text { and personnel (perfor- } \\
\text { mance bias) }\end{array}$ & Low risk & $\begin{array}{l}\text { Quote from publication: "Double blind (subject, investigator)". "Matching } \\
\text { placebos for albiglutide, sitagliptin, and glimepiride were used to maintain } \\
\text { blinding to treatment". }\end{array}$ \\
\hline
\end{tabular}


Ahrén 2014 (Continued)

hypoglycaemia
Comment: investigator-assessed or self-reported outcome measurement. Blinding of participants and key study personnel ensured

\begin{tabular}{|c|c|c|}
\hline $\begin{array}{l}\text { Blinding of participants } \\
\text { and personnel (perfor- } \\
\text { mance bias) } \\
\text { non-fatal myocardial in- } \\
\text { farction/heart failure/non- } \\
\text { fatal stroke }\end{array}$ & Low risk & $\begin{array}{l}\text { Quote from publication: "Double blind (subject, investigator)." "Matching } \\
\text { placebos for albiglutide, sitagliptin, and glimepiride were used to maintain } \\
\text { blinding to treatment". "Possible cardiovascular events were prospectively } \\
\text { recorded and adjudicated by an independent clinical endpoint committee } \\
\text { masked to treatment allocation" }\end{array}$ \\
\hline
\end{tabular}

fatal stroke

Comment: adjudicated outcome measurement. Blinding of key study personnel ensured

\begin{tabular}{|c|c|c|}
\hline $\begin{array}{l}\text { Blinding of participants } \\
\text { and personnel (perfor- } \\
\text { mance bias) } \\
\text { non-serious adverse } \\
\text { events }\end{array}$ & Low risk & $\begin{array}{l}\text { Quote from publication: "Double blind (subject, investigator)." "Matching } \\
\text { placebos for albiglutide, sitagliptin, and glimepiride were used to maintain } \\
\text { blinding to treatment". } \\
\text { Comment: self-reported outcome measurement. Blinding of participants en- } \\
\text { sured }\end{array}$ \\
\hline $\begin{array}{l}\text { Blinding of participants } \\
\text { and personnel (perfor- } \\
\text { mance bias) } \\
\text { serious adverse events }\end{array}$ & Low risk & $\begin{array}{l}\text { Quote from publication: "Double blind (subject, investigator)." "Matching } \\
\text { placebos for albiglutide, sitagliptin, and glimepiride were used to maintain } \\
\text { blinding to treatment". "Possible cardiovascular events were prospectively } \\
\text { recorded and adjudicated by an independent clinical endpoint committee } \\
\text { masked to treatment allocation". "An independent, blinded pancreatitis adju- } \\
\text { dication committee comprising three gastro intestinal specialists adjudicated } \\
\text { adverse events suggesting pancreatitis and all laboratory elevations of lipase } \\
\text { and/or amylase more than or equal to three times the ULN. The pancreatitis } \\
\text { adjudication committee adjudicated both the probability of events being pan- } \\
\text { creatitis (definite, probable, possible, not likely) and the likelihood of a rela- } \\
\text { tionship to the study drug (definite, probable, possible, unlikely alternate eti- } \\
\text { ology)" }\end{array}$ \\
\hline
\end{tabular}

Comment: adjudicated/investigator-assessed outcome measurement. Blinding of key study personnel ensured

\begin{tabular}{|c|c|c|}
\hline $\begin{array}{l}\text { Blinding of participants } \\
\text { and personnel (perfor- } \\
\text { mance bias) }\end{array}$ & I rw risk & $\begin{array}{l}\text { Quote from publication: "Double blind (subject, investigator)." "Matching } \\
\text { placebos for albiglutide, sitagliptin, and glimepiride were used to maintain }\end{array}$ \\
\hline
\end{tabular}

weight $(\mathrm{kg})$

Comment: investigator-assessed or self-reported outcome measurement. Blinding of participants and key study personnel ensured

\begin{tabular}{|c|c|c|}
\hline $\begin{array}{l}\text { Blinding of participants } \\
\text { and personnel (perfor- } \\
\text { mance bias) }\end{array}$ & Low risk & $\begin{array}{l}\text { Quote from publication: "Double blind (subject, investigator)." "Matching } \\
\text { placebos for albiglutide, sitagliptin, and glimepiride were used to maintain } \\
\text { blinding to treatment" }\end{array}$ \\
\hline
\end{tabular}

HbAlc

Comment: investigator-assessed outcome measurement. Blinding of key study personnel ensured

$\begin{array}{lll}\text { Blinding of outcome as- } & \text { Low risk } & \text { Quote from publication: "Double blind (subject, investigator)". "Matching } \\ \text { sessment (detection bias) } & & \text { placebos for albiglutide, sitagliptin, and glimepiride were used to maintain } \\ \text { all-cause/cardiovascular } & \text { blinding to treatment." "Possible cardiovascular events were prospectively } \\ \text { mortality } & \text { recorded and adjudicated by an independent clinical endpoint committee } \\ & \text { masked to treatment allocation" }\end{array}$

Comment: adjudicated/investigator-assessed outcome measurement. Blinding of key study personnel ensured

Blinding of outcome as-
sessment (detection bias)
hypoglycaemia

Quote from publication: "Double blind (subject, investigator)". "Matching placebos for albiglutide, sitagliptin, and glimepiride were used to maintain blinding to treatment"

Metformin and second- or third-generation sulphonylurea combination therapy for adults with type 2 diabetes mellitus (Review) 
Comment: investigator-assessed or self-reported outcome measurement. Blinding of participants and key study personnel ensured

Blinding of outcome as- Low risk sessment (detection bias) non-fatal myocardial infarction/heart failure/nonfatal stroke
Quote from publication: "Double blind (subject, investigator)." "Matching placebos for albiglutide, sitagliptin, and glimepiride were used to maintain blinding to treatment". "Possible cardiovascular events were prospectively recorded and adjudicated by an independent clinical endpoint committee masked to treatment allocation"

Comment: adjudicated outcome measurement. Blinding of key study personnel ensured

\begin{tabular}{|c|c|c|}
\hline $\begin{array}{l}\text { Blinding of outcome as- } \\
\text { sessment (detection bias) } \\
\text { non-serious adverse }\end{array}$ & Low risk & $\begin{array}{l}\text { Quote from publication: "Double blind (subject, investigator)." "Matching } \\
\text { placebos for albiglutide, sitagliptin, and glimepiride were used to maintain } \\
\text { blinding to treatment" }\end{array}$ \\
\hline
\end{tabular}

Comment: self-reported outcome measurement. Blinding of participants ensured

\section{Blinding of outcome as- Low risk sessment (detection bias)} serious adverse events
Quote from publication: "Double blind (subject, investigator)." "Matching placebos for albiglutide, sitagliptin, and glimepiride were used to maintain blinding to treatment". "Possible cardiovascular events were prospectively recorded and adjudicated by an independent clinical endpoint committee masked to treatment allocation". "An independent, blinded pancreatitis adjudication committee comprising three gastro intestinal specialists adjudicated adverse events suggesting pancreatitis and all laboratory elevations of lipase and/or amylase more than or equal to three times the ULN. The pancreatitis adjudication committee adjudicated both the probability of events being pancreatitis (definite, probable, possible, not likely) and the likelihood of a relationship to the study drug (definite, probable, possible, unlikely alternate etiology)"

Comment: adjudicated/investigator-assessed outcome measurement. Blinding of key study personnel ensured

\begin{tabular}{lll}
\hline Blinding of outcome as- & Low risk & Quote from publication: "Double blind (subject, investigator)." "Matching \\
sessment (detection bias) & & placebos for albiglutide, sitagliptin, and glimepiride were used to maintain \\
weight $(\mathrm{kg})$ & blinding to treatment"
\end{tabular}

Comment: investigator-assessed or self-reported outcome measurement. Blinding of participants and key study personnel ensured
Blinding of outcome as- Low risk sessment (detection bias) HbA1c
Quote from publication: "Double blind (subject, investigator)." "Matching placebos for albiglutide, sitagliptin, and glimepiride were used to maintain blinding to treatment"

Comment: investigator-assessed outcome measurement. Blinding of key study personnel ensured
Incomplete outcome data Low risk (attrition bias) all-cause/cardiovascular mortality
Quote from publication: "SAEs and non-serious AEs are reported for members of the Safety Population, comprised of all participants who received at least one dose of study treatment."

Comment: $>95 \%$ of the randomised participants were included in the analyses. There was a high dropout rate (53\%-61\% of the participants completed the study), however, the dropout rate was balanced between groups. We assumed that mortality status was searched in registers at the end of the trial. About $5 \%$ of the participants in each intervention group were lost to follow-up. Not clarified how these missing data were imputed. The proportion of missingness is small and judged not to give raise to risk of attrition bias 
Ahrén 2014 (Continued)

Incomplete outcome data High risk (attrition bias)

hypoglycaemia

Quote from publication: "SAEs and non-serious AEs are reported for members of the Safety Population, comprised of all participants who received at least one dose of study treatment."

Comment: $>95 \%$ of the participants in all treatment groups were included in the analyses. There was a high dropout rate (53\%-61\% of the participants completed the study), however, the dropout rate was balanced between groups. We do not know how the trial authors imputed missing data from the participants not completing the study. The reasons for dropouts were balanced among the intervention groups. The proportion of missing outcomes compared with observed event risk enough to induce clinically relevant bias in intervention effect estimate

Incomplete outcome data High risk (attrition bias) non-fatal myocardial infarction/heart failure/nonfatal stroke

Quote from publication: "SAEs and non-serious AEs are reported for members of the Safety Population, comprised of all participants who received at least one dose of study treatment."

Comment: $>95 \%$ of the participants in all treatment groups were included in the analyses. There was a high dropout rate (53\%-61\% of the participants completed the study), however, the dropout rate was balanced between groups. We do not know how the trial authors imputed missing data from the participants not completing the study. The reasons for dropouts were balanced among the intervention groups. The proportion of missing outcomes compared with observed event risk enough to induce clinically relevant bias in intervention effect estimate

Incomplete outcome data Low risk (attrition bias) non-serious adverse events
Quote from publication: "SAEs and non-serious AEs are reported for members of the Safety Population, comprised of all participants who received at least one dose of study treatment."

Comment: $>95 \%$ of the participants in all treatment groups were included in the analyses. There was a high dropout rate (53\%-61\% of the participants completed the study), however, the dropout rate was balanced between groups. We do not know how the trial authors imputed missing data from the participants not completing the study. The reasons for dropouts were balanced among the intervention groups. The proportion of missing outcomes compared with observed event risk was not enough to induce clinically relevant bias in intervention effect estimate

Quote from publication: "SAEs and non-serious AEs are reported for members of the Safety Population, comprised of all participants who received at least one dose of study treatment."

Comment: $>95 \%$ of the participants in all treatment groups were included in the analyses. There was a high dropout rate (53\%-61\% of the participants completed the study), however, the dropout rate was balanced between groups. We do not know how the trial authors imputed missing data from the participants not completing the study. The reasons for dropouts were balanced among the intervention groups. The proportion of missing outcomes compared with observed event risk enough to induce clinically relevant bias in intervention effect estimate

Quote from publication: "ITT Population with observed values. Only those participants who were available at the indicated time points were analyzed."

Comment: $15 \%-37 \%$ of the randomised participants were included in the analyses. There was a high dropout rate $53 \%-61 \%$ of the participants completed the study), however, the dropout rate was balanced between groups. The reasons for dropouts were balanced among the intervention groups. Only participants who were available at the indicated time points were analysed. 
Ahrén 2014 (Continued)

Plausible effect size among missing outcomes enough to induce clinically relevant bias in observed effect size

Incomplete outcome data High risk (attrition bias)

HbA1c
Quote from publication: "Intent-to-Treat (ITT) Population with observed values. Only those par. with a value at Baseline and at the specified visit were analyzed."

Comment: $15 \%-37 \%$ of the randomised participants were included in the analyses. There was a high dropout rate $53 \%-61 \%$ of the participants completed the study), however, the dropout rate was balanced between groups. The reasons for dropouts were balanced among the intervention groups. Only participants with a value at baseline and at the specified visit were analysed. Plausible effect size among missing outcomes enough to induce clinically relevant bias in observed effect size
Selective reporting (re- Low risk porting bias)
Comment: all of the trial's primary and secondary outcomes as specified in the protocol have been reported

Other bias Unclear risk

Quote from publication: "The sponsor of the study participated in the study design, data collection, data review, data analysis, and writing of the report."

Comment: The sponsor was a pharmaceutical company

\section{Charbonnel 2005}

Methods Study design: randomised, double-blind, double-dummy, parallel-group study

Randomisation ratio: 1:1 to receive pioglitazone or gliclazide in addition to metformin

Inclusion criteria: T2DM, inadequately managed with metformin alone (at $\geq 50 \%$ of the maximum rec-
ommended dose or at the MTD for $\geq 3$ months), aged 35-75 years, HbAlc of $\geq 7.5 \%$ or $\leq 11.0 \%$, fasting
C-peptide of $\geq 1.5 \mathrm{ng} / \mathrm{mL}(0.50 \mathrm{nmol} / \mathrm{L})$, stable or worsening glycaemic control for $\geq 3$ months prior to
screening
Exclusion criteria: T1DM, ketoacidosis, MI, transient ischaemic attacks or stroke in the previous 6
months, symptomatic heart failure, acute malabsorption or chronic pancreatitis, familial polyposis
coli, malignant disease in the previous 10 years or substance abuse, female participants had to be post-
menopausal, sterilised or using satisfactory contraception, pregnant or breastfeeding women were ex-
cluded, previous treatment with insulin, gliclazide, pioglitazone or other sulphonylureas or TZDs

Diagnostic criteria: not reported

\begin{tabular}{ll}
\hline Interventions & $\begin{array}{l}\text { Number of study centres: } 75 \\
\text { Run-in period: no } \\
\text { Extension period: yes, the study was continued to } 2 \text { years }\end{array}$ \\
\hline Outcomes & Composite outcome measures reported: no \\
\hline Study details & Trial terminated early: no \\
& Trial ID: - \\
\hline Publication details & Language of publication: English \\
& Funding: the work was supported by Takeda Europe R\&D Centre and Eli Lilly and Company, USA \\
& Publication status: peer-reviewed journal
\end{tabular}


Charbonnel 2005 (Continued)

Stated aim for study Quote from publication: "The aim of this analysis was to examine the long-term effects of pioglitazone or gliclazide addition to failing metformin monotherapy..."

Notes

\section{Risk of bias}

Bias Authors' judgement Support for judgement

Random sequence genera- Low risk tion (selection bias)

Quote from publication: "Patients were randomised using block randomisation via a central telephone system (QTONE)"

Comment: adequate generation of random sequence ensured

\begin{tabular}{|c|c|c|}
\hline $\begin{array}{l}\text { Allocation concealment } \\
\text { (selection bias) }\end{array}$ & Low risk & $\begin{array}{l}\text { Quote from publication: "Patients were randomised using block randomisa- } \\
\text { tion via a central telephone system (QTONE)" }\end{array}$ \\
\hline
\end{tabular}

Comment: adequate concealment ensured

\begin{tabular}{|c|c|c|}
\hline $\begin{array}{l}\text { Blinding of participants } \\
\text { and personnel (perfor- } \\
\text { mance bias) } \\
\text { all-cause/cardiovascular }\end{array}$ & Low risk & $\begin{array}{l}\text { Quote from publication: "double-blind..., double dummy" } \\
\text { Comment: investigator-assessed outcome measurement. Blinding of study } \\
\text { personnel ensured }\end{array}$ \\
\hline
\end{tabular}
mortality

\begin{tabular}{|c|c|c|}
\hline $\begin{array}{l}\text { Blinding of participants } \\
\text { and personnel (perfor- } \\
\text { mance bias) } \\
\text { hypoglycaemia }\end{array}$ & Low risk & $\begin{array}{l}\text { Quote from publication: "double-blind..., double dummy" } \\
\text { Comment: investigator-assessed/self-reported outcome measurement. Blind- } \\
\text { ing of participant and study personnel ensured. }\end{array}$ \\
\hline
\end{tabular}

\begin{tabular}{|c|c|c|}
\hline $\begin{array}{l}\text { Blinding of participants } \\
\text { and personnel (perfor- } \\
\text { mance bias) } \\
\text { non-fatal myocardial in- }\end{array}$ & Low risk & $\begin{array}{l}\text { Quote from publication: "double-blind..., double dummy" } \\
\text { Comment: investigator-assessed outcome measurement. Blinding of study } \\
\text { personnel ensured }\end{array}$ \\
\hline
\end{tabular}

farction/heart failure/non-

fatal stroke

\begin{tabular}{|c|c|c|}
\hline $\begin{array}{l}\text { Blinding of participants } \\
\text { and personnel (perfor- } \\
\text { mance bias) } \\
\text { serious adverse events }\end{array}$ & Low risk & $\begin{array}{l}\text { Quote from publication: "double-blind..., double dummy" } \\
\text { Comment: investigator-assessed outcome measurement. Blinding of study } \\
\text { personnel ensured }\end{array}$ \\
\hline
\end{tabular}

\begin{tabular}{|c|c|c|}
\hline $\begin{array}{l}\text { Blinding of participants } \\
\text { and personnel (perfor- } \\
\text { mance bias) } \\
\text { weight (kg) }\end{array}$ & Low risk & $\begin{array}{l}\text { Quote from publication: "double-blind..., double dummy" } \\
\text { Comment: investigator-assessed/self-reported outcome measurement. Blind- } \\
\text { ing of participants and study personnel ensured. }\end{array}$ \\
\hline $\begin{array}{l}\text { Blinding of participants } \\
\text { and personnel (perfor- } \\
\text { mance bias) } \\
\text { HbA1c }\end{array}$ & Low risk & $\begin{array}{l}\text { Quote from publication: "double-blind..., double dummy" } \\
\text { Comment: investigator-assessed outcome measurement. Blinding of study } \\
\text { personnel ensured }\end{array}$ \\
\hline
\end{tabular}

\begin{tabular}{ll}
\hline $\begin{array}{l}\text { Blinding of outcome as- } \\
\text { sessment (detection bias) } \\
\text { all-cause/cardiovascular }\end{array}$ & Low risk \\
mortality & Quote from publication: "double-blind..., double dummy" \\
& $\begin{array}{l}\text { Comment: investigator-assessed outcome measurement. Blinding of study } \\
\text { personnel ensured }\end{array}$ \\
\hline
\end{tabular}

$\begin{aligned} & \text { Blinding of outcome as- } \\ & \text { sessment (detection bias) }\end{aligned}$
Low risk Quote from publication: "double-blind..., double dummy"


Charbonnel 2005 (Continued)

hypoglycaemia
Comment: investigator-assessed/self-reported outcome measurement. Blinding of participants and study personnel ensured

\begin{tabular}{|c|c|c|}
\hline $\begin{array}{l}\text { Blinding of outcome as- } \\
\text { sessment (detection bias) } \\
\text { non-fatal myocardial in- } \\
\text { farction/heart failure/non- }\end{array}$ & Low risk & $\begin{array}{l}\text { Quote from publication: "double-blind..., double dummy" } \\
\text { Comment: investigator-assessed outcome measurement. Blinding of study } \\
\text { personnel ensured. }\end{array}$ \\
\hline
\end{tabular}

fatal stroke

\begin{tabular}{lll}
\hline $\begin{array}{l}\text { Blinding of outcome as- } \\
\text { sessment (detection bias) } \\
\text { serious adverse events }\end{array}$ & Low risk & Quote from publication: "double-blind..., double dummy" \\
& $\begin{array}{l}\text { Comment: investigator-assessed outcome measurement. Blinding of study } \\
\text { personnel ensured }\end{array}$
\end{tabular}

\begin{tabular}{|c|c|c|}
\hline $\begin{array}{l}\text { Blinding of outcome as- } \\
\text { sessment (detection bias) } \\
\text { weight }(\mathrm{kg})\end{array}$ & Low risk & $\begin{array}{l}\text { Quote from publication: "double-blind..., double dummy" } \\
\text { Comment: investigator-assessed/self-reported outcome measurement. Blind- } \\
\text { ing of participants and study personnel ensured }\end{array}$ \\
\hline
\end{tabular}

\begin{tabular}{|c|c|c|}
\hline $\begin{array}{l}\text { Blinding of outcome as- } \\
\text { sessment (detection bias) } \\
\text { HbA1c }\end{array}$ & Low risk & $\begin{array}{l}\text { Quote from publication: "double-blind..., double dummy" } \\
\text { Comment: investigator-assessed outcome measurement. Blinding of study } \\
\text { personnel ensured }\end{array}$ \\
\hline
\end{tabular}

\begin{tabular}{ll}
\hline $\begin{array}{l}\text { Incomplete outcome data } \\
\text { (attrition bias) }\end{array}$ & Low risk \\
$\begin{array}{l}\text { all-cause/cardiovascular } \\
\text { mortality }\end{array}$ & $\begin{array}{l}\text { Quote from publication: "All patients who had taken at least one dose of } \\
\text { study medication were included in the safety analysis." }\end{array}$ \\
& $\begin{array}{l}\text { Comment: }>99 \% \text { of randomised participants were included in the analyses. } \\
\text { There was a high dropout rate }(74 \%-76 \% \text { of randomised participants complet- }\end{array}$ \\
& $\begin{array}{l}\text { ed the study), however, the dropout rate was balanced between groups. We } \\
\text { assumed that trial authors searched registers for mortality status at the end of } \\
\text { the trial. }\end{array}$
\end{tabular}

$\begin{array}{lll}\begin{array}{l}\text { Incomplete outcome data } \\ \text { (attrition bias) }\end{array} & \text { High risk } & \begin{array}{l}\text { Quote from publication: "All patients who had taken at least one dose of } \\ \text { study medication were included in the safety analysis." }\end{array}\end{array}$

hypoglycaemia

\begin{abstract}
Comment: $>99 \%$ of the participants were included in the analyses. There was a high dropout rate (74\%-76\% of the participants completed the study), however, the dropout rate was balanced between groups. We do not know how the trial authors imputed missing data from the participants not completing the study. The reasons for dropouts were balanced. The proportion of missing outcomes compared with observed event risk enough to induce clinically relevant bias in intervention effect estimate.
\end{abstract}

Incomplete outcome data High risk
(attrition bias)
non-fatal myocardial in-
farction/heart failure/non-
fatal stroke

Quote from publication: "All patients who had taken at least one dose of study medication were included in the safety analysis."
Comment: $>99 \%$ of the participants were included in the analyses. There was a high dropout rate (74\%-76\% of the participants completed the study), how- ever, the dropout rate was balanced between groups. We do not know how the trial authors imputed missing data from the participants not completing the study. The reasons for dropouts were balanced. The proportion of missing out- comes compared with observed event risk enough to induce clinically relevant bias in intervention effect estimate.

Incomplete outcome data High risk
(attrition bias)

serious adverse events

Quote from publication: "All patients who had taken at least one dose of study medication were included in the safety analysis."

Comment: > 99\% of the participants were included in the analyses. There was a high dropout rate (74\%-76\% of the participants completed the study), however, the dropout rate was balanced between groups. We do not know how the

Metformin and second- or third-generation sulphonylurea combination therapy for adults with type 2 diabetes mellitus (Review) Copyright (c) 2019 The Cochrane Collaboration. Published by John Wiley \& Sons, Ltd. 
trial authors imputed missing data from the participants not completing the study. The reasons for dropouts were balanced. The proportion of missing outcomes compared with observed event risk enough to induce clinically relevant bias in intervention effect estimate.

Incomplete outcome data Unclear risk
(attrition bias) weight $(\mathrm{kg})$
Quote from publication: "All patients who had taken at least one dose of study medication were included in the safety analysis."

Comment: $>99 \%$ of the participants were included in the analyses. There was a high dropout rate (74\%-76\% of the participants completed the study), however, the dropout rate was balanced between groups. We do not know how the trial authors imputed missing data from the participants not completing the study. The reasons for dropouts were balanced.
Incomplete outcome data Unclear risk (attrition bias)

HbA1c
Quote from publication: "The analysis was carried out on the intention-totreat (ITT) population, which included all patients who had taken at least one dose of study medication and had $\mathrm{HbAlc}$ recorded at baseline and at least once post-baseline."

Comment: $>99 \%$ of the participants were included in the analyses. There was a high dropout rate (74\%-76\% of the participants completed the study), however, the dropout rate was balanced between groups. We do not know how the trial authors imputed missing data from the participants not completing the study. The reasons for dropouts were balanced.

\begin{tabular}{ll}
\hline $\begin{array}{l}\text { Selective reporting (re- } \\
\text { porting bias) }\end{array}$ & Low risk \\
(methods section) have been reported
\end{tabular}

Other bias Unclear risk Comment: the trial was supported by pharmaceutical companies

\section{Dei Cas 2017}

Methods Study design: randomised, open-label, active-controlled parallel-group study

Randomisation ratio: 1:2

Participants

Inclusion criteria: men and women aged $\geq 35$ years, T2DM (ADA 1997 criteria) with at least 1 year of disease duration at the time of the screening visit, in metformin failure (HbA1c 7.0\%-9.0\%; 53-75 mmol/ $\mathrm{mol}$ ) and $\mathrm{BMI} \geq 20$ or $\leq 40 \mathrm{~kg} / \mathrm{m}^{2}$; treatment with metformin in monotherapy at a stable dose of at least $1.5 \mathrm{~g} /$ day (or MTD) in the 3 months prior to the screening visit

Exclusion criteria: T1DM or secondary diabetes, significant progression of diabetic macro- (acute cerebro-vascular event or any revascularisation procedure, clinically relevant peripheral artery disease, onset of a diabetic foot) or micro- (retinopathy progression, increase of $\geq 0.5 \mathrm{mg} / \mathrm{dL}$ of plasma creatinine or progression to macro-proteinuria, onset of clinically relevant neuropathy) angiopathy in the 6 months prior to study visit; history of acute or chronic pancreatitis, pancreatectomy, gastric surgery, inflammatory bowel disease, organ failure or other severe diseases limiting life expectancy; drugs interfering with glucose levels (i.e. corticosteroids) or acute diseases (i.e. infections) in the 3 months before screening visit, history of inflammatory/infective/autoimmune chronic disease, contraindications to the maintenance of the background therapy (metformin), including-but not limited to-chronic kidney failure or plasma creatinine concentrations $>1.5 \mathrm{mg} / \mathrm{dL}$, severe respiratory failure, etc.; contraindications to the use of a sulphonylurea or DPP-4 inhibitors, clinically relevant psychiatric disorders, any clinically significant abnormality identified in physical examination, laboratory tests (known chronic liver diseases) or vital signs at screening, pregnancy, uncontrolled or inadequately controlled hypertension at screening and history of low compliance

Diagnostic criteria: ADA 1997 criteria 
Dei Cas 2017 (Continued)

Interventions
Number of study centres: 1

Run-in period: none

Extension period: none

\begin{tabular}{ll}
\hline Outcomes & Composite outcome measures reported: no \\
\hline Study details & Trial terminated early: no \\
& Trial ID: NCT01822548 \\
\hline
\end{tabular}

Publication details Language of publication: English

Funding: non-commercial funding; Research program Regione-Emilia Romagna-University 2007-2009; Italian Ministry of Health Ricerca Finalizzata GR-2011-02347600

Commercial funding: unconditional grant from Novartis Italia

Publication status: peer-reviewed journal

Stated aim for study

Quote from publication: "The purpose of this study is to evaluate the effect of DPP-IV inhibitor Vildagliptin vs. Glibenclamide on circulating endothelial progenitor cells (EPCs) number in type 2 diabetes patients in metformin failure."

Notes -

\section{Risk of bias}

Bias Authors' judgement Support for judgement

Random sequence genera- Low risk tion (selection bias)
Quote from publication: "Randomization was based on random numbers generated by a statistical software. Randomization numbers and sequence were kept by the data manager. Randomization list was concealed to study investigators. Principal investigator and collaborators investigator enrolled the participants."

Comment: adequate generation of random sequence ensured

Allocation concealment Low risk
(selection bias)

Quote from publication: "Randomization was based on random numbers generated by a statistical software. Randomization numbers and sequence were kept by the data manager. Randomization list was concealed to study investigators. Principal investigator and collaborators investigator enrolled the participants."

Comment: adequate concealment ensured

\begin{tabular}{|c|c|c|}
\hline $\begin{array}{l}\text { Blinding of participants } \\
\text { and personnel (perfor- } \\
\text { mance bias) } \\
\text { all-cause/cardiovascular } \\
\text { mortality }\end{array}$ & Low risk & $\begin{array}{l}\text { Quote from publication: "... open-label..." } \\
\text { Comment: investigator-assessed outcome measurement. Incomplete blind- } \\
\text { ing, but outcome unlikely to be influenced by lack of blinding }\end{array}$ \\
\hline $\begin{array}{l}\text { Blinding of participants } \\
\text { and personnel (perfor- } \\
\text { mance bias) } \\
\text { amputation of lower ex- } \\
\text { tremity/blindness or se- } \\
\text { vere vision loss/end-stage } \\
\text { renal disease }\end{array}$ & Low risk & $\begin{array}{l}\text { Quote from publication: "... open-label..." } \\
\text { Comment: investigator-assessed outcome measurement. Incomplete blind- } \\
\text { ing, but outcome unlikely to be influenced by lack of blinding }\end{array}$ \\
\hline
\end{tabular}

Metformin and second- or third-generation sulphonylurea combination therapy for adults with type 2 diabetes mellitus (Review) 
Dei Cas 2017 (Continued)

Blinding of participants and personnel (performance bias)

hypoglycaemia
High risk

Quote from publication: "....on the basis of the clinical judgment of their physician, owing to hypoglycemic (4 mild and 1 severe) events."

Comment: investigator-assessed/self-reported outcome measurement. No blinding of participants or investigators. Outcome likely to be influenced by lack of blinding

\section{Blinding of participants and personnel (perfor- mance bias) non-fatal myocardial in- farction/heart failure/non- fatal stroke}

Quote from publication: "... open-label..."

Comment: investigator-assessed outcome measurement. Incomplete blinding, but outcome unlikely to be influenced by lack of blinding
Blinding of participants and personnel (performance bias) non-serious adverse events
Low risk

\begin{tabular}{ll}
\hline $\begin{array}{l}\text { Blinding of participants } \\
\text { and personnel (perfor- }\end{array}$ & Low risk \\
$\begin{array}{l}\text { mance bias) } \\
\text { serious adverse events }\end{array}$ & $\begin{array}{l}\text { Quote from publication: "No adverse events were reported in the V arm dur- } \\
\text { ing the whole study period..." }\end{array}$ \\
& $\begin{array}{l}\text { Comment: investigator-assessed/self-reported outcome measurement. No } \\
\text { blinding of participants or investigators. Outcome unlikely to be influenced by } \\
\text { lack of blinding }\end{array}$
\end{tabular}

\begin{tabular}{|c|c|c|}
\hline $\begin{array}{l}\text { Blinding of participants } \\
\text { and personnel (perfor- } \\
\text { mance bias) } \\
\text { weight (kg) }\end{array}$ & High risk & $\begin{array}{l}\text { Quote from publication: "...open-label..." } \\
\text { Comment: investigator-assessed/self-reported outcome measurement. No } \\
\text { blinding of participants or investigators. Outcome likely to be influenced by } \\
\text { lack of blinding }\end{array}$ \\
\hline
\end{tabular}

Quote from publication: "..No adverse events were reported in the V arm during the whole study period..."

Comment: investigator-assessed/self-reported outcome measurement. No blinding of participants or investigators. Outcome likely to be influenced by lack of blinding

\begin{tabular}{ll}
\hline $\begin{array}{l}\text { Blinding of participants } \\
\text { and personnel (perfor- }\end{array}$ & Quote from publication: "... open-label..." \\
mance bias) & \\
HbAlc & Comment: investigator-assessed outcome measurement. Incomplete blind- \\
\hline
\end{tabular}

\begin{tabular}{|c|c|c|}
\hline $\begin{array}{l}\text { Blinding of outcome as- } \\
\text { sessment (detection bias) } \\
\text { all-cause/cardiovascular } \\
\text { mortality }\end{array}$ & Low risk & $\begin{array}{l}\text { Quote from publication: "... open-label..." } \\
\text { Comment: investigator-assessed outcome measurement. Incomplete blind- } \\
\text { ing, but outcome unlikely to be influenced by lack of blinding }\end{array}$ \\
\hline
\end{tabular}

\begin{tabular}{|c|c|c|}
\hline $\begin{array}{l}\text { Blinding of outcome as- } \\
\text { sessment (detection bias) } \\
\text { amputation of lower ex- } \\
\text { tremity/blindness or se- } \\
\text { vere vision loss/end-stage } \\
\text { renal disease }\end{array}$ & Low risk & $\begin{array}{l}\text { Quote from publication: "... open-label..." } \\
\text { Comment: investigator-assessed outcome measurement. Incomplete blind- } \\
\text { ing, but outcome unlikely to be influenced by lack of blinding }\end{array}$ \\
\hline
\end{tabular}

\begin{tabular}{|c|c|c|}
\hline $\begin{array}{l}\text { Blinding of outcome as- } \\
\text { sessment (detection bias) } \\
\text { hypoglycaemia }\end{array}$ & High risk & $\begin{array}{l}\text { Quote from publication: "....on the basis of the clinical judgment of their } \\
\text { physician, owing to hypoglycemic ( } 4 \text { mild and } 1 \text { severe) events." } \\
\text { Comment: investigator-assessed/self-reported outcome measurement. No } \\
\text { blinding of participants or investigators. Outcome likely to be influenced by } \\
\text { lack of blinding }\end{array}$ \\
\hline
\end{tabular}

Blinding of outcome as-
sessment (detection bias) $\quad$ Low risk $\quad$ Quote from publication: "... open-label..."


Dei Cas 2017 (Continued) non-fatal myocardial infarction/heart failure/nonfatal stroke
Comment: investigator-assessed outcome measurement. Incomplete blinding, but outcome unlikely to be influenced by lack of blinding
Blinding of outcome as- High risk sessment (detection bias) non-serious adverse events
Quote from publication: "No adverse events were reported in the V arm during the whole study period..."

Comment: investigator-assessed/self-reported outcome measurement. No blinding of participants or investigators. Outcome likely to be influenced by lack of blinding

\section{Blinding of outcome as- Low risk} sessment (detection bias) serious adverse events
Quote from publication: "No adverse events were reported in the V arm during the whole study period..."

Comment: investigator-assessed/self-reported outcome measurement. No blinding of participants or investigators. Outcome likely to be influenced by lack of blinding
Quote from publication: "...open-label..."

Comment: investigator-assessed/self-reported outcome measurement. No blinding of participants or investigators. Outcome likely to be influenced by lack of blinding

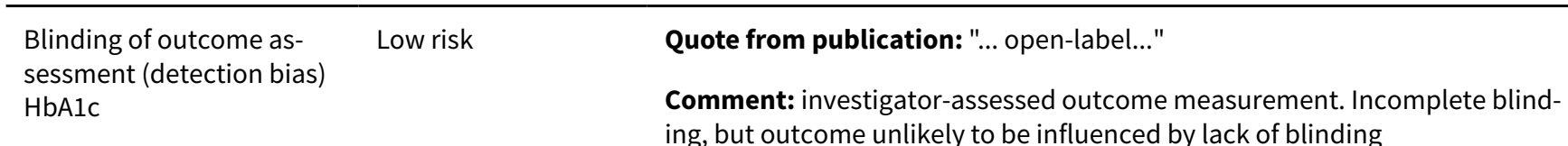
ing, but outcome unlikely to be influenced by lack of blinding

Incomplete outcome data Low risk
(attrition bias)
all-cause/cardiovascular
mortality

Incomplete outcome data Low risk (attrition bias) amputation of lower extremity/blindness or severe vision loss/end-stage renal disease
Comment: data obtained from trial author. Data were available on all randomised participants, all randomised participants were analysed in the intervention group they had been randomised to initially.

\begin{tabular}{|c|c|c|}
\hline $\begin{array}{l}\text { Incomplete outcome data } \\
\text { (attrition bias) } \\
\text { hypoglycaemia }\end{array}$ & Low risk & $\begin{array}{l}\text { Comment: data were available on all randomised participants, all randomised } \\
\text { participants were analysed in the intervention group they had been ran- } \\
\text { domised to initially }\end{array}$ \\
\hline $\begin{array}{l}\text { Incomplete outcome data } \\
\text { (attrition bias) } \\
\text { non-fatal myocardial in- } \\
\text { farction/heart failure/non- } \\
\text { fatal stroke }\end{array}$ & Low risk & $\begin{array}{l}\text { Comment: data obtained from author. Data were available on all randomised } \\
\text { participants, all randomised participants were analysed in the intervention } \\
\text { group they had been randomised to initially }\end{array}$ \\
\hline $\begin{array}{l}\text { Incomplete outcome data } \\
\text { (attrition bias) } \\
\text { non-serious adverse } \\
\text { events }\end{array}$ & Low risk & $\begin{array}{l}\text { Comment: data were available on all randomised participants, all randomised } \\
\text { participants were analysed in the intervention group they had been ran- } \\
\text { domised to initially }\end{array}$ \\
\hline $\begin{array}{l}\text { Incomplete outcome data } \\
\text { (attrition bias) } \\
\text { serious adverse events }\end{array}$ & Low risk & $\begin{array}{l}\text { Comment: data were available on all randomised participants, all randomised } \\
\text { participants were analysed in the intervention group they had been ran- } \\
\text { domised to initially }\end{array}$ \\
\hline
\end{tabular}


Dei Cas 2017 (Continued)

Incomplete outcome data Low risk (attrition bias)

weight $(\mathrm{kg})$
Comment: data obtained from author. Data were available on all randomised participants, all randomised participants were analysed in the intervention group they had been randomised to initially

\begin{tabular}{|c|c|c|}
\hline $\begin{array}{l}\text { Incomplete outcome data } \\
\text { (attrition bias) } \\
\text { HbA1c }\end{array}$ & Low risk & $\begin{array}{l}\text { Comment: data obtained from author. Data were available on all randomisec } \\
\text { participants, all randomised participants were analysed in the intervention } \\
\text { group they had been randomised to initially }\end{array}$ \\
\hline
\end{tabular}

\begin{tabular}{ll}
\hline $\begin{array}{l}\text { Selective reporting (re- } \\
\text { porting bias) }\end{array}$ & Low risk \\
\end{tabular}

Other bias Unclear risk Comment: the trial received funding from a pharmaceutical company

\section{Del Prato 2014}

Study design: double-blind, randomised, active-controlled, 3-arm, parallel-group study
Randomisation ratio: $1: 1: 1$ to receive 104 weeks of double-blind treatment with either alogliptin 12.5
mg once daily, alogliptin $25 \mathrm{mg}$ once daily or glipizide $5 \mathrm{mg}$ once daily (with titration up to $20 \mathrm{mg}$ once
daily up to week 20 as needed, based on the predefined hyperglycaemia criteria detailed below) in ad-
dition to metformin
Non-inferiority design and superiority design: non-inferiority of alogliptin doses to glipizide was
established sequentially if the upper limit of the one-sided $98.75 \%$ confidence interval for the differ-
ence in change from baseline between alogliptin $25 \mathrm{mg}$ and glipizide, then alogliptin $12.5 \mathrm{mg}$ and glip-
izide, was $<0.3 \%$. Superiority of either dose of alogliptin to glipizide was established if the upper lim-
it of the one-sided $98.75 \%$ confidence interval for the difference in change from baseline between that
alogliptin dose and glipizide was < $0 \%$

Participants Inclusion criteria and diagnostic criteria: adults aged 18-80 years with a historical diagnosis of T2DM, $\mathrm{BMI} \geq 23$ and $\leq 45 \mathrm{~kg} / \mathrm{m}^{2}$ (if Asian, $\geq 20$ and $\leq 35 \mathrm{~kg} / \mathrm{m}^{2}$ ), and inadequate glycaemic control defined in one of two ways: (i) HbA1c $7 \%-9 \%$ with FPG $<15.3 \mathrm{mmol} / \mathrm{L}$ on stable metformin ( $\geq 1500 \mathrm{mg}$ or MTD), or (ii) $\mathrm{HbA1c}$ of $7.5 \%-10 \%$ on metformin $<1500 \mathrm{mg}$ without documented MTD, with $\mathrm{HbA} 1 \mathrm{c} 7.0 \%-9.0 \%$ and FPG $<15.3 \mathrm{mmol} / \mathrm{L}$ after metformin stabilization ( $\geq 1500 \mathrm{mg}$ or MTD) for 8 weeks

Exclusion criteria: treatment with other antidiabetic agents within the previous 2 months; $\mathrm{SBP} \geq 150$ $\mathrm{mmHg}$ and/or DBP $\geq 90 \mathrm{mmHg}$; history of cancer (other than squamous cell or basal cell carcinoma of the skin in full remission for $\geq 5$ years); NYHA III-IV; receiving alogliptin in a previous investigational study; and history of coronary angioplasty, coronary stent placement, coronary bypass surgery, MI, stroke or transient ischaemic attack in the previous 3 months

Interventions Number of study centres: 310

Run-in period: each participant's schedule depended on the metformin dosage at screening: schedule A (participants with $\mathrm{HbA1c} 7.0 \%-9.0 \%$ on stable metformin at $\geq 1500 \mathrm{mg}$ or MTD) consisted of screening (up to 2 weeks), stabilisation (4 weeks). Schedule B (participants with HbA1c $7.5 \%-10.0 \%$ on metformin $<1500 \mathrm{mg}$ and below MTD) consisted of pre-screening (up to 2 weeks), titration (to metformin $\geq$ $1500 \mathrm{mg}$ or MTD, 8 weeks), screening (up to 1 week), stabilisation (4 weeks)

Extension period: no

\begin{tabular}{ll}
\hline Outcomes & Composite outcome measures reported: no \\
\hline Study details & Trial terminated early: no \\
& Trial ID: NCT00856284 \\
\hline Publication details & Language of publication: English \\
\hline
\end{tabular}


Publication status: peer-reviewed journal

Stated aim for study

Quote from publication: "To evaluate the long-term durability of the efficacy of alogliptin compared with glipizide in combination with metformin in people with type 2 diabetes inadequately controlled on stable-dose metformin"

Notes

\section{Risk of bias}

\begin{tabular}{lll}
\hline Bias & Authors' judgement & Support for judgement \\
\hline $\begin{array}{l}\text { Random sequence genera- } \\
\text { tion (selection bias) }\end{array}$ & Unclear risk & $\begin{array}{l}\text { Quote from publication: "... patients were randomized ..." "Demographic and } \\
\text { other baseline characteristics of randomized patients were similar among the } \\
\text { treatment groups" }\end{array}$
\end{tabular}

Comment: insufficient information about the sequence generation process

\begin{tabular}{lll}
\hline $\begin{array}{l}\text { Allocation concealment } \\
\text { (selection bias) }\end{array}$ & Unclear risk & $\begin{array}{l}\text { Quote from publication: "Demographic and other baseline characteristics of } \\
\text { randomized patients were similar among the treatment groups" }\end{array}$
\end{tabular}

Comment: insufficient information about the allocation concealment

\section{Blinding of participants Low risk} and personnel (performance bias)

all-cause/cardiovascular mortality

Quote from publication: "Double blind (subject, investigator)." "The occurrence of major adverse cardiovascular events (MACE) was assessed by an independent adjudication committee comprising three independent subject-area experts. The members blindly reviewed and adjudicated all deaths, all serious cardiovascular events, and selected non-serious potential cardiovascular events. MACE was defined according to standard criteria as cardiovascular death, non-fatal myocardial infarction and non-fatal stroke"

Comment: adjudicator-assessed outcome measurement. Blinding of participant and key study personnel ensured

Blinding of participants Low risk and personnel (performance bias) hypoglycaemia
Quote from publication: "Double blind (subject, investigator)" "Episodes of hypoglycaemia were monitored by use of glucometers and diaries issued to all patients. For each incident, patients were asked to record any signs or symptoms of hypoglycaemia, blood glucose at the time of the event and any assistance needed to treat the event. For recurrent episodes, the patient was instructed to notify the investigator as soon as possible. Patients were also encouraged to measure their blood glucose at least once per day"

Comment: self-reported outcome measurement. Blinding of participants ensured

$\begin{array}{ll}\begin{array}{l}\text { Blinding of participants } \\ \text { and personnel (perfor- }\end{array} & \text { Low risk } \\ \text { mance bias) } & \begin{array}{l}\text { Quote from publication: "Double blind (subject, investigator)." "The occur- } \\ \text { rence of major adverse cardiovascular events (MACE) was assessed by an inde- } \\ \text { non-fatal myocardial in- }\end{array} \\ \begin{array}{l}\text { pendent adjudication committee comprising three independent subject-area } \\ \text { farction/heart failure/non- }\end{array} & \begin{array}{l}\text { experts. The members blindly reviewed and adjudicated all deaths, all seri- } \\ \text { fatal stroke }\end{array} \\ \begin{array}{l}\text { ous cardiovascular events, and selected non-serious potential cardiovascu- } \\ \text { lar events. MACE was defined according to standard criteria as cardiovascular } \\ \text { death, non-fatal myocardial infarction and non-fatal stroke" }\end{array}\end{array}$

Comment: adjudicator-assessed outcome measurement. Blinding of participant and key study personnel ensured

Blinding of participants Low risk
and personnel (perfor-
mance bias)

Quote from publication: "Double blind (subject, investigator)" 
Del Prato 2014 (Continued) non-serious adverse events
Comment: self-reported outcome measurement. Blinding of participants ensured
Blinding of participants Low risk and personnel (performance bias)

serious adverse events
Quote from publication: "Double blind (subject, investigator)" "Patients were carefully monitored for pancreatitis, and urged to seek follow-up if experiencing persistent nausea and/or vomiting for $\geq 3$ days, with or without abdominal pain. Diagnosis was established by central laboratory analysis of pancreatic enzymes (repeated until resolution) and appropriate imaging tests"

Comment: investigator-assessed outcome measurement. Blinding of participants and key study personnel ensured

\begin{tabular}{|c|c|c|}
\hline $\begin{array}{l}\text { Blinding of participants } \\
\text { and personnel (perfor- } \\
\text { mance bias) } \\
\text { weight (kg) }\end{array}$ & Low risk & $\begin{array}{l}\text { Quote from publication: "Double blind (subject, investigator)" } \\
\text { Comment: investigator-assessed or self-reported outcome measurement. } \\
\text { Blinding of participants and key study personnel ensured }\end{array}$ \\
\hline
\end{tabular}

Low risk

Blinding of participants and personnel (performance bias)

$\mathrm{HbA1c}$
Quote from publication: "Double blind (subject, investigator)" "Antihyperglycaemic efficacy was assessed using standard laboratory measures and assessments of weight throughout the study"

Comment: investigator-assessed outcome measurement. Blinding of investigators ensured

\section{Blinding of outcome as- Low risk sessment (detection bias) all-cause/cardiovascular mortality \\ Quote from publication: "Double blind (subject, investigator)." "The occur- rence of major adverse cardiovascular events (MACE) was assessed by an inde- pendent adjudication committee comprising three independent subject-area experts. The members blindly reviewed and adjudicated all deaths, all seri- ous cardiovascular events, and selected non-serious potential cardiovascu- lar events. MACE was defined according to standard criteria as cardiovascular death, non-fatal myocardial infarction and non-fatal stroke"}

Comment: adjudicator-assessed outcome measurement. Blinding of outcome assessment ensured

Blinding of outcome as- Low risk sessment (detection bias) hypoglycaemia
Quote from publication: "Double blind (subject, investigator)" "Episodes of hypoglycaemia were monitored by use of glucometers and diaries issued to all patients. For each incident, patients were asked to record any signs or symptoms of hypoglycaemia, blood glucose at the time of the event and any assistance needed to treat the event. For recurrent episodes, the patient was instructed to notify the investigator as soon as possible. Patients were also encouraged to measure their blood glucose at least once per day"

Comment: self-reported outcome measurement. Blinding of outcome assessment ensured
Quote from publication: "Double blind (subject, investigator)." "The occurrence of major adverse cardiovascular events (MACE) was assessed by an independent adjudication committee comprising three independent subject-area experts. The members blindly reviewed and adjudicated all deaths, all serious cardiovascular events, and selected non-serious potential cardiovascular events. MACE was defined according to standard criteria as cardiovascular death, non-fatal myocardial infarction and non-fatal stroke"

Comment: adjudicator-assessed outcome measurement. Blinding of outcome assessment ensured

Quote from publication: "Double blind (subject, investigator)"

Comment: self-reported outcome measurement. Blinding of outcome assessment ensured 
Del Prato 2014 (Continued) non-serious adverse

events

Blinding of outcome as- Low risk sessment (detection bias) serious adverse events
Quote from publication: "Double blind (subject, investigator)" "Patients were carefully monitored for pancreatitis, and urged to seek follow-up if experiencing persistent nausea and/or vomiting for $\geq 3$ days, with or without abdominal pain. Diagnosis was established by central laboratory analysis of pancreatic enzymes (repeated until resolution) and appropriate imaging tests"

Comment: investigator-assessed outcome measurement. Blinding of outcome assessment ensured

$\begin{array}{lll}\begin{array}{l}\text { Blinding of outcome as- } \\ \text { sessment (detection bias) }\end{array} & \text { Low risk } & \text { Quote from publication: "Double blind (subject, investigator)" } \\ \text { weight }(\mathrm{kg}) & \begin{array}{l}\text { Comment: investigator-assessed or self-reported outcome measurement. } \\ \text { Blinding of outcome assessment ensured }\end{array}\end{array}$

Blinding of outcome as- Low risk sessment (detection bias) HbA1c

Quote from publication: "Double blind (subject, investigator)" "Antihyperglycaemic efficacy was assessed using standard laboratory measures and assessments of weight throughout the study"

Comment: investigator-assessed outcome measurement. Blinding of outcome assessment ensured (attrition bias) all-cause/cardiovascular mortality
Incomplete outcome data Low risk

Quote from publication: "The safety set used for safety endpoints included all patients who took at least one dose of study medication".

Comment: $>99 \%$ of the randomised participants were included in the analyses. There was a high dropout rate (49\%-56\% of the participants completed the study), however, the dropout rate was balanced between groups. We assumed that trial authors searched registers for mortality status at the end of the trial. About $2.5 \%$ of the participants in each intervention group were lost to follow-up. Not clarified how these missing data were imputed. The proportion of missingness is small and judged not to give rise to risk of attrition bias
Incomplete outcome data High risk (attrition bias)

hypoglycaemia
Quote from publication: "The safety set used for safety endpoints included all patients who took at least one dose of study medication".

Comment: $>99 \%$ of the participants in all treatment groups were included in the analyses. There was a high dropout rate (49\%-56\% of the participants completed the study), however, the dropout rate was balanced between groups. We do not know how the trial authors imputed missing data from the participants not completing the study. The reasons for dropouts were balanced among the intervention groups. The proportion of missing outcomes compared with observed event risk was enough to induce clinically relevant bias in intervention effect estimate

Quote from publication: "The safety set used for safety endpoints included all patients who took at least one dose of study medication".

Comment: > 99\% of the participants in all treatment groups were included in the analyses. There was a high dropout rate (49\%-56\% of the participants completed the study), however, the dropout rate was balanced between groups. We do not know how the trial authors imputed missing data from the participants not completing the study. The reasons for dropouts were balanced among the intervention groups. The proportion of missing outcomes compared with observed event risk was enough to induce clinically relevant bias in intervention effect estimate

Quote from publication: "The safety set used for safety endpoints included all patients who took at least one dose of study medication".

\footnotetext{
Incomplete outcome data Low risk (attrition bias)
} 
Del Prato 2014 (Continued) non-serious adverse events
Comment: $>99 \%$ of the participants in all treatment groups were included in the analyses. There was a high dropout rate (49\%-56\% of the participants completed the study), however, the dropout rate was balanced between groups. We do not know how the trial authors imputed missing data from the participants not completing the study. The reasons for dropouts were balanced among the intervention groups.

The proportion of missing outcomes compared with observed event risk was not enough to induce clinically relevant bias in intervention effect estimate
Incomplete outcome data High risk (attrition bias)

serious adverse events
Quote from publication: "The safety set used for safety endpoints included all patients who took at least one dose of study medication".

Comment: $>99 \%$ of the participants in all treatment groups were included in the analyses. There was a high dropout rate (49\%-56\% of the participants completed the study), however, the dropout rate was balanced between groups. We do not know how the trial authors imputed missing data from the participants not completing the study. The reasons for dropouts were balanced among the intervention groups.

The proportion of missing outcomes compared with observed event risk was enough to induce clinically relevant bias in intervention effect estimate

\section{Incomplete outcome data High risk} (attrition bias) weight $(\mathrm{kg})$

Quote from publication: "... secondary and exploratory analyses used the full analysis set. For any given variable, the full analysis set included all patients receiving study medication with a baseline and at least one post-baseline assessment." "Missing values were extrapolated using the last observation carried forward in all efficacy analyses except PPG, for which no extrapolation was done."

Comment: $>98 \%$ of the participants were included in the analyses. There was a high dropout rate (49\%-56\% of the participants completed the study), however, the dropout rate was balanced between groups. The reasons for dropouts were balanced among the intervention groups. Inappropriate method of imputation for missing data was used (LOCF). Plausible effect size among missing outcomes was enough to induce clinically relevant bias in observed effect size

Incomplete outcome data High risk (attrition bias)

Quote from publication: "The primary efficacy analysis was performed in the per-protocol set..." "The per-protocol set included all randomized patients who took at least 1 dose of double-blind study drug, with a baseline assessment and at least 1 post-baseline assessment for that variable and who had no major protocol violations. Last observation carried forward was used."

Comment: around $40 \%$ of the participants were included in the analyses. There was a high dropout rate ( $49 \%-56 \%$ of the participants completed the study), however, the dropout rate was balanced between groups. The reasons for dropouts were balanced among the intervention groups. Inappropriate method of imputation for missing data was used (LOCF). Plausible effect size among missing outcomes was enough to induce clinically relevant bias in observed effect size

Selective reporting (re- Low risk porting bias)

Comment: all of the trial's primary and secondary outcomes as specified in the protocol have been reported
Quote from publication: "This study was sponsored by Takeda Development Center Americas, Inc., Deerfield, IL, USA. Manuscript writing and editorial assistance was provided by Lyndsey Wood and Clare Gurton (on behalf of Rx Communications, UK). Support for this assistance was funded by Takeda."

Comment: the trial received funding from a pharmaceutical company 
Randomisation ratio: 1:1 to receive double-blind treatment with dapagliflozin or glipizide in addition to metformin

Non-inferiority and superiority design: "A hierarchic closed-testing procedure was used to control the type I error rate across the primary and key secondary end points at the 0.05 level (two-sided). Thus, if non-inferiority was established for the primary end point at a one-sided 0.025 significance level, then key secondary end point testing for superiority could proceed in the sequence described previously. If the first key secondary end point was significant at a two-sided 0.05 significance level, then the second secondary end point could be evaluated, and so forth."

Participants

Inclusion criteria: men and women aged $\geq 18$ years, inadequately controlled T2DM ( $\mathrm{HbA1C}>6.5$ and $\leq$ $10 \%)$, receiving metformin or metformin and one other OAD administered up to half-maximal dose for at least 8 weeks before enrolment. Further criteria included FPG $\leq 15 \mathrm{mmol} / \mathrm{L}$ and C-peptide concentration of $\geq 0.33 \mathrm{nmol} / \mathrm{L}$

Exclusion criteria: T1DM; diabetes insipidus; corticosteroid-induced T2DM; a history of diabetic ketoacidosis or hyperosmolar non-ketotic coma; poorly controlled diabetes characterised by polyuria/ polydipsia with $>10 \%$ weight loss; use of insulin within 1 year of enrolment, except in the case of hospitalisation or use in gestational diabetes, $\mathrm{BMI}>45.0 \mathrm{~kg} / \mathrm{m}^{2}$; calculated creatinine clearance $<60 \mathrm{~mL} /$ min; urine albumin:creatinine ratio $>203.4 \mathrm{mg} / \mathrm{mmol}$; AST and/or AST and/or creatine kinase $\geq 3 \times$ ULN range; serum total bilirubin $>34 \mu \mathrm{mol} / \mathrm{L}$; haemoglobin $\leq 11 \mathrm{~g} / \mathrm{dL}$ for men and $\leq 10 \mathrm{~g} / \mathrm{dL}$ for women; abnormal TSH; systolic BP $\geq 180 \mathrm{mmHg}$ and/or diastolic BP $\geq 110 \mathrm{mmHg}$; CV within 6 months of enrolment; congestive heart failure; congenital renal glycosuria; significant renal, hepatic, respiratory, haematological, oncological, endocrine, immunological (including hypersensitivity to study medications), and alcohol and/or substance misuse disorders; pregnancy and/or lactation; use of systemic corticosteroids equivalent to $>10 \mathrm{mg}$ of oral prednisolone within 30 days of enrolment; a history of bariatric surgery; and use of weight loss medication within 30 days of enrolment

Diagnostic criteria: not reported

\section{Number of study centres: 95}

Run-in period: eligible participants receiving metformin monotherapy at a stable dose of $<1500 \mathrm{mg} /$ day or at a variable dose, or combined with another $\mathrm{OAD}$, entered an 8-week stabilisation period during which other OADs were discontinued and the metformin dose was stabilised to $1500-2500 \mathrm{mg} / \mathrm{day}$ in all participants. Participants who were already receiving a stable dose of metformin monotherapy (1500-2500 mg/day) for at least 8 weeks before enrolment skipped the dose-stabilisation period. A 2week, single-blind, placebo-lead in period followed the stabilisation period.

Extension period: yes, 156-week extension period

Outcomes Composite outcome measures reported: no

Study details Trial terminated early: no

Trial ID: NCT00660907

Publication details Language of publication: English and Spanish

Funding: commercial funding by AstraZeneca and Bristol-Myers Squibb

Publication status: peer-reviewed journal

Stated aim for study

Quote from publication: "To assess the long-term glycaemic durability, safety and tolerability of dapagliflozin versus glipizide as add-on therapies in patients with type 2 diabetes inadequately controlled by metformin alone" 
Del Prato 2015 (Continued)

Notes

\section{Risk of bias}

\begin{tabular}{lll}
\hline Bias & Authors' judgement & Support for judgement \\
\hline $\begin{array}{ll}\text { Random sequence genera- } \\
\text { tion (selection bias) }\end{array}$ & Low risk & $\begin{array}{l}\text { Quote from publication: "Patients were randomized sequentially at study lev- } \\
\text { el according to a predefined computer-generated randomization scheme pro- } \\
\text { vided by AstraZeneca." }\end{array}$
\end{tabular}

Comment: adequate generation of random sequence ensured

\begin{tabular}{ll}
\hline $\begin{array}{l}\text { Allocation concealment } \\
\text { (selection bias) }\end{array}$ & Low risk \\
& $\begin{array}{l}\text { Quote from publication: "Allocation of study treatments was performed via } \\
\text { an Interactive Web Response System in balanced block sizes of } 4 \text { to ensure ap- } \\
\text { proximate balance among treatment groups." }\end{array}$
\end{tabular}

Comment: adequate concealment ensured

\begin{tabular}{ll}
\hline $\begin{array}{l}\text { Blinding of participants } \\
\text { and personnel (perfor- } \\
\text { mance bias) }\end{array}$ & Low risk \\
$\begin{array}{l}\text { all-cause/cardiovascular } \\
\text { mortality }\end{array}$ & $\begin{array}{l}\text { Quote from publication: "Blinding of patients and investigators to study } \\
\text { treatment was achieved using a double-dummy technique. Metformin was ad- } \\
\text { ministered as an open-label treatment throughout the study." }\end{array}$ \\
& $\begin{array}{l}\text { Comment: investigator-assessed outcome measurement. Blinding of investi- } \\
\text { gator ensured }\end{array}$
\end{tabular}

\begin{tabular}{ll}
\hline $\begin{array}{l}\text { Blinding of participants } \\
\begin{array}{l}\text { and personnel (perfor- } \\
\text { mance bias) }\end{array}\end{array}$ & $\begin{array}{l}\text { Quote from publication: "Blinding of patients and investigators to study } \\
\text { treatment was achieved using a double-dummy technique. Metformin was ad- } \\
\text { hypoglycaemia }\end{array}$ \\
& $\begin{array}{l}\text { ministered as an open-label treatment throughout the study." } \\
\text { Comment: investigator-assessed/self-reported outcome measurement. Blind- } \\
\text { ing of investigator and participant ensured }\end{array}$
\end{tabular}

\begin{tabular}{|c|c|c|}
\hline $\begin{array}{l}\text { Blinding of participants } \\
\text { and personnel (perfor- } \\
\text { mance bias) } \\
\text { non-fatal myocardial in- } \\
\text { farction/heart failure/non- } \\
\text { fatal stroke }\end{array}$ & Low risk & $\begin{array}{l}\text { Quote from publication: "Blinding of patients and investigators to study } \\
\text { treatment was achieved using a double-dummy technique. Metformin was ad } \\
\text { ministered as an open-label treatment throughout the study." } \\
\text { Comment: investigator-assessed outcome measurement. Blinding of investi- } \\
\text { gator ensured }\end{array}$ \\
\hline
\end{tabular}

\begin{tabular}{ll}
\hline $\begin{array}{l}\text { Blinding of participants } \\
\begin{array}{l}\text { and personnel (perfor- } \\
\text { mance bias) }\end{array}\end{array}$ & Low risk \\
$\begin{array}{l}\text { non-serious adverse } \\
\text { events }\end{array}$ & $\begin{array}{l}\text { Quote from publication: "Blinding of patients and investigators to study } \\
\text { treatment was achieved using a double-dummy technique. Metformin was ad- } \\
\text { ministered as an open-label treatment throughout the study." }\end{array}$ \\
& $\begin{array}{l}\text { Comment: investigator-assessed/self-reported outcome measurement. Blind- } \\
\text { ing of investigator and participant ensured }\end{array}$
\end{tabular}

\begin{tabular}{|c|c|c|}
\hline $\begin{array}{l}\text { Blinding of participants } \\
\text { and personnel (perfor- } \\
\text { mance bias) }\end{array}$ & Low risk & $\begin{array}{l}\text { Quote from publication: "Blinding of patients and investigators to study } \\
\text { treatment was achieved using a double-dummy technique. Metformin was ad- } \\
\text { ministered as an open-label treatment throughout the study." }\end{array}$ \\
\hline
\end{tabular}

Comment: investigator-assessed outcome measurement. Blinding of investigator ensured

\begin{tabular}{|c|c|c|}
\hline $\begin{array}{l}\text { Blinding of participants } \\
\text { and personnel (perfor- } \\
\text { mance bias) }\end{array}$ & Low risk & $\begin{array}{l}\text { Quote from publication: "Blinding of patients and investigators to study } \\
\text { treatment was achieved using a double-dummy technique. Metformin was ad- } \\
\text { ministered as an open-label treatment throughout the study." }\end{array}$ \\
\hline
\end{tabular}

weight $(\mathrm{kg})$

Comment: investigator-assessed/self-reported outcome measurement. Blinding of investigator and participant ensured 


\section{Del Prato 2015 (Continued)}

Blinding of participants and personnel (performance bias)

HbA1c
Low risk

Quote from publication: "Blinding of patients and investigators to study treatment was achieved using a double-dummy technique. Metformin was administered as an open-label treatment throughout the study."

Comment: investigator-assessed outcome measurement. Blinding of investigator ensured

\section{Blinding of outcome as- Low risk} sessment (detection bias) all-cause/cardiovascular mortality
Quote from publication: "Blinding of patients and investigators to study treatment was achieved using a double-dummy technique. Metformin was administered as an open-label treatment throughout the study."

Comment: investigator-assessed outcome measurement. Blinding of investigator ensured

$\begin{array}{lll}\begin{array}{l}\text { Blinding of outcome as- } \\ \text { sessment (detection bias) }\end{array} & \text { Low risk } & \begin{array}{l}\text { Quote from publication: "Blinding of patients and investigators to study } \\ \text { treatment was achieved using a double-dummy technique. Metformin was ad- } \\ \text { hypoglycaemia }\end{array} \\ \text { ministered as an open-label treatment throughout the study." }\end{array}$

hypoglycaemia

Comment: investigator-assessed/self-reported outcome measurement. Blinding of investigator and participant ensured

\author{
Blinding of outcome as- \\ sessment (detection bias) \\ non-fatal myocardial in- \\ farction/heart failure/non- \\ fatal stroke
}

Low risk

Quote from publication: "Blinding of patients and investigators to study treatment was achieved using a double-dummy technique. Metformin was administered as an open-label treatment throughout the study."

Comment: investigator-assessed outcome measurement. Blinding of investigator ensured

$\begin{array}{lll}\text { Blinding of outcome as- } & \text { Low risk } & \text { Quote from publication: "Blinding of patients and investigators to study } \\ \text { sessment (detection bias) } & & \text { treatment was achieved using a double-dummy technique. Metformin was ad- } \\ \text { non-serious adverse } & \text { ministered as an open-label treatment throughout the study." }\end{array}$

events

Comment: investigator-assessed/self-reported outcome measurement. Blinding of investigator and participant ensured
Blinding of outcome as- Low risk sessment (detection bias) serious adverse events
Quote from publication: "Blinding of patients and investigators to study treatment was achieved using a double-dummy technique. Metformin was administered as an open-label treatment throughout the study."

Comment: investigator-assessed outcome measurement. Blinding of investigator ensured
Quote from publication: "Blinding of patients and investigators to study treatment was achieved using a double-dummy technique. Metformin was administered as an open-label treatment throughout the study."

Comment: investigator-assessed/self-reported outcome measurement. Blinding of investigator and participant ensured

\begin{tabular}{|c|c|c|}
\hline $\begin{array}{l}\text { Blinding of outcome as- } \\
\text { sessment (detection bias) } \\
\text { HbAlc }\end{array}$ & Low risk & $\begin{array}{l}\text { Quote from publication: "Blinding of patients and investigators to study } \\
\text { treatment was achieved using a double-dummy technique. Metformin was ad- } \\
\text { ministered as an open-label treatment throughout the study." }\end{array}$ \\
\hline
\end{tabular}

Comment: investigator-assessed outcome measurement. Blinding of investigator ensured

\begin{tabular}{|c|c|c|}
\hline $\begin{array}{l}\text { Incomplete outcome data } \\
\text { (attrition bias) } \\
\text { all-cause/cardiovascular } \\
\text { mortality }\end{array}$ & Low risk & $\begin{array}{l}\text { Quote from publication: "All analyses during the extension periods were con- } \\
\text { sidered exploratory." "Rates of AEs were evaluated using the safety popula- } \\
\text { tion, which comprised all patients who had received } \geq 1 \text { dose of study medica- } \\
\text { tion." }\end{array}$ \\
\hline
\end{tabular}


Comment: $>99 \%$ of the randomised participants were included in the analyses. There was a high dropout rate $(34 \%-40 \%$ of randomised participants completed the study), however, the dropout rate was balanced between groups. No information on method to impute missing data. We assumed that trial authors searched registers for mortality status at the end of the trial. The proportion of missingness is small and judged not to give rise to risk of attrition bias

Incomplete outcome data Low risk (attrition bias)

hypoglycaemia
Quote from publication: "All analyses during the extension periods were considered exploratory." "Rates of AEs were evaluated using the safety population, which comprised all patients who had received $\geq 1$ dose of study medication."

Comment: $>99 \%$ of the randomised participants were included in the analyses. There was a high dropout rate (34\%- $40 \%$ of randomised participants completed the study), however, the dropout rate was balanced between groups. The reasons for dropouts were balanced among the intervention groups. No information on method to impute missing data. The proportion of missing outcomes compared with observed event risk was not enough to induce clinically relevant bias in intervention effect estimate
Incomplete outcome data High risk (attrition bias) non-fatal myocardial infarction/heart failure/nonfatal stroke
Quote from publication: "... the safety analysis set, consisting of all patients who received one or more doses of the investigational product..." "Missing values at week 52 were replaced using the LOCF method"

Comment: $>99 \%$ of the randomised participants were included in the analyses. There was a high dropout rate (to week $52,77 \%-79 \%$ of randomised participants completed the study), however, the dropout rate was balanced between groups. The reasons for dropouts were balanced among the intervention groups. Inappropiate method of imputing missing data was used (LOCF). The proportion of missing outcomes compared with observed event risk was enough to induce clinically relevant bias in intervention effect estimate

Quote from publication: "... the safety analysis set, consisting of all patients who received one or more doses of the investigational product..." "Missing values at week 52 were replaced using the LOCF method"

Comment: $>99 \%$ of the randomised participants were included in the analyses. There was a high dropout rate (to week 52 , 77\%-79\% of randomised participants completed the study), however, the dropout rate was balanced between groups. The reasons for dropouts were balanced among the intervention groups. Inappropiate method of imputing missing data was used (LOCF). The proportion of missing outcomes compared with observed event risk was not enough to induce clinically relevant bias in intervention effect estimate
Incomplete outcome data High risk (attrition bias)

serious adverse events
Quote from publication: "All analyses during the extension periods were considered exploratory." "Rates of AEs were evaluated using the safety population, which comprised all patients who had received $\geq 1$ dose of study medication."

Comment: $>99 \%$ of the randomised participants were included in the analyses. There was a high dropout rate (34\%- $40 \%$ of randomised participants completed the study), however, the dropout rate was balanced between groups. The reasons for dropouts were balanced among the intervention groups. No information on method to impute missing data. The proportion of missing outcomes compared with observed event risk was enough to induce clinically relevant bias in intervention effect estimate

Quote from publication: "All analyses during the extension periods were considered exploratory" "Efficacy was evaluated using the full analysis set, which comprised all randomized patients who received at least one dose of study medication, and who had a non-missing baseline value and a value for $\geq 1$ of 
repeated-measures analysis, with the fixed categorical effects of treatment, week and treatment-by-week interaction, as well as the continuous fixed covariates of baseline value and baseline value-by-week interaction."

Comment: $34 \%-39 \%$ of the randomised participants were included in the analyses. There was a high dropout rate (34\%- $40 \%$ of randomised participants completed the study), however, the dropout rate was balanced between groups. The reasons for dropouts were balanced among the intervention groups. Only participants who completed the study (including participants receiving rescue therapy) were included in the analysis. Plausible effect size among missing outcomes was enough to induce clinically relevant bias in observed effect size

\section{Incomplete outcome data High risk} (attrition bias)

HbA1c
Quote from publication: "All analyses during the extension periods were considered exploratory" "Efficacy was evaluated using the full analysis set, which comprised all randomized patients who received at least one dose of study medication, and who had a non-missing baseline value and a value for $\geq 1$ of the efficacy variables. Primary and continuous key efficacy endpoints were analysed using longitudinal

repeated-measures analysis, with the fixed categorical effects of treatment, week and treatment-by-week interaction, as well as the continuous fixed covariates of baseline value and baseline value-by-week interaction."

Comment: $17 \%-20 \%$ of the randomised participants were included in the analyses. There was a high dropout rate (34\%- $40 \%$ of randomised participants completed the study), however, the dropout rate was balanced between groups. The reasons for dropouts were balanced among the intervention groups. Only participants who completed the study (excluding participants receiving rescue therapy) were included in the analysis. Plausible effect size among missing outcomes was enough to induce clinically relevant bias in observed effect size

\begin{tabular}{|c|c|c|}
\hline $\begin{array}{l}\text { Selective reporting (re- } \\
\text { porting bias) }\end{array}$ & Low risk & $\begin{array}{l}\text { Comment: all of the trial's primary and secondary outcomes as specified ir } \\
\text { the protocol have been reported }\end{array}$ \\
\hline
\end{tabular}

Unclear risk

Comment: the trial received funding from a pharmaceutical company

Randomisation ratio: 1:1 to receive glimepiride or rosiglitazone in addition to metformin

Participants

Inclusion criteria: T2DM for at least 6 months and inadequate glycaemic control with diet and oral glucose-lowering drugs such as sulphonylureas or metformin, both to the MTD. No participants were taking glimepiride or thiazolidinediones. All participants had a fasting C-peptide level $>1.0 \mathrm{ng} / \mathrm{mL}$, were overweight (BMI mean $25.3 \mathrm{~kg} / \mathrm{m}^{2}$ ), hypertensive (according to WHO 1999) and had triglyceridaemia (according to National Cholesterol Education Program Expert Panel 2001)

Exclusion criteria: history of ketoacidosis or unstable or rapidly progressive diabetic background retinopathy, nephropathy (microalbuminuria, evaluated by proteinuria $<300 \mathrm{mg} / 24 \mathrm{~h}$ ) or neuropathy (evaluated by electromyography). Participants with impaired liver function (transaminases $>40 \mathrm{U} / \mathrm{L}$ ), impaired kidney function (creatinine $>1.5 \mathrm{mg} / \mathrm{dL}$ ) or anaemia $(\mathrm{Hb}<115 \mathrm{gm} / \mathrm{L})$. Participants with unstable cardiovascular conditions (e.g. NYHA class III-IV or a history of MI or stroke) or cerebrovascular conditions within 6 months of study enrolment. Women who were pregnant, lactating, or of child-bearing potential while not taking adequate contraceptive precautions 
Derosa 2005 (Continued)

Diagnostic criteria: ADA 2001

Interventions Number of study centres: 2

Run-in period: 1 week of washout from other previous different treatments

Extension period: no

Outcomes Composite outcome measures reported: no

Study details Trial terminated early: no

Trial ID: -

Publication details Language of publication: English

Funding: non-commercial funding

Publication status: peer-reviewed journal

Stated aim for study $\quad$ Quote from publication: "... to evaluate the differential effect on glucose and lipid parameters of the association between glimepiride plus metformin and rosiglitazone plus metformin in patients affected by type 2 diabetes and metabolic syndrome"; "To evaluate the differential effect on coagulation and fibrinolysis parameters of combination therapy with glimepiride-metformin and with rosiglitazone-metformin beyond their effect on glucose metabolism in patients with type 2 diabetes and metabolic syndrome"; "... to compare the effect of long-term (12-month) combination treatment with glimepiride or rosiglitazone plus metformin on blood pressure in patients with type 2 diabetes mellitus (DM-2) and the metabolic syndrome. Secondary end points were glycemic control and improvement in insulin sensitivity"; "... to evaluate the differential effect on a wide range of metabolic parameters and non-conventional cardiovascular risk factors (plasma Lp[a] levels and basal homocysteinaemia) of glimepiride and rosiglitazone in such patients"

Notes

\section{Risk of bias}

\section{Bias}

Authors' judgement Support for judgement

Random sequence genera- Low risk tion (selection bias)
Quote from publication: "Randomization was done by drawing envelopes containing randomization codes prepared by an independent statistician. The envelopes were then further mixed and distributed to the investigators, who consecutively assigned the randomization codes to the enrolled patients. A copy of the code was provided only to the statistician. The code was not to be broken until database lock or in case of emergency. Glimepiride and rosiglitazone were supplied as matching opaque white capsules in bottles coded to ensure the double-blind status of the study."

Comment: adequate generation of random sequence ensured
Allocation concealment Low risk (selection bias)
Quote from publication: "Randomization was done by drawing envelopes containing randomization codes prepared by an independent statistician. The envelopes were then further mixed and distributed to the investigators, who consecutively assigned the randomization codes to the enrolled patients. A copy of the code was provided only to the statistician. The code was not to be broken until database lock or in case of emergency. Glimepiride and rosiglitazone were supplied as matching opaque white capsules in bottles coded to ensure the double-blind status of the study."

Comment: adequate concealment of allocation prior to assignment ensured 
Derosa 2005 (Continued)

Blinding of participants and personnel (performance bias) all-cause/cardiovascular mortality
Low risk

Quote from publication: "double-blind", "Glimepiride and rosiglitazone were supplied as matching opaque white capsules in coded bottles to ensure the double-blind status of the study"

Comment: investigator-assessed outcome measurement. Blinding of key study personnel ensured

\section{Blinding of participants Low risk} and personnel (performance bias) amputation of lower extremity/blindness or severe vision loss/end-stage renal disease
Quote from publication: "double-blind", "Glimepiride and rosiglitazone were supplied as matching opaque white capsules in coded bottles to ensure the double-blind status of the study"

Comment: investigator-assessed outcome measurement. Blinding of key study personnel ensured

\begin{tabular}{|c|c|c|}
\hline $\begin{array}{l}\text { Blinding of participants } \\
\text { and personnel (perfor- } \\
\text { mance bias) }\end{array}$ & Low risk & $\begin{array}{l}\text { Quote from publication: "double-blind", "Glimepiride and rosiglitazone were } \\
\text { supplied as matching opaque white capsules in coded bottles to ensure the } \\
\text { double-blind status of the study" }\end{array}$ \\
\hline
\end{tabular}

hypoglycaemia

Comment: investigator-assessed/self-reported outcome measurement. Blinding of participants and key study personnel ensured

\begin{tabular}{|c|c|c|}
\hline $\begin{array}{l}\text { Blinding of participants } \\
\text { and personnel (perfor- } \\
\text { mance bias) }\end{array}$ & Low risk & $\begin{array}{l}\text { Quote from publication: "double-blind", "Glimepiride and rosiglitazone were } \\
\text { supplied as matching opaque white capsules in coded bottles to ensure the } \\
\text { double-blind status of the study" }\end{array}$ \\
\hline
\end{tabular}

non-fatal myocardial in-

farction/heart failure/non-

fatal stroke

Comment: investigator-assessed outcome measurement. Blinding of key study personnel ensured

\begin{tabular}{|c|c|c|}
\hline $\begin{array}{l}\text { Blinding of participants } \\
\text { and personnel (perfor- } \\
\text { mance bias) }\end{array}$ & Low risk & $\begin{array}{l}\text { Quote from publication: "double-blind", "Glimepiride and rosiglitazone were } \\
\text { supplied as matching opaque white capsules in coded bottles to ensure the } \\
\text { double-blind status of the study" }\end{array}$ \\
\hline
\end{tabular}

non-serious adverse

events

Comment: investigator-assessed/self-reported outcome measurement. Blinding of participants and key study personnel ensured

Blinding of participants Low risk and personnel (performance bias)

serious adverse events
Quote from publication: "double-blind", "Glimepiride and rosiglitazone were supplied as matching opaque white capsules in coded bottles to ensure the double-blind status of the study"

Comment: investigator-assessed outcome measurement. Blinding of key study personnel ensured

\begin{tabular}{|c|c|c|}
\hline $\begin{array}{l}\text { Blinding of participants } \\
\text { and personnel (perfor- } \\
\text { mance bias) }\end{array}$ & Low risk & $\begin{array}{l}\text { Quote from publication: "double-blind", "Glimepiride and rosiglitazone were } \\
\text { supplied as matching opaque white capsules in coded bottles to ensure the } \\
\text { double-blind status of the study" }\end{array}$ \\
\hline
\end{tabular}

weight (kg)

Comment: investigator-assessed outcome measurement. Blinding of key study personnel ensured

$\begin{array}{ll}\text { Blinding of participants } & \text { Low risk } \\ \begin{array}{l}\text { and personnel (perfor- } \\ \text { mance bias) }\end{array} & \begin{array}{l}\text { Quote from publication: "double-blind", "Glimepiride and rosiglitazone were } \\ \text { supplied as matching opaque white capsules in coded bottles to ensure the } \\ \text { double-blind status of the study" }\end{array}\end{array}$

HbAlc

Comment: investigator-assessed outcome measurement. Blinding of key study personnel ensured

Blinding of outcome as- $\quad$ Low risk
sessment (detection bias)

Quote from publication: "double-blind", "Glimepiride and rosiglitazone were supplied as matching opaque white capsules in coded bottles to ensure the double-blind status of the study" 
Derosa 2005 (Continued) all-cause/cardiovascular mortality
Comment: investigator-assessed outcome measurement. Blinding of outcome assessor ensured

$\begin{array}{lll}\text { Blinding of outcome as- } & \text { Low risk } & \begin{array}{l}\text { Quote from publication: "double-blind", "Glimepiride and rosiglitazone were } \\ \text { sessment (detection bias) } \\ \text { smputation of lower ex- }\end{array} \\ \text { double-blind status of the study" }\end{array}$
amputation of lower extremity/blindness or severe vision loss/end-stage renal disease double-blind status of the study"

Comment: investigator-assessed outcome measurement. Blinding of outcome assessor ensured

Blinding of outcome as- $\quad$ Low risk
sessment (detection bias)

hypoglycaemia

Quote from publication: "double-blind", "Glimepiride and rosiglitazone were supplied as matching opaque white capsules in coded bottles to ensure the double-blind status of the study"

Comment: investigator-assessed/self-reported outcome measurement. Blinding of outcome assessor ensured

$\begin{array}{lll}\text { Blinding of outcome as- } & \text { Low risk } & \begin{array}{l}\text { Quote from publication: "double-blind", "Glimepiride and rosiglitazone were } \\ \text { sessment (detection bias) }\end{array} \\ \text { non-fatal myocardial in- } & \text { double-blind status of the study" }\end{array}$

farction/heart failure/non-

fatal stroke

Comment: investigator-assessed outcome measurement. Blinding of outcome assessor ensured

$\begin{array}{lll}\text { Blinding of outcome as- } & \text { Low risk } & \begin{array}{l}\text { Quote from publication: "double-blind", "Glimepiride and rosiglitazone were } \\ \text { sessment (detection bias) } \\ \text { non-serious adverse }\end{array}\end{array}$

events

Comment: investigator-assessed/self-reported outcome measurement. Blinding of outcome assessor ensured

$\begin{array}{lll}\begin{array}{l}\text { Blinding of outcome as- } \\ \text { sessment (detection bias) } \\ \text { serious adverse events }\end{array} & \text { Low risk } & \begin{array}{l}\text { Quote from publication: "double-blind", "Glimepiride and rosiglitazone were } \\ \text { supplied as matching opaque white capsules in coded bottles to ensure the } \\ \text { double-blind status of the study" }\end{array}\end{array}$

Comment: investigator-assessed outcome measurement. Blinding of outcome assessor ensured

\begin{tabular}{|c|c|c|}
\hline $\begin{array}{l}\text { Blinding of outcome as- } \\
\text { sessment (detection bias) } \\
\text { weight }(\mathrm{kg})\end{array}$ & Low risk & $\begin{array}{l}\text { Quote from publication: "double-blind", "Glimepiride and rosiglitazone were } \\
\text { supplied as matching opaque white capsules in coded bottles to ensure the } \\
\text { double-blind status of the study" }\end{array}$ \\
\hline
\end{tabular}

Comment: investigator-assessed outcome measurement. Blinding of investigator ensured

\begin{tabular}{|c|c|c|}
\hline $\begin{array}{l}\text { Blinding of outcome as- } \\
\text { sessment (detection bias) } \\
\text { HbAlc }\end{array}$ & Low risk & $\begin{array}{l}\text { Quote from publication: "double-blind", "Glimepiride and rosiglitazone were } \\
\text { supplied as matching opaque white capsules in coded bottles to ensure the } \\
\text { double-blind status of the study" }\end{array}$ \\
\hline
\end{tabular}

Comment: investigator-assessed outcome measurement. Blinding of investigator ensured

$\begin{array}{ll}\begin{array}{l}\text { Incomplete outcome data } \\ \text { (attrition bias) } \\ \text { all-cause/cardiovascular } \\ \text { mortality }\end{array} & \begin{array}{l}\text { Quote from publication: "Patients were included in the safety analysis if } \\ \text { they had received at least } 1 \text { dose of trial medication and tolerability data were } \\ \text { available from at least } 1 \text { follow-up visit" }\end{array} \\ & \begin{array}{l}\text { Comment: } 96 \% \text { of randomised participants completed the study. } 96 \% \text { of the } \\ \text { randomised participants were included in the analysis. Only participants who } \\ \text { completed the study were included in the analysis. The number of participants } \\ \text { who dropped out was balanced between groups. The reasons for dropouts }\end{array}\end{array}$


Incomplete outcome data Low risk (attrition bias) amputation of lower extremity/blindness or severe vision loss/end-stage renal disease
Quote from publication: "Patients were included in the safety analysis if they had received at least 1 dose of trial medication and tolerability data were available from at least 1 follow-up visit"

Comment: $96 \%$ of randomised participants completed the study. $96 \%$ of the randomised participants were included in the analysis. Only participants who completed the study were included in the analysis. The number of participants who dropped out was balanced between groups. The reasons for dropouts were balanced among the intervention groups. No information on imputation. The proportion of missing outcomes compared with observed event risk was not enough to have a clinically relevant impact on the intervention effect estimate

Incomplete outcome data Low risk
(attrition bias)

hypoglycaemia

Quote from publication: "Patients were included in the safety analysis if they had received at least 1 dose of trial medication and tolerability data were available from at least 1 follow-up visit"

Comment: $96 \%$ of randomised participants completed the study. $96 \%$ of the randomised participants were included in the analysis. Only participants who completed the study were included in the analysis. The number of participants who dropped out was balanced between groups. The reasons for dropouts were balanced among the intervention groups. No information on imputation. The proportion of missing outcomes compared with observed event risk was not enough to have a clinically relevant impact on the intervention effect estimate

\begin{tabular}{|c|c|c|}
\hline $\begin{array}{l}\text { Incomplete outcome data } \\
\text { (attrition bias) } \\
\text { non-fatal myocardial in- }\end{array}$ & Low risk & $\begin{array}{l}\text { Quote from publication: "Patients were included in the safety analysis if } \\
\text { they had received at least } 1 \text { dose of trial medication and tolerability data were } \\
\text { available from at least } 1 \text { follow-up visit" }\end{array}$ \\
\hline
\end{tabular}

farction/heart failure/nonfatal stroke

Comment: $96 \%$ of randomised participants completed the study. $96 \%$ of the randomised participants were included in the analysis. Only participants who completed the study were included in the analysis. The number of participants who dropped out was balanced between groups. The reasons for dropouts were balanced among the intervention groups. No information on imputation. The proportion of missing outcomes compared with observed event risk was not enough to have a clinically relevant impact on the intervention effect estimate

Incomplete outcome data Low risk (attrition bias) non-serious adverse events

Quote from publication: "Patients were included in the safety analysis if they had received at least 1 dose of trial medication and tolerability data were available from at least 1 follow-up visit"

Comment: $96 \%$ of randomised participants completed the study. $96 \%$ of the randomised participants were included in the analysis. Only participants who completed the study were included in the analysis. The number of participants who dropped out was balanced between groups. The reasons for dropouts were balanced among the intervention groups. No information on imputation. The proportion of missing outcomes compared with observed event risk was not enough to have a clinically relevant impact on the intervention effect estimate

Incomplete outcome data Low risk (attrition bias)

serious adverse events
Quote from publication: "Patients were included in the safety analysis if they had received at least 1 dose of trial medication and tolerability data were available from at least 1 follow-up visit"

Comment: $96 \%$ of randomised participants completed the study. $96 \%$ of the randomised participants were included in the analysis. Only participants who 
completed the study were included in the analysis. The number of participants who dropped out was balanced between groups. The reasons for dropouts were balanced among the intervention groups. No information on imputation. The proportion of missing outcomes compared with observed event risk was not enough to have a clinically relevant impact on the intervention effect estimate

Incomplete outcome data Low risk (attrition bias) weight $(\mathrm{kg})$
Quote from publication: "An intention-to-treat analysis was conducted in patients who had received at least one dose of study medication and had a subsequent efficacy observation"

Comment: $96 \%$ of randomised participants completed the study. $96 \%$ of the randomised participants were included in the analysis. Only participants who completed the study were included in the analysis. The number of participants who dropped out was balanced between groups. The reasons for dropouts were balanced among the intervention groups. Plausible effect size among missing outcomes was not enough to have a clinically relevant impact on observed effect size

\section{Incomplete outcome data Low risk} (attrition bias)

HbA1c
Quote from publication: "An intention-to-treat analysis was conducted in patients who had received at least one dose of study medication and had a subsequent efficacy observation"

Comment: $96 \%$ of randomised participants completed the study. $96 \%$ of the randomised participants were included in the analysis. Only participants who completed the study were included in the analysis. The number of participants who dropped out was balanced between groups. The reasons for dropouts were balanced among the intervention groups. Plausible effect size among missing outcomes was not enough to have a clinically relevant impact on observed effect size

Selective reporting (re- High risk
porting bias)

Comment: no protocol available. All of the trial's prespecified primary and secondary outcomes (methods section) have been reported. Unclear whether common outcomes (all-cause mortality, hypoglycaemia) were measured; not mentioned, but clinical judgement says likely to have been measured and analysed but not reported on the basis of non-significant results

\section{Derosa 2009a}

Methods Study design: multicentre, double-blind, RCT

Randomisation ratio: 1:1:1:1 to receive pioglitazone, metformin, pioglitazone + metformin or glimepiride + metformin

\section{Participants}

Inclusion criteria: white people, $\geq 18$ years of age with T2DM according to the ESC and the EASD guidelines criteria 2007 who were naive and with poor glycaemic control, expressed as $\mathrm{HbA1c}$ level $>6.5 \%$, and were overweight $\mathrm{BMI} \geq 25$ and $<30 \mathrm{~kg} / \mathrm{m}^{2}$

Exclusion criteria: history of ketoacidosis or unstable or rapidly progressive diabetic retinopathy, nephropathy, or neuropathy; impaired hepatic function (defined as plasma aminotransferase and/or $\gamma$-glutamyltransferase level higher than the ULN for age and sex); impaired renal function (defined as serum creatinine level > ULN for age and sex); or severe anaemia. Participants with serious cardiovascular disease (e.g. NYHA class I-IV or a history of MI or stroke) or cerebrovascular conditions within 6 months before study enrolment. Women who were pregnant, breastfeeding, or of childbearing potential and not taking adequate contraceptive precautions

Diagnostic criteria: ESC and the EASD 2007

Interventions Number of study centres: 2


Derosa 2009a (Continued)

Run-in period: no

Extension period: no

\begin{tabular}{ll}
\hline Outcomes & Composite outcome measures reported: no \\
\hline Study details & Trial terminated early: no \\
& Trial ID: - \\
\hline Publication details & Language of publication: English \\
& Funding: not reported \\
& Publication status: peer-reviewed journal \\
\hline Stated aim for study & $\begin{array}{l}\text { Quote from publication: "The aim of this study was to directly compare the longterm effect of } 4 \text { an- } \\
\text { tidiabetic treatment protocols on insulin resistance evaluated by euglycemic hyperinsulinemic clamp } \\
\text { in type } 2 \text { diabetes mellitus patients. In particular, we aimed to evaluate if the combination of } 2 \text { in- } \\
\text { sulin-sensitizing agents (pioglitazone and metformin) could significantly improve the insulin resistance } \\
\text { when compared with single agent-based protocols and with a protocol including an insulin secreta- } \\
\text { gogue (glimepiride)." }\end{array}$ \\
\hline Notes & \begin{tabular}{l} 
- \\
\hline
\end{tabular}
\end{tabular}

Risk of bias

\begin{tabular}{lll}
\hline Bias $\quad$ Authors' judgement & Support for judgement \\
\hline
\end{tabular}

Random sequence genera- Low risk

tion (selection bias)

\begin{abstract}
Quote from publication: "Randomization was done using a drawing of envelopes containing randomization codes prepared by a statistician. A copy of the code was provided only to the responsible person performing the statistical analysis. The code was only broken after database lock, but could have been broken for individual subjects in cases of an emergency."
\end{abstract}

"The treatments were supplied as matching opaque white capsules in coded bottles to ensure the double-blind status of the study."

Comment: adequate generation of random sequence ensured
Allocation concealment Low risk (selection bias)
Quote from publication: "Randomization was done using a drawing of envelopes containing randomization codes prepared by a statistician. A copy of the code was provided only to the responsible person performing the statistical analysis. The code was only broken after database lock, but could have been broken for individual subjects in cases of an emergency."

"The treatments were supplied as matching opaque white capsules in coded bottles to ensure the double-blind status of the study."

Comment: adequate concealment of allocation ensured
Comment: no trial protocol was available. All of the trial's prespecified primary and secondary outcomes (methods section) have been reported. Unclear whether common outcomes (all-cause mortality) were measured; not mentioned, but clinical judgement says likely to have been measured and analysed but not reported on the basis of non-significant results. Incomplete reporting of adverse events and hypoglycaemia, only events leading to discontinuation are mentioned. Incomplete reporting of $\mathrm{HbAlc}$ due to missing information of participants included in analysis 
Derosa 2009a (Continued)

Other bias Unclear risk Comment: the primary author had performed a similar study four years earlier (Derosa 2005)

Derosa 2009b

Methods Study design: multicentre, double-blind, parallel-group RCT

Randomisation ratio: 1:1 to receive nateglinide or glibenclamide in addition to metformin

Participants

Inclusion criteria: white, $\geq 18$ years of age with T2DM according to ADA criteria (duration $\geq 6$ months), poor glycaemic control ( $\mathrm{HbAlc}$ level $>7.0 \%$ ), hypertension according to the WHO criteria (systolic/diastolic BP, $\geq 130 / \geq 85 \mathrm{mmHg}$ ), overweight (BMI $25.0-28.0 \mathrm{~kg} / \mathrm{m}^{2}$ ). None of the participants were taking hypolipidaemic drugs, diuretics, beta-blockers or thyroxin

Exclusion criteria: history of ketoacidosis or unstable or rapidly progressive diabetic retinopathy, nephropathy, or neuropathy; impaired hepatic function (defined as plasma aminotransferase and/or gamma-glutamyltransferase level $>$ ULN for age and sex), impaired renal function (defined as serum creatinine level > ULN for age and sex), or severe anaemia. People with serious cardiovascular disease (e.g. NYHA class I-IV or a history of MI or stroke) or cerebrovascular conditions within 6 months before study enrolment. Women who were pregnant or breastfeeding or of childbearing potential and not taking adequate contraceptive precautions

Diagnostic criteria: ADA 2001, WHO 1999

Interventions Number of study centres: 3

Run-in period: yes, 6-month of run-in which nateglinide and glibenclamide were titrated. Metformin was added in each arm after 1-month of run-in

Extension period: no

Outcomes Composite outcome measures reported: no

Study details Trial terminated early: no

Trial ID: -

Publication details Language of publication: English

Funding: not reported

Publication status: peer-reviewed journal

Stated aim for study Quote from publication: Derosa 2009: "The aim of our study is to directly compare the long-term metabolic effects of nateglinide and glibenclamide in naïve type 2 diabetic patients treated with metformin"; Derosa 2007: "... the aim of our study is to evaluate the differential effect on coagulation and fibrinolysis parameters and on non conventional cardiovascular risk factors of the association between metformin plus nateglinide or glibenclamide in naïve type 2 diabetes patients"

Notes

\section{Risk of bias}

\begin{tabular}{lll}
\hline Bias & Authors' judgement & Support for judgement \\
\hline $\begin{array}{ll}\text { Random sequence genera- } \\
\text { tion (selection bias) }\end{array}$ & Low risk & $\begin{array}{l}\text { Quote from publication: "Patients were randomized using envelopes contain- } \\
\text { ing randomization codes prepared by a statistician. A copy of the randomiza- } \\
\text { tion code was provided only to the person responsible for performing the sta- }\end{array}$ \\
\hline
\end{tabular}


tistical analysis. The code was only broken after database lock, but could have been broken for individual patients in cases of emergency, such as hospitalization or suspect of a serious adverse event. Nateglinide, glibenclamide and metformin were supplied as identical, opaque, white capsules in coded bottles to ensure the double-blind status of the study."

Comment: adequate generation of random sequence ensured

Allocation concealment Low risk
(selection bias)

Quote from publication: "Patients were randomized using envelopes contain(selection bias) ing randomization codes prepared by a statistician. A copy of the randomization code was provided only to the person responsible for performing the statistical analysis. The code was only broken after database lock, but could have been broken for individual patients in cases of emergency, such as hospitalization or suspect of a serious adverse event. Nateglinide, glibenclamide and metformin were supplied as identical, opaque, white capsules in coded bottles to ensure the double-blind status of the study."

Comment: adequate concealment of allocation ensured

\begin{tabular}{|c|c|c|}
\hline $\begin{array}{l}\text { Blinding of participants } \\
\text { and personnel (perfor- } \\
\text { mance bias) }\end{array}$ & Low risk & $\begin{array}{l}\text { Quote from publication: "... double-blind.. " "Nateglinide, glibenclamide and } \\
\text { metformin were supplied as identical, opaque, white capsules in coded bottles } \\
\text { to ensure the double-blind status of the study." }\end{array}$ \\
\hline
\end{tabular}

HbAlc

Comment: investigator-assessed outcome measurement. Blinding of key study personnel ensured

$\begin{array}{lll}\begin{array}{l}\text { Blinding of outcome as- } \\ \text { sessment (detection bias) }\end{array} & \text { Low risk } & \begin{array}{l}\text { Quote from publication: "... double-blind.. " "Nateglinide, glibenclamide and } \\ \text { metformin were supplied as identical, opaque, white capsules in coded bottles } \\ \text { HbAlc }\end{array} \\ \text { to ensure the double-blind status of the study." }\end{array}$

Comment: investigator-assessed outcome measurement. Blinding of investigator ensured

\begin{tabular}{|c|c|c|}
\hline $\begin{array}{l}\text { Incomplete outcome data } \\
\text { (attrition bias) } \\
\text { HbAlc }\end{array}$ & Low risk & $\begin{array}{l}\text { Quote from publication: "An intention-to-treat analysis was conducted in pa- } \\
\text { tients who had received } \geq 1 \text { dose of study medication } \\
\text { and had a subsequent efficacy observation." }\end{array}$ \\
\hline
\end{tabular}

HbAlc and had a subsequent efficacy observation."

Comment: $94 \%$ of randomised participants completed the study. $94 \%$ of the randomised participants were included in the analysis. Only participants who completed the study were included in the analysis. The number of participants who dropped out was balanced between groups. The reasons for dropouts were balanced among the intervention groups. Plausible effect size among missing outcomes was not enough to have a clinically relevant impact on observed effect size

Selective reporting (re- High risk
porting bias)

Comment: no protocol available. All of the trial's prespecified primary and secondary outcomes (methods section) have been reported. Unclear whether common outcomes (all-cause mortality, hypoglycaemia) were measured; not mentioned, but clinical judgement says likely to have been measured and analysed but not reported on the basis of non-significant results. Clear that outcomes (non-serious adverse events, serious adverse events) were measured but was not necessarily analysed; judgement says likely to have been analysed but not reported because of non-significant results 
Participants Inclusion criteria: T2DM, white, $\geq 18$ years of age with poor glycaemic control (expressed as $\mathrm{HbA1C}$ level $>8.0 \%$ ) and overweight (BMI $\geq 25$ and $<30 \mathrm{~kg} / \mathrm{m}^{2}$ ) receiving therapy with metformin at the mean dosage of $1500 \pm 500 \mathrm{mg} /$ day. They were intolerant to metformin at maximum dosage $(3000 \mathrm{mg} /$ day) with the onset of gastrointestinal disorders like diarrhoea and significant meteorism when metformin was titrated to the maximum level

Exclusion criteria: history of ketoacidosis or unstable or rapidly progressive diabetic retinopathy, nephropathy, or neuropathy, impaired hepatic function (defined as plasma aminotransferase and/or $\gamma^{-}$ glutamyltransferase level > ULN for age and sex), impaired renal function (defined as serum creatinine level > ULN for age and sex), or severe anaemia. Participants with serious cardiovascular disease (e.g. NYHA class I-IV or a history of MI or stroke) or cerebrovascular conditions within 6 months before study enrolment. Women who were pregnant or breastfeeding or of childbearing potential and not taking adequate contraceptive precautions

Diagnostic criteria: ESC and EASD 2007

Interventions

\section{Number of study centres: 8}

Run-in period: no

Extension period: no

Outcomes Composite outcome measures reported: no

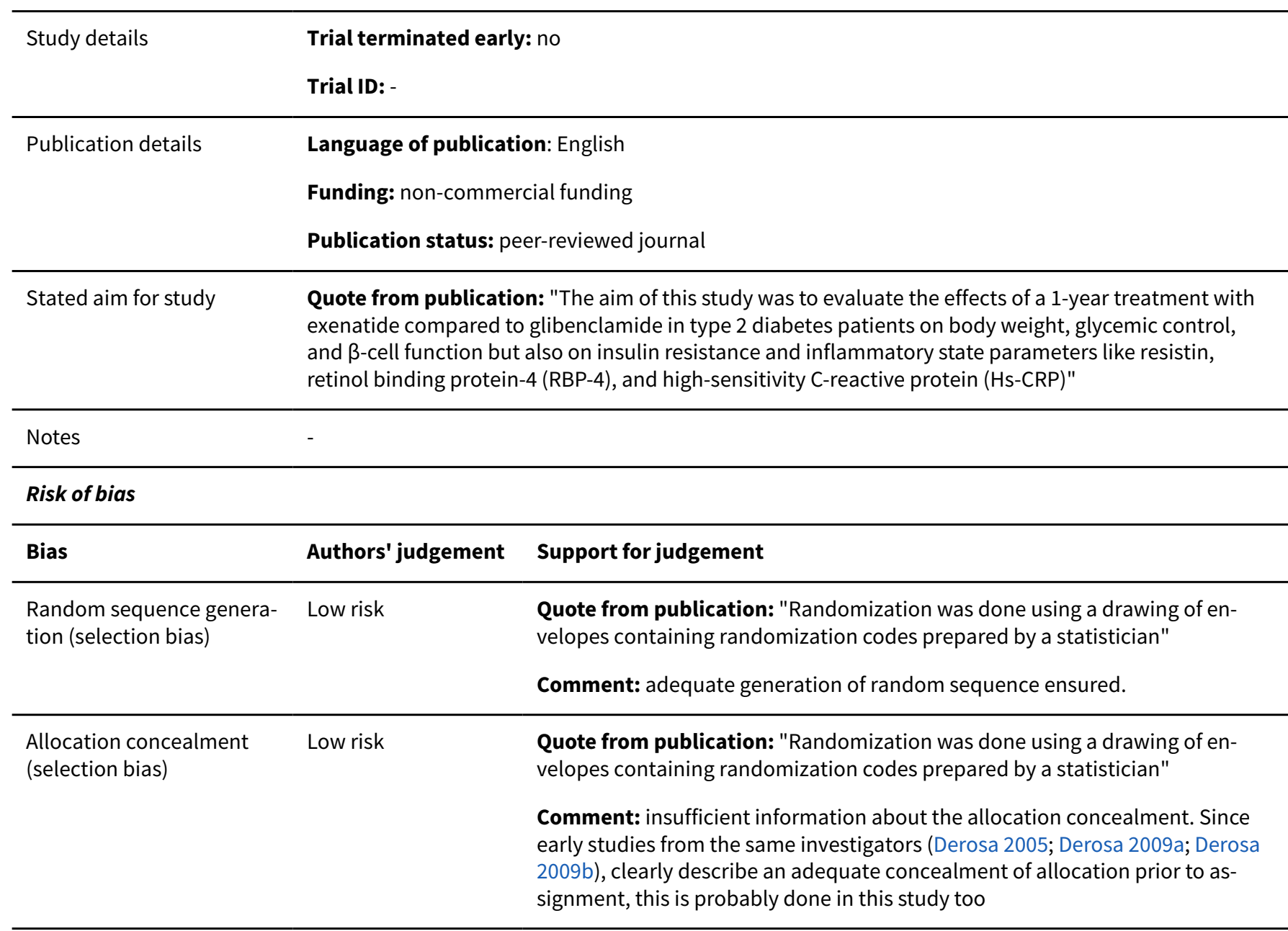


Derosa 2010 (Continued)
Blinding of participants
Low risk
Quote from publication: "single-blind" and personnel (perfor- mance bias) weight $(\mathrm{kg})$
Comment: investigator-assessed outcome measurement. Blinding of investi- gator ensured

\begin{tabular}{|c|c|c|}
\hline $\begin{array}{l}\text { Blinding of participants } \\
\text { and personnel (perfor- } \\
\text { mance bias) } \\
\text { HbAlC }\end{array}$ & Low risk & $\begin{array}{l}\text { Quote from publication: "single-blind" } \\
\text { Comment: investigator-assessed outcome measurement. Blinding of investi- } \\
\text { gator ensured }\end{array}$ \\
\hline
\end{tabular}

\begin{tabular}{ll}
\hline $\begin{array}{l}\text { Blinding of outcome as- } \\
\text { sessment (detection bias) } \\
\text { weight }(\mathrm{kg})\end{array}$ & Low risk
\end{tabular}$\quad \begin{aligned} & \text { Quote from publication: "single-blind" } \\
& \end{aligned}$

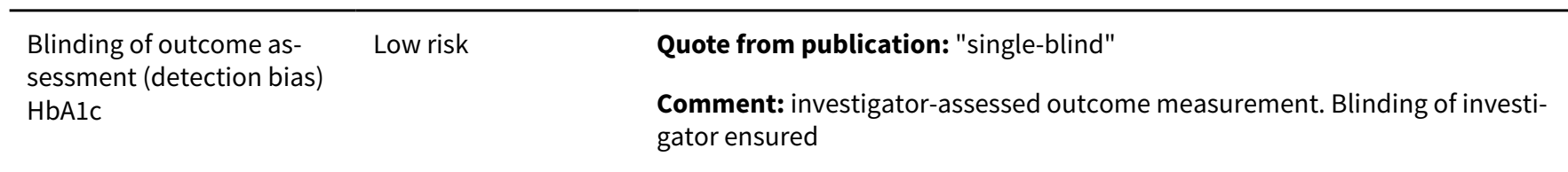

Incomplete outcome data Low risk (attrition bias)

weight $(\mathrm{kg})$
Quote from publication: "Every patient who had received at least one dose of the study medication underwent a tolerability observation to exclude the presence of acute adverse reactions. After that an intention-to-treat analysis was conducted in patients who had received one or more doses of study medication, did not show any acute adverse reaction, and had a subsequent efficacy observation"

Comment: $87 \%$ of randomised participants completed the study. $87 \%$ of the randomised participants were included in the analysis. Only participants who completed the study were included in the analysis. The number of participants who dropped out was balanced between groups. The reasons for dropouts were balanced among the intervention groups. Plausible effect size among missing outcomes was not enough to have a clinically relevant impact on observed effect size

\section{Incomplete outcome data Low risk} (attrition bias)

HbA1c
Quote from publication: "Every patient who had received at least one dose of the study medication underwent a tolerability observation to exclude the presence of acute adverse reactions. After that an intention-to-treat analysis was conducted in patients who had received one or more doses of study medication, did not show any acute adverse reaction, and had a subsequent efficacy observation"

Comment: $87 \%$ of randomised participants completed the study. $87 \%$ of the randomised participants were included in the analysis. Only participants who completed the study were included in the analysis. The number of participants who dropped out was balanced between groups. The reasons for dropouts were balanced among the intervention groups. Plausible effect size among missing outcomes was not enough to have a clinically relevant impact on observed effect size

$\begin{array}{ll}\begin{array}{l}\text { Selective reporting (re- } \\ \text { porting bias) }\end{array} & \text { High risk } \\ & \begin{array}{l}\text { Comment: no protocol available. All of the trial's prespecified primary and } \\ \text { secondary outcomes (methods section) have been reported. Unclear whether } \\ \text { common outcomes (all-cause mortality) were measured; not mentioned, but } \\ \text { clinical judgement says likely to have been measured and analysed but not re- } \\ \text { ported on the basis of non-significant results. Incomplete reporting of adverse } \\ \text { events and hypoglycaemia, only events leading to discontinuation are men- } \\ \text { tioned }\end{array}\end{array}$


Derosa 2011a

$\begin{array}{ll}\text { Methods } & \text { Study design: multicentre, randomised, single-blind, controlled study } \\ \text { Randomisation ratio: } 1: 1 \text { to receive exenatide or glimepiride in addition to metformin }\end{array}$

Participants

Inclusion criteria: white, $\mathrm{T} 2 \mathrm{DM}, \geq 18$ years of age with poor glycaemic control, expressed as HbA1c level $>8.0 \%$, and overweight $\left(\mathrm{BMI} \geq 25\right.$, and $\left.<30 \mathrm{~kg} / \mathrm{m}^{2}\right)$. They were taking metformin at various different doses (1000-2000 mg/day) and were intolerant to metformin at the highest dosages (2500-3000 mg/ day)

Exclusion criteria: history of ketoacidosis or unstable or rapidly progressive diabetic retinopathy, nephropathy, or neuropathy; impaired hepatic function (defined as plasma aminotransferase and/or gamma-glutamyltransferase level > ULN for age and sex), impaired renal function (defined as serum creatinine level > ULN for age and sex), or severe anaemia. Participants with serious cardiovascular disease (e.g. NYHA classes I-IV or a history of MI or stroke) or cerebrovascular conditions within 6 months before study enrolment. Women who were pregnant or breastfeeding or of childbearing potential and not taking adequate contraceptive precautions

Diagnostic criteria: ESC and EASD 2007

\begin{tabular}{ll}
\hline Interventions & $\begin{array}{l}\text { Number of study centres: } 7 \\
\text { Run-in period: not reported } \\
\text { Extension period: no }\end{array}$ \\
\hline Outcomes & Composite outcome measures reported: \\
\hline Study details & Trial terminated early: no \\
& Trial ID: - \\
\hline Publication details & Language of publication: English \\
& Funding: non-commercial funding \\
& Publication status: peer-reviewed journal
\end{tabular}

Stated aim for study

Quote from publication: "The aim of this study was to evaluate the effect of exenatide compared to glimepiride on body weight, glycemic control and insulin resistance in type 2 diabetic patients taking metformin"

\begin{tabular}{|c|c|c|}
\hline Notes & - & \\
\hline \multicolumn{3}{|l|}{ Risk of bias } \\
\hline Bias & Authors' judgement & Support for judgement \\
\hline \multirow[t]{2}{*}{$\begin{array}{l}\text { Random sequence genera- } \\
\text { tion (selection bias) }\end{array}$} & Low risk & $\begin{array}{l}\text { Quote from publication: "Randomization was done using a drawing of en- } \\
\text { velopes containing randomization codes prepared by a statistician" }\end{array}$ \\
\hline & & Comment: adequate generation of random sequence ensured \\
\hline \multirow[t]{2}{*}{$\begin{array}{l}\text { Allocation concealment } \\
\text { (selection bias) }\end{array}$} & Low risk & $\begin{array}{l}\text { Quote from publication: "Randomization was done using a drawing of en- } \\
\text { velopes containing randomization codes prepared by a statistician" }\end{array}$ \\
\hline & & $\begin{array}{l}\text { Comment: insufficient information about the allocation concealment. Since } \\
\text { an early study from the same investigators (Derosa 2005; Derosa 2009a; Derosa } \\
2009 \text { b) clearly describes an adequate concealment of allocation prior to as- } \\
\text { signment, this is probably done in this study too }\end{array}$ \\
\hline
\end{tabular}


Derosa 2011a (Continued)
Blinding of participants
Low risk
Quote from publication: "single-blind" and personnel (perfor- mance bias) weight $(\mathrm{kg})$
Comment: investigator-assessed outcome measurement. Blinding of investi- gator ensured

\begin{tabular}{|c|c|c|}
\hline $\begin{array}{l}\text { Blinding of participants } \\
\text { and personnel (perfor- } \\
\text { mance bias) } \\
\text { HbA1C }\end{array}$ & Low risk & $\begin{array}{l}\text { Quote from publication: "single-blind" } \\
\text { Comment: investigator-assessed outcome measurement. Blinding of investi- } \\
\text { gator ensured }\end{array}$ \\
\hline
\end{tabular}

\begin{tabular}{|c|c|c|}
\hline $\begin{array}{l}\text { Blinding of outcome as- } \\
\text { sessment (detection bias) } \\
\text { weight (kg) }\end{array}$ & Low risk & $\begin{array}{l}\text { Quote from publication: "single-blind" } \\
\text { Comment: investigator-assessed outcome measurement. Blinding of investi- } \\
\text { gator ensured }\end{array}$ \\
\hline
\end{tabular}

\begin{tabular}{|c|c|c|}
\hline $\begin{array}{l}\text { Blinding of outcome as- } \\
\text { sessment (detection bias) }\end{array}$ & Low risk & Quote from publication: "single-blind" \\
\hline HbAlc & & $\begin{array}{l}\text { Comment: investigator-assessed outcome measurement. Blinding of investi- } \\
\text { gator ensured }\end{array}$ \\
\hline
\end{tabular}

Incomplete outcome data Low risk (attrition bias)

weight $(\mathrm{kg})$
Quote from publication: "Every patient who had received at least one dose of the study medication underwent a tolerability observation to exclude the pres- ence of acute adverse reactions ... After that an intention-to-treat analysis was conducted in patients who had received one or more doses of study medica- tion, did not show any acute adverse reaction, and had a subsequent efficacy observation."
Comment: $91 \%$ of randomised participants completed the study. $91 \%$ of ran- domised participants were included in the analysis. Only participants who completed the study were included in the analysis. The number of participants who dropped out was balanced between groups. The reasons for dropouts were balanced among the intervention groups. Plausible effect size among missing outcomes was not enough to have a clinically relevant impact on ob- served effect size

Incomplete outcome data Low risk
(attrition bias)

$\mathrm{HbA} 1 \mathrm{c}$

Quote from publication: "Every patient who had received at least one dose of the study medication underwent a tolerability observation to exclude the presence of acute adverse reactions ... After that an intention-to-treat analysis was conducted in patients who had received one or more doses of study medication, did not show any acute adverse reaction, and had a subsequent efficacy observation."

Comment: $91 \%$ of randomised participants completed the study. $91 \%$ of randomised participants were included in the analysis. Only participants who completed the study were included in the analysis. The number of participants who dropped out was balanced between groups. The reasons for dropouts were balanced among the intervention groups. Plausible effect size among missing outcomes was not enough to have a clinically relevant impact on observed effect size

\begin{tabular}{|c|c|c|}
\hline $\begin{array}{l}\text { Selective reporting (re- } \\
\text { porting bias) }\end{array}$ & High risk & $\begin{array}{l}\text { Comment: no trial protocol available. All of the trial's prespecified prima- } \\
\text { ry and secondary outcomes (methods section) have been reported. Unclear } \\
\text { whether common outcomes (all-cause mortality) were measured; not men- } \\
\text { tioned, but clinical judgement says likely to have been measured and analysed } \\
\text { but not reported on the basis of non-significant results. Incomplete reporting } \\
\text { of adverse events and hypoglycaemia, only events leading to discontinuation } \\
\text { are mentioned }\end{array}$ \\
\hline
\end{tabular}

\begin{tabular}{ll}
\hline Other bias $\quad$ Unclear risk & $\begin{array}{l}\text { Comment: one year earlier the primary author had performed a similar study } \\
\text { (Derosa 2010) }\end{array}$ \\
\hline \hline
\end{tabular}


Derosa 2011b

Methods

Study design: multicentre, randomised, double-blind, controlled study

Randomisation ratio: 1:1 to receive pioglitazone or glibenclamide in addition to metformin

Participants

Inclusion criteria: white, $\geq 18$ years of age with uncontrolled T2DM ( $\mathrm{HbAlc}>62 \mathrm{mmol} / \mathrm{mol}$ or $7.0 \%)$ with diet, physical activity, and metformin (mean dosage: $1700 \pm 850 \mathrm{mg} /$ day)

Exclusion criteria: history of ketoacidosis or unstable or rapidly progressive diabetic retinopathy, nephropathy (defined by onset of albumin excretion $>300 \mathrm{mg} / 24 \mathrm{~h}$ or albumin excretion rate $>200 \mu \mathrm{g}$ / min over a 6-month period), or neuropathy; impaired hepatic function (defined as plasma aminotransferase and/or gamma-glutamyltransferase level > ULN for age and sex), impaired renal function (defined as serum creatinine level > ULN for age and sex), or severe anaemia (defined as haemoglobin lev$\mathrm{el}<8 \mathrm{~g} / \mathrm{dL}$ ), serious cardiovascular disease or cardiac failure or history of cardiac failure (NYHA Class IIV) or a history of MI or stroke or cerebrovascular conditions (stroke or transient ischaemic attack) within 6 months before study enrolment. Post-menopausal women with a history of osteoporosis for the increased risk of distal upper limb (forearm, hand, and wrist) or distal lower limb (foot, ankle, fibula and tibia) fractures reported with pioglitazone. Women who were pregnant or breastfeeding or of childbearing potential and not taking adequate contraceptive precautions

Diagnostic criteria: ESC and EASD 2007

\begin{tabular}{|c|c|c|}
\hline \multirow[t]{3}{*}{ Interventions } & \multicolumn{2}{|c|}{ Number of study centres: 2} \\
\hline & \multicolumn{2}{|l|}{ Run-in period: no } \\
\hline & \multicolumn{2}{|l|}{ Extension period: no } \\
\hline Outcomes & \multicolumn{2}{|c|}{ Composite outcome measures reported: no } \\
\hline \multirow[t]{2}{*}{ Study details } & \multicolumn{2}{|c|}{ Trial terminated early: no } \\
\hline & \multicolumn{2}{|l|}{ Trial ID: - } \\
\hline \multirow[t]{3}{*}{ Publication details } & \multirow{3}{*}{\multicolumn{2}{|c|}{$\begin{array}{l}\text { Language of publication: English } \\
\text { Funding: not reported } \\
\text { Publication status: peer-reviewed journal }\end{array}$}} \\
\hline & & \\
\hline & & \\
\hline Stated aim for study & \multicolumn{2}{|c|}{$\begin{array}{l}\text { Quote from publication: "The aim of the study was to evaluate the effect of pioglitazone and gliben- } \\
\text { clamide on lipid profile and inflammatory parameters during an oral fat load (OFL)." }\end{array}$} \\
\hline Notes & \multicolumn{2}{|l|}{-} \\
\hline \multicolumn{3}{|l|}{ Risk of bias } \\
\hline Bias & Authors' judgement & Support for judgement \\
\hline $\begin{array}{l}\text { Random sequence genera- } \\
\text { tion (selection bias) }\end{array}$ & Low risk & $\begin{array}{l}\text { Quote from publication: "Pioglitazone and glibenclamide were supplied as } \\
\text { identical, opaque, white capsules in coded bottles to ensure the blind status of } \\
\text { the study. Randomization was done using a drawing of envelopes containing } \\
\text { randomization codes prepared by a statistician. A copy of the code was provid- } \\
\text { ed only to the responsible person performing the statistical analysis. The code } \\
\text { was only broken after database lock, but could have been broken for individ- } \\
\text { ual subjects in cases of an emergency." }\end{array}$ \\
\hline & & Comment: adequate generation of random sequence ensured \\
\hline
\end{tabular}


Derosa 2011b (Continued)

Allocation concealment (selection bias)

Low risk

Quote from publication: "Pioglitazone and glibenclamide were supplied as identical, opaque, white capsules in coded bottles to ensure the blind status of the study. Randomization was done using a drawing of envelopes containing randomization codes prepared by a statistician. A copy of the code was provided only to the responsible person performing the statistical analysis. The code was only broken after database lock, but could have been broken for individual subjects in cases of an emergency."

Comment: adequate concealment of allocation prior to assignment ensured

\begin{tabular}{ll}
\hline $\begin{array}{l}\text { Blinding of participants } \\
\text { and personnel (perfor- }\end{array}$ & Low risk \\
$\begin{array}{l}\text { mance bias) } \\
\text { weight }(\mathrm{kg})\end{array}$ & $\begin{array}{l}\text { Comment: investigator-assessed outcome measurement. Blinding of investi- } \\
\text { gator ensured }\end{array}$ \\
\hline
\end{tabular}

\begin{tabular}{|c|c|c|}
\hline $\begin{array}{l}\text { Blinding of participants } \\
\text { and personnel (perfor- } \\
\text { mance bias) } \\
\text { HbAlc }\end{array}$ & Low risk & $\begin{array}{l}\text { Quote from publication: "double-blind" } \\
\text { Comment: investigator-assessed outcome measurement. Blinding of investi- } \\
\text { gator ensured }\end{array}$ \\
\hline
\end{tabular}

\begin{tabular}{|c|c|c|}
\hline $\begin{array}{l}\text { Blinding of outcome as- } \\
\text { sessment (detection bias) } \\
\text { weight }(\mathrm{kg})\end{array}$ & Low risk & $\begin{array}{l}\text { Quote from publication: "double-blind" } \\
\text { Comment: investigator-assessed outcome measurement. Blinding of investi- } \\
\text { gator ensured }\end{array}$ \\
\hline
\end{tabular}

\begin{tabular}{|c|c|c|}
\hline $\begin{array}{l}\text { Blinding of outcome as- } \\
\text { sessment (detection bias) } \\
\text { HbA1c }\end{array}$ & Low risk & $\begin{array}{l}\text { Quote from publication: "double-blind" } \\
\text { Comment: investigator-assessed outcome measurement. Blinding of investi- } \\
\text { gator ensured }\end{array}$ \\
\hline
\end{tabular}

Incomplete outcome data Low risk (attrition bias) weight $(\mathrm{kg})$
Quote from publication: "An intention to treat analysis was conducted in patients who had received $\geq 1$ dose of study medication and had a subsequent efficacy observation"

Comment: $97 \%$ of randomised participants completed the study. $97 \%$ of the randomised participants were included in the analysis. Only participants who completed the study were included in the analysis. The number of participants who dropped out was balanced between groups. The reasons for dropouts were balanced among the intervention groups. Plausible effect size among missing outcomes was not enough to have a clinically relevant impact on observed effect size

Incomplete outcome data Low risk (attrition bias)

HbAlc
Quote from publication: "An intention to treat analysis was conducted in patients who had received $\geq 1$ dose of study medication and had a subsequent efficacy observation"

Comment: $97 \%$ of randomised participants completed the study. $97 \%$ of the randomised participants were included in the analysis. Only participants who completed the study were included in the analysis. The number of participants who dropped out was balanced between groups. The reasons for dropouts were balanced among the intervention groups. Plausible effect size among missing outcomes was not enough to have a clinically relevant impact on observed effect size

Comment: no protocol available. All of the trial's prespecified primary and secondary outcomes (methods section) have been reported. Unclear whether common outcomes (all-cause mortality) were measured; not mentioned, but clinical judgement says likely to have been measured and analysed but not reported on the basis of non-significant results. Incomplete reporting of adverse 


\begin{tabular}{ll}
\hline Other bias $\quad$ Unclear risk & $\begin{array}{l}\text { Comment: one year earlier the primary author had performed a similar study } \\
\text { (Derosa 2005) }\end{array}$ \\
\hline
\end{tabular}

Filozof 2010

Study design: multicentre, randomised, double-blind, active-controlled study
Randomisation ratio: $1: 1$ to receive vildagliptin or gliclazide in addition to metformin
Non-inferiority design: 1-sided confidence interval
Inclusion criteria: non-fertile or using a medically approved birth control method, 18-78 years with
T2DM and HbA1c 7.5\%-11\%, who had received metformin for at least 3 months and were on a stable
dose of $\geq 1500$ mg daily for $\geq 4$ weeks prior to visit 1
Exclusion criteria: history of T1DM, diabetes as a result of pancreatic injury or secondary forms of di-
abetes (Cushing's syndrome and acromegaly) and participants experiencing acute metabolic diabet-
ic complications (ketoacidosis or hyperosmolar state) within the past 6 months. Participants with seri-
ous cardiac conditions (torsades de pointes, sustained and clinically relevant ventricular tachycardia
or ventricular fibrillation, percutaneous coronary intervention within the past 3 months, MI, coronary
artery bypass surgery, unstable angina; or stroke within the last 6 months and congestive heart fail-
ure requiring pharmacological treatment, second- or third-degree atrioventricular block or prolonged
QTC) or clinically significant renal or liver disease ). ALT or AST $>2 \times$ ULN range, total bilirubin $>2 \times$ ULN
range, positive hepatitis B surface antigen and/or hepatitis C antibody, serum creatinine $\geq 132 \mu$ mol/L
in male participants and $\geq 123 \mu$ mol/L in female participants, or a history of abnormal creatinine clear-
ance, clinically significant TSH values outside of normal range at screening, or fasting triglycerides $>7.9$
mmol/L at screening

Diagnostic criteria: not reported

Interventions Number of study centres: 220

Run-in period: 4 weeks of run-in with metformin $\geq 1500 \mathrm{mg}$ daily at stable dose

Extension period: no

\begin{tabular}{ll}
\hline Outcomes & Composite outcome measures reported: yes, cardiovascular and cerebrovascular outcomes \\
\hline Study details & Trial terminated early: no \\
& Trial ID: NCT00102466 \\
\hline
\end{tabular}

Publication details Language of publication: English

Funding: commercial funding (vildagliptin), Novartis Pharma AG

Publication status: peer-reviewed journal

$\begin{array}{ll}\text { Stated aim for study } & \text { Quote from publication: "To demonstrate non-inferiority of vildagliptin compared with gliclazide, as } \\ \text { an add-on therapy, in patients with Type } 2 \text { diabetes inadequately controlled with metformin in a 52- } \\ \text { week, randomized, double-blind, active-controlled study." }\end{array}$

Notes

\section{Risk of bias}


Filozof 2010 (Continued)
Bias
Authors' judgement Support for judgement

Random sequence genera- Unclear risk tion (selection bias)

Quote from publication: "Eligible patients were randomized..."

Comment: insufficient information about the sequence generation process to permit judgement of 'low risk or 'high risk'

\begin{tabular}{|c|c|c|}
\hline $\begin{array}{l}\text { Allocation concealment } \\
\text { (selection bias) }\end{array}$ & Unclear risk & $\begin{array}{l}\text { Quote from publication: "Eligible patients were randomized..." } \\
\text { Comment: insufficient information about the allocation concealment to per- } \\
\text { mit judgement of 'low risk' or 'high risk' }\end{array}$ \\
\hline
\end{tabular}

Blinding of participants Low risk and personnel (performance bias)

all-cause/cardiovascular mortality
Quote from publication: "... double blind..." "All the randomized patients were blinded using a double-dummy design." "The number and percentage of AEs confirmed by the Cardiovascular and Cerebrovascular Safety Committee and the Internal Medicine Committee were summarized by treatment. Both of these committees independently and blindly reviewed, assessed and categorized cardiovascular and cerebrovascular events and prespecified clinical events (as defined by the panel) that might have been observed during the study."

Comment: adjudicated/investigator-assessed outcome measurement. Blinding of participants and study personnel ensured

Blinding of participants Low risk and personnel (performance bias) serious adverse events
Quote from publication: "... double blind..." "All the randomized patients were blinded using a double-dummy design." "The number and percentage of AEs confirmed by the Cardiovascular and Cerebrovascular Safety Committee and the Internal Medicine Committee were summarized by treatment. Both of these committees independently and blindly reviewed, assessed and categorized cardiovascular and cerebrovascular events and prespecified clinical events (as defined by the panel) that might have been observed during the study."

Comment: adjudicated/investigator-assessed outcome measurement. Blinding of participants and study personnel ensured

\begin{tabular}{|c|c|c|}
\hline $\begin{array}{l}\text { Blinding of participants } \\
\text { and personnel (perfor- } \\
\text { mance bias) } \\
\text { weight (kg) }\end{array}$ & Unclear risk & $\begin{array}{l}\text { Quote from publication: "... double blind..." "All the randomized patients we } \\
\text { blinded using a double-dummy design." } \\
\text { Comment: investigator-assessed outcome measurement. Blinding of study } \\
\text { personnel ensured }\end{array}$ \\
\hline
\end{tabular}

Blinding of participants Low risk
and personnel (performance bias)

HbA1c
Quote from publication: "... double blind..." "All the randomized patients were blinded using a double-dummy design."

Comment: investigator-assessed outcome measurement. Blinding of study personnel ensured
Blinding of outcome as- Low risk sessment (detection bias) all-cause/cardiovascular mortality

Quote from publication: "... double blind..." "The number and percentage of AEs confirmed by the Cardiovascular and Cerebrovascular Safety Committee and the Internal Medicine Committee were summarized by treatment. Both of these committees independently and blindly reviewed, assessed and categorized cardiovascular and cerebrovascular events and prespecified clinical events (as defined by the panel) that might have been observed during the study."

Comment: adjudicated/investigator-assessed outcome measurement. Blinding of outcome assessor ensured

Blinding of outcome as- Low risk sessment (detection bias)
Quote from publication: "... double blind..." "The number and percentage of AEs confirmed by the Cardiovascular and Cerebrovascular Safety Committee 
Filozof 2010 (Continued) serious adverse events and the Internal Medicine Committee were summarized by treatment. Both of these committees independently and blindly reviewed, assessed and categorized cardiovascular and cerebrovascular events and prespecified clinical events (as defined by the panel) that might have been observed during the study."

Comment: adjudicated/investigator-assessed outcome measurement. Blinding of outcome assessor ensured
Blinding of outcome as- Unclear risk $\quad$ Quote from publication: "... double blind..." sessment (detection bias) weight $(\mathrm{kg})$
Blinding of outcome as- Low risk $\quad$ Quote from publication: "... double blind..." sessment (detection bias) HbAlc
Incomplete outcome data Low risk (attrition bias) all-cause/cardiovascular mortality

Comment: investigator-assessed outcome measurement. Blinding of outcome assessor ensured

Comment: investigator-assessed outcome measurement. Blinding of outcome assessor ensured

Quote from publication: "Safety (SAF) population: patients who received at least one dose of study drug and had at least one post-baseline safety assessment." "Safety summaries were tabulated using data from the SAF population." "For analysis, the last available post-baseline assessment (last observation carried forward; LOCF) was used."

Comment: $>99 \%$ of the randomised participants were included in the analyses. There was a high dropout rate (79\%-83\% of randomised participants completed the study), however, the dropout rate was balanced between groups. Inappropriate method of imputing missing data was used (LOCF). We assumed that trial authors searched registers for mortality status at the end of the trial

Incomplete outcome data High risk (attrition bias)

serious adverse events

Quote from publication: "Safety (SAF) population: patients who received at least one dose of study drug and had at least one post-baseline safety assessment." "Safety summaries were tabulated using data from the SAF population." "For analysis, the last available post-baseline assessment (last observation carried forward; LOCF) was used."

Comment: $>99 \%$ of the randomised participants were included in the analyses. There was a high dropout rate $(79 \%-83 \%$ of randomised participants completed the study), however, the dropout rate was balanced between groups. The reasons for dropouts were balanced among the intervention groups. Inappropriate method of imputing missing data was used (LOCF). The proportion of missing outcomes compared with observed event risk was enough to induce clinically relevant bias in intervention effect estimate

Incomplete outcome data High risk (attrition bias) weight $(\mathrm{kg})$
Quote from publication: "Per protocol (PP) population: included patients in the ITT population with more than 24 weeks of treatment, with no major protocol violations, and who underwent the final valid assessment of the primary efficacy variable $\mathrm{HbA1 \textrm {c }}$ within 7 days after the last dose of study drug and either (i) completed more than 48 weeks of treatment or (ii) had $<48$ weeks of treatment but discontinued from study drug because of unsatisfactory therapeutic response." "The analysis of the primary and secondary efficacy variables were based on the PP population." "For analysis, the last available postbaseline assessment (last observation carried forward; LOCF) was used."

Comment: $75 \%-80 \%$ of the randomised participants were included in the analyses. There was a high dropout rate ( $79 \%-83 \%$ of randomised participants completed the study), however, the dropout rate was balanced between groups. The reasons for dropouts were balanced among the intervention groups. Inappropriate method of imputing missing data was used (LOCF). Analyses based on per protocol population 
Filozof 2010 (Continued)

Incomplete outcome data High risk (attrition bias)

HbA1c
Quote from publication: "Per protocol (PP) population: included patients in the ITT population with more than 24 weeks of treatment, with no major protocol violations, and who underwent the final valid assessment of the primary efficacy variable $\mathrm{HbAlc}$ within 7 days after the last dose of study drug and either (i) completed more than 48 weeks of treatment or (ii) had $<48$ weeks of treatment but discontinued from study drug because of unsatisfactory therapeutic response." "The analysis of the primary and secondary efficacy variables were based on the PP population." "For analysis, the last available postbaseline assessment (last observation carried forward; LOCF) was used."

Comment: $75 \%-80 \%$ of the randomised participants were included in the analyses. There was a high dropout rate $(79 \%-83 \%$ of randomised participants completed the study), however, the dropout rate was balanced between groups. The reasons for dropouts were balanced among the intervention groups. Inappropriate method of imputing missing data was used (LOCF). Analyses based on per protocol population

Selective reporting (re- High risk
porting bias)

Comment: discrepancy between protocol (ClinicalTrials.gov) and full article. Of outcomes of interest in the review, the full article has change in body weight as a secondary outcome. Change in body weight is not mentioned in the protocol

Other bias Unclear risk

Comment: the trial received funding from a pharmaceutical company and some authors have received payment from a pharmaceutical company

Study design: open-label, randomised, controlled study
Randomisation ratio: $1: 1$ to receive exenatide or glimepiride in addition to metformin
Non-inferiority design and superiority design: non-inferiority of exenatide to glimepiride if the $97.5 \%$
Cl for the hazard ratio, with a Cox proportional hazards model with baseline $\mathrm{HbAlc}$ as covariate, ex-
cluded 1.25, thus rejecting the hypothesis that risk of treatment failure with exenatide was $>25 \%$
greater than that with glimepiride. If non-inferiority was shown, we tested superiority with $95 \% \mathrm{Cl}$ (ex-
cluding 1 )

Participants

Inclusion criteria: aged 18-85 years of age, T2DM as defined by WHO criteria, have been on stable metformin MTD (either immediate or extended-release), with suboptimal glycaemic control evident from $\mathrm{HbAl} \geq 6.5 \%$ and $\leq 9.0 \%$. Body weight had to be stable (not $>10 \%$ variation) for the previous 3 months and BMI had to be $\geq 25 \mathrm{~kg} / \mathrm{m}^{2}$ and $<40 \mathrm{~kg} / \mathrm{m}^{2}$

Exclusion criteria: previous or current malignancy, active, symptomatic proliferative retinopathy or macular oedema, liver or gastrointestinal disease, renal failure, previously been treated with TZDs, insulin, alpha-glucosidase inhibitors, SUs or meglitinides

Diagnostic criteria: WHO criteria

Interventions
Number of study centres: 128
Run-in period: no
Extension period: yes, participants who experience treatment failure and, thus, reach the primary end point will be entered into an extension period of the study. Participants who were initially randomised to exenatide will be re-randomised to further add-on treatment (third-line) with either glimepiride or pioglitazone, and participants initially randomised to glimepiride will receive add-on treatment with exenatide 
Gallwitz 2012a (Continued)

Outcomes Composite outcome measures reported: no

$$
\text { Study details }
$$

Trial terminated early: no

Trial ID: NCT00359762

\begin{tabular}{ll}
\hline Stated aim for study & $\begin{array}{l}\text { Quote from publication: "We aimed to assess durability of glycaemic control achieved with GLP-1 re- } \\
\text { ceptor agonist exenatide twice a day and sulphonylurea glimepiride in patients with type } 2 \text { diabetes in- } \\
\text { adequately controlled by metformin alone." }\end{array}$ \\
\hline Notes & -
\end{tabular}

\section{Risk of bias}

Bias Authors' judgement Support for judgement

Random sequence genera- Low risk tion (selection bias)

\author{
Language of publication: English
}

Funding: commercial funding by Eli Lilly and Co; Amylin Pharmaceuticals

Publication status: peer-reviewed journal ceptor agonist exenatide twice a day and sulphonylurea glimepiride in patients with type 2 diabetes inadequately controlled by metformin alone."

Quote from publication: "We used a computer-generated randomisation sequence to randomly assign patients... Before database lock the study team were masked to group assignment and statistical analyses were planned with no knowledge of groups."

Comment: adequate generation of random sequence ensured
Allocation concealment Low risk (selection bias)
Quote from publication: "We used a computer-generated randomisation sequence to randomly assign patients... Before database lock the study team were masked to group assignment and statistical analyses were planned with no knowledge of groups."

Comment: adequate concealment ensured

\begin{tabular}{|c|c|c|}
\hline $\begin{array}{l}\text { Blinding of participants } \\
\text { and personnel (perfor- } \\
\text { mance bias) } \\
\text { all-cause/cardiovascular }\end{array}$ & Low risk & $\begin{array}{l}\text { Quote from publication: "... open-label..." } \\
\text { Comment: investigator-assessed outcome measurement. Incomplete blind- } \\
\text { ing, the outcome is unlikely to be influenced by lack of blinding }\end{array}$ \\
\hline
\end{tabular}
mortality

\begin{tabular}{|c|c|c|}
\hline $\begin{array}{l}\text { Blinding of participants } \\
\text { and personnel (perfor- } \\
\text { mance bias) } \\
\text { hypoglycaemia }\end{array}$ & High risk & $\begin{array}{l}\text { Quote from publication: "... open-label..." } \\
\text { Comment: self-reported outcome measurement. Incomplete blinding, the } \\
\text { outcome is likely to be influenced by lack of blinding }\end{array}$ \\
\hline
\end{tabular}

\begin{tabular}{|c|c|c|}
\hline $\begin{array}{l}\text { Blinding of participants } \\
\text { and personnel (perfor- } \\
\text { mance bias) } \\
\text { non-fatal myocardial in- } \\
\text { farction/heart failure/non- } \\
\text { fatal stroke }\end{array}$ & Low risk & $\begin{array}{l}\text { Quote from publication: "... open-label..." } \\
\text { Comment: investigator-assessed outcome measurement. Incomplete blind- } \\
\text { ing, the outcome is unlikely to be influenced by lack of blinding }\end{array}$ \\
\hline
\end{tabular}

\begin{tabular}{|c|c|c|}
\hline $\begin{array}{l}\text { Blinding of participants } \\
\text { and personnel (perfor- } \\
\text { mance bias) } \\
\text { non-serious adverse } \\
\text { events }\end{array}$ & High risk & $\begin{array}{l}\text { Quote from publication: "... open-label..." } \\
\text { Comment: self-reported outcome measurement. Incomplete blinding, the } \\
\text { outcome is likely to be influenced by lack of blinding }\end{array}$ \\
\hline
\end{tabular}


Gallwitz 2012a (Continued)
Blinding of participants
Low risk
Quote from publication: "... open-label..." and personnel (perfor- mance bias)
Comment: investigator-assessed outcome measurement. Incomplete blind- serious adverse events ing, the outcome is unlikely to be influenced by lack of blinding

Blinding of participants High risk
and personnel (perfor-
mance bias)
weight $(\mathrm{kg})$

Quote from publication: "... open-label..."

Comment: investigator-assessed outcome measurement. Incomplete blinding, the outcome is likely to be influenced by lack of blinding

\begin{tabular}{ll}
\hline $\begin{array}{l}\text { Blinding of participants } \\
\text { and personnel (perfor- }\end{array}$ & Quw risk \\
$\begin{array}{l}\text { mance bias) } \\
\text { HbAlc }\end{array}$ & Comment: investigator-assessed outcome measurement. Incomplete blind- \\
\end{tabular}

\begin{tabular}{|c|c|c|}
\hline $\begin{array}{l}\text { Blinding of outcome as- } \\
\text { sessment (detection bias) } \\
\text { all-cause/cardiovascular } \\
\text { mortality }\end{array}$ & Low risk & $\begin{array}{l}\text { Quote from publication: "... open-label..." } \\
\text { Comment: investigator-assessed outcome measurement. Incomplete blind- } \\
\text { ing, the outcome is unlikely to be influenced by lack of blinding }\end{array}$ \\
\hline
\end{tabular}

\begin{tabular}{|c|c|c|}
\hline $\begin{array}{l}\text { Blinding of outcome as- } \\
\text { sessment (detection bias) } \\
\text { hypoglycaemia }\end{array}$ & High risk & $\begin{array}{l}\text { Quote from publication: "... open-label..." } \\
\text { Comment: self-reported outcome measurement. Incomplete blinding, the } \\
\text { outcome is likely to be influenced by lack of blinding }\end{array}$ \\
\hline
\end{tabular}

\begin{tabular}{|c|c|c|}
\hline $\begin{array}{l}\text { Blinding of outcome as- } \\
\text { sessment (detection bias) } \\
\text { non-fatal myocardial in- } \\
\text { farction/heart failure/non- } \\
\text { fatal stroke }\end{array}$ & Low risk & $\begin{array}{l}\text { Quote from publication: "... open-label..." } \\
\text { Comment: investigator-assessed outcome measurement. Incomplete blind- } \\
\text { ing, the outcome is unlikely to be influenced by lack of blinding }\end{array}$ \\
\hline
\end{tabular}

\begin{tabular}{|c|c|c|}
\hline $\begin{array}{l}\text { Blinding of outcome as- } \\
\text { sessment (detection bias) } \\
\text { non-serious adverse } \\
\text { events }\end{array}$ & High risk & $\begin{array}{l}\text { Quote from publication: "... open-label..." } \\
\text { Comment: self-reported outcome measurement. Incomplete blinding, the } \\
\text { outcome is likely to be influenced by lack of blinding }\end{array}$ \\
\hline
\end{tabular}

\begin{tabular}{|c|c|c|}
\hline $\begin{array}{l}\text { Blinding of outcome as- } \\
\text { sessment (detection bias) } \\
\text { serious adverse events }\end{array}$ & Low risk & $\begin{array}{l}\text { Quote from publication: "... open-label..." } \\
\text { Comment: investigator-assessed outcome measurement. Incomplete blind- } \\
\text { ing, the outcome is unlikely to be influenced by lack of blinding }\end{array}$ \\
\hline
\end{tabular}

\begin{tabular}{|c|c|c|}
\hline $\begin{array}{l}\text { Blinding of outcome as- } \\
\text { sessment (detection bias) } \\
\text { weight (kg) }\end{array}$ & High risk & $\begin{array}{l}\text { Quote from publication: "... open-label..." } \\
\text { Comment: investigator-assessed outcome measurement. Incomplete blind- } \\
\text { ing, the outcome is likely to be influenced by lack of blinding }\end{array}$ \\
\hline $\begin{array}{l}\text { Blinding of outcome as- } \\
\text { sessment (detection bias) } \\
\text { HbAlc }\end{array}$ & Low risk & $\begin{array}{l}\text { Quote from publication: "... open-label..." } \\
\text { Comment: investigator-assessed outcome measurement. Incomplete blind- } \\
\text { ing, the outcome is unlikely to be influenced by lack of blinding }\end{array}$ \\
\hline $\begin{array}{l}\text { Incomplete outcome data } \\
\text { (attrition bias) } \\
\text { all-cause/cardiovascular } \\
\text { mortality }\end{array}$ & Low risk & $\begin{array}{l}\text { Quote from publication: "ITT Safety Population: Enrolled patients receiving } \\
\text { at least one dose of study medication..." } \\
\text { Comment: > } 99 \% \text { of randomised participants were included in the analyses. } \\
\text { There was a high dropout rate ( } 66 \%-75 \% \text { of randomised participants complet- } \\
\text { ed the study), however, the dropout rate was balanced between groups. We } \\
\text { assumed that trial authors searched registers for mortality status at the end of } \\
\text { the trial }\end{array}$ \\
\hline
\end{tabular}


Gallwitz 2012a (Continued)

Incomplete outcome data Low risk (attrition bias)

hypoglycaemia
Quote from publication: "ITT Safety Population: Enrolled patients receiving at least one dose of study medication..."

Comment: $>99 \%$ of the randomised participants were included in the analyses. There was a high dropout rate $66 \%-75 \%$ of randomised participants completed the study), however, the dropout rate was balanced between groups. The reasons for dropouts were not balanced among the intervention groups (in the exenatide group more discontinued the trial due to adverse events). No information on imputation method. The proportion of missing outcomes compared with observed event risk was not enough to induce clinically relevant bias in intervention effect estimate

\section{Incomplete outcome data High risk} (attrition bias) non-fatal myocardial infarction/heart failure/nonfatal stroke
Quote from publication: "ITT Safety Population: Enrolled patients receiving at least one dose of study medication..."

Comment: $>99 \%$ of the randomised participants were included in the analyses. There was a high dropout rate (66\%-75\% of randomised participants completed the study), however, the dropout rate was balanced between groups. The reasons for dropouts were not balanced among the intervention groups (in the exenatide group more discontinued trial due to adverse events). No information on imputation method. The proportion of missing outcomes compared with observed event risk was enough to induce clinically relevant bias in intervention effect estimate
Incomplete outcome data Low risk (attrition bias)

non-serious adverse

events

Quote from publication: "ITT Safety Population: Enrolled patients receiving at least one dose of study medication..."

Comment: $>99 \%$ of the randomised participants were included in the analyses. There was a high dropout rate $(66 \%-75 \%$ of randomised participants completed the study), however, the dropout rate was balanced between groups. The reasons for dropouts were not balanced among the intervention groups (in the exenatide group more discontinued trial due to adverse events). No information on imputation method. The proportion of missing outcomes compared with observed event risk was not enough to induce clinically relevant bias in intervention effect estimate

Quote from publication: "ITT Safety Population: Enrolled patients receiving at least one dose of study medication..."

Comment: $>99 \%$ of the randomised participants were included in the analyses. There was a high dropout rate $(66 \%-75 \%$ of randomised participants completed the study), however, the dropout rate was balanced between groups. The reasons for dropouts were not balanced among the intervention groups (in the exenatide group more discontinued trial due to adverse events). No information on imputation method. The proportion of missing outcomes compared with observed event risk was enough to induce clinically relevant bias in intervention effect estimate

Incomplete outcome data High risk (attrition bias) weight $(\mathrm{kg})$
Quote from publication: "ITT Safety Population: Enrolled patients receiving at least one dose of study medication in Study Period II with patients analyzed according to treatment actually received. The analysis included only time points up to that week where at least $25 \%$ of the originally enrolled population was still in the study. Missing data at Year 3 was not imputed."

Comment: $35 \%-39 \%$ of the randomised participants were included in the analyses. There was a high dropout rate $(66 \%-75 \%$ of randomised participants completed the study), however, the dropout rate was balanced between groups. The reasons for dropouts were not balanced among the intervention groups (in the exenatide group more discontinued trial due to adverse 
events). No imputation method. Plausible effect size among missing outcomes is enough to induce clinically relevant bias in observed effect size

Incomplete outcome data Unclear risk (attrition bias)

HbA1c
Quote from publication: "Intention to treat... only randomly assigned patients receiving at least one dose of study treatment, and with baseline and at least one post-baseline $\mathrm{HbA1c}$ measurement were included." "Change in $\mathrm{HbAlc}$ from baseline to endpoint. Endpoint for $\mathrm{HbAlc}$ was defined as the $\mathrm{HbA1C}$ measured at the treatment failure for patients reaching primary endpoint and was the last observation in study period II for other patients (either followed until the end of the study period II or discontinuing the study)."

Comment: $94 \%$ of the randomised participants were included in the analyses. There was a high dropout rate $(66 \%-75 \%$ of randomised participants completed the study), however, the dropout rate was balanced between groups. The reasons for dropouts were not balanced among the intervention groups (in the exenatide group more discontinued trial due to adverse events). LOCF used. Plausible effect size among missing outcomes is not enough to induce clinically relevant bias in observed effect size

\begin{tabular}{lll}
\hline $\begin{array}{l}\text { Selective reporting (re- } \\
\text { porting bias) }\end{array}$ & Low risk & $\begin{array}{l}\text { Comment: all of the trial's primary and secondary outcomes as specified in } \\
\text { the protocol have been reported }\end{array}$ \\
\hline Other bias & Unclear risk & Comment: the trial was funded by a pharmaceutical company \\
\hline
\end{tabular}

\section{Gallwitz 2012b}

\section{Methods} Study design: randomised, double-blind, double-dummy, parallel-group, active-controlled, non-inferiority trial

Randomisation ratio: 1:1 to receive linagliptin or glimepiride in addition to metformin

Non-inferiority design: 1-sided confidence interval
Inclusion criteria: aged $18-80$ years, T2DM, receiving metformin at a stable dose of $\geq 1500 \mathrm{mg} /$ day (or a MTD $<1500 \mathrm{mg} /$ day) alone or with one other oral antidiabetic drug, $\mathrm{HbA1c} 6.5 \%-10 \%$ (on metformin alone) or $6 \%-9 \%$ (on metformin and 1 additional oral antidiabetic drug), BMI $\leq 40 \mathrm{~kg} / \mathrm{m}^{2}$ irrespective of ethnicity

Exclusion criteria: diagnoses of MI, stroke, or transient ischaemic attack in the 6 months before screening, impaired hepatic function at screening, and treatment with rosiglitazone, pioglitazone, a glucagon-like peptide 1 analogue or agonist, insulin, or an anti-obesity drug in the 3 months before screening

Diagnostic criteria: not reported

Interventions Number of study centres: 209

Run-in period: participants receiving metformin monotherapy entered a 2-week open-label placebo run-in period. Those receiving metformin and 1 additional oral antidiabetic drug entered a 6 -week washout period followed by the 2-week open-label placebo run-in

Extension period: no

Outcomes

Composite outcome measures reported: yes, composite outcome of target $\mathrm{HbA} 1 \mathrm{c}<7 \%$ with no hypoglycaemia and no weight gain over 2 years 
Gallwitz 2012b (Continued)

Trial ID: NCT00622284

$\begin{array}{ll}\text { Publication details } & \text { Language of publication: English } \\ \text { Funding: commercial funding by Boehringer Ingelheim } \\ \text { Publication status: peer-reviewed journal }\end{array}$

Stated aim for study

Quote from publication: "The aim of this hypothesis-driven study was to assess the long-term efficacy and safety of linagliptin compared with a commonly used sulphonylurea (glimepiride) as second-line treatment in participants with type 2 diabetes inadequately controlled on metformin. Additionally, as part of a large phase 3 programme, this study prospectively assessed cardiovascular safety."

\section{Notes}

\section{Risk of bias}

\begin{tabular}{lll}
\hline Bias & Authors' judgement & Support for judgement \\
\hline $\begin{array}{l}\text { Random sequence genera- } \\
\text { tion (selection bias) }\end{array}$ & Low risk & $\begin{array}{l}\text { Quote from publication: "Treatment assignment was done with a computer } \\
\text { generated random sequence... Assignment used a central interactive voice or } \\
\text { web response system with randomisation codes generated by the study spon- } \\
\text { sor. Study investigators and participants were masked to treatment assign- } \\
\text { ment for the duration of the trial. Placebo and active treatments were identi- } \\
\text { cal in appearance. Only dedicated personnel could access the randomisation } \\
\text { codes for treatment assignment, and could provide access in an emergency } \\
\text { only" } \\
\text { Comment: adequate generation of random sequence ensured }\end{array}$ \\
\hline
\end{tabular}

\begin{tabular}{ll}
\hline $\begin{array}{l}\text { Allocation concealment } \\
\text { (selection bias) }\end{array}$ & Quote from publication: "Treatment assignment was done with a computer \\
& generated random sequence... Assignment used a central interactive voice or \\
& web response system with randomisation codes generated by the study spon- \\
& sor. Study investigators and participants were masked to treatment assign- \\
& ment for the duration of the trial. Placebo and active treatments were identi- \\
& cal in appearance. Only dedicated personnel could access the randomisation \\
codes for treatment assignment, and could provide access in an emergency & \\
only"
\end{tabular}

Comment: adequate concealment ensured

\begin{tabular}{|c|c|c|}
\hline $\begin{array}{l}\text { Blinding of participants } \\
\text { and personnel (perfor- } \\
\text { mance bias) } \\
\text { all-cause/cardiovascular } \\
\text { mortality }\end{array}$ & Low risk & $\begin{array}{l}\text { Quote from publication: "...Double blind, double dummy..." "Additionally, } \\
\text { a masked independent clinical event committee prospectively reviewed all } \\
\text { reported treatment-emergent fatal events, suspected events of stroke, my- } \\
\text { ocardial ischaemia (including myocardial infarction), admission to hospital for } \\
\text { heart failure, stent thrombosis, and re-vascularisation procedures. The com- } \\
\text { mittee members evaluated whether prespecified criteria for adjudication end- } \\
\text { points (cardiovascular death, stroke, myocardial infarction, and admission to } \\
\text { hospital for unstable angina) were met." }\end{array}$ \\
\hline
\end{tabular}

Comment: adjudicated/investigator-assessed outcome measurement. Blinding of study personnel ensured

\begin{tabular}{ll}
\hline $\begin{array}{l}\text { Blinding of participants } \\
\text { and personnel (perfor- }\end{array}$ & Low risk \\
$\begin{array}{l}\text { mance bias) } \\
\text { hypoglycaemia }\end{array}$ & $\begin{array}{l}\text { Comment: investigator-assessed/self-reported outcome measurement. Blind- } \\
\text { ing of participant and study personnel ensured }\end{array}$ \\
\hline
\end{tabular}


Gallwitz 2012b (Continued)

Blinding of participants and personnel (performance bias) non-fatal myocardial infarction/heart failure/nonfatal stroke
Low risk

Quote from publication: "...Double blind, double dummy..." "Additionally, a masked independent clinical event committee prospectively reviewed all reported treatment-emergent fatal events, suspected events of stroke, myocardial ischaemia (including myocardial infarction), admission to hospital for heart failure, stent thrombosis, and re-vascularisation procedures. The committee members evaluated whether prespecified criteria for adjudication endpoints (cardiovascular death, stroke, myocardial infarction, and admission to hospital for unstable angina) were met."

Comment: adjudicated/investigator-assessed outcome measurement. Blinding of study personnel ensured

events
Quote from publication: "...Double blind, double dummy..."

Comment: self-reported outcome measurement. Blinding of participant en-

non-serious adverse sured

Low risk

\begin{tabular}{|c|c|c|}
\hline $\begin{array}{l}\text { Blinding of participants } \\
\text { and personnel (perfor- } \\
\text { mance bias) } \\
\text { non-serious adverse }\end{array}$ & Low risk & $\begin{array}{l}\text { Quote from publication: "...Double blind, double dummy..." } \\
\text { Comment: self-reported outcome measurement. Blinding of participant en- } \\
\text { sured }\end{array}$ \\
\hline
\end{tabular}

Blinding of participants Low risk and personnel (performance bias)

serious adverse events
Quote from publication: "...Double blind, double dummy..." "Additionally, a masked independent clinical event committee prospectively reviewed all reported treatment-emergent fatal events, suspected events of stroke, myocardial ischaemia (including myocardial infarction), admission to hospital for heart failure, stent thrombosis, and re-vascularisation procedures. The committee members evaluated whether prespecified criteria for adjudication endpoints (cardiovascular death, stroke, myocardial infarction, and admission to hospital for unstable angina) were met."

Comment: adjudicated/investigator-assessed outcome measurement. Blinding of study personnel ensured

\begin{tabular}{ll}
\hline $\begin{array}{l}\text { Blinding of participants } \\
\text { and personnel (perfor- }\end{array}$ & Low risk \\
$\begin{array}{l}\text { mance bias) } \\
\text { weight }(\mathrm{kg})\end{array}$ & $\begin{array}{l}\text { Quote from publication: "...Double blind, double dummy..." } \\
\text { ing of participant and study personnel ensured }\end{array}$
\end{tabular}

\begin{tabular}{|c|c|c|}
\hline $\begin{array}{l}\text { Blinding of participants } \\
\text { and personnel (perfor- } \\
\text { mance bias) } \\
\text { HbAlc }\end{array}$ & Low risk & $\begin{array}{l}\text { Quote from publication: "...Double blind, double dummy..." } \\
\text { Comment: investigator-assessed. Blinding of study personnel ensured }\end{array}$ \\
\hline $\begin{array}{l}\text { Blinding of outcome as- } \\
\text { sessment (detection bias) } \\
\text { all-cause/cardiovascular } \\
\text { mortality }\end{array}$ & Low risk & $\begin{array}{l}\text { Quote from publication: "...Double blind, double dummy..." "Additionally, } \\
\text { a masked independent clinical event committee prospectively reviewed all } \\
\text { reported treatment-emergent fatal events, suspected events of stroke, my- } \\
\text { ocardial ischaemia (including myocardial infarction), admission to hospital for } \\
\text { heart failure, stent thrombosis, and re-vascularisation procedures. The com- } \\
\text { mittee members evaluated whether prespecified criteria for adjudication end- } \\
\text { points (cardiovascular death, stroke, myocardial infarction, and admission to } \\
\text { hospital for unstable angina) were met." }\end{array}$ \\
\hline
\end{tabular}

Comment: adjudicated/investigator-assessed outcome measurement. Blinding of study personnel ensured

\begin{tabular}{lll}
\hline $\begin{array}{l}\text { Blinding of outcome as- } \\
\text { sessment (detection bias) } \\
\text { hypoglycaemia }\end{array}$ & Low risk & $\begin{array}{l}\text { Quote from publication: "...Double blind, double dummy..." } \\
\text { Comment: investigator-assessed/self-reported outcome measurement. Blind- } \\
\text { ing of participant and study personnel ensured }\end{array}$ \\
\hline $\begin{array}{l}\text { Blinding of outcome as- } \\
\text { sessment (detection bias) }\end{array}$ & Low risk & $\begin{array}{l}\text { Quote from publication: "...Double blind, double dummy..." "Additionally, } \\
\text { a masked independent clinical event committee prospectively reviewed all } \\
\text { reported treatment-emergent fatal events, suspected events of stroke, my- }\end{array}$
\end{tabular}


Gallwitz 2012b (Continued) non-fatal myocardial infarction/heart failure/nonfatal stroke ocardial ischaemia (including myocardial infarction), admission to hospital for heart failure, stent thrombosis, and re-vascularisation procedures. The committee members evaluated whether prespecified criteria for adjudication endpoints (cardiovascular death, stroke, myocardial infarction, and admission to hospital for unstable angina) were met."

Comment: adjudicated/investigator-assessed outcome measurement. Blinding of study personnel ensured

Quote from publication: "...Double blind, double dummy..."

Comment: self-reported outcome measurement. Blinding of participant ensured merious adverse events
Low risk
Blinding of outcome as- Low risk sessment (detection bias) serious adverse events
Quote from publication: "...Double blind, double dummy..." "Additionally, a masked independent clinical event committee prospectively reviewed all reported treatment-emergent fatal events, suspected events of stroke, myocardial ischaemia (including myocardial infarction), admission to hospital for heart failure, stent thrombosis, and re-vascularisation procedures. The committee members evaluated whether prespecified criteria for adjudication endpoints (cardiovascular death, stroke, myocardial infarction, and admission to hospital for unstable angina) were met."

Comment: adjudicated/investigator-assessed outcome measurement. Blinding of study personnel ensured

Quote from publication: "...Double blind, double dummy..."

Comment: investigator-assessed/self-reported outcome measurement. Blinding of participant and study personnel ensured

Quote from publication: "...Double blind, double dummy..."

Comment: investigator-assessed. Blinding of study personnel ensured

HbA1c

Quote from publication: "Safety analyses were done on the treated set with descriptive statistics" "Treated set included randomised patients who received at least one dose of treatment."

Comment: $>99 \%$ of randomised participants were included in the analyses. There was a high dropout rate ( $77 \%$ of randomised participants completed the study), however, the dropout rate was balanced between groups. We assumed that trial authors searched registers for mortality status at the end of the trial
Incomplete outcome data High risk (attrition bias)

hypoglycaemia
Quote from publication: "Safety analyses were done on the treated set with descriptive statistics" "Treated set included randomised patients who received at least one dose of treatment."

Comment: $>99 \%$ of the participants were included in the analyses. There was a high dropout rate ( $77 \%$ of the participants completed the study), however, the dropout rate was balanced between groups. We do not know how the trial authors imputed missing data from the participants not completing the study. The reasons for dropouts were not balanced (more dropouts in the linagliptin group due to lack of efficacy and more dropouts in the glimepiride group due to adverse events)

\footnotetext{
Incomplete outcome data High risk (attrition bias) non-fatal myocardial infarction/heart failure/nonfatal stroke
}

Quote from publication: "Safety analyses were done on the treated set with descriptive statistics" "Treated set included randomised patients who received at least one dose of treatment." 
Comment: > 99\% of the participants were included in the analyses. There was a high dropout rate ( $77 \%$ of the participants completed the study), however, the dropout rate was balanced between groups. We do not know how the trial authors imputed missing data from the participants not completing the study. The reasons for dropouts were not balanced (more dropouts in the linagliptin group due to lack of efficacy and more dropouts in the glimepiride group due to adverse events). The proportion of missing outcomes compared with observed event risk was enough to induce clinically relevant bias in intervention effect estimate

Incomplete outcome data Low risk (attrition bias) non-serious adverse events
Quote from publication: "Safety analyses were done on the treated set with descriptive statistics" "Treated set included randomised patients who received at least one dose of treatment."

Comment: > 99\% of the participants were included in the analyses. There was a high dropout rate ( $77 \%$ of the participants completed the study), however, the dropout rate was balanced between groups. We do not know how the trial authors imputed missing data from the participants not completing the study. The reasons for dropouts were not balanced (more dropouts in the linagliptin group due to lack of efficacy and more dropouts in the glimepiride group due to adverse events). The proportion of missing outcomes compared with observed event risk was not enough to induce clinically relevant bias in intervention effect estimate
Incomplete outcome data High risk (attrition bias)

serious adverse events

Quote from publication: "Safety analyses were done on the treated set with descriptive statistics" "Treated set included randomised patients who received at least one dose of treatment."

Comment: $>99 \%$ of the participants were included in the analyses. There was a high dropout rate ( $77 \%$ of the participants completed the study), however, the dropout rate was balanced between groups. We do not know how the trial authors imputed missing data from the participants not completing the study. The reasons for dropouts were not balanced (more dropouts in the linagliptin group due to lack of efficacy and more dropouts in the glimepiride group due to adverse events). The proportion of missing outcomes compared with observed event risk was enough to induce clinically relevant bias in intervention effect estimate

Quote from publication: "This population includes the FAS (full analysis set) further restricted to patients with a baseline body weight and one on-treatment body weight measurement. Last observation carried forward (LOCF) was used as imputation rule."

Comment: $>93 \%$ of the participants were included in the analyses. There was a high dropout rate ( $77 \%$ of the participants completed the study), however, the dropout rate was balanced between groups. The reasons for dropouts were not balanced (more dropouts in the linagliptin group due to lack of efficacy and more dropouts in the glimepiride group due to adverse events). Inappropriate method of imputation missing data was used (LOCF). Plausible effect size among missing outcomes was not enough to induce clinically relevant bias in observed effect size

Incomplete outcome data High risk (attrition bias)

HbA1c
Quote from publication: "The Full Analysis Set (FAS) included all treated and randomized patients with a baseline and at least one on-treatment $\mathrm{HbA1C}$ measurement. Last observation carried forward (LOCF) was used as imputation rule."

Comment: $>97 \%$ of the participants were included in the analyses. There was a high dropout rate ( $77 \%$ of the participants completed the study), however, the dropout rate was balanced between groups. The reasons for dropouts were not balanced (more dropouts in the linagliptin group due to lack of effi- 
Gallwitz 2012b (Continued)

cacy and more dropouts in the glimepiride group due to adverse events). Inappropriate method of imputation missing data was used (LOCF).

\begin{tabular}{lll}
\hline $\begin{array}{l}\text { Selective reporting (re- } \\
\text { porting bias) }\end{array}$ & Low risk & $\begin{array}{l}\text { Comment: all of the trial's primary and secondary outcomes as specified in } \\
\text { the protocol have been reported }\end{array}$ \\
\hline Other bias & High risk & $\begin{array}{l}\text { Comment: the trial was funded by a pharmaceutical company. The sponsor } \\
\text { was involved in study design, data collection, data review, and data analysis }\end{array}$ \\
\hline
\end{tabular}

Gerich 2005

Study design: randomised, multicentre, double-blind, active-controlled study
Randomisation ratio: $1: 1$ to receive glyburide or nateglinide in addition to metformin

Participants

Inclusion criteria: men and women, T2DM, inadequately controlled by diet and exercise, drug naive, aged $18-77$ years, baseline $\mathrm{hbA} 1 \mathrm{C} \geq 7.0 \%$ and $\leq 11.0 \%, \mathrm{FPG} \leq 15 \mathrm{mmol} / \mathrm{L}$, BMI between $22-45 \mathrm{~kg} / \mathrm{m}^{2}$. Women of childbearing potential were required to practice a medically approved birth control method

Exclusion criteria: T1DM or any secondary forms of diabetes, symptomatic hyperglycaemia with $>10 \%$ weight loss in the previous 8 weeks, abnormal renal function or significant diabetes complications, history of lactic acidosis or congestive heart failure requiring pharmacologic treatment, liver disease or persistent elevations ( 2 x ULN) of liver enzymes or other medical conditions that could interfere with interpretation of results or pose significant risk to the participant

Diagnostic criteria: not reported

\begin{tabular}{ll}
\hline Interventions & Number of study centres: 102 \\
Run-in period: 4 weeks maintenance period \\
Extension period: no
\end{tabular}

Outcomes Composite outcome measures reported: no

Study details

Trial terminated early: no

Trial ID: CDJN608A US07

\begin{tabular}{ll}
\hline Publication details & Language of publication: English \\
& Funding: commercial funding, Novartis Pharmaceuticals \\
& Publication status: peer-reviewed journal
\end{tabular}

Stated aim for study

Quote from publication: "To compare long-term efficacy and safety of initial combination therapy with nateglinide/metformin versus glyburide/metformin."

\begin{tabular}{lll}
\hline Notes & - \\
\hline Risk of bias & \\
\hline Bias & Authors' judgement & Support for judgement \\
\hline $\begin{array}{l}\text { Random sequence genera- } \\
\text { tion (selection bias) }\end{array}$ & Unclear risk & Quote from publication: "Patients were then randomized..." \\
& & Comment: insufficient information about the sequence generation process \\
\hline
\end{tabular}


Gerich 2005 (Continued)
Allocation concealment
Unclear risk
Quote from publication: "Patients were then randomized..." (selection bias)

Comment: insufficient information about the allocation concealment

\begin{tabular}{|c|c|c|}
\hline $\begin{array}{l}\text { Blinding of participants } \\
\text { and personnel (perfor- }\end{array}$ & Low risk & $\begin{array}{l}\text { Quote from publication: "double-blind" "The blind was maintained by the } \\
\text { use of matching placebo for nateglinide and glyburide" }\end{array}$ \\
\hline
\end{tabular}

mance bias)

all-cause/cardiovascular

Comment: investigator-assessed outcome measurement. Blinding of key mortality study personnel ensured

Blinding of participants Low risk
and personnel (perfor-
mance bias)
hypoglycaemia

Quote from publication: "double-blind" "The blind was maintained by the use of matching placebo for nateglinide and glyburide"

Comment: investigator-assessed/self-reported outcome measurement. Blinding of participant and key study personnel ensured

\begin{tabular}{lll}
\hline $\begin{array}{l}\text { Blinding of participants } \\
\text { and personnel (perfor- } \\
\text { mance bias) } \\
\text { serious adverse events }\end{array}$ & Low risk & $\begin{array}{l}\text { Quote from publication: "double-blind" "The blind was maintained by the } \\
\text { use of matching placebo for nateglinide and glyburide" }\end{array}$ \\
\hline $\begin{array}{l}\text { Blinding of participants } \\
\text { and personnel (perfor- } \\
\text { mance bias) }\end{array}$ & Low risk & $\begin{array}{l}\text { Comment: investigator-assessed outcome measurement. Blinding of key } \\
\text { study personnel ensured }\end{array}$ \\
weight (kg) & $\begin{array}{l}\text { Quote from publication: "double-blind" "The blind was maintained by the } \\
\text { use of matching placebo for nateglinide and glyburide" }\end{array}$ \\
& $\begin{array}{l}\text { Comment: investigator-assessed/self-reported outcome measurement. Blind- } \\
\text { ing of participant and key study personnel ensured }\end{array}$
\end{tabular}

\begin{tabular}{|c|c|c|}
\hline $\begin{array}{l}\text { Blinding of participants } \\
\text { and personnel (perfor- }\end{array}$ & Low risk & $\begin{array}{l}\text { Quote from publication: "double-blind" "The blind was maintained by the } \\
\text { use of matching placebo for nateglinide and glyburide" }\end{array}$ \\
\hline HbAlc & & $\begin{array}{l}\text { Comment: investigator-assessed outcome measurement. Blinding of key } \\
\text { study personnel ensured }\end{array}$ \\
\hline
\end{tabular}

\begin{tabular}{|c|c|c|}
\hline $\begin{array}{l}\text { Blinding of outcome as- } \\
\text { sessment (detection bias) } \\
\text { all-cause/cardiovascular } \\
\text { mortality }\end{array}$ & Low risk & $\begin{array}{l}\text { Quote from publication: "double-blind" "The blind was maintained by the } \\
\text { use of matching placebo for nateglinide and glyburide" } \\
\text { Comment: investigator-assessed outcome measurement. Blinding of out- } \\
\text { come assessor ensured }\end{array}$ \\
\hline
\end{tabular}

\begin{tabular}{lll}
\hline $\begin{array}{l}\text { Blinding of outcome as- } \\
\text { sessment (detection bias) }\end{array}$ & Low risk & $\begin{array}{l}\text { Quote from publication: "double-blind" "The blind was maintained by the } \\
\text { use of matching placebo for nateglinide and glyburide" }\end{array}$
\end{tabular}

hypoglycaemia

Comment: investigator-assessed/self-reported outcome measurement. Blinding of outcome assessor ensured

$\begin{array}{lll}\begin{array}{l}\text { Blinding of outcome as- } \\ \text { sessment (detection bias) }\end{array} & \text { Low risk } & \begin{array}{l}\text { Quote from publication: "double-blind" "The blind was maintained by the } \\ \text { use of matching placebo for nateglinide and glyburide" }\end{array}\end{array}$
serious adverse events

Comment: investigator-assessed outcome measurement. Blinding of outcome assessor ensured

\begin{tabular}{lll}
\hline Blinding of outcome as- & Low risk & Quote from publication: "double-blind" "The blind was maintained by the \\
sessment (detection bias) & use of matching placebo for nateglinide and glyburide"
\end{tabular}
weight $(\mathrm{kg})$

Comment: investigator-assessed/self-reported outcome measurement. Blinding of outcome assessor ensured

\begin{tabular}{|c|c|c|}
\hline $\begin{array}{l}\text { Blinding of outcome as- } \\
\text { sessment (detection bias) } \\
\text { HbA1C }\end{array}$ & Low risk & $\begin{array}{l}\text { Quote from publication: "double-blind" "The blind was maintained by the } \\
\text { use of matching placebo for nateglinide and glyburide" }\end{array}$ \\
\hline
\end{tabular}


Comment: investigator-assessed outcome measurement. Blinding of outcome assessor ensured

\begin{tabular}{ll}
\hline $\begin{array}{l}\text { Incomplete outcome data } \\
\text { (attrition bias) }\end{array}$ & Quote from publication: "Received study drug (safety population)" \\
all-cause/cardiovascular & Comment: $100 \%$ of randomised participants were included in the analysis. \\
mortality & There was a high dropout rate $(58 \%-64 \%$ of randomised participants complet- \\
& $\begin{array}{l}\text { ed the study), however, the dropout rate was balanced between groups. No in- } \\
\text { formation on imputation method. We assumed that trial authors searched reg- } \\
\text { isters for mortality status at the end of the trial }\end{array}$
\end{tabular}

Incomplete outcome data High risk
(attrition bias)

hypoglycaemia

\section{Quote from publication: "Received study drug (safety population)"}

Comment: $100 \%$ of randomised participants were included in the analysis. There was a high dropout rate (58\%-64\% of randomised participants completed the study), however, the dropout rate was balanced between groups. The reasons for dropouts were balanced among the intervention groups. No information on imputation method. The proportion of missing outcomes compared with observed event risk was enough to induce clinically relevant bias in intervention effect estimate

\begin{tabular}{ll}
\hline $\begin{array}{l}\text { Incomplete outcome data } \\
\text { (attrition bias) }\end{array}$ & Quote from publication: "Received study drug (safety population)" \\
serious adverse events & Comment: $100 \%$ of randomised participants were included in the analysis. \\
& There was a high dropout rate $(58 \%-64 \%$ of randomised participants complet- \\
& ed the study), however, the dropout rate was balanced between groups. The \\
& reasons for dropouts were balanced among the intervention groups. No in- \\
formation on imputation method. The proportion of missing outcomes com- \\
pared with observed event risk was enough to induce clinically relevant bias in \\
intervention effect estimate
\end{tabular}

Incomplete outcome data High risk (attrition bias) weight (kg)
Quote from publication: "Secondary efficacy variables... in the ITT population, with last observation carried forward."

Comment: $95 \%$ of the randomised participants were included in the analyses. There was a high dropout rate (58\%-64\% of randomised participants completed the study), however, the dropout rate was balanced between groups. The reasons for dropouts were balanced among the intervention groups. Inappropriate method of imputing missing data was used (LOCF). Plausible effect size among missing outcomes was enough to induce clinically relevant bias in observed effect size

Incomplete outcome data High risk (attrition bias)

HbAlc
Quote from publication: "The primary efficacy variable... in the intent-totreat (ITT) population with last observation carried forward."

Comment: $95 \%$ of the randomised participants were included in the analyses. There was a high dropout rate (58\%-64\% of randomised participants completed the study), however, the dropout rate was balanced between groups. The reasons for dropouts were balanced among the intervention groups. Inappropriate method of imputing missing data was used (LOCF). Plausible effect size among missing outcomes was enough to induce clinically relevant bias in observed effect size
Selective reporting (re- High risk porting bias)
Comment: no protocol available. According to Novartis trial document the safety assessments consisted of recording major adverse cardiac events (MACE). No results for MACE are reported

Other bias Unclear risk Comment: the trial received funding from a pharmaceutical company


Methods Study design: phase 3b, international, multicentre, randomised, parallel-group, active-controlled, double-blind, double-dummy, non-inferiority trial

Randomisation ratio: 1:1 to receive saxagliptin or glipizide in addition to metformin

\section{Non-inferiority design}

Participants Inclusion criteria: men and women aged $\geq 18$ years with T2DM and HbA1c $>6.5 \%-10.0 \%$ on a stable dose of metformin monotherapy $\geq 1500 \mathrm{mg} /$ day for at least 8 weeks prior to enrolment

Exclusion criteria: T1DM; history of diabetic ketoacidosis or hyperosmolar non-ketotic coma; insulin therapy within 1 year of enrolment; treatment with a thiazolidinedione within the 12 weeks prior to enrolment; treatment with systemic glucocorticoids other than replacement therapy; previous DPP-4 inhibitor treatment; donation of blood, plasma or platelets within the 3 months prior to enrolment; congestive heart failure defined as NYHA class III-IV and/or known left ventricular ejection fraction $\leq 40 \%$; significant cardiovascular history within the past 6 months defined as MI, coronary angioplasty or bypass graft(s), valvular disease or repair, unstable angina pectoris, transient ischaemic attack or cerebrovascular accident; history of haemoglobinopathies; significant alcohol or drug abuse within the year prior to enrolment; treatment with HIV/antiviral drugs or cytochrome P450 3A4-inducers; serum creatinine $\geq 1.5 \mathrm{mg} / \mathrm{dL}$ ( $\geq 133 \mu \mathrm{mol} / \mathrm{L}$ ) for men or $\geq 1.4 \mathrm{mg} / \mathrm{dL}(\geq 124 \mu \mathrm{mol} / \mathrm{L}$ ) for women; active liver disease and/or significant abnormal liver function (AST > $2 \times$ ULN and/or ALT $>2 \times$ ULN and/or total bilirubin $>2.0 \mathrm{mg} / \mathrm{dL}(>34 \mu \mathrm{mol} / \mathrm{L})$ ) or any clinically significant laboratory abnormality upon screening

Diagnostic criteria: not reported

Interventions Number of study centres: 130

Run-in period: 2-week single-blind, placebo-controlled lead-in period that included advice on diet and exercise

Extension period: yes, 52-week extension receiving the same double-blind and background treatment

Composite outcome measures reported: yes, composite outcome measure of participants reaching
the therapeutic goal ( $\mathrm{HbA} 1 \mathrm{c}<7 \%$ observed at week 104 in participants with $\mathrm{HbA} 1 \mathrm{c} \geq 7 \%$ at baseline)
without hypoglycaemic episodes and without weight gain (i.e. weight increase $<2 \%$ from baseline, cal-
culated using LOCF methods)

Study details

\section{Trial terminated early: no}

Trial ID: NCT00575588

$\begin{array}{ll}\text { Publication details } & \text { Language of publication: English } \\ \text { Funding: commercial funding by Bristol-Myers Squibb and AstraZeneca } \\ \text { Publication status: peer-reviewed journal }\end{array}$

Stated aim for study

Quote from publication: "To compare the long-term safety, tolerability and efficacy of saxagliptin vs. glipizide as add-on therapy to metformin"

Notes -

\section{Risk of bias}

\begin{tabular}{lll}
\hline Bias & Authors' judgement & Support for judgement \\
\hline $\begin{array}{l}\text { Random sequence genera- } \\
\text { tion (selection bias) }\end{array}$ & Low risk & $\begin{array}{l}\text { Quote from publication: "... eligible patients were randomised (1 : } 1) \text { via an in- } \\
\text { teractive Web-response system using a balanced block randomisation sched- } \\
\text { ule... " }\end{array}$
\end{tabular}


Göke 2013 (Continued)

Comment: adequate generation of random sequence ensured

\begin{tabular}{|c|c|c|}
\hline $\begin{array}{l}\text { Allocation concealment } \\
\text { (selection bias) }\end{array}$ & Low risk & $\begin{array}{l}\text { Quote from publication: "... eligible patients were randomised }(1: 1) \text { via an in- } \\
\text { teractive Web-response system using a balanced block randomisation sched- } \\
\text { ule..." }\end{array}$ \\
\hline
\end{tabular}

Comment: adequate concealment ensured

\begin{tabular}{ll}
\hline $\begin{array}{l}\text { Blinding of participants } \\
\text { and personnel (perfor- }\end{array}$ & Low risk \\
$\begin{array}{l}\text { mance bias) } \\
\text { all-cause/cardiovascular } \\
\text { mortality }\end{array}$ & $\begin{array}{l}\text { Quote from publication: "... double-blind..." "Blinding was ensured using a } \\
\text { double-dummy technique." }\end{array}$ \\
\end{tabular}

\begin{tabular}{|c|c|c|}
\hline $\begin{array}{l}\text { Blinding of participants } \\
\text { and personnel (perfor- } \\
\text { mance bias) } \\
\text { hypoglycaemia }\end{array}$ & Low risk & $\begin{array}{l}\text { Quote from publication: "... double-blind..." "Blinding was ensured using a } \\
\text { double-dummy technique." } \\
\text { Comment: self-reported outcome measurement. Blinding of participant en- } \\
\text { sured }\end{array}$ \\
\hline
\end{tabular}

\begin{tabular}{ll}
\hline $\begin{array}{l}\text { Blinding of participants } \\
\begin{array}{l}\text { and personnel (perfor- } \\
\text { mance bias) }\end{array}\end{array}$ & $\begin{array}{l}\text { Quote from publication: "... double-blind..." "Blinding was ensured using a } \\
\text { double-dummy technique." }\end{array}$ \\
$\begin{array}{l}\text { non-fatal myocardial in- } \\
\text { farction/heart failure/non- }\end{array}$ & $\begin{array}{l}\text { Comment: investigator-assessed outcome measurement. Blinding of study } \\
\text { personnel ensured }\end{array}$
\end{tabular}
fatal stroke

\begin{tabular}{|c|c|c|}
\hline $\begin{array}{l}\text { Blinding of participants } \\
\text { and personnel (perfor- } \\
\text { mance bias) } \\
\text { non-serious adverse } \\
\text { events }\end{array}$ & Low risk & $\begin{array}{l}\text { Quote from publication: "... double-blind..." "Blinding was ensured using a } \\
\text { double-dummy technique." } \\
\text { Comment: self-reported outcome measurement. Blinding of participant en- } \\
\text { sured }\end{array}$ \\
\hline
\end{tabular}

\begin{tabular}{ll}
\hline $\begin{array}{l}\text { Blinding of participants } \\
\text { and personnel (perfor- }\end{array}$ & Low risk \\
$\begin{array}{l}\text { mance bias) } \\
\text { serious adverse events }\end{array}$ & $\begin{array}{l}\text { Quote from publication: "... double-blind..." "Blinding was ensured using a } \\
\text { double-dummy technique." }\end{array}$ \\
& $\begin{array}{l}\text { Comment: investigator-assessed outcome measurement. Blinding of study } \\
\text { personnel ensured }\end{array}$ \\
\hline
\end{tabular}

\begin{tabular}{|c|c|c|}
\hline $\begin{array}{l}\text { Blinding of participants } \\
\text { and personnel (perfor- } \\
\text { mance bias) } \\
\text { weight (kg) }\end{array}$ & Low risk & $\begin{array}{l}\text { Quote from publication: "... double-blind..." "Blinding was ensured using a } \\
\text { double-dummy technique." } \\
\text { Comment: investigator-assessed. Blinding of study personnel ensured }\end{array}$ \\
\hline $\begin{array}{l}\text { Blinding of participants } \\
\text { and personnel (perfor- } \\
\text { mance bias) } \\
\text { HbAlc }\end{array}$ & Low risk & $\begin{array}{l}\text { Quote from publication: "... double-blind..." "Blinding was ensured using a } \\
\text { double-dummy technique." } \\
\text { Comment: investigator-assessed outcome measurement. Blinding of study } \\
\text { personnel ensured }\end{array}$ \\
\hline
\end{tabular}

\begin{tabular}{|c|c|c|}
\hline $\begin{array}{l}\text { Blinding of outcome as- } \\
\text { sessment (detection bias) } \\
\text { all-cause/cardiovascular } \\
\text { mortality }\end{array}$ & Low risk & $\begin{array}{l}\text { Quote from publication: "... double-blind..." "Blinding was ensured using a } \\
\text { double-dummy technique." } \\
\text { Comment: investigator-assessed outcome measurement. Blinding of study } \\
\text { personnel ensured }\end{array}$ \\
\hline
\end{tabular}

\begin{tabular}{ll}
\hline $\begin{array}{l}\text { Blinding of outcome as- } \\
\text { sessment (detection bias) } \\
\text { hypoglycaemia }\end{array}$ & Low risk
\end{tabular}$\quad \begin{aligned} & \text { Quote from publication: "... double-blind..." "Blinding was ensured using a } \\
& \text { double-dummy technique." }\end{aligned}$


Comment: self-reported outcome measurement. Blinding of participant ensured

\begin{tabular}{|c|c|c|}
\hline $\begin{array}{l}\text { Blinding of outcome as- } \\
\text { sessment (detection bias) } \\
\text { non-fatal myocardial in- } \\
\text { farction/heart failure/non- } \\
\text { fatal stroke }\end{array}$ & Low risk & $\begin{array}{l}\text { Quote from publication: "... double-blind..." "Blinding was ensured using a } \\
\text { double-dummy technique." } \\
\text { Comment: investigator-assessed outcome measurement. Blinding of study } \\
\text { personnel ensured }\end{array}$ \\
\hline
\end{tabular}

\begin{tabular}{ll}
\hline $\begin{array}{l}\text { Blinding of outcome as- } \\
\text { sessment (detection bias) } \\
\text { non-serious adverse }\end{array}$ & Low risk \\
events & $\begin{array}{l}\text { Quote from publication: "... double-blind..." "Blinding was ensured using a } \\
\text { double-dummy technique." }\end{array}$ \\
& $\begin{array}{l}\text { Comment: self-reported outcome measurement. Blinding of participant en- } \\
\text { sured }\end{array}$
\end{tabular}

\begin{tabular}{|c|c|c|}
\hline $\begin{array}{l}\text { Blinding of outcome as- } \\
\text { sessment (detection bias) }\end{array}$ & Low risk & $\begin{array}{l}\text { Quote from publication: "... double-blind..." "Blinding was ensured using a } \\
\text { double-dummy technique." }\end{array}$ \\
\hline
\end{tabular}

serious adverse events

Comment: investigator-assessed outcome measurement. Blinding of study personnel ensured

\begin{tabular}{lll}
\hline $\begin{array}{l}\text { Blinding of outcome as- } \\
\begin{array}{l}\text { sessment (detection bias) } \\
\text { weight }(\mathrm{kg})\end{array}\end{array}$ & Low risk & $\begin{array}{l}\text { Quote from publication: "... double-blind..." "Blinding was ensured using a } \\
\text { double-dummy technique." }\end{array}$ \\
\hline $\begin{array}{l}\text { Blinding of outcome as- } \\
\text { sessment (detection bias) } \\
\text { HbAlc }\end{array}$ & Low risk & $\begin{array}{l}\text { Quote from publication: "... double-blind..." "Blinding was ensured using a } \\
\text { double-dummy technique." }\end{array}$ \\
& $\begin{array}{l}\text { Comment: investigator-assessed outcome measurement. Blinding of study } \\
\text { personnel ensured }\end{array}$
\end{tabular}

Incomplete outcome data Low risk Quote from publication: "Safety and tolerability were analysed using descrip-
(attrition bias) tive statistics in all patients who took $\geq 1$ dose of study drug"

all-cause/cardiovascular mortality
Comment: $100 \%$ of randomised participants were included in the analyses. There was a high dropout rate (34\%-37\% of randomised participants completed the study), however, the dropout rate was balanced between groups. We assumed that trial authors searched registers for mortality status at the end of the trial
Incomplete outcome data High risk (attrition bias)

hypoglycaemia
Quote from publication: "Safety and tolerability were analysed using descriptive statistics in all patients who took $\geq 1$ dose of study drug"

Comment: $100 \%$ of the participants were included in the analyses. There was a high dropout rate (34\%-37\% of the participants completed the study), however, the dropout rate was balanced between groups. We do not know how the trial authors imputed missing data from the participants not completing the study. The reasons for dropouts were balanced. The proportion of missing outcomes compared with observed event risk was enough to induce clinically relevant bias in intervention effect estimate

Quote from publication: "Safety and tolerability were analysed using descriptive statistics in all patients who took $\geq 1$ dose of study drug"

Comment: $100 \%$ of the participants were included in the analyses. There was a high dropout rate (34\%-37\% of the participants completed the study), however, the dropout rate was balanced between groups. We do not know how the trial authors imputed missing data from the participants not completing the study. The reasons for dropouts were balanced. The proportion of missing out- 
Incomplete outcome data High risk (attrition bias)

non-serious adverse

events

Quote from publication: "Safety and tolerability were analysed using descriptive statistics in all patients who took $\geq 1$ dose of study drug"

Comment: $100 \%$ of the participants were included in the analyses. There was a high dropout rate (34\%-37\% of the participants completed the study), however, the dropout rate was balanced between groups. We do not know how the trial authors imputed missing data from the participants not completing the study. The reasons for dropouts were balanced. The proportion of missing outcomes compared with observed event risk was enough to induce clinically relevant bias in intervention effect estimate

Incomplete outcome data High risk (attrition bias)

serious adverse events
Quote from publication: "Safety and tolerability were analysed using descriptive statistics in all patients who took $\geq 1$ dose of study drug"

Comment: $100 \%$ of the participants were included in the analyses. There was a high dropout rate (34\%-37\% of the participants completed the study), however, the dropout rate was balanced between groups. We do not know how the trial authors imputed missing data from the participants not completing the study. The reasons for dropouts were balanced. The proportion of missing outcomes compared with observed event risk was enough to induce clinically relevant bias in intervention effect estimate
Incomplete outcome data High risk (attrition bias)

weight (kg)
Quote from publication: "Efficacy variables were analysed in the full analysis set, defined as all randomised patients who received $\geq 1$ dose of randomised study drug and had $\geq 1$ non-missing baseline and $\geq 1$ post baseline efficacy data assessment." "Number of subjects with observed values at week 104 was $\mathrm{N}=186$ for saxagliptin + metformin and N=165 for glipizide + metformin"

Comment: $>99 \%$ of the randomised participants were included in the analyses. $38 \%-43 \%$ of the randomised participants had observed values at week 104. Unknown, how the missing data were imputed. Plausible effect size among missing outcomes was enough to induce clinically relevant bias in observed effect size

Incomplete outcome data High risk (attrition bias)

Quote from publication: "Efficacy variables were analysed in the full analysis set, defined as all randomised patients who received $\geq 1$ dose of randomised study drug and had $\geq 1$ non-missing baseline and $\geq 1$ post baseline efficacy data assessment." "Number of subjects with observed values at week 104 was $\mathrm{N}=184$ for saxagliptin + metformin and N=160 for glipizide + metformin"

Comment: $>99 \%$ of the randomised participants were included in the analyses. $37 \%-43 \%$ of the randomised participants had observed values at week 104. Unknown, how the missing data were imputed. Plausible effect size among missing outcomes enough to induce clinically relevant bias in observed effect size

\begin{tabular}{|c|c|}
\hline $\begin{array}{l}\text { Selective reporting (re- } \\
\text { porting bias) }\end{array}$ & Low risk \\
\hline
\end{tabular}

\begin{tabular}{ll}
\hline Other bias Unclear risk Comment: the trial was funded by a pharmaceutical company \\
\hline
\end{tabular}


Randomisation ratio: 2:1:1 to receive rosiglitazone/metformin fixed-dose combination, glibenclamide + metformin and gliclazide + metformin

\section{Non-inferiority design}

\section{Participants}

Inclusion criteria: $\mathrm{BMI} \geq 25 \mathrm{~kg} / \mathrm{m}^{2}, \mathrm{~T} 2 \mathrm{DM}, \mathrm{HbAlC} \geq 7 \%$ and $\leq 10 \%$ having received metformin ( $\geq 0.85 \mathrm{~g}$ / day) for at least 8 weeks prior to screening

Exclusion criteria: had used any oral antidiabetic drug other than metformin in the prior 12 weeks, or insulin at any time other than during pregnancy or for emergency treatment, history of metabolic acidosis, oedema requiring pharmacological treatment (either ongoing or within the prior 12 months), anaemia (haemoglobin $<11.0 \mathrm{~g} / \mathrm{dL}$ for men and $<10.0 \mathrm{~g} / \mathrm{dL}$ for women), renal or hepatic disease, known congestive heart failure (all NYHA grades), unstable or severe angina, or a history of MI, percutaneous transluminal coronary angioplasty, coronary artery bypass graft or cerebrovascular accident within 3 months, or left ventricular dysfunction within 6 months, of screening, fasting C-peptide $\leq 0.5$ $\mathrm{nmol} / \mathrm{L}$, or with systolic $\mathrm{BP}>170 \mathrm{mmHg}$ or diastolic $\mathrm{BP}>100 \mathrm{mmHg}$ while on antihypertensive medication

Diagnostic criteria: WHO 1999 criteria

Interventions Number of study centres: 118

Run-in period: at screening, eligible individuals currently receiving $\geq 1.5 \mathrm{~g} /$ day metformin entered a 4week, single-blind run-in period during which they received metformin $2 \mathrm{~g} /$ day. Those currently receiving $<1.5 \mathrm{~g} /$ day metformin underwent an additional 1-week titration step, during which they received metformin $1.5 \mathrm{~g} /$ day before entering the 4 -week run-in period of treatment with metformin $2 \mathrm{~g} /$ day

Extension period: no

\begin{tabular}{ll}
\hline Outcomes & Composite outcome measures reported: no \\
\hline Study details & Trial terminated early: no \\
& Trial ID: NCT00359112 \\
\hline Publication details & Language of publication: English \\
& Funding: commercial funding by GlaxoSmithKline \\
& Publication status: peer-reviewed journal \\
\hline Stated aim for study & $\begin{array}{l}\text { Quote from publication: "... to compare rosiglitazone / metformin fixed-dose combination therapy } \\
\text { with combination sulphonylurea plus metformin therapy in overweight individuals with inadequately } \\
\text { controlled type 2 diabetes mellitus." }\end{array}$ \\
\hline
\end{tabular}

Notes -

\section{Risk of bias}

Bias Authors' judgement Support for judgement

Random sequence genera- Low risk tion (selection bias)
Quote from publication: "Investigators used RAMOS, a telephone-based interactive voice response system involving computer-generated randomization."

Comment: adequate generation of random sequence ensured
Allocation concealment Low risk (selection bias)
Quote from publication: "Investigators used RAMOS, a telephone-based interactive voice response system involving computer-generated randomization." 
Comment: adequate concealment ensured

\begin{tabular}{|c|c|c|}
\hline $\begin{array}{l}\text { Blinding of participants } \\
\text { and personnel (perfor- } \\
\text { mance bias) } \\
\text { all-cause/cardiovascular } \\
\text { mortality }\end{array}$ & Low risk & $\begin{array}{l}\text { Quote from publication: "double-blind" } \\
\text { Comment: investigator-assessed outcome measurement. Blinding of study } \\
\text { personnel ensured }\end{array}$ \\
\hline
\end{tabular}

\begin{tabular}{ll}
\hline $\begin{array}{l}\text { Blinding of participants } \\
\text { and personnel (perfor- }\end{array}$ & Low risk \\
$\begin{array}{l}\text { mance bias) } \\
\text { hypoglycaemia }\end{array}$ & $\begin{array}{l}\text { Quote from publication: "double blind" } \\
\text { sured }\end{array}$ \\
\hline
\end{tabular}

Blinding of participants Low risk and personnel (performance bias) non-fatal myocardial infarction/heart failure/nonfatal stroke
Quote from publication: "double-blind"

Comment: investigator-assessed outcome measurement. Blinding of study personnel ensured

\begin{tabular}{|c|c|c|}
\hline $\begin{array}{l}\text { Blinding of participants } \\
\text { and personnel (perfor- } \\
\text { mance bias) } \\
\text { non-serious adverse } \\
\text { events }\end{array}$ & Low risk & $\begin{array}{l}\text { Quote from publication: "double blind" } \\
\text { Comment: self-reported outcome measurement. Blinding of participant en- } \\
\text { sured }\end{array}$ \\
\hline
\end{tabular}

\begin{tabular}{|c|c|c|}
\hline $\begin{array}{l}\text { Blinding of participants } \\
\text { and personnel (perfor- } \\
\text { mance bias) } \\
\text { serious adverse events }\end{array}$ & Low risk & $\begin{array}{l}\text { Quote from publication: "double-blind" } \\
\text { Comment: investigator-assessed outcome measurement. Blinding of study } \\
\text { personnel ensured }\end{array}$ \\
\hline
\end{tabular}

\begin{tabular}{|c|c|c|}
\hline $\begin{array}{l}\text { Blinding of participants } \\
\text { and personnel (perfor- } \\
\text { mance bias) } \\
\text { weight (kg) }\end{array}$ & Low risk & $\begin{array}{l}\text { Quote from publication: "double-blind" } \\
\text { Comment: investigator-assessed/self-reported outcome measurement. Blind- } \\
\text { ing of participant and study personnel ensured }\end{array}$ \\
\hline
\end{tabular}

\begin{tabular}{|c|c|c|}
\hline $\begin{array}{l}\text { Blinding of participants } \\
\text { and personnel (perfor- } \\
\text { mance bias) } \\
\text { HbAlc }\end{array}$ & Low risk & $\begin{array}{l}\text { Quote from publication: "double-blind" } \\
\text { Comment: investigator-assessed. Blinding of study personnel ensured }\end{array}$ \\
\hline $\begin{array}{l}\text { Blinding of outcome as- } \\
\text { sessment (detection bias) } \\
\text { all-cause/cardiovascular } \\
\text { mortality }\end{array}$ & Low risk & $\begin{array}{l}\text { Quote from publication: "double-blind" } \\
\text { Comment: investigator-assessed outcome measurement. Blinding of study } \\
\text { personnel ensured }\end{array}$ \\
\hline $\begin{array}{l}\text { Blinding of outcome as- } \\
\text { sessment (detection bias) } \\
\text { hypoglycaemia }\end{array}$ & Low risk & $\begin{array}{l}\text { Quote from publication: "double blind" } \\
\text { Comment: self-reported outcome measurement. Blinding of participant en- } \\
\text { sured }\end{array}$ \\
\hline
\end{tabular}

\begin{tabular}{|c|c|c|}
\hline $\begin{array}{l}\text { Blinding of outcome as- } \\
\text { sessment (detection bias) } \\
\text { non-fatal myocardial in- } \\
\text { farction/heart failure/non- } \\
\text { fatal stroke }\end{array}$ & Low risk & $\begin{array}{l}\text { Quote from publication: "double-blind" } \\
\text { Comment: investigator-assessed outcome measurement. Blinding of study } \\
\text { personnel ensured }\end{array}$ \\
\hline
\end{tabular}

$\begin{array}{ll}\text { Blinding of outcome as- } & \text { Low risk }\end{array}$ Quote from publication: "double blind"


Hamann 2008 (Continued) non-serious adverse events
Comment: self-reported outcome measurement. Blinding of participant ensured

\begin{tabular}{|c|c|c|}
\hline $\begin{array}{l}\text { Blinding of outcome as- } \\
\text { sessment (detection bias) } \\
\text { serious adverse events }\end{array}$ & Low risk & $\begin{array}{l}\text { Quote from publication: "double-blind" } \\
\text { Comment: investigator-assessed outcome measurement. Blinding of study } \\
\text { personnel ensured }\end{array}$ \\
\hline
\end{tabular}
personnel ensured

\begin{tabular}{lll}
\hline $\begin{array}{l}\text { Blinding of outcome as- } \\
\text { sessment (detection bias) } \\
\text { weight }(\mathrm{kg})\end{array}$ & Low risk & $\begin{array}{l}\text { Quote from publication: "double-blind" } \\
\text { Comment: investigator-assessed/self-reported outcome measurement } \\
\text { ing of participant and study personnel ensured }\end{array}$ \\
\hline $\begin{array}{l}\text { Blinding of outcome as- } \\
\begin{array}{l}\text { sessment (detection bias) } \\
\text { HbAlc }\end{array}\end{array}$ & Low risk & Quote from publication: "double-blind" \\
\hline
\end{tabular}

Incomplete outcome data Low risk (attrition bias)

all-cause/cardiovascular mortality
Quote from publication: "The safety population consisted of all randomized subjects who received at least one dose of double-blind study medication."

Comment: $>99 \%$ of randomised participants were included in the analyses. There was a high dropout rate $(76 \%-79 \%$ of randomised participants completed the study), however, the dropout rate was balanced between groups. We assumed that trial authors searched registers for mortality status at the end of the trial

Incomplete outcome data Low risk (attrition bias)

hypoglycaemia

Quote from publication: "The safety population consisted of all randomized subjects who received at least one dose of double-blind study medication."
Comment: $>99 \%$ of the participants were included in the analyses. There was a high dropout rate (76\%-79\% of the participants completed the study), however, the dropout rate was balanced between groups. We do not know how the trial authors imputed missing data from the participants not completing the study. The reasons for dropouts were balanced. The proportion of missing outcomes compared with observed event risk was not enough to induce clinically relevant bias in intervention effect estimate

Quote from publication: "The safety population consisted of all randomized subjects who received at least one dose of double-blind study medication."

Comment: $>99 \%$ of the participants were included in the analyses. There was a high dropout rate (76\%-79\% of the participants completed the study), however, the dropout rate was balanced between groups. We do not know how the trial authors imputed missing data from the participants not completing the study. The reasons for dropouts were balanced. The proportion of missing outcomes compared with observed event risk was enough to induce clinically relevant bias in intervention effect estimate

Quote from publication: "The safety population consisted of all randomized subjects who received at least one dose of double-blind study medication."

Incomplete outcome data Low risk (attrition bias) non-serious adverse events
Comment: > 99\% of the participants were included in the analyses. There was a high dropout rate (76\%-79\% of the participants completed the study), however, the dropout rate was balanced between groups. We do not know how the trial authors imputed missing data from the participants not completing the study. The reasons for dropouts were balanced. The proportion of missing outcomes compared with observed event risk was not enough to induce clinically relevant bias in intervention effect estimate

Quote from publication: "The safety population consisted of all randomized subjects who received at least one dose of double-blind study medication."
Incomplete outcome data High risk (attrition bias)

righ risk


Hamann 2008 (Continued) serious adverse events
Comment: $>99 \%$ of the participants were included in the analyses. There was a high dropout rate (76\%-79\% of the participants completed the study), however, the dropout rate was balanced between groups. We do not know how the trial authors imputed missing data from the participants not completing the study. The reasons for dropouts were balanced. The proportion of missing outcomes compared with observed event risk was enough to induce clinically relevant bias in intervention effect estimate $\begin{array}{lll}\begin{array}{l}\text { Incomplete outcome data } \\ \text { (attrition bias) }\end{array} & \text { Unclear risk } & \begin{array}{l}\text { Quote from publication: "The safety population consisted of all randomized } \\ \text { subjects who received at least one dose of double-blind study medication." }\end{array}\end{array}$

weight $(\mathrm{kg})$
Comment: $>99 \%$ of the participants were included in the analyses. There was a high dropout rate ( $76 \%-79 \%$ of the participants completed the study), however, the dropout rate was balanced between groups. We do not know how the trial authors imputed missing data from the participants not completing the study. The reasons for dropouts were balanced. Not sure if plausible effect size among missing outcomes is enough to induce clinically relevant bias in observed effect size

\section{Incomplete outcome data Unclear risk} (attrition bias)

HbAlc

Quote from publication: "The intention-to-treat (ITT) population consisted of all randomized subjects who received at least one dose of study medication, had a baseline assessment and at least one corresponding on-therapy assessment for HbA 1c, FPG, C-peptide, insulin or proinsulin." "The primary efficacy endpoint, biomarker and health outcome endpoints were evaluated in ITT without LOCF population..."

Comment: $76 \%-78 \%$ of the participants were included in the analyses. There was a high dropout rate ( $76 \%-79 \%$ of the participants completed the study), however, the dropout rate was balanced between groups. No imputation used. The reasons for dropouts were balanced. Not sure if plausible effect size among missing outcomes is enough to induce clinically relevant bias in observed effect size

$\begin{array}{ll}\begin{array}{l}\text { Selective reporting (re- } \\ \text { porting bias) }\end{array} & \text { Low risk } \\ \end{array}$

Handelsman 2017

\section{Methods}

Comment: the trial was funded by a pharmaceutical company 
Handelsman 2017 (Continued)

ed GFR (based on modification of diet in renal disease formula) $<60 \mathrm{~mL} / \mathrm{min} / 1.73 \mathrm{~m}^{2}$, or creatinine $\geq 1.4$ $\mathrm{mg} / \mathrm{dL}(123.8 \mu \mathrm{mol} / \mathrm{L})$ (men) or $\geq 1.3 \mathrm{mg} / \mathrm{dL}$ (114.9 $\mu \mathrm{mol} / \mathrm{L})$ (women) were also excluded

Diagnostic criteria: not reported

\begin{tabular}{ll}
\hline Interventions & Number of study centres: 127 \\
& Run-in period: 2 -week single-blind placebo run-in \\
& Extension period: no \\
\hline Outcomes & $\begin{array}{l}\text { Composite outcome measures reported: yes, composite end-point of an HbAlc decrease }>0.5 \% \text { with } \\
\text { no symptomatic hypoglycaemia and no body weight gain, after } 54 \text { weeks of treatment }\end{array}$
\end{tabular}

Study details Trial terminated early: no

Trial ID: NCT01682759

Publication details Language of publication: English

Funding: commercial funding by Merck \& Co

Publication status: peer-reviewed journal

Stated aim for study Quote from publication: "The primary objectives of this study were to assess the safety and tolerabili-
ty of omarigliptin and to compare its efficacy with glimepiride after 54 weeks of treatment"

\begin{tabular}{ll}
\hline Notes & - \\
\hline
\end{tabular}

\section{Risk of bias}

\begin{tabular}{lll}
\hline Bias & Authors' judgement & Support for judgement \\
\hline $\begin{array}{l}\text { Random sequence genera- } \\
\text { tion (selection bias) }\end{array}$ & Low risk & $\begin{array}{l}\text { Quote from publication: "...patients were randomised centrally, using an in- } \\
\text { teractive voice response system..." }\end{array}$ \\
& Comment: adequate generation of random sequence ensured
\end{tabular}

$\begin{array}{ll}\begin{array}{l}\text { Allocation concealment } \\ \text { (selection bias) }\end{array} & \text { Low risk } \\ & \begin{array}{l}\text { Quote from publication: "...patients were randomised centrally, using an in- } \\ \text { teractive voice response system..." }\end{array} \\ \text { Comment: adequate concealment ensured }\end{array}$

\begin{tabular}{|c|c|c|}
\hline $\begin{array}{l}\text { Blinding of participants } \\
\text { and personnel (perfor- } \\
\text { mance bias) } \\
\text { all-cause/cardiovascular } \\
\text { mortality }\end{array}$ & Low risk & $\begin{array}{l}\text { Quote from publication: "...double-blind, double-dummy..." } \\
\text { Comment: investigator-assessed outcome measurement. Blinding ensured }\end{array}$ \\
\hline $\begin{array}{l}\text { Blinding of participants } \\
\text { and personnel (perfor- } \\
\text { mance bias) } \\
\text { hypoglycaemia }\end{array}$ & Low risk & $\begin{array}{l}\text { Quote from publication: "...double-blind, double-dummy..." } \\
\text { Comment: investigator-assessed/self-reported outcome measurement. Blind- } \\
\text { ing ensured }\end{array}$ \\
\hline
\end{tabular}

\begin{tabular}{ll}
\hline $\begin{array}{l}\text { Blinding of participants } \\
\text { and personnel (perfor- }\end{array}$ & Low risk \\
$\begin{array}{l}\text { mance bias) } \\
\text { non-fatal myocardial in- }\end{array}$ & Comment: investigator-assessed outcome measurement. Blinding ensured \\
farction/heart failure/non- & \\
fatal stroke &
\end{tabular}


Handelsman 2017 (Continued)
Blinding of participants
Low risk
Quote from publication: "...double-blind, double-dummy..." and personnel (perfor-
Comment: self-reported outcome measurement. Blinding ensured
mance bias)
non-serious adverse
events

\begin{tabular}{ll}
\hline $\begin{array}{l}\text { Blinding of participants } \\
\text { and personnel (perfor- }\end{array}$ & Low risk \\
$\begin{array}{l}\text { mance bias) } \\
\text { serious adverse events }\end{array}$ & Quote from publication: "...double-blind, double-dummy..." \\
\hline
\end{tabular}

\begin{tabular}{|c|c|c|}
\hline $\begin{array}{l}\text { Blinding of participants } \\
\text { and personnel (perfor- } \\
\text { mance bias) } \\
\text { weight (kg) }\end{array}$ & Low risk & $\begin{array}{l}\text { Quote from publication: "...double-blind, double-dummy..." } \\
\text { Comment: investigator-assessed outcome measurement. Blinding ensured }\end{array}$ \\
\hline
\end{tabular}

\begin{tabular}{|c|c|c|}
\hline $\begin{array}{l}\text { Blinding of participants } \\
\text { and personnel (perfor- } \\
\text { mance bias) } \\
\text { HbAlc }\end{array}$ & Low risk & $\begin{array}{l}\text { Quote from publication: "...double-blind, double-dummy..." } \\
\text { Comment: investigator-assessed outcome measurement. Blinding ensured }\end{array}$ \\
\hline
\end{tabular}

\begin{tabular}{|c|c|c|}
\hline $\begin{array}{l}\text { Blinding of outcome as- } \\
\text { sessment (detection bias) } \\
\text { all-cause/cardiovascular } \\
\text { mortality }\end{array}$ & Low risk & $\begin{array}{l}\text { Quote from publication: "...double-blind, double-dummy..." } \\
\text { Comment: investigator-assessed outcome measurement. Blinding ensured }\end{array}$ \\
\hline
\end{tabular}

\begin{tabular}{ll}
\hline $\begin{array}{l}\text { Blinding of outcome as- } \\
\text { sessment (detection bias) } \\
\text { hypoglycaemia }\end{array}$ & Low risk
\end{tabular}$\quad \begin{aligned} & \text { Quote from publication: "...double-blind, double-dummy..." } \\
& \end{aligned}$

\begin{tabular}{|c|c|c|}
\hline $\begin{array}{l}\text { Blinding of outcome as- } \\
\text { sessment (detection bias) } \\
\text { non-fatal myocardial in- } \\
\text { farction/heart failure/non- } \\
\text { fatal stroke }\end{array}$ & Low risk & $\begin{array}{l}\text { Quote from publication: "...double-blind, double-dummy..." } \\
\text { Comment: investigator-assessed outcome measurement. Blinding ensured }\end{array}$ \\
\hline
\end{tabular}

\begin{tabular}{|c|c|c|}
\hline $\begin{array}{l}\text { Blinding of outcome as- } \\
\text { sessment (detection bias) } \\
\text { non-serious adverse } \\
\text { events }\end{array}$ & Low risk & $\begin{array}{l}\text { Quote from publication: "...double-blind, double-dummy..." } \\
\text { Comment: self-reported outcome measurement. Blinding ensured }\end{array}$ \\
\hline $\begin{array}{l}\text { Blinding of outcome as- } \\
\text { sessment (detection bias) } \\
\text { serious adverse events }\end{array}$ & Low risk & $\begin{array}{l}\text { Quote from publication: "...double-blind, double-dummy..." } \\
\text { Comment: investigator-assessed outcome measurement. Blinding ensured }\end{array}$ \\
\hline $\begin{array}{l}\text { Blinding of outcome as- } \\
\text { sessment (detection bias) } \\
\text { weight }(\mathrm{kg})\end{array}$ & Low risk & $\begin{array}{l}\text { Quote from publication: "...double-blind, double-dummy..." } \\
\text { Comment: investigator-assessed outcome measurement. Blinding ensured }\end{array}$ \\
\hline $\begin{array}{l}\text { Blinding of outcome as- } \\
\text { sessment (detection bias) } \\
\text { HbA1c }\end{array}$ & Low risk & $\begin{array}{l}\text { Quote from publication: "...double-blind, double-dummy..." } \\
\text { Comment: investigator-assessed outcome measurement. Blinding ensured }\end{array}$ \\
\hline $\begin{array}{l}\text { Incomplete outcome data } \\
\text { (attrition bias) } \\
\text { all-cause/cardiovascular } \\
\text { mortality }\end{array}$ & Low risk & $\begin{array}{l}\text { Quote from publication: "Analysis of safety data used the population of all } \\
\text { randomised patients who received at least one dose of study treatment." "Se- } \\
\text { rious adverse events are presented, regardless of time from last dose of blind- } \\
\text { ed study medication, including data after glycaemic rescue. Non-serious ad- } \\
\text { verse events are presented, regardless of time from last dose of blinded study }\end{array}$ \\
\hline
\end{tabular}



ed in the treatment group corresponding to the study treatment they actually received."

Comment: $>99 \%$ of randomised participants were included in the analyses. There was a high dropout rate ( $76 \%-77 \%$ of randomised participants completed the study), however, the dropout rate was balanced between groups. We assumed that trial authors searched registers for mortality status at the end of the trial

\section{Incomplete outcome data Low risk} (attrition bias)

hypoglycaemia

Quote from publication: "Analysis of safety data used the population of all randomised patients who received at least one dose of study treatment." "Serious adverse events are presented, regardless of time from last dose of blinded study medication, including data after glycaemic rescue. Non-serious adverse events are presented, regardless of time from last dose of blinded study medication, excluding data after glycaemic rescue." "Participants were included in the treatment group corresponding to the study treatment they actually received."

Comment: $>99 \%$ of randomised participants were included in the analyses. There was a high dropout rate ( $76 \%-77 \%$ of randomised participants completed the study), however, the dropout rate was balanced between the intervention groups. We do not know how the trial authors imputed missing data from the participants not completing the study. The reasons for dropouts were balanced. The proportion of missing outcomes compared with observed event risk was not enough to induce clinically relevant bias in intervention effect estimate

Incomplete outcome data High risk (attrition bias) non-fatal myocardial infarction/heart failure/nonfatal stroke
Quote from publication: "Analysis of safety data used the population of all randomised patients who received at least one dose of study treatment." "Serious adverse events are presented, regardless of time from last dose of blinded study medication, including data after glycaemic rescue. Non-serious adverse events are presented, regardless of time from last dose of blinded study medication, excluding data after glycaemic rescue." "Participants were included in the treatment group corresponding to the study treatment they actually received."

Comment: $>99 \%$ of randomised participants were included in the analyses. There was a high dropout rate ( $76 \%-77 \%$ of randomised participants completed the study), however, the dropout rate was balanced between groups. We do not know how the trial authors imputed missing data from the participants not completing the study. The reasons for dropouts were balanced. The proportion of missing outcomes compared with observed event risk was enough to induce clinically relevant bias in intervention effect estimate

Quote from publication: "Analysis of safety data used the population of all randomised patients who received at least one dose of study treatment." "Serious adverse events are presented, regardless of time from last dose of blinded study medication, including data after glycaemic rescue. Non-serious adverse events are presented, regardless of time from last dose of blinded study medication, excluding data after glycaemic rescue." "Participants were included in the treatment group corresponding to the study treatment they actually received."

Comment: $>99 \%$ of randomised participants were included in the analyses. There was a high dropout rate ( $76 \%-77 \%$ of randomised participants completed the study), however, the dropout rate was balanced between groups. We do not know how the trial authors imputed missing data from the participants not completing the study. The reasons for dropouts were balanced. The proportion of missing outcomes compared with observed event risk was not enough to induce clinically relevant bias in intervention effect estimate 
Handelsman 2017 (Continued)

Incomplete outcome data High risk (attrition bias)

serious adverse events
Quote from publication: "Analysis of safety data used the population of all randomised patients who received at least one dose of study treatment." "Serious adverse events are presented, regardless of time from last dose of blinded study medication, including data after glycaemic rescue. Non-serious adverse events are presented, regardless of time from last dose of blinded study medication, excluding data after glycaemic rescue." "Participants were included in the treatment group corresponding to the study treatment they actually received."

Comment: $>99 \%$ of randomised participants were included in the analyses. There was a high dropout rate (76\%-77\% of randomised participants completed the study), however, the dropout rate was balanced between groups. We do not know how the trial authors imputed missing data from the participants not completing the study. The reasons for dropouts were balanced. The proportion of missing outcomes compared with observed event risk was enough to induce clinically relevant bias in intervention effect estimate
Incomplete outcome data Unclear risk (attrition bias)

weight $(\mathrm{kg})$

Quote from publication: "Analysis of safety data used the population of all randomised patients who received at least one dose of study treatment." "Serious adverse events are presented, regardless of time from last dose of blinded study medication, including data after glycaemic rescue. Non-serious adverse events are presented, regardless of time from last dose of blinded study medication, excluding data after glycaemic rescue." "Participants were included in the treatment group corresponding to the study treatment they actually received."

Comment: $>99 \%$ of the randomised participants were included in the analyses. There was a high dropout rate $(76 \%-77 \%$ of randomised participants completed the study), however, the dropout rate was balanced between groups. The reasons for dropouts were balanced among the intervention groups. No information on imputation method. Not sure if plausible effect size among missing outcomes was enough to induce clinically relevant bias in observed effect size

\section{Incomplete outcome data Unclear risk} (attrition bias)

HbA1c
Quote from publication: "The population of all randomised patients who received at least one dose of study treatment and had a baseline or a post-randomisation measurement served as the primary population for efficacy analyses."

Comment: $>99 \%$ of the randomised participants were included in the analyses. There was a high dropout rate (76\%- $77 \%$ of randomised participants completed the study), however, the dropout rate was balanced between groups. The reasons for dropouts were balanced among the intervention groups. No information on imputation method. Not sure if plausible effect size among missing outcomes was enough to induce clinically relevant bias in observed effect size

Comment: all of the trial's primary and secondary outcomes as specified in the protocol have been reported
Selective reporting (re- Low risk porting bias)
Comment: the trial was funded by a pharmaceutical company

Hollander 2017

Methods

Study design: multicentre, randomised, double-blind, active-controlled, parallel-group, phase lll clinical study 
Hollander 2017 (Continued)

Randomisation ratio: 1:1:1 to receive ertugliflozin $5 \mathrm{mg}$, ertugliflozin $15 \mathrm{mg}$ or glimepiride $1 \mathrm{mg}-8 \mathrm{mg}$ in addition to metformin

\section{Non-inferiority design}

Participants

Inclusion criteria: age $\geq 18$ years, $\mathrm{T} 2 \mathrm{DM}, \mathrm{HbAlc} \geq 53$ and $\leq 75 \mathrm{mmol} / \mathrm{mol}(\geq 7.0 \%$ and $\leq 9.0 \%)$, on $\geq 1500$ $\mathrm{mg} /$ day of metformin monotherapy for at least 8 weeks at screening. Participants on this regimen for $<8$ weeks, on lower doses of metformin, or on any dose of metformin with another glucose-lowering agent at screening were eligible if they met the above criteria after the appropriate dose/medication adjustment, stabilisation, or washout period

Exclusion criteria: history of T1DM or ketoacidosis; $\geq 5 \%$ change in body weight in previous 6 months; treatment in previous 12 weeks with insulin or any other type of injectable a glucose-lowering agent, pioglitazone or rosiglitazone, other SGLT2 inhibitors, bromocriptine, or colesevelam, or any other glucose-lowering agents, with the exceptions of SUs administered at $<50 \%$ of the maximum approved dose, DPP-4 inhibitors, meglitinides, and alpha-glucosidase inhibitors; history of myocardial infarction, unstable angina, arterial revascularization, stroke, transient ischaemic attack, or NYHA class III-IV heart failure within 3 months of screening; any active, obstructive uropathy or indwelling urinary catheter; mean value for triplicate sitting systolic BP > $160 \mathrm{mmHg}$ and/or diastolic BP >90 mmHg (participants on BP medication must have been on a stable regimen for at least 4 weeks prior to randomisation); estimated GFR $<55 \mathrm{~mL} / \mathrm{min} / 1.73 \mathrm{~m}^{2}$; serum creatinine $\geq 115 \mu \mathrm{mol} / \mathrm{L}(1.3 \mathrm{mg} / \mathrm{dL})$ in men or $\geq 106 \mu \mathrm{mol} / \mathrm{L}$ $(1.2 \mathrm{mg} / \mathrm{dL})$ in women

Diagnostic criteria: ADA guidelines

Interventions Number of study centres: 232

Run-in period: 2-week single-blind placebo run-in

Extension period: the study was conducted over 104 weeks in two 52-week phases. 104-week results not available yet

Outcomes

Composite outcome measures reported: yes, $\mathrm{HbAlc}$ reduction $>5.5 \mathrm{mmol} / \mathrm{mol}(0.5 \%)$ with no symptomatic hypoglycaemia or body weight gain, and $\mathrm{HbAlc}<53 \mathrm{mmol} / \mathrm{mol}(7.0 \%)$ with no symptomatic hypoglycaemia

Study details

Trial terminated early: no

Trial ID: NCT01999218

Publication details Language of publication: English

Funding: commercial funding by Merck Sharp \& Dohme Corp.

Publication status: peer-reviewed journal

Stated aim for study

Quote from publication: "The primary hypothesis was that the glycemic efficacy of ertugliflozin $15 \mathrm{mg}$, as an add-on to metformin, was non-inferior to that of glimepiride after 52 weeks of treatment."

\section{Notes}

\section{Risk of bias}

\begin{tabular}{lll}
\hline Bias & Authors' judgement & Support for judgement \\
\hline $\begin{array}{ll}\text { Random sequence genera- } \\
\text { tion (selection bias) }\end{array}$ & Low risk & $\begin{array}{l}\text { Quote from publication: "Randomization was performed using a central elec- } \\
\text { tronic randomization system." }\end{array}$ \\
& & Comment: adequate generation of random sequence ensured \\
\hline
\end{tabular}


Hollander 2017 (Continued)

Allocation concealment (selection bias)
Low risk tronic randomization system."

Comment: adequate concealment ensured
Blinding of participants Low risk and personnel (performance bias) all-cause/cardiovascular mortality

Quote from publication: "Ertugliflozin and glimepiride tablets were packaged identically relative to their matching placebos. Study personnel, including patients, investigators, study site, central laboratory, and the sponsor remained blinded throughout the 52-week phase A treatment period."

Comment: investigator-assessed outcome measurement. Blinding of participant and study personnel ensured

\begin{tabular}{|c|c|c|}
\hline $\begin{array}{l}\text { Blinding of participants } \\
\text { and personnel (perfor- } \\
\text { mance bias) } \\
\text { amputation of lower ex- }\end{array}$ & Low risk & $\begin{array}{l}\text { Quote from publication: "Ertugliflozin and glimepiride tablets were packaged } \\
\text { identically relative to their matching placebos. Study personnel, including pa- } \\
\text { tients, investigators, study site, central laboratory, and the sponsor remained } \\
\text { blinded throughout the } 52 \text {-week phase A treatment period }\end{array}$ \\
\hline
\end{tabular}
tremity/blindness or severe vision loss/end-stage renal disease

Comment: investigator-assessed outcome measurement. Blinding of participant and study personnel ensured

Blinding of participants Low risk and personnel (performance bias)

hypoglycaemia
Quote from publication: "Ertugliflozin and glimepiride tablets were packaged identically relative to their matching placebos. Study personnel, including patients, investigators, study site, central laboratory, and the sponsor remained blinded throughout the 52-week phase A treatment period."

Comment: investigator-assessed/self-reported outcome measurement. Blinding of participant and study personnel ensured

\begin{tabular}{|c|c|c|}
\hline $\begin{array}{l}\text { Blinding of participants } \\
\text { and personnel (perfor- } \\
\text { mance bias) } \\
\text { non-fatal myocardial in- } \\
\text { farction/heart failure/non- } \\
\text { fatal stroke }\end{array}$ & Low risk & $\begin{array}{l}\text { Quote from publication: "Ertugliflozin and glimepiride tablets were package } \\
\text { identically relative to their matching placebos. Study personnel, including pa- } \\
\text { tients, investigators, study site, central laboratory, and the sponsor remained } \\
\text { blinded throughout the } 52 \text {-week phase A treatment period." } \\
\text { Comment: investigator-assessed outcome measurement. Blinding of partici- } \\
\text { pant and study personnel ensured }\end{array}$ \\
\hline
\end{tabular}

Blinding of participants Low risk and personnel (performance bias)

serious adverse events identically relative to their matching placebos. Study personnel, including patients, investigators, study site, central laboratory, and the sponsor remained blinded throughout the 52-week phase A treatment period."
Quote from publication: "Ertugliflozin and glimepiride tablets were packaged

Comment: investigator-assessed outcome measurement. Blinding of participant and study personnel ensured

\begin{tabular}{|c|c|c|}
\hline $\begin{array}{l}\text { Blinding of participants } \\
\text { and personnel (perfor- } \\
\text { mance bias) } \\
\text { HbAlc }\end{array}$ & Low risk & $\begin{array}{l}\text { Quote from publication: "Ertugliflozin and glimepiride tablets were packaged } \\
\text { identically relative to their matching placebos. Study personnel, including pa- } \\
\text { tients, investigators, study site, central laboratory, and the sponsor remained } \\
\text { blinded throughout the } 52 \text {-week } \\
\text { phase A treatment period." }\end{array}$ \\
\hline
\end{tabular}

Comment: investigator-assessed outcome measurement. Blinding of participant and study personnel ensured

\begin{tabular}{lll}
\hline $\begin{array}{l}\text { Blinding of outcome as- } \\
\text { sessment (detection bias) }\end{array}$ & Low risk & $\begin{array}{l}\text { Quote from publication: "Ertugliflozin and glimepiride tablets were packaged } \\
\text { identically relative to their matching placebos. Study personnel, including pa- }\end{array}$ \\
$\begin{array}{l}\text { all-cause/cardiovascular } \\
\text { mortality }\end{array}$ & $\begin{array}{l}\text { tients, investigators, study site, central laboratory, and the sponsor remained } \\
\text { blinded throughout the 52-week phase A treatment period." }\end{array}$
\end{tabular}


Comment: investigator-assessed outcome measurement. Blinding of investigator ensured

Blinding of outcome as-
sessment (detection bias)
amputation of lower ex-
tremity/blindness or se-
vere vision loss/end-stage
renal disease

Low risk

Ler

Quote from publication: "Ertugliflozin and glimepiride tablets were packaged identically relative to their matching placebos. Study personnel, including patients, investigators, study site, central laboratory, and the sponsor remained blinded throughout the 52-week phase A treatment period."

Comment: investigator-assessed outcome measurement. Blinding of investigator ensured

Blinding of outcome as- $\quad$ Low risk
sessment (detection bias)
hypoglycaemia
Quote from publication: "Ertugliflozin and glimepiride tablets were packaged identically relative to their matching placebos. Study personnel, including pa- tients, investigators, study site, central laboratory, and the sponsor remained blinded throughout the 52-week phase A treatment period."

Comment: investigator-assessed/self-reported outcome measurement. Blinding of participant and investigator ensured

\begin{tabular}{|c|c|c|}
\hline $\begin{array}{l}\text { Blinding of outcome as- } \\
\text { sessment (detection bias) } \\
\text { non-fatal myocardial in- } \\
\text { farction/heart failure/non- } \\
\text { fatal stroke }\end{array}$ & Low risk & $\begin{array}{l}\text { Quote from publication: "Ertugliflozin and glimepiride tablets were packaged } \\
\text { identically relative to their matching placebos. Study personnel, including pa- } \\
\text { tients, investigators, study site, central laboratory, and the sponsor remained } \\
\text { blinded throughout the } 52 \text {-week phase A treatment period." }\end{array}$ \\
\hline
\end{tabular}

Comment: investigator-assessed outcome measurement. Blinding of investigator ensured

$\begin{array}{lll}\begin{array}{l}\text { Blinding of outcome as- } \\ \text { sessment (detection bias) }\end{array} & \text { Low risk } & \begin{array}{l}\text { Quote from publication: "Ertugliflozin and glimepiride tablets were packaged } \\ \text { identically relative to their matching placebos. Study personnel, including pa- } \\ \text { serious adverse events }\end{array} \\ \begin{array}{l}\text { tients, investigators, study site, central laboratory, and the sponsor remained } \\ \text { blinded throughout the 52-week phase A treatment period." }\end{array}\end{array}$

Comment: investigator-assessed outcome measurement. Blinding of investigator ensured

\begin{tabular}{|c|c|c|}
\hline $\begin{array}{l}\text { Blinding of outcome as- } \\
\text { sessment (detection bias) } \\
\text { HbA1c }\end{array}$ & Low risk & $\begin{array}{l}\text { Quote from publication: "Ertugliflozin and glimepiride tablets were packaged } \\
\text { identically relative to their matching placebos. Study personnel, including pa- } \\
\text { tients, investigators, study site, central laboratory, and the sponsor remained } \\
\text { blinded throughout the 52-week phase A treatment period." }\end{array}$ \\
\hline
\end{tabular}

Comment: investigator-assessed outcome measurement. Blinding of investigator ensured

Incomplete outcome data Low risk
(attrition bias)
all-cause/cardiovascular
mortality
Quote from publication: "Safety analyses included all randomized, treated patients. Data following initiation of glycemic rescue were included for the analysis of SAEs, discontinuations due to AEs, and excluded for the other end- points."

Comment: $>99 \%$ of randomised participants were included in the analyses. There was a high dropout rate $(76 \%-81 \%$ of randomised participants completed the study), however, the dropout rate was balanced between groups. We assumed that trial authors searched registers for mortality status at the end of the trial

\author{
Incomplete outcome data High risk \\ (attrition bias) \\ amputation of lower ex- \\ tremity/blindness or se- \\ vere vision loss/end-stage \\ renal disease
}
Quote from publication: "Safety analyses included all randomized, treated patients. Data following initiation of glycemic rescue were included for the analysis of SAEs, discontinuations due to AEs, and excluded for the other end- points."

Comment: $>99 \%$ of randomised participants were included in the analyses. There was a high dropout rate $(76 \%-81 \%$ of randomised participants complet- 
Hollander 2017 (Continued)

ed the study), however, the dropout rate was balanced between groups. We do not know how the trial authors imputed missing data from the participants not completing the study. The reasons for dropouts were balanced. The proportion of missing outcomes compared with observed event risk was enough to induce clinically relevant bias in intervention effect estimate

Incomplete outcome data High risk (attrition bias)

hypoglycaemia

Quote from publication: "Safety analyses included all randomized, treated patients. Data following initiation of glycemic rescue were included for the analysis of SAEs, discontinuations due to AEs, and excluded for the other endpoints."

Comment: $>99 \%$ of randomised participants were included in the analyses. There was a high dropout rate ( $76 \%-81 \%$ of randomised participants completed the study), however, the dropout rate was balanced between groups. We do not know how the trial authors imputed missing data from the participants not completing the study. The reasons for dropouts were balanced. The proportion of missing outcomes compared with observed event risk was enough to induce clinically relevant bias in intervention effect estimate

Incomplete outcome data High risk (attrition bias) non-fatal myocardial infarction/heart failure/nonfatal stroke
Quote from publication: "Safety analyses included all randomized, treated patients. Data following initiation of glycemic rescue were included for the analysis of SAEs, discontinuations due to AEs, and excluded for the other endpoints."

Comment: $>99 \%$ of randomised participants were included in the analyses. There was a high dropout rate $(76 \%-81 \%$ of randomised participants completed the study), however, the dropout rate was balanced between groups. We do not know how the trial authors imputed missing data from the participants not completing the study. The reasons for dropouts were balanced. The proportion of missing outcomes compared with observed event risk was enough to induce clinically relevant bias in intervention effect estimate

\section{Incomplete outcome data High risk} (attrition bias) serious adverse events
Quote from publication: "Safety analyses included all randomized, treated patients. Data following initiation of glycemic rescue were included for the analysis of SAEs, discontinuations due to AEs, and excluded for the other endpoints."

Comment: $>99 \%$ of randomised participants were included in the analyses. There was a high dropout rate ( $76 \%-81 \%$ of randomised participants completed the study), however, the dropout rate was balanced between groups. We do not know how the trial authors imputed missing data from the participants not completing the study. The reasons for dropouts were balanced. The proportion of missing outcomes compared with observed event risk was enough to induce clinically relevant bias in intervention effect estimate

Incomplete outcome data Unclear risk
(attrition bias)
HbA1c

Incomplete outcome data Unclear risk HbA1c
Quote from publication: "Efficacy analyses excluded results following initiation of glycemic rescue therapy to avoid the confounding influence of rescue therapy. The full analysis set (FAS; all randomized patients who took at least one dose of study drug and had at least one measurement of the respective endpoint) was the primary population for efficacy analyses." "HbAlc reduction from baseline at week 52 was assessed in categorical subgroups using a repeated measures analysis of covariance (ANCOVA) model."

Comment: $77 \%-81 \%$ of the participants were included in the analyses. There was a high dropout rate ( $76 \%-81 \%$ of the participants completed the study), however, the dropout rate was balanced between groups. No information on imputation method. The reasons for dropouts were balanced. Not sure if plausible effect size among missing outcomes was enough to induce clinically relevant bias in observed effect size 
Hollander 2017 (Continued)

Selective reporting (re- Low risk Comment: all of the trial's primary and secondary outcomes as specified in porting bias)

the protocol have been reported

Other bias

Unclear risk

Comment: the trial was funded by a pharmaceutical company

Home 2009

Methods Study design: prospective, multicentre, randomised, open-label trial

Randomisation ratio: 1:1 to receive rosiglitazone or sulphonylurea (glibenclamide, gliclazide or glimepiride) in addition to metformin

\section{Non-inferiority design}

Participants

Inclusion criteria: T2DM, age 40-75 years, $\mathrm{BMI}>25.0 \mathrm{~kg} / \mathrm{m}^{2}, \mathrm{HbA} 1 \mathrm{c}>7.0 \%$ and $\leq 9.0 \%$, on maximum tolerated dose/MPD of background monotherapy of metformin, on oral glucose-lowering drugs for $\geq 6$ months and on current drug at the maximum tolerated dose/MPD for $\geq 2$ months. If female, then postmenopausal, sterilised or using effective contraceptive measures

Exclusion criteria: using other glucose-lowering therapies; use of a combination of $\geq 2$ oral glucose-lowering agents within 6 months; use of insulin, except for pregnancy, intercurrent illness or stabilisation; previous use of any PPAR- $\gamma$ agonist; hospitalisation for a major CV event in the last 3 months; scheduled major $\mathrm{CV}$ intervention, or gangrene; diagnosed or receiving medication specifically for heart failure (except diuretics alone); systolic or diastolic BP $>180 / 105 \mathrm{mmHg}$, on therapy if used; fasting serum triglycerides $>12.0 \mathrm{mmol} / \mathrm{L}$; serum creatinine $>130 \mu \mathrm{mol} / \mathrm{L}(>1.47 \mathrm{mg} / \mathrm{dL})$; ALT, AST, total bilirubin or alkaline phosphatase $\geq 2.5 \times$ ULN; haemoglobin $<11.0 \mathrm{~g} / \mathrm{dL}$ for men or $<10.0 \mathrm{~g} / \mathrm{dL}$ for women or haemoglobinopathy interfering with valid $\mathrm{HbAlc}$ assay; contraindication/intolerance to metformin, glyburide, gliclazide or glimepiride; pre-existing medical condition judged to preclude safe participation in the study; abuse of alcohol or drugs, or presence of any condition that may lead to poor adherence to study protocols; recent use of an investigational drug; pregnancy, breast feeding or planning pregnancy

\section{Diagnostic criteria: 1999 WHO criteria}

\section{Number of study centres: 364}

Run-in period: 4-week run-in period that included reinforcement of lifestyle education. Participants continued to take metformin

Extension period: yes, 4 years of observational follow-up was added to the study to monitor the occurrence of cancer and bone fractures. At the end of the main study, all study medication was stopped. Participants were not provided with study medication in the observational follow-up; instead, anti-diabetic treatment was prescribed at the investigator's discretion.

Outcomes Composite outcome measures reported: no

Study details Trial terminated early: no

Trial ID: NCT00379769

Publication details Language of publication: English

Funding: commercial funding by GlaxoSmithKline plc, UK

Publication status: peer-reviewed journal 
Home 2009 (Continued)

Stated aim for study

Quote from publication: "Our aim was to assess non-inferiority of rosiglitazone in combination with metformin or sulfonylurea compared with metformin and sulfonylurea dual therapy for cardiovascular outcomes."

Notes -

\section{Risk of bias}

Bias Authors' judgement Support for judgement

Random sequence genera- Low risk tion (selection bias)
Quote from publication: "The treatment allocation schedule was computer generated in blocks and stratified according to background glucose-lowering medication (metformin or sulphonylurea). Participants were randomised centrally using an interactive voice response telephone system."

Comment: adequate generation of random sequence ensured

Allocation concealment Low risk
(selection bias)

Quote from publication: "The treatment allocation schedule was computer generated in blocks and stratified according to background glucose-lowering medication (metformin or sulphonylurea). Participants were randomised centrally using an interactive voice response telephone system."

Comment: adequate concealment ensured
Low risk and personnel (performance bias) all-cause/cardiovascular mortality inding of participants

Quote from publication: "...open-label trial..." "The study was monitored by a clinical trials organisation (Quintiles, Bracknell, UK), which also coordinated data collection." "An independent data safety and monitoring board reviewed conduct of the study and unblinded data at about 6-month intervals." "Deaths and investigator-diagnosed cardiovascular events were identified through adverse-event reporting, direct questioning, or both, at study visits with trial record forms. Data from all relevant clinical sources were obtained by Quintiles and provided to an independent clinical endpoints committee who were blind to treatment allocation. All deaths were adjudicated with predefined criteria."

Comment: adjudicated outcome measurement. Incomplete blinding of participant and investigator, but the outcome is not likely to be influenced by lack of blinding

Quote from publication: "...open-label trial..."

Blinding of participants and personnel (perfor-

High risk

mance bias)

hypoglycaemia

Comment: self-reported outcome measurement. Incomplete blinding, the outcome is likely to be influenced by lack of blinding
Blinding of participants Low risk and personnel (performance bias) non-fatal myocardial infarction/heart failure/nonfatal stroke
Quote from publication: "...open-label trial..." "The study was monitored by a clinical trials organisation (Quintiles, Bracknell, UK), which also coordinated data collection." "An independent data safety and monitoring board reviewed conduct of the study and unblinded data at about 6-month intervals." "Deaths and investigator-diagnosed cardiovascular events were identified through adverse-event reporting, direct questioning, or both, at study visits with trial record forms. Data from all relevant clinical sources were obtained by Quintiles and provided to an independent clinical endpoints committee who were blind to treatment allocation. All deaths were adjudicated with predefined criteria."

Comment: adjudicated outcome measurement. Incomplete blinding of participant and investigator, but the outcome is not likely to be influenced by lack of blinding

Blinding of participants $\quad$ High risk $\quad$ Quote from publication: "...open-label trial..."
$\begin{aligned} & \text { and personnel (perfor- } \\ & \text { mance bias) }\end{aligned}$


Home 2009 (Continued) non-serious adverse events
Comment: self-reported outcome measurement. Incomplete blinding, the outcome is likely to be influenced by lack of blinding
Blinding of participants and personnel (performance bias)

serious adverse events
Low risk

Quote from publication: "...open-label trial..." "The study was monitored by a clinical trials organisation (Quintiles, Bracknell, UK), which also coordinated data collection." "An independent data safety and monitoring board reviewed conduct of the study and unblinded data at about 6-month intervals." "Deaths and investigator-diagnosed cardiovascular events were identified through adverse-event reporting, direct questioning, or both, at study visits with trial record forms. Data from all relevant clinical sources were obtained by Quintiles and provided to an independent clinical endpoints committee who were blind to treatment allocation. All deaths were adjudicated with predefined criteria."

Comment: adjudicated/investigator-assessed outcome measurement. Incomplete blinding of participant and investigator, but the outcome is not likely to be influenced by lack of blinding

\begin{tabular}{|c|c|c|}
\hline $\begin{array}{l}\text { Blinding of participants } \\
\text { and personnel (perfor- } \\
\text { mance bias) } \\
\text { weight (kg) }\end{array}$ & High risk & $\begin{array}{l}\text { Quote from publication: "...open-label trial..." } \\
\text { Comment: investigator-assessed/self-reported outcome measurement. In- } \\
\text { complete blinding of participant and investigator, the outcome is likely to be } \\
\text { influenced by lack of blinding }\end{array}$ \\
\hline
\end{tabular}

\begin{tabular}{|c|c|c|}
\hline $\begin{array}{l}\text { Blinding of participants } \\
\text { and personnel (perfor- } \\
\text { mance bias) } \\
\text { HbA1C }\end{array}$ & Low risk & $\begin{array}{l}\text { Quote from publication: "...open-label trial..." } \\
\text { Comment: investigator-assessed outcome measurement. Incomplete blinding } \\
\text { of participant and investigator, but the outcome is not likely to be influenced } \\
\text { by lack of blinding }\end{array}$ \\
\hline
\end{tabular}

\section{Blinding of outcome as- Low risk} sessment (detection bias) all-cause/cardiovascular mortality of participant and investigator, but the outcome is not likely to be influenced by lack of blinding

Quote from publication: "...open-label trial..." "The study was monitored by a clinical trials organisation (Quintiles, Bracknell, UK), which also coordinated data collection." "An independent data safety and monitoring board reviewed conduct of the study and unblinded data at about 6-month intervals." "Deaths and investigator-diagnosed cardiovascular events were identified through adverse-event reporting, direct questioning, or both, at study visits with trial record forms. Data from all relevant clinical sources were obtained by Quintiles and provided to an independent clinical endpoints committee who were blind to treatment allocation. All deaths were adjudicated with predefined criteria."

Comment: adjudicated outcome measurement. Blinding of clinical endpoint committee ensured

$\begin{array}{lll}\begin{array}{l}\text { Blinding of outcome as- } \\ \text { sessment (detection bias) } \\ \text { hypoglycaemia }\end{array} & \text { High risk } & \text { Quote from publication: "...open-label trial..." } \\ & \begin{array}{l}\text { Comment: self-reported outcome measurement. Incomplete blinding of out- } \\ \text { come assessor, the outcome is likely to be influenced by lack of blinding }\end{array}\end{array}$

Blinding of outcome as- Low risk sessment (detection bias) non-fatal myocardial infarction/heart failure/nonfatal stroke 
Home 2009 (Continued)

\section{Blinding of outcome as- sessment (detection bias) non-serious adverse} events
High risk Quote from publication: "...open-label trial..."

Comment: self-reported outcome measurement. Incomplete blinding, the outcome is likely to be influenced by lack of blinding
Blinding of outcome as- Low risk sessment (detection bias) serious adverse events

Quote from publication: "...open-label trial..." "The study was monitored by a clinical trials organisation (Quintiles, Bracknell, UK), which also coordinated data collection." "An independent data safety and monitoring board reviewed conduct of the study and unblinded data at about 6-month intervals." "Deaths and investigator-diagnosed cardiovascular events were identified through adverse-event reporting, direct questioning, or both, at study visits with trial record forms. Data from all relevant clinical sources were obtained by Quintiles and provided to an independent clinical endpoints committee who were blind to treatment allocation. All deaths were adjudicated with predefined criteria."

Comment: adjudicated/investigator-assessed outcome measurement. Blinding of clinical endpoint committee ensured

\begin{tabular}{lll}
\hline $\begin{array}{l}\text { Blinding of outcome as- } \\
\text { sessment (detection bias) } \\
\text { weight }(\mathrm{kg})\end{array}$ & High risk & Quote from publication: "...open-label trial..." \\
& $\begin{array}{l}\text { Comment: investigator-assessed/self-reported outcome measurement. In- } \\
\text { complete blinding of outcome assessor, the outcome is likely to be influenced } \\
\text { by lack of blinding }\end{array}$
\end{tabular}

Blinding of outcome as- Low risk sessment (detection bias) HbAlc

\section{Quote from publication: "...open-label trial..."}

Comment: investigator-assessed outcome measurement. Incomplete blinding of outcome assessor, but the outcome is not likely to be influenced by lack of blinding
Incomplete outcome data Low risk all-cause/cardiovascular mortality (attrition bias)

Quote from publication: "All individuals who receive at least one dose of randomised study medication will be assessed for clinical safety and tolerability" "The primary endpoint is the time to first CV hospitalisation or death ... Participants who do not achieve the endpoint during their time in the study will have their data censored based on the date of final observed contact."

Comment: $>99 \%$ of the randomised participants were included in the analyses. There was a high dropout rate $(82 \%-84 \%$ of randomised participants completed the study), however, the dropout rate was balanced between groups. We assumed that trial authors searched registers for mortality status at the end of the trial. Data were censored

\section{Incomplete outcome data High risk} (attrition bias)

hypoglycaemia
Quote from publication: "All individuals who receive at least one dose of randomised study medication will be assessed for clinical safety and tolerability"

Comment: $>99 \%$ of the randomised participants were included in the analyses. There was a high dropout rate ( $82 \%-84 \%$ of randomised participants completed the study), however, the dropout rate was balanced between groups. The reasons for dropouts were balanced among the intervention groups. No information on imputation method. The proportion of missing outcomes compared with observed event risk was enough to induce clinically relevant bias in intervention effect estimate

$\begin{array}{ll}\begin{array}{l}\text { Incomplete outcome data } \\ \text { (attrition bias) }\end{array} & \text { Quote from publication: "All individuals who receive at least one dose of ran- } \\ \text { non-fatal myocardial in- } & \text { domised study medication will be assessed for clinical safety and tolerability" } \\ \text { farction/heart failure/non- } & \text { "The primary endpoint is the time to first CV hospitalisation or death ... Partici- } \\ \text { fatal stroke } & \text { pants who do not achieve the endpoint during their time in the study will have } \\ \text { their data censored based on the date of final observed contact." }\end{array}$

Comment: $>99 \%$ of the randomised participants were included in the analyses. There was a high dropout rate $(82 \%-84 \%$ of randomised participants com-

Metformin and second- or third-generation sulphonylurea combination therapy for adults with type 2 diabetes mellitus (Review) Copyright (c) 2019 The Cochrane Collaboration. Published by John Wiley \& Sons, Ltd. 
Incomplete outcome data Low risk (attrition bias)

non-serious adverse

events
Quote from publication: "All individuals who receive at least one dose of randomised study medication will be assessed for clinical safety and tolerability"

Comment: $>99 \%$ of the randomised participants were included in the analyses. There was a high dropout rate $(82 \%-84 \%$ of randomised participants completed the study), however, the dropout rate was balanced between groups. The reasons for dropouts were balanced among the intervention groups. No information on imputation method. The proportion of missing outcomes compared with observed event risk was not enough to induce clinically relevant bias in intervention effect estimate

Incomplete outcome data Low risk (attrition bias)

serious adverse events

Quote from publication: "All individuals who receive at least one dose of randomised study medication will be assessed for clinical safety and tolerability" "The primary endpoint is the time to first CV hospitalisation or death ... Participants who do not achieve the endpoint during their time in the study will have their data censored based on the date of final observed contact."

Comment: $>99 \%$ of the randomised participants were included in the analyses. There was a high dropout rate $(82 \%-84 \%$ of randomised participants completed the study), however, the dropout rate was balanced between groups. The reasons for dropouts were balanced among the intervention groups. No information on imputation method. The proportion of missing outcomes compared with observed event risk was not enough to induce clinically relevant bias in intervention effect estimate. Data for death and CV hospitalisation were censored

Incomplete outcome data Unclear risk (attrition bias)

weight $(\mathrm{kg})$

Quote from publication: "Analyses of changes in $\mathrm{HbA1c}$ and other quantitative measures at 5 years used a repeated measures model (based on all available data and assuming data were missing at random), including terms for baseline and baseline by visit interaction, with an unstructured covariance matrix for the within-patient variability in each treatment group."

Comment: $>97 \%$ of the randomised participants were included in the analyses. There was a high dropout rate $(82 \%-84 \%$ of randomised participants completed the study), however, the dropout rate was balanced between groups. The reasons for dropouts were balanced among the intervention groups. Imputation by repeated measures model. Not sure if plausible effect size among missing outcomes is enough to induce clinically relevant bias in observed effect size

Incomplete outcome data Unclear risk (attrition bias)

HbA1c
Quote from publication: "Analyses of changes in $\mathrm{HbAlc}$ and other quantitative measures at 5 years used a repeated measures model (based on all available data and assuming data were missing at random), including terms for baseline and baseline by visit interaction, with an unstructured covariance matrix for the within-patient variability in each treatment group."

Comment: $>97 \%$ of the randomised participants were included in the analyses. There was a high dropout rate $(82 \%-84 \%$ of randomised participants completed the study), however, the dropout rate was balanced between groups. The reasons for dropouts were balanced among the intervention groups. Imputation by repeated measures model. Not sure if plausible effect size among missing outcomes is enough to induce clinically relevant bias in observed effect size

\begin{tabular}{|c|c|c|}
\hline $\begin{array}{l}\text { Selective reporting (re- } \\
\text { porting bias) }\end{array}$ & Low risk & $\begin{array}{l}\text { Comment: all of the trial's primary and secondary outcomes as specified in } \\
\text { the protocol have been reported }\end{array}$ \\
\hline
\end{tabular}


Home 2009 (Continued)

Other bias

Unclear risk
Comment: the trial received funding from a pharmaceutical company. Sponsor statisticians were involved in the design, reporting plan, and data analysis. The steering committee had responsibility for study conduct, data collected, data analysis, the writing of reports, and the decision to publish

Leiter 2015

Methods Study design: randomised, double-blind, active-controlled, phase 3, non-inferiority trial

Randomisation ratio: 1:1:1 to receive canagliflozin $100 \mathrm{mg}$, canagliflozin $300 \mathrm{mg}$ or glimepiride in addition to metformin

Non-inferiority and superiority design: non-inferiority of canagliflozin $100 \mathrm{mg}$ or $300 \mathrm{mg}$, or both, to glimepiride for $\mathrm{HbA1}$ c reduction at week 52 . Assessment of non-inferiority of canagliflozin to glimepiride was based on a prespecified non-inferiority margin of $0.3 \%$. If non-inferiority was shown, the protocol specified a step-down assessment of superiority, on the basis of an upper bound of the $95 \% \mathrm{Cl}$ for the difference of each canagliflozin dose versus glimepiride of $<0.0 \%$. All statistical tests were interpreted at a two-sided significance level of $5 \%$, and all $\mathrm{Cls}$ at a two-sided confidence level of $95 \%$.

For body composition descriptive statistics and $95 \% \mathrm{Cls}$ for changes from baseline were provided

Participants

Inclusion criteria: $18-80$ years, $\mathrm{T} 2 \mathrm{DM}, \mathrm{HbA1c}$ of $7.0 \%-9.5 \%$, receiving stable metformin therapy $(\geq 2000$ $\mathrm{mg} /$ day or $\geq 1500 \mathrm{mg} /$ day if unable to tolerate a higher dose) for at least 10 weeks. Participants who were receiving metformin in combination with 1 other oral non-thiazolidinedione antihyperglycaemic drug at screening discontinued the second antihyperglycaemic drug and, if needed, had their metformin dose increased

Exclusion criteria: history of $>1$ severe hypoglycaemic episode (within 6 months); repeated measurements of FPG or fasting self-monitored blood glucose, or both, of $15.0 \mathrm{mmol} / \mathrm{L}$ or more during the pretreatment phase; an estimated GFR of $<55 \mathrm{~mL} / \mathrm{min} / 1.73 \mathrm{~m}^{2}$ (or $<60 \mathrm{~mL} / \mathrm{min} / 1.73 \mathrm{~m}^{2}$ if based on restriction of metformin use in local label) or serum creatinine concentrations of $\geq 124 \mu \mathrm{mol} / \mathrm{L}$ for men and $\geq$ $115 \mu \mathrm{mol} / \mathrm{L}$ for women; or were given thiazolidinedione within 16 weeks before screening

Diagnostic criteria: not reported

Interventions Number of study centres: 157

Run-in period: 2-week, single-blind, placebo run-in period. Participants who were receiving metformin at doses lower than specified in the protocol had their metformin dose increased before entering an up to 12 week metformin dose-stable run-in period before the 2-week, placebo run-in period

Extension period: yes, 52-week, double-blind extension period

Outcomes

Composite outcome measures reported: $\mathrm{HbAlc}$ and weight are reported as separate and as composite outcome measures

\title{
Study details
}

\author{
Trial terminated early: no
}

Trial ID: NCT00968812

\section{Publication details Language of publication: English}

Funding: commercial funding, canagliflozin is marketed under license by Janssen Research \& Development, LLC

Publication status: peer-reviewed journal 
Leiter 2015 (Continued)

Stated aim for study

Quote from publication: "... we assessed the efficacy and safety of canagliflozin compared with glimepiride as add-on therapy in patients with type 2 diabetes inadequately controlled with metformin."

Notes

\section{Risk of bias}

Bias Authors' judgement Support for judgement

Random sequence genera- Low risk tion (selection bias)
Quote from publication: "The sponsor prepared the computer-generated randomisation schedule before the study. Randomisation was balanced with the use of permuted blocks of three patients per block and stratified by whether the patient was taking a stable, protocol-specified dose of metformin before screening versus whether they had either undergone metformin dose adjustment or discontinued use of a second antihyperglycaemic drug, or both, and by country."

Comment: adequate generation of random sequence ensured

\begin{tabular}{ll}
\hline $\begin{array}{l}\text { Allocation concealment } \\
\text { (selection bias) }\end{array}$ & Low risk \\
& $\begin{array}{l}\text { Q } 1: 1: 1 \text { ratio, by an interactive voice or web response system to be given } \\
\text { canagliflozin } 100 \mathrm{mg} \text { or } 300 \mathrm{mg} \text { or glimepiride." }\end{array}$
\end{tabular}

Comment: adequate concealment ensured

\begin{abstract}
Quote from publication: "... double-blind..." "After randomisation, HbA1c and fasting plasma glucose values were masked to staff at the study centres unless values met glycaemic rescue criteria (and were subsequently provided unmasked). Patients, study investigators, and local sponsor personnel were masked to treatment assignment until final database lock. To maintain masked treatment, study drug was supplied in levels (levels one to five) to allow for masked increases and decreases of glimepiride throughout the double-blind treatment period."
\end{abstract}

Comment: investigator-assessed outcome measurement. Blinding of investigator ensured

\begin{abstract}
Blinding of participants Low risk and personnel (performance bias) hypoglycaemia

Quote from publication: "... double-blind..." "After randomisation, HbA1c and fasting plasma glucose values were masked to staff at the study centres unless values met glycaemic rescue criteria (and were subsequently provided unmasked). Patients, study investigators, and local sponsor personnel were masked to treatment assignment until final database lock. To maintain masked treatment, study drug was supplied in levels (levels one to five) to allow for masked increases and decreases of glimepiride throughout the double-blind treatment period."
\end{abstract}

Comment: investigator-assessed/self-reported outcome measurement. Blinding of investigator and participant ensured

\begin{tabular}{|c|c|c|}
\hline $\begin{array}{l}\text { Blinding of participants } \\
\text { and personnel (perfor- } \\
\text { mance bias) } \\
\text { non-fatal myocardial in- } \\
\text { farction/heart failure/non- } \\
\text { fatal stroke }\end{array}$ & Low risk & $\begin{array}{l}\text { Quote from publication: "... double-blind..." "After randomisation, HbA1c } \\
\text { and fasting plasma glucose values were masked to staff at the study centres } \\
\text { unless values met glycaemic rescue criteria (and were subsequently provid- } \\
\text { ed unmasked). Patients, study investigators, and local sponsor personnel } \\
\text { were masked to treatment assignment until final database lock. To maintain } \\
\text { masked treatment, study drug was supplied in levels (levels one to five) to al-- } \\
\text { low for masked increases and decreases of glimepiride throughout the dou- } \\
\text { ble-blind treatment period." }\end{array}$ \\
\hline
\end{tabular}


Comment: investigator-assessed outcome measurement. Blinding of investigator ensured

Blinding of participants Low risk
and personnel (perfor-
mance bias)
non-serious adverse
events

Quote from publication: "... double-blind..." "After randomisation, HbA1c and fasting plasma glucose values were masked to staff at the study centres unless values met glycaemic rescue criteria (and were subsequently provided unmasked). Patients, study investigators, and local sponsor personnel were masked to treatment assignment until final database lock. To maintain masked treatment, study drug was supplied in levels (levels one to five) to allow for masked increases and decreases of glimepiride throughout the double-blind treatment period."

Comment: investigator-assessed/self-reported outcome measurement. Blinding of investigator and participant ensured

Blinding of participants Low risk and personnel (performance bias) serious adverse events
Quote from publication: "... double-blind..." "After randomisation, HbA1c and fasting plasma glucose values were masked to staff at the study centres unless values met glycaemic rescue criteria (and were subsequently provided unmasked). Patients, study investigators, and local sponsor personnel were masked to treatment assignment until final database lock. To maintain masked treatment, study drug was supplied in levels (levels one to five) to allow for masked increases and decreases of glimepiride throughout the double-blind treatment period."

Comment: investigator-assessed outcome measurement. Blinding of investigator ensured

Blinding of participants Low risk
and personnel (performance bias) weight $(\mathrm{kg})$
Quote from publication: "... double-blind..." "After randomisation, HbA1c and fasting plasma glucose values were masked to staff at the study centres unless values met glycaemic rescue criteria (and were subsequently provided unmasked). Patients, study investigators, and local sponsor personnel were masked to treatment assignment until final database lock. To maintain masked treatment, study drug was supplied in levels (levels one to five) to allow for masked increases and decreases of glimepiride throughout the double-blind treatment period."

Comment: investigator-assessed/self-reported outcome measurement. Blinding of investigator and participant ensured

$\begin{array}{ll}\begin{array}{l}\text { Blinding of participants } \\ \text { and personnel (perfor- }\end{array} & \text { Quote from publication: "... double-blind..." "After randomisation, HbA1c } \\ \text { mance bias) } & \begin{array}{l}\text { and fasting plasma glucose values were masked to staff at the study centres } \\ \text { HbAlc }\end{array} \\ & \begin{array}{l}\text { unless values met glycaemic rescue criteria (and were subsequently provid- } \\ \text { ed unmasked). Patients, study investigators, and local sponsor personnel }\end{array} \\ & \text { were masked to treatment assignment until final database lock. To maintain } \\ & \text { masked treatment, study drug was supplied in levels (levels one to five) to al- } \\ & \text { low for masked increases and decreases of glimepiride throughout the dou- } \\ & \text { ble-blind treatment period." }\end{array}$

Comment: investigator-assessed outcome measurement. Blinding of investigator ensured 
Comment: investigator-assessed outcome measurement. Blinding of investigator ensured

Blinding of outcome as- $\quad$ Low risk
sessment (detection bias)

hypoglycaemia
Quote from publication: "... double-blind..." "After randomisation, HbA1c and fasting plasma glucose values were masked to staff at the study centres unless values met glycaemic rescue criteria (and were subsequently provided unmasked). Patients, study investigators, and local sponsor personnel were masked to treatment assignment until final database lock. To maintain masked treatment, study drug was supplied in levels (levels one to five) to allow for masked increases and decreases of glimepiride throughout the double-blind treatment period."

Comment: investigator-assessed/self-reported outcome measurement. Blinding of investigator and participant ensured
Blinding of outcome as- Low risk sessment (detection bias) non-fatal myocardial infarction/heart failure/nonfatal stroke
Quote from publication: "... double-blind..." "After randomisation, HbA1c and fasting plasma glucose values were masked to staff at the study centres unless values met glycaemic rescue criteria (and were subsequently provided unmasked). Patients, study investigators, and local sponsor personnel were masked to treatment assignment until final database lock. To maintain masked treatment, study drug was supplied in levels (levels one to five) to allow for masked increases and decreases of glimepiride throughout the double-blind treatment period."

Comment: investigator-assessed outcome measurement. Blinding of investigator ensured

\section{Blinding of outcome as- Low risk} sessment (detection bias) non-serious adverse events
Quote from publication: "... double-blind..." "After randomisation, HbA1c and fasting plasma glucose values were masked to staff at the study centres unless values met glycaemic rescue criteria (and were subsequently provided unmasked). Patients, study investigators, and local sponsor personnel were masked to treatment assignment until final database lock. To maintain masked treatment, study drug was supplied in levels (levels one to five) to allow for masked increases and decreases of glimepiride throughout the double-blind treatment period."

Comment: investigator-assessed/self-reported outcome measurement. Blinding of investigator and participant ensured

Blinding of outcome as- $\quad$ Low risk
sessment (detection bias)

serious adverse events
Quote from publication: "... double-blind..." "After randomisation, HbA1c and fasting plasma glucose values were masked to staff at the study centres unless values met glycaemic rescue criteria (and were subsequently provided unmasked). Patients, study investigators, and local sponsor personnel were masked to treatment assignment until final database lock. To maintain masked treatment, study drug was supplied in levels (levels one to five) to allow for masked increases and decreases of glimepiride throughout the double-blind treatment period."

Comment: investigator-assessed outcome measurement. Blinding of investigator ensured

Quote from publication: "... double-blind..." "After randomisation, HbA1c and fasting plasma glucose values were masked to staff at the study centres unless values met glycaemic rescue criteria (and were subsequently provided unmasked). Patients, study investigators, and local sponsor personnel were masked to treatment assignment until final database lock. To maintain masked treatment, study drug was supplied in levels (levels one to five) to allow for masked increases and decreases of glimepiride throughout the double-blind treatment period." 
Comment: investigator-assessed/self-reported outcome measurement. Blinding of investigator and participant ensured

Blinding of outcome as- $\quad$ Low risk
sessment (detection bias)
HbAlc

\begin{abstract}
Quote from publication: "... double-blind..." "After randomisation, HbA1c and fasting plasma glucose values were masked to staff at the study centres unless values met glycaemic rescue criteria (and were subsequently provided unmasked). Patients, study investigators, and local sponsor personnel were masked to treatment assignment until final database lock. To maintain masked treatment, study drug was supplied in levels (levels one to five) to allow for masked increases and decreases of glimepiride throughout the double-blind treatment period."
\end{abstract}

Comment: investigator-assessed outcome measurement. Blinding of investigator ensured

\begin{abstract}
Incomplete outcome data Low risk (attrition bias) all-cause/cardiovascular mortality

Quote from publication: "We did primary efficacy analyses in the modified intention-to-treat population, according to randomised treatment assignment. We did safety analyses in the same population according to the predominant treatment received (no patients received treatment other than that to which they were randomly assigned, so the modified intention-to-treat and safety analysis populations were identical). Missing data were imputed with the LOCF approach; in patients given glycaemic rescue drug, the last observation before rescue initiation was used." "The proportions of patients who received glycemic rescue therapy during the entire 104-week treatment period were $19.9 \%, 13.0 \%$, and $20.9 \%$, respectively, in the canagliflozin 100 and $300 \mathrm{mg}$ and glimepiride groups therapy."
\end{abstract}

Comment: $>99 \%$ of the randomised participants were included in the analyses. There was a high dropout rate (65\%-71\% of randomised participants completed the study), however, the dropout rate was balanced between groups. Inappropriate method of imputing missing data was used (LOCF). We assumed that trial authors searched registers for mortality status at the end of the trial
Incomplete outcome data Low risk (attrition bias)

hypoglycaemia
Quote from publication: "We did primary efficacy analyses in the modified intention-to-treat population, according to randomised treatment assignment. We did safety analyses in the same population according to the predominant treatment received (no patients received treatment other than that to which they were randomly assigned, so the modified intention-to-treat and safety analysis populations were identical). Missing data were imputed with the LOCF approach; in patients given glycaemic rescue drug, the last observation before rescue initiation was used." "The proportions of patients who received glycemic rescue therapy during the entire 104-week treatment period were $19.9 \%, 13.0 \%$, and $20.9 \%$, respectively, in the canagliflozin 100 and $300 \mathrm{mg}$ and glimepiride groups therapy."

Comment: $>99 \%$ of the randomised participants were included in the analyses. There was a high dropout rate $(65 \%-71 \%$ of randomised participants completed the study), however, the dropout rate was balanced between groups. The reasons for dropouts were balanced among the intervention groups. Inappropriate method of imputing missing data was used (LOCF). The proportion of missing outcomes compared with observed event risk was not enough to have a clinically relevant impact on the intervention effect estimate
Incomplete outcome data High risk (attrition bias) non-fatal myocardial infarction/heart failure/nonfatal stroke
Quote from publication: "We did primary efficacy analyses in the modified intention-to-treat population, according to randomised treatment assignment. We did safety analyses in the same population according to the predominant treatment received (no patients received treatment other than that to which they were randomly assigned, so the modified intention-to-treat and safety analysis populations were identical). Missing data were imputed with the LOCF approach; in patients given glycaemic rescue drug, the 
Leiter 2015 (Continued)

last observation before rescue initiation was used." "The proportions of patients who received glycemic rescue therapy during the entire 104-week treatment period were $19.9 \%, 13.0 \%$, and $20.9 \%$, respectively, in the canagliflozin 100 and $300 \mathrm{mg}$ and glimepiride groups therapy."

Comment: $>99 \%$ of the randomised participants were included in the analyses. There was a high dropout rate $(65 \%-71 \%$ of randomised participants completed the study), however, the dropout rate was balanced between groups. The reasons for dropouts were balanced among the intervention groups. Inappropriate method of imputing missing data was used (LOCF). The proportion of missing outcomes compared with observed event risk was enough to induce clinically relevant bias in intervention effect estimate

Incomplete outcome data Low risk (attrition bias) non-serious adverse events
Quote from publication: "We did primary efficacy analyses in the modified intention-to-treat population, according to randomised treatment assignment. We did safety analyses in the same population according to the predominant treatment received (no patients received treatment other than that to which they were randomly assigned, so the modified intention-to-treat and safety analysis populations were identical). Missing data were imputed with the LOCF approach; in patients given glycaemic rescue drug, the last observation before rescue initiation was used." "The proportions of patients who received glycemic rescue therapy during the entire 104-week treatment period were $19.9 \%, 13.0 \%$, and $20.9 \%$, respectively, in the canagliflozin 100 and $300 \mathrm{mg}$ and glimepiride groups therapy."

Comment: $>99 \%$ of the randomised participants were included in the analyses. There was a high dropout rate $65 \%-71 \%$ of randomised participants completed the study), however, the dropout rate was balanced between groups. The reasons for dropouts were balanced among the intervention groups. Inappropriate method of imputing missing data was used (LOCF). The proportion of missing outcomes compared with observed event risk was not enough to have a clinically relevant impact on the intervention effect estimate
Incomplete outcome data High risk (attrition bias)

serious adverse events
Quote from publication: "We did primary efficacy analyses in the modified intention-to-treat population, according to randomised treatment assignment. We did safety analyses in the same population according to the predominant treatment received (no patients received treatment other than that to which they were randomly assigned, so the modified intention-to-treat and safety analysis populations were identical). Missing data were imputed with the LOCF approach; in patients given glycaemic rescue drug, the last observation before rescue initiation was used." "The proportions of patients who received glycemic rescue therapy during the entire 104-week treatment period were $19.9 \%, 13.0 \%$, and $20.9 \%$, respectively, in the canagliflozin 100 and $300 \mathrm{mg}$ and glimepiride groups therapy."

Comment: $>99 \%$ of the randomised participants were included in the analyses. There was a high dropout rate $(65 \%-71 \%$ of randomised participants completed the study), however, the dropout rate was balanced between groups. The reasons for dropouts were balanced among the intervention groups. Inappropriate method of imputing missing data was used (LOCF). The proportion of missing outcomes compared with observed event risk was enough to induce clinically relevant bias in intervention effect estimate

Quote from publication: "We did primary efficacy analyses in the modified intention-to-treat population, according to randomised treatment assignment. We did safety analyses in the same population according to the predominant treatment received (no patients received treatment other than that to which they were randomly assigned, so the modified intention-to-treat and safety analysis populations were identical). Missing data were imputed with the LOCF approach; in patients given glycaemic rescue drug, the last observation before rescue initiation was used." "The proportions of patients who received 
Leiter 2015 (Continued)

glycemic rescue therapy during the entire 104-week treatment period were $19.9 \%, 13.0 \%$, and $20.9 \%$, respectively, in the canagliflozin 100 and $300 \mathrm{mg}$ and glimepiride groups therapy."

Comment: $>99 \%$ of the randomised participants were included in the analyses. There was a high dropout rate $(65 \%-71 \%$ of randomised participants completed the study), however, the dropout rate was balanced between groups. The reasons for dropouts were balanced among the intervention groups. Inappropriate method of imputing missing data was used (LOCF). Not sure if plausible effect size among missing outcomes is enough to induce clinically relevant bias in observed effect size

Incomplete outcome data Unclear risk (attrition bias)

HbAlc
Quote from publication: "We did primary efficacy analyses in the modified intention-to-treat population, according to randomised treatment assignment. We did safety analyses in the same population according to the predominant treatment received (no patients received treatment other than that to which they were randomly assigned, so the modified intention-to-treat and safety analysis populations were identical). Missing data were imputed with the LOCF approach; in patients given glycaemic rescue drug, the last observation before rescue initiation was used." "The proportions of patients who received glycemic rescue therapy during the entire 104-week treatment period were $19.9 \%, 13.0 \%$, and $20.9 \%$, respectively, in the canagliflozin 100 and $300 \mathrm{mg}$ and glimepiride groups therapy."

Comment: $>99 \%$ of the randomised participants were included in the analyses. There was a high dropout rate $65 \%-71 \%$ of randomised participants completed the study), however, the dropout rate was balanced between groups. The reasons for dropouts were balanced among the intervention groups. Inappropriate method of imputing missing data was used (LOCF). Not sure if plausible effect size among missing outcomes is enough to induce clinically relevant bias in observed effect size

\begin{tabular}{lll}
\hline $\begin{array}{l}\text { Selective reporting (re- } \\
\text { porting bias) }\end{array}$ & Low risk & $\begin{array}{l}\text { Comment: all of the trial's primary and secondary outcomes as specified in } \\
\text { the protocol have been reported }\end{array}$ \\
\hline Other bias & Unclear risk & Comment: the trial received funding from a pharmaceutical company \\
\hline
\end{tabular}

\section{Maffioli 2013}

\begin{tabular}{ll}
\hline Methods & Study design: randomised, double-blind, parallel-study \\
& Randomisation ratio: $1: 1$ to receive pioglitazone or glibenclamide alone or in combination with rosu- \\
vastatin in addition to metformin therapy
\end{tabular}


Maffioli 2013 (Continued)

cations to pioglitazone (osteoporosis and heart failure) or glibenclamide (frequent hypoglycaemia) or HMG-CoA inhibitors (previous rhabdomyolysis, muscular pathologies)

Diagnostic criteria: ESC and EASD 2007

\begin{tabular}{ll}
\hline Interventions & Number of study centres: 1 \\
Run-in period: yes, 3-month run-in period with metformin $850 \mathrm{mg} 3 \times /$ day \\
Extension period: no
\end{tabular}

Outcomes Composite outcome measures reported: no

Study details Trial terminated early: no

Trial ID: -

\begin{tabular}{ll}
\hline Publication details & $\begin{array}{l}\text { Language of publication: English } \\
\text { Funding: non-commercial funding } \\
\text { Publication status: peer-reviewed journal }\end{array}$ \\
\hline Stated aim for study & $\begin{array}{l}\text { Quote from publication: "... the aim of this study was to evaluate the effects of pioglitazone or gliben- } \\
\text { clamide alone and in combination with rosuvastatin on hepatic steatosis, evaluated by abdominal ul- } \\
\text { trasonography, in type } 2 \text { diabetic patients." }\end{array}$
\end{tabular}

Notes -

\section{Risk of bias}

\begin{tabular}{ll}
\hline Bias $\quad$ Authors' judgement & Support for judgement \\
\hline
\end{tabular}

Random sequence genera- Low risk tion (selection bias)

\begin{abstract}
Quote from publication: "Pioglitazone, glibenclamide, and rosuvastatin were supplied as identical, opaque, white capsules in coded bottles to ensure the blind status of the study. Randomization was carried out using a drawing of envelopes containing randomization codes prepared by a statistician. A copy of the code was provided only to the responsible individual carrying out the statistical analysis. The code was only broken after database lock, but could have been broken for individual patients in cases of an emergency."
\end{abstract}

Comment: adequate generation of random sequence ensured
Quote from publication: "Pioglitazone, glibenclamide, and rosuvastatin were supplied as identical, opaque, white capsules in coded bottles to ensure the blind status of the study. Randomization was carried out using a drawing of envelopes containing randomization codes prepared by a statistician. A copy of the code was provided only to the responsible individual carrying out the statistical analysis. The code was only broken after database lock, but could have been broken for individual patients in cases of an emergency."

Comment: adequate concealment of allocation ensured 
Maffioli 2013 (Continued)

Blinding of participants and personnel (perfor-

Low risk mance bias)

HbA1c
Quote from publication: "double-blind". "Pioglitazone, glibenclamide, and rosuvastatin were supplied as identical, opaque, white capsules in coded bottles to ensure the blind status of the study."

Comment: investigator-assessed outcome measurement. Blinding of investigator ensured

\section{Blinding of outcome as- Low risk} sessment (detection bias) weight $(\mathrm{kg})$
Quote from publication: "double-blind". "Pioglitazone, glibenclamide, and rosuvastatin were supplied as identical, opaque, white capsules in coded bottles to ensure the blind status of the study."

Comment: investigator-assessed outcome measurement. Blinding of investigator ensured
Blinding of outcome as- Low risk sessment (detection bias) HbA1c
Quote from publication: "double-blind". "Pioglitazone, glibenclamide, and rosuvastatin were supplied as identical, opaque, white capsules in coded bottles to ensure the blind status of the study."

Comment: investigator-assessed outcome measurement. Blinding of investigator ensured

Incomplete outcome data Low risk (attrition bias)

weight $(\mathrm{kg})$
Quote from publication: "Every patient who had received at least one dose of the study medication underwent a tolerability observation to exclude the presence of acute adverse reactions"; "Then, an intention-to-treat analysis was carried out in patients who had received one or more doses of study medication, did not show any acute adverse reaction, and had undergone a subsequent efficacy observation."

Comment: $94 \%$ of randomised participants completed the study. $94 \%$ of randomised participants were included in the analysis. Only participants who completed the study were included in the analysis. The number of participants who dropped out was balanced between groups. The reasons for dropouts were balanced among the intervention groups. Plausible effect size among missing outcomes was not enough to have a clinically relevant impact on observed effect size

\section{Incomplete outcome data Low risk} (attrition bias)

$\mathrm{HbA1c}$
Quote from publication: "Every patient who had received at least one dose of the study medication underwent a tolerability observation to exclude the presence of acute adverse reactions"; "Then, an intention-to-treat analysis was carried out in patients who had received one or more doses of study medication, did not show any acute adverse reaction, and had undergone a subsequent efficacy observation."

Comment: $94 \%$ of randomised participants completed the study. $94 \%$ of randomised participants were included in the analysis. Only participants who completed the study were included in the analysis. The number of participants who dropped out was balanced between groups. The reasons for dropouts were balanced among the intervention groups. Plausible effect size among missing outcomes was not enough to have a clinically relevant impact on observed effect size
Selective reporting (re- High risk porting bias)
Comment: no trial protocol available. All of the trial's prespecified primary and secondary outcomes (methods section) have been reported. Unclear whether common outcomes (all-cause mortality) were measured; not mentioned, but clinical judgement says likely to have been measured and analysed but not reported on the basis of non-significant results. Incomplete reporting of adverse events and hypoglycaemia, only events leading to discontinuation are mentioned 
Maffioli 2013 (Continued)

Other bias Unclear risk Comment: in 2011 the primary author performed a similar study (Derosa 2011b)

Matthews 2010

Methods Study design: multicentre, randomised, double-blind, double-dummy, active-controlled study

Randomisation ratio: 1:1 to receive glimepiride or vildagliptin in addition to metformin

Non-inferiority design: 1-sided confidence interval

Participants

Inclusion criteria: male and female participants (non-fertile or using a medically approved birth control method) with T2DM and HbA1c of $6.5 \%-8.5 \%$, who had received metformin for $\geq 3$ months and were on a stable dose of $\geq 1500 \mathrm{mg}$ daily for a minimum of $\geq 4$ weeks prior to visit 1 , aged $18-73$ years, BMI of $22-45 \mathrm{~kg} / \mathrm{m}^{2}$

Exclusion criteria: T1DM or secondary forms of diabetes, acute metabolic diabetic complications in the past 6 months, acute infections that might affect blood glucose control in the 4 weeks prior to visit 1 , serious cardiac conditions (history of torsades de pointes or ventricular tachycardia; percutaneous coronary intervention in the past 3 months; myocardial infarction, coronary artery bypass surgery, unstable angina or stroke in the past 6 months; congestive heart failure requiring pharmacological treatment; second- or third-degree atrioventricular block or prolonged QTc) or clinically significant liver or renal disease, ALT or AST > $3 \times$ ULN, direct bilirubin $>1.3 \times$ ULN, serum creatinine levels $\geq 132 \mathrm{mmol} / \mathrm{L}$ in men or $\geq 123 \mathrm{mmol} / \mathrm{L}$ in women, clinically significant TSH outside of normal range at screening; or fasting triglycerides $>7.9 \mathrm{mmol} / \mathrm{L}$

Diagnostic criteria: not reported

Interventions

\section{Number of study centres: 402}

Run-in period: no

Extension period: no

\section{Outcomes}

Composite outcome measures reported: yes, composite outcome measure of cardiovascular and cerebrovascular outcomes

Study details

Trial terminated early: yes. "The study was originally planned to last for up to 5 years to assess treatment durability, and had a primary endpoint of risk of failure of glycaemic control defined as $\mathrm{HbAl}$ $>8 \%$. However, the scope of the study was later modified because of a much higher than expected discontinuation rate and fewer participants than expected reaching the endpoint of $\mathrm{HbAlc}>8 \%$ (because of a change in standards of care, meaning that participants dropped out of the study to receive additional therapy before reaching this endpoint). The power was re-estimated using a blinded sample population and found to be insufficient. The study purpose was therefore amended to a 2-year non-inferiority study and the primary endpoint altered to 'change in $\mathrm{HbA1c}$ from baseline to week 104 endpoint'. The trial ended when the last randomized participant had been in the study for 2 years."

Trial ID: NCT00106340, EudraCT2004-004559-21, CLAF237A2308

\section{Language of publication: English}

Funding: commercial funding by Novartis Pharmaceuticals Corporation

Publication status: peer-reviewed journal
Quote from publication: "To show that vildagliptin added to metformin is non-inferior to glimepiride in reducing HbA1c levels from baseline over 2 years" 
Matthews 2010 (Continued)

Notes

\section{Risk of bias}

\begin{tabular}{lll}
\hline Bias & Authors' judgement & Support for judgement \\
\hline $\begin{array}{l}\text { Random sequence genera- } \\
\text { tion (selection bias) }\end{array}$ & Unclear risk & Quote from publication: "...patients were randomized..." \\
& Comment: insufficient information about the sequence generation \\
$\begin{array}{l}\text { Allocation concealment } \\
\text { (selection bias) }\end{array}$ & Unclear risk & Quote from publication: "...patients were randomized..." \\
& & $\begin{array}{l}\text { Comment: insufficient information about the allocation concealment to per- } \\
\text { mit judgement of 'low risk' or 'high risk' }\end{array}$
\end{tabular}

\begin{tabular}{|c|c|c|}
\hline $\begin{array}{l}\text { Blinding of participants } \\
\text { and personnel (perfor- } \\
\text { mance bias) } \\
\text { all-cause/cardiovascular }\end{array}$ & Low risk & $\begin{array}{l}\text { Quote from publication: "Masking: Double Blind (Subject, Caregiver, Investi- } \\
\text { gator, Outcomes Assessor)" "...double-dummy..." "An independent cardiovas- } \\
\text { cular and cerebrovascular (CCV) adjudication committee reviewed all occur- } \\
\text { rences af CCV events in a blinded fashion." }\end{array}$ \\
\hline
\end{tabular}

all-cause/cardiovascula

mortality

Comment: adjudicated and investigator-assessed outcome measurement. Blinding of adjudication committee and study personnel ensured

\begin{tabular}{|c|c|c|}
\hline $\begin{array}{l}\text { Blinding of participants } \\
\text { and personnel (perfor- } \\
\text { mance bias) }\end{array}$ & Low risk & $\begin{array}{l}\text { Quote from publication: "Masking: Double Blind (Subject, Caregiver, Inves- } \\
\text { tigator, Outcomes Assessor)" "...double-dummy..." "...self-monitored plasma } \\
\text { glucose..." }\end{array}$ \\
\hline
\end{tabular}

hypoglycaemia

Comment: investigator-assessed and self-reported outcome measurement. Blinding of participant and study personnel ensured

Blinding of participants Low risk
and personnel (perfor-
mance bias)
non-fatal myocardial in-
farction/heart failure/non-
fatal stroke

Blinding of participants Low risk
and personnel (performance bias) serious adverse events
Quote from publication: "Masking: Double Blind (Subject, Caregiver, Investigator, Outcomes Assessor)" "...double-dummy..." "An independent cardiovascular and cerebrovascular (CCV) adjudication committee reviewed all occurrences af CCV events in a blinded fashion."

Comment: adjudicated outcome measurement. Blinding of adjudication committee ensured
Quote from publication: "Masking: Double Blind (Subject, Caregiver, Investigator, Outcomes Assessor)" "...double-dummy..." "An independent cardiovascular and cerebrovascular (CCV) adjudication committee reviewed all occurrences af CCV events in a blinded fashion."

Comment: adjudicated and investigator-assessed outcome measurement. Blinding of adjudication committee and study personnel ensured

\begin{tabular}{|c|c|c|}
\hline $\begin{array}{l}\text { Blinding of participants } \\
\text { and personnel (perfor- } \\
\text { mance bias) } \\
\text { weight (kg) }\end{array}$ & Low risk & $\begin{array}{l}\text { Quote from publication: "Masking: Double Blind (Subject, Caregiver, Investi- } \\
\text { gator, Outcomes Assessor)" "...double-dummy..." } \\
\text { Comment: investigator-assessed and self-reported outcome measurement. } \\
\text { Blinding of participant and study personnel ensured }\end{array}$ \\
\hline
\end{tabular}

Blinding of participants Low risk and personnel (performance bias)

HbAlc
Quote from publication: "Masking: Double Blind (Subject, Caregiver, Investi-
gator, Outcomes Assessor)" "...double-dummy..."

Comment: investigator-assessed outcome measurement. Blinding of study personnel ensured

$\begin{array}{ll}\begin{array}{l}\text { Blinding of outcome as- } \\ \text { sessment (detection bias) }\end{array} \quad \text { Low risk } & \begin{array}{l}\text { Quote from publication: "Masking: Double Blind (Subject, Caregiver, Investi- } \\ \text { gator, Outcomes Assessor)" "...double-dummy..." "An independent cardiovas- }\end{array}\end{array}$


Matthews 2010 (Continued) all-cause/cardiovascular mortality cular and cerebrovascular (CCV) adjudication committee reviewed all occurrences af CCV events in a blinded fashion."

Comment: adjudicated and investigator-assessed outcome measurement. Blinding of outcome assessor ensured

\begin{tabular}{|c|c|c|}
\hline $\begin{array}{l}\text { Blinding of outcome as- } \\
\text { sessment (detection bias) } \\
\text { hypoglycaemia }\end{array}$ & Low risk & $\begin{array}{l}\text { Quote from publication: "Masking: Double Blind (Subject, Caregiver, Inves- } \\
\text { tigator, Outcomes Assessor)" "...double-dummy..." "...self-monitored plasma } \\
\text { glucose..." }\end{array}$ \\
\hline
\end{tabular}

Comment: investigator-assessed and self-reported outcome measurement. Blinding of outcome assessor ensured
Low risk

Blinding of outcome as sessment (detection bias) non-fatal myocardial infarction/heart failure/nonfatal stroke
Quote from publication: "Masking: Double Blind (Subject, Caregiver, Investigator, Outcomes Assessor)" "...double-dummy..." "An independent cardiovascular and cerebrovascular (CCV) adjudication committee reviewed all occurrences af CCV events in a blinded fashion."

Comment: adjudicated outcome measurement. Blinding of outcome assessor ensured
Blinding of outcome as- Low risk sessment (detection bias) serious adverse events
Quote from publication: "Masking: Double Blind (Subject, Caregiver, Investigator, Outcomes Assessor)" "...double-dummy..." "An independent cardiovascular and cerebrovascular (CCV) adjudication committee reviewed all occurrences af CCV events in a blinded fashion."

Comment: adjudicated and investigator-assessed outcome measurement. Blinding of outcome assessor ensured
Blinding of outcome as- Low risk sessment (detection bias) weight $(\mathrm{kg})$
Quote from publication: "Masking: Double Blind (Subject, Caregiver, Investigator, Outcomes Assessor)" "...double-dummy..."

Comment: investigator-assessed and self-reported outcome measurement. Blinding of outcome assessor ensured

Quote from publication: "Masking: Double Blind (Subject, Caregiver, Investigator, Outcomes Assessor)" "...double-dummy..."

Comment: investigator-assessed outcome measurement. Blinding of outcome assessor ensured

Blinding of outcome as- Low risk sessment (detection bias) HbA1c

\section{Incomplete outcome data Low risk} (attrition bias) all-cause/cardiovascular mortality
Quote from publication: "Safety population: All patients who received at least one dose of study drug and had at least one post baseline safety assessment (including patients on rescue medication)."

Comment: $>99 \%$ of the randomised participants were included in the analyses. There was a high dropout rate (61\%-64\% of randomised participants completed the study), however, the dropout rate was balanced between groups. No information on imputation method. We assumed that trial authors searched registers for mortality status at the end of the trial

Incomplete outcome data High risk
(attrition bias)
hypoglycaemia

Quote from publication: "Safety population: All patients who received at least one dose of study drug and had at least one post baseline safety assessment (including patients on rescue medication)."

Comment: $>99 \%$ of the randomised participants were included in the analyses. There was a high dropout rate $(61 \%-64 \%$ of randomised participants completed the study), however, the dropout rate was balanced between groups. The reasons for dropouts were balanced among the intervention groups. No information on imputation method. The proportion of missing outcomes com- 
Incomplete outcome data High risk (attrition bias)

non-fatal myocardial infatal stroke farction/heart failure/non-

Quote from publication: "Safety population: All patients who received at least one dose of study drug and had at least one post baseline safety assessment (including patients on rescue medication)."

Comment: $>99 \%$ of the randomised participants were included in the analyses. There was a high dropout rate (61\%-64\% of randomised participants completed the study), however, the dropout rate was balanced between groups. The reasons for dropouts were balanced among the intervention groups. No information on imputation method. The proportion of missing outcomes compared with observed event risk was enough to induce clinically relevant bias in intervention effect estimate

Incomplete outcome data High risk (attrition bias)

serious adverse events

Quote from publication: "Safety population: All patients who received at least one dose of study drug and had at least one post baseline safety assessment (including patients on rescue medication)."
Comment: $>99 \%$ of the randomised participants were included in the analyses. There was a high dropout rate $(61 \%-64 \%$ of randomised participants completed the study), however, the dropout rate was balanced between groups. The reasons for dropouts were balanced among the intervention groups. No information on imputation method. The proportion of missing outcomes compared with observed event risk was enough to induce clinically relevant bias in intervention effect estimate

\section{Incomplete outcome data Unclear risk} (attrition bias) weight $(\mathrm{kg})$

Quote from publication: "Intent-to- treat (ITT) population: All patients who received at least one dose of study drug and had at least one post baseline efficacy assessment (primary or secondary). Assessments made while on rescue medication were not included."

Comment: $>97 \%$ of the randomised participants were included in the analyses. There was a high dropout rate $(61 \%-64 \%$ of randomised participants completed the study), however, the dropout rate was balanced between groups. The reasons for dropouts were balanced among the intervention groups. No information on imputation method. Not sure if plausible effect size among missing outcomes is enough to induce clinically relevant bias in observed effect size

\section{Incomplete outcome data Unclear risk} (attrition bias)

HbAlc
Quote from publication: "Intent-to- treat (ITT) population: All patients who received at least one dose of study drug and had at least one post baseline efficacy assessment (primary or secondary). Assessments made while on rescue medication were not included." "The primary efficacy assessment was change from baseline in $\mathrm{HbAlc}$ at study end point, using last observation carried forward for patients who discontinued early. For patients who received rescue medication, the week 104 endpoint was defined as the measurement obtained at the last visit before rescue medication."

Comment: $>94 \%$ of the randomised participants were included in the analyses. There was a high dropout rate $(61 \%-64 \%$ of randomised participants completed the study), however, the dropout rate was balanced between groups. The reasons for dropouts were balanced among the intervention groups. Inappropriate method of imputing missing data was used (LOCF). Not sure if plausible effect size among missing outcomes is enough to induce clinically relevant bias in observed effect size

Comment: all of the trial's primary and secondary outcomes as specified in the protocol have been reported 
Matthews 2010 (Continued)

Other bias Unclear risk Comment: the trial received funding from a pharmaceutical company

Nauck 2013

Methods Study design: double-blind, double-dummy, active-control, parallel-group, multicentre, multinational trial

Randomisation ratio: 2:2:2:1:2 to receive one of three doses of liraglutide $(0.6,1.2$ or $1.8 \mathrm{mg} / \mathrm{day})$ in addition to metformin, metformin plus placebo (liraglutide or glimepiride) or glimepiride in addition to metformin

Superiority design or non-inferiority design: superiority of glycaemic control with liraglutide was determined if the upper limit of the two-sided $95 \%$ confidence interval for the treatment difference was < $0 \%$, non-inferiority was concluded if $<0.4 \%$. Significance was $\mathrm{P}<0.05$

\section{Participants}

Inclusion criteria: T2DM, 18-80 years of age, $\mathrm{HbA1c}$ between $7 \%$ and $11 \%$ (prestudy OAD monotherapy for $\geq 3$ months) or between $7 \%$ and $10 \%$ (prestudy combination OAD therapy for $\geq 3$ months), $B M I \leq 40$ $\mathrm{kg} / \mathrm{m}^{2}$

Exclusion criteria: used insulin during the previous 3 months (except short-term treatment), any serious medical condition, women of child bearing potential who were pregnant, breast-feeding or intending to become pregnant or not using adequate contraceptive methods, participants using any drug (except for OADs), which in the Investigator's opinion could interfere with the glucose level (e.g. systemic corticosteroids)

Diagnostic criteria: not reported

Interventions Number of study centres: 170

Run-in period: 3-week forced titration period followed by a 3-week metformin maintenance period. Particiapnts taking metformin at enrolment could go through a modified titration period or advance directly to the metformin maintenance period. After randomisation participants underwent a 2- and 3week titration period of for liraglutide and glimepiride

Extension period: yes, participants completing the 26-week double-blind core period could enrol in an 18-month open-label extension period

Outcomes Composite outcome measures reported: yes, composite endpoint of $\mathrm{HbA} 1 \mathrm{c}<7.0 \%(<53 \mathrm{mmol} / \mathrm{mol})$,
no weight gain and no hypoglycaemia

Study details Trial terminated early: no

Trial ID: NCT00318461

\section{Publication details Language of publication: English}

Funding: commercial funding, sponsored by Novo Nordisk A/S

Publication status: peer-reviewed journal

$$
\begin{aligned}
& \text { Quote from publication: "To investigate efficacy and safety of dual therapy with liraglutide and met- } \\
& \text { formin in comparison to glimepiride and metformin, and metformin monotherapy over } 2 \text { years in pa- } \\
& \text { tients with type } 2 \text { diabetes." }
\end{aligned}
$$

$$
\text { Notes }
$$

\section{Risk of bias}


Nauck 2013 (Continued)

Bias Authors' judgement Support for judgement

Random sequence genera- Low risk tion (selection bias)
Quote from publication: "Randomization was performed using a telephone-based or web-based randomization system. Subjects were randomly assigned to the lowest available randomization number and stratified with respect to their previous use of OAD monotherapy or combination therapy."

Comment: adequate generation of random sequence ensured
Allocation concealment Low risk (selection bias)

Quote from publication: "Randomization was performed using a telephone-based or web-based randomization system. Subjects were randomly assigned to the lowest available randomization number and stratified with respect to their previous use of OAD monotherapy or combination therapy."

Comment: adequate concealment ensured

Blinding of participants and personnel (performance bias) all-cause/cardiovascular mortality
Low risk

Quote from publication: "... double-dummy... The double-dummy design required that subjects in the liraglutide and placebo groups received a glimepiride placebo, whereas subjects in the glimepiride and placebo groups received an injection of liraglutide placebo." "Subjects completing the study could enroll in an 18-month open-label extension period."

Comment: investigator-assessed outcome measurement. Incomplete blinding, but the outcome is not likely to be influenced by lack of blinding due to objective assessment of the outcome

\begin{tabular}{|c|c|c|}
\hline $\begin{array}{l}\text { Blinding of participants } \\
\text { and personnel (perfor- } \\
\text { mance bias) } \\
\text { amputation of lower ex- } \\
\text { tremity/blindness or se- }\end{array}$ & Low risk & $\begin{array}{l}\text { Quote from publication: "... double-dummy... The double-dummy design re- } \\
\text { quired that subjects in the liraglutide and placebo groups received a glimepiri- } \\
\text { de placebo, whereas subjects in the glimepiride and placebo groups received } \\
\text { an injection of liraglutide placebo." "Subjects completing the study could en- } \\
\text { roll in an 18-month open-label extension period." }\end{array}$ \\
\hline
\end{tabular}
vere vision loss/end-stage renal disease
Comment: investigator-assessed outcome measurement. Incomplete blinding, but the outcome is not likely to be influenced by lack of blinding due to objective assessment of the outcome

$\begin{array}{ll}\begin{array}{l}\text { Blinding of participants } \\ \text { and personnel (perfor- }\end{array} & \begin{array}{l}\text { Quote from publication: "... double-dummy... The double-dummy design re- } \\ \text { quired that subjects in the liraglutide and placebo groups received a glimepiri- } \\ \text { mance bias) }\end{array} \\ \begin{array}{l}\text { de placebo, whereas subjects in the glimepiride and placebo groups received } \\ \text { anpoglycaemia }\end{array} & \begin{array}{l}\text { anjection of liraglutide placebo." "Subjects completing the study could en- } \\ \text { roll in an 18-month open-label extension period." "Hypoglycaemic episodes }\end{array} \\ & \text { were defined as major, minor, or symptoms only. Major if the subject was un- } \\ & \text { able to treat her/himself. Minor if subject was able to treat her/himself and } \\ \text { plasma glucose was below } 3.1 \mathrm{mmol} / \mathrm{L} . "\end{array}$

Comment: self-reported outcome measurement. Incomplete blinding, but the outcome is not likely to be influenced by lack of blinding due to objective assessment of the outcome

\author{
Blinding of participants Low risk \\ and personnel (perfor- \\ mance bias) \\ non-fatal myocardial in- \\ farction/heart failure/non- \\ fatal stroke
}

\begin{abstract}
Quote from publication: "... double-dummy... The double-dummy design required that subjects in the liraglutide and placebo groups received a glimepiride placebo, whereas subjects in the glimepiride and placebo groups received an injection of liraglutide placebo." "Subjects completing the study could enroll in an 18-month open-label extension period."
\end{abstract}

Comment: investigator-assessed outcome measurement. Incomplete blinding, but the outcome is not likely to be influenced by lack of blinding due to objective assessment of the outcome 
Nauck 2013 (Continued)

Blinding of participants and personnel (performance bias) non-serious adverse events
High risk

Quote from publication: "... double-dummy... The double-dummy design required that subjects in the liraglutide and placebo groups received a glimepiride placebo, whereas subjects in the glimepiride and placebo groups received an injection of liraglutide placebo." "Subjects completing the study could enroll in an 18-month open-label extension period."

Comment: self-reported outcome measurement. Incomplete blinding, the outcome is likely to be influenced by lack of blinding due to subjective assessment of the outcome and personnel (performance bias) serious adverse events
Blinding of participants Low risk

Quote from publication: "... double-dummy... The double-dummy design required that subjects in the liraglutide and placebo groups received a glimepiride placebo, whereas subjects in the glimepiride and placebo groups received an injection of liraglutide placebo." "Subjects completing the study could enroll in an 18-month open-label extension period."

Comment: investigator-assessed outcome measurement. Incomplete blinding, but the outcome is not likely to be influenced by lack of blinding due to objective assessment of the outcome

\section{Blinding of participants Low risk} and personnel (perfor-

Quote from publication: "... double-dummy... The double-dummy design remance bias) weight $(\mathrm{kg})$ quired that subjects in the liraglutide and placebo groups received a glimepiride placebo, whereas subjects in the glimepiride and placebo groups received an injection of liraglutide placebo." "Subjects completing the study could enroll in an 18-month open-label extension period."

Comment: self-reported or investigator-assessed outcome measurement. Incomplete blinding, but the outcome is not likely to be influenced by lack of blinding due to objective assessment of the outcome
Blinding of participants Low risk and personnel (performance bias)

HbAlc
Quote from publication: "... double-dummy... The double-dummy design required that subjects in the liraglutide and placebo groups received a glimepiride placebo, whereas subjects in the glimepiride and placebo groups received an injection of liraglutide placebo." "Subjects completing the study could enroll in an 18-month open-label extension period."

Comment: investigator-assessed outcome measurement. Incomplete blinding, but the outcome is not likely to be influenced by lack of blinding due to objective assessment of the outcome
Blinding of outcome as- Low risk sessment (detection bias) all-cause/cardiovascular mortality

Quote from publication: "... double-dummy... The double-dummy design required that subjects in the liraglutide and placebo groups received a glimepiride placebo, whereas subjects in the glimepiride and placebo groups received an injection of liraglutide placebo." "Subjects completing the study could enroll in an 18-month open-label extension period."

Comment: investigator-assessed outcome measurement. Incomplete blinding, but the outcome is not likely to be influenced by lack of blinding due to objective assessment of the outcome

Blinding of outcome as- Low risk sessment (detection bias) amputation of lower extremity/blindness or severe vision loss/end-stage renal disease
Quote from publication: "... double-dummy... The double-dummy design required that subjects in the liraglutide and placebo groups received a glimepiride placebo, whereas subjects in the glimepiride and placebo groups received an injection of liraglutide placebo." "Subjects completing the study could enroll in an 18-month open-label extension period."

Comment: investigator-assessed outcome measurement. Incomplete blinding, but the outcome is not likely to be influenced by lack of blinding due to objective assessment of the outcome 
Nauck 2013 (Continued)

Blinding of outcome as- Low risk sessment (detection bias) hypoglycaemia

Quote from publication: "... double-dummy... The double-dummy design required that subjects in the liraglutide and placebo groups received a glimepiride placebo, whereas subjects in the glimepiride and placebo groups received an injection of liraglutide placebo." "Subjects completing the study could enroll in an 18-month open-label extension period." "Hypoglycaemic episodes were defined as major, minor, or symptoms only. Major if the subject was unable to treat her/himself. Minor if subject was able to treat her/himself and plasma glucose was below $3.1 \mathrm{mmol} / \mathrm{L}$."

Comment: self-reported outcome measurement. Incomplete blinding, but the outcome is not likely to be influenced by lack of blinding due to objective assessment of the outcome

Blinding of outcome as- Low risk sessment (detection bias) non-fatal myocardial infarction/heart failure/nonfatal stroke
Quote from publication: "... double-dummy... The double-dummy design required that subjects in the liraglutide and placebo groups received a glimepiride placebo, whereas subjects in the glimepiride and placebo groups received an injection of liraglutide placebo." "Subjects completing the study could enroll in an 18-month open-label extension period."

Comment: investigator-assessed outcome measurement. Incomplete blinding, but the outcome is not likely to be influenced by lack of blinding due to objective assessment of the outcome

Blinding of outcome as- $\quad$ High risk
sessment (detection bias)
non-serious adverse
events

Quote from publication: "... double-dummy... The double-dummy design required that subjects in the liraglutide and placebo groups received a glimepiride placebo, whereas subjects in the glimepiride and placebo groups received an injection of liraglutide placebo." "Subjects completing the study could enroll in an 18-month open-label extension period."

Comment: self-reported outcome measurement. Incomplete blinding, the outcome is likely to be influenced by lack of blinding due to subjective assessment of the outcome

Blinding of outcome as- Low risk sessment (detection bias) serious adverse events
Quote from publication: "... double-dummy... The double-dummy design required that subjects in the liraglutide and placebo groups received a glimepiride placebo, whereas subjects in the glimepiride and placebo groups received an injection of liraglutide placebo." "Subjects completing the study could enroll in an 18-month open-label extension period."

Comment: investigator-assessed outcome measurement. Incomplete blinding, but the outcome is not likely to be influenced by lack of blinding due to objective assessment of the outcome
Blinding of outcome as- Low risk sessment (detection bias) weight $(\mathrm{kg})$
Quote from publication: "... double-dummy... The double-dummy design required that subjects in the liraglutide and placebo groups received a glimepiride placebo, whereas subjects in the glimepiride and placebo groups received an injection of liraglutide placebo." "Subjects completing the study could enroll in an 18-month open-label extension period."

Comment: self-reported or investigator-assessed outcome measurement. Incomplete blinding, but the outcome is not likely to be influenced by lack of blinding due to objective assessment of the outcome 
Comment: investigator-assessed outcome measurement. Incomplete blinding, but the outcome is not likely to be influenced by lack of blinding due to objective assessment of the outcome

Incomplete outcome data Low risk (attrition bias) all-cause/cardiovascular mortality

Quote from publication: "Safety analysis set is all randomised subjects who were exposed to at least one dose of study product."

Comment: $>99 \%$ of the randomised participants were included in the analyses. There was a high dropout rate and it was not balanced ( $25 \%-57 \%$ of randomised participants completed the study). No information on imputation method. We assumed that trial authors searched registers for mortality status at the end of the trial

Incomplete outcome data High risk (attrition bias) amputation of lower extremity/blindness or severe vision loss/end-stage renal disease
Quote from publication: "Safety analysis set is all randomised subjects who were exposed to at least one dose of study product."

Comment: $>99 \%$ of the randomised participants were included in the analyses. There was a high dropout rate and it was not balanced (25\% to $57 \%$ of randomised participants completed the study). The reasons for dropouts were not balanced among the intervention groups (a difference of $>10 \%$ in dropouts due to adverse events between the liraglutide $1.8 \mathrm{mg}$ group and metformin + placebo group and a difference of $>10 \%$ in dropouts due to lack of efficacy between the metformin + placebo group compared to all other groups and between the glimepiride group and the liraglutide $1.2 \mathrm{mg}$ group). No information on imputation method. The proportion of missing outcomes compared with observed event risk was enough to induce clinically relevant bias in intervention effect estimate

Incomplete outcome data High risk (attrition bias)

Quote from publication: "Safety analysis set is all randomised subjects who were exposed to at least one dose of study product."

hypoglycaemia
Comment: $>99 \%$ of the randomised participants were included in the analyses. There was a high dropout rate and it was not balanced $(25 \%-57 \%$ of randomised participants completed the study). The reasons for dropouts were not balanced among the intervention groups (a difference of $>10 \%$ in dropouts due to adverse events between the liraglutide $1.8 \mathrm{mg}$ group and metformin + placebo group and a difference of $>10 \%$ in dropouts due to lack of efficacy between the metformin + placebo group compared to all other groups and between the glimepiride group and the liraglutide $1.2 \mathrm{mg}$ group). No information on imputation method. The proportion of missing outcomes compared with observed event risk was enough to induce clinically relevant bias in intervention effect estimate

\section{Incomplete outcome data High risk} (attrition bias) non-fatal myocardial infarction/heart failure/nonfatal stroke
Quote from publication: "Safety analysis set is all randomised subjects who were exposed to at least one dose of study product."

Comment: $>99 \%$ of the randomised participants were included in the analyses. There was a high dropout rate and it was not balanced ( $25 \%-57 \%$ of randomised participants completed the study). The reasons for dropouts were not balanced among the intervention groups (a difference of $>10 \%$ in dropouts due to adverse events between the lira 1.8-group and metformin + placebo group and a difference of $>10 \%$ in dropouts due to lack of efficacy between the metformin + placebo group compared to all other groups and between the glimepiride group and the lira $1.2 \mathrm{mg}$ group). No information on imputation method. The proportion of missing outcomes compared with observed event risk was enough to induce clinically relevant bias in intervention effect estimate

Quote from publication: "Safety analysis set is all randomised subjects who were exposed to at least one dose of study product."
Incomplete outcome data High risk (attrition bias) 
Nauck 2013 (Continued) non-serious adverse events
Comment: $>99 \%$ of the randomised participants were included in the analyses. There was a high dropout rate and it was not balanced (25\%-57\% of randomised participants completed the study). The reasons for dropouts were not balanced among the intervention groups (a difference of $>10 \%$ in dropouts due to adverse events between the liraglutide $1.8 \mathrm{mg}$ group and metformin + placebo group and a difference of $>10 \%$ in dropouts due to lack of efficacy between the metformin + placebo group compared to all other groups and between the glimepiride group and the liraglutide $1.2 \mathrm{mg}$ group). No information on imputation method. The proportion of missing outcomes compared with observed event risk was enough to induce clinically relevant bias in intervention effect estimate

\section{Incomplete outcome data High risk} (attrition bias)

serious adverse events
Quote from publication: "Safety analysis set is all randomised subjects who were exposed to at least one dose of study product."

Comment: $>99 \%$ of the randomised participants were included in the analyses. There was a high dropout rate and it was not balanced (25\%-57\% of randomised participants completed the study). The reasons for dropouts were not balanced among the intervention groups (a difference of $>10 \%$ in dropouts due to adverse events between the liraglutide $1.8 \mathrm{mg}$ group and metformin + placebo group and a difference of $>10 \%$ in dropouts due to lack of efficacy between the metformin + placebo group compared to all other groups and between the glimepiride group and the liraglutide $1.2 \mathrm{mg}$ group). No information on imputation method. The proportion of missing outcomes compared with observed event risk was enough to induce clinically relevant bias in intervention effect estimate

Incomplete outcome data High risk (attrition bias)

weight $(\mathrm{kg})$

Quote from publication: "Unless specified, efficacy endpoints were analysed using the intent-to-treat (ITT) population (all randomized patients exposed to drug). Missing data were imputed using last observation carried forward (LOCF)"

Comment: $>97 \%$ of the randomised participants were included in the analyses. There was a high dropout rate and it was not balanced ( $25 \%-57 \%$ of randomised participants completed the study). The reasons for dropouts were not balanced among the intervention groups (a difference of $>10 \%$ in dropouts due to adverse events between the liraglutide $1.8 \mathrm{mg}$ group and metformin + placebo group and a difference of $>10 \%$ in dropouts due to lack of efficacy between the metformin + placebo group compared to all other groups and between the glimepiride group and the liraglutide $1.2 \mathrm{mg}$ group). Inapropriate method to impute missing data (LOCF). Plausible effect size among missing outcomes enough to induce clinically relevant bias in observed effect size

Quote from publication: "Unless specified, efficacy endpoints were analysed using the intent-to-treat (ITT) population (all randomized patients exposed to drug). Missing data were imputed using last observation carried forward (LOCF)"

Comment: $>95 \%$ of the randomised participants were included in the analyses. There was a high dropout rate and it was not balanced $(25 \%-57 \%$ of randomised participants completed the study). The reasons for dropouts were not balanced among the intervention groups (a difference of $>10 \%$ in dropouts due to adverse events between the liraglutide $1.8 \mathrm{mg}$ group and metformin + placebo group and a difference of $>10 \%$ in dropouts due to lack of efficacy between the metformin + placebo group compared to all other groups and between the glimepiride group and the liraglutide $1.2 \mathrm{mg}$ group). Inapropriate method to impute missing data (LOCF). Plausible effect size among missing outcomes enough to induce clinically relevant bias in observed effect size 
Nauck 2013 (Continued)

\begin{tabular}{|c|c|c|}
\hline $\begin{array}{l}\text { Selective reporting (re- } \\
\text { porting bias) }\end{array}$ & Low risk & $\begin{array}{l}\text { Comment: all of the trial's primary and secondary outcomes as specified in } \\
\text { the protocol have been reported }\end{array}$ \\
\hline
\end{tabular}

Other bias Unclear risk Comment: the trial received funding from a pharmaceutical company

Study design: open, national, randomised, multi-centre, parallel-group, controlled study
Randomisation ratio: 1:1 to receive fixed association of rosiglitazone-metformin or free association of
gliclazide in addition to metformin

Participants

Inclusion criteria: adults aged 40-75 years, T2DM diagnosed at $\geq 1$ year previously, treated with metformin at a minimum dose of $1.5 \mathrm{~g} /$ day and maximum dose of $3 \mathrm{~g} /$ day and at stable dose for at least 8 weeks before selection visit, $\mathrm{HbA} 1 \mathrm{c}$ level $>6.5 \%$ and $\leq 8.5 \%$, BMI $>25$ and $<35$

Exclusion criteria: T1DM, treatment with other hypoglycaemic agents than metformin in the last 3 months, FPG $>200 \mathrm{mg} / \mathrm{dL}$ at visit 2, hypersensitivity to the studied treatments (rosiglitazone, metformin chlor hydrate, gliclazide), congestive heart failure, unstable or severe angina, recent $\mathrm{MI}$, respiratory insufficiency, use of insulin for glycaemic control in the past 6 months prior to visit 1 (except during pregnancy or acute episodes such as hospitalisation, trauma or infection), history of metabolic acidosis including diabetic ketoacidosis, anaemia defined by haemoglobin concentration $<11.0 \mathrm{~g} /$ $\mathrm{dL}$ for men and $<10.0 \mathrm{~g} / \mathrm{dL}$ for women, renal disease or renal dysfunction, e.g. as suggested by serum creatinine levels $\geq 135.0 \mu \mathrm{mol} / \mathrm{L}$ in men and $\geq 110.0 \mu \mathrm{mol} / \mathrm{L}$ in women and/or creatinine clearance $<40$ $\mathrm{mL} / \mathrm{min}$, presence of clinically significant hepatic disease, with ALT, AST, total bilirubin, alkaline phosphatase $>2.5 \times$ ULN, chronic diseases requiring periodic or intermittent treatment with oral or IV corticosteroids, participants receiving danazol, miconazole or phenylbutazone, active alcohol, drug or medication abuse within the last 6 months or any condition that would indicate the likelihood of poor participant compliance, women who were lactating, pregnant or planning to become pregnant, any clinically significant abnormality identified at screening which, in the investigator's judgement, makes the patient unsuitable for inclusion in the study, use of any other investigational agent within 30 days or 5 half-lives (whichever is longer) prior to visit 1 , received or anticipate receiving radiocontrast dye during the study

Diagnostic criteria: WHO criteria

\begin{tabular}{ll}
\hline Interventions & Number of study centres: 94 \\
& Run-in period: no \\
& Extension period: no \\
\hline Outcomes & Composite outcome measures reported: no \\
\hline Study details & Trial terminated early: no \\
& Trial ID: NCT00367055 \\
\hline Publication details & Language of publication: English \\
& Funding: commercial funding by GlaxoSmithKline \\
& Publication status: other; Scientific result summary and clinicaltrials.gov \\
\hline Stated aim for study & $\begin{array}{l}\text { Quote from publication: "The objective of the study was to demonstrate that rosiglitazone offers bet- } \\
\text { mer protection of b-cell function than gliclazide when these substances are given in association with } \\
\text { metform in type } 2 \text { diabetic patients not controlled by metformin alone" }\end{array}$
\end{tabular}


NCT00367055 (Continued)

Notes

\section{Risk of bias}

\begin{tabular}{lll}
\hline Bias & Authors' judgement & Support for judgement \\
\hline $\begin{array}{l}\text { Random sequence genera- } \\
\text { tion (selection bias) }\end{array}$ & Unclear risk & Quote from publication: "... randomised..." \\
& & $\begin{array}{l}\text { Comment: insufficient information about the sequence generation process to } \\
\text { permit judgement of 'low risk or 'high risk' }\end{array}$
\end{tabular}

\begin{tabular}{|c|c|c|}
\hline $\begin{array}{l}\text { Allocation concealment } \\
\text { (selection bias) }\end{array}$ & Unclear risk & $\begin{array}{l}\text { Quote from publication: "... randomised..." } \\
\text { Comment: insufficient information about the allocation concealment to per- } \\
\text { mit judgement of 'low risk' or 'high risk' }\end{array}$ \\
\hline
\end{tabular}

\begin{tabular}{|c|c|c|}
\hline $\begin{array}{l}\text { Blinding of participants } \\
\text { and personnel (perfor- } \\
\text { mance bias) } \\
\text { all-cause/cardiovascular } \\
\text { mortality }\end{array}$ & Low risk & $\begin{array}{l}\text { Quote from publication: "Open..." } \\
\text { Comment: investigator-assessed outcome measurement. Incomplete blinding } \\
\text { of participant and investigator, but the outcome is not likely to be influenced } \\
\text { by lack of blinding }\end{array}$ \\
\hline
\end{tabular}

\begin{tabular}{|c|c|c|}
\hline $\begin{array}{l}\text { Blinding of participants } \\
\text { and personnel (perfor- } \\
\text { mance bias) } \\
\text { hypoglycaemia }\end{array}$ & Unclear risk & $\begin{array}{l}\text { Quote from publication: "Open..." } \\
\text { Comment: investigator-assessed/self-reported outcome measurement. In- } \\
\text { complete blinding of participant and investigator. Not defined if outcome } \\
\text { measurement is self-reported or investigator-assessed }\end{array}$ \\
\hline
\end{tabular}

\begin{tabular}{|c|c|c|}
\hline $\begin{array}{l}\text { Blinding of participants } \\
\text { and personnel (perfor- } \\
\text { mance bias) } \\
\text { non-serious adverse } \\
\text { events }\end{array}$ & High risk & $\begin{array}{l}\text { Quote from publication: "Open..." } \\
\text { Comment: self-reported outcome measurement. Incomplete blinding, the } \\
\text { outcome is likely to be influenced by lack of blinding }\end{array}$ \\
\hline $\begin{array}{l}\text { Blinding of participants } \\
\text { and personnel (perfor- } \\
\text { mance bias) } \\
\text { serious adverse events }\end{array}$ & Low risk & $\begin{array}{l}\text { Quote from publication: "Open..." } \\
\text { Comment: investigator-assessed outcome measurement. Incomplete blinding } \\
\text { of participant and investigator, but the outcome is not likely to be influenced } \\
\text { by lack of blinding }\end{array}$ \\
\hline
\end{tabular}

\begin{tabular}{|c|c|c|}
\hline $\begin{array}{l}\text { Blinding of participants } \\
\text { and personnel (perfor- } \\
\text { mance bias) } \\
\text { HbA1C }\end{array}$ & Low risk & $\begin{array}{l}\text { Quote from publication: "Open..." } \\
\text { Comment: investigator-assessed outcome measurement. Incomplete blinding } \\
\text { of participant and investigator, but the outcome is not likely to be influenced } \\
\text { by lack of blinding }\end{array}$ \\
\hline
\end{tabular}

\begin{tabular}{|c|c|c|}
\hline $\begin{array}{l}\text { Blinding of outcome as- } \\
\text { sessment (detection bias) } \\
\text { all-cause/cardiovascular } \\
\text { mortality }\end{array}$ & Low risk & $\begin{array}{l}\text { Quote from publication: "Open..." } \\
\text { Comment: investigator-assessed outcome measurement. Incomplete blinding } \\
\text { of participant and investigator, but the outcome is not likely to be influenced } \\
\text { by lack of blinding }\end{array}$ \\
\hline
\end{tabular}

\begin{tabular}{|c|c|c|}
\hline $\begin{array}{l}\text { Blinding of outcome as- } \\
\text { sessment (detection bias) } \\
\text { hypoglycaemia }\end{array}$ & Unclear risk & $\begin{array}{l}\text { Quote from publication: "Open..." } \\
\text { Comment: investigator-assessed/self-reported outcome measurement. In- } \\
\text { complete blinding of participant and investigator. Not defined if outcome } \\
\text { measurement is self-reported or investigator-assessed }\end{array}$ \\
\hline
\end{tabular}

\begin{tabular}{ll}
\hline Blinding of outcome as- \\
sessment (detection bias)
\end{tabular}$\quad$ High risk Quote from publication: "Open..."


NCT00367055 (Continued) non-serious adverse events
Comment: self-reported outcome measurement. Incomplete blinding, the outcome is likely to be influenced by lack of blinding

$\begin{array}{ll}\begin{array}{l}\text { Blinding of outcome as- } \\ \text { sessment (detection bias) } \\ \text { serious adverse events }\end{array} & \text { Low risk }\end{array} \quad \begin{aligned} & \text { Quote from publication: "Open..." } \\ & \begin{array}{l}\text { Comment: investigator-assessed outcome measurement. Incomplete blinding } \\ \text { of participant and investigator, but the outcome is not likely to be influenced } \\ \text { by lack of blinding }\end{array}\end{aligned}$

\begin{tabular}{|c|c|c|}
\hline $\begin{array}{l}\text { Blinding of outcome as- } \\
\text { sessment (detection bias) } \\
\text { HbA1c }\end{array}$ & Low risk & $\begin{array}{l}\text { Quote from publication: "Open..." } \\
\text { Comment: investigator-assessed outcome measurement. Incomplete blinding } \\
\text { of participant and investigator, but the outcome is not likely to be influenced } \\
\text { by lack of blinding }\end{array}$ \\
\hline
\end{tabular}

Incomplete outcome data Low risk (attrition bias) all-cause/cardiovascular mortality
Quote from publication: "Analyses for clinical safety and tolerability were conducted using the all randomized population, comprised of all subjects who received a randomization number and received at least one dose of study medication."

Comment: $93 \%-96 \%$ of the randomised participants were included in the analyses. There was a high dropout rate $(68 \%-71 \%$ of randomised participants completed the study), however, the dropout rate was balanced between groups. We assumed that trial authors searched registers for mortality status at the end of the trial

Incomplete outcome data High risk
(attrition bias)

hypoglycaemia

Quote from publication: "Analyses for clinical safety and tolerability were conducted using the all randomized population, comprised of all subjects who received a randomization number and received at least one dose of study medication."

Comment: $93 \%-96 \%$ of the randomised participants were included in the analyses. There was a high dropout rate $(68 \%-71 \%$ of randomised participants completed the study), however, the dropout rate was balanced between groups. The reasons for dropouts were balanced among the intervention groups. No information on imputation method. The proportion of missing outcomes compared with observed event risk was enough to induce clinically relevant bias in intervention effect estimate

Incomplete outcome data Low risk (attrition bias) non-serious adverse events
Quote from publication: "Analyses for clinical safety and tolerability were conducted using the all randomized population, comprised of all subjects who received a randomization number and received at least one dose of study medication."

Comment: $93 \%-96 \%$ of the randomised participants were included in the analyses. There was a high dropout rate $(68 \%-71 \%$ of randomised participants completed the study), however, the dropout rate was balanced between groups. The reasons for dropouts were balanced among the intervention groups. No information on imputation method. The proportion of missing outcomes compared with observed event risk was not enough to induce clinically relevant bias in intervention effect estimate
Incomplete outcome data High risk

(attrition bias)

serious adverse events
Quote from publication: "Analyses for clinical safety and tolerability were conducted using the all randomized population, comprised of all subjects who received a randomization number and received at least one dose of study medication."

Comment: $93 \%-96 \%$ of the randomised participants were included in the analyses. There was a high dropout rate $(68 \%-71 \%$ of randomised participants completed the study), however, the dropout rate was balanced between groups. The reasons for dropouts were balanced among the interven- 
tion groups. No information on imputation method. The proportion of missing outcomes compared with observed event risk was enough to induce clinically relevant bias in intervention effect estimate

Incomplete outcome data High risk (attrition bias)

HbAlc
Quote from publication: "All efficacy analyses were performed on the ITT population according to the randomized treatment group."

Comment: $71 \%$ of the randomised participants were included in the analyses. There was a high dropout rate $(68 \%-71 \%$ of randomised participants completed the study), however, the dropout rate was balanced between groups. The reasons for dropouts were balanced among the intervention groups. No information on imputation method. Plausible effect size among missing outcomes is enough to induce clinically relevant bias in observed effect size

Selective reporting (re- Low risk
porting bias)
porting bias)
Comment: all of the trial's prespecified primary and secondary outcomes (methods section) have been reported in the results section

\begin{tabular}{ll}
\hline Other bias Unclear risk Comment: sponsored by a pharmaceutical company \\
\hline
\end{tabular}

\section{Petrica 2009}

$\begin{array}{ll}\text { Methods } & \text { Study design: open-label, randomised, controlled clinical trial } \\ & \text { Randomisation ratio: } 1: 1 \text { to receive rosiglitazone or glimepiride in addition to metformin }\end{array}$

Inclusion criteria: T2DM ( $>5$ years), normoalbuminuria at the time of enrolment, absence of microan-
giopathic complications, HbA1c $>7 \%$ with previous medication (stable therapy with metformin for at
least 6 months), requiring other antidiabetic agents, no chronic kidney disease of non-diabetic origin

Exclusion criteria: symptoms and/or history of cerebrovascular disease (transient ischaemic attack, stroke), micro/macroalbuminuria and thyroid dysfunction

Diagnostic criteria: not reported

\begin{tabular}{ll}
\hline Interventions & Number of study centres: not reported \\
& Run-in period: no \\
& Extension period: no \\
\hline Outcomes & Composite outcome measures reported: no \\
\hline Study details & Trial terminated early: no \\
& Trial ID: - \\
\hline Publication details & Language of publication: English \\
& $\begin{array}{l}\text { Funding: non-commercial funding: Victor Babes University of Medicine and Pharmacy, County Emer- } \\
\text { gency Hospital Timisoara, Romania } \\
\text { Publication status: peer-reviewed journal }\end{array}$ \\
\hline Stated aim for study & $\begin{array}{l}\text { Quote from publication: "The aim of our study was to demonstrate the renal and cerebral protective } \\
\text { effects of the thiazolidinedione rosiglitazone vs. glimepiride, a sulfonylurea compound, in normoalbu- } \\
\text { minuric type 2 DM patients with no history or symptoms of cerebrovascular disease." }\end{array}$
\end{tabular}


Petrica 2009 (Continued)

Risk of bias

\begin{tabular}{lll}
\hline Bias & Authors' judgement & Support for judgement \\
\hline $\begin{array}{l}\text { Random sequence genera- } \\
\text { tion (selection bias) }\end{array}$ & Unclear risk & Quote from publication: "Patients were randomly assigned..." \\
& & $\begin{array}{l}\text { Comment: insufficient information about the sequence generation process to } \\
\text { permit judgement of 'low risk or 'high risk' }\end{array}$
\end{tabular}

\begin{tabular}{|c|c|c|}
\hline $\begin{array}{l}\text { Allocation concealment } \\
\text { (selection bias) }\end{array}$ & Unclear risk & $\begin{array}{l}\text { Quote from publication: "Patients were randomly assigned..." } \\
\text { Comment: insufficient information about the allocation concealment to per- } \\
\text { mit judgement of 'low risk' or 'high risk' }\end{array}$ \\
\hline
\end{tabular}

Blinding of participants Low risk and personnel (performance bias)

HbAlc

Quote from publication: "open label" "The laboratory staff who performed the assessments were blinded as to the treatments the patients were receiving"

Comment: investigator-assessed outcome measurement. Incomplete blinding, the outcome is unlikely to be influenced by lack of blinding

\begin{tabular}{|c|c|c|}
\hline $\begin{array}{l}\text { Blinding of outcome as- } \\
\text { sessment (detection bias) } \\
\text { HbAlc }\end{array}$ & Low risk & $\begin{array}{l}\text { Quote from publication: "open label" "The laboratory staff who performed } \\
\text { the assessments were blinded as to the treatments the patients were receiv- } \\
\text { ing" }\end{array}$ \\
\hline
\end{tabular}

Comment: investigator-assessed outcome measurement. Incomplete blinding, the outcome is unlikely to be influenced by lack of blinding

$\begin{array}{ll}\begin{array}{l}\text { Incomplete outcome data Low risk } \\ \text { (attrition bias) }\end{array} & \begin{array}{l}\text { Comment: } 77 \% \text { of the randomised participants were included in the analy- } \\ \text { HbAlc }\end{array} \\ & \begin{array}{l}\text { ses. There was a high dropout rate ( } 77 \% \text { of the participants completed the } \\ \text { for dropouts were not balanced (3 participants in the rosiglitazone group dis- } \\ \text { continued due to weight gain compared to } 0 \text { participants in the glimepiride } \\ \text { group). Plausible effect size among missing outcomes was not enough to have } \\ \text { a clinically relevant impact on observed effect size }\end{array}\end{array}$

Selective reporting (re- High risk porting bias)
Comment: no protocol available. All of the trial's prespecified primary and secondary outcomes (methods section) have been reported. Unclear whether common outcomes (all-cause mortality, hypoglycaemia) were measured; not mentioned, but clinical judgement says likely to have been measured and analysed but not reported on the basis of non-significant results. Clear that outcomes (non-serious adverse events, serious adverse events) were measured but not necessarily analysed; judgement says likely to have been analysed but not reported because of non-significant results

\section{Petrica 2011}

\begin{tabular}{|c|c|}
\hline Methods & $\begin{array}{l}\text { Study design: open-label, RCT } \\
\text { Randomisation ratio: 1:1 to receive pioglitazone or glimepiride in addition to metformin }\end{array}$ \\
\hline Participants & $\begin{array}{l}\text { Inclusion criteria: T2DM (> } 5 \text { years), normoalbuminuria at the time of enrolment, absence of microan- } \\
\text { giopathic complications, no chronic kidney disease of non-diabetic origin, } \mathrm{HbA} 1 \mathrm{c}>7 \% \text { with previous } \\
\text { medication (stable therapy with metformin for at least } 6 \text { months), a fact which required association of } \\
\text { other antidiabetic agents }\end{array}$ \\
\hline & $\begin{array}{l}\text { Exclusion criteria: symptoms and/or history of cerebrovascular disease (transient ischaemic attack, } \\
\text { stroke), and micro/macroalbuminuria }\end{array}$ \\
\hline
\end{tabular}


Petrica 2011 (Continued)

Diagnostic criteria: not reported

\begin{tabular}{ll}
\hline Interventions & Number of study centres: not reported \\
& Run-in period: no \\
& Extension period: no \\
\hline Outcomes & Composite outcome measures reported: no \\
\hline Study details & Trial terminated early: no \\
& Trial ID: - \\
\hline
\end{tabular}

Publication details Language of publication: English

Funding: non-commercial funding: Victor Babes University of Medicine and Pharmacy, County Emergency Hospital, Timisoara, Romania

Publication status: peer-reviewed journal

Stated aim for study Quote from publication: "The aim of our work was to validate our previous observations in a longitudinal study and to demonstrate that PT dysfunction occurs before the stage of albuminuria. Moreover, we attempted to document the different patterns of endothelial behaviour in two distinct vascular segments, the kidney and the brain. In addition, we assessed the renal and cerebral protective effects of pioglitazone versus glimepiride, a sulphonylurea compound, in normoalbuminuric patients with type 2 DM."

Notes -

\section{Risk of bias}

\begin{tabular}{lll}
\hline Bias & Authors' judgement & Support for judgement \\
\hline $\begin{array}{l}\text { Random sequence genera- } \\
\text { tion (selection bias) }\end{array}$ & Unclear risk & Quote from publication: "Patients were randomly assigned..." \\
& & $\begin{array}{l}\text { Comment: insufficient information about the sequence generation process to } \\
\text { permit judgement of 'low risk or 'high risk' }\end{array}$ \\
\hline
\end{tabular}

\begin{tabular}{|c|c|c|}
\hline $\begin{array}{l}\text { Allocation concealment } \\
\text { (selection bias) }\end{array}$ & Unclear risk & $\begin{array}{l}\text { Quote from publication: "Patients were randomly assigned..." } \\
\text { Comment: insufficient information about the allocation concealment to per- } \\
\text { mit judgement of 'low risk' or 'high risk' }\end{array}$ \\
\hline
\end{tabular}

$\begin{array}{ll}\begin{array}{l}\text { Blinding of participants } \\ \text { and personnel (perfor- }\end{array} & \text { Low risk } \\ \begin{array}{l}\text { mance bias) } \\ \text { HbAlc }\end{array} & \begin{array}{l}\text { Quote from publication: "open label" "The laboratory staff who performed } \\ \text { the assessments were blinded to the medical treatment of the patients en- } \\ \text { rolled in the study" }\end{array} \\ & \begin{array}{l}\text { Comment: investigator-assessed outcome measurement. Incomplete blind- } \\ \text { ing, the outcome is unlikely to be influenced by lack of blinding }\end{array}\end{array}$

\begin{tabular}{lll}
\hline $\begin{array}{l}\text { Blinding of outcome as- } \\
\text { sessment (detection bias) } \\
\text { HbAlc }\end{array}$ & Low risk & $\begin{array}{l}\text { Quote from publication: "open label" "The laboratory staff who performed } \\
\text { the assessments were blinded to the medical treatment of the patients en- } \\
\text { rolled in the study" }\end{array}$ \\
& $\begin{array}{l}\text { Comment: investigator-assessed outcome measurement. Incomplete blind- } \\
\text { ing, the outcome is unlikely to be influenced by lack of blinding }\end{array}$ \\
\hline $\begin{array}{l}\text { Incomplete outcome data } \\
\text { (attrition bias) } \\
\text { HbAlc }\end{array}$ & Low risk & $\begin{array}{l}\text { Comment: } 87 \% \text { of the randomised participants were included in the analyses. } \\
\text { There was a low dropout rate }(87 \% \text { of the participants completed the study). } \\
\text { The dropout rate and the reason for dropout were balanced between groups. }\end{array}$
\end{tabular}


Petrica 2011 (Continued)

Plausible effect size among missing outcomes was not enough to have a clinically relevant impact on observed effect size

Selective reporting (re- High risk porting bias)
Comment: no protocol available. All of the trial's prespecified primary and secondary outcomes (methods section) have been reported. Unclear whether common outcomes (all-cause mortality, hypoglycaemia) were measured; not mentioned, but clinical judgement says likely to have been measured and analysed but not reported on the basis of non-significant results. Clear that outcomes (non-serious adverse events, serious adverse events) were measured but not necessarily analysed; judgement says likely to have been analysed but not reported because of non-significant results

Ridderstråle 2014

Study design: randomised, double-blind, double-dummy, active-controlled, parallel-group study
Randomisation ratio: $1: 1$ to receive empagliflozin or glimepiride in addition to metformin
Non-inferiority and superiority design: the non-inferiority of empagliflozin to glimepiride for the pri-
mary endpoint of change from baseline in HbAlc concentration was tested at weeks 52 and 104 . Key
secondary endpoints were tested for superiority at weeks 52 and 104 . At week 104 , if non-inferiority for
HbAlc was established, tests for the superiority of empagliflozin versus glimepiride were to be done
in a hierarchical order: first, change in bodyweight; second, occurrence of confirmed hypoglycaemic
adverse events; third, superiority of empagliflozin versus glimepiride in change in HbA1c concentra-
tion; fourth, change in systolic BP; fifth, change in diastolic BP. The non-inferiority of empagliflozin to
glimepiride for the primary endpoint was based on a one-sided significance level of $1.25 \%$ (adjusted
for repeated testing at weeks 52 and 104). Superiority tests were based on a significance level of $2.5 \%$
(two-sided)

Participants

Inclusion criteria: age $\geq 18$ years, T2DM with insufficient glycaemic control with diet, exercise and metformin immediate release ( $\geq 1500 \mathrm{mg} /$ day or MTD, or maximum dose according to local label, with dose unchanged for 12 weeks prior to randomisation), $\mathrm{HbA} 1 \mathrm{c} \geq 7 \%$ and $\leq 10 \%$ at screening $\mathrm{BMI} \leq 45 \mathrm{~kg} / \mathrm{m}^{2}$ at screening. Female participants: post-menopausal, or pre-menopausal and using appropriate contraception; not pregnant/breastfeeding

Exclusion criteria: blood glucose level $>13.3 \mathrm{mmol} / \mathrm{L}$ after an overnight fast during placebo run-in, use of any glucose-lowering drugs other than metformin immediate release $\leq 12$ weeks prior to randomisation, bariatric surgery within 2 years; treatment with anti-obesity drugs within 3 months of screening; any treatment leading to unstable body weight, estimated GFR $<60 \mathrm{~mL} / \mathrm{min} / 1.73 \mathrm{~m}^{2}$ (modified diet renal disease) during screening or placebo run-in, indication of liver disease (ALT, AST or alkaline phosphatase $>3 \times$ ULN) during screening or placebo run-in, history of cancer within 5 years (except basal cell carcinoma), acute coronary syndrome, stroke or transient ischaemic attack within 3 months of informed consent, disorders causing unstable red blood cells; treatment with systemic steroids; change in dose of thyroid hormones within 6 weeks of screening; any uncontrolled endocrine condition (except T2DM), alcohol or drug abuse within 3 months of informed consent, taking an investigational drug $\leq 30$ days prior to receiving study drug

Diagnostic criteria: not reported

Interventions

\section{Number of study centres: 173}

Run-in period: yes, 2-week open-label placebo run-in

Extension period: yes, participants who completed the 104-week randomised treatment were eligible to participate in the 104-week extension, during which they would continue to receive the treatment allocated at randomisation in a double-blind manner. However, some sites did not participate in the 2year extension, and so considered participants to have completed treatment after 2 years. 
Ridderstråle 2014 (Continued)

Outcomes

Composite outcome measures reported: yes, $\mathrm{HbAlc}<7.0 \%$ or $\mathrm{HbAlc}$ reduction $\geq 1.0 \%$, no confirmed hypoglycaemia, and weight loss $>2 \%$ after 52,104 and 208 weeks of treatment and $\mathrm{HbA1C}<6.5 \%$ or $\mathrm{HbA} 1 \mathrm{c}$ reduction $\geq 1.0 \%$, no confirmed hypoglycaemia, and weight loss $>2 \%$ after 52,104 and 208 weeks of treatment
Trial terminated early: no

Trial ID: NCT01167881

$\begin{array}{ll}\text { Publication details } & \text { Language of publication: English } \\ \text { Funding: commercial funding by Boehringer Ingelheim and Eli Lilly } \\ \text { Publication status: peer-reviewed journal }\end{array}$

Stated aim for study

Quote from publication: "The objective in this trial (EMPA-REG H2H-SU) was to compare the efficacy and safety of empagliflozin and the sulfonylurea glimepiride as a second-line therapy in patients with type 2 diabetes with inadequate glycaemic control on metformin."

Notes -

\section{Risk of bias}

\begin{tabular}{lll}
\hline Bias & Authors' judgement & Support for judgement \\
\hline $\begin{array}{ll}\text { Random sequence genera- } \\
\text { tion (selection bias) }\end{array}$ & Low risk & $\begin{array}{l}\text { Quote from publication: "The study sponsor did the randomisation, stratified } \\
\text { by HbAlc concentration at screening, eGFR, and region, using an interactive } \\
\text { response system with a computer-generated random sequence" }\end{array}$
\end{tabular}

Comment: adequate generation of random sequence ensured

\begin{tabular}{ll}
\hline $\begin{array}{l}\text { Allocation concealment } \\
\text { (selection bias) }\end{array}$ & Low risk \\
& $\begin{array}{l}\text { Quote from publication: "The study sponsor did the randomisation, stratified } \\
\text { by HbAlc concentration at screening, eGFR, and region, using an interactive } \\
\text { response system with a computer-generated random sequence" }\end{array}$ \\
Comment: adequate concealment ensured
\end{tabular}

\begin{tabular}{|c|c|c|}
\hline $\begin{array}{l}\text { Blinding of participants } \\
\text { and personnel (perfor- } \\
\text { mance bias) } \\
\text { all-cause/cardiovascular } \\
\text { mortality }\end{array}$ & Low risk & $\begin{array}{l}\text { Quote from publication: "double-blind, double-dummy" "patients and inves- } \\
\text { tigators were masked to treatment assignment" "An independent data moni- } \\
\text { toring committee monitored safety of the patients throughout the trial." } \\
\text { Comment: adjudicated outcome measurement. Blinding ensured }\end{array}$ \\
\hline $\begin{array}{l}\text { Blinding of participants } \\
\text { and personnel (perfor- } \\
\text { mance bias) } \\
\text { hypoglycaemia }\end{array}$ & Low risk & $\begin{array}{l}\text { Quote from publication: "double-blind, double-dummy" "patients and inves- } \\
\text { tigators were masked to treatment assignment" "An independent data moni- } \\
\text { toring committee monitored safety of the patients throughout the trial." } \\
\text { Comment: adjudicated outcome measurement. Blinding ensured }\end{array}$ \\
\hline
\end{tabular}

\begin{tabular}{|c|c|c|}
\hline $\begin{array}{l}\text { Blinding of participants } \\
\text { and personnel (perfor- } \\
\text { mance bias) }\end{array}$ & Low risk & $\begin{array}{l}\text { Quote from publication: "double-blind, double-dummy" "patients and inves- } \\
\text { tigators were masked to treatment assignment" "An independent data moni- } \\
\text { toring committee monitored safety of the patients throughout the trial." }\end{array}$ \\
\hline
\end{tabular}

non-fatal myocardial infarction/heart failure/nonfatal stroke

Comment: adjudicated outcome measurement. Blinding ensured

Blinding of participants Low risk
and personnel (perfor-
mance bias)

Quote from publication: "double-blind, double-dummy" "patients and investigators were masked to treatment assignment" "An independent data monitoring committee monitored safety of the patients throughout the trial." 
Ridderstråle 2014 (Continued) non-serious adverse events
Comment: adjudicated outcome measurement. Blinding ensured

Quote from publication: "double-blind, double-dummy" "patients and investigators were masked to treatment assignment" "An independent data monitoring committee monitored safety of the patients throughout the trial."

Comment: adjudicated outcome measurement. Blinding ensured

Quote from publication: "double-blind, double-dummy" "patients and investigators were masked to treatment assignment"

and personnel (perfor-

Low risk

mance bias

weight $(\mathrm{kg})$

Comment: investigator-assessed/self-reported outcome measurement. Blinding ensured
Blinding of participants Low risk Quote from publication: "double-blind, double-dummy" "patients and invesand personnel (perfor-

mance bias)

HbA1c tigators were masked to treatment assignment"

Comment: investigator-assessed outcome measurement. Blinding ensured
Blinding of outcome as- Low risk sessment (detection bias) all-cause/cardiovascular mortality
Quote from publication: "double-blind, double-dummy" "patients and investigators were masked to treatment assignment" "An independent data monitoring committee monitored safety of the patients throughout the trial."

Comment: adjudicated outcome measurement. Blinding ensured
Blinding of outcome as- Low risk sessment (detection bias) hypoglycaemia
Quote from publication: "double-blind, double-dummy" "patients and investigators were masked to treatment assignment" "An independent data monitoring committee monitored safety of the patients throughout the trial."

Comment: adjudicated outcome measurement. Blinding ensured

\begin{tabular}{lll}
\hline Blinding of outcome as- & Low risk & Quote from publication: "double-blind, double-dummy" "patients and inves- \\
sessment (detection bias) & & tigators were masked to treatment assignment" "An independent data moni- \\
non-fatal myocardial in- & toring committee monitored safety of the patients throughout the trial."
\end{tabular}
toring committee monitored safety of the patients throughout the trial."

farction/heart failure/nonfatal stroke

Comment: adjudicated outcome measurement. Blinding ensured

$\begin{array}{lll}\text { Blinding of outcome as- } & \text { Low risk } & \text { Quote from publication: "double-blind, double-dummy" "patients and inves- } \\ \text { sessment (detection bias) } & \text { tigators were masked to treatment assignment" "An independent data moni- } \\ \text { non-serious adverse } & \text { toring committee monitored safety of the patients throughout the trial." }\end{array}$

events

Comment: adjudicated outcome measurement. Blinding ensured.

Blinding of outcome as- Low risk sessment (detection bias) serious adverse events
Quote from publication: "double-blind, double-dummy" "patients and investigators were masked to treatment assignment" "An independent data monitoring committee monitored safety of the patients throughout the trial."

\begin{tabular}{|c|c|c|}
\hline $\begin{array}{l}\text { Blinding of outcome as- } \\
\text { sessment (detection bias) } \\
\text { weight (kg) }\end{array}$ & Low risk & $\begin{array}{l}\text { Quote from publication: "double-blind, double-dummy" "patients and inves- } \\
\text { tigators were masked to treatment assignment" } \\
\text { Comment: investigator-assessed/self-reported outcome measurement. Blind- } \\
\text { ing ensured }\end{array}$ \\
\hline
\end{tabular}

Blinding of outcome as- $\quad$ Low risk
sessment (detection bias)
HbA1c

Quote from publication: "double-blind, double-dummy" "patients and investigators were masked to treatment assignment"

Comment: investigator-assessed outcome measurement. Blinding ensured 
Ridderstråle 2014 (Continued)

Incomplete outcome data Low risk (attrition bias)

all-cause/cardiovascular mortality
Quote from publication: "Safety was analysed in the treated set (patients given at least one dose of study drug)"

Comment: $>99 \%$ of randomised participants were included in the analyses. There was a high dropout rate ( $83 \%-85 \%$ of randomised participants completed 2 years of treatment), however, the dropout rate was balanced between groups. We assumed that trial authors searched registers for mortality status at the end of the trial

\section{Incomplete outcome data Low risk} (attrition bias)

hypoglycaemia

Quote from publication: "Safety was analysed in the treated set (patients given at least one dose of study drug)"
Comment: > 99\% of the participants were included in the analyses. There was a high dropout rate (76\%-79\% of the participants completed 4 years of treatment), however, the dropout rate was balanced between groups. We do not know how the trial authors imputed missing data from the participants not completing the study. The reasons for dropouts were balanced. The proportion of missing outcomes compared with observed event risk was not enough to induce clinically relevant bias in intervention effect estimate

\section{Incomplete outcome data High risk (attrition bias) \\ Quote from publication: "Safety was analysed in the treated set (patients giv- en at least one dose of study drug)"} non-fatal myocardial infarction/heart failure/nonfatal stroke

Comment: $>99 \%$ of the participants were included in the analyses. There was a high dropout rate (76\%-79\% of the participants completed 4 years of treatment), however, the dropout rate was balanced between groups. We do not know how the trial authors imputed missing data from the participants not completing the study. The reasons for dropouts were balanced. The proportion of missing outcomes compared with observed event risk was enough to induce clinically relevant bias in intervention effect estimate

Quote from publication: "Safety was analysed in the treated set (patients given at least one dose of study drug)"

Comment: $>99 \%$ of the participants were included in the analyses. There was a high dropout rate (76\%-79\% of the participants completed 4 years of treatment), however, the dropout rate was balanced between groups. We do not know how the trial authors imputed missing data from the participants not completing the study. The reasons for dropouts were balanced. The proportion of missing outcomes compared with observed event risk was not enough to induce clinically relevant bias in intervention effect estimate

Quote from publication: "Safety was analysed in the treated set (patients given at least one dose of study drug)"

Comment: $>99 \%$ of the participants were included in the analyses. There was a high dropout rate (76\%-79\% of the participants completed 4 years of treatment), however, the dropout rate was balanced between groups. We do not know how the trial authors imputed missing data from the participants not completing the study. The reasons for dropouts were balanced. The proportion of missing outcomes compared with observed event risk was enough to induce clinically relevant bias in intervention effect estimate

Quote from publication: "The efficacy analyses will be performed on the full analysis set (FAS), i.e. all randomized patients who received $\geq 1$ dose of study drug and had a baseline $\mathrm{HbAlc}$ assessment, using the last observation carried forward (LOCF) methodology for imputation of missing data." "Secondary endpoints will be analyzed using the same model as the primary endpoint..."

Comment: $>99 \%$ of the participants were included in the analyses. There was a high dropout rate $(83 \%-85 \%$ of the participants completed 2 years of treat- 

(LOCF)

Incomplete outcome data Unclear risk (attrition bias)

HbAlc
Quote from publication: "The efficacy analyses will be performed on the full analysis set (FAS), i.e. all randomized patients who received $\geq 1$ dose of study drug and had a baseline $\mathrm{HbAlc}$ assessment, using the last observation carried forward (LOCF) methodology for imputation of missing data."

Comment: $>99 \%$ of the participants were included in the analyses. There was a high dropout rate ( $83 \%-85 \%$ of the participants completed 2 years of treatment), however, the dropout rate was balanced between groups. The reasons for dropouts were balanced. Inappropriate method for imputing missing data (LOCF)

\begin{tabular}{lll}
\hline $\begin{array}{l}\text { Selective reporting (re- } \\
\text { porting bias) }\end{array}$ & Low risk & $\begin{array}{l}\text { Comment: all of the trial's primary and secondary outcomes as specified in } \\
\text { the protocol have been reported }\end{array}$ \\
\hline Other bias & Unclear risk & Comment: the trial was funded by pharmaceutical companies \\
\hline
\end{tabular}

Ristic 2007

Methods Study design: double-blind, double-dummy, parallel-group, randomised study

Randomisation ratio: 1:1 to receive nateglinide or gliclazide in addition to metformin

Participants

Inclusion criteria: $\geq 18$ years of age, T2DM for $\geq 6$ months, received metformin monotherapy for $\geq 3$ months on metformin dose of $1000 \mathrm{mg}$ /day continuously for $\geq 2$ months prior to study entry, but remain inadequately controlled by medication, diet and physical exercise, baseline $\mathrm{HbA1c} 6.8 \%-9.0 \%$, BMI between 20 and $35 \mathrm{~kg} / \mathrm{m}^{2}$

Exclusion criteria: T1DM, diabetes that is a result of pancreatic injury or secondary forms of diabetes, history of acute metabolic diabetic complications, significant diabetic complications, chronic insulin treatment, pregnant or lactating women, any oral anti-diabetic treatment, other than metformin within 3 months prior to week 0 , treatment with any drug with a known frequent toxicity to a major organ system within the past 3 months, any of the following significant medical history: Ml, coronary surgery, ventricular tachycardia or ventricular fibrillation within the past 6 months. Liver disease such as cirrhosis or chronic active hepatitis or persistent ALT, AST or alkaline phosphatase increases $>3 \times$ ULN, FPG $\geq$ $11.1 \mathrm{mmol} / \mathrm{L}$, fasting triglycerides $>750 \mathrm{mg} / \mathrm{dL}$ at week -2 , total bilirubin $>2 \times$ ULN at week -2

Diagnostic criteria: not reported

Interventions Number of study centres: 26

Run-in period: no

Extension period: yes, 6 months double-blind extension after 24 weeks treatment

\begin{tabular}{ll}
\hline Outcomes & Composite outcome measures reported: no \\
\hline Study details & Trial terminated early: no \\
& Trial ID: CDJN608A 2308E1 \\
\hline
\end{tabular}

\section{Publication details Language of publication: English}

Funding: commercial funding by Novartis Pharma 
Ristic 2007 (Continued)

Publication status: peer-reviewed journal

Stated aim for study

Quote from publication: "... to evaluate the effect of nateglinide compared with that of gliclazide in combination with metformin on $\mathrm{HbA1c}$, fasting plasma glucose (FPG), body weight and postprandial insulin and glucose, after 12 months of treatment."

\section{Notes}

\section{Risk of bias}

\begin{tabular}{lll}
\hline Bias & Authors' judgement & Support for judgement \\
\hline $\begin{array}{ll}\text { Random sequence genera- } \\
\text { tion (selection bias) }\end{array}$ & Low risk & $\begin{array}{l}\text { Quote from publication: "Randomization to treatment was by a comput- } \\
\text { er-generated schedule via an interactive voice-responding system that as- } \\
\text { signed randomization on a study-centre basis with a block size of 4." }\end{array}$ \\
& Comment: adequate generation of random sequence ensured
\end{tabular}

\begin{tabular}{ll}
\hline $\begin{array}{l}\text { Allocation concealment } \\
\text { (selection bias) }\end{array}$ & Low risk \\
& $\begin{array}{l}\text { Quote from publication: "Randomization to treatment was by a comput- } \\
\text { er-generated schedule via an interactive voice-responding system that as- } \\
\text { signed randomization on a study-centre basis with a block size of } 4 . "\end{array}$
\end{tabular}

Comment: adequate concealment ensured

Blinding of participants Low risk
and personnel (perfor-
mance bias)
all-cause/cardiovascular
mortality

Quote from publication: "double-blind" "A double-dummy technique, using identical-looking nateglinide and placebo tablets and identical-looking gliclazide and placebo capsules, was used to blind study medication assignment."

Comment: investigator-assessed outcome measurement. Blinding of study personnel ensured

\begin{tabular}{|c|c|c|}
\hline $\begin{array}{l}\text { Blinding of participants } \\
\text { and personnel (perfor- } \\
\text { mance bias) } \\
\text { weight (kg) }\end{array}$ & Low risk & $\begin{array}{l}\text { Quote from publication: "double-blind" "A double-dummy technique, us- } \\
\text { ing identical-looking nateglinide and placebo tablets and identical-looking } \\
\text { gliclazide and placebo capsules, was used to blind study medication assign- } \\
\text { ment." }\end{array}$ \\
\hline
\end{tabular}

Comment: self-reported or investigator-assessed outcome measurement. Blinding ensured

\begin{tabular}{|c|c|c|}
\hline $\begin{array}{l}\text { Blinding of participants } \\
\text { and personnel (perfor- } \\
\text { mance bias) } \\
\text { HbA1C }\end{array}$ & Low risk & $\begin{array}{l}\text { Quote from publication: "double-blind" "A double-dummy technique, us- } \\
\text { ing identical-looking nateglinide and placebo tablets and identical-looking } \\
\text { gliclazide and placebo capsules, was used to blind study medication assign- } \\
\text { ment." }\end{array}$ \\
\hline
\end{tabular}

Comment: investigator-assessed outcome measurement. Blinding ensured

\begin{tabular}{|c|c|c|}
\hline $\begin{array}{l}\text { Blinding of outcome as- } \\
\text { sessment (detection bias) } \\
\text { all-cause/cardiovascular } \\
\text { mortality }\end{array}$ & Low risk & $\begin{array}{l}\text { Quote from publication: "double-blind" "A double-dummy technique, us- } \\
\text { ing identical-looking nateglinide and placebo tablets and identical-looking } \\
\text { gliclazide and placebo capsules, was used to blind study medication assign- } \\
\text { ment." }\end{array}$ \\
\hline
\end{tabular}

Comment: investigator-assessed outcome measurement. Blinding of study personnel ensured

\begin{tabular}{|c|c|c|}
\hline $\begin{array}{l}\text { Blinding of outcome as- } \\
\text { sessment (detection bias) } \\
\text { weight (kg) }\end{array}$ & Low risk & $\begin{array}{l}\text { Quote from publication: "double-blind" "A double-dummy technique, us- } \\
\text { ing identical-looking nateglinide and placebo tablets and identical-looking } \\
\text { gliclazide and placebo capsules, was used to blind study medication assign- } \\
\text { ment." }\end{array}$ \\
\hline
\end{tabular}


Comment: self-reported or investigator-assessed outcome measurement. Blinding ensured

Blinding of outcome as- $\quad$ Low risk
sessment (detection bias)
HbAlc

\begin{abstract}
Quote from publication: "double-blind" "A double-dummy technique, using identical-looking nateglinide and placebo tablets and identical-looking gliclazide and placebo capsules, was used to blind study medication assignment."
\end{abstract}

Comment: investigator-assessed outcome measurement. Blinding ensured

\begin{tabular}{ll}
\hline $\begin{array}{l}\text { Incomplete outcome data } \\
\text { (attrition bias) }\end{array}$ & Low risk \\
$\begin{array}{l}\text { all-cause/cardiovascular } \\
\text { mortality }\end{array}$ & $\begin{array}{l}\text { Quote from publication: "safety was assessed for all randomized patients } \\
\text { with a post-baseline safety assessment." }\end{array}$ \\
& $\begin{array}{l}\text { Comment: } 78 \%-84 \% \text { of randomised participants were included in the analy- } \\
\text { ses. We assumed that trial authors searched registers for mortality status at } \\
\text { the end of the trial }\end{array}$
\end{tabular}

\begin{tabular}{|c|c|c|}
\hline $\begin{array}{l}\text { Incomplete outcome data } \\
\text { (attrition bias) } \\
\text { weight (kg) }\end{array}$ & Unclear risk & $\begin{array}{l}\text { Quote from publication: "Eficacy analyses used the intention-to-treat popula- } \\
\text { tion which included all randomized patients with at least one post-baseline ef- } \\
\text { ficacy evaluation..." }\end{array}$ \\
\hline
\end{tabular}
Incomplete outcome data Unclear risk (attrition bias)

Comment: $78 \%-84 \%$ of randomised participants were included in the analyses. There was a high dropout rate $(76 \%-81 \%$ of the randomised participants completed the study), however, the dropout rate was balanced between groups. No information on imputation method

Incomplete outcome data Unclear risk
(attrition bias)

$\mathrm{HbA} 1 \mathrm{c}$
Quote from publication: "The primary efficacy evaluation was based on $\mathrm{HbA1C}$ changes from baseline to end point at week 52 or the final visit, using the last observation carried forward (LOCF) approach. Baseline was calculated as the average of the measurements obtained from the evaluations for $\mathrm{HbAlC}$ on weeks 2 and 0 . If one of these measurements was missing, the remaining measurement was used as the baseline; if both were missing, then the patient was excluded from the analysis. The primary population in this assessment was the extension ITT population."

Comment: $78 \%-84 \%$ of randomised participants were included in the analyses. There was a high dropout rate $(76 \%-81 \%$ of the randomised participants completed the trial), however, the dropout rate was balanced between groups. The reasons for dropouts were balanced. Inappropriate method for imputing missing data (LOCF)

\begin{tabular}{|c|c|c|}
\hline $\begin{array}{l}\text { Selective reporting (re- } \\
\text { porting bias) }\end{array}$ & Low risk & $\begin{array}{l}\text { Comment: all of the trial's primary and secondary outcomes as specified in } \\
\text { the protocol have been reported }\end{array}$ \\
\hline
\end{tabular}

Other bias Unclear risk Comment: the study was sponsored by a pharmaceutical company

\section{Schernthaner 2015}

$\begin{array}{ll}\text { Methods } & \text { Study design: multinational, randomised, double-blind, active-controlled, parallel-arm, phase IIIb/IV } \\ \text { study }\end{array}$

Randomisation ratio: $1: 1$ to receive glimepiride or saxagliptin in addition to metformin

Participants

Inclusion criteria: T2DM aged $\geq 65$ years, who were on stable metformin monotherapy at any dose for
$\geq 8$ weeks before enrolment and had an $\mathrm{HbA1c}$ concentration of $7.0 \%-9.0 \%$

Exclusion criteria: T1DM; treatment with any antihyperglycaemic therapy other than metformin monotherapy < 8 weeks before enrolment; treatment with systemic glucocorticoids (except for replacement therapy) or cytochrome P450 3A4-inducers; history of ketoacidosis or hyperosmolar non- 
Schernthaner 2015 (Continued)

ketonic coma; history of haemoglobinopathies; renal impairment (creatinine clearance $<60 \mathrm{~mL} / \mathrm{min}$ ); cognitive function problems; alcohol or illegal drug abuse for $\leq 12$ months before enrolment; and history of hypersensitivity or contraindication to the study drugs; AST levels $>3 x$ ULN and/or ALT levels $>3 x$ ULN and/or total bilirubin $>34 \mu \mathrm{mol} / \mathrm{L}$; and creatine kinase $>10 \times$ ULN. All participants abstained from donating blood, plasma or platelets during the study

Diagnostic criteria: not reported

\begin{tabular}{ll}
\hline Interventions & Number of study centres: 152 \\
Run-in period: yes, 2-week single-blind (to participants only) placebo lead-in period \\
Extension period: no
\end{tabular}

Outcomes Composite outcome measures reported: no

\begin{tabular}{ll}
\hline Study details & Trial terminated early: no \\
& Trial ID: NCT01006603 \\
\hline Publication details & Language of publication: English \\
& Funding: commercial funding by AstraZeneca and Bristol-Myers Squibb \\
& Publication status: peer-reviewed journal
\end{tabular}

\begin{tabular}{ll}
\hline Stated aim for study & $\begin{array}{l}\text { Quote from publication: "To assess the efficacy and safety of adjunctive saxagliptin vs glimepiride in } \\
\text { elderly patients with type } 2 \text { diabetes (T2D) and inadequate glycaemic control." }\end{array}$
\end{tabular}

\begin{tabular}{ll}
\hline Notes & - \\
\hline Risk of bias &
\end{tabular}

\begin{tabular}{lll}
\hline Bias & Authors' judgement & Support for judgement \\
\hline $\begin{array}{ll}\text { Random sequence genera- } \\
\text { tion (selection bias) }\end{array}$ & Low risk & $\begin{array}{l}\text { Quote from publication: "Randomization was carried out via an interactive } \\
\text { web response system..." } \\
\text { Comment: adequate generation of random sequence ensured }\end{array}$ \\
& & Com \\
\hline
\end{tabular}

\begin{tabular}{|c|c|c|}
\hline $\begin{array}{l}\text { Allocation concealment } \\
\text { (selection bias) }\end{array}$ & Low risk & $\begin{array}{l}\text { Quote from publication: "Randomization was carried out via an interactive } \\
\text { web response system..." } \\
\text { Comment: adequate concealment ensured }\end{array}$ \\
\hline $\begin{array}{l}\text { Blinding of participants } \\
\text { and personnel (perfor- } \\
\text { mance bias) } \\
\text { all-cause/cardiovascular } \\
\text { mortality }\end{array}$ & Low risk & $\begin{array}{l}\text { Quote from publication: "double-blind" } \\
\text { Comment: investigator-assessed outcome measurement. Blinding ensured }\end{array}$ \\
\hline
\end{tabular}

\begin{tabular}{|c|c|c|}
\hline $\begin{array}{l}\text { Blinding of participants } \\
\text { and personnel (perfor- } \\
\text { mance bias) } \\
\text { hypoglycaemia }\end{array}$ & Low risk & $\begin{array}{l}\text { Quote from publication: "double-blind" } \\
\text { Comment: investigator-assessed or self-reported outcome measurement. } \\
\text { Blinding ensured }\end{array}$ \\
\hline
\end{tabular}

\begin{tabular}{|c|c|c|}
\hline $\begin{array}{l}\text { Blinding of participants } \\
\text { and personnel (perfor- } \\
\text { mance bias) }\end{array}$ & Low risk & $\begin{array}{l}\text { Quote from publication: "double-blind" } \\
\text { Comment: investigator-assessed outcome measurement. Blinding ensured }\end{array}$ \\
\hline
\end{tabular}


Schernthaner 2015 (Continued)

non-fatal myocardial in-

farction/heart failure/non-

fatal stroke

\begin{tabular}{ll}
\hline $\begin{array}{l}\text { Blinding of participants } \\
\text { and personnel (perfor- }\end{array}$ & Quote from publication: "double-blind" \\
mance bias) & Comment: self-reported outcome measurement. Blinding ensured \\
non-serious adverse & \\
events &
\end{tabular}

\begin{tabular}{|c|c|c|}
\hline $\begin{array}{l}\text { Blinding of participants } \\
\text { and personnel (perfor- } \\
\text { mance bias) } \\
\text { serious adverse events }\end{array}$ & Low risk & $\begin{array}{l}\text { Quote from publication: "double-blind" } \\
\text { Comment: investigator-assessed outcome measurement. Blinding ensured }\end{array}$ \\
\hline
\end{tabular}

serious adverse events

\begin{tabular}{|c|c|c|}
\hline $\begin{array}{l}\text { Blinding of participants } \\
\text { and personnel (perfor- } \\
\text { mance bias) } \\
\text { weight (kg) }\end{array}$ & Low risk & $\begin{array}{l}\text { Quote from publication: "double-blind" } \\
\text { Comment: investigator-assessed or self-reported outcome measurement } \\
\text { Blinding ensured }\end{array}$ \\
\hline
\end{tabular}

\begin{tabular}{|c|c|c|}
\hline $\begin{array}{l}\text { Blinding of participants } \\
\text { and personnel (perfor- } \\
\text { mance bias) } \\
\text { HbA1c }\end{array}$ & Low risk & $\begin{array}{l}\text { Quote from publication: "double-blind" } \\
\text { Comment: investigator-assessed outcome measurement. Blinding ensured }\end{array}$ \\
\hline
\end{tabular}

\begin{tabular}{|c|c|c|}
\hline $\begin{array}{l}\text { Blinding of outcome as- } \\
\text { sessment (detection bias) } \\
\text { all-cause/cardiovascular } \\
\text { mortality }\end{array}$ & Low risk & $\begin{array}{l}\text { Quote from publication: "double-blind" } \\
\text { Comment: investigator-assessed outcome measurement. Blinding ensured }\end{array}$ \\
\hline
\end{tabular}

\begin{tabular}{|c|c|c|}
\hline $\begin{array}{l}\text { Blinding of outcome as- } \\
\text { sessment (detection bias) } \\
\text { hypoglycaemia }\end{array}$ & Low risk & $\begin{array}{l}\text { Quote from publication: "double-blind" } \\
\text { Comment: investigator-assessed or self-reported outcome measurement. } \\
\text { Blinding ensured }\end{array}$ \\
\hline
\end{tabular}

\begin{tabular}{|c|c|c|}
\hline $\begin{array}{l}\text { Blinding of outcome as- } \\
\text { sessment (detection bias) } \\
\text { non-fatal myocardial in- } \\
\text { farction/heart failure/non- } \\
\text { fatal stroke }\end{array}$ & Low risk & $\begin{array}{l}\text { Quote from publication: "double-blind" } \\
\text { Comment: investigator-assessed outcome measurement. Blinding ensured }\end{array}$ \\
\hline
\end{tabular}

\begin{tabular}{lll}
\hline $\begin{array}{l}\text { Blinding of outcome as- } \\
\text { sessment (detection bias) } \\
\text { non-serious adverse } \\
\text { events }\end{array}$ & Low risk & Quote from publication: "double-blind" \\
\hline $\begin{array}{l}\text { Blinding of outcome as- } \\
\text { sessment (detection bias) } \\
\text { serious adverse events }\end{array}$ & Low risk & Quote from publication: "double-blind" \\
\hline $\begin{array}{l}\text { Blinding of outcome as- } \\
\text { sessment (detection bias) } \\
\text { weight (kg) }\end{array}$ & Low risk & Comment: investigator-assessed outcome measurement. Blinding ensured \\
\hline $\begin{array}{l}\text { Blinding of outcome as- } \\
\text { sessment (detection bias) } \\
\text { HbAlc }\end{array}$ & Low risk & Quote from publication: "double-blind" \\
\hline
\end{tabular}


Schernthaner 2015 (Continued)

Incomplete outcome data Low risk (attrition bias) mortality all-cause/cardiovascular

Quote from publication: "The safety population, which included all randomized patients who took $\geq 1$ dose of the study medication, was used for reporting safety and tolerability results and for primary, key secondary and post hoc efficacy assessments."

Comment: $>99 \%$ of randomised participants were included in the analyses. There was a high dropout rate ( $79 \%-80 \%$ of randomised participants completed the study), however, the dropout rate was balanced between groups. We assumed that trial authors searched registers for mortality status at the end of the trial

Incomplete outcome data High risk (attrition bias)

hypoglycaemia

Quote from publication: "The safety population, which included all randomized patients who took $\geq 1$ dose of the study medication, was used for reporting safety and tolerability results and for primary, key secondary and post hoc efficacy assessments."

Comment: $>99 \%$ of the participants were included in the analyses. There was a high dropout rate $(79 \%-80 \%$ of the randomised participants completed the study), however, the dropout rate was balanced between groups. We do not know how the trial authors imputed missing data from the participants not completing the study. The reasons for dropouts were balanced. The proportion of missing outcomes compared with observed event risk was enough to induce clinically relevant bias in intervention effect estimate

Incomplete outcome data High risk (attrition bias) non-fatal myocardial infarction/heart failure/nonfatal stroke

Quote from publication: "The safety population, which included all randomized patients who took $\geq 1$ dose of the study medication, was used for reporting safety and tolerability results and for primary, key secondary and post hoc efficacy assessments."

Comment: $>99 \%$ of the participants were included in the analyses. There was a high dropout rate (79\%-80\% of the randomised participants completed the study), however, the dropout rate was balanced between groups. We do not know how the trial authors imputed missing data from the participants not completing the study. The reasons for dropouts were balanced. The proportion of missing outcomes compared with observed event risk was enough to induce clinically relevant bias in intervention effect estimate

Incomplete outcome data Low risk (attrition bias) non-serious adverse events
Quote from publication: "The safety population, which included all randomized patients who took $\geq 1$ dose of the study medication, was used for reporting safety and tolerability results and for primary, key secondary and post hoc efficacy assessments."

Comment: $>99 \%$ of the participants were included in the analyses. There was a high dropout rate (79\%-80\% of the randomised participants completed the study), however, the dropout rate was balanced between groups. We do not know how the trial authors imputed missing data from the participants not completing the study. The reasons for dropouts were balanced. The proportion of missing outcomes compared with observed event risk was not enough to induce clinically relevant bias in intervention effect estimate

\section{Incomplete outcome data High risk} (attrition bias)

serious adverse events
Quote from publication: "The safety population, which included all randomized patients who took $\geq 1$ dose of the study medication, was used for reporting safety and tolerability results and for primary, key secondary and post hoc efficacy assessments."

Comment: $>99 \%$ of the participants were included in the analyses. There was a high dropout rate $(79 \%-80 \%$ of the randomised participants completed the study), however, the dropout rate was balanced between groups. We do not know how the trial authors imputed missing data from the participants not completing the study. The reasons for dropouts were balanced. The propor- 
Incomplete outcome data Unclear risk (attrition bias)

weight $(\mathrm{kg})$
Quote from publication: "Safety and tolerability assessments included adverse events (AEs) and body weight." "The safety population, which included all randomized patients who took $\geq 1$ dose of the study medication, was used for reporting safety and tolerability results and for primary, key secondary and post hoc efficacy assessments."

Comment: $>99 \%$ of the randomised participants were included in the analyses. There was a high dropout rate $(79 \%-80 \%$ of the randomised participants completed the study), however, the dropout rate was balanced between groups. We do not know how the trial authors imputed missing data from the participants not completing the study. The reasons for dropouts were balanced

\section{Incomplete outcome data Unclear risk} (attrition bias)

HbA1c
Quote from publication: "The number of subjects with non-missing baseline and week 52 (LOCF) values in the full analysis set (defined as the subset of patients in the randomized analysis set who took at least one randomised IP dose and have non-missing baseline and post-baseline efficacy data for at least one variable). "

Comment: $>95 \%$ of the participants were included in the analyses. There was a high dropout rate $(79 \%-80 \%$ of the randomised participants completed the study), however, the dropout rate was balanced between groups. The reasons for dropouts were balanced. Inapropriate method for imputing missing data (LOCF)

\begin{tabular}{|c|c|c|}
\hline $\begin{array}{l}\text { Selective reporting (re- } \\
\text { porting bias) }\end{array}$ & Low risk & $\begin{array}{l}\text { Comment: all of the trial's primary and secondary outcomes as specified in } \\
\text { the protocol have been reported }\end{array}$ \\
\hline
\end{tabular}

Other bias Unclear risk Comment: the trial was funded by pharmaceutical companies

\section{Seck 2010}

Methods Study design: multinational, randomised, parallel-group, non-inferiority study with an active-controlled, double-blind treatment period

Randomisation ratio: 1:1 to receive sitagliptin or glipizide in addition to metformin monotherapy

\section{Non-inferiority design}

Participants

Inclusion criteria: age 18-78 years, T2DM, not currently on an $\mathrm{OHA}$, were taking any $\mathrm{OHA}$ in monotherapy or were taking metformin in combination with another $\mathrm{OHA}$

Exclusion criteria: history of T1DM, insulin use within 8 weeks of screening, renal function impairment inconsistent with the use of metformin or a FPG (or a fasting fingerstick glucose) at or just prior to randomisation $>15.0 \mathrm{mmol} / \mathrm{L}(270 \mathrm{mg} / \mathrm{dL})$

\section{Diagnostic criteria: not reported}

\section{Number of study centres: 173}

Run-in period: yes. Participants who were already on metformin $\geq 1500 \mathrm{mg} /$ day and had an $\mathrm{HbAlc} \geq$ $6.5 \%$ and $\leq 10 \%$ directly entered a 2 -week placebo run-in period and were eligible to be randomised. Participants not currently on an OHA, participants on an OHA other than metformin monotherapy at a dose $\geq 1500 \mathrm{mg} /$ day or participants on metformin in combination with another OHA entered a metformin monotherapy treatment titration and dose-stable period of at least 8 weeks. Participants with 
Extension period: yes, the study continued as a randomised, double-blind, active-controlled study for an additional year

$\begin{array}{ll}\text { Outcomes } & \begin{array}{l}\text { Composite outcome measures reported: yes, composite endpoint for HbA1c reduction, lack of hypo- } \\ \text { glycaemia and no body weight gain }\end{array}\end{array}$

Study details

Trial terminated early: no

Trial ID: NCT00094770

\begin{tabular}{ll}
\hline Stated aim for study & $\begin{array}{l}\text { Quote from publication: "To evaluate the 2-year safety and efficacy of adding sitagliptin or glipizide to } \\
\text { ongoing metformin in patients with type } 2 \text { diabetes" }\end{array}$ \\
\hline Notes & - \\
\hline
\end{tabular}

\section{Risk of bias}

Bias Authors' judgement Support for judgement

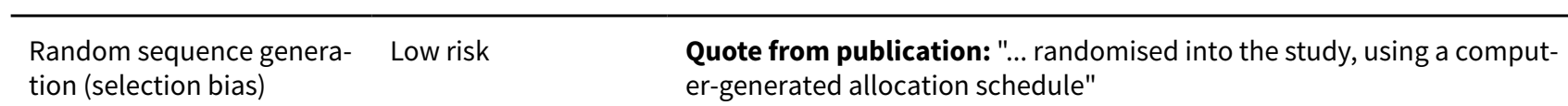
$\begin{array}{ll}\text { tion (selection bias) } & \text { er-generated allocation schedule" }\end{array}$

Comment: adequate generation of random sequence ensured

\begin{tabular}{|c|c|c|}
\hline $\begin{array}{l}\text { Allocation concealment } \\
\text { (selection bias) }\end{array}$ & Low risk & $\begin{array}{l}\text { Quote from publication: "... randomised into the study, using a comput- } \\
\text { er-generated allocation schedule" } \\
\text { Comment: adequate concealment ensured }\end{array}$ \\
\hline $\begin{array}{l}\text { Blinding of participants } \\
\text { and personnel (perfor- } \\
\text { mance bias) } \\
\text { all-cause/cardiovascular } \\
\text { mortality }\end{array}$ & Low risk & $\begin{array}{l}\text { Quote from publication: "double-blind" "All adverse experiences were rated } \\
\text { by the study site investigators for intensity and relationship to study drug" } \\
\text { Comment: investigator-assessed outcome measurement. Blinding of study } \\
\text { personnel ensured }\end{array}$ \\
\hline
\end{tabular}

Blinding of participants Low risk
and personnel (performance bias)

hypoglycaemia

Quote from publication: "double-blind" "Patients experiencing symptoms of hypoglycaemia were instructed to obtain a fingerstick glucose, record the value in a log book and contact their study site" All adverse experiences were rated by the study site investigators for intensity and relationship to study drug"

Comment: investigator-assessed/self-reported outcome measurement. Blinding of participants and study personnel ensured

\begin{tabular}{ll}
\hline $\begin{array}{l}\text { Blinding of participants } \\
\text { and personnel (perfor- }\end{array}$ & Low risk \\
$\begin{array}{l}\text { mance bias) } \\
\text { non-fatal myocardial in- }\end{array}$ & $\begin{array}{l}\text { Quote from publication: "double-blind" "All adverse experiences were rated } \\
\text { by the study site investigators for intensity and relationship to study drug" }\end{array}$ \\
$\begin{array}{l}\text { farction/heart failure/non- } \\
\text { fatal stroke }\end{array}$ & $\begin{array}{l}\text { Comment: investigator-assessed outcome measurement. Blinding of study } \\
\text { personnel ensured }\end{array}$ \\
\hline
\end{tabular}


Seck 2010 (Continued)

Blinding of participants Low risk Quote from publication: "double-blind" "All adverse experiences were rated and personnel (perforby the study site investigators for intensity and relationship to study drug" mance bias) non-serious adverse events

Comment: investigator-assessed/self-reported outcome measurement. Blinding of participants and study personnel ensured

Blinding of participants Low risk and personnel (performance bias)

serious adverse events
Quote from publication: "double-blind" "All adverse experiences were rated by the study site investigators for intensity and relationship to study drug"

Comment: investigator-assessed outcome measurement. Blinding of study personnel ensured

Quote from publication: "double-blind"

$\begin{array}{ll}\begin{array}{l}\text { Blinding of participants } \\ \text { and personnel (perfor- }\end{array} & \text { Low risk } \\ \begin{array}{l}\text { mance bias) } \\ \text { weight }(\mathrm{kg})\end{array} & \begin{array}{l}\text { Comment: investigator-assessed/self-reported outcome measurement. Blind- } \\ \text { ing of participants and study personnel ensured }\end{array}\end{array}$

\begin{tabular}{|c|c|c|}
\hline $\begin{array}{l}\text { Blinding of participants } \\
\text { and personnel (perfor- } \\
\text { mance bias) } \\
\text { HbA1c }\end{array}$ & Low risk & $\begin{array}{l}\text { Quote from publication: "double-blind" } \\
\text { Comment: investigator-assessed outcome measurement. Blinding of study } \\
\text { personnel ensured }\end{array}$ \\
\hline
\end{tabular}

\begin{tabular}{lll}
\hline $\begin{array}{l}\text { Blinding of outcome as- } \\
\text { sessment (detection bias) }\end{array}$ & Low risk & $\begin{array}{l}\text { Quote from publication: "double-blind" "All adverse experiences were rated } \\
\text { by the study site investigators for intensity and relationship to study drug" }\end{array}$
\end{tabular}
all-cause/cardiovascular mortality

Comment: investigator-assessed outcome measurement. Blinding of study personnel ensured

\section{Blinding of outcome as- Low risk} sessment (detection bias) hypoglycaemia

Quote from publication: "double-blind" "Patients experiencing symptoms of hypoglycaemia were instructed to obtain a fingerstick glucose, record the value in a log book and contact their study site" All adverse experiences were rated by the study site investigators for intensity and relationship to study drug"

Comment: investigator-assessed/self-reported outcome measurement. Blinding of participants and study personnel ensured

\begin{tabular}{|c|c|c|}
\hline $\begin{array}{l}\text { Blinding of outcome as- } \\
\text { sessment (detection bias) } \\
\text { non-fatal myocardial in- } \\
\text { farction/heart failure/non- } \\
\text { fatal stroke }\end{array}$ & Low risk & $\begin{array}{l}\text { Quote from publication: "double-blind" "All adverse experiences were rated } \\
\text { by the study site investigators for intensity and relationship to study drug" } \\
\text { Comment: investigator-assessed outcome measurement. Blinding of study } \\
\text { personnel ensured }\end{array}$ \\
\hline $\begin{array}{l}\text { Blinding of outcome as- } \\
\text { sessment (detection bias) } \\
\text { non-serious adverse } \\
\text { events }\end{array}$ & Low risk & $\begin{array}{l}\text { Quote from publication: "double-blind" "All adverse experiences were rated } \\
\text { by the study site investigators for intensity and relationship to study drug" } \\
\text { Comment: investigator-assessed/self-reported outcome measurement. Blind- } \\
\text { ing of participants and study personnel ensured }\end{array}$ \\
\hline $\begin{array}{l}\text { Blinding of outcome as- } \\
\text { sessment (detection bias) } \\
\text { serious adverse events }\end{array}$ & Low risk & $\begin{array}{l}\text { Quote from publication: "double-blind" "All adverse experiences were rated } \\
\text { by the study site investigators for intensity and relationship to study drug" } \\
\text { Comment: investigator-assessed outcome measurement. Blinding of study } \\
\text { personnel ensured }\end{array}$ \\
\hline $\begin{array}{l}\text { Blinding of outcome as- } \\
\text { sessment (detection bias) } \\
\text { weight (kg) }\end{array}$ & Low risk & $\begin{array}{l}\text { Quote from publication: "double-blind" } \\
\text { Comment: investigator-assessed/self-reported outcome measurement. Blind- } \\
\text { ing of participants and study personnel ensured }\end{array}$ \\
\hline
\end{tabular}


Seck 2010 (Continued)

Blinding of outcome as- Low risk Quote from publication: "double-blind" sessment (detection bias) HbA1c

Comment: investigator-assessed outcome measurement. Blinding of study personnel ensured

Incomplete outcome data Low risk

(attrition bias)

Quote from publication: "Safety and tolerability were evaluated over the 2-

all-cause/cardiovascular year treatment period by a review of safety parameters including... data from mortality the all-patients-as-treated (APaT) cohort, which was defined as all randomised patients who received at least one dose of study medication." "In the analyses of safety parameters, missing values were not imputed."

Comment: $100 \%$ of randomised participants were included in the analyses. There was a high dropout rate ( $43 \%-45 \%$ of randomised participants completed the study), however, the dropout rate was balanced between groups. We assumed that trial authors searched registers for mortality status at the end of the trial

Incomplete outcome data High risk (attrition bias)

hypoglycaemia
Quote from publication: "Safety and tolerability were evaluated over the 2year treatment period by a review of safety parameters including... data from the all-patients-as-treated (APaT) cohort, which was defined as all randomised patients who received at least one dose of study medication." "In the analyses of safety parameters, missing values were not imputed."

Comment: $100 \%$ of the randomised participants were included in the analyses. There was a high dropout rate $(43 \%-45 \%$ of the participants completed the study), however, the dropout rate was balanced between groups. No imputation of data from the participants not completing the study. The reasons for dropouts were balanced. The proportion of missing outcomes compared with observed event risk was enough to induce clinically relevant bias in intervention effect estimate

\section{Incomplete outcome data High risk} (attrition bias) non-fatal myocardial infarction/heart failure/nonfatal stroke

Quote from publication: "Safety and tolerability were evaluated over the 2year treatment period by a review of safety parameters including... data from the all-patients-as-treated (APaT) cohort, which was defined as all randomised patients who received at least one dose of study medication." "In the analyses of safety parameters, missing values were not imputed."

Comment: $100 \%$ of the randomised participants were included in the analyses. There was a high dropout rate (43\%- $45 \%$ of the participants completed the study), however, the dropout rate was balanced between groups. No imputation of data from the participants not completing the study. The reasons for dropouts were balanced. The proportion of missing outcomes compared with observed event risk was enough to induce clinically relevant bias in intervention effect estimate

Quote from publication: "Safety and tolerability were evaluated over the 2year treatment period by a review of safety parameters including... data from the all-patients-as-treated (APaT) cohort, which was defined as all randomised patients who received at least one dose of study medication." "In the analyses of safety parameters, missing values were not imputed."

Comment: $100 \%$ of the randomised participants were included in the analyses. There was a high dropout rate ( $43 \%-45 \%$ of the participants completed the study), however, the dropout rate was balanced between groups. No imputation of data from the participants not completing the study. The reasons for dropouts were balanced. The proportion of missing outcomes compared with observed event risk was not enough to induce clinically relevant bias in intervention effect estimate

Incomplete outcome data High risk (attrition bias)
Quote from publication: "Safety and tolerability were evaluated over the 2year treatment period by a review of safety parameters including... data from 
Seck 2010 (Continued) serious adverse events the all-patients-as-treated (APaT) cohort, which was defined as all randomised patients who received at least one dose of study medication." "In the analyses of safety parameters, missing values were not imputed."

Comment: $100 \%$ of the randomised participants were included in the analyses. There was a high dropout rate (43\%- $45 \%$ of the participants completed the study), however, the dropout rate was balanced between groups. No imputation of data from the participants not completing the study. The reasons for dropouts were balanced. The proportion of missing outcomes compared with observed event risk was enough to induce clinically relevant bias in intervention effect estimate
Incomplete outcome data High risk (attrition bias) weight $(\mathrm{kg})$
Quote from publication: "Safety and tolerability were evaluated over the 2year treatment period by a review of safety parameters including... data from the all-patients-as-treated (APaT) cohort, which was defined as all randomised patients who received at least one dose of study medication." "In the analyses of safety parameters, missing values were not imputed."

Comment: $43 \%-45 \%$ of the randomised participants were included in the analyses. There was a high dropout rate ( $43 \%-45 \%$ of the participants completed the study), however, the dropout rate was balanced between groups. No imputation used. The reasons for dropouts were balanced. Plausible effect size among missing outcomes enough to induce clinically relevant bias in observed effect size

Incomplete outcome data High risk (attrition bias)

Quote from publication: "To support the findings in the analysis of the PP population, additional efficacy analyses were performed for key endpoints (HbAlc and FPG) on the all-patients-treated (APT) cohort that consisted of all randomised patients who received at least one dose of study treatment and who had both a baseline and at least one post-baseline measurement. Missing values in the APT analysis were imputed by the last observation carried forward approach over the 2-year study."

Comment: $>95 \%$ of the randomised participants were included in the analyses. There was a high dropout rate (43\%- $45 \%$ of the participants completed the study), however, the dropout rate was balanced between groups. The reasons for dropouts were balanced. Inapropiate imputation method used (LOCF)

Comment: all of the trial's primary and secondary outcomes as specified in the protocol have been reported
Selective reporting (re- Low risk porting bias)
Comment: the trial was funded by a pharmaceutical company

Other bias Unclear risk Comment: the trial was funded by a pharmaceutical company

Vaccaro 2017

\begin{tabular}{|c|c|}
\hline Methods & $\begin{array}{l}\text { Study design: multicentre, randomised, open-label, parallel trial } \\
\text { Randomisation ratio: 1:1 to receive pioglitazone or a sulphonylurea in addition to metformin }\end{array}$ \\
\hline \multirow[t]{2}{*}{ Participants } & $\begin{array}{l}\text { Inclusion criteria: age } 50-75 \text { years, T2DM of at least } 2 \text { years duration, } \mathrm{BMI} 20-45 \mathrm{~kg} / \mathrm{m}^{2} \text {, stable treat- } \\
\text { ment for the last } 2 \text { months with metformin in monotherapy (least } 2-3 \mathrm{~g} / \text { day), } \mathrm{HbA} 1 \mathrm{c} \text { of } 7.0 \%-9.0 \%\end{array}$ \\
\hline & $\begin{array}{l}\text { Exclusion criteria: T1DM, previous treatment with thiazolidinediones in the last six months, con- } \\
\text { traindication/intolerance to metformin or sulphonylureas or thiazolidinediones, documented coro- } \\
\text { nary or cerebrovascular events in the previous } 3 \text { months, serum creatinine }>1.5 \mathrm{mg} / \mathrm{dL} \text {, history of con- } \\
\text { gestive heart failure, NYHA } \geq \mathrm{I} \text {, chronic use of glucocorticoids, ischaemic ulcer or gangrene, liver cirrho- } \\
\text { sis or severe hepatic dysfunction (ALT increase of } 2.5 \times \mathrm{ULN} \text { ), pregnancy or breast feeding, cancer, sub- } \\
\text { stance abuse or any health problem that may interfere with the compliance to the study protocol or } \\
\text { limit life expectancy }\end{array}$ \\
\hline
\end{tabular}


Vaccaro 2017 (Continued)

Diagnostic criteria: not reported

\begin{tabular}{|c|c|}
\hline Interventions & $\begin{array}{l}\text { Number of study centres: } 57 \\
\text { Run-in period: no }\end{array}$ \\
\hline Outcomes & $\begin{array}{l}\text { Composite outcome measures reported: "The primary efficacy outcome is a composite of all-cause } \\
\text { mortality, nonfatal myocardial infarction (including silent myocardial infarction), nonfatal stroke, un- } \\
\text { planned coronary revascularization. The principal secondary outcome is a composite ischemic end } \\
\text { point of sudden death, fatal and non-fatal myocardial infarction (including silent myocardial infarc- } \\
\text { tion), fatal and nonfatal stroke, major leg amputation (above the ankle), endovascular or surgical in- } \\
\text { terventions on the coronary, leg or carotid arteries. Other secondary outcomes are: } 1 \text { ) A composite CV } \\
\text { endpoint including the primary endpoint plus hospitalization for heart failure, endovascular or surgi- } \\
\text { cal intervention on the coronary, leg or carotid arteries, incident angina or intermittent claudication; } 2 \text { ) } \\
\text { All cases of heart failure; } 3 \text { ) A microvascular composite endpoint including: incident macroalbuminuria, } \\
\text { or doubling of baseline plasma creatinine, or a creatinine clearance reduction of } 20 \mathrm{ml} / \mathrm{min} / 1.73 \mathrm{~m}^{2} \text { or } \\
\text { plasma creatinine }>3.3 \mathrm{mg} / \mathrm{dl} \text {, or dialysis..." }\end{array}$ \\
\hline
\end{tabular}

Study details

Trial terminated early: "The observed event rate during follow-up was lower than expected, with 213 adjudicated primary endpoint events in total. Following the data and safety monitoring board's recommendation, a futility analysis for the primary endpoint using a frequentist approach was done in March 31,2017 . The results of this analysis showed that, if the future data distribution followed the current trend (the most plausible hypothesis), the probability of observing a significant positive result (ie, an HR of $0 \cdot 80$, two-sided log-rank test) at the planned end of follow-up would be as low as $5 \%$. On the basis of the futility analysis, the study was discontinued on May 23, 2017."

Trial ID: NCT00700856

\begin{tabular}{ll}
\hline Publication details & Language of publication: English \\
& Funding: non-commercial funding (the Italian Medicines Agency, the Italian Diabetes Society, and Dia- \\
bete Ricerca (a non-profit foundation))
\end{tabular}

Publication status: peer-reviewed journal

Stated aim for study

Quote from publication: "The aim of the study was to compare the long-term effect of these two therapeutic options with respect to incidence of cardiovascular events, as well as their effects on glucose control and safety"

Notes -

\section{Risk of bias}

\begin{tabular}{lll}
\hline Bias & Authors' judgement & Support for judgement \\
\hline $\begin{array}{l}\text { Random sequence genera- } \\
\text { tion (selection bias) }\end{array}$ & Low risk & $\begin{array}{l}\text { Quote from publication: "Permuted blocks randomisation (block size 10) was } \\
\text { done centrally via an interactive telephone system..." }\end{array}$
\end{tabular}

Comment: adequate generation of random sequence ensured

\begin{tabular}{lll}
\hline $\begin{array}{l}\text { Allocation concealment } \\
\text { (selection bias) }\end{array}$ & Low risk & $\begin{array}{l}\text { Quote from publication: "Permuted blocks randomisation (block size 10) was } \\
\text { done centrally via an interactive telephone system..." } \\
\text { Comment: adequate concealment ensured }\end{array}$ \\
\hline $\begin{array}{l}\text { Blinding of participants } \\
\text { and personnel (perfor- }\end{array}$ & Low risk & $\begin{array}{l}\text { Quote from publication: "Participants and investigators were aware of treat- } \\
\text { mance bias) }\end{array}$ \\
$\begin{array}{l}\text { all-cause/cardiovascular } \\
\text { mortality }\end{array}$ & $\begin{array}{l}\text { ed adverse events of particular interest with respect to the study drugs (heart } \\
\text { failure, pathological fractures, macular oedema, and neoplasms) were adjudi- } \\
\text { cated by an independent endpoint committee unaware of treatment group as- } \\
\text { signment." }\end{array}$ \\
\hline
\end{tabular}


Comment: adjudicated outcome measurement. Incomplete blinding, the outcome is unlikely to be influenced by lack of blinding

Blinding of participants Low risk
and personnel (perfor-
mance bias)
amputation of lower ex-
tremity/blindness or se-
vere vision loss/end-stage
renal disease

renal disease

\begin{abstract}
Quote from publication: "Participants and investigators were aware of treatment assignment. The components of the primary outcome and some selected adverse events of particular interest with respect to the study drugs (heart failure, pathological fractures, macular oedema, and neoplasms) were adjudicated by an independent endpoint committee unaware of treatment group assignment."
\end{abstract}

Comment: investigator-assessed outcome measurement. Incomplete blinding, the outcome is unlikely to be influenced by lack of blinding
Blinding of participants High risk and personnel (performance bias)

hypoglycaemia
Quote from publication: "Participants and investigators were aware of treatment assignment. The components of the primary outcome and some selected adverse events of particular interest with respect to the study drugs (heart failure, pathological fractures, macular oedema, and neoplasms) were adjudicated by an independent endpoint committee unaware of treatment group assignment."

Comment: investigator-assessed/self-reported outcome measurement. Incomplete blinding, the outcome is likely to be influenced by lack of blinding

$\begin{array}{ll}\begin{array}{l}\text { Blinding of participants } \\ \text { and personnel (perfor- }\end{array} & \text { Quote from publication: "Participants and investigators were aware of treat- } \\ \text { mance bias) } & \begin{array}{l}\text { ment assignment. The components of the primary outcome and some select- } \\ \text { ed adverse events of particular interest with respect to the study drugs (heart } \\ \text { non-fatal myocardial in- }\end{array} \\ \begin{array}{l}\text { farction/heart failure/non- } \\ \text { fatal stroke }\end{array} & \begin{array}{l}\text { cated by an independent endpoint committee unaware of treatment group as- } \\ \text { signment." }\end{array}\end{array}$

Comment: adjudicated outcome measurement. Incomplete blinding, the outcome is unlikely to be influenced by lack of blinding

Blinding of participants High risk and personnel (performance bias) non-serious adverse events

Quote from publication: "Participants and investigators were aware of treatment assignment. The components of the primary outcome and some selected adverse events of particular interest with respect to the study drugs (heart failure, pathological fractures, macular oedema, and neoplasms) were adjudicated by an independent endpoint committee unaware of treatment group assignment."

Comment: self-reported outcome measurement. Incomplete blinding, the outcome is likely to be influenced by lack of blinding

\begin{tabular}{|c|c|c|}
\hline $\begin{array}{l}\text { Blinding of participants } \\
\text { and personnel (perfor- } \\
\text { mance bias) } \\
\text { serious adverse events }\end{array}$ & Low risk & $\begin{array}{l}\text { Quote from publication: "Participants and investigators were aware of treat- } \\
\text { ment assignment. The components of the primary outcome and some select- } \\
\text { ed adverse events of particular interest with respect to the study drugs (heart } \\
\text { failure, pathological fractures, macular oedema, and neoplasms) were adjudi- } \\
\text { cated by an independent endpoint committee unaware of treatment group as- } \\
\text { signment." }\end{array}$ \\
\hline
\end{tabular}

Comment: adjudicated/investigator-assessed outcome measurement. Incomplete blinding, the outcome is unlikely to be influenced by lack of blinding

\begin{tabular}{|c|c|c|}
\hline $\begin{array}{l}\text { Blinding of participants } \\
\text { and personnel (perfor- } \\
\text { mance bias) } \\
\text { weight (kg) }\end{array}$ & High risk & $\begin{array}{l}\text { Quote from publication: "Participants and investigators were aware of treat- } \\
\text { ment assignment. The components of the primary outcome and some select- } \\
\text { ed adverse events of particular interest with respect to the study drugs (heart } \\
\text { failure, pathological fractures, macular oedema, and neoplasms) were adjudi- } \\
\text { cated by an independent endpoint committee unaware of treatment group as- } \\
\text { signment." }\end{array}$ \\
\hline
\end{tabular}


Comment: investigator-assessed/self-reported outcome measurement. Incomplete blinding, the outcome is likely to be influenced by lack of blinding

Blinding of participants Low risk
and personnel (perfor-
mance bias)
HbAlc

Quote from publication: "Participants and investigators were aware of treatment assignment. The components of the primary outcome and some selected adverse events of particular interest with respect to the study drugs (heart failure, pathological fractures, macular oedema, and neoplasms) were adjudicated by an independent endpoint committee unaware of treatment group assignment."

Comment: adjudicated/investigator-assessed outcome measurement. Incomplete blinding, the outcome is unlikely to be influenced by lack of blinding

\begin{tabular}{|c|c|c|}
\hline $\begin{array}{l}\text { Blinding of outcome as- } \\
\text { sessment (detection bias) } \\
\text { all-cause/cardiovascular } \\
\text { mortality }\end{array}$ & Low risk & $\begin{array}{l}\text { Quote from publication: "Participants and investigators were aware of treat- } \\
\text { ment assignment. The components of the primary outcome and some select- } \\
\text { ed adverse events of particular interest with respect to the study drugs (heart } \\
\text { failure, pathological fractures, macular oedema, and neoplasms) were adjudi- } \\
\text { cated by an independent endpoint committee unaware of treatment group as- } \\
\text { signment." }\end{array}$ \\
\hline
\end{tabular}

Comment: adjudicated outcome measurement. Blinding of outcome committee ensured

\begin{tabular}{|c|c|c|}
\hline $\begin{array}{l}\text { Blinding of outcome as- } \\
\text { sessment (detection bias) } \\
\text { amputation of lower ex- } \\
\text { tremity/blindness or se- } \\
\text { vere vision loss/end-stage } \\
\text { renal disease }\end{array}$ & Low risk & $\begin{array}{l}\text { Quote from publication: "Participants and investigators were aware of treat- } \\
\text { ment assignment. The components of the primary outcome and some select- } \\
\text { ed adverse events of particular interest with respect to the study drugs (heart } \\
\text { failure, pathological fractures, macular oedema, and neoplasms) were adjudi- } \\
\text { cated by an independent endpoint committee unaware of treatment group as- } \\
\text { signment." }\end{array}$ \\
\hline
\end{tabular}

Comment: investigator-assessed outcome measurement. Incomplete blinding, the outcome is unlikely to be influenced by lack of blinding

\begin{abstract}
Blinding of outcome as- Low risk sessment (detection bias) non-fatal myocardial infarction/heart failure/nonfatal stroke ment assignment. The components of the primary outcome and some selected adverse events of particular interest with respect to the study drugs (heart failure, pathological fractures, macular oedema, and neoplasms) were adjudicated by an independent endpoint committee unaware of treatment group assignment."
\end{abstract}

Quote from publication: "Participants and investigators were aware of treatment assignment. The components of the primary outcome and some selected adverse events of particular interest with respect to the study drugs (heart failure, pathological fractures, macular oedema, and neoplasms) were adjudicated by an independent endpoint committee unaware of treatment group assignment."

Comment: investigator-assessed/self-reported outcome measurement. Incomplete blinding, the outcome is likely to be influenced by lack of blinding
High risk

Quote from publication: "Participants and investigators were aware of treat-

Comment: adjudicated outcome measurement. Blinding of outcome committee ensured

\begin{tabular}{lll}
\hline $\begin{array}{l}\text { Blinding of outcome as- } \\
\text { sessment (detection bias) }\end{array}$ & High risk & Quote from publication: "Participants and investigators were aware of treat- \\
non-serious adverse & ment assignment. The components of the primary outcome and some select- \\
events & ed adverse events of particular interest with respect to the study drugs (heart \\
& failure, pathological fractures, macular oedema, and neoplasms) were adjudi- \\
& cated by an independent endpoint committee unaware of treatment group as- \\
signment."
\end{tabular}


Comment: self-reported outcome measurement. Incomplete blinding, the outcome is likely to be influenced by lack of blinding

Blinding of outcome as- Low risk sessment (detection bias) serious adverse events
Quote from publication: "Participants and investigators were aware of treatment assignment. The components of the primary outcome and some selected adverse events of particular interest with respect to the study drugs (heart failure, pathological fractures, macular oedema, and neoplasms) were adjudicated by an independent endpoint committee unaware of treatment group assignment."

Comment: adjudicated/investigator-assessed outcome measurement. Incomplete blinding, the outcome is unlikely to be influenced by lack of blinding

\section{Blinding of outcome as- High risk} sessment (detection bias) weight $(\mathrm{kg})$

Quote from publication: "Participants and investigators were aware of treatment assignment. The components of the primary outcome and some selected adverse events of particular interest with respect to the study drugs (heart failure, pathological fractures, macular oedema, and neoplasms) were adjudicated by an independent endpoint committee unaware of treatment group assignment."

Comment: investigator-assessed/self-reported outcome measurement. Incomplete blinding, the outcome is likely to be influenced by lack of blinding

Blinding of outcome as- Low risk sessment (detection bias) HbAlc
Quote from publication: "Participants and investigators were aware of treatment assignment. The components of the primary outcome and some selected adverse events of particular interest with respect to the study drugs (heart failure, pathological fractures, macular oedema, and neoplasms) were adjudicated by an independent endpoint committee unaware of treatment group assignment."

Comment: adjudicated/investigator-assessed outcome measurement. Incomplete blinding, the outcome is unlikely to be influenced by lack of blinding
Incomplete outcome data Low risk (attrition bias) all-cause/cardiovascular mortality
Quote from publication: "The safety analysis set includes only participants exposed to the trial medications. Participants were regarded as exposed to the trial medications as long as they had taken at least one dose of pioglitazone or sulfonylurea." "Patients who prematurely discontinued the study drugs were followed up for ascertainment of cardiovascular outcomes and information on vital status was obtained from the national health registry using the patient's fiscal code as identifier for patients lost to follow-up."

Comment: $>99 \%$ of the randomised participants were included in the analyses. There was a high dropout rate ( $72 \%-84 \%$ of randomised participants completed the study). The dropout rate was not balanced between groups, mostly due to decision from participants to leave trial. Vital status was searched in registers.
Incomplete outcome data High risk (attrition bias) amputation of lower extremity/blindness or severe vision loss/end-stage renal disease
Quote from publication: "The safety analysis set includes only participants exposed to the trial medications. Participants were regarded as exposed to the trial medications as long as they had taken at least one dose of pioglitazone or sulfonylurea."

Comment: $>99 \%$ of the randomised participants were included in the analyses. There was a high dropout rate ( $72 \%-84 \%$ of randomised participants completed the study). The dropout rate was not balanced between groups, mostly due to decision from participants to leave trial. No information on imputation method. The proportion of missing outcomes compared with observed event risk was enough to induce clinically relevant bias in intervention effect estimate. 
Vaccaro 2017 (Continued)

Incomplete outcome data Low risk (attrition bias)

hypoglycaemia

Quote from publication: "The safety analysis set includes only participants exposed to the trial medications. Participants were regarded as exposed to the trial medications as long as they had taken at least one dose of pioglitazone or sulfonylurea."

Comment: $>99 \%$ of the randomised participants were included in the analyses. There was a high dropout rate ( $72 \%-84 \%$ of randomised participants completed the study). The dropout rate was not balanced between groups, mostly due to decision from participants to leave trial. No information on imputation method. The proportion of missing outcomes compared with observed event risk was not enough to induce clinically relevant bias in intervention effect estimate.

Incomplete outcome data Low risk (attrition bias)

non-fatal myocardial in-

farction/heart failure/non-

fatal stroke
Quote from publication: "The safety analysis set includes only participants exposed to the trial medications. Participants were regarded as exposed to the trial medications as long as they had taken at least one dose of pioglitazone or sulfonylurea." "Patients who prematurely discontinued the study drugs were followed up for ascertainment of cardiovascular outcomes and information on vital status was obtained from the national health registry using the patient's fiscal code as identifier for patients lost to follow-up."

Comment: $>99 \%$ of the randomised participants were included in the analyses. There was a high dropout rate $(72 \%-84 \%$ of randomised participants completed the study). The dropout rate was not balanced between groups, mostly due to decision from participants to leave trial. Participants who prematurely discontinued the study drugs were followed up for ascertainment of cardiovascular outcomes

\section{Incomplete outcome data Low risk} (attrition bias) non-serious adverse events

Quote from publication: "The safety analysis set includes only participants exposed to the trial medications. Participants were regarded as exposed to the trial medications as long as they had taken at least one dose of pioglitazone or sulfonylurea."

Comment: $>99 \%$ of the randomised participants were included in the analyses. There was a high dropout rate $(72 \%-84 \%$ of randomised participants completed the study). The dropout rate was not balanced between groups, mostly due to decision from participants to leave trial. No information on imputation method. The proportion of missing outcomes compared with observed event risk was not enough to induce clinically relevant bias in intervention effect estimate

Incomplete outcome data High risk (attrition bias)

serious adverse events
Quote from publication: "The safety analysis set includes only participants exposed to the trial medications. Participants were regarded as exposed to the trial medications as long as they had taken at least one dose of pioglitazone or sulfonylurea."

Comment: $>99 \%$ of the randomised participants were included in the analyses. There was a high dropout rate $(72 \%-84 \%$ of randomised participants completed the study). The dropout rate was not balanced between groups, mostly due to decision from participants to leave trial. No information on imputation method. The proportion of missing outcomes compared with observed event risk was enough to induce clinically relevant bias in intervention effect estimate
Incomplete outcome data Unclear risk (attrition bias)

weight $(\mathrm{kg})$
Quote from publication: "The trial efficacy analysis was done in the modified intention-to-treat population, which included all randomly assigned participants with baseline data available and without any protocol violations in relation to inclusion or exclusion criteria. Data from the patients who completed or discontinued the trial without having an outcome were censored from the day of their last visit; events occurring after that visit were not included" 
Comment: $>99 \%$ of the randomised participants were included in the analyses. There was a high dropout rate $(72 \%-84 \%$ of randomised participants completed the study). The dropout rate was not balanced between groups, mostly due to decision from participants to leave trial. No information on imputation method

Incomplete outcome data Unclear risk (attrition bias)

HbA1c
Quote from publication: "The trial efficacy analysis was done in the modified intention-to-treat population, which included all randomly assigned participants with baseline data available and without any protocol violations in relation to inclusion or exclusion criteria. Data from the patients who completed or discontinued the trial without having an outcome were censored from the day of their last visit; events occurring after that visit were not included"

Comment: $>99 \%$ of the randomised participants were included in the analyses. There was a high dropout rate $(72 \%-84 \%$ of randomised participants completed the study). The dropout rate was not balanced between groups, mostly due to decision from participants to leave trial. No information on imputation method

Comment: all of the trial's primary and secondary outcomes as specified in the protocol have been reported

Selective reporting (re- Low risk
porting bias)

ADA: American Diabetes Association; AE: adverse event; ALT: alanine amino transferase; AST: aspartate aminotransferase; BMI: body mass index; BP: blood pressure; CDM: core diabetes model; CV: cardiovascular event; CI: confidence interval; DBP: diastolic blood pressure; DPP-4: dipeptidyl peptidase-4; EASD: European Association for the Study of Diabetes; eGFR: estimated glomerular filtration rate; ESC: European Society of Cardiology; FPG: fasting plasma glucose; GFR: glomerular filtration rate; GLP-1: glucagon-like peptide 1; HbA1C: glycosylated haemoglobin A1c; LOCF: last observation carried forward; MI: myocardial infarction; MTD: maximum tolerated dose; NYHA: New York Heart Association; OAD: oral antidiabetic drug; OHA: oral antihyperglycaemic agent; PPAR -ү: peroxisome proliferator-activated receptor gamma; PT: proximal tubule; RCT: randomised controlled trial; SAE: serious adverse event; SBP: systolic blood pressure; SGLT-2: sodium-glucose co-transporter 2; SU: sulphonylurea; T1DM: type 1 diabetes mellitus; T2DM: type 2 diabetes mellitus; TSH: thyroidstimulating hormone; TZD: thiazolidinediones; ULN: upper limit of normal; WHO: World Health Organization

\section{Characteristics of excluded studies [ordered by study ID]}

\begin{tabular}{ll}
\hline Study & Reason for exclusion \\
\hline ACCORD 2007 & Did not compare interventions of interest \\
\hline Alsharidah 2018 & Not a randomised clinical trial \\
\hline Araki 2015 & Did not compare interventions of interest \\
\hline Bermudez-Pirela 2007 & Duration of the intervention < 52 weeks \\
\hline Berndt-Zipfel 2013 & Duration of the intervention $<52$ weeks \\
\hline Bode 2013 & Did not compare interventions of interest \\
\hline Bruce 2006 & Duration of the intervention < 52 weeks \\
\hline Charbonnel 2006 & Did not compare interventions of interest. Duration of the intervention <52 weeks \\
\hline Cryer 2005 & $\begin{array}{l}\text { The trial compared interventions of interest (metformin vs metformin plus sulphonylurea), but on- } \\
\text { ly reported metformin vs usual care. We contacted the trial authors to get separate data, but have } \\
\text { not had a reply }\end{array}$ \\
\hline \hline CTRI/2013/02/003417 & Did not compare interventions of interest \\
\hline \hline
\end{tabular}




\begin{tabular}{|c|c|}
\hline Study & Reason for exclusion \\
\hline Derosa 2015 & Not a randomised clinical trial \\
\hline EUCTR2004-002549-11-FI & Did not compare interventions of interest. Investigating an investigational drug (tesaglitazar) \\
\hline EUCTR2006-001240-30-BE & The trial was cancelled in 2010 \\
\hline EUCTR2009-014727-23-IT & Prematurely ended, no study results \\
\hline EUCTR2009-017524-36-HU & Did not compare interventions of interest \\
\hline Gregorio 1989 & Did not compare interventions of interest \\
\hline Haering 2015 & Not a randomised clinical trial \\
\hline Hassanein 2014 & Duration of the intervention $<52$ weeks \\
\hline Heller 2018 & Did not compare interventions of interest \\
\hline Hermann 2001 & Did not compare interventions of interest \\
\hline Inagaki 2013 & Did not compare interventions of interest \\
\hline Iqbal 2014 & Did not compare interventions of interest \\
\hline ISRCTN19750520 & Did not compare interventions of interest \\
\hline ISRCTN41840459 & Did not compare interventions of interest \\
\hline Jackson 1987 & Did not compare interventions of interest. Duration of the intervention $<52$ weeks \\
\hline Javaid 2007 & Not a randomised clinical trial \\
\hline Johansen 2007 & Did not compare interventions of interest \\
\hline JPRN-UMIN000005327 & Did not compare interventions of interest \\
\hline Kala 2017 & Duration of the intervention $<52$ weeks \\
\hline Malha 2014 & Duration of the intervention $<52$ weeks \\
\hline Marre 2002 & Duration of the intervention $<52$ weeks \\
\hline Meneghini 2010 & Did not compare interventions of interest. Duration of the intervention $<52$ weeks \\
\hline Moon 2014 & Duration of the intervention $<52$ weeks \\
\hline Morikawa 2011 & Did not compare interventions of interest \\
\hline Nauck 2006 & Duration of the intervention $<52$ weeks \\
\hline NCT00269061 & Did not compare interventions of interest \\
\hline NCT00449605 & Study was cancelled \\
\hline NCT00518882 & Did not compare interventions of interest \\
\hline
\end{tabular}




\begin{tabular}{|c|c|}
\hline Study & Reason for exclusion \\
\hline NCT00543751 & Study was cancelled \\
\hline NCT00839527 & Did not compare interventions of interest \\
\hline NCT00909597 & Did not compare interventions of interest \\
\hline NCT00947557 & Duration of the intervention $<52$ weeks \\
\hline NCT01087567 & Did not compare interventions of interest \\
\hline NCT01106625 & Did not compare interventions of interest \\
\hline NCT01426737 & Protocol for a trial which is never completed \\
\hline NCT01455883 & Study withdrawn \\
\hline NCT01481116 & Did not compare interventions of interest. Comparator is an investigational drug (fasiglifam) \\
\hline NCT01593137 & Study withdrawn \\
\hline NCT02244164 & Not a randomised clinical trial \\
\hline NCT02462369 & Did not compare interventions of interest \\
\hline NCT02587741 & Did not compare interventions of interest \\
\hline NCT02616666 & Did not compare interventions of interest \\
\hline NCT03060980 & Study was cancelled \\
\hline Onuchin 2010 & Duration of the intervention $<52$ weeks \\
\hline Rosenstock 2006 & Did not compare interventions of interest. Duration of the intervention $<52$ weeks \\
\hline Rosenstock 2018 & The duration of the intervention was not identical in the intervention groups \\
\hline Rubin 2008 & $\begin{array}{l}\text { Did not compare interventions of interest. Comparator was a drug no longer approved for use } \\
\text { (muraglitazar) }\end{array}$ \\
\hline Shankar 2017 & The duration of the intervention was not identical in the intervention groups \\
\hline Tolman 2009 & Did not compare interventions of interest \\
\hline UKPDS 1998 & Did not compare interventions of interest \\
\hline Weissman 2014 & Did not compare interventions of interest \\
\hline Yki-Järvinen 1999 & Did not compare interventions of interest \\
\hline
\end{tabular}

Characteristics of studies awaiting assessment [ordered by study ID] 
Müller-Wieland 2018

Methods Randomised clinical trial

Participants People with T2DM treated with metformin

Estimated number of participants: 1359

\begin{tabular}{ll}
\hline Interventions & Metformin + dapagliflozin, metformin + saxagliptin and dapagliflozin, metformin + glimepiride \\
\hline Outcomes & Primary outcome: change in HbAlc from baseline to end of treatment \\
& Relevant proposed outcome measures for 'Summary of findings' table: none \\
\hline Study details & Trial identifier: NCT02471404 \\
& Completion date: March 2017 \\
\hline Publication details & Marked as 'completed' in ClinicalTrials.gov and recently published in a peer-reviewed journal \\
\hline Stated aim of study & $\begin{array}{l}\text { "This study is being carried out to see if dapagliflozin and dapagliflozin plus saxagliptin as an addi- } \\
\text { tion to metformin is effective and safe in treating patients with type 2 diabetes when compared to } \\
\text { glimepiride (sulphonylurea) as an addition to metformin treatment" }\end{array}$ \\
\hline Notes & $\begin{array}{l}\text { Classified as awaiting classification because the study was only published shortly before the publi- } \\
\text { cation of this review and does not report outcomes for 'Summary of findings' table }\end{array}$ \\
\hline
\end{tabular}

\section{NCT02564926}

\begin{tabular}{ll}
\hline Methods & Randomised clinical trial \\
\hline Participants & People with T2DM treated with metformin \\
& Estimated number of participants: 125 \\
\hline Interventions & Metformin + dapagliflozin, metformin + glimepiride
\end{tabular}

\begin{tabular}{ll}
\hline Outcomes & $\begin{array}{l}\text { Primary outcome: changes in total body fat mass from baseline using DXA scan } 52 \text { weeks after the } \\
\text { start of the treatment } \\
\text { Secondary outcomes: including change in HbA1c levels from baseline to } 52 \text { weeks } \\
\text { Relevant proposed outcome measures for 'Summary of findings' table: none }\end{array}$ \\
\hline Study details & Trial identifier: NCT02564926 \\
\hline Completion date: January 2018 \\
\hline Publication details & Marked as 'completed' in ClinicalTrials.gov \\
\hline Notes & "To evaluate the effect of dapagliflozin on body composition in Korean T2DM partici- \\
pants.12-month, randomised, open-label, parallel-group, multi-centre phase IV study"
\end{tabular}

DXA: dual X-ray absorptiometry; HbA1c: glycosylated haemoglobin A1c; T2DM: type 2 diabetes mellitus 
Characteristics of ongoing studies [ordered by study ID]

\section{EUCTR2011-003335-63-IT}

Trial name or title

Effects of liraglutide on pancreatic function in type 2 diabetic patients with secondary failure to oral hypoglycemic agents

\begin{tabular}{ll}
\hline Methods & Randomised clinical trial \\
\hline Participants & People with T2DM treated with metformin and sulphonylurea
\end{tabular}

Estimated number of participants: 80

\begin{tabular}{ll}
\hline Interventions & Liraglutide, glimepiride, insulin, metformin \\
\hline Outcomes & $\begin{array}{l}\text { Primary endpoint: ß-cell glucose sensitivity as derived from the OGTT } \\
\text { Secondary objective: to compare the effects of liraglutide and sulphonylurea on glycaemic control } \\
\text { (fasting plasma glucose, HbA1C) }\end{array}$ \\
\hline Starting date & June 2012 \\
\hline Contact information & stefano.delprato@med.unipi.it \\
\hline $\begin{array}{l}\text { Study identifier } \\
\text { Official title and purpose of }\end{array}$ & $\begin{array}{l}\text { Effects of liraglutide on pancreatic function in type 2 diabetic patients with secondary failure to } \\
\text { oral hypoglycemic agents } \\
\text { study }\end{array}$ \\
$\begin{array}{l}\text { "To determine to which extent liraglutide can maintain B-cell function in type 2 diabetic patients } \\
\text { with secondary failure after an adequate period of good glycaemic control" }\end{array}$ \\
$\begin{array}{l}\text { Relevant proposed outcome } \\
\text { findings' table }\end{array}$ & \begin{tabular}{l} 
None \\
\hline Notes
\end{tabular} \\
\hline
\end{tabular}

\section{EUCTR2012-000152-34-IT}

$\begin{array}{ll}\text { Trial name or title } & \begin{array}{l}\text { Evaluation of the effect of a new drug for diabetes on atherosclerosis in patients with primary fail- } \\ \text { ure metformin }\end{array}\end{array}$

Methods Randomised clinical trial

Participants People with T2DM on metformin treatment

\section{Estimated number of participants: 64}

\begin{tabular}{ll}
\hline Interventions & Sitagliptin, sulphonylurea \\
\hline Outcomes & Primary endpoint: evaluation of endothelial function and oxidative stress \\
& Secondary objective: evaluation of the role of DPP-4 inhibitors on lipid and glycaemic control
\end{tabular}

Starting date February 2012


EUCTR2012-000152-34-IT (Continued)

\begin{tabular}{ll} 
Contact information & maria.delben@uniroma1.it \\
\hline Study identifier & EUCTR2012-000152-34-IT \\
\hline $\begin{array}{l}\text { Official title and purpose of } \\
\text { study }\end{array}$ & $\begin{array}{l}\text { Evaluation of the effect of a new drug for diabetes on atherosclerosis in patients with primary fail- } \\
\text { ure metformin } \\
\text { "Main objective: evaluate the role of DPP4 inhibitors on endothelial function and oxidative stress" }\end{array}$
\end{tabular}

Relevant proposed outcome None

measures for 'Summary of

findings' table

Notes Study completion date: not specified (EudraCT end of trial status: ongoing)

\section{JPRN-UMIN000008815}

Trial name or title

The effect of DPP-4 inhibitor on pancreatic beta cell function and renal function in type 2 diabetic patients

\begin{tabular}{ll}
\hline Methods & Randomised clinical trial \\
\hline Participants & People with T2DM treated with metformin
\end{tabular}

Estimated number of participants: 200

\begin{tabular}{|c|c|}
\hline Interventions & Sitagliptin, glimepiride \\
\hline \multirow[t]{2}{*}{ Outcomes } & $\begin{array}{l}\text { Primary outcomes: the ratio and amount of change of proinsulin/IRI ratio in } 12 \text { months from the } \\
\text { start of treatment; the ratio and amount of change of urinary microalbuminuria in the 3-month av- } \\
\text { erage of } 10,11,12 \text { months after the start of treatment }\end{array}$ \\
\hline & Secondary outcomes include change from baseline to week 52 in $\mathrm{HbA1c}$ \\
\hline Starting date & April 2011 \\
\hline Contact information & ehirata11@gmail.com \\
\hline Study identifier & JPRN-UMIN000008815 \\
\hline $\begin{array}{l}\text { Official title and purpose of } \\
\text { study }\end{array}$ & $\begin{array}{l}\text { The effect of DPP- } 4 \text { inhibitor on pancreatic beta cell function and renal function in type } 2 \text { diabetic } \\
\text { patients }\end{array}$ \\
\hline $\begin{array}{l}\text { Relevant proposed outcome } \\
\text { measures for 'Summary of } \\
\text { findings' table }\end{array}$ & None \\
\hline
\end{tabular}

Notes Study completion date: not specified (UMIN-CTR last follow-up date: June 2014)

\section{NCT01243424}

Trial name or title

CAROLINA: Cardiovascular outcome study of linagliptin versus glimepiride in patients with type 2 diabetes 
NCT01243424 (Continued)

Methods Randomised clinical trial

Participants

People with T2DM at high risk of cardiovascular disease receiving usual care

Estimated number of participants: 6000

\begin{tabular}{ll}
\hline Interventions & Linagliptin, glimepiride \\
\hline Outcomes & Primary outcomes: cardiovascular death, non-fatal myocardial infarction, non-fatal stroke \\
\hline Starting date & October 2010 \\
\hline Contact information & Boehringer Ingelheim \\
\hline Study identifier & NCT01243424 \\
\hline $\begin{array}{l}\text { Official title and purpose of } \\
\text { study }\end{array}$ & $\begin{array}{l}\text { A multicentre, international, randomised, parallel group, double blind study to evaluate cardiovas- } \\
\text { cular safety of linagliptin versus glimepiride in participants with type 2 diabetes mellitus at high } \\
\text { cardiovascular risk } \\
\text { "... to investigate the long term impact on cardiovascular morbidity and mortality, relevant efficacy } \\
\text { parameters (e.g., glycaemic parameters) and safety (e.g., weight and hypoglycaemia) of treatment } \\
\text { with linagliptin in patients with type 2 diabetes at elevated cardiovascular risk receiving usual care, } \\
\text { and compare outcome against glimepiride" }\end{array}$ \\
\hline
\end{tabular}

Relevant proposed outcome measures for 'Summary of findings' table
Yes: time to first occurrence of cardiovascular death (including fatal stroke and fatal MI), non-fatal MI (excluding silent MI) or non-fatal stroke

Notes Study completion date: August 2018

\section{NCT01794143}

Trial name or title

\begin{tabular}{ll}
\hline Methods & Randomised clinical trial \\
\hline Participants & People with T2DM treated with metformin
\end{tabular}

Estimated number of participants: 5000

\begin{tabular}{ll}
\hline Interventions & Glimepiride, sitagliptin, liraglutide, insulin \\
\hline Outcomes & $\begin{array}{l}\text { Primary endpoint: time to } \mathrm{HbAlc} \geq 7 \%, \text { while receiving metformin and the randomly assigned } \\
\text { study medication }\end{array}$ \\
\hline Starting date & May 2013 \\
\hline Contact information & David M Nathan, MD, Massachusetts General Hospital \\
\hline Study identifier & NCT01794143 \\
\hline
\end{tabular}

Official title and purpose of study

A comparative effectiveness study of major glycemia-lowering medications for treatment of type 2 diabetes (GRADE)

Glycemia reduction approaches in diabetes: a comparative effectiveness study 
NCT01794143 (Continued)

"The GRADE study is a pragmatic, unmasked clinical trial that will compare commonly used diabetes medications, when combined with metformin, on glycemia-lowering effectiveness and patient-centered outcomes"

None

Relevant proposed outcome measures for 'Summary of findings' table
Notes Estimated study completion date: July 2021

NCT02142309

\section{Trial name or title}

Participants

Methods

Glycemic durability after metformin failure (AMAZING)

Randomised clinical trial

People with newly-diagnosed T2DM, failing to diet

Estimated number of participants: 450

\begin{tabular}{ll}
\hline Interventions & Glimepiride, vildagliptin, pioglitazone, canagliflozin \\
\hline Outcomes & Primary outcome: time to primary failure with a HbA1c value > 7\% on MTDs of the assigned drug \\
\hline Starting date & October 2005 \\
\hline Contact information & Dario Giugliano, MD dario.giugliano@unina2.it \\
\hline Study identifier & NCT02142309 \\
\hline $\begin{array}{l}\text { Official title and purpose of } \\
\text { study }\end{array}$ & $\begin{array}{l}\text { Effect of glimepiride, vildagliptin, pioglitazone and canagliflozin on durability of glycemic control } \\
\text { after metformin failure in type 2 diabetes } \\
\text { "To compare commonly used oral diabetes medications, when combined with metformin, on } \\
\text { glycemia-lowering effectiveness" }\end{array}$ \\
\hline
\end{tabular}

Relevant proposed outcome None

measures for 'Summary of

findings' table

\section{NCT02730377}

\section{Trial name or title}

Efficacy in controlling glycaemia with Victoza (liraglutide) as add-on to metformin vs. OADs as addon to metformin after up to 104 weeks of treatment in participants with type 2 diabetes (LIRAPRIME)

\begin{tabular}{ll}
\hline Methods & Randomised clinical trial \\
\hline Participants & People with T2DM treated with metformin
\end{tabular}

Estimated number of participants: 1994 
NCT02730377 (Continued)

Interventions Liraglutide, alpha-glucosidase inhibitor, DPP-4 inhibitor, meglitinide, SGLT-2 inhibitor, sulphonylurea, thiazolidinediones

Outcomes

Primary outcome: time to inadequate glycaemic control defined as $\mathrm{HbA} 1 \mathrm{c}>7.0 \%$

Secondary outcomes include change in body weight

\begin{tabular}{|c|c|}
\hline Starting date & March 2016 \\
\hline Contact information & Novo Nordisk A/S \\
\hline Study identifier & NCT02730377 \\
\hline $\begin{array}{l}\text { Official title and purpose of } \\
\text { study }\end{array}$ & $\begin{array}{l}\text { Efficacy in controlling glycaemia with Victoza (liraglutide) as add-on to metformin vs. OADs as add- } \\
\text { on to metformin after up to } 104 \text { weeks of treatment in participants with type } 2 \text { diabetes inade- } \\
\text { quately controlled with metformin monotherapy and treated in a primary care setting }\end{array}$ \\
\hline & $\begin{array}{l}\text { "The aim of the trial is to investigate efficacy in controlling glycaemia with Victoza (liraglutide) as } \\
\text { add-on to metformin background treatment vs. OADs as add-on to metformin background treat- } \\
\text { ment for } 104 \text { weeks of treatment in subjects with type } 2 \text { diabetes" }\end{array}$ \\
\hline
\end{tabular}

Relevant proposed outcome

None

measures for 'Summary of

findings' table

Notes Estimated study completion date: August 2019

\section{NCT02769481}

\section{Trial name or title}

Participants

\section{Methods}

Safety and efficacy of bexagliflozin compared to glimepiride as add-on therapy to metformin in type 2 diabetes participants

Randomised controlled trial

People with T2DM currently taking metformin or taking metformin and 1 additional oral medication for diabetes

\section{Estimated number of participants: 429}

\begin{tabular}{ll}
\hline Interventions & Bexagliflozin, glimepiride \\
\hline Outcomes & Primary outcome: change in HbAlc from baseline to week 60 \\
\hline Starting date & August 2016 \\
\hline Contact information & J. Paul Lock, MD, Theracos Sub, LLC \\
\hline Study identifier & NCT02769481 \\
\hline $\begin{array}{l}\text { Official title and purpose of } \\
\text { study }\end{array}$ & $\begin{array}{l}\text { A phase 3, randomized, double-blind, active-controlled study to evaluate the effects of } \\
\text { bexagliflozin versus glimepiride in subjects with type 2 diabetes mellitus who have inadequate } \\
\text { glycemic control by metformin } \\
\text { "The purpose of this study is to investigate the effect of bexagliflozin compared to glimepiride as an } \\
\text { add-on therapy to metformin in lowering hemoglobin A1c (HbA1c) levels in subjects with type } 2 \text { di- } \\
\text { abetes mellitus (T2DM)" }\end{array}$ \\
\hline
\end{tabular}

Metformin and second- or third-generation sulphonylurea combination therapy for adults with type 2 diabetes mellitus (Review) Copyright @ 2019 The Cochrane Collaboration. Published by John Wiley \& Sons, Ltd. 
NCT02769481 (Continued)

Relevant proposed outcome None measures for 'Summary of

findings' table

Notes $\quad$ Estimated study completion date: December 2019

\section{NCT03332771}

Trial name or title

Efficacy and safety of sotagliflozin versus glimepiride and placebo in participants with type 2 diabetes mellitus that are taking metformin monotherapy (SOTA-GLIM)

\begin{tabular}{ll}
\hline Methods & Randomised clinical trial \\
\hline Participants & People with T2DM treated with metformin
\end{tabular}

Estimated number of participants: 930

\begin{tabular}{ll}
\hline Interventions & Sotagliflozin, glimepiride \\
\hline Outcomes & Primary outcome: change from baseline to week 52 in haemoglobin A1c \\
\hline Starting date & December 2017 \\
\hline Contact information & Contact-Us@sanofi.com \\
\hline Study identifier & NCT03332771 \\
\hline $\begin{array}{l}\text { Official title and purpose of } \\
\text { study }\end{array}$ & $\begin{array}{l}\text { A 52-week randomized, double-blind, double-dummy, active and placebo-controlled, paral- } \\
\text { lel-group, multicentre study to evaluate the efficacy and safety of sotagliflozin compared to } \\
\text { glimepiride or placebo added to metformin in patients with type 2 diabetes who have inadequate } \\
\text { glycemic control with metformin monotherapy } \\
\text { "To demonstrate the non-inferiority of sotagliflozin dose } 1 \text { compared to glimepiride on HbA1c (gly- } \\
\text { cosylated A1c) reduction in patients with T2D (type 2 diabetes) who have inadequate glycemic con- } \\
\text { trol with metformin" }\end{array}$ \\
\hline
\end{tabular}

Relevant proposed outcome None

measures for 'Summary of

findings' table

Notes Estimated study completion date: August 2019

DPP-4: dipeptidyl pepdiase-4; HbA1c: glycated haemoglobin A1c; IRI: immunoreactive insulin; MI: myocardial infarction; MTD: maximum tolerated dose; OAD: oral antidiabetic drug; OGTT: oral glucose tolerance test; SGLT-2: sodium-glucose co-transporter-2; SoF: summary of findings; T2DM: type 2 diabetes mellitus

\section{DATA AND ANALYSES}


Comparison 1. Metformin plus sulphonylurea vs metformin plus placebo

\begin{tabular}{|c|c|c|c|c|}
\hline Outcome or subgroup title & No. of studies & $\begin{array}{l}\text { No. of partici- } \\
\text { pants }\end{array}$ & Statistical method & Effect size \\
\hline 1 All-cause mortality & 2 & & Risk Ratio (M-H, Fixed, 95\% Cl) & Subtotals only \\
\hline 2 Serious adverse events & 2 & 771 & Risk Ratio (M-H, Random, 95\% Cl) & $0.97[0.59,1.61]$ \\
\hline 3 Cardiovascular mortality & 2 & & Risk Ratio (M-H, Fixed, 95\% Cl) & Subtotals only \\
\hline $\begin{array}{l}4 \text { Non-fatal myocardial in- } \\
\text { farction }\end{array}$ & 2 & 771 & Risk Ratio (M-H, Random, 95\% Cl) & $0.63[0.08,5.10]$ \\
\hline 5 Heart failure & 2 & & Risk Ratio (M-H, Fixed, 95\% Cl) & Subtotals only \\
\hline $\begin{array}{l}6 \text { Non-serious adverse } \\
\text { events }\end{array}$ & 2 & 771 & Risk Ratio (M-H, Random, 95\% Cl) & $1.25[0.96,1.64]$ \\
\hline $\begin{array}{l}7 \text { Mild/moderate hypogly- } \\
\text { caemia }\end{array}$ & 2 & 771 & Risk Ratio (M-H, Random, 95\% Cl) & $3.93[0.71,21.88]$ \\
\hline 8 Serious hypoglycaemia & 2 & & Risk Ratio (M-H, Random, 95\% Cl) & Subtotals only \\
\hline 9 Weight change & 2 & 476 & $\begin{array}{l}\text { Mean Difference (IV, Random, 95\% } \\
\text { Cl) }\end{array}$ & $3.37[1.35,5.39]$ \\
\hline 10 Change in $\mathrm{HbAlc}$ & 2 & 472 & $\begin{array}{l}\text { Mean Difference (IV, Random, 95\% } \\
\mathrm{Cl} \text { ) }\end{array}$ & $-0.47[-1.07,0.14]$ \\
\hline
\end{tabular}

Analysis 1.1. Comparison 1 Metformin plus sulphonylurea vs metformin plus placebo, Outcome 1 All-cause mortality.

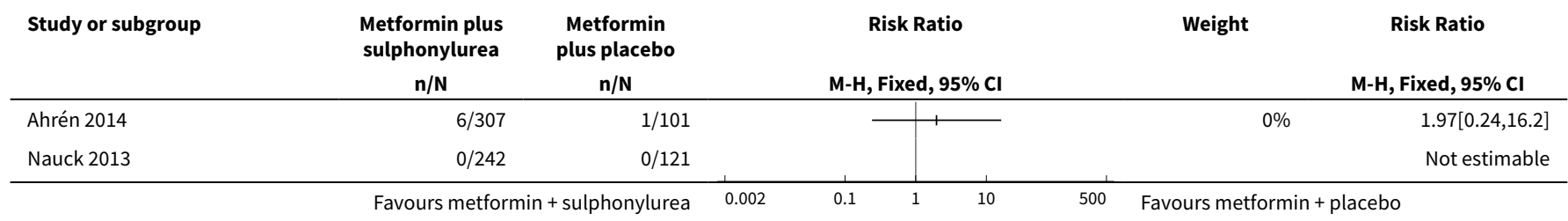

Analysis 1.2. Comparison 1 Metformin plus sulphonylurea vs metformin plus placebo, Outcome 2 Serious adverse events.

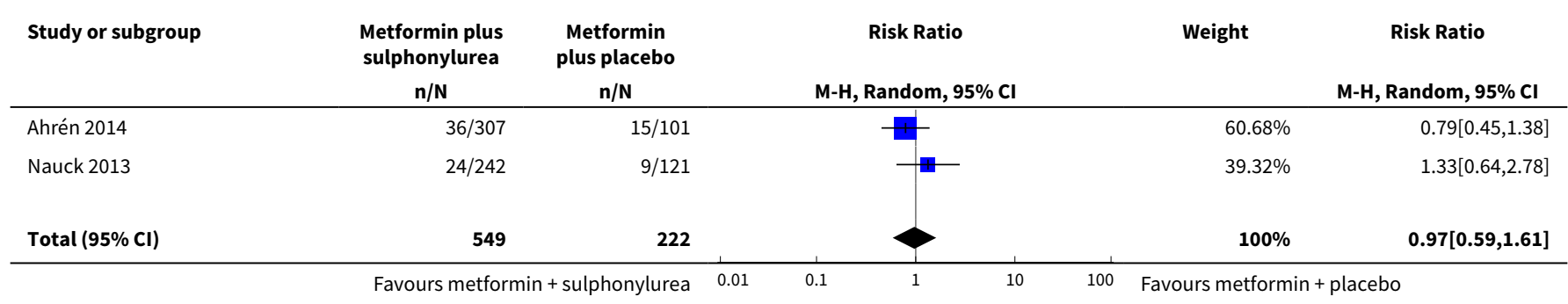




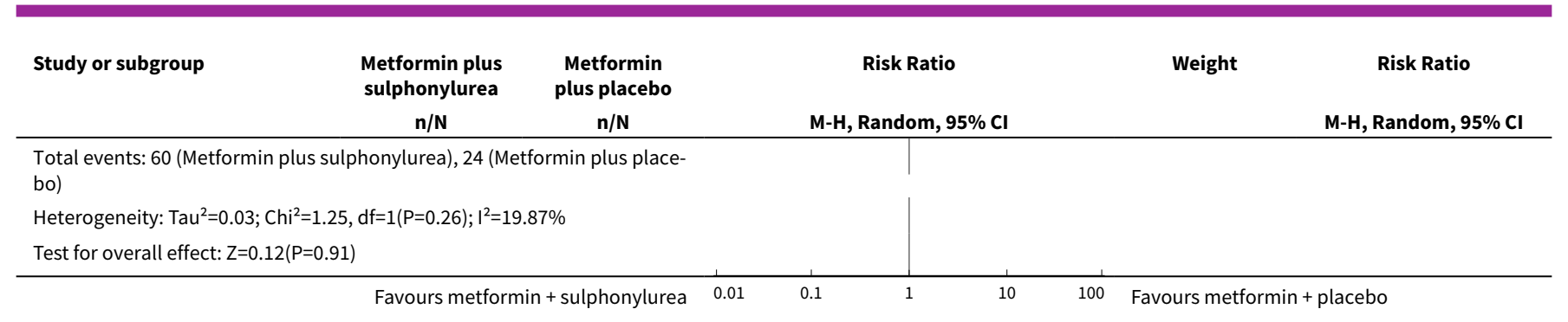

Analysis 1.3. Comparison 1 Metformin plus sulphonylurea vs metformin plus placebo, Outcome 3 Cardiovascular mortality.

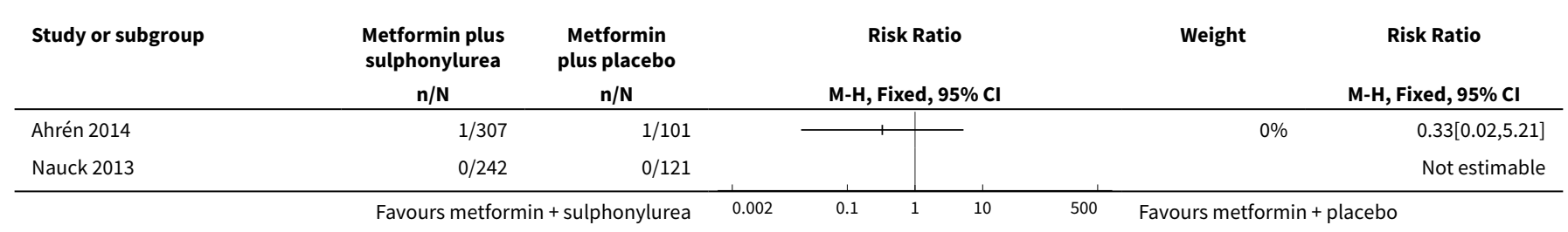

Analysis 1.4. Comparison 1 Metformin plus sulphonylurea vs metformin plus placebo, Outcome 4 Non-fatal myocardial infarction.

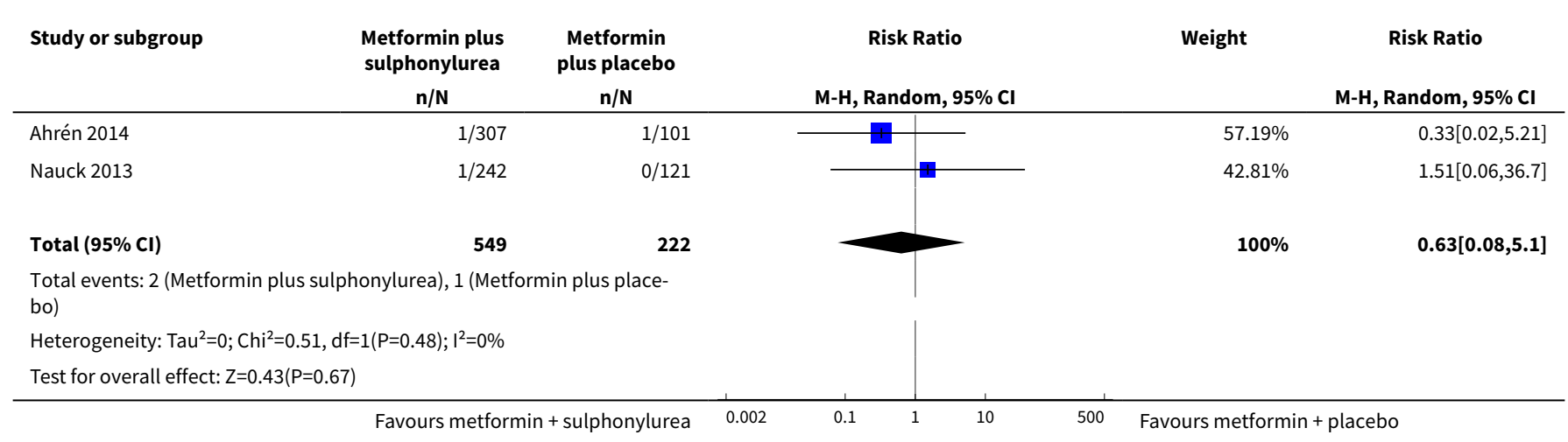

\section{Analysis 1.5. Comparison 1 Metformin plus sulphonylurea vs metformin plus placebo, Outcome 5 Heart failure.}

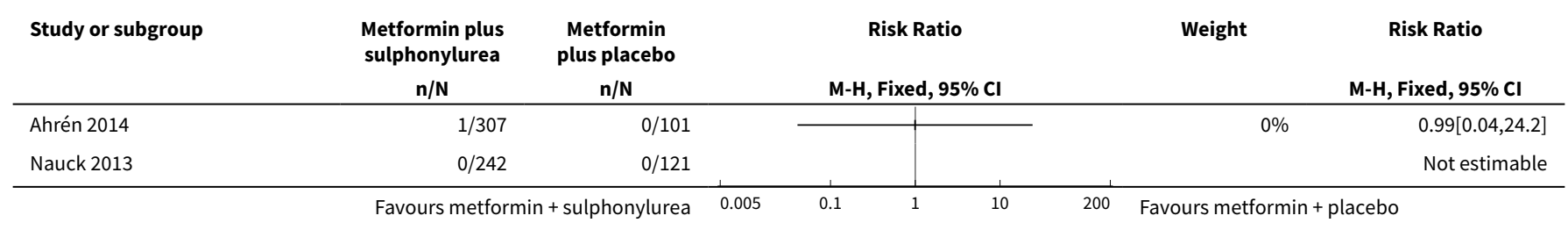


Analysis 1.6. Comparison 1 Metformin plus sulphonylurea vs metformin plus placebo, Outcome 6 Non-serious adverse events.

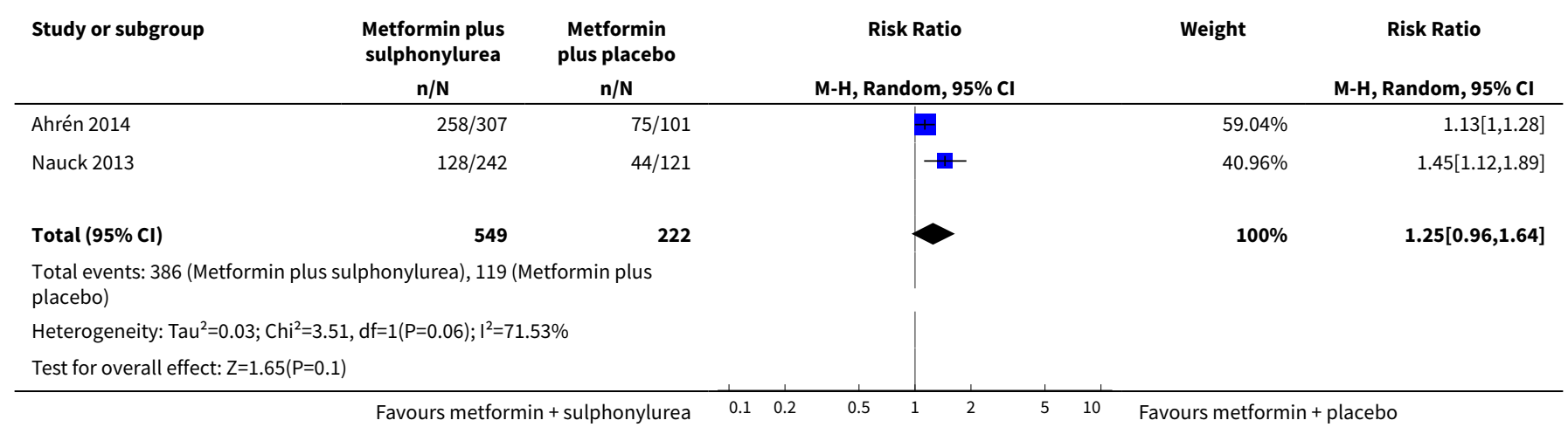

Analysis 1.7. Comparison 1 Metformin plus sulphonylurea vs metformin plus placebo, Outcome $7 \mathrm{Mild} /$ moderate hypoglycaemia.

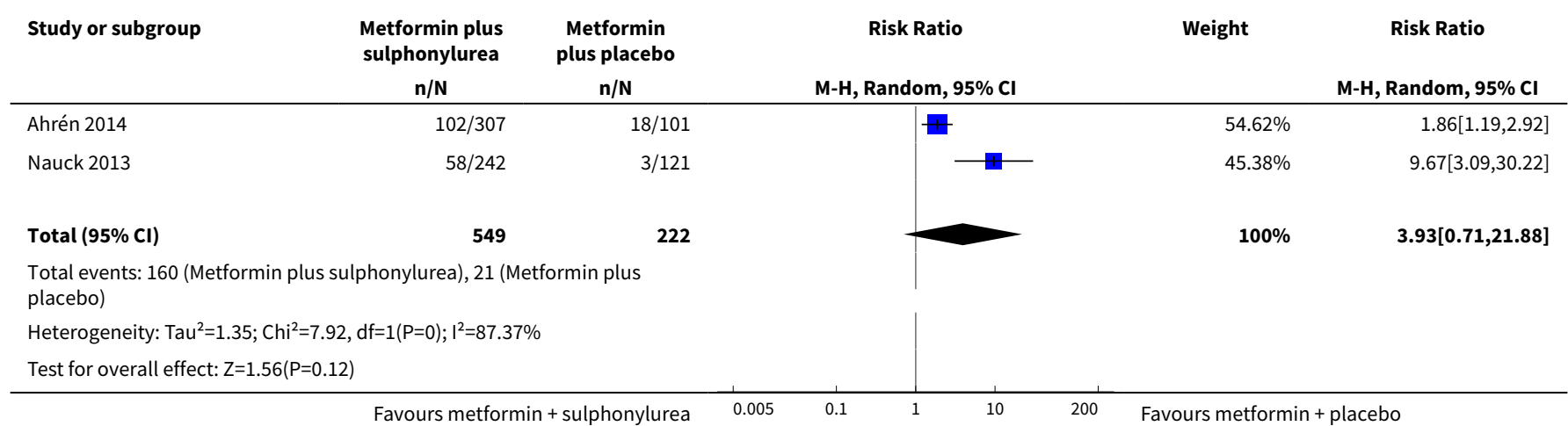

Analysis 1.8. Comparison 1 Metformin plus sulphonylurea vs metformin plus placebo, Outcome 8 Serious hypoglycaemia.

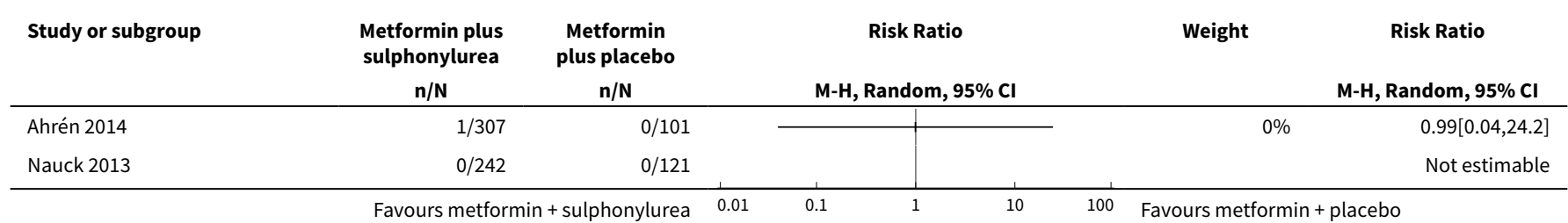

Analysis 1.9. Comparison 1 Metformin plus sulphonylurea vs metformin plus placebo, Outcome 9 Weight change.

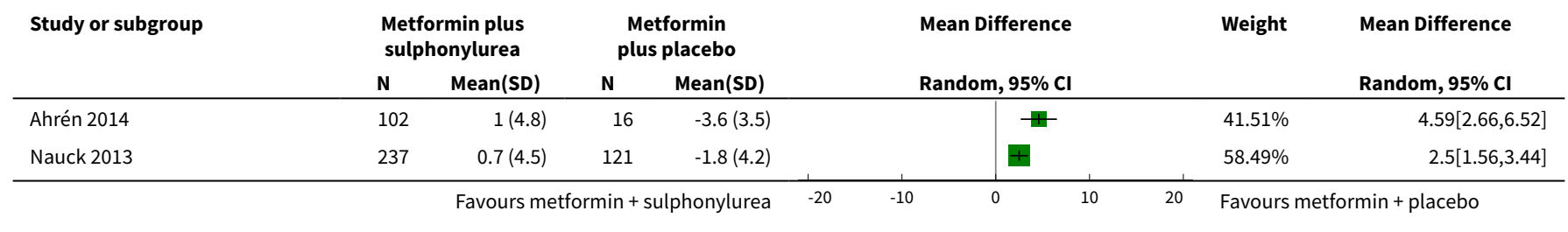




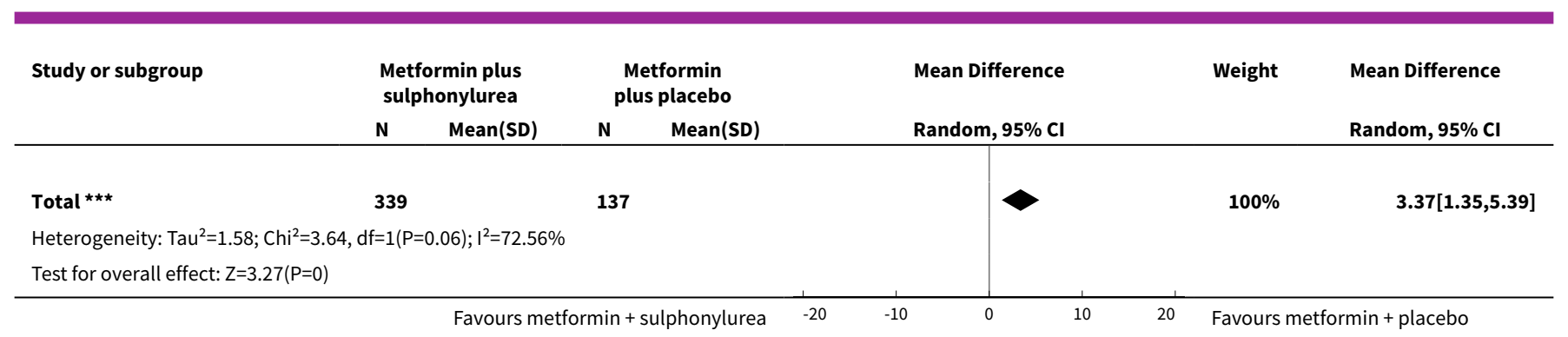

\section{Analysis 1.10. Comparison 1 Metformin plus sulphonylurea vs metformin plus placebo, Outcome 10 Change in HbA1c.}

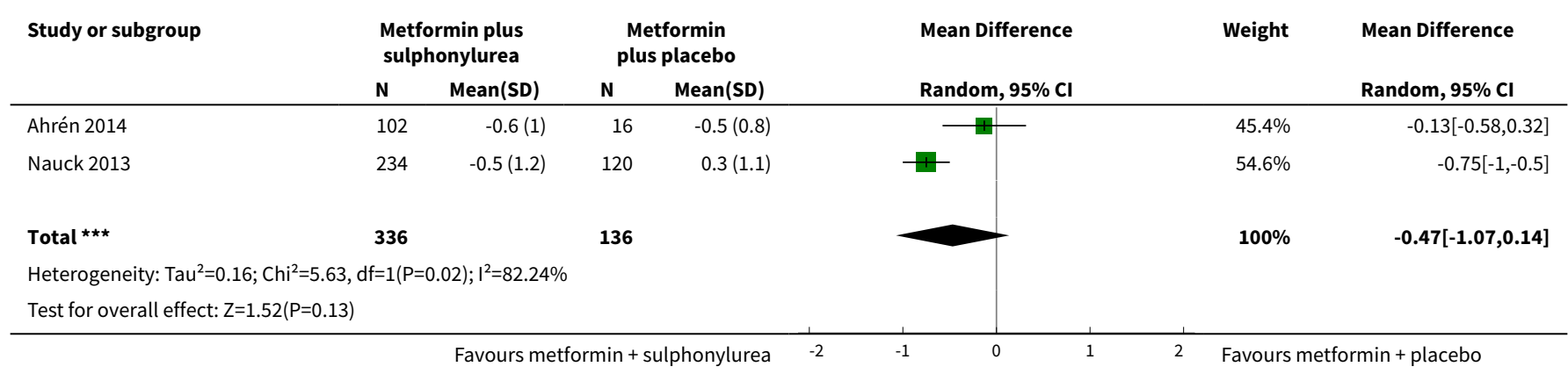

\section{Comparison 2. Metformin plus sulphonylurea vs metformin plus GLP-1 analogue}

\begin{tabular}{|c|c|c|c|c|}
\hline $\begin{array}{l}\text { Outcome or subgroup ti- } \\
\text { tle }\end{array}$ & No. of studies & $\begin{array}{l}\text { No. of partici- } \\
\text { pants }\end{array}$ & Statistical method & Effect size \\
\hline 1 All-cause mortality & 3 & 2594 & Risk Ratio (M-H, Random, 95\% Cl) & $1.15[0.49,2.67]$ \\
\hline 2 Serious adverse events & 3 & 2594 & Risk Ratio (M-H, Random, 95\% Cl) & $0.90[0.73,1.11]$ \\
\hline 3 Cardiovascular mortality & 1 & & Risk Ratio (M-H, Fixed, 95\% Cl) & Totals not selected \\
\hline $\begin{array}{l}4 \text { Non-fatal myocardial in- } \\
\text { farction }\end{array}$ & 2 & 1575 & Risk Ratio (M-H, Random, 95\% Cl) & $0.57[0.12,2.82]$ \\
\hline 5 Heart failure & 3 & 2594 & Risk Ratio (M-H, Random, 95\% Cl) & $0.54[0.10,2.77]$ \\
\hline 6 End-stage renal disease & 1 & & Risk Ratio (M-H, Fixed, 95\% Cl) & Totals not selected \\
\hline $\begin{array}{l}7 \text { Non-serious adverse } \\
\text { events }\end{array}$ & 3 & & Risk Ratio (M-H, Random, 95\% Cl) & Totals not selected \\
\hline $\begin{array}{l}8 \mathrm{Mild} / \text { moderate hypogly- } \\
\text { caemia }\end{array}$ & 3 & 2594 & Risk Ratio (M-H, Random, 95\% Cl) & $3.24[2.05,5.13]$ \\
\hline 9 Serious hypoglycaemia & 3 & 2594 & Risk Ratio (M-H, Random, 95\% Cl) & $1.00[0.16,6.30]$ \\
\hline 10 Weight (change) & 5 & 1777 & Mean Difference (IV, Random, 95\% CI) & $5.54[3.62,7.46]$ \\
\hline 11 Change in $\mathrm{HbAlc}$ & 5 & 2346 & Mean Difference (IV, Random, 95\% CI) & $0.01[-0.15,0.17]$ \\
\hline
\end{tabular}


Analysis 2.1. Comparison 2 Metformin plus sulphonylurea vs metformin plus GLP-1 analogue, Outcome 1 All-cause mortality.

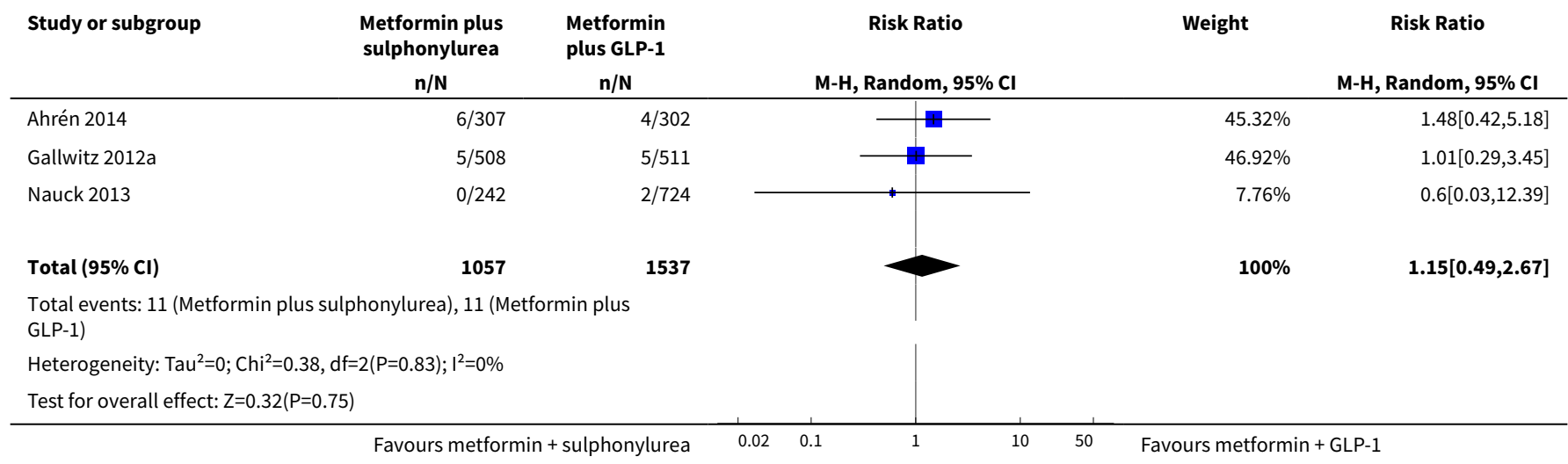

Analysis 2.2. Comparison 2 Metformin plus sulphonylurea vs metformin plus GLP-1 analogue, Outcome 2 Serious adverse events.

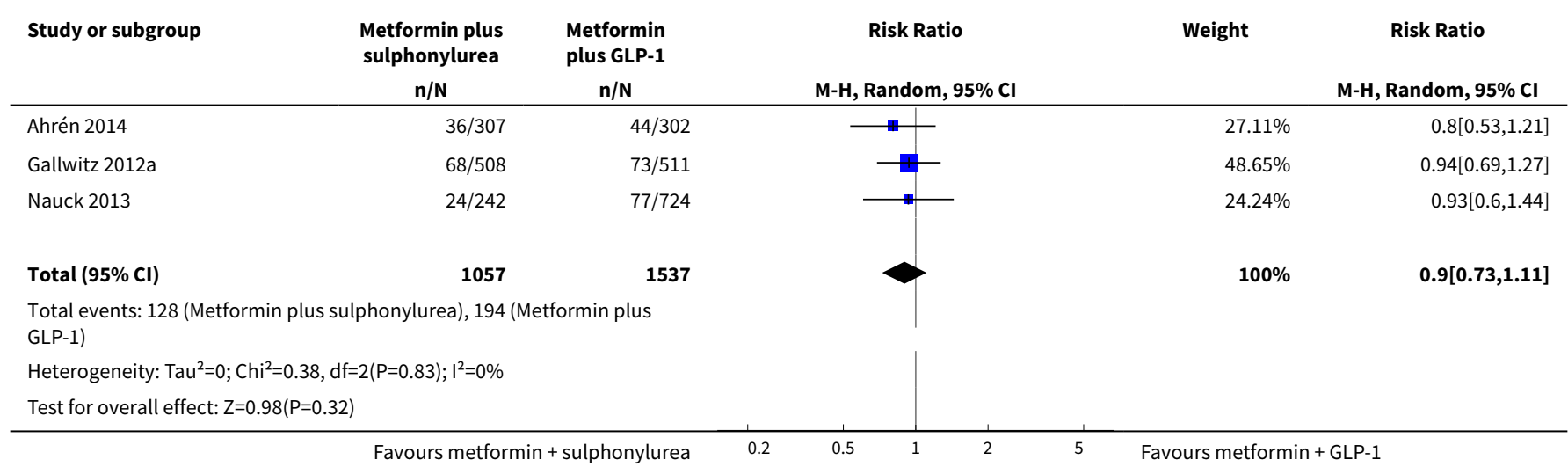

Analysis 2.3. Comparison 2 Metformin plus sulphonylurea vs metformin plus GLP-1 analogue, Outcome 3 Cardiovascular mortality.

\begin{tabular}{|c|c|c|c|c|c|c|c|}
\hline \multirow[t]{2}{*}{ Study or subgroup } & Metformin plus & Metformin plus GLP-1 & \multicolumn{4}{|c|}{ Risk Ratio } & \multirow{2}{*}{$\begin{array}{c}\text { Risk Ratio } \\
\text { M-H, Fixed, } 95 \% \mathrm{Cl}\end{array}$} \\
\hline & $n / N$ & $\mathrm{n} / \mathrm{N}$ & & M-H & xed, $95 \% \mathrm{Cl}$ & & \\
\hline \multirow[t]{2}{*}{ Ahrén 2014} & $1 / 307$ & $1 / 302$ & & 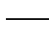 & — & & $0.98[0.06,15.66]$ \\
\hline & Favo & etformin + sulphonylurea & 0.005 & 0.1 & 10 & 200 & $\begin{array}{l}\text { Favours metformin + } \\
\text { GLP-1 }\end{array}$ \\
\hline
\end{tabular}


Analysis 2.4. Comparison 2 Metformin plus sulphonylurea vs metformin plus GLP-1 analogue, Outcome 4 Non-fatal myocardial infarction.

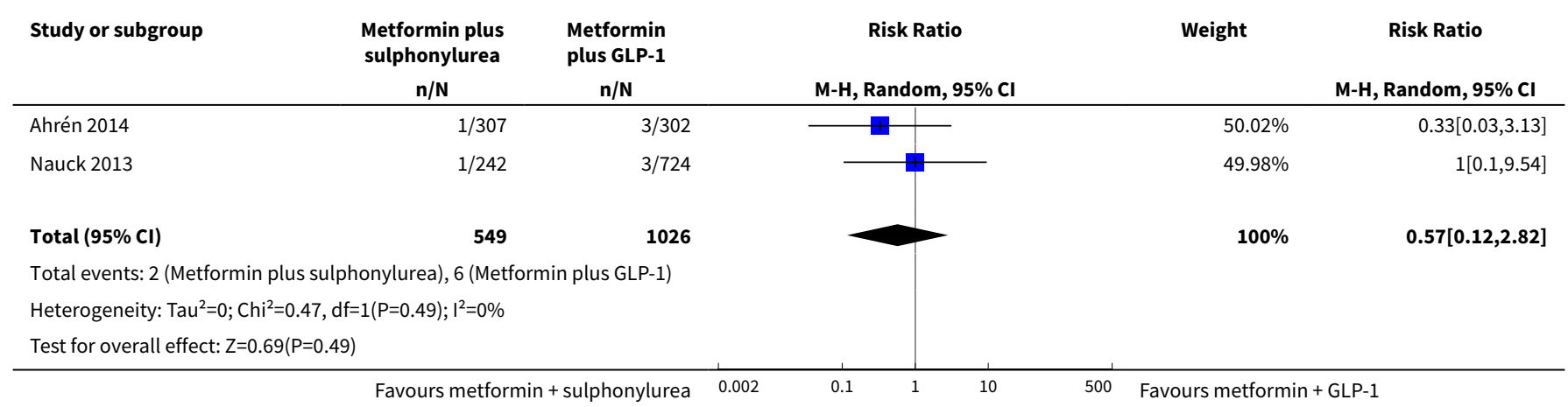

Analysis 2.5. Comparison 2 Metformin plus sulphonylurea vs metformin plus GLP-1 analogue, Outcome 5 Heart failure.

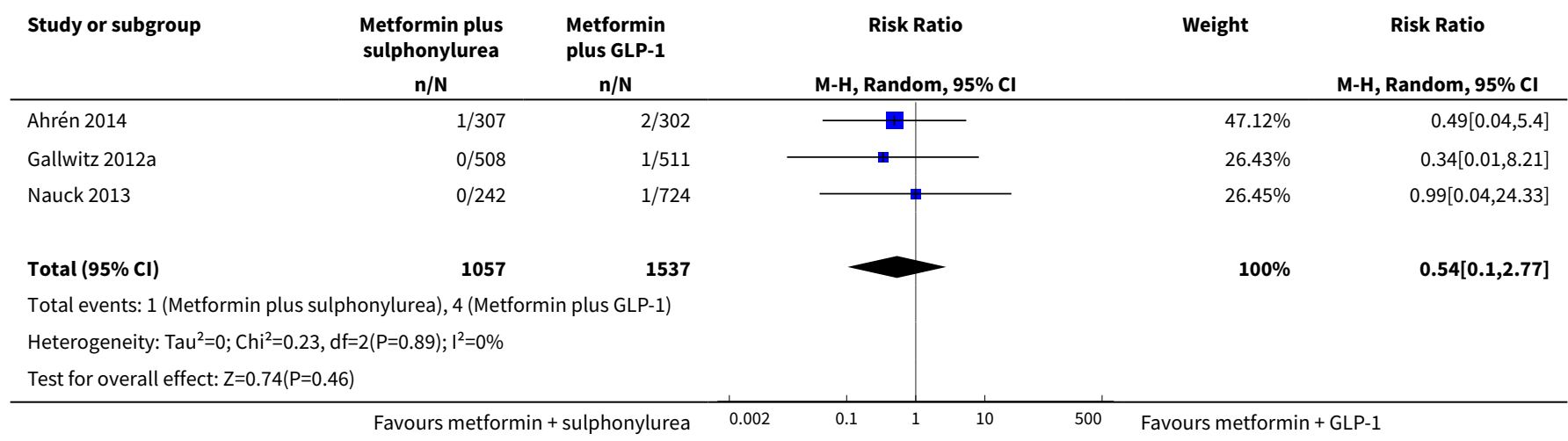

Analysis 2.6. Comparison 2 Metformin plus sulphonylurea vs metformin plus GLP-1 analogue, Outcome 6 End-stage renal disease.

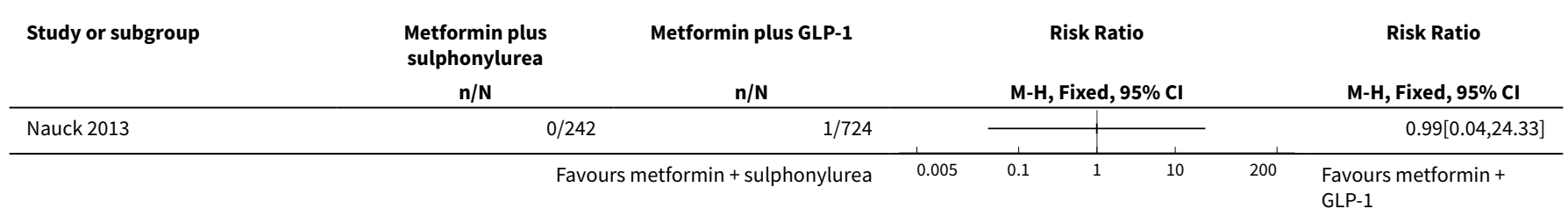

Analysis 2.7. Comparison 2 Metformin plus sulphonylurea vs metformin plus GLP-1 analogue, Outcome 7 Non-serious adverse events.

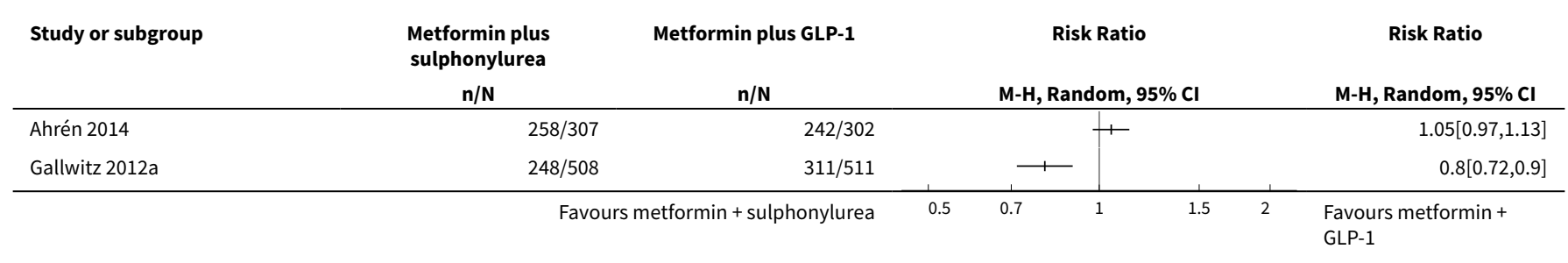




\begin{tabular}{|c|c|c|c|c|c|c|}
\hline \multirow[t]{2}{*}{ Study or subgroup } & Metformin plus & \multirow[t]{2}{*}{ Metformin plus GLP-1 } & \multicolumn{3}{|c|}{ Risk Ratio } & \multirow{2}{*}{$\begin{array}{c}\text { Risk Ratio } \\
\text { M-H, Random, } 95 \% \mathrm{CI}\end{array}$} \\
\hline & $\mathrm{n} / \mathrm{N}$ & & & \multicolumn{2}{|c|}{ M-H, Random, 95\% Cl } & \\
\hline \multirow[t]{2}{*}{ Nauck 2013} & $128 / 242$ & $434 / 724$ & & 1 & & $0.88[0.77,1.01]$ \\
\hline & Favou & etformin + sulphonylurea & 0.5 & 0.7 & 1.5 & $\begin{array}{l}\text { Favours metformin }+ \\
\text { GLP-1 }\end{array}$ \\
\hline
\end{tabular}

Analysis 2.8. Comparison 2 Metformin plus sulphonylurea vs metformin plus GLP-1 analogue, Outcome $8 \mathrm{Mild} / \mathrm{moderate}$ hypoglycaemia.

\begin{tabular}{|c|c|c|c|c|c|c|}
\hline \multirow[t]{2}{*}{ Study or subgroup } & \multirow{2}{*}{$\begin{array}{c}\text { Metformin plus } \\
\text { sulphonylurea } \\
n / N\end{array}$} & \multirow{2}{*}{$\begin{array}{c}\text { Metformin } \\
\text { plus GLP-1 } \\
\mathrm{n} / \mathrm{N} \\
\end{array}$} & \multicolumn{2}{|r|}{ Risk Ratio } & \multirow[t]{2}{*}{ Weight } & \multirow{2}{*}{$\begin{array}{c}\text { Risk Ratio } \\
\text { M-H, Random, 95\% Cl }\end{array}$} \\
\hline & & & & M-H, Random, 95\% Cl & & \\
\hline Ahrén 2014 & $102 / 307$ & $35 / 302$ & & $\rightarrow-$ & $32.41 \%$ & $2.87[2.02,4.07]$ \\
\hline Gallwitz 2012a & $240 / 508$ & $102 / 511$ & & 典 & $37.19 \%$ & $2.37[1.94,2.88]$ \\
\hline Nauck 2013 & $58 / 242$ & $32 / 724$ & & $\rightarrow$ & $30.4 \%$ & $5.42[3.61,8.14]$ \\
\hline Total $(95 \% \mathrm{Cl})$ & 1057 & 1537 & & & $100 \%$ & $3.24[2.05,5.13]$ \\
\hline \multicolumn{7}{|c|}{$\begin{array}{l}\text { Total events: } 400 \text { (Metformin plus sulphonylurea), } 169 \text { (Metformin plus } \\
\text { GLP-1) }\end{array}$} \\
\hline \multicolumn{7}{|c|}{ Heterogeneity: $\mathrm{Tau}^{2}=0.14 ; \mathrm{Chi}^{2}=12.99, \mathrm{df}=2(\mathrm{P}=0) ; \mathrm{I}^{2}=84.61 \%$} \\
\hline \multicolumn{7}{|c|}{ Test for overall effect: $Z=5.02(P<0.0001)$} \\
\hline
\end{tabular}

Analysis 2.9. Comparison 2 Metformin plus sulphonylurea vs metformin plus GLP-1 analogue, Outcome 9 Serious hypoglycaemia.

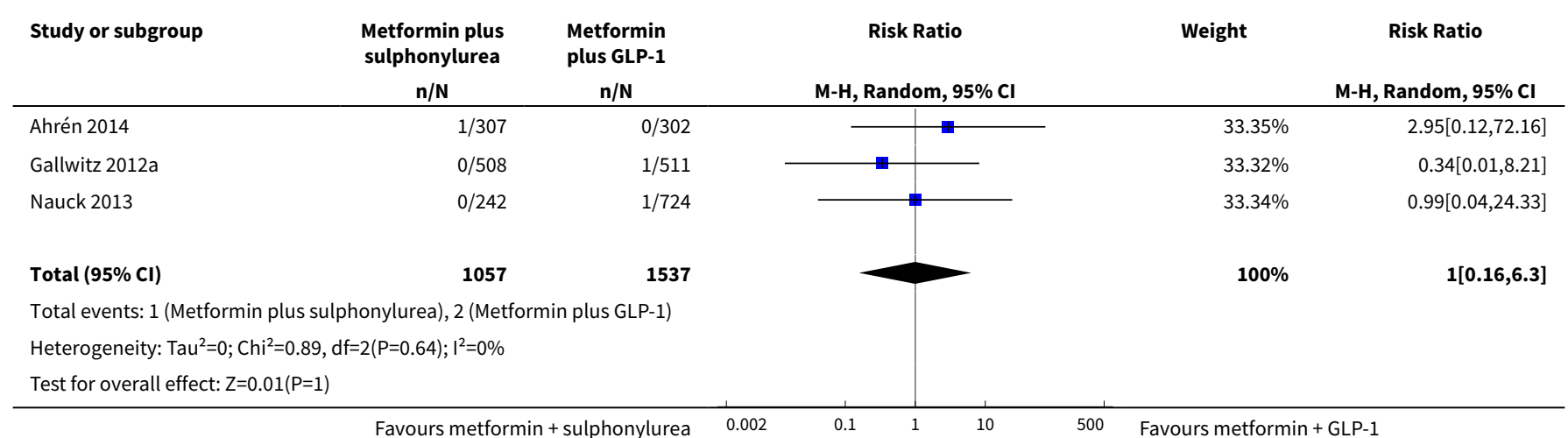

Analysis 2.10. Comparison 2 Metformin plus sulphonylurea vs metformin plus GLP-1 analogue, Outcome 10 Weight (change).

\begin{tabular}{|c|c|c|c|c|c|c|c|c|c|}
\hline \multirow[t]{2}{*}{ Study or subgroup } & \multicolumn{2}{|c|}{$\begin{array}{l}\text { Metformin plus } \\
\text { sulphonylurea }\end{array}$} & \multicolumn{2}{|c|}{$\begin{array}{l}\text { Metformin } \\
\text { plus GLP-1 }\end{array}$} & \multicolumn{3}{|c|}{ Mean Difference } & \multirow[t]{2}{*}{ Weight } & \multirow{2}{*}{$\begin{array}{l}\text { Mean Difference } \\
\text { Random, 95\% Cl }\end{array}$} \\
\hline & $\mathbf{N}$ & Mean(SD) & $\mathbf{N}$ & Mean(SD) & & Ran & , $95 \% \mathrm{Cl}$ & & \\
\hline Ahrén 2014 & 102 & $1(4.8)$ & 116 & $-2.3(5.1)$ & & & $*$ & $21.95 \%$ & $3.29[1.98,4.6]$ \\
\hline Derosa 2010 & 57 & $86.7(11.2)$ & 59 & $74(4.1)$ & & & $\rightarrow$ & $14.96 \%$ & $12.7[9.61,15.79]$ \\
\hline Derosa 2011a & 49 & $80.5(7.7)$ & 52 & $75.1(6.5)$ & & & $\rightarrow$ & $16.12 \%$ & $5.4[2.61,8.19]$ \\
\hline
\end{tabular}




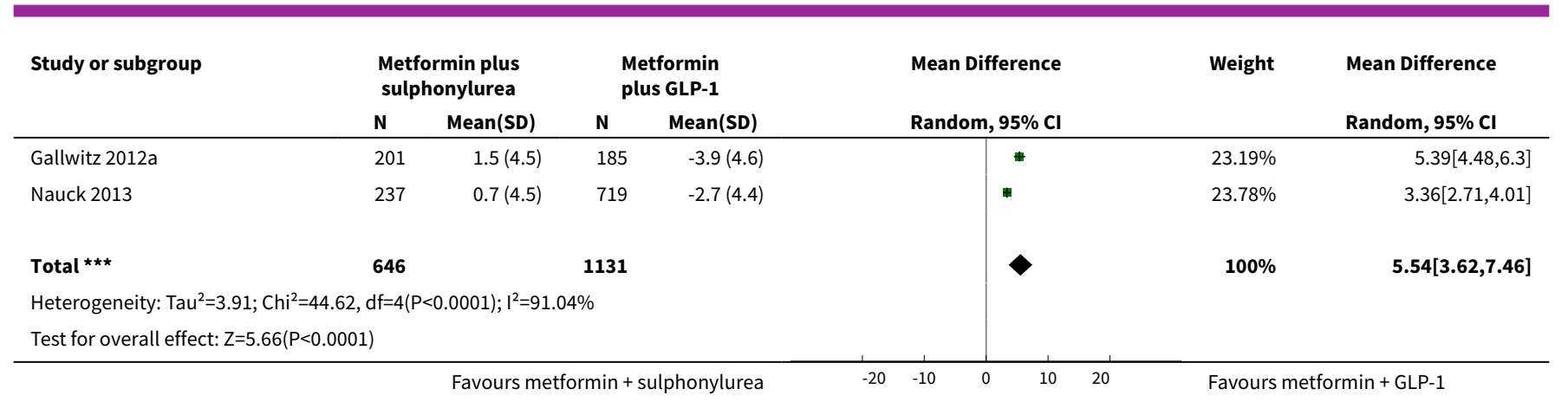

Analysis 2.11. Comparison 2 Metformin plus sulphonylurea vs metformin plus GLP-1 analogue, Outcome 11 Change in HbA1c.

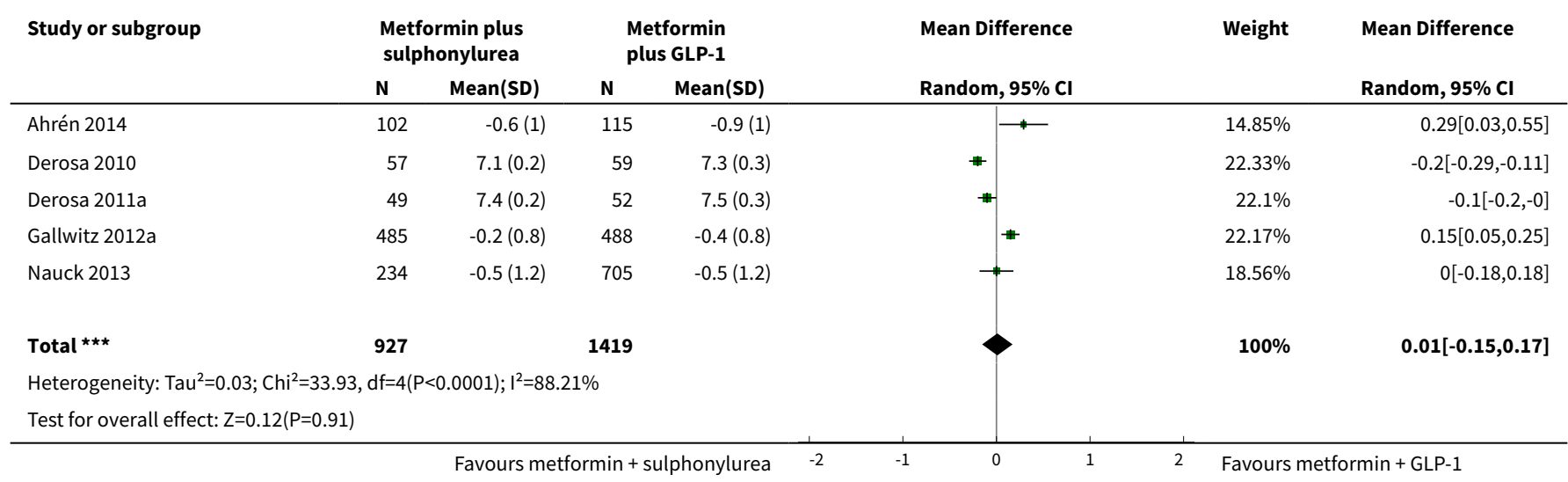

\section{Comparison 3. Metformin plus sulphonylurea vs metformin plus DPP-4 inhibitor}

\begin{tabular}{|c|c|c|c|c|}
\hline Outcome or subgroup title & No. of studies & $\begin{array}{l}\text { No. of partici- } \\
\text { pants }\end{array}$ & Statistical method & Effect size \\
\hline 1 All-cause mortality & 9 & 11694 & $\begin{array}{l}\text { Risk Ratio (M-H, Random, 95\% } \\
\text { Cl) }\end{array}$ & $1.32[0.76,2.28]$ \\
\hline $\begin{array}{l}1.1 \text { Trials with long duration ( } \geq 2 \\
\text { years) }\end{array}$ & 6 & 9909 & $\begin{array}{l}\text { Risk Ratio (M-H, Random, 95\% } \\
\mathrm{Cl} \text { ) }\end{array}$ & $1.38[0.72,2.64]$ \\
\hline $\begin{array}{l}1.2 \text { Trials with short duration }(<2 \\
\text { years) }\end{array}$ & 3 & 1785 & $\begin{array}{l}\text { Risk Ratio (M-H, Random, 95\% } \\
\mathrm{Cl} \text { ) }\end{array}$ & $1.02[0.14,7.20]$ \\
\hline 2 Serious adverse events & 9 & 11694 & $\begin{array}{l}\text { Risk Ratio (M-H, Random, 95\% } \\
\mathrm{Cl} \text { ) }\end{array}$ & $1.07[0.97,1.18]$ \\
\hline $\begin{array}{l}2.1 \text { Trials with long duration ( } \geq 2 \\
\text { years) }\end{array}$ & 6 & 9909 & $\begin{array}{l}\text { Risk Ratio (M-H, Random, 95\% } \\
\mathrm{Cl} \text { ) }\end{array}$ & $1.08[0.97,1.19]$ \\
\hline $\begin{array}{l}2.2 \text { Trials with short duration }(<2 \\
\text { years) }\end{array}$ & 3 & 1785 & $\begin{array}{l}\text { Risk Ratio (M-H, Random, 95\% } \\
\mathrm{Cl} \text { ) }\end{array}$ & $1.01[0.61,1.68]$ \\
\hline
\end{tabular}




\begin{tabular}{|c|c|c|c|c|}
\hline Outcome or subgroup title & No. of studies & $\begin{array}{l}\text { No. of partici- } \\
\text { pants }\end{array}$ & Statistical method & Effect size \\
\hline 3 Cardiovascular mortality & 6 & 6874 & $\begin{array}{l}\text { Risk Ratio (M-H, Random, 95\% } \\
\text { Cl) }\end{array}$ & $1.54[0.63,3.79]$ \\
\hline $\begin{array}{l}3.1 \text { Trials with long duration ( } \geq 2 \\
\text { years) }\end{array}$ & 5 & 6810 & $\begin{array}{l}\text { Risk Ratio (M-H, Random, 95\% } \\
\text { Cl) }\end{array}$ & $1.54[0.63,3.79]$ \\
\hline $\begin{array}{l}3.2 \text { Trials with short duration }(<2 \\
\text { years) }\end{array}$ & 1 & 64 & $\begin{array}{l}\text { Risk Ratio (M-H, Random, 95\% } \\
\mathrm{Cl} \text { ) }\end{array}$ & $0.0[0.0,0.0]$ \\
\hline 4 Non-fatal myocardial infarction & 6 & 6874 & $\begin{array}{l}\text { Risk Ratio (M-H, Random, 95\% } \\
\text { Cl) }\end{array}$ & $1.45[0.69,3.07]$ \\
\hline $\begin{array}{l}4.1 \text { Trials with long duration ( } \geq 2 \\
\text { years) }\end{array}$ & 5 & 6810 & $\begin{array}{l}\text { Risk Ratio (M-H, Random, 95\% } \\
\text { Cl) }\end{array}$ & $1.45[0.69,3.07]$ \\
\hline $\begin{array}{l}4.2 \text { Trials with short duration }(<2 \\
\text { years) }\end{array}$ & 1 & 64 & $\begin{array}{l}\text { Risk Ratio (M-H, Random, 95\% } \\
\text { Cl) }\end{array}$ & $0.0[0.0,0.0]$ \\
\hline 5 Heart failure & 8 & 10691 & $\begin{array}{l}\text { Risk Ratio (M-H, Random, 95\% } \\
\text { Cl) }\end{array}$ & $1.05[0.47,2.34]$ \\
\hline $\begin{array}{l}5.1 \text { Trials with long duration ( } \geq 2 \\
\text { years) }\end{array}$ & 6 & 9909 & $\begin{array}{l}\text { Risk Ratio (M-H, Random, 95\% } \\
\text { Cl) }\end{array}$ & $0.78[0.33,1.86]$ \\
\hline $\begin{array}{l}5.2 \text { Trials with short duration }(<2 \\
\text { years) }\end{array}$ & 2 & 782 & $\begin{array}{l}\text { Risk Ratio (M-H, Random, 95\% } \\
\mathrm{Cl} \text { ) }\end{array}$ & $6.00[0.73,49.59]$ \\
\hline 6 Non-fatal stroke & 4 & 5093 & $\begin{array}{l}\text { Risk Ratio (M-H, Random, 95\% } \\
\mathrm{Cl} \text { ) }\end{array}$ & $2.21[0.74,6.58]$ \\
\hline $\begin{array}{l}6.1 \text { Trials with long duration ( } \geq 2 \\
\text { years) }\end{array}$ & 3 & 5029 & $\begin{array}{l}\text { Risk Ratio (M-H, Random, 95\% } \\
\text { Cl) }\end{array}$ & $2.21[0.74,6.58]$ \\
\hline $\begin{array}{l}6.2 \text { Trials with short duration }(<2 \\
\text { years) }\end{array}$ & 1 & 64 & $\begin{array}{l}\text { Risk Ratio (M-H, Random, 95\% } \\
\text { Cl) }\end{array}$ & $0.0[0.0,0.0]$ \\
\hline 7 Non-serious adverse events & 7 & 7592 & $\begin{array}{l}\text { Risk Ratio (M-H, Random, 95\% } \\
\text { Cl) }\end{array}$ & $1.18[1.03,1.35]$ \\
\hline $\begin{array}{l}7.1 \text { Trials with long duration ( } \geq 2 \\
\text { years) }\end{array}$ & 5 & 6810 & $\begin{array}{l}\text { Risk Ratio (M-H, Random, 95\% } \\
\text { Cl) }\end{array}$ & $1.21[1.04,1.42]$ \\
\hline $\begin{array}{l}7.2 \text { Trials with short duration }(<2 \\
\text { years) }\end{array}$ & 2 & 782 & $\begin{array}{l}\text { Risk Ratio (M-H, Random, 95\% } \\
\text { Cl) }\end{array}$ & $0.99[0.82,1.21]$ \\
\hline 8 Mild/moderate hypoglycaemia & 7 & 9973 & $\begin{array}{l}\text { Risk Ratio (M-H, Random, 95\% } \\
\text { Cl) }\end{array}$ & $7.42[4.77,11.53]$ \\
\hline $\begin{array}{l}8.1 \text { Trials with long duration ( } \geq 2 \\
\text { years) }\end{array}$ & 5 & 9051 & $\begin{array}{l}\text { Risk Ratio (M-H, Random, 95\% } \\
\text { Cl) }\end{array}$ & $6.67[4.32,10.28]$ \\
\hline $\begin{array}{l}8.2 \text { Trials with short duration }(<2 \\
\text { years) }\end{array}$ & 2 & 922 & $\begin{array}{l}\text { Risk Ratio (M-H, Random, 95\% } \\
\mathrm{Cl} \text { ) }\end{array}$ & $39.09[7.69,198.82]$ \\
\hline
\end{tabular}




\begin{tabular}{|c|c|c|c|c|}
\hline Outcome or subgroup title & No. of studies & $\begin{array}{l}\text { No. of partici- } \\
\text { pants }\end{array}$ & Statistical method & Effect size \\
\hline 9 Serious hypoglycaemia & 8 & 10691 & $\begin{array}{l}\text { Risk Ratio (M-H, Random, 95\% } \\
\mathrm{Cl} \text { ) }\end{array}$ & $8.04[3.31,19.53]$ \\
\hline $\begin{array}{l}9.1 \text { Trials with long duration ( } \geq 2 \\
\text { years) }\end{array}$ & 5 & 9051 & $\begin{array}{l}\text { Risk Ratio (M-H, Random, 95\% } \\
\text { Cl) }\end{array}$ & $8.66[3.10,24.16]$ \\
\hline $\begin{array}{l}9.2 \text { Trials with short duration }(<2 \\
\text { years) }\end{array}$ & 3 & 1640 & $\begin{array}{l}\text { Risk Ratio (M-H, Random, 95\% } \\
\mathrm{Cl})\end{array}$ & $6.46[1.10,37.85]$ \\
\hline 10 Weight change (kg) & 9 & 10228 & $\begin{array}{l}\text { Mean Difference (IV, Random, } \\
95 \% \mathrm{CI} \text { ) }\end{array}$ & $2.15[1.71,2.58]$ \\
\hline $\begin{array}{l}10.1 \text { Trials with long duration ( } \geq 2 \\
\text { years) }\end{array}$ & 6 & 8667 & $\begin{array}{l}\text { Mean Difference (IV, Random, } \\
95 \% \mathrm{CI} \text { ) }\end{array}$ & $2.25[1.71,2.78]$ \\
\hline $\begin{array}{l}10.2 \text { Trials with short duration }(<2 \\
\text { years) }\end{array}$ & 3 & 1561 & $\begin{array}{l}\text { Mean Difference (IV, Random, } \\
95 \% \mathrm{Cl} \text { ) }\end{array}$ & $1.78[1.27,2.30]$ \\
\hline 11 Change in $\mathrm{HbA} 1 \mathrm{c}$ & 9 & 9320 & $\begin{array}{l}\text { Mean Difference (IV, Random, } \\
95 \% \mathrm{CI} \text { ) }\end{array}$ & $-0.05[-0.13,0.03]$ \\
\hline $\begin{array}{l}11.1 \text { Trials with long duration ( } \geq 2 \\
\text { years) }\end{array}$ & 6 & 7779 & $\begin{array}{l}\text { Mean Difference (IV, Random, } \\
95 \% \mathrm{CI} \text { ) }\end{array}$ & $-0.03[-0.14,0.07]$ \\
\hline $\begin{array}{l}11.2 \text { Trials with short duration }(<2 \\
\text { years) }\end{array}$ & 3 & 1541 & $\begin{array}{l}\text { Mean Difference (IV, Random, } \\
95 \% \mathrm{Cl} \text { ) }\end{array}$ & $-0.09[-0.25,0.07]$ \\
\hline 12 Fasting plasma glucose & 1 & & $\begin{array}{l}\text { Mean Difference (IV, Fixed, 95\% } \\
\mathrm{CI} \text { ) }\end{array}$ & Totals not selected \\
\hline $13 \mathrm{BMI}$ & 1 & & $\begin{array}{l}\text { Mean Difference (IV, Fixed, 95\% } \\
\mathrm{Cl} \text { ) }\end{array}$ & Totals not selected \\
\hline
\end{tabular}

\section{Analysis 3.1. Comparison 3 Metformin plus sulphonylurea vs metformin plus DPP-4 inhibitor, Outcome 1 All-cause mortality.}

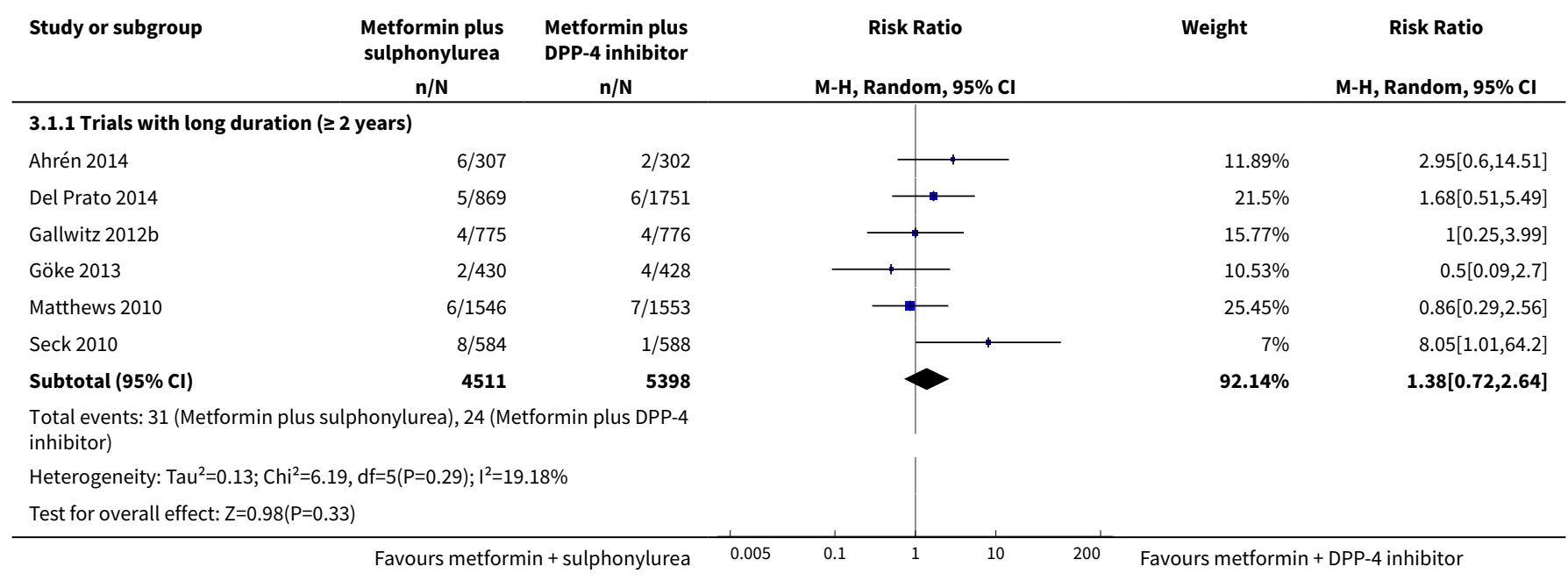




\begin{tabular}{|c|c|c|c|c|c|}
\hline Study or subgroup & $\begin{array}{l}\text { Metformin plus } \\
\text { sulphonylurea }\end{array}$ & $\begin{array}{l}\text { Metformin plus } \\
\text { DPP-4 inhibitor }\end{array}$ & Risk Ratio & Weight & Risk Ratio \\
\hline & $n / N$ & $n / N$ & M-H, Random, $95 \% \mathrm{Cl}$ & & M-H, Random, $95 \% \mathrm{Cl}$ \\
\hline
\end{tabular}

3.1.2 Trials with short duration ( $<2$ years)

$\begin{array}{lrr}\text { Dei Cas } 2017 & 0 / 24 & 0 / 40 \\ \text { Filozof } 2010 & 1 / 493 & 1 / 510 \\ \text { Schernthaner } 2015 & 1 / 359 & 1 / 359 \\ \text { Subtotal (95\% Cl) } & \mathbf{8 7 6} & \mathbf{9 0 9}\end{array}$

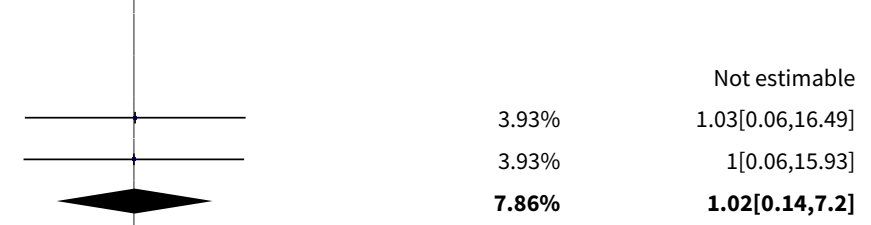

Total events: 2 (Metformin plus sulphonylurea), 2 (Metformin plus DPP-4 inhibitor)

Heterogeneity: $\mathrm{Tau}^{2}=0 ; \mathrm{Chi}^{2}=0, \mathrm{df}=1(\mathrm{P}=0.99) ; \mathrm{I}^{2}=0 \%$

Test for overall effect: $Z=0.02(P=0.99)$

Total $(95 \% \mathrm{Cl})$

Total events: 33 (Metformin plus sulphonylurea), 26 (Metformin plus DPP-4 inhibitor)

Heterogeneity: $\mathrm{Tau}^{2}=0 ; \mathrm{Chi}^{2}=6.26, \mathrm{df}=7(\mathrm{P}=0.51) ; \mathrm{I}^{2}=0 \%$

Test for overall effect: $Z=0.99(P=0.32)$

Test for subgroup differences: $\mathrm{Chi}^{2}=0.08, \mathrm{df}=1(\mathrm{P}=0.77), \mathrm{I}^{2}=0 \%$

Analysis 3.2. Comparison 3 Metformin plus sulphonylurea vs metformin plus DPP-4 inhibitor, Outcome 2 Serious adverse events.

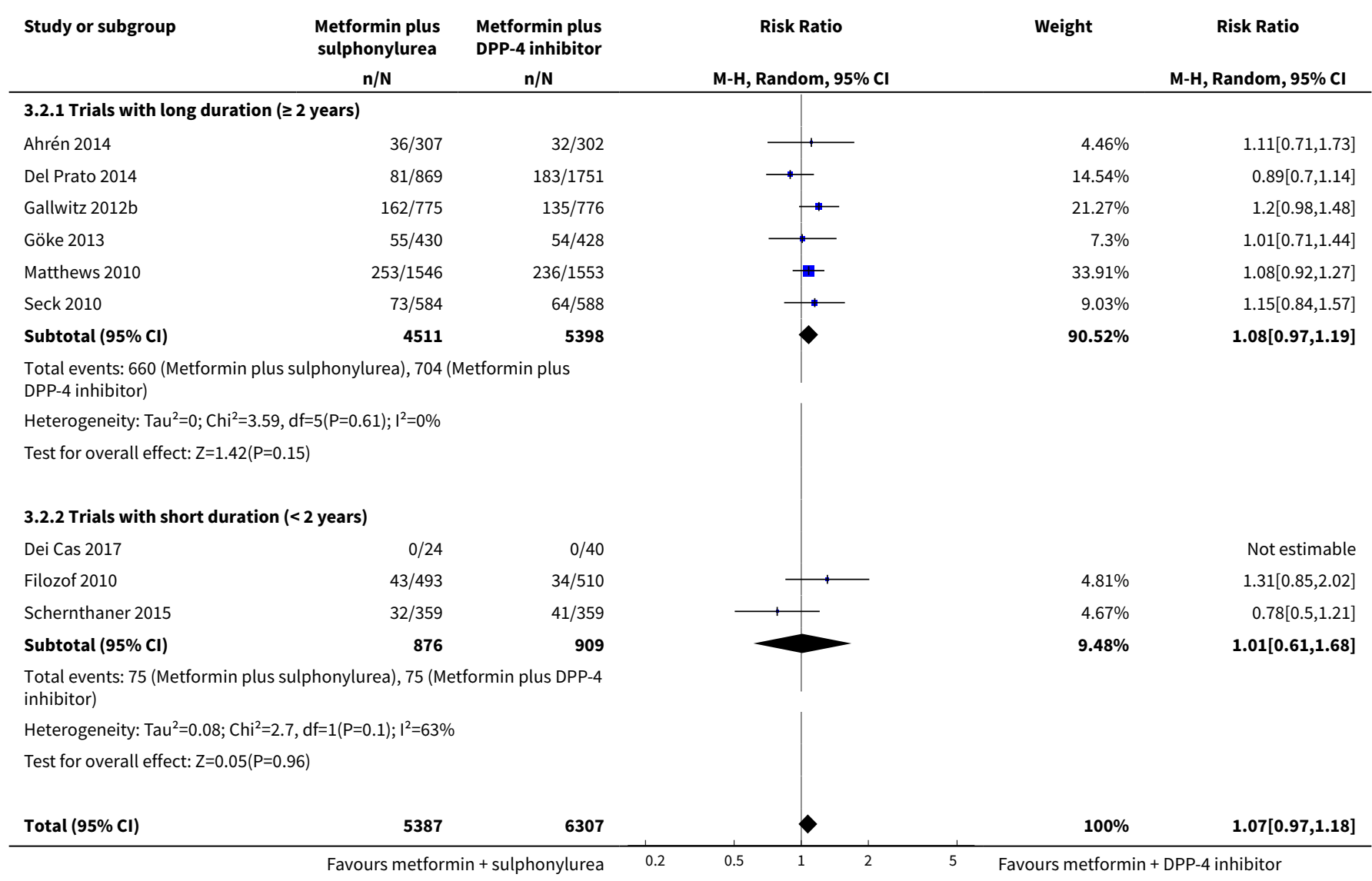




\begin{tabular}{|c|c|c|c|c|c|c|}
\hline Study or subgroup & $\begin{array}{c}\text { Metformin plus } \\
\text { sulphonylurea } \\
n / N\end{array}$ & $\begin{array}{l}\text { Metformin plus } \\
\text { DPP-4 inhibitor } \\
n \mathbf{n} / \mathbf{N} \\
\end{array}$ & \multicolumn{2}{|r|}{$\begin{array}{c}\text { Risk Ratio } \\
\text { M-H, Random, } 95 \% \mathrm{Cl}\end{array}$} & Weight & $\begin{array}{c}\text { Risk Ratio } \\
\text { M-H, Random, } 95 \% \mathrm{CI}\end{array}$ \\
\hline \multicolumn{7}{|c|}{$\begin{array}{l}\text { Total events: } 735 \text { (Metformin plus sulphonylurea), } 779 \text { (Metformin plus } \\
\text { DPP-4 inhibitor) }\end{array}$} \\
\hline \multicolumn{7}{|c|}{ Heterogeneity: $\mathrm{Tau}^{2}=0 ; \mathrm{Chi}^{2}=6.42, \mathrm{df}=7(\mathrm{P}=0.49) ; \mathrm{I}^{2}=0 \%$} \\
\hline \multicolumn{7}{|c|}{ Test for overall effect: $Z=1.38(P=0.17)$} \\
\hline \multicolumn{7}{|c|}{ Test for subgroup differences: $\mathrm{Chi}^{2}=0.05, \mathrm{df}=1(\mathrm{P}=0.82), \mathrm{I}^{2}=0 \%$} \\
\hline
\end{tabular}

Analysis 3.3. Comparison 3 Metformin plus sulphonylurea vs metformin plus DPP-4 inhibitor, Outcome 3 Cardiovascular mortality.

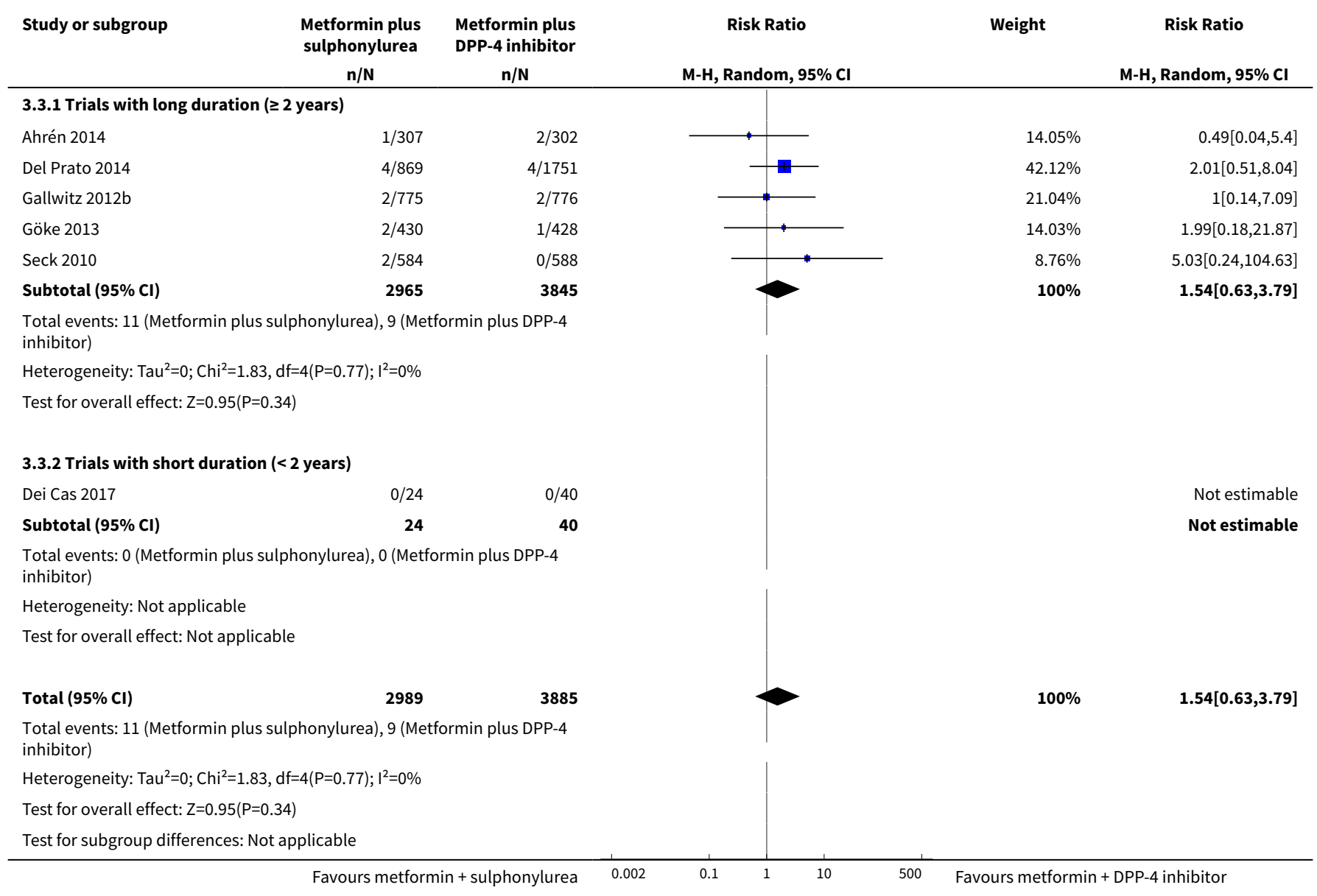

Analysis 3.4. Comparison 3 Metformin plus sulphonylurea vs metformin plus DPP-4 inhibitor, Outcome 4 Non-fatal myocardial infarction.

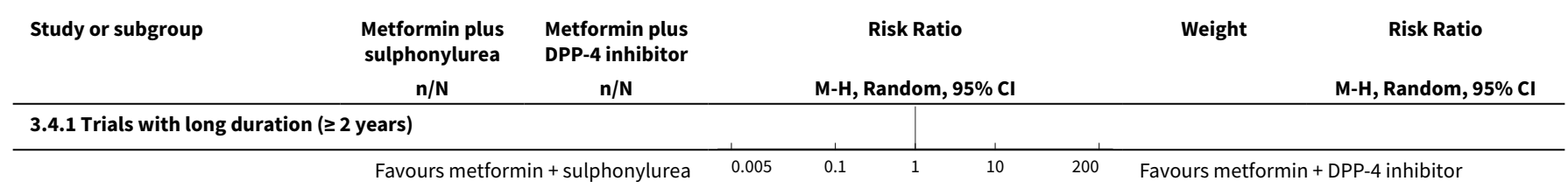




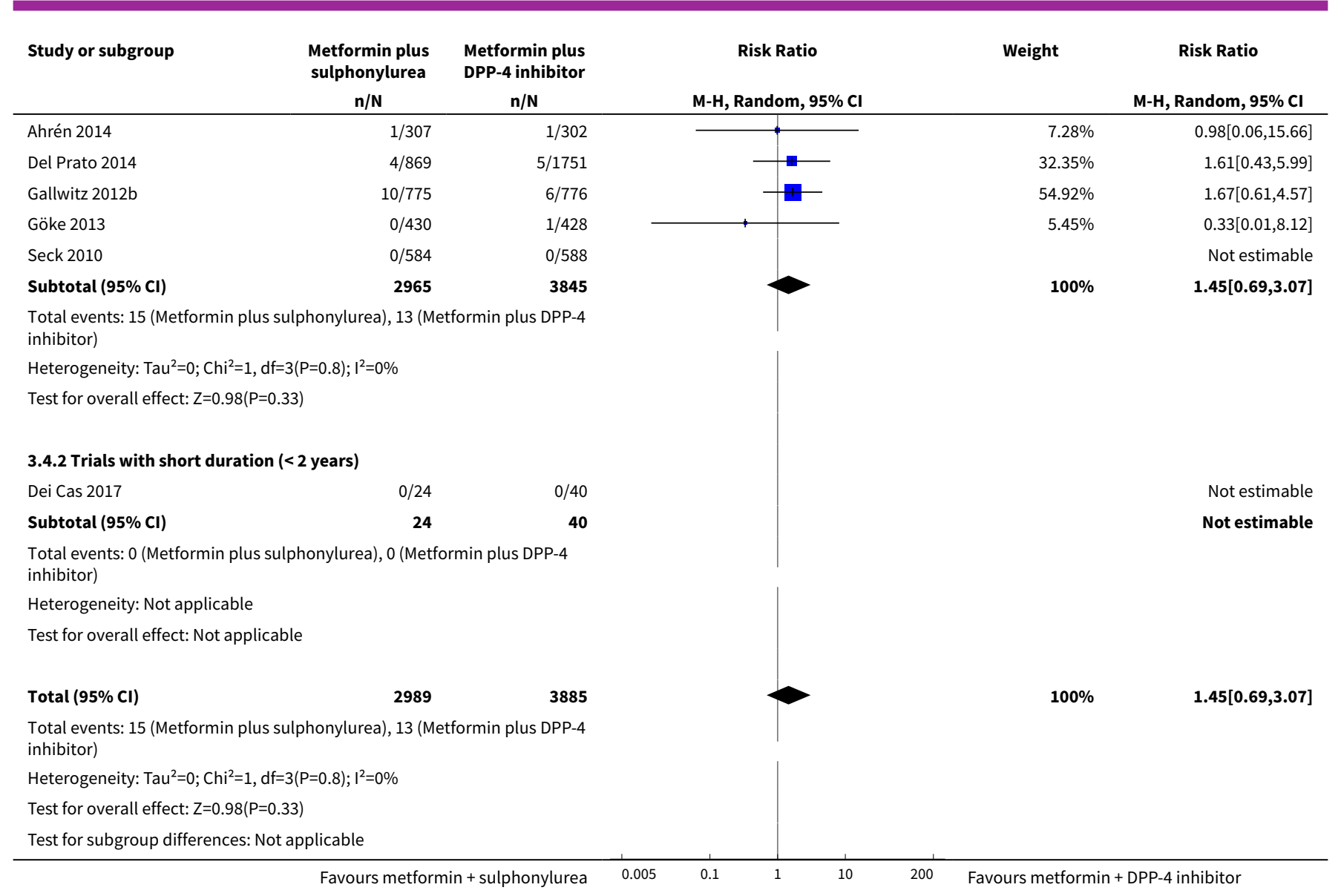

Analysis 3.5. Comparison 3 Metformin plus sulphonylurea
vs metformin plus DPP-4 inhibitor, Outcome 5 Heart failure.

Study or subgroup

Metformin plus

Metformin plus DPP-4 inhibitor

Weight

Risk Ratio sulphonylure $\mathrm{n} / \mathrm{N}$ M-H, Random, 95\% CI M-H, Random, $95 \% \mathrm{CI}$

$\begin{array}{lrr}\text { 3.5.1 Trials with long duration ( } \geq \mathbf{2} \text { years) } & & \\ \text { Ahrén } 2014 & 1 / 307 & 1 / 302 \\ \text { Del Prato 2014 } & 1 / 869 & 3 / 1751 \\ \text { Gallwitz 2012b } & 2 / 775 & 3 / 776 \\ \text { Göke 2013 } & 1 / 430 & 1 / 428 \\ \text { Matthews } 2010 & 3 / 1546 & 3 / 1553 \\ \text { Seck 2010 } & 1 / 584 & 2 / 588 \\ \text { Subtotal }(\mathbf{9 5} \% \mathbf{C l}) & \mathbf{4 5 1 1} & \mathbf{5 3 9 8}\end{array}$

Total events: 9 (Metformin plus sulphonylurea), 13 (Metformin plus DPP-4 inhibitor)

Heterogeneity: $\mathrm{Tau}^{2}=0 ; \mathrm{Chi}^{2}=0.33, \mathrm{df}=5(\mathrm{P}=1) ; \mathrm{I}^{2}=0 \%$

Test for overall effect: $\mathrm{Z}=0.55(\mathrm{P}=0.58)$

3.5.2 Trials with short duration ( $<2$ years)

Dei Cas 2017

Schernthaner 2015

$0 / 24 \quad 0 / 40$

Subtotal $(95 \% \mathrm{CI})$

$6 / 359$

383

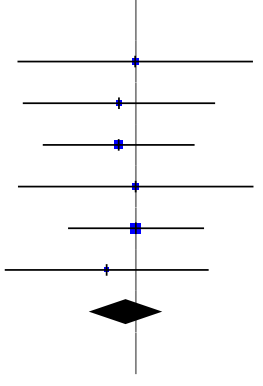

$8.37 \%$

$0.98[0.06,15.66]$

$12.54 \%$

$0.67[0.07,6.45]$

$20.1 \%$

$0.67[0.11,3.98]$

$8.37 \%$

$1[0.06,15.86]$

$25.09 \%$

$1[0.2,4.97]$

$11.16 \%$

$0.5[0.05,5.54]$

$85.62 \%$

$\mathbf{0 . 7 8}[0.33,1.86]$

Favours metformin + sulphonylurea $\quad 0.005$

399

$14.38 \%$

Not estimable

$14.38 \%$

$6[0.73,49.59]$ 


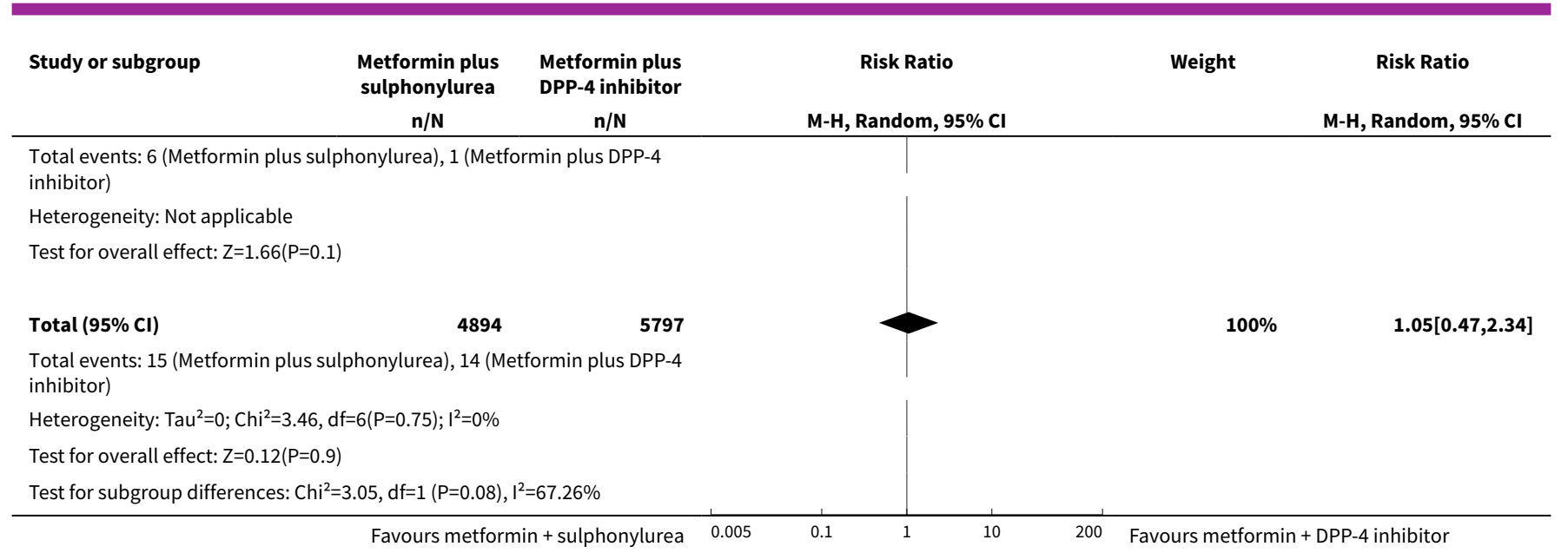

\section{Analysis 3.6. Comparison 3 Metformin plus sulphonylurea vs metformin plus DPP-4 inhibitor, Outcome 6 Non-fatal stroke.}

\begin{tabular}{|c|c|c|c|c|c|}
\hline Study or subgroup & $\begin{array}{c}\text { Metformin plus } \\
\text { sulphonylurea } \\
\mathrm{n} / \mathrm{N} \\
\end{array}$ & $\begin{array}{c}\text { Metformin plus } \\
\text { DPP-4 inhibitor } \\
\mathrm{n} / \mathrm{N} \\
\end{array}$ & M-H, Random, $95 \% \mathrm{Cl}$ & Weight & $\begin{array}{c}\text { Risk Ratio } \\
\text { M-H, Random, } 95 \% \mathrm{Cl} \\
\end{array}$ \\
\hline \multicolumn{6}{|c|}{ 3.6.1 Trials with long duration ( $\geq 2$ years) } \\
\hline Del Prato 2014 & $3 / 869$ & $5 / 1751$ & & $45.59 \%$ & $1.21[0.29,5.05]$ \\
\hline Gallwitz 2012b & $11 / 775$ & $3 / 776$ & & $54.41 \%$ & $3.67[1.03,13.11]$ \\
\hline Göke 2013 & $0 / 430$ & $0 / 428$ & & & Not estimable \\
\hline \multicolumn{6}{|c|}{$\begin{array}{l}\text { Total events: } 14 \text { (Metformin plus sulphonylurea), } 8 \text { (Metformin plus DPP-4 } \\
\text { inhibitor) }\end{array}$} \\
\hline \multicolumn{6}{|c|}{ Heterogeneity: Tau $^{2}=0.15 ; \mathrm{Chi}^{2}=1.31, \mathrm{df}=1(\mathrm{P}=0.25) ; \mathrm{I}^{2}=23.56 \%$} \\
\hline \multicolumn{6}{|c|}{ Test for overall effect: $Z=1.43(P=0.15)$} \\
\hline \multicolumn{6}{|c|}{ 3.6.2 Trials with short duration ( $<2$ years) } \\
\hline Subtotal $(95 \% \mathrm{Cl})$ & 24 & 40 & & & Not estimable \\
\hline \multicolumn{6}{|c|}{$\begin{array}{l}\text { Total events: } 0 \text { (Metformin plus sulphonylurea), } 0 \text { (Metformin plus DPP-4 } \\
\text { inhibitor) }\end{array}$} \\
\hline \multicolumn{6}{|c|}{ Heterogeneity: Not applicable } \\
\hline \multicolumn{6}{|c|}{ Test for overall effect: Not applicable } \\
\hline Total $(95 \% \mathrm{Cl})$ & 2098 & 2995 & & $100 \%$ & $2.21[0.74,6.58]$ \\
\hline \multicolumn{6}{|c|}{$\begin{array}{l}\text { Total events: } 14 \text { (Metformin plus sulphonylurea), } 8 \text { (Metformin plus DPP-4 } \\
\text { inhibitor) }\end{array}$} \\
\hline \multicolumn{6}{|c|}{ Heterogeneity: $\operatorname{Tau}^{2}=0.15 ; \mathrm{Chi}^{2}=1.31, \mathrm{df}=1(\mathrm{P}=0.25) ; \mathrm{I}^{2}=23.56 \%$} \\
\hline \multicolumn{6}{|c|}{ Test for overall effect: $Z=1.43(P=0.15)$} \\
\hline Test for subgroup dif & oplicable & & & & \\
\hline
\end{tabular}


Analysis 3.7. Comparison 3 Metformin plus sulphonylurea vs metformin plus DPP-4 inhibitor, Outcome 7 Non-serious adverse events.

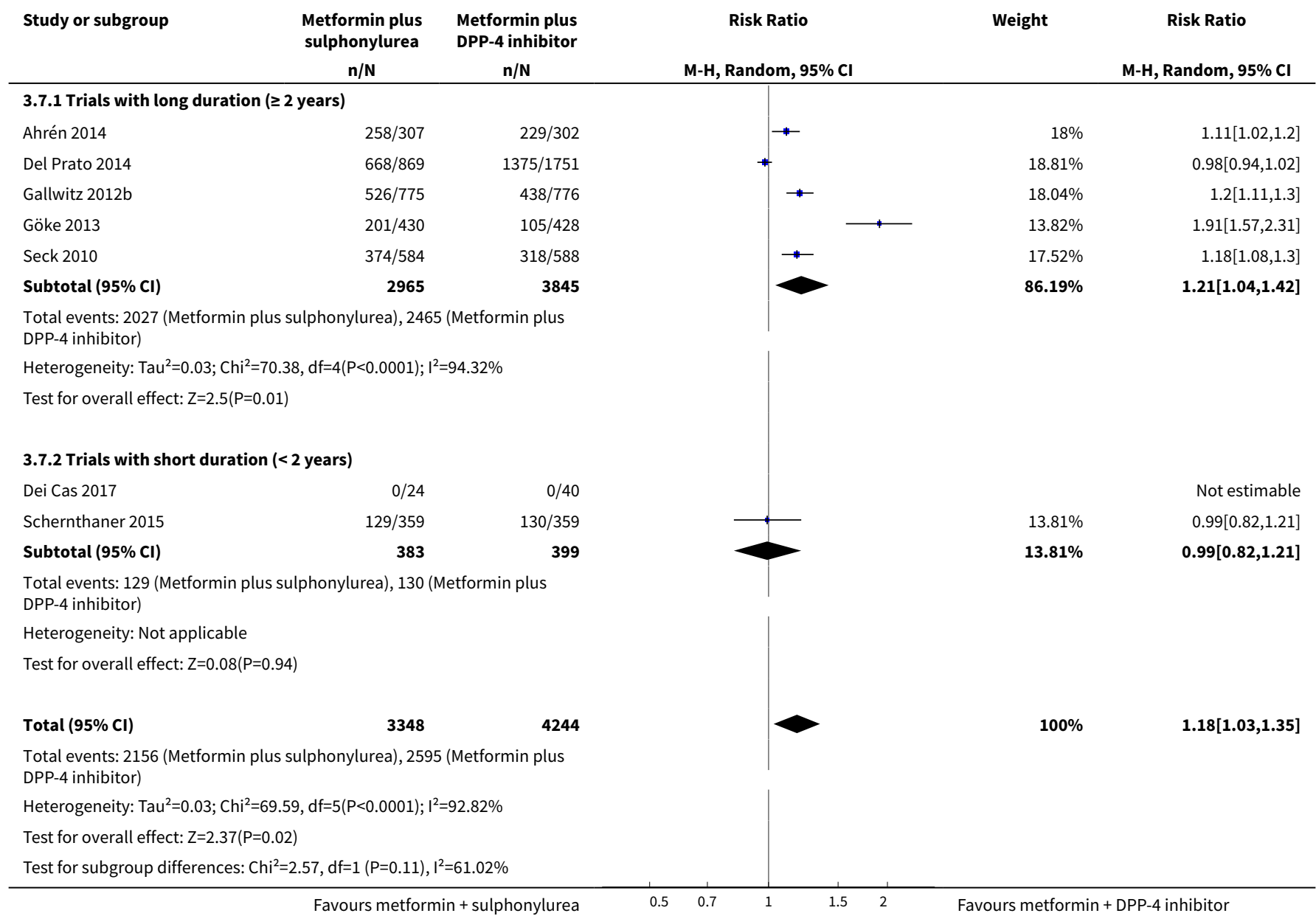

Analysis 3.8. Comparison 3 Metformin plus sulphonylurea vs metformin plus DPP-4 inhibitor, Outcome 8 Mild/moderate hypoglycaemia.

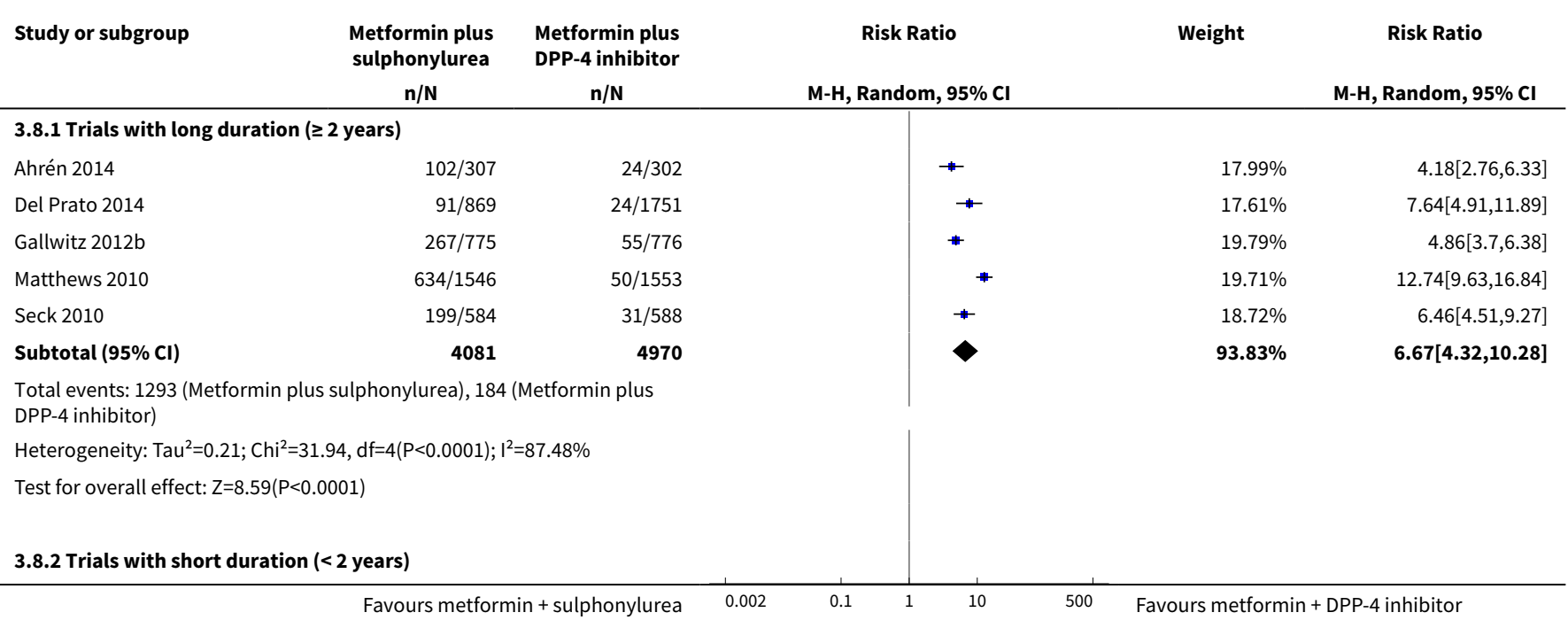




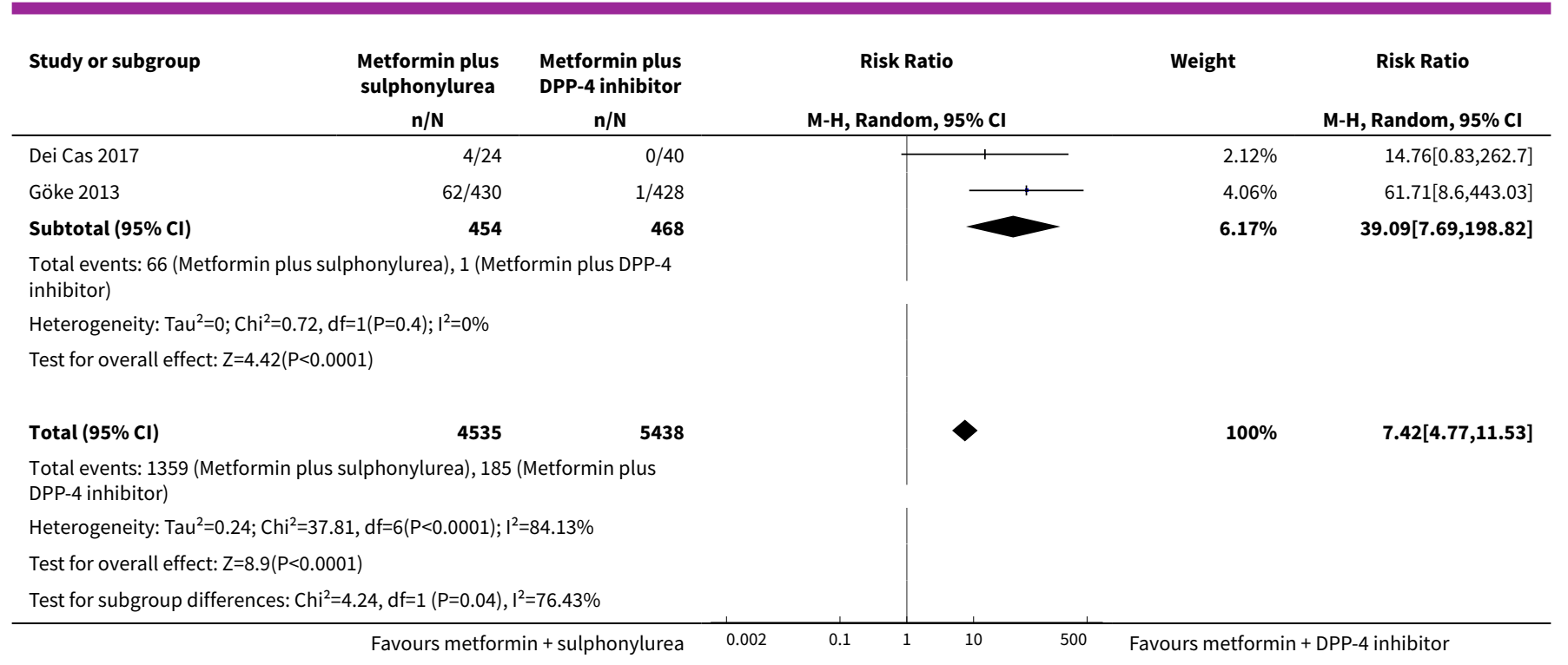

\section{Analysis 3.9. Comparison 3 Metformin plus sulphonylurea vs metformin plus DPP-4 inhibitor, Outcome 9 Serious hypoglycaemia.}

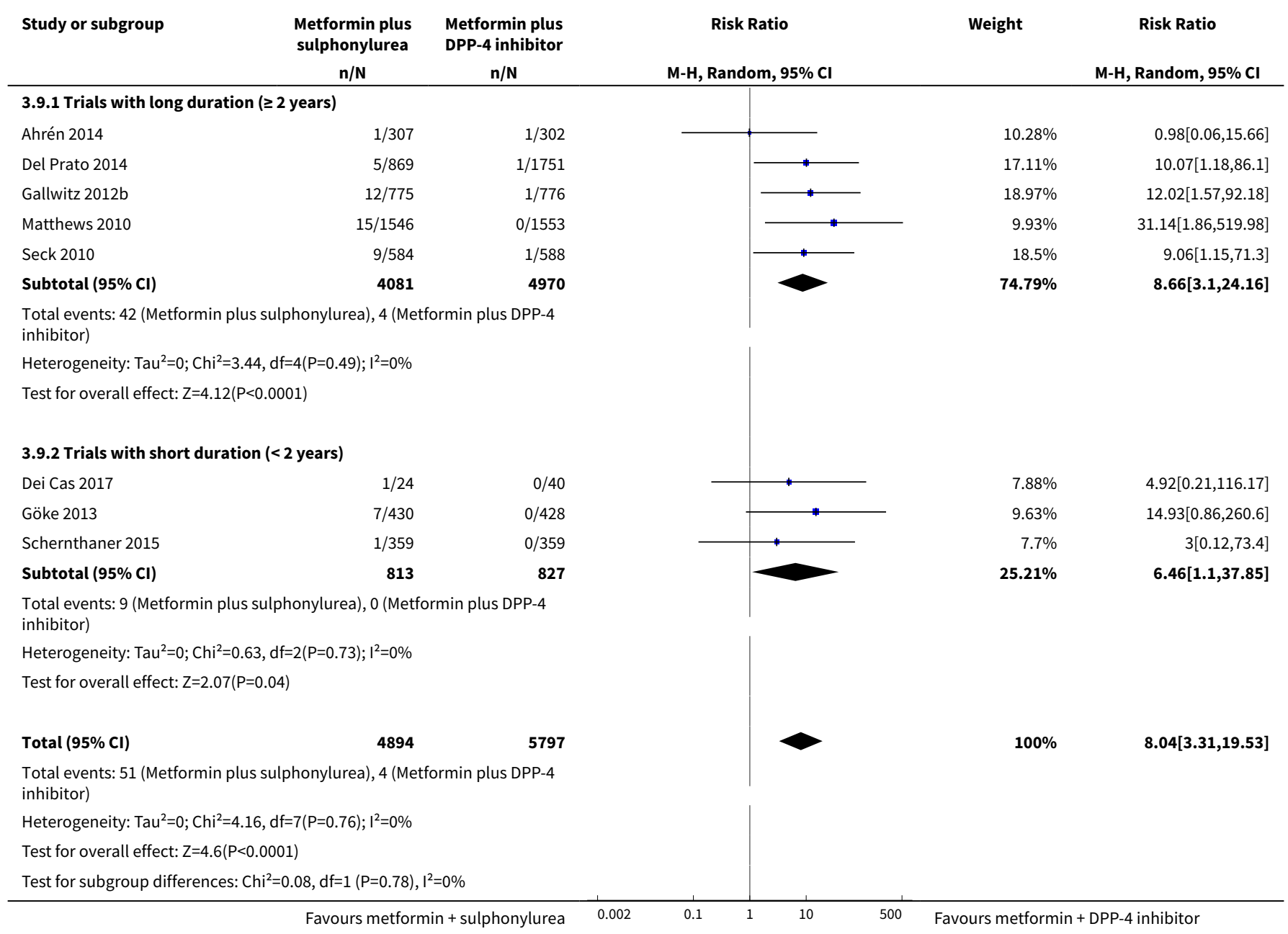


Analysis 3.10. Comparison 3 Metformin plus sulphonylurea vs metformin plus DPP-4 inhibitor, Outcome 10 Weight change (kg).

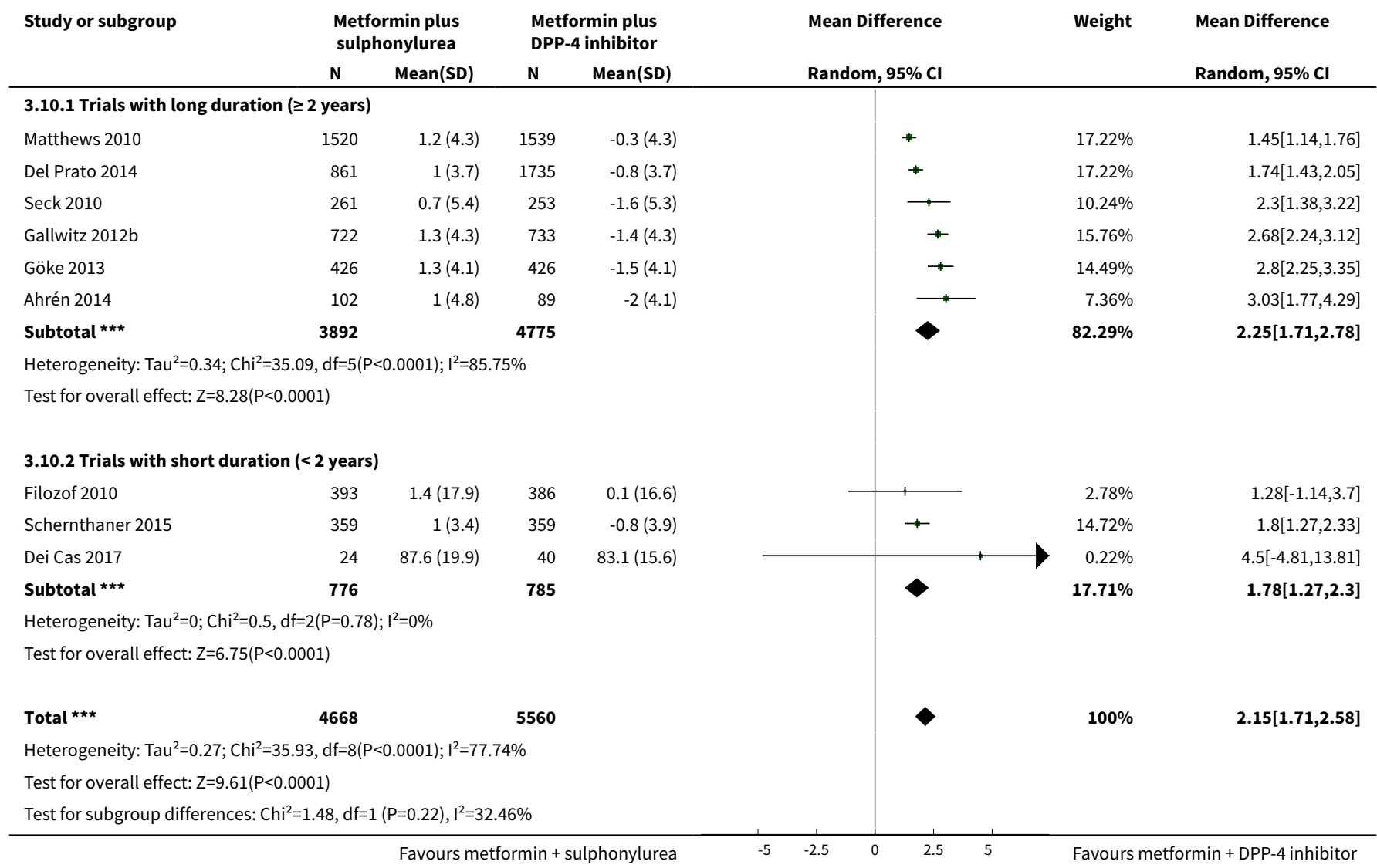

\section{Analysis 3.11. Comparison 3 Metformin plus sulphonylurea vs metformin plus DPP-4 inhibitor, Outcome 11 Change in HbA1c.}

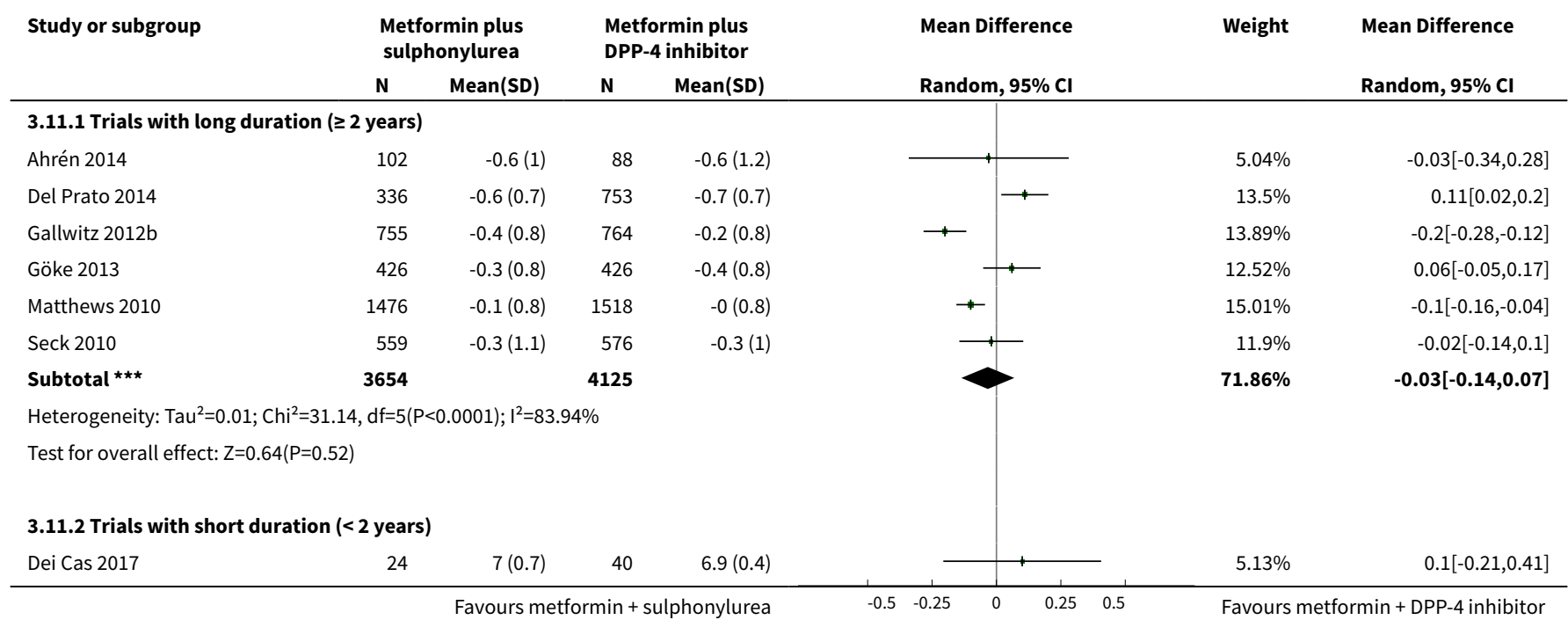




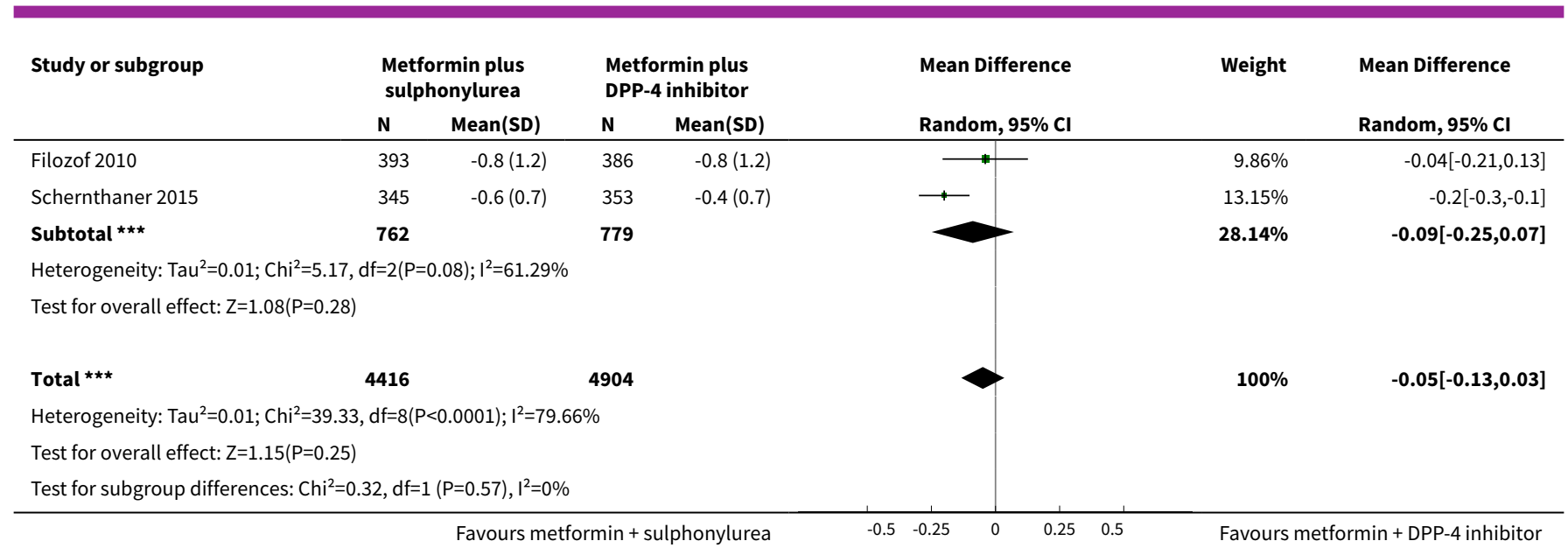

Analysis 3.12. Comparison 3 Metformin plus sulphonylurea vs metformin plus DPP-4 inhibitor, Outcome 12 Fasting plasma glucose.

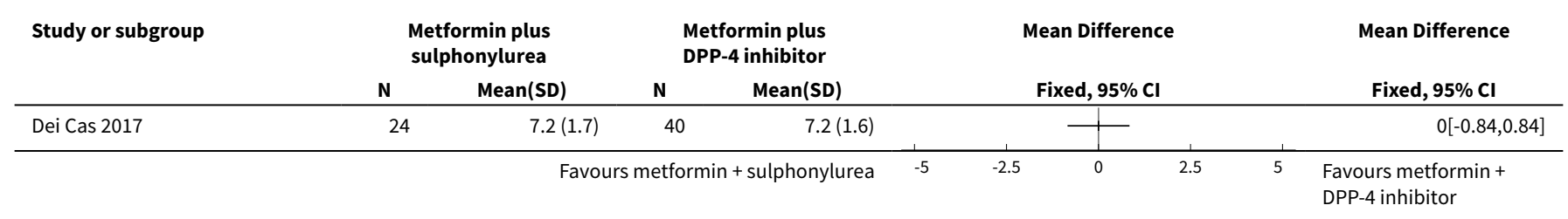

Analysis 3.13. Comparison 3 Metformin plus sulphonylurea vs metformin plus DPP-4 inhibitor, Outcome 13 BMI.

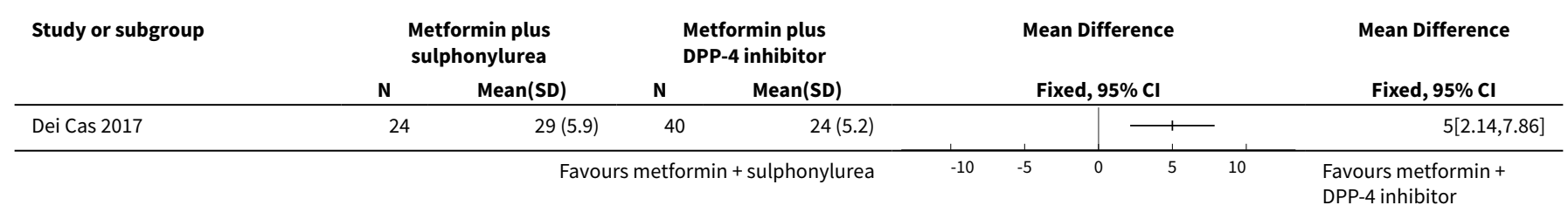

Comparison 4. Metformin plus sulphonylurea vs metformin plus long-acting DPP-4 inhibitor

\begin{tabular}{lllll}
\hline Outcome or subgroup title & No. of studies & $\begin{array}{l}\text { No. of partici- } \\
\text { pants }\end{array}$ & Statistical method & Effect size \\
\hline 1 All-cause mortality & 1 & Risk Ratio (M-H, Fixed, 95\% Cl) & Totals not selected \\
\hline 2 Serious adverse events & 1 & Risk Ratio (M-H, Fixed, 95\% Cl) & Totals not selected \\
\hline $\begin{array}{l}3 \text { Cardiovascular mortality } \\
\text { 4 Non-fatal myocardial in- }\end{array}$ & 1 & Risk Ratio (M-H, Fixed, 95\% Cl) & Totals not selected \\
\hline $\begin{array}{l}\text { farction } \\
\text { 5 Non-serious adverse }\end{array}$ & 1 & Risk Ratio (M-H, Fixed, 95\% Cl) & Totals not selected \\
\hline
\end{tabular}




\begin{tabular}{lllll}
\hline Outcome or subgroup title & No. of studies & $\begin{array}{l}\text { No. of partici- } \\
\text { pants }\end{array}$ & Statistical method & Effect size \\
\hline $\begin{array}{l}6 \text { Mild/moderate hypogly- } \\
\text { caemia }\end{array}$ & 1 & Risk Ratio (M-H, Fixed, 95\% Cl) & Totals not selected \\
\hline 7 Serious hypoglycaemia & 1 & Risk Ratio (M-H, Fixed, 95\% Cl) & Totals not selected \\
\hline 8 Weight change (kg) & 1 & Mean Difference (IV, Fixed, 95\% Cl) & Totals not selected \\
\hline 9 Change in HbA1c (\%) & 1 & Mean Difference (IV, Fixed, 95\% Cl) & Totals not selected \\
\hline
\end{tabular}

Analysis 4.1. Comparison 4 Metformin plus sulphonylurea vs metformin plus long-acting DPP-4 inhibitor, Outcome 1 All-cause mortality.

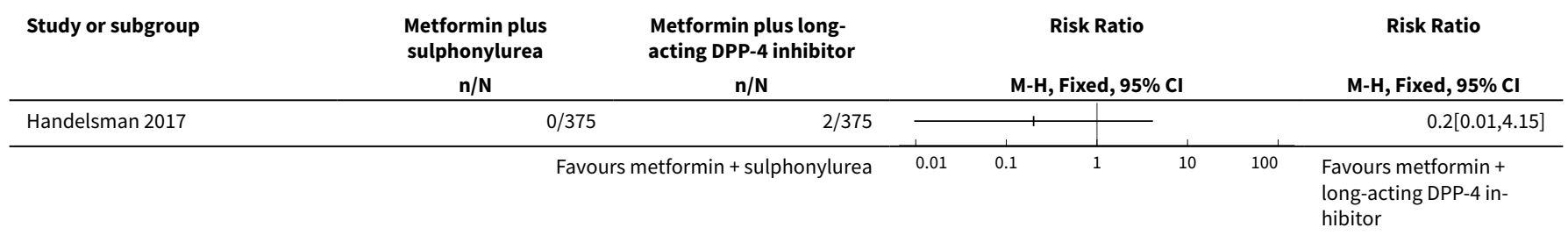

Analysis 4.2. Comparison 4 Metformin plus sulphonylurea vs metformin plus long-acting DPP-4 inhibitor, Outcome 2 Serious adverse events.

\begin{tabular}{|c|c|c|c|c|c|c|c|}
\hline \multirow[t]{2}{*}{ Study or subgroup } & $\begin{array}{l}\text { Metformin plus } \\
\text { sulphonylurea }\end{array}$ & \multirow[t]{2}{*}{$\begin{array}{l}\text { Metformin plus long- } \\
\text { acting DPP-4 inhibitor } \\
\qquad n / N\end{array}$} & \multicolumn{4}{|c|}{ Risk Ratio } & Risk Ratio \\
\hline & $n / N$ & & \multicolumn{4}{|c|}{ M-H, Fixed, 95\% Cl } & M-H, Fixed, 95\% Cl \\
\hline Handelsman 2017 & $18 / 375$ & $24 / 375$ & & & & & $0.75[0.41,1.36]$ \\
\hline & Favol & netformin + sulphonylurea & 0.01 & 0.1 & 10 & 100 & $\begin{array}{l}\text { Favours metformin + } \\
\text { long-acting DPP-4 in- } \\
\text { hibitor }\end{array}$ \\
\hline
\end{tabular}

Analysis 4.3. Comparison 4 Metformin plus sulphonylurea vs metformin plus long-acting DPP-4 inhibitor, Outcome 3 Cardiovascular mortality.

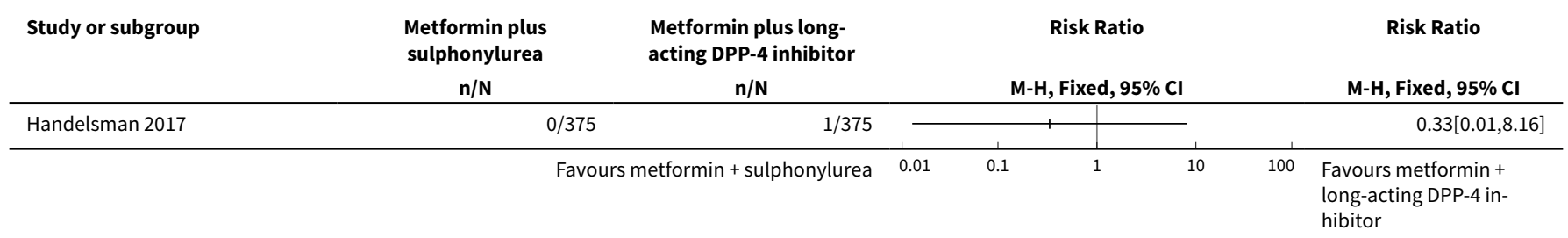


Analysis 4.4. Comparison 4 Metformin plus sulphonylurea vs metformin plus long-acting DPP-4 inhibitor, Outcome 4 Non-fatal myocardial infarction.

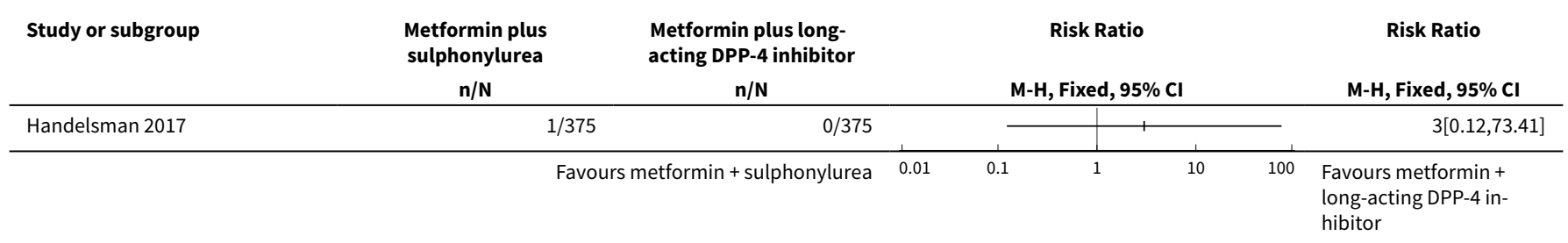

Analysis 4.5. Comparison 4 Metformin plus sulphonylurea vs metformin plus long-acting DPP-4 inhibitor, Outcome 5 Non-serious adverse events.

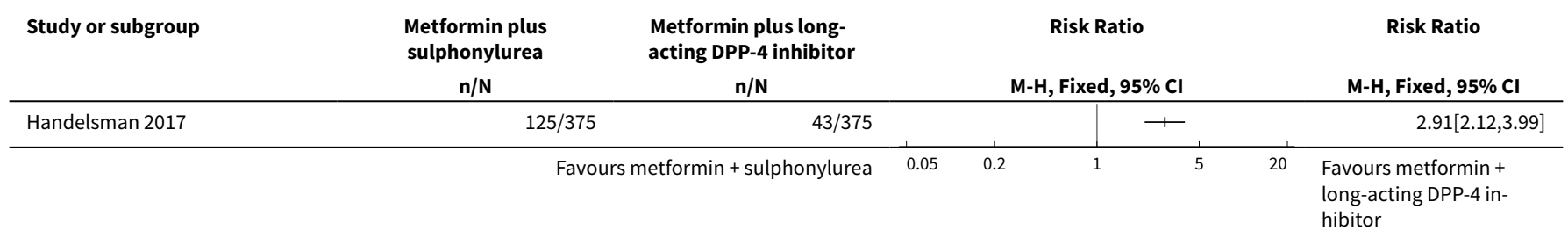

Analysis 4.6. Comparison 4 Metformin plus sulphonylurea vs metformin plus long-acting DPP-4 inhibitor, Outcome 6 Mild/moderate hypoglycaemia.

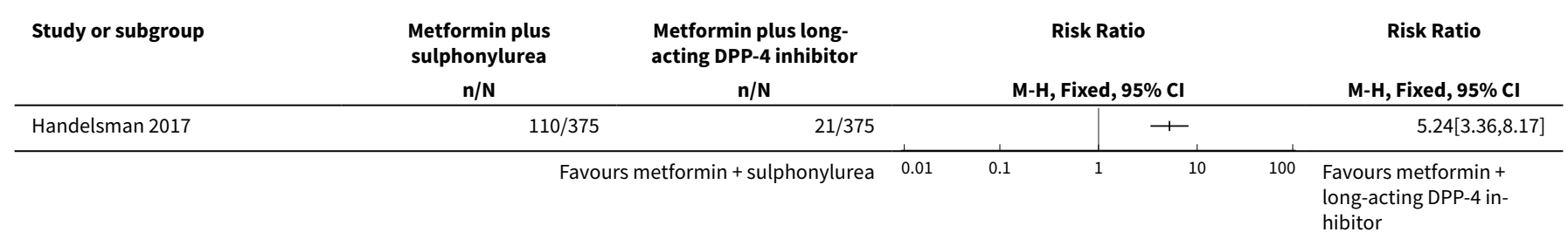

Analysis 4.7. Comparison 4 Metformin plus sulphonylurea vs metformin plus long-acting DPP-4 inhibitor, Outcome 7 Serious hypoglycaemia.

\begin{tabular}{|c|c|c|c|c|c|c|c|c|}
\hline \multirow[t]{2}{*}{ Study or subgroup } & Metformin plus & $\begin{array}{l}\text { Metformin plus long- } \\
\text { acting DPP-4 inhibitor }\end{array}$ & \multicolumn{5}{|c|}{ Risk Ratio } & \multirow{2}{*}{$\begin{array}{c}\text { Risk Ratio } \\
\text { M-H, Fixed, } 95 \% \mathrm{Cl}\end{array}$} \\
\hline & $\mathrm{n} / \mathrm{N}$ & $n / N$ & \multicolumn{4}{|c|}{ M-H, Fixed, $95 \% \mathrm{Cl}$} & & \\
\hline \multirow[t]{2}{*}{ Handelsman 2017} & $6 / 375$ & $1 / 375$ & & & & & & $6[0.73,49.6]$ \\
\hline & Favou & netformin + sulphonylurea & 0.01 & 0.1 & 1 & 10 & 100 & $\begin{array}{l}\text { Favours metformin + } \\
\text { long-acting DPP-4 in- } \\
\text { hibitor }\end{array}$ \\
\hline
\end{tabular}

Analysis 4.8. Comparison 4 Metformin plus sulphonylurea vs metformin plus long-acting DPP-4 inhibitor, Outcome 8 Weight change (kg).

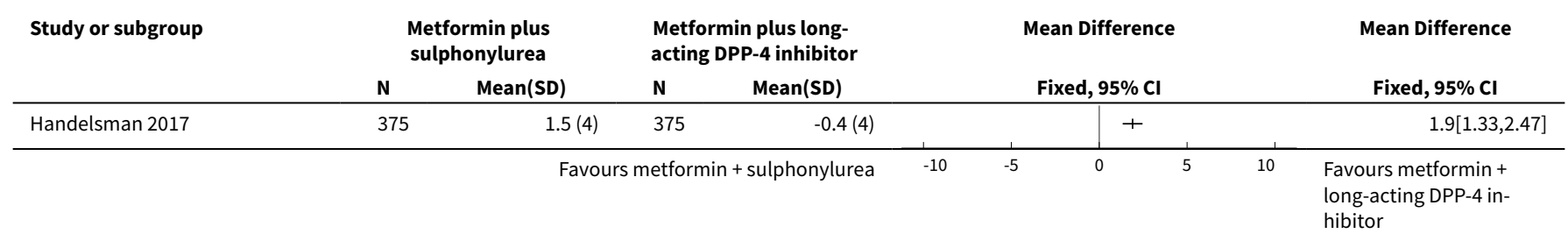


Analysis 4.9. Comparison 4 Metformin plus sulphonylurea vs metformin plus long-acting DPP-4 inhibitor, Outcome 9 Change in HbA1c (\%).

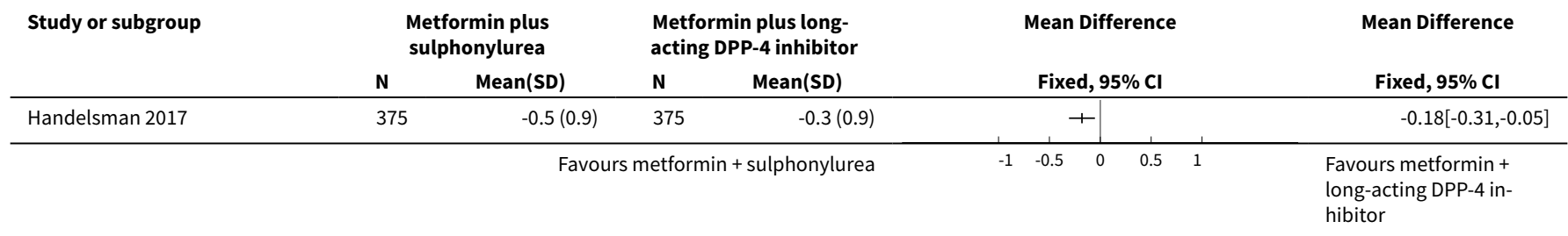

\section{Comparison 5. Metformin plus sulphonylurea vs metformin plus thiazolidinedione}

\begin{tabular}{|c|c|c|c|c|}
\hline $\begin{array}{l}\text { Outcome or subgroup } \\
\text { title }\end{array}$ & No. of studies & $\begin{array}{l}\text { No. of partici- } \\
\text { pants }\end{array}$ & Statistical method & Effect size \\
\hline 1 All-cause mortality & 6 & 6654 & Risk Ratio (M-H, Random, 95\% Cl) & $1.09[0.85,1.40]$ \\
\hline 1.1 Rosiglitazone & 4 & 2996 & Risk Ratio (M-H, Random, 95\% Cl) & $1.20[0.86,1.68]$ \\
\hline 1.2 Pioglitazone & 2 & 3658 & Risk Ratio (M-H, Random, 95\% Cl) & $1.09[0.42,2.80]$ \\
\hline 2 Serious adverse events & 6 & 6654 & Risk Ratio (M-H, Random, 95\% Cl) & $1.01[0.93,1.11]$ \\
\hline 2.1 Rosiglitazone & 4 & 2996 & Risk Ratio (M-H, Random, 95\% Cl) & $1.02[0.81,1.29]$ \\
\hline 2.2 Pioglitazone & 2 & 3658 & Risk Ratio (M-H, Random, 95\% Cl) & $0.99[0.83,1.18]$ \\
\hline $\begin{array}{l}3 \text { Cardiovascular mortal- } \\
\text { ity }\end{array}$ & 4 & 5940 & Risk Ratio (M-H, Random, 95\% Cl) & $0.78[0.36,1.67]$ \\
\hline 3.1 Rosiglitazone & 3 & 2912 & Risk Ratio (M-H, Random, 95\% Cl) & $0.91[0.30,2.72]$ \\
\hline 3.2 Pioglitazone & 1 & 3028 & Risk Ratio (M-H, Random, 95\% Cl) & $0.46[0.14,1.48]$ \\
\hline $\begin{array}{l}4 \text { Non-fatal myocardial } \\
\text { infarction }\end{array}$ & 3 & 3718 & Risk Ratio (M-H, Random, 95\% Cl) & $1.21[0.68,2.14]$ \\
\hline 4.1 Rosiglitazone & 2 & 690 & Risk Ratio (M-H, Random, 95\% Cl) & $2.93[0.12,71.65]$ \\
\hline 4.2 Pioglitazone & 1 & 3028 & Risk Ratio (M-H, Random, 95\% Cl) & $1.18[0.66,2.10]$ \\
\hline 5 Heart failure & 5 & 6570 & Risk Ratio (M-H, Random, 95\% Cl) & $0.67[0.43,1.04]$ \\
\hline 5.1 Rosiglitazone & 3 & 2912 & Risk Ratio (M-H, Random, 95\% Cl) & $0.74[0.41,1.33]$ \\
\hline 5.2 Pioglitazone & 2 & 3658 & Risk Ratio (M-H, Random, 95\% Cl) & $0.60[0.31,1.16]$ \\
\hline 6 Non-fatal stroke & 2 & 3123 & Risk Ratio (M-H, Random, 95\% Cl) & $1.29[0.67,2.47]$ \\
\hline 6.1 Rosiglitazone & 1 & 95 & Risk Ratio (M-H, Random, 95\% Cl) & $0.0[0.0,0.0]$ \\
\hline 6.2 Pioglitazone & 1 & 3028 & Risk Ratio (M-H, Random, 95\% Cl) & $1.29[0.67,2.47]$ \\
\hline
\end{tabular}




\begin{tabular}{|c|c|c|c|c|}
\hline $\begin{array}{l}\text { Outcome or subgroup } \\
\text { title }\end{array}$ & No. of studies & $\begin{array}{l}\text { No. of partici- } \\
\text { pants }\end{array}$ & Statistical method & Effect size \\
\hline $\begin{array}{l}7 \text { Amputation of lower } \\
\text { extremity }\end{array}$ & 2 & 3123 & Risk Ratio (M-H, Random, 95\% Cl) & $0.0[0.0,0.0]$ \\
\hline 7.1 Rosiglitazone & 1 & 95 & Risk Ratio (M-H, Random, 95\% Cl) & $0.0[0.0,0.0]$ \\
\hline 7.2 Pioglitazone & 1 & 3028 & Risk Ratio (M-H, Random, 95\% Cl) & $0.0[0.0,0.0]$ \\
\hline $\begin{array}{l}8 \text { Blindness or severe vi- } \\
\text { sion loss }\end{array}$ & 1 & & Risk Ratio (M-H, Fixed, 95\% Cl) & Totals not selected \\
\hline 9 End-stage renal disease & 1 & & Risk Ratio (M-H, Fixed, 95\% Cl) & Totals not selected \\
\hline $\begin{array}{l}10 \text { Non-serious adverse } \\
\text { events }\end{array}$ & 5 & 6024 & Risk Ratio (M-H, Random, 95\% Cl) & $0.94[0.44,2.01]$ \\
\hline 10.1 Rosiglitazone & 4 & 2996 & Risk Ratio (M-H, Random, 95\% Cl) & $1.00[0.92,1.08]$ \\
\hline 10.2 Pioglitazone & 1 & 3028 & Risk Ratio (M-H, Random, 95\% Cl) & $0.93[0.82,1.05]$ \\
\hline $\begin{array}{l}11 \text { Mild/moderate hypo- } \\
\text { glycaemia }\end{array}$ & 5 & 6059 & Risk Ratio (M-H, Random, 95\% Cl) & $3.63[2.98,4.44]$ \\
\hline 11.1 Rosiglitazone & 3 & 2401 & Risk Ratio (M-H, Random, 95\% Cl) & $3.76[2.81,5.02]$ \\
\hline 11.2 Pioglitazone & 2 & 3658 & Risk Ratio (M-H, Random, 95\% Cl) & $4.78[1.93,11.87]$ \\
\hline $\begin{array}{l}12 \text { Serious hypogly- } \\
\text { caemia }\end{array}$ & 5 & 6570 & Risk Ratio (M-H, Random, 95\% Cl) & $3.98[0.34,46.01]$ \\
\hline 12.1 Rosiglitazone & 3 & 2912 & Risk Ratio (M-H, Random, 95\% Cl) & $1.16[0.37,3.68]$ \\
\hline 12.2 Pioglitazone & 2 & 3658 & Risk Ratio (M-H, Random, 95\% Cl) & $24.68[3.34,182.16]$ \\
\hline 13 Weight (change) & 7 & 6877 & Mean Difference (IV, Random, 95\% CI) & $-0.55[-2.75,1.64]$ \\
\hline 13.1 Rosiglitazone & 3 & 2865 & Mean Difference (IV, Random, 95\% Cl) & $-0.96[-4.77,2.86]$ \\
\hline 13.2 Pioglitazone & 4 & 4012 & Mean Difference (IV, Random, 95\% Cl) & $-0.44[-1.36,0.47]$ \\
\hline 14 Change in $\mathrm{HbA} 1 \mathrm{c}$ & 10 & 7020 & Mean Difference (IV, Random, 95\% CI) & $0.17[0.04,0.30]$ \\
\hline 14.1 Rosiglitazone & 5 & 2940 & Mean Difference (IV, Random, 95\% CI) & $0.20[-0.02,0.42]$ \\
\hline 14.2 Pioglitazone & 5 & 4080 & Mean Difference (IV, Random, 95\% CI) & $0.15[-0.04,0.34]$ \\
\hline
\end{tabular}


Analysis 5.1. Comparison 5 Metformin plus sulphonylurea vs metformin plus thiazolidinedione, Outcome 1 All-cause mortality.

\begin{tabular}{|c|c|c|c|c|}
\hline \multirow[t]{2}{*}{ Study or subgroup } & \multirow{2}{*}{$\begin{array}{c}\text { Metformin } \\
\text { plus thiazo- } \\
\text { lidinedione } \\
\mathbf{n} / \mathbf{N} \\
\end{array}$} & \multirow[b]{2}{*}{ M-H, Random, $95 \% \mathrm{Cl}$} & \multirow[t]{2}{*}{ Weight } & \multirow{2}{*}{$\begin{array}{c}\text { Risk Ratio } \\
\text { M-H, Random, } 95 \% \mathrm{Cl} \\
\end{array}$} \\
\hline & & & & \\
\hline Derosa 2005 & $0 / 48$ & & & Not estimable \\
\hline Hamann 2008 & $2 / 294$ & & $1.63 \%$ & $0.98[0.14,6.89]$ \\
\hline Home 2009 & $57 / 1117$ & & $52.95 \%$ & $1.19[0.84,1.67]$ \\
\hline NCT00367055 & $0 / 43$ & 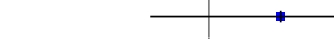 & $0.69 \%$ & $5.24[0.26,105.93]$ \\
\hline Subtotal $(95 \% \mathrm{CI})$ & 1502 & & $55.27 \%$ & $1.2[0.86,1.68]$ \\
\hline \multicolumn{5}{|c|}{$\begin{array}{l}\text { Total events: } 71 \text { (Metformin plus sulphonylurea), } 59 \text { (Metformin plus thia- } \\
\text { zolidinedione) }\end{array}$} \\
\hline \multicolumn{5}{|c|}{ Heterogeneity: Tau $^{2}=0 ; \mathrm{Chi}^{2}=0.97, \mathrm{df}=2(\mathrm{P}=0.62) ; \mathrm{I}^{2}=0 \%$} \\
\hline \multicolumn{5}{|c|}{ Test for overall effect: $Z=1.08(P=0.28)$} \\
\hline Charbonnel 2005 & $0 / 317$ & " & $0.68 \%$ & $5.06[0.24,105.05]$ \\
\hline Vaccaro 2017 & $55 / 1535$ & & $44.05 \%$ & $0.93[0.64,1.36]$ \\
\hline Subtotal $(95 \% \mathrm{Cl})$ & 1852 & & $44.73 \%$ & $1.09[0.42,2.8]$ \\
\hline \multicolumn{5}{|c|}{$\begin{array}{l}\text { Total events: } 52 \text { (Metformin plus sulphonylurea), } 55 \text { (Metformin plus thia- } \\
\text { zolidinedione) }\end{array}$} \\
\hline \multicolumn{5}{|c|}{ Heterogeneity: $\operatorname{Tau}^{2}=0.22 ; \mathrm{Chi}^{2}=1.18, \mathrm{df}=1(\mathrm{P}=0.28) ; \mathrm{I}^{2}=15.24 \%$} \\
\hline \multicolumn{5}{|c|}{ Test for overall effect: $Z=0.17(P=0.86)$} \\
\hline Total $(95 \% \mathrm{Cl})$ & 3354 & & $100 \%$ & $1.09[0.85,1.4]$ \\
\hline \multicolumn{5}{|c|}{$\begin{array}{l}\text { Total events: } 123 \text { (Metformin plus sulphonylurea), } 114 \text { (Metformin plus thi- } \\
\text { azolidinedione) }\end{array}$} \\
\hline \multicolumn{5}{|c|}{ Heterogeneity: $\operatorname{Tau}^{2}=0 ; \mathrm{Chi}^{2}=2.93, \mathrm{df}=4(\mathrm{P}=0.57) ; \mathrm{I}^{2}=0 \%$} \\
\hline \multicolumn{5}{|c|}{ Test for overall effect: $Z=0.66(P=0.51)$} \\
\hline
\end{tabular}

Analysis 5.2. Comparison 5 Metformin plus sulphonylurea vs metformin plus thiazolidinedione, Outcome 2 Serious adverse events.

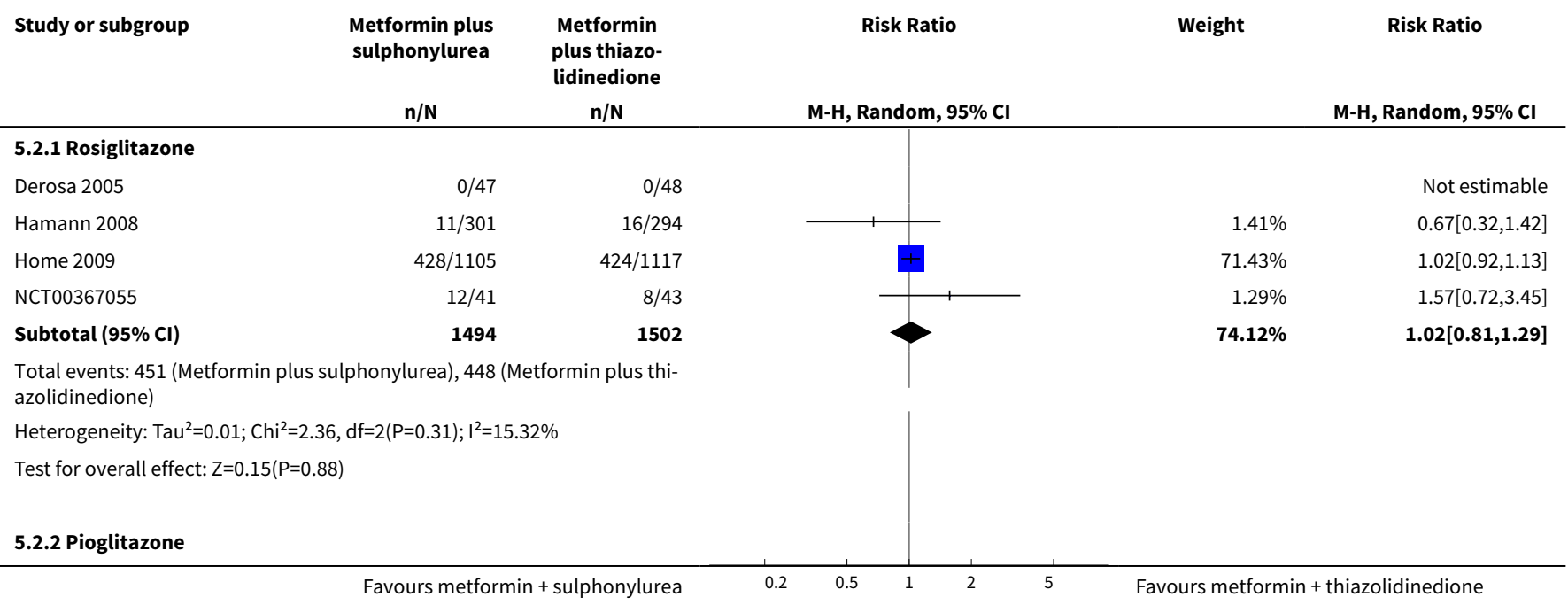




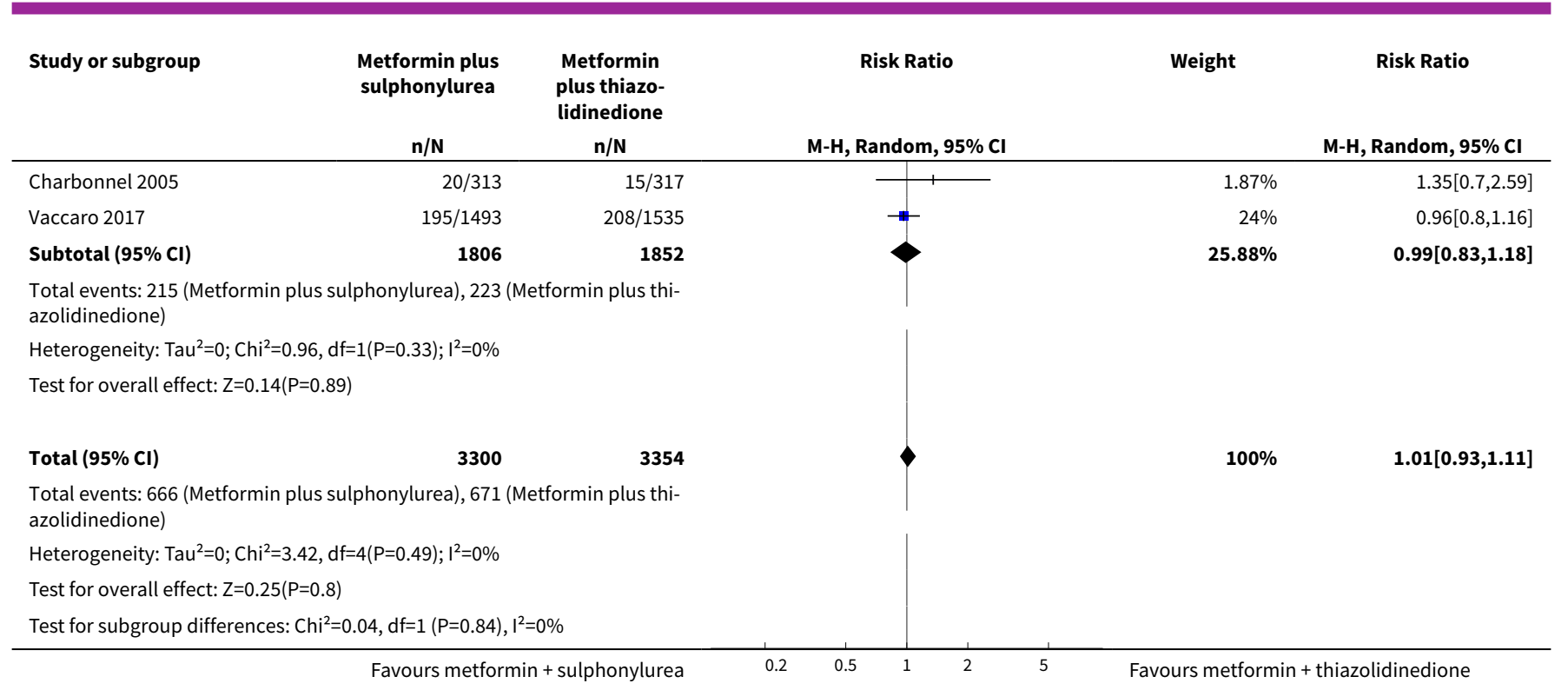

Analysis 5.3. Comparison 5 Metformin plus sulphonylurea vs metformin plus thiazolidinedione, Outcome 3 Cardiovascular mortality.

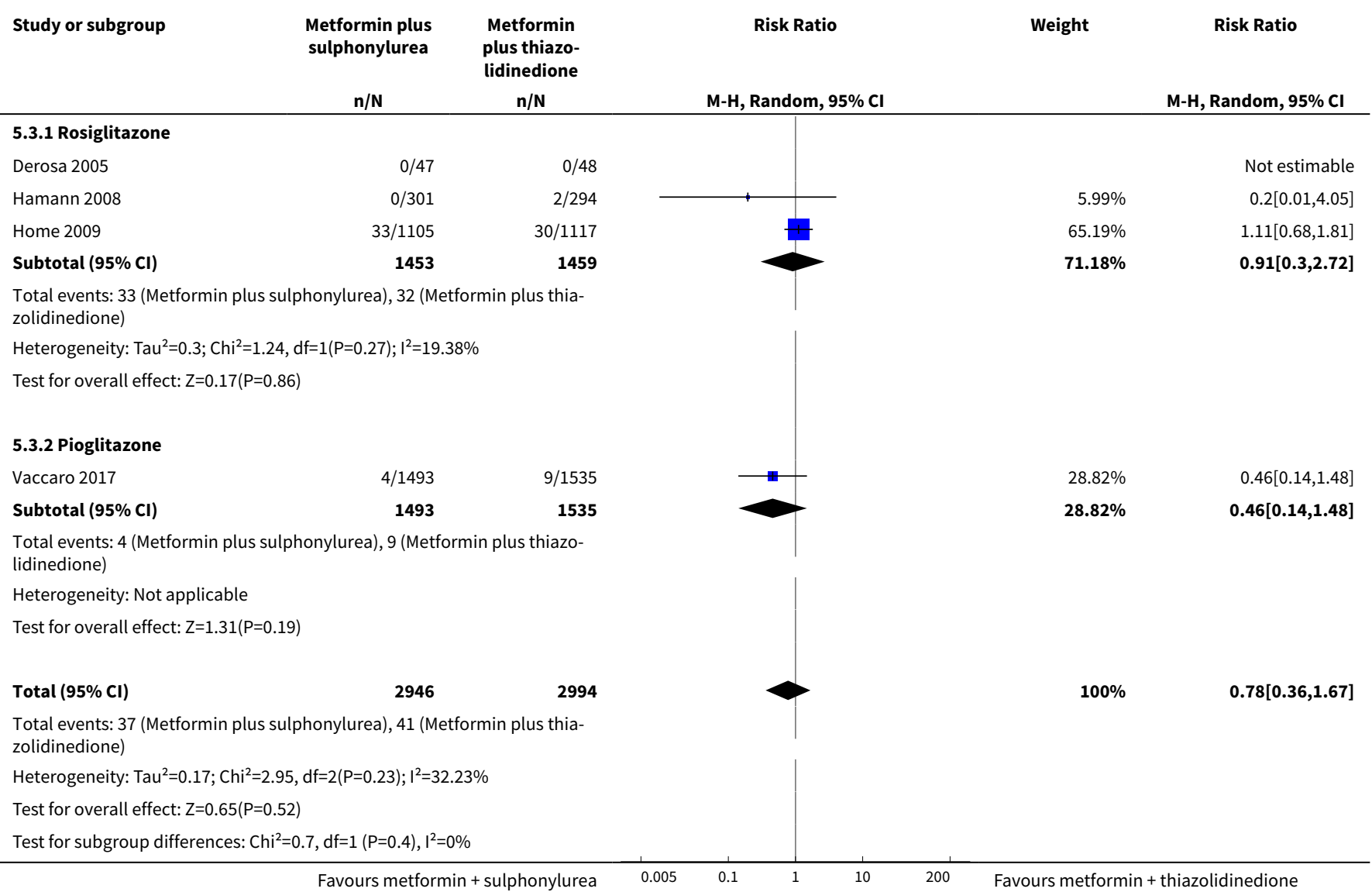


Analysis 5.4. Comparison 5 Metformin plus sulphonylurea vs metformin plus thiazolidinedione, Outcome 4 Non-fatal myocardial infarction.

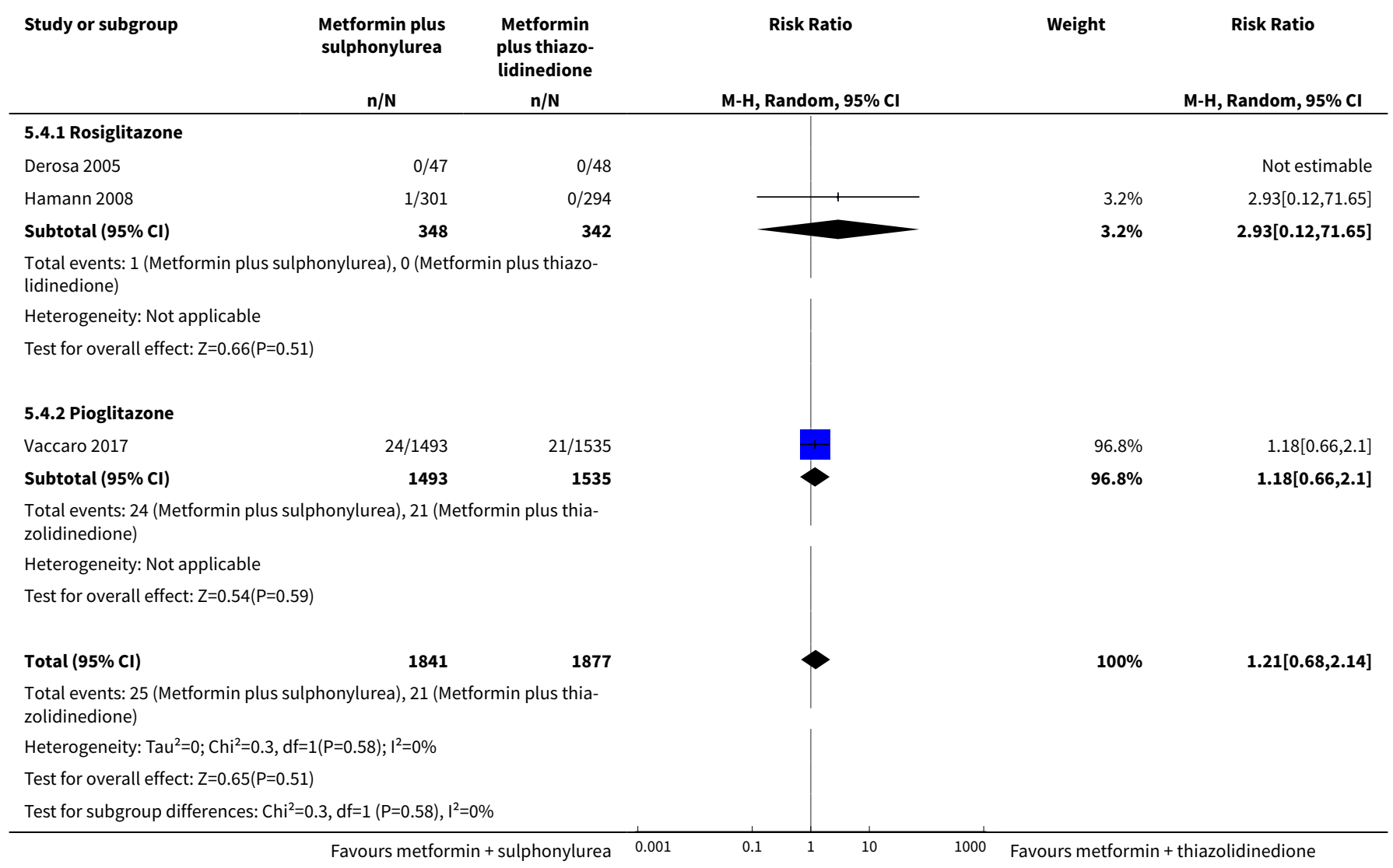

\section{Analysis 5.5. Comparison 5 Metformin plus sulphonylurea vs} metformin plus thiazolidinedione, Outcome 5 Heart failure.

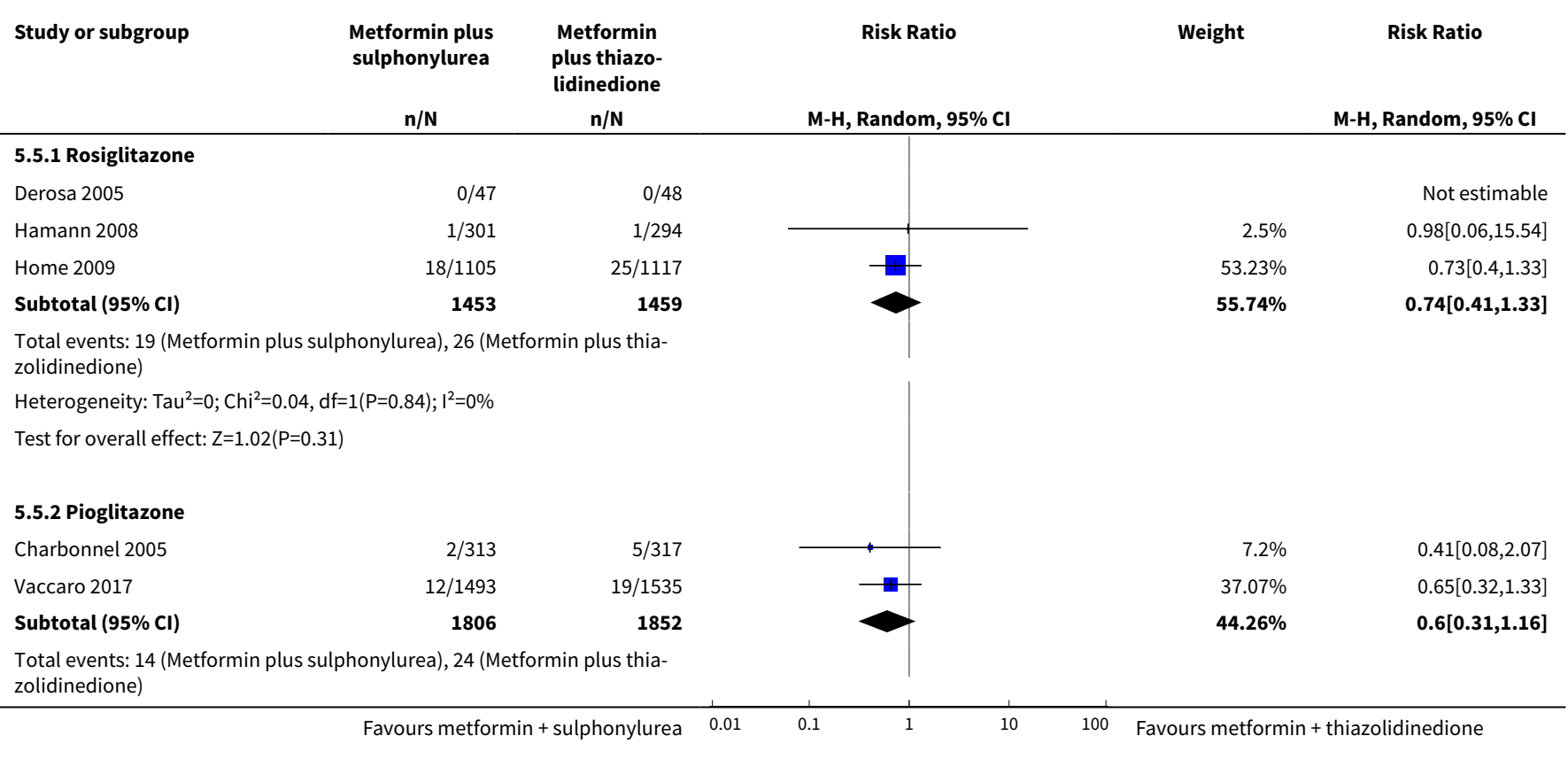




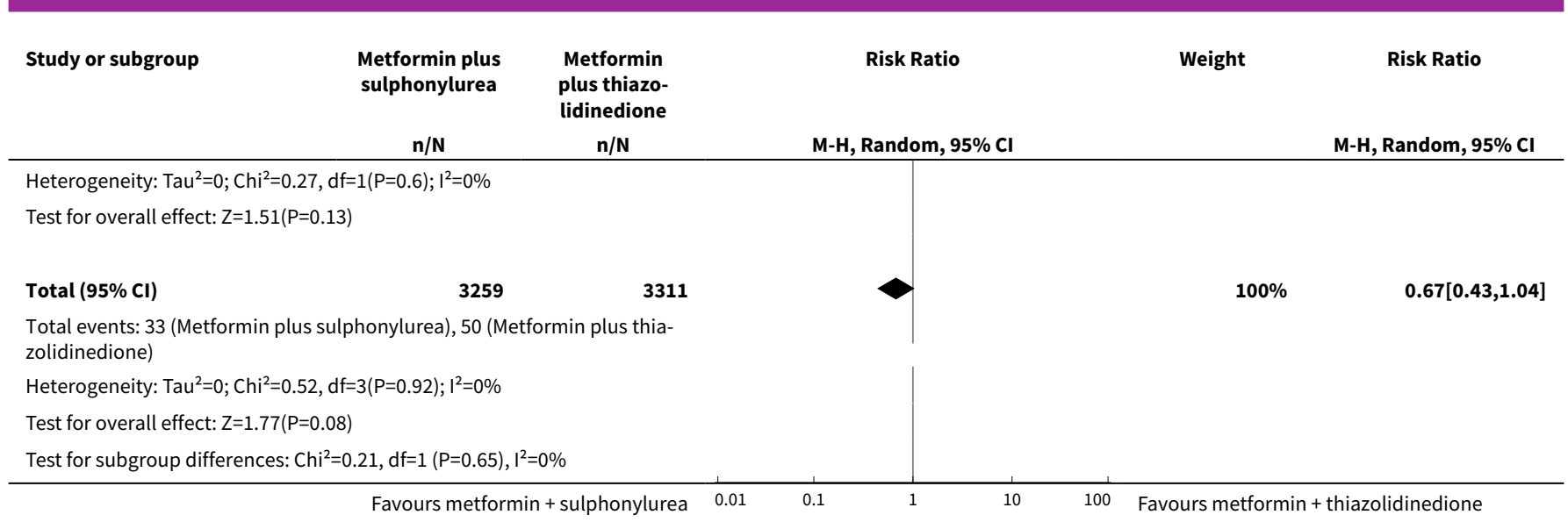

\section{Analysis 5.6. Comparison 5 Metformin plus sulphonylurea vs metformin plus thiazolidinedione, Outcome 6 Non-fatal stroke.}

\begin{tabular}{|c|c|c|c|c|c|}
\hline \multirow[t]{2}{*}{ Study or subgroup } & $\begin{array}{l}\text { Metformin plus } \\
\text { sulphonylurea }\end{array}$ & $\begin{array}{l}\text { Metformin } \\
\text { plus thiazo- } \\
\text { lidinedione }\end{array}$ & \multirow[b]{2}{*}{ M-H, Random, 95\% Cl } & \multirow[t]{2}{*}{ Weight } & \multirow[b]{2}{*}{ M-H, Random, $95 \% \mathrm{Cl}$} \\
\hline & $\mathrm{n} / \mathrm{N}$ & $\mathrm{n} / \mathrm{N}$ & & & \\
\hline Derosa 2005 & $0 / 47$ & $0 / 48$ & & & Not estimable \\
\hline Subtotal $(95 \% \mathrm{CI})$ & 47 & 48 & & & Not estimable \\
\hline \multicolumn{6}{|c|}{$\begin{array}{l}\text { Total events: } 0 \text { (Metformin plus sulphonylurea), } 0 \text { (Metformin plus thiazo- } \\
\text { lidinedione) }\end{array}$} \\
\hline \multicolumn{6}{|c|}{ Heterogeneity: Not applicable } \\
\hline \multicolumn{6}{|c|}{ Test for overall effect: Not applicable } \\
\hline \multicolumn{6}{|l|}{ 5.6.2 Pioglitazone } \\
\hline Vaccaro 2017 & $20 / 1493$ & $16 / 1535$ & - & $100 \%$ & $1.29[0.67,2.47]$ \\
\hline Subtotal $(95 \% \mathrm{CI})$ & 1493 & 1535 & & $100 \%$ & $1.29[0.67,2.47]$ \\
\hline \multicolumn{6}{|c|}{ Heterogeneity: Not applicable } \\
\hline \multicolumn{6}{|c|}{ Test for overall effect: $\mathrm{Z}=0.75(\mathrm{P}=0.45)$} \\
\hline Total $(95 \% \mathrm{Cl})$ & 1540 & 1583 & & $100 \%$ & $1.29[0.67,2.47]$ \\
\hline \multicolumn{6}{|c|}{$\begin{array}{l}\text { Total events: } 20 \text { (Metformin plus sulphonylurea), } 16 \text { (Metformin plus thia- } \\
\text { zolidinedione) }\end{array}$} \\
\hline \multicolumn{6}{|c|}{ Heterogeneity: Not applicable } \\
\hline \multicolumn{6}{|c|}{ Test for overall effect: $\mathrm{Z}=0.75(\mathrm{P}=0.45)$} \\
\hline Test for subgroup dif & plicable & & & & \\
\hline
\end{tabular}


Analysis 5.7. Comparison 5 Metformin plus sulphonylurea vs metformin plus thiazolidinedione, Outcome 7 Amputation of lower extremity.

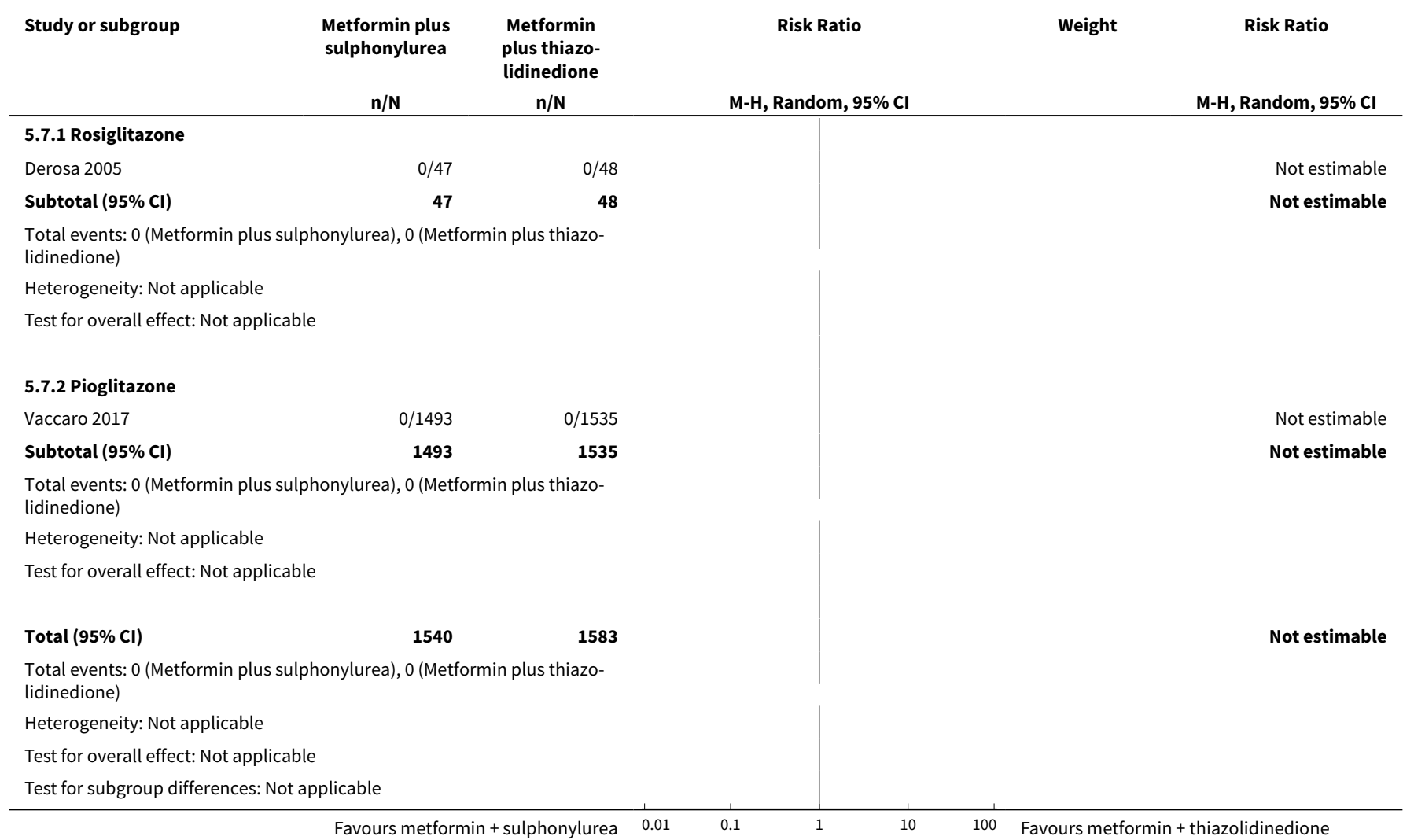

Analysis 5.8. Comparison 5 Metformin plus sulphonylurea vs metformin plus thiazolidinedione, Outcome 8 Blindness or severe vision loss.

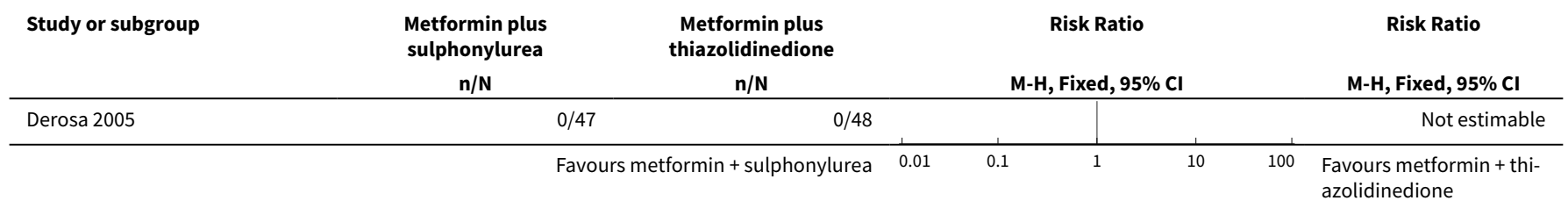

Analysis 5.9. Comparison 5 Metformin plus sulphonylurea vs metformin plus thiazolidinedione, Outcome 9 End-stage renal disease.

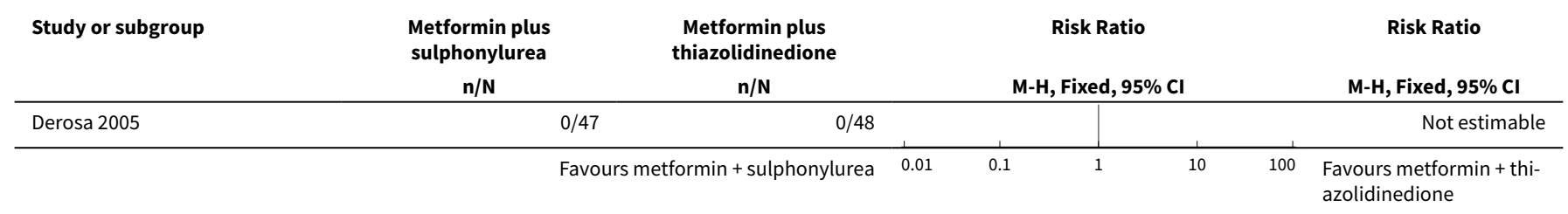


Analysis 5.10. Comparison 5 Metformin plus sulphonylurea vs metformin plus thiazolidinedione, Outcome 10 Non-serious adverse events.

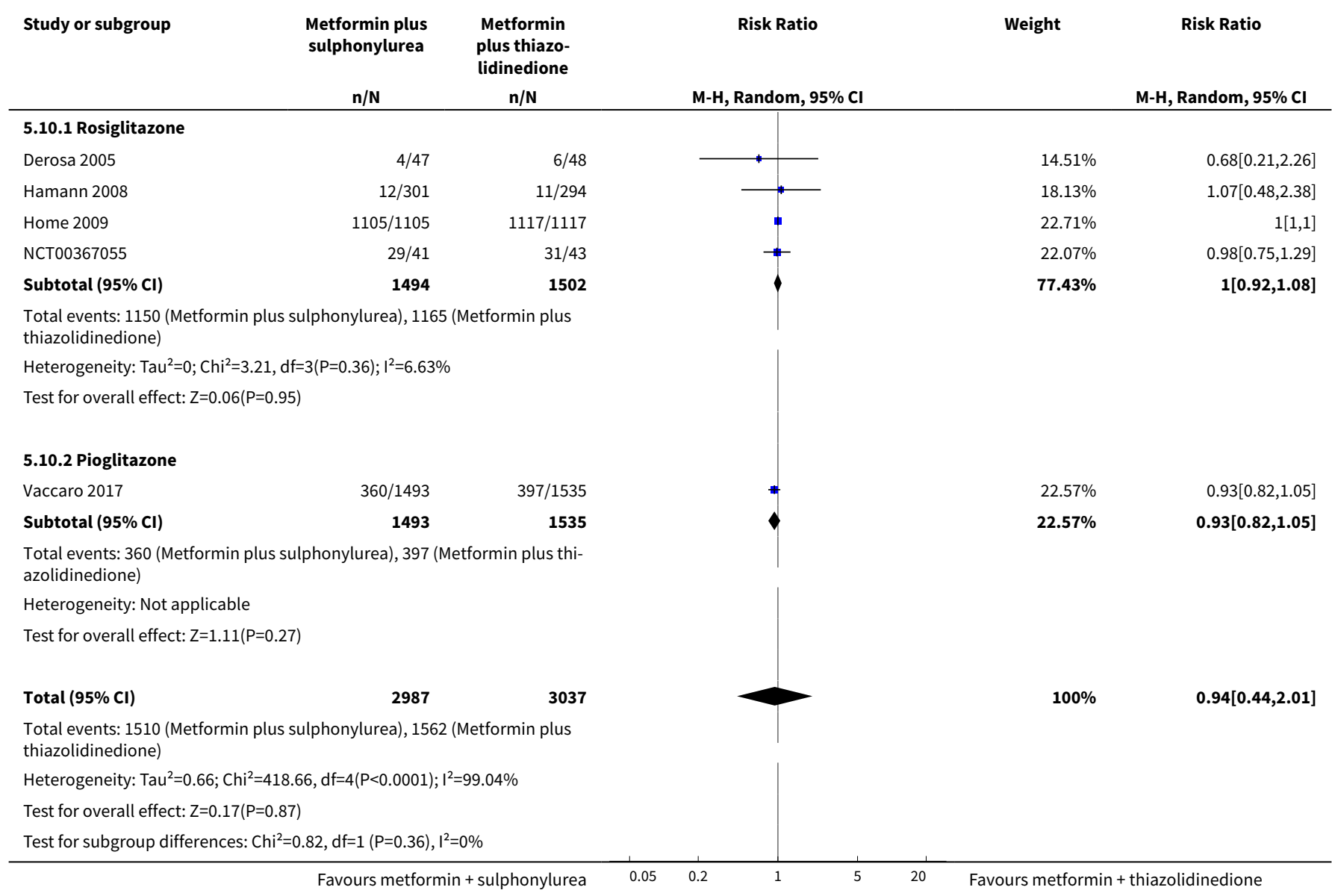

\section{Analysis 5.11. Comparison 5 Metformin plus sulphonylurea vs metformin plus thiazolidinedione, Outcome 11 Mild/moderate hypoglycaemia.}

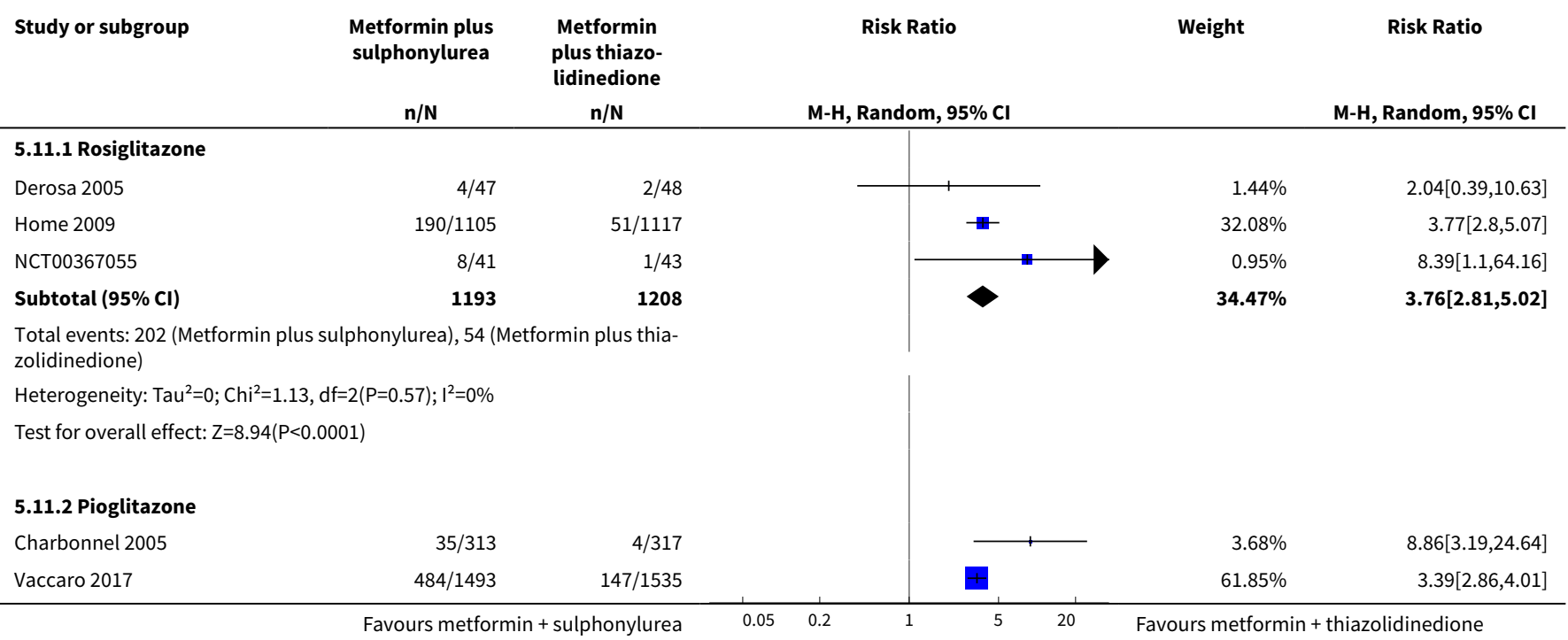




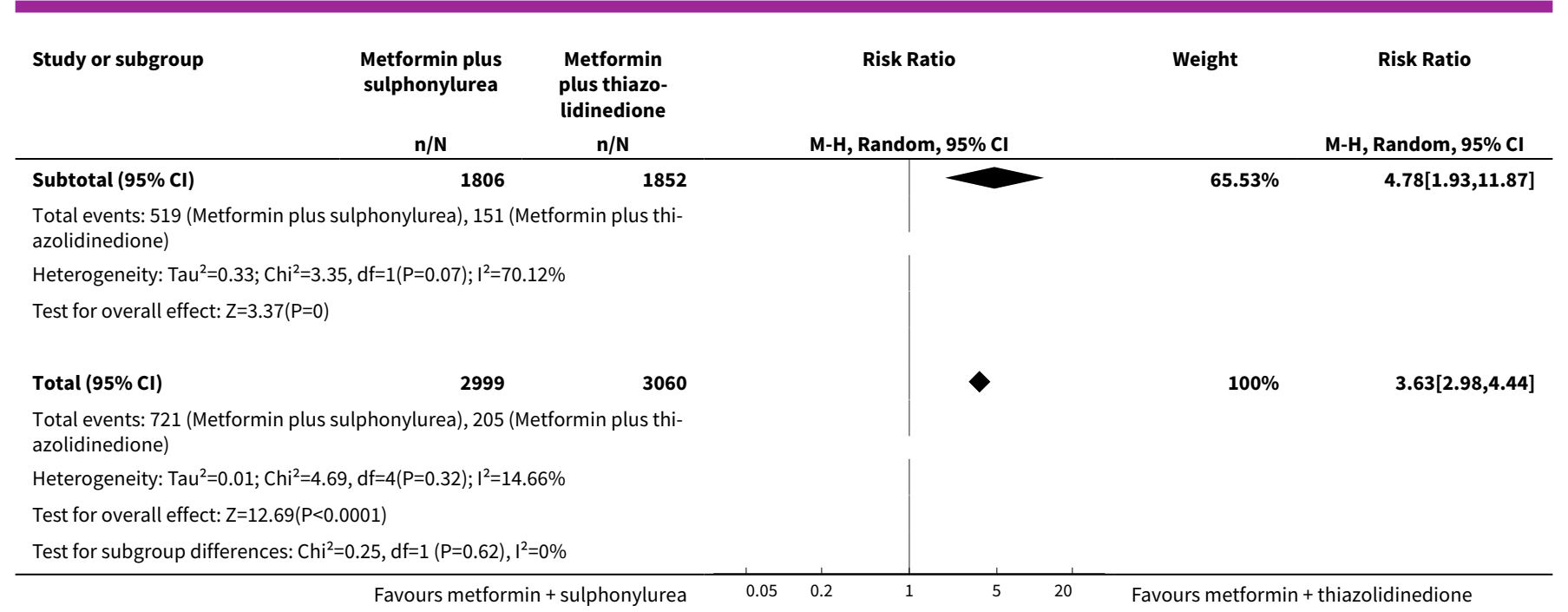

Analysis 5.12. Comparison 5 Metformin plus sulphonylurea vs metformin plus thiazolidinedione, Outcome 12 Serious hypoglycaemia.

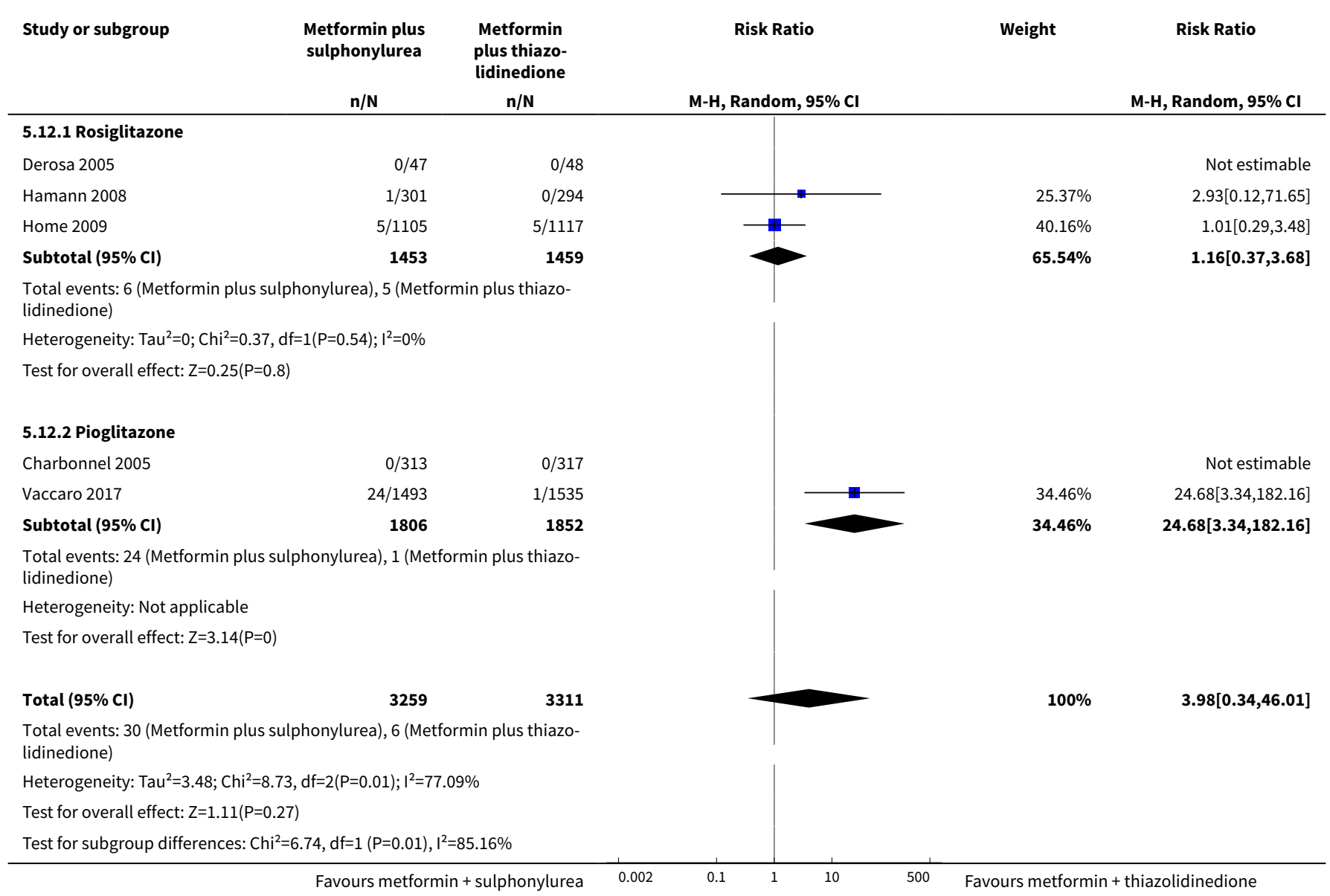


Analysis 5.13. Comparison 5 Metformin plus sulphonylurea vs metformin plus thiazolidinedione, Outcome 13 Weight (change).

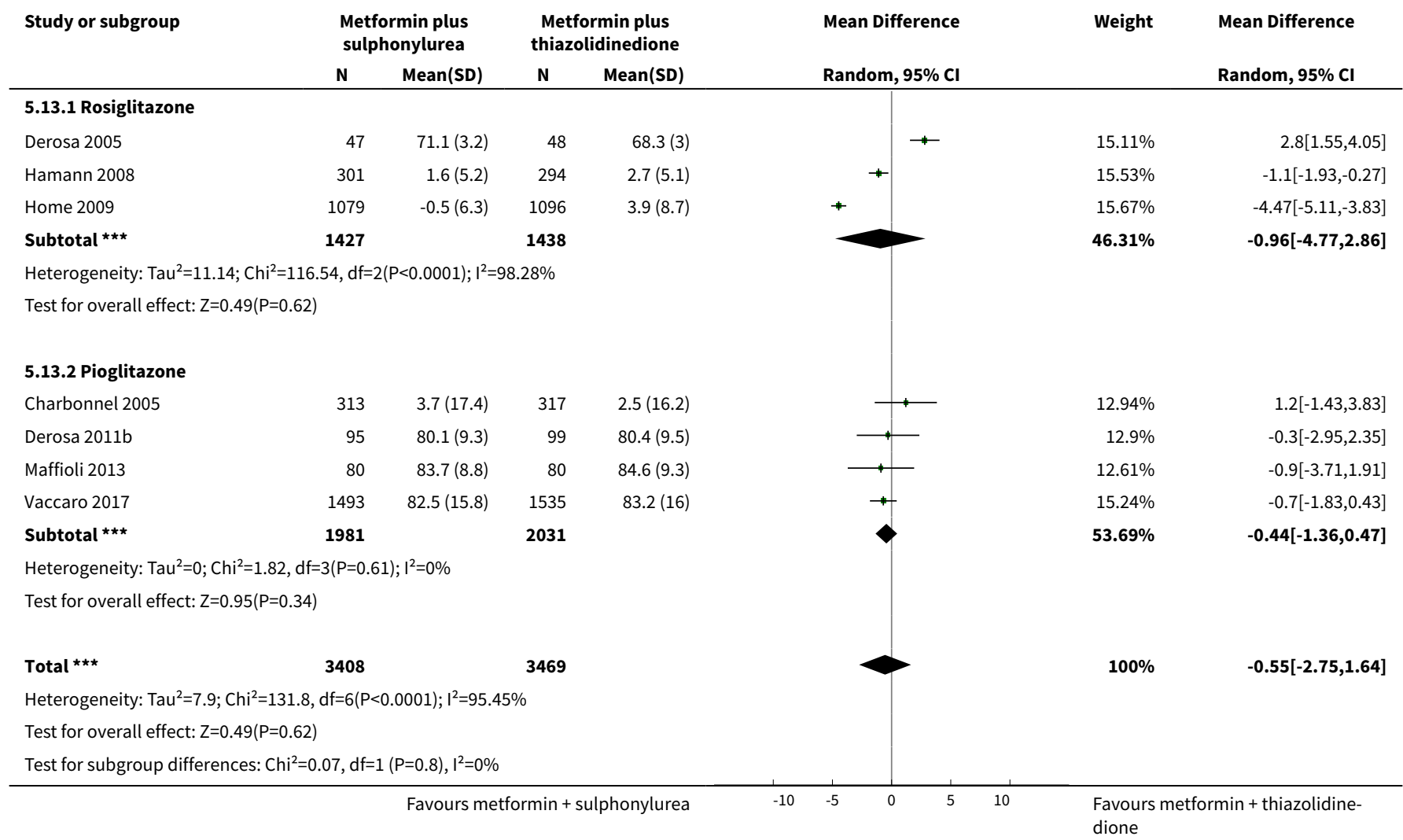

Analysis 5.14. Comparison 5 Metformin plus sulphonylurea vs metformin plus thiazolidinedione, Outcome 14 Change in HbA1c.

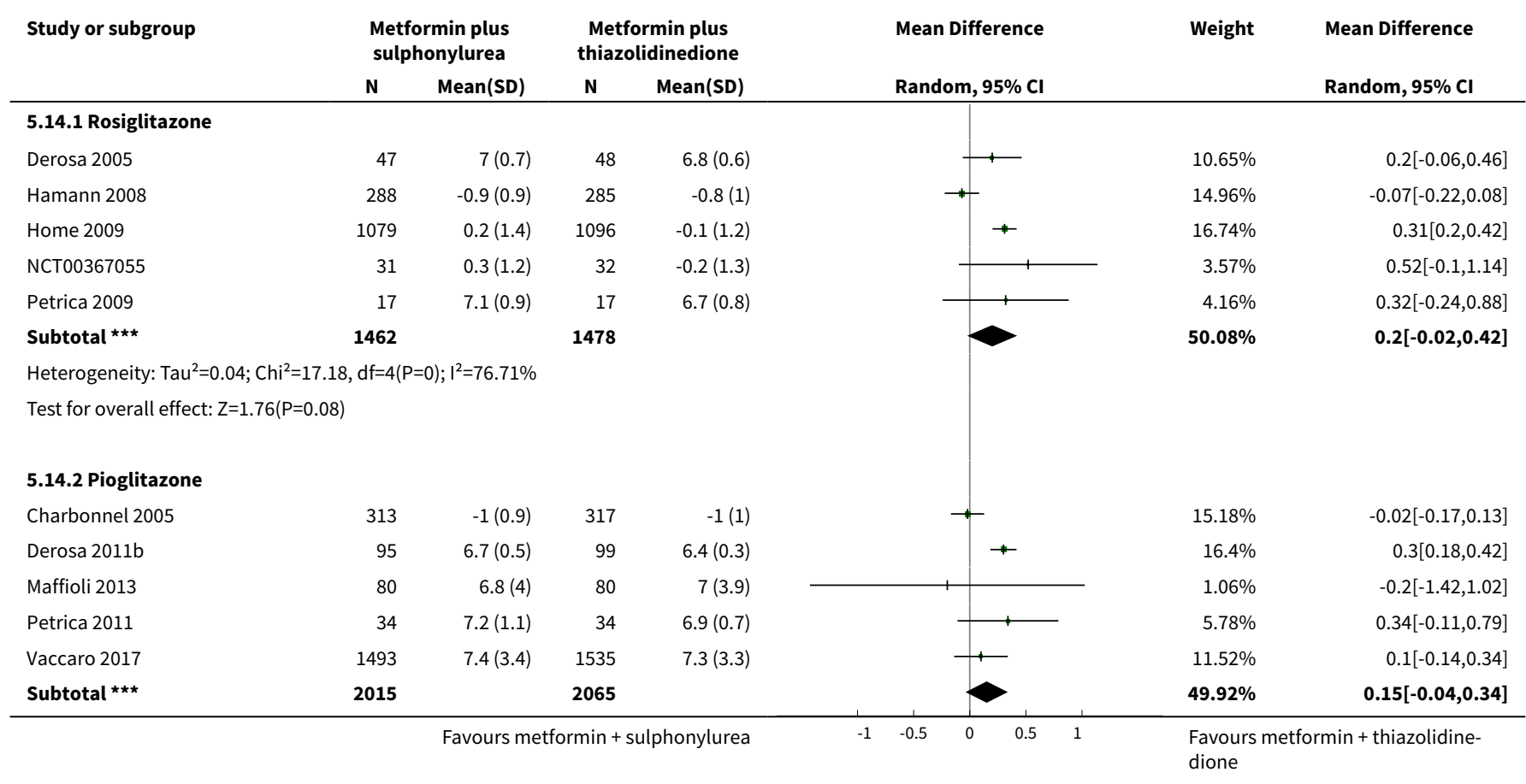




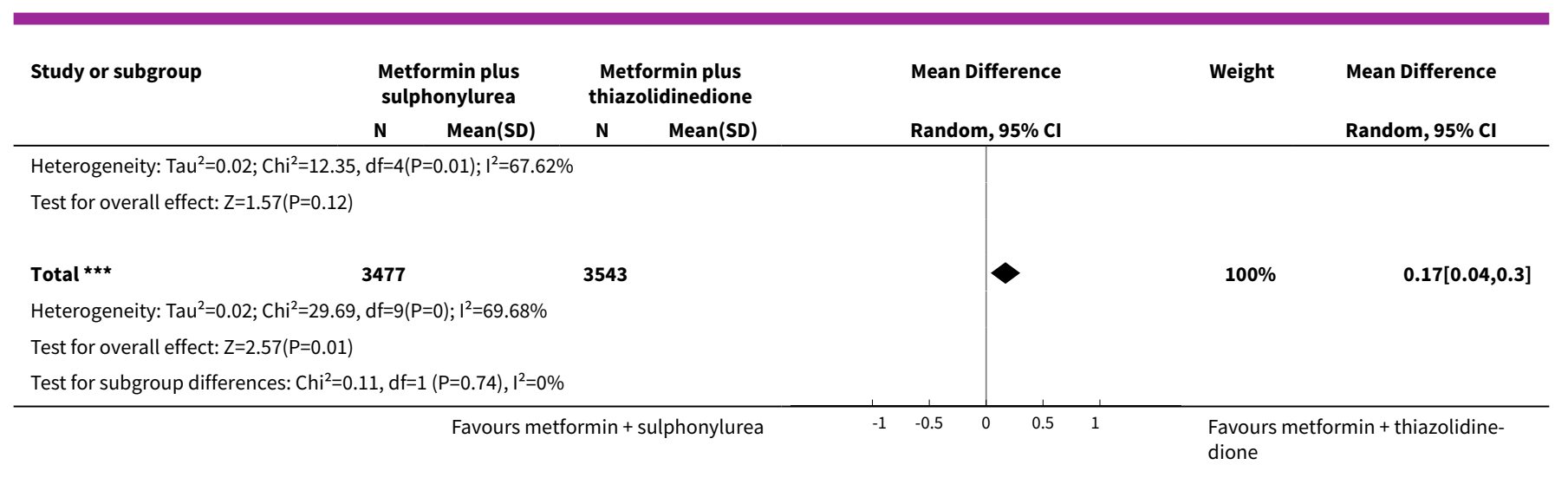

\section{Comparison 6. Metformin plus sulphonylurea vs metformin plus thiazolidinedione (subgroups duration of intervention)}

\begin{tabular}{|c|c|c|c|c|}
\hline Outcome or subgroup title & No. of studies & $\begin{array}{l}\text { No. of partici- } \\
\text { pants }\end{array}$ & Statistical method & Effect size \\
\hline 1 All-cause mortality & 6 & 6654 & $\begin{array}{l}\text { Risk Ratio (M-H, Random, 95\% } \\
\text { Cl) }\end{array}$ & $1.09[0.85,1.40]$ \\
\hline $\begin{array}{l}1.1 \text { Trials with long duration ( } \geq 2 \\
\text { years) }\end{array}$ & 4 & 5964 & $\begin{array}{l}\text { Risk Ratio (M-H, Random, 95\% } \\
\text { Cl) }\end{array}$ & $1.09[0.85,1.40]$ \\
\hline $\begin{array}{l}1.2 \text { Trials with short duration }(<2 \\
\text { years) }\end{array}$ & 2 & 690 & $\begin{array}{l}\text { Risk Ratio (M-H, Random, 95\% } \\
\text { Cl) }\end{array}$ & $0.98[0.14,6.89]$ \\
\hline 2 Serious adverse events & 6 & 6654 & $\begin{array}{l}\text { Risk Ratio (M-H, Random, 95\% } \\
\text { Cl) }\end{array}$ & $1.01[0.93,1.11]$ \\
\hline $\begin{array}{l}2.1 \text { Trials with long duration ( } \geq 2 \\
\text { years) }\end{array}$ & 4 & 5964 & $\begin{array}{l}\text { Risk Ratio (M-H, Random, 95\% } \\
\text { Cl) }\end{array}$ & $1.02[0.93,1.11]$ \\
\hline $\begin{array}{l}2.2 \text { Trials with short duration }(<2 \\
\text { years) }\end{array}$ & 2 & 690 & $\begin{array}{l}\text { Risk Ratio (M-H, Random, 95\% } \\
\mathrm{Cl} \text { ) }\end{array}$ & $0.67[0.32,1.42]$ \\
\hline 3 Cardiovascular mortality & 4 & 5940 & $\begin{array}{l}\text { Risk Ratio (M-H, Random, 95\% } \\
\mathrm{Cl} \text { ) }\end{array}$ & $0.78[0.36,1.67]$ \\
\hline $\begin{array}{l}3.1 \text { Trials with long duration ( } \geq 2 \\
\text { years) }\end{array}$ & 2 & 5250 & $\begin{array}{l}\text { Risk Ratio (M-H, Random, 95\% } \\
\text { Cl) }\end{array}$ & $0.84[0.38,1.89]$ \\
\hline $\begin{array}{l}3.2 \text { Trials with short duration }(<2 \\
\text { years) }\end{array}$ & 2 & 690 & $\begin{array}{l}\text { Risk Ratio (M-H, Random, 95\% } \\
\text { Cl) }\end{array}$ & $0.20[0.01,4.05]$ \\
\hline 4 Non-fatal myocardial infarction & 3 & 3718 & $\begin{array}{l}\text { Risk Ratio (M-H, Random, 95\% } \\
\text { Cl) }\end{array}$ & $1.21[0.68,2.14]$ \\
\hline $\begin{array}{l}4.1 \text { Trials with long duration ( } \geq 2 \\
\text { years) }\end{array}$ & 1 & 3028 & $\begin{array}{l}\text { Risk Ratio (M-H, Random, 95\% } \\
\text { Cl) }\end{array}$ & $1.18[0.66,2.10]$ \\
\hline $\begin{array}{l}4.2 \text { Trials with short duration }(<2 \\
\text { years) }\end{array}$ & 2 & 690 & $\begin{array}{l}\text { Risk Ratio (M-H, Random, 95\% } \\
\text { Cl) }\end{array}$ & $2.93[0.12,71.65]$ \\
\hline
\end{tabular}




\begin{tabular}{|c|c|c|c|c|}
\hline Outcome or subgroup title & No. of studies & $\begin{array}{l}\text { No. of partici- } \\
\text { pants }\end{array}$ & Statistical method & Effect size \\
\hline 5 Heart failure & 5 & 6570 & $\begin{array}{l}\text { Risk Ratio (M-H, Random, 95\% } \\
\text { Cl) }\end{array}$ & $0.67[0.43,1.04]$ \\
\hline $\begin{array}{l}5.1 \text { Trials with long duration ( } \geq 2 \\
\text { years) }\end{array}$ & 3 & 5880 & $\begin{array}{l}\text { Risk Ratio (M-H, Random, 95\% } \\
\text { Cl) }\end{array}$ & $0.67[0.43,1.04]$ \\
\hline $\begin{array}{l}5.2 \text { Trials with short duration }(<2 \\
\text { years) }\end{array}$ & 2 & 690 & $\begin{array}{l}\text { Risk Ratio (M-H, Random, 95\% } \\
\text { Cl) }\end{array}$ & $0.98[0.06,15.54]$ \\
\hline 6 Non-fatal stroke & 2 & & $\begin{array}{l}\text { Risk Ratio (M-H, Random, 95\% } \\
\text { Cl) }\end{array}$ & Totals not selected \\
\hline $\begin{array}{l}6.1 \text { Trials with long duration ( } \geq 2 \\
\text { years) }\end{array}$ & 1 & & $\begin{array}{l}\text { Risk Ratio (M-H, Random, 95\% } \\
\mathrm{Cl} \text { ) }\end{array}$ & $0.0[0.0,0.0]$ \\
\hline $\begin{array}{l}6.2 \text { Trials with short duration }(<2 \\
\text { years) }\end{array}$ & 1 & & $\begin{array}{l}\text { Risk Ratio (M-H, Random, 95\% } \\
\mathrm{Cl} \text { ) }\end{array}$ & $0.0[0.0,0.0]$ \\
\hline 7 Non-serious adverse events & 5 & 6024 & $\begin{array}{l}\text { Risk Ratio (M-H, Random, 95\% } \\
\text { Cl) }\end{array}$ & $0.94[0.44,2.01]$ \\
\hline $\begin{array}{l}7.1 \text { Trials with long duration ( } \geq 2 \\
\text { years) }\end{array}$ & 3 & 5334 & $\begin{array}{l}\text { Risk Ratio (M-H, Random, 95\% } \\
\text { Cl) }\end{array}$ & $0.97[0.39,2.42]$ \\
\hline $\begin{array}{l}7.2 \text { Trials with short duration }(<2 \\
\text { years) }\end{array}$ & 2 & 690 & $\begin{array}{l}\text { Risk Ratio (M-H, Random, 95\% } \\
\text { Cl) }\end{array}$ & $0.93[0.48,1.81]$ \\
\hline 8 Mild/moderate hypoglycaemia & 5 & 6059 & $\begin{array}{l}\text { Risk Ratio (M-H, Random, 95\% } \\
\text { Cl) }\end{array}$ & $3.63[2.98,4.44]$ \\
\hline $\begin{array}{l}8.1 \text { Trials with long duration ( } \geq 2 \\
\text { years) }\end{array}$ & 4 & 5964 & $\begin{array}{l}\text { Risk Ratio (M-H, Random, 95\% } \\
\text { Cl) }\end{array}$ & $3.73[2.95,4.72]$ \\
\hline $\begin{array}{l}8.2 \text { Trials with short duration }(<2 \\
\text { years) }\end{array}$ & 1 & 95 & $\begin{array}{l}\text { Risk Ratio (M-H, Random, 95\% } \\
\text { Cl) }\end{array}$ & $2.04[0.39,10.63]$ \\
\hline 9 Serious hypoglycaemia & 5 & 6570 & $\begin{array}{l}\text { Risk Ratio (M-H, Random, 95\% } \\
\mathrm{Cl} \text { ) }\end{array}$ & $3.98[0.34,46.01]$ \\
\hline $\begin{array}{l}9.1 \text { Trials with long duration ( } \geq 2 \\
\text { years) }\end{array}$ & 3 & 5880 & $\begin{array}{l}\text { Risk Ratio (M-H, Random, 95\% } \\
\mathrm{Cl} \text { ) }\end{array}$ & $4.61[0.14,149.68]$ \\
\hline $\begin{array}{l}9.2 \text { Trials with short duration }(<2 \\
\text { years) }\end{array}$ & 2 & 690 & $\begin{array}{l}\text { Risk Ratio (M-H, Random, 95\% } \\
\mathrm{Cl} \text { ) }\end{array}$ & $2.93[0.12,71.65]$ \\
\hline 10 Weight change & 7 & 6877 & $\begin{array}{l}\text { Mean Difference (IV, Random, } \\
95 \% \mathrm{CI} \text { ) }\end{array}$ & $-0.55[-2.75,1.64]$ \\
\hline $\begin{array}{l}10.1 \text { Trials with long duration ( } \geq 2 \\
\text { years) }\end{array}$ & 3 & 5833 & $\begin{array}{l}\text { Mean Difference (IV, Random, } \\
95 \% \mathrm{CI} \text { ) }\end{array}$ & $-1.49[-4.79,1.81]$ \\
\hline $\begin{array}{l}10.2 \text { Trials with short duration }(<2 \\
\text { years) }\end{array}$ & 4 & 1044 & $\begin{array}{l}\text { Mean Difference (IV, Random, } \\
95 \% \mathrm{CI} \text { ) }\end{array}$ & $0.20[-2.15,2.56]$ \\
\hline
\end{tabular}




\begin{tabular}{lllll}
\hline Outcome or subgroup title & No. of studies & $\begin{array}{l}\text { No. of partici- } \\
\text { pants }\end{array}$ & Statistical method & Effect size \\
\hline 11 Change in HbA1c & 10 & 7020 & $\begin{array}{l}\text { Mean Difference (IV, Random, } \\
95 \% \mathrm{Cl})\end{array}$ & $0.17[0.04,0.30]$ \\
\hline $\begin{array}{l}11.1 \text { Trials with long duration }(\geq 2 \\
\text { years) }\end{array}$ & 4 & 5896 & $\begin{array}{l}\text { Mean Difference (IV, Random, } \\
95 \% \mathrm{Cl})\end{array}$ & $0.17[-0.04,0.39]$ \\
\hline $\begin{array}{l}11.2 \text { Trials with short duration }(<2 \\
\text { years) }\end{array}$ & 6 & 1124 & $\begin{array}{l}\text { Mean Difference (IV, Random, } \\
95 \% \text { Cl) }\end{array}$ & $0.17[-0.02,0.37]$ \\
\hline
\end{tabular}

Analysis 6.1. Comparison 6 Metformin plus sulphonylurea vs metformin plus thiazolidinedione (subgroups duration of intervention), Outcome 1 All-cause mortality.

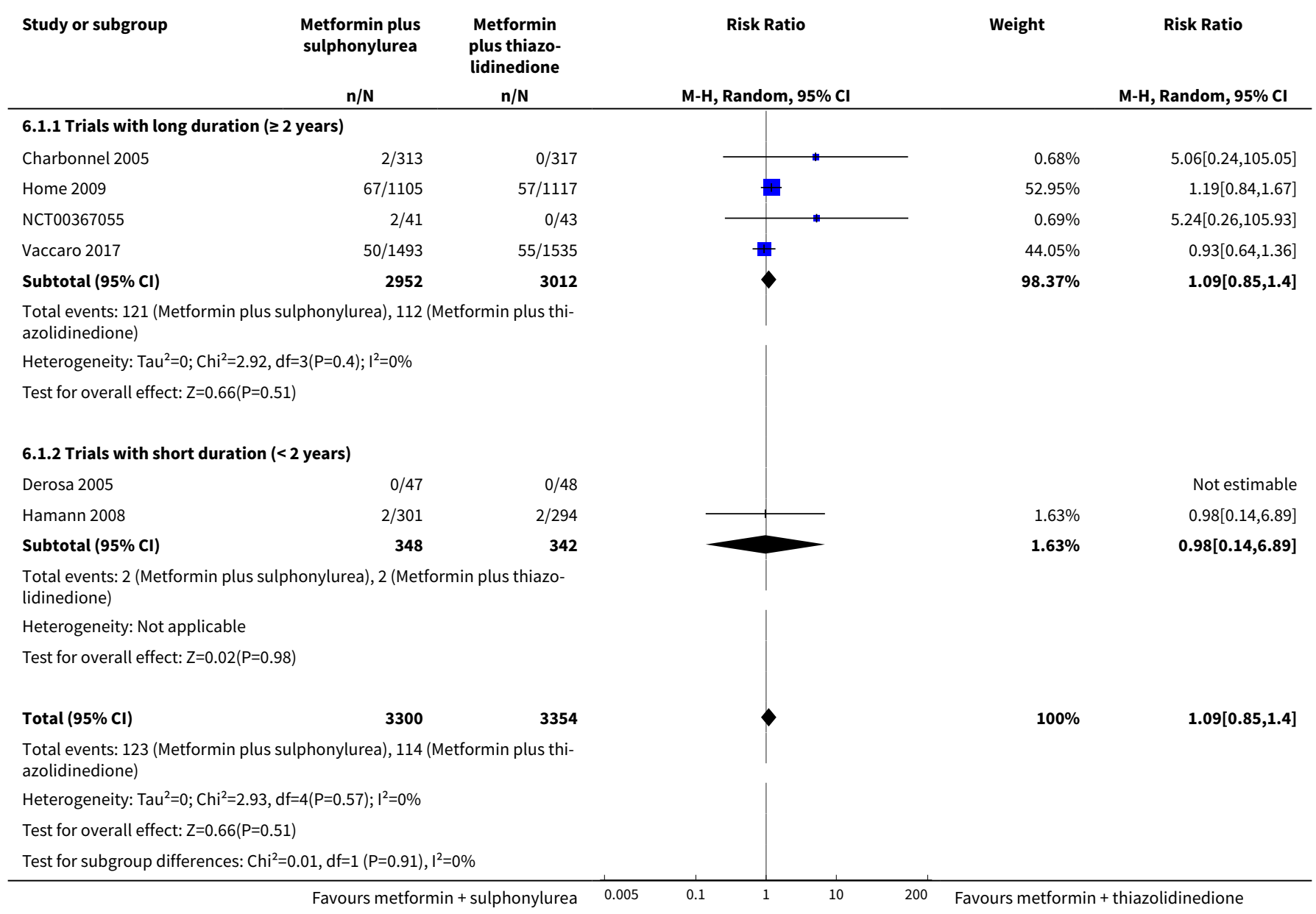


Analysis 6.2. Comparison 6 Metformin plus sulphonylurea vs metformin plus thiazolidinedione (subgroups duration of intervention), Outcome 2 Serious adverse events.

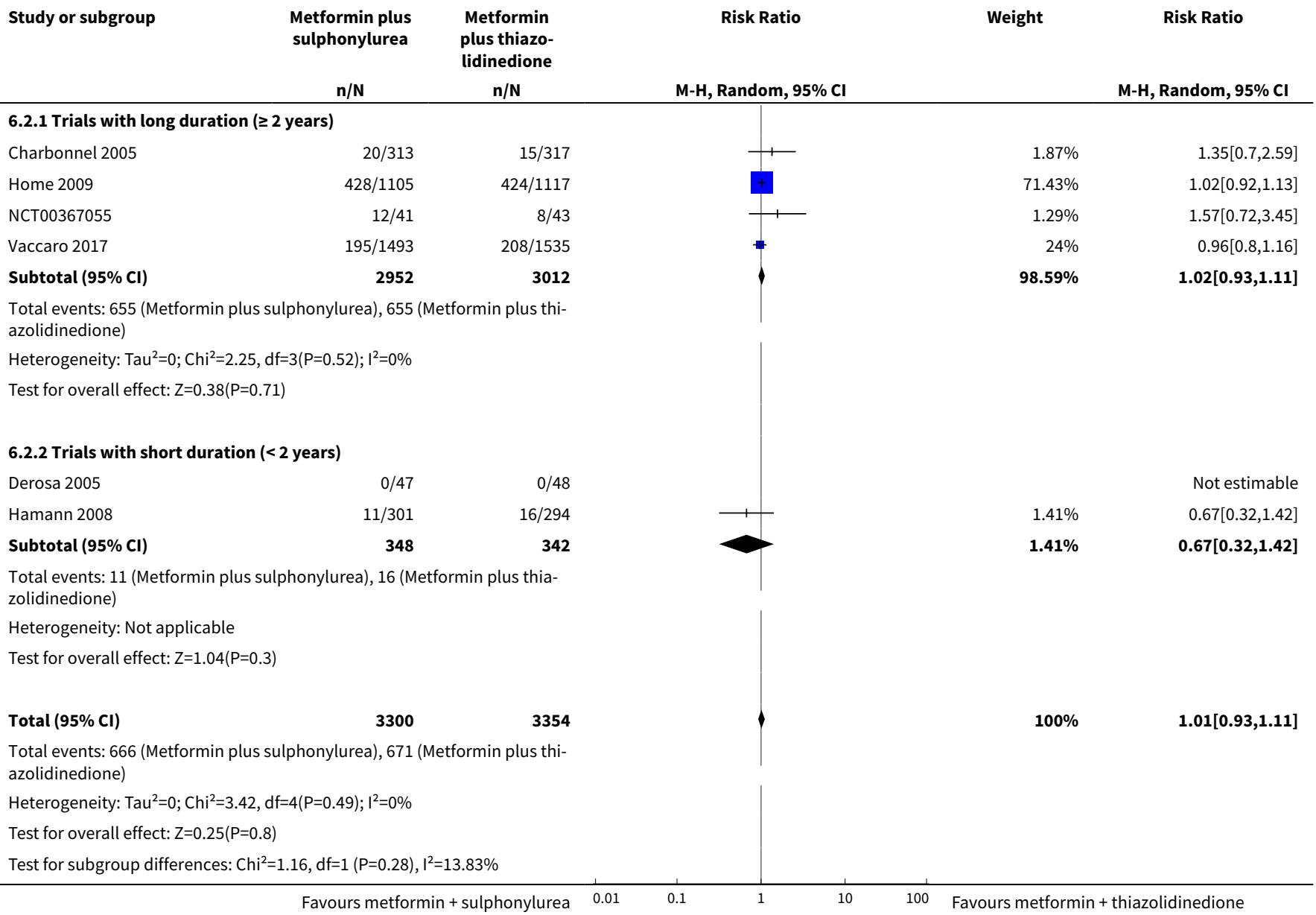

Analysis 6.3. Comparison 6 Metformin plus sulphonylurea vs metformin plus thiazolidinedione (subgroups duration of intervention), Outcome 3 Cardiovascular mortality.

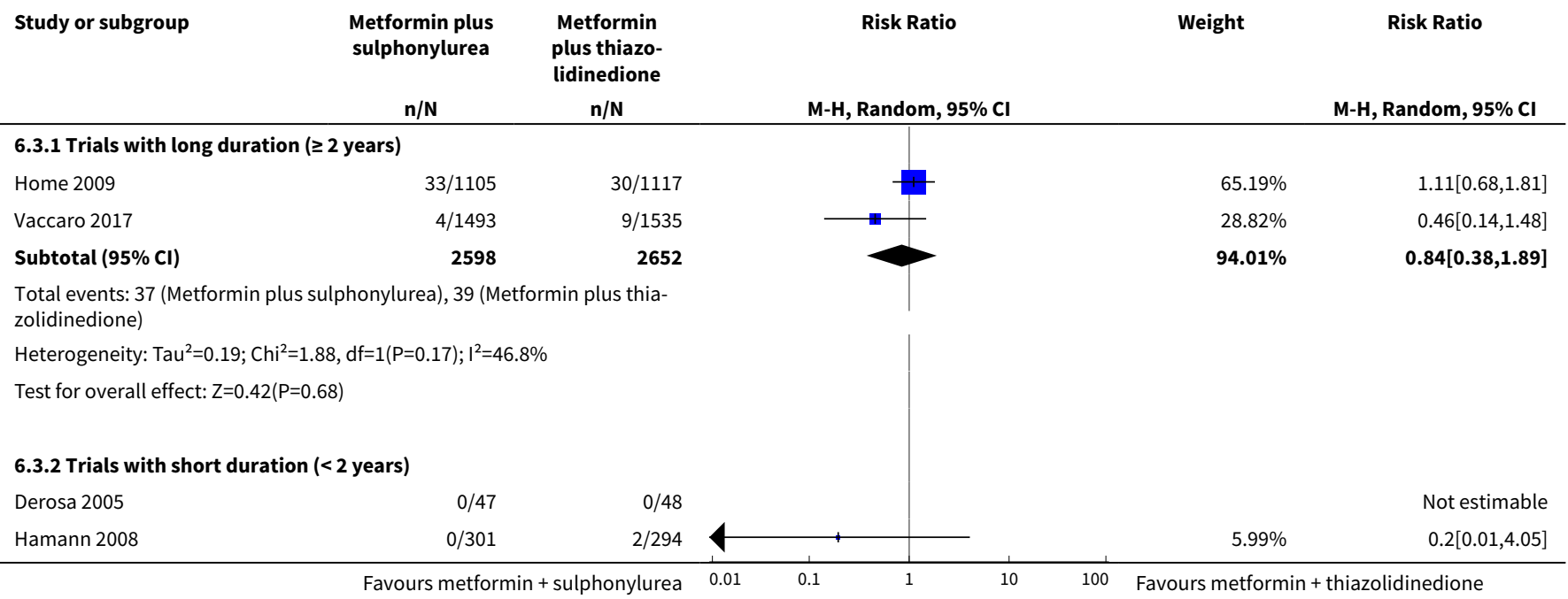




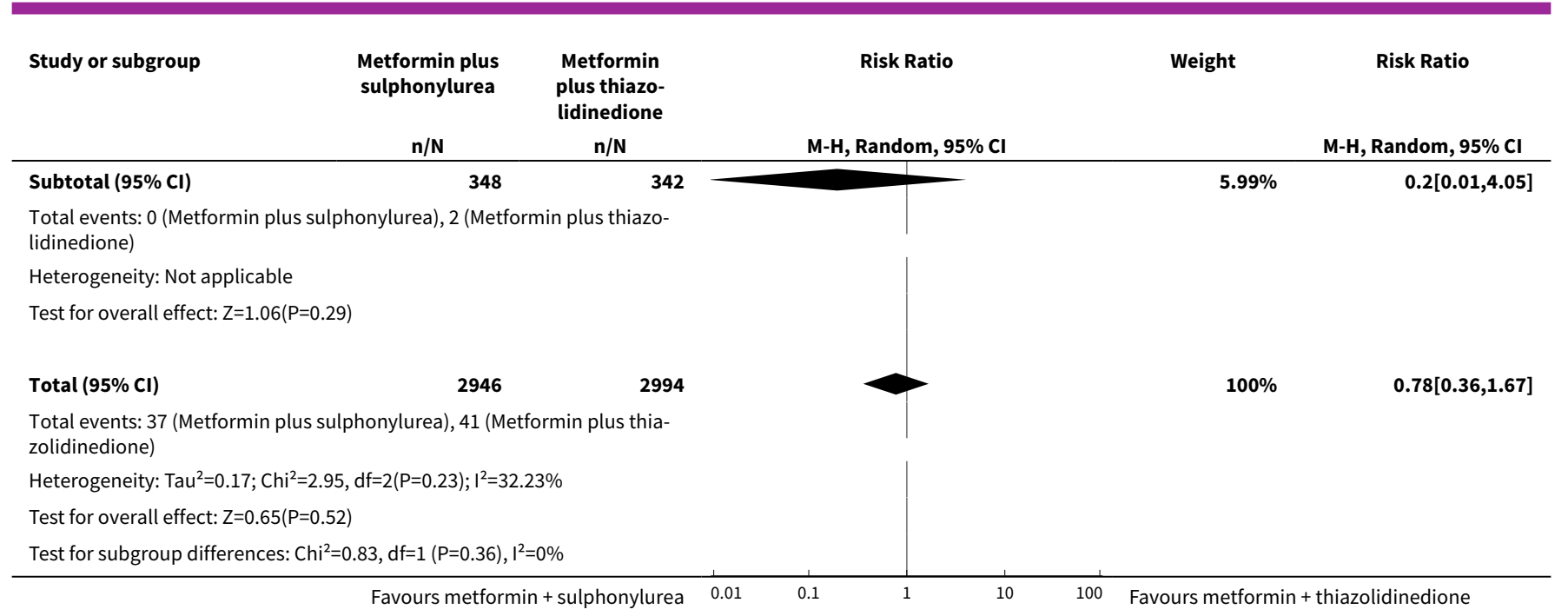

\section{Analysis 6.4. Comparison 6 Metformin plus sulphonylurea vs metformin plus thiazolidinedione (subgroups duration of intervention), Outcome 4 Non-fatal myocardial infarction.}

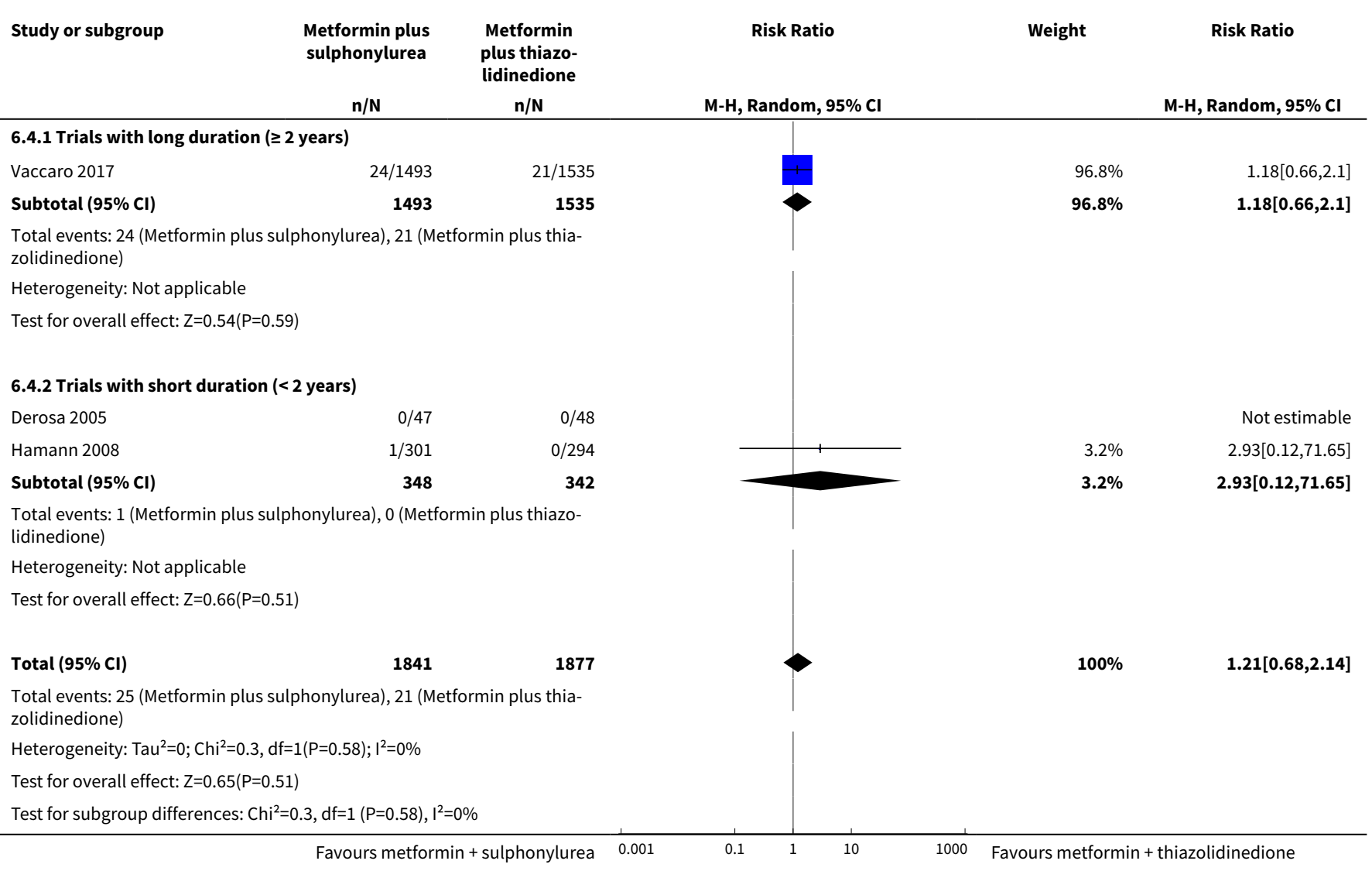


Analysis 6.5. Comparison 6 Metformin plus sulphonylurea vs metformin plus thiazolidinedione (subgroups duration of intervention), Outcome 5 Heart failure.

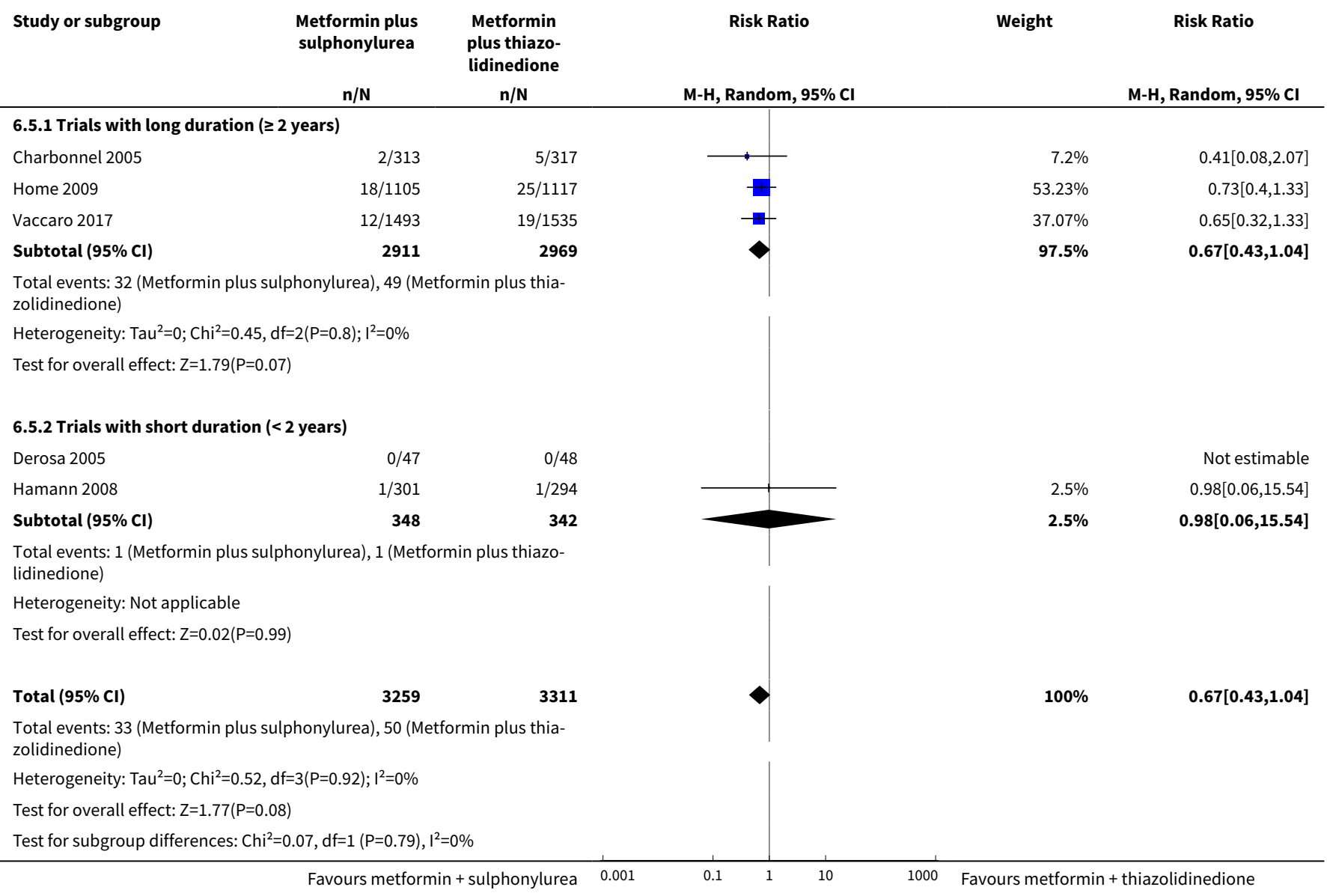

Analysis 6.6. Comparison 6 Metformin plus sulphonylurea vs metformin plus thiazolidinedione (subgroups duration of intervention), Outcome 6 Non-fatal stroke.

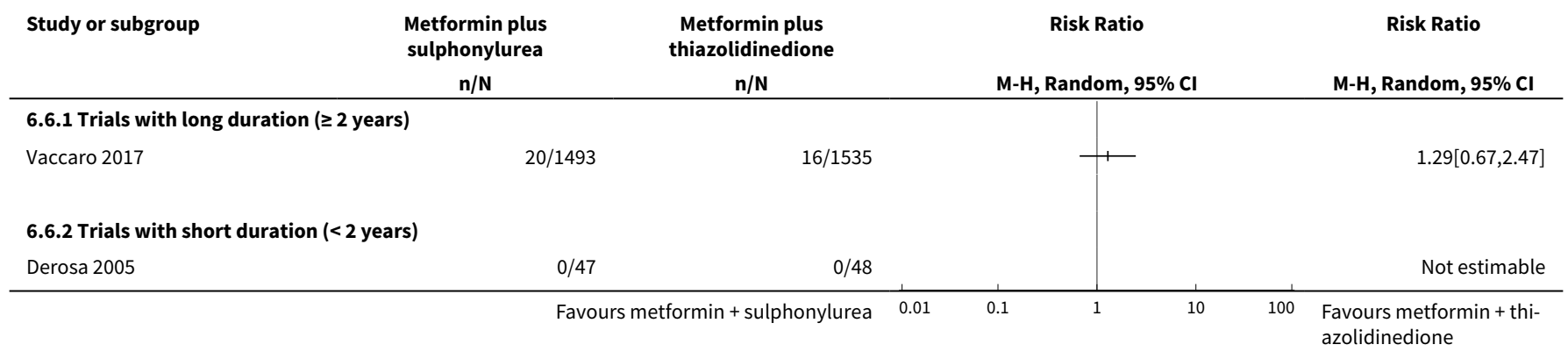


Analysis 6.7. Comparison 6 Metformin plus sulphonylurea vs metformin plus thiazolidinedione (subgroups duration of intervention), Outcome 7 Non-serious adverse events.

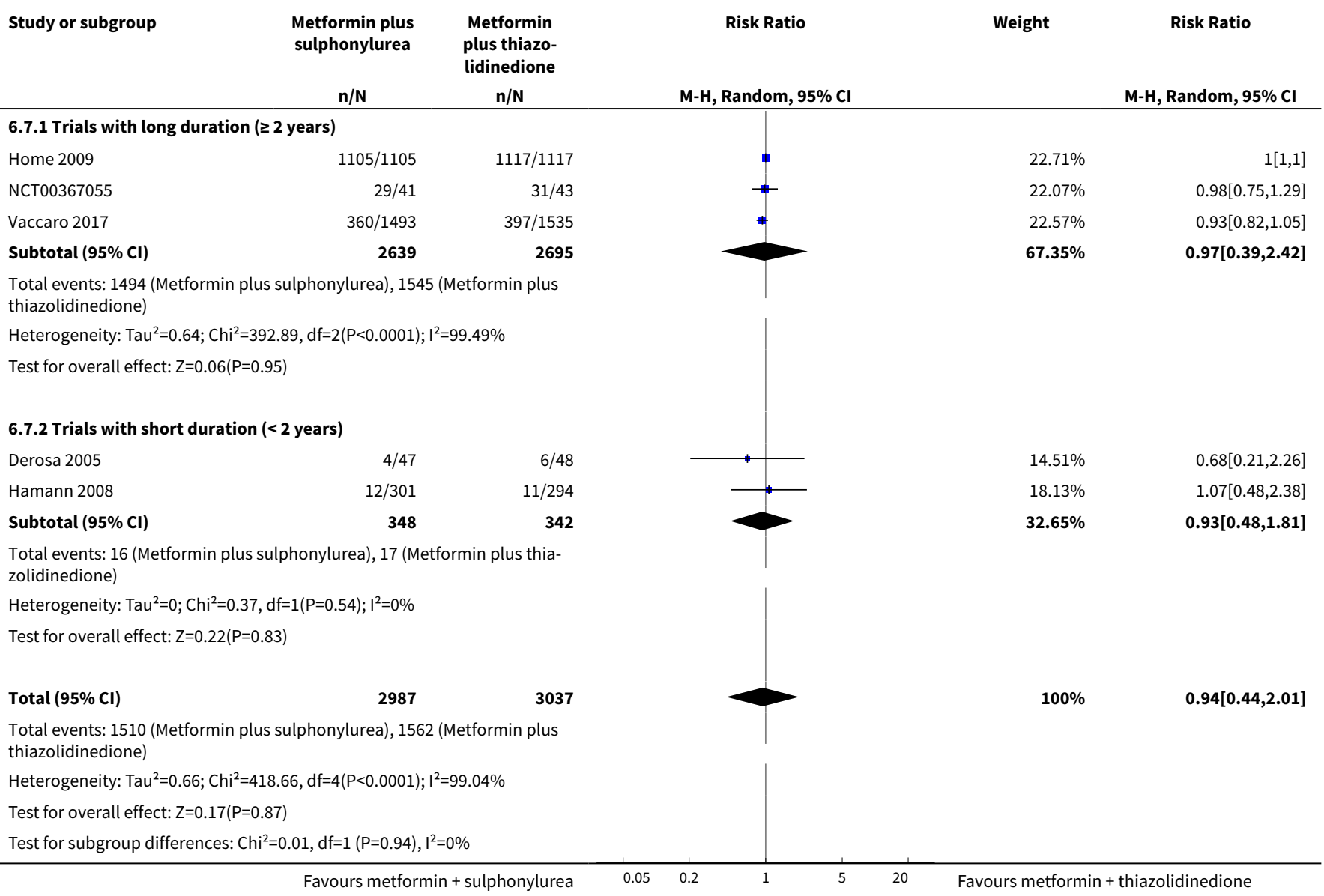
Analysis 6.8. Comparison 6 Metformin plus sulphonylurea vs metformin plus thiazolidinedione
(subgroups duration of intervention), Outcome 8 Mild/moderate hypoglycaemia.

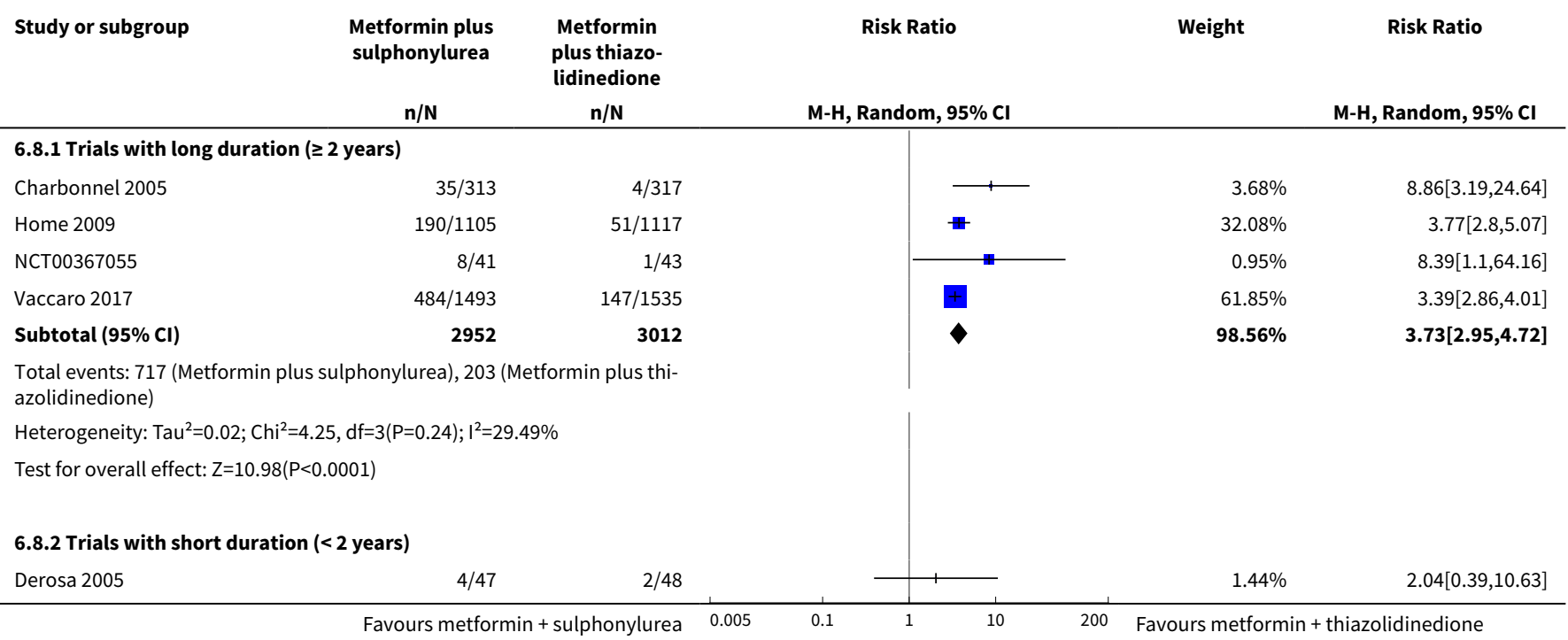




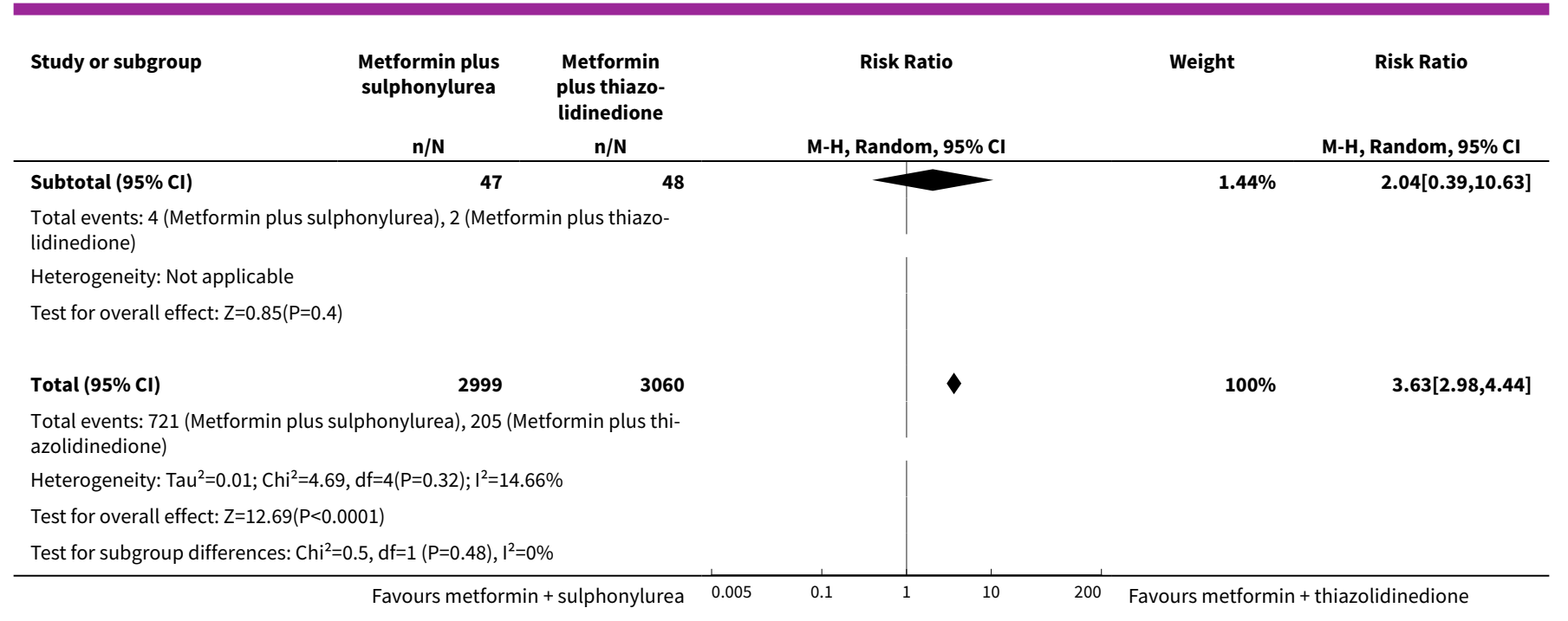

Analysis 6.9. Comparison 6 Metformin plus sulphonylurea vs metformin plus thiazolidinedione (subgroups duration of intervention), Outcome 9 Serious hypoglycaemia.

\begin{tabular}{|c|c|c|c|c|c|}
\hline \multirow[t]{2}{*}{ Study or subgroup } & \multirow{2}{*}{$\begin{array}{c}\text { Metformin plus } \\
\text { sulphonylurea } \\
n / N\end{array}$} & \multirow{2}{*}{$\begin{array}{c}\text { Metformin } \\
\text { plus thiazo- } \\
\text { lidinedione } \\
\mathrm{n} / \mathrm{N}\end{array}$} & Risk Ratio & \multirow[t]{2}{*}{ Weight } & \multirow{2}{*}{$\begin{array}{c}\text { Risk Ratio } \\
\text { M-H, Random, 95\% Cl }\end{array}$} \\
\hline & & & M-H, Random, $95 \% \mathrm{Cl}$ & & \\
\hline \multicolumn{3}{|c|}{ 6.9.1 Trials with long duration ( $\geq 2$ years) } & & & \\
\hline Charbonnel 2005 & $0 / 313$ & $0 / 317$ & & & Not estimable \\
\hline Home 2009 & $5 / 1105$ & $5 / 1117$ & + & $40.16 \%$ & $1.01[0.29,3.48]$ \\
\hline Subtotal $(95 \% \mathrm{Cl})$ & 2911 & 2969 & & $74.63 \%$ & $4.61[0.14,149.68]$ \\
\hline \multicolumn{3}{|c|}{$\begin{array}{l}\text { Total events: } 29 \text { (Metformin plus sulphonylurea), } 6 \text { (Metformin plus thiazo- } \\
\text { lidinedione) }\end{array}$} & & & \\
\hline \multicolumn{3}{|c|}{ Heterogeneity: $\operatorname{Tau}^{2}=5.61 ; \mathrm{Chi}^{2}=8.8, \mathrm{df}=1(\mathrm{P}=0) ; \mathrm{I}^{2}=88.63 \%$} & & & \\
\hline \multicolumn{3}{|c|}{ Test for overall effect: $Z=0.86(P=0.39)$} & & & \\
\hline \multicolumn{3}{|c|}{ 6.9.2 Trials with short duration ( $<2$ years) } & & & \\
\hline Hamann 2008 & $1 / 301$ & $0 / 294$ & $\longrightarrow$ & $25.37 \%$ & $2.93[0.12,71.65]$ \\
\hline Subtotal $(95 \% \mathrm{Cl})$ & 348 & 342 & & $25.37 \%$ & $2.93[0.12,71.65]$ \\
\hline \multicolumn{3}{|c|}{$\begin{array}{l}\text { Total events: } 1 \text { (Metformin plus sulphonylurea), } 0 \text { (Metformin plus thiazo- } \\
\text { lidinedione) }\end{array}$} & & & \\
\hline \multicolumn{3}{|c|}{ Heterogeneity: Not applicable } & & & \\
\hline \multicolumn{3}{|c|}{ Test for overall effect: $\mathrm{Z}=0.66(\mathrm{P}=0.51)$} & & & \\
\hline Total $(95 \% \mathrm{Cl})$ & 3259 & 3311 & & $100 \%$ & $3.98[0.34,46.01]$ \\
\hline \multicolumn{3}{|c|}{$\begin{array}{l}\text { Total events: } 30 \text { (Metformin plus sulphonylurea), } 6 \text { (Metformin plus thiazo- } \\
\text { lidinedione) }\end{array}$} & & & \\
\hline \multicolumn{3}{|c|}{ Heterogeneity: Tau $^{2}=3.48 ; \mathrm{Chi}^{2}=8.73, \mathrm{df}=2(\mathrm{P}=0.01) ; \mathrm{I}^{2}=77.09 \%$} & & & \\
\hline \multicolumn{3}{|c|}{ Test for overall effect: $Z=1.11(P=0.27)$} & & & \\
\hline \multicolumn{3}{|c|}{ Test for subgroup differences: $\mathrm{Chi}^{2}=0.04, \mathrm{df}=1(\mathrm{P}=0.85), \mathrm{I}^{2}=0 \%$} & & & \\
\hline
\end{tabular}


Analysis 6.10. Comparison 6 Metformin plus sulphonylurea vs metformin plus thiazolidinedione (subgroups duration of intervention), Outcome 10 Weight change.

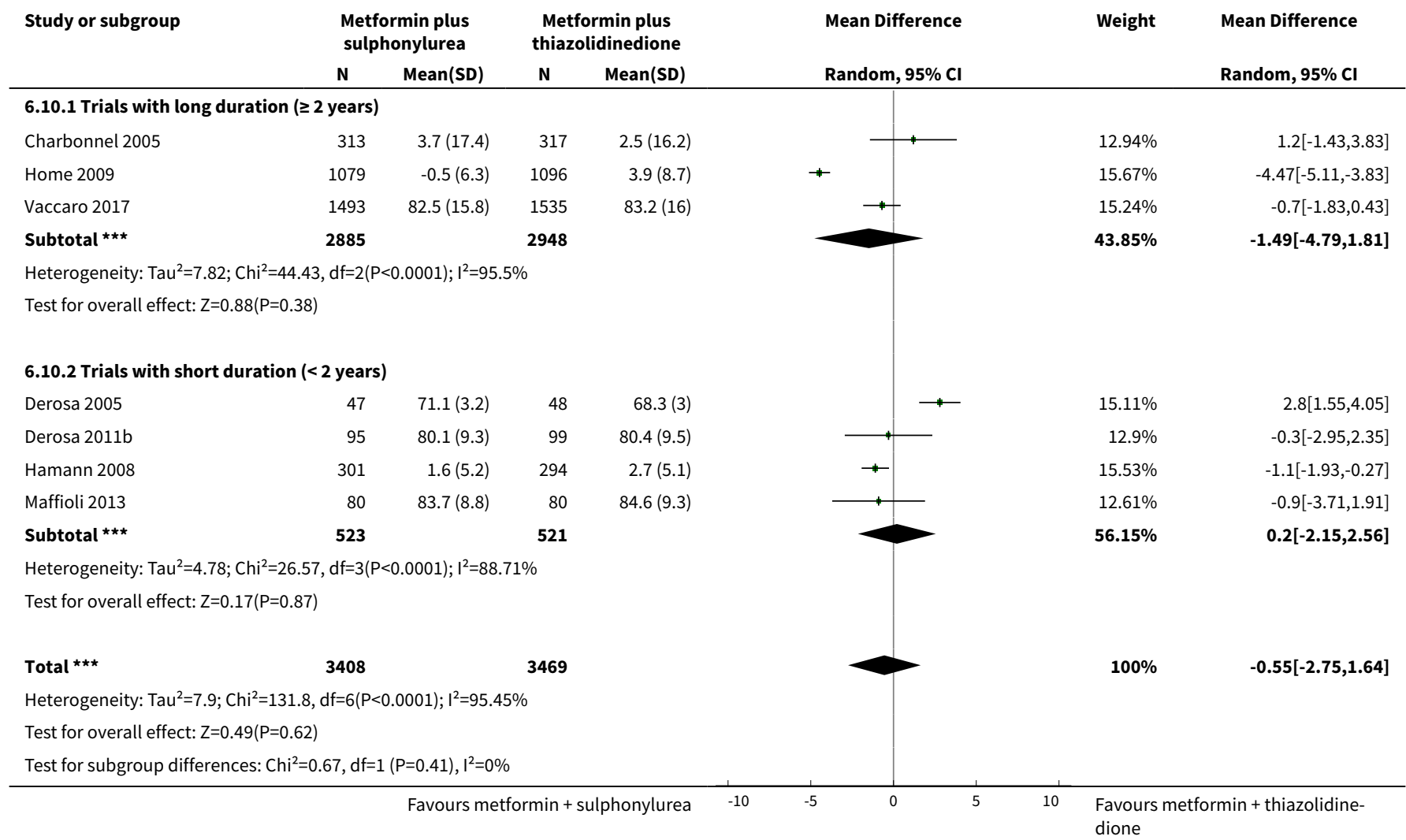

Analysis 6.11. Comparison 6 Metformin plus sulphonylurea vs metformin plus thiazolidinedione (subgroups duration of intervention), Outcome 11 Change in HbA1c.

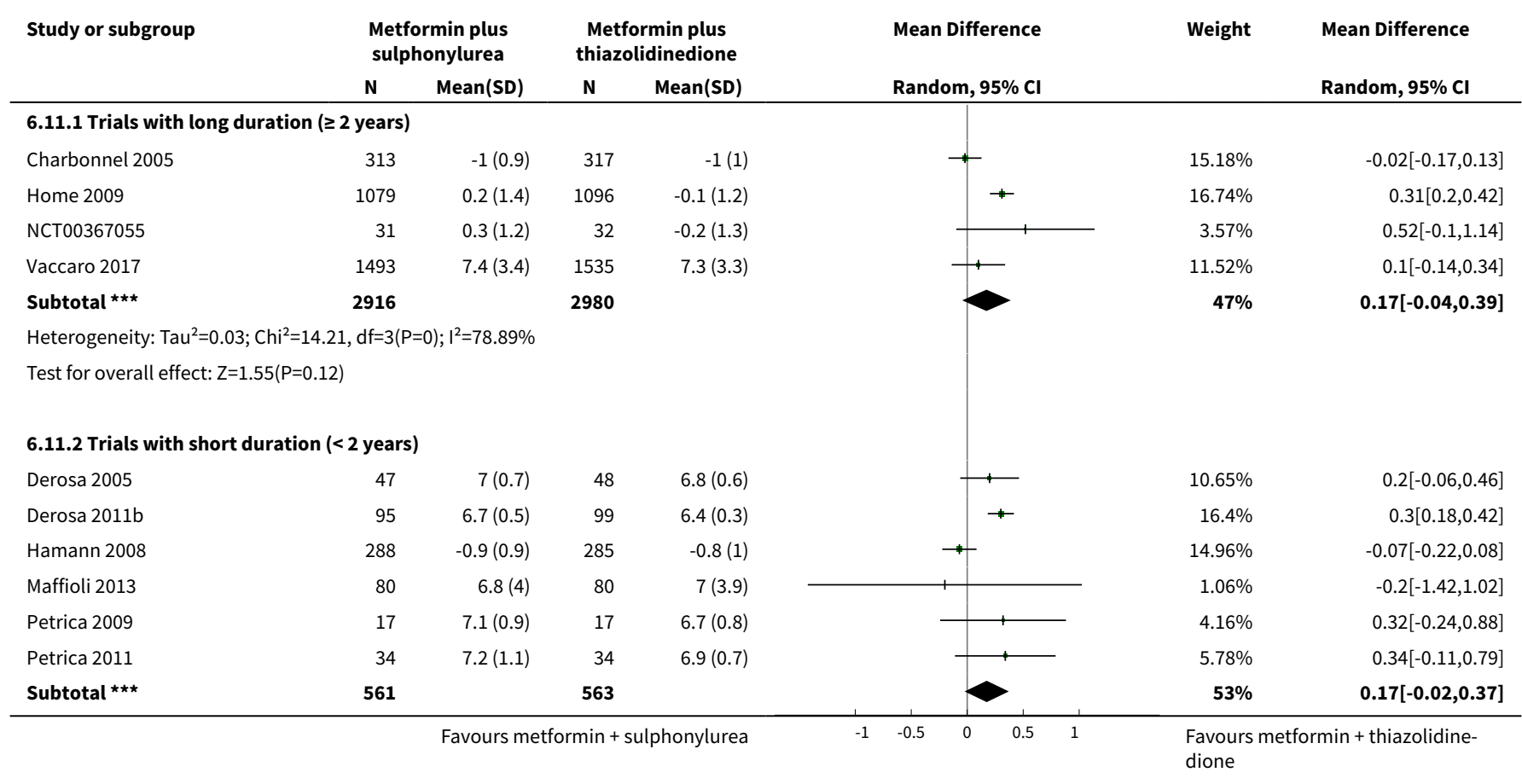




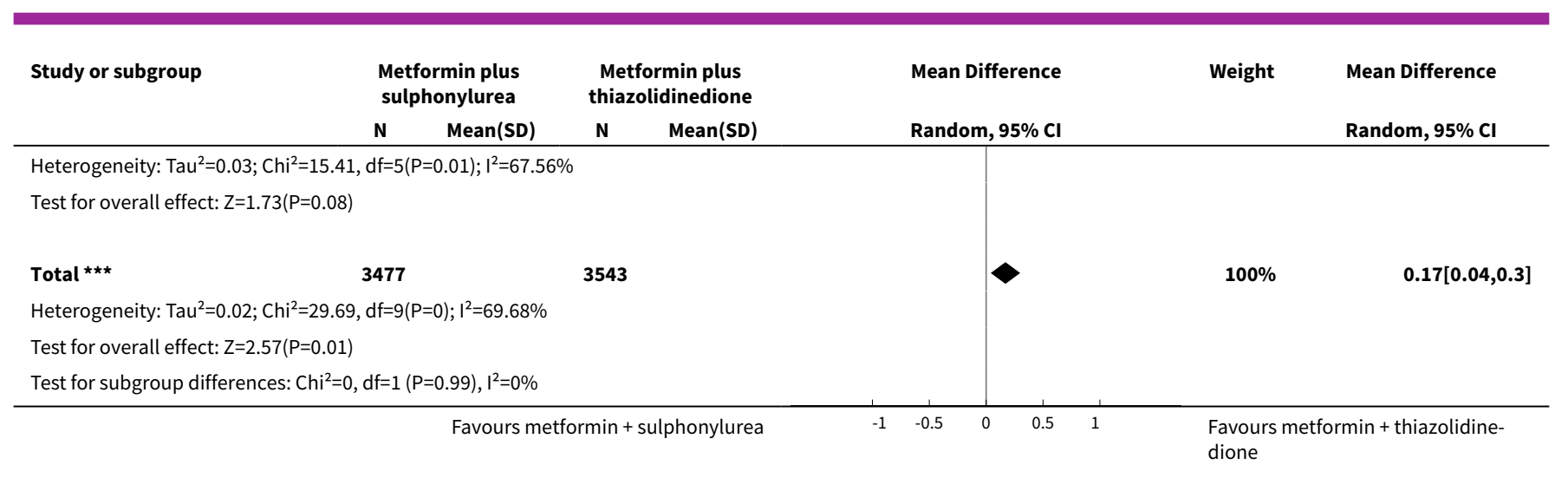

\section{Comparison 7. Metformin plus sulphonylurea vs metformin plus glinide}

\begin{tabular}{lllll}
\hline $\begin{array}{l}\text { Outcome or subgroup ti- } \\
\text { tle }\end{array}$ & No. of studies & $\begin{array}{l}\text { No. of partici- } \\
\text { pants }\end{array}$ & Statistical method & Effect size \\
\hline 1 All-cause mortality & 3 & & Risk Ratio (M-H, Fixed, 95\% Cl) & Totals not selected \\
\hline 2 Serious adverse events & 3 & 874 & Risk Ratio (M-H, Random, 95\% Cl) & $1.68[0.54,5.21]$ \\
\hline 3 Cardiovascular mortality & 2 & 446 & Risk Ratio (M-H, Fixed, 95\% Cl) & $0.0[0.0,0.0]$ \\
\hline $\begin{array}{l}4 \text { Mild/moderate hypogly- } \\
\text { caemia }\end{array}$ & 1 & Risk Ratio (M-H, Fixed, 95\% Cl) & Totals not selected \\
\hline 5 Serious hypoglycaemia & 2 & & Risk Ratio (M-H, Fixed, 95\% Cl) & Totals not selected \\
\hline $\begin{array}{l}6 \text { Weight change } \\
7 \text { Change in HbAlc }\end{array}$ & 2 & Mean Difference (IV, Fixed, 95\% Cl) & $1.11[-0.06,2.29]$ \\
\hline
\end{tabular}

Analysis 7.1. Comparison 7 Metformin plus sulphonylurea vs metformin plus glinide, Outcome 1 All-cause mortality.

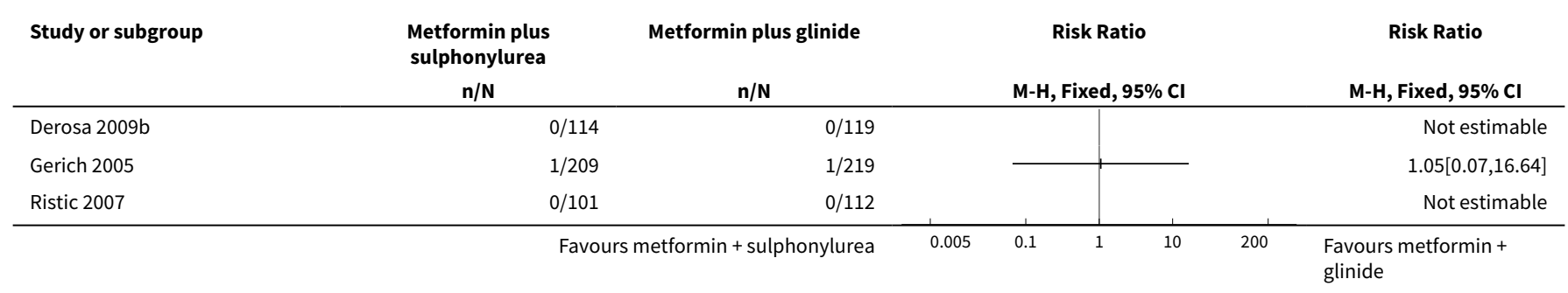


Analysis 7.2. Comparison 7 Metformin plus sulphonylurea vs metformin plus glinide, Outcome 2 Serious adverse events.

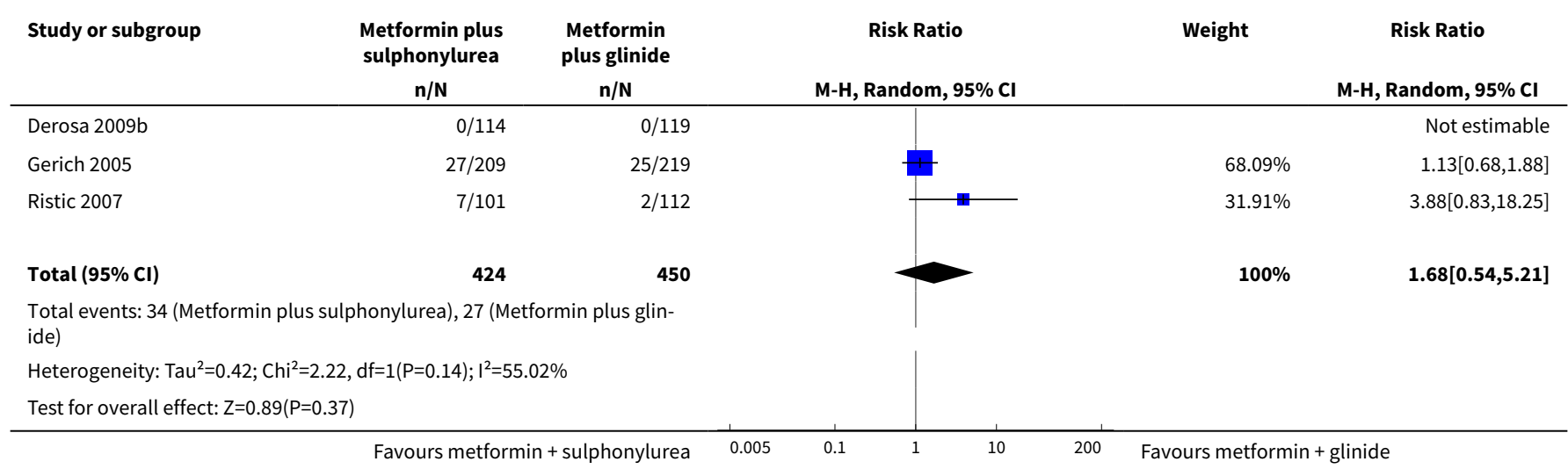

Analysis 7.3. Comparison 7 Metformin plus sulphonylurea vs metformin plus glinide, Outcome 3 Cardiovascular mortality.

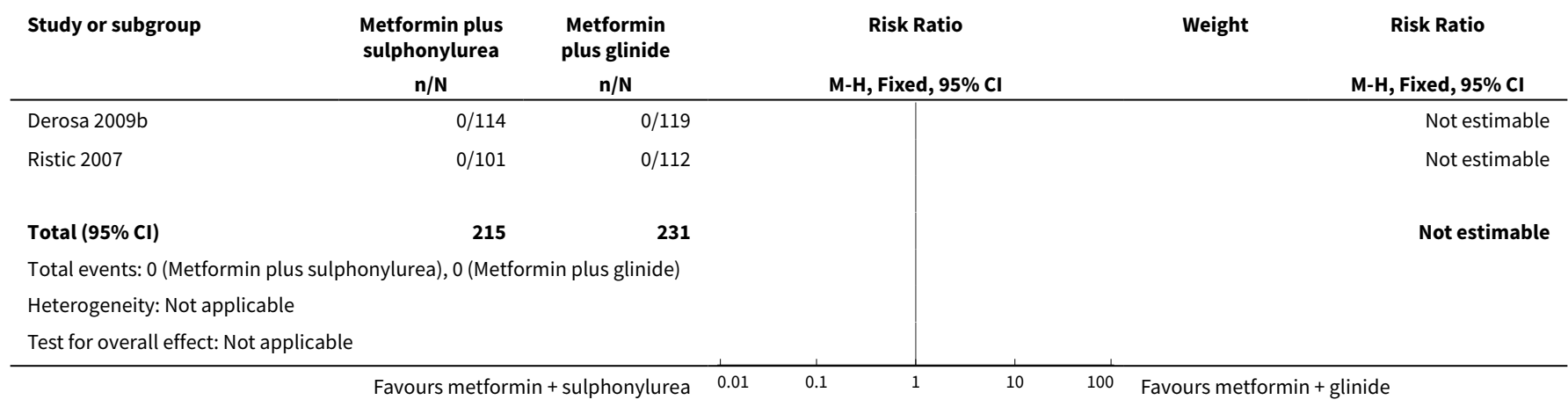

Analysis 7.4. Comparison 7 Metformin plus sulphonylurea vs metformin plus glinide, Outcome $4 \mathrm{Mild} /$ moderate hypoglycaemia.

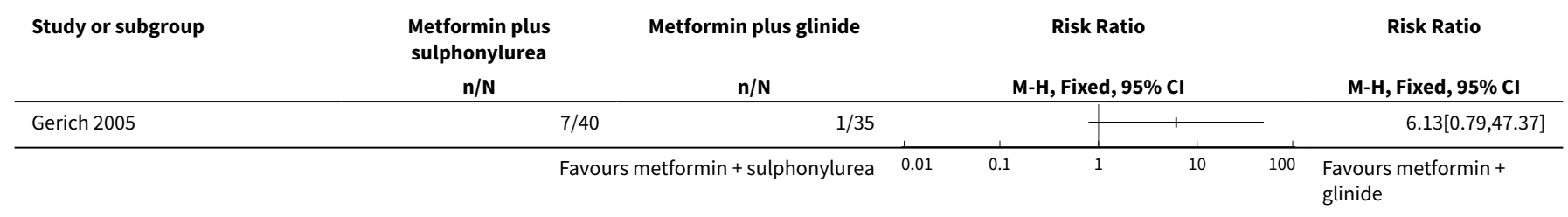

Analysis 7.5. Comparison 7 Metformin plus sulphonylurea vs metformin plus glinide, Outcome 5 Serious hypoglycaemia.

\begin{tabular}{|c|c|c|c|c|c|c|c|}
\hline \multirow[t]{2}{*}{ Study or subgroup } & Metformin plus & Metformin plus glinide & \multicolumn{4}{|c|}{ Risk Ratio } & \multirow{2}{*}{$\begin{array}{c}\text { Risk Ratio } \\
\text { M-H, Fixed, } 95 \% \mathrm{Cl}\end{array}$} \\
\hline & $n / N$ & $n / N$ & \multicolumn{4}{|c|}{ M-H, Fixed, 95\% Cl } & \\
\hline \multirow[t]{2}{*}{ Derosa 2009b } & $0 / 114$ & $0 / 119$ & & & & & Not estimable \\
\hline & Favol & netformin + sulphonylurea & 0.01 & 0.1 & 10 & 100 & $\begin{array}{l}\text { Favours metformin + } \\
\text { glinide }\end{array}$ \\
\hline
\end{tabular}




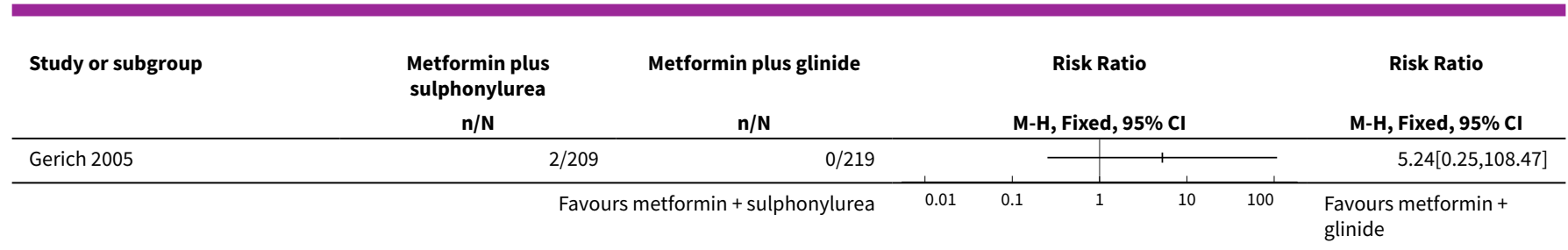

Analysis 7.6. Comparison 7 Metformin plus sulphonylurea vs metformin plus glinide, Outcome 6 Weight change.

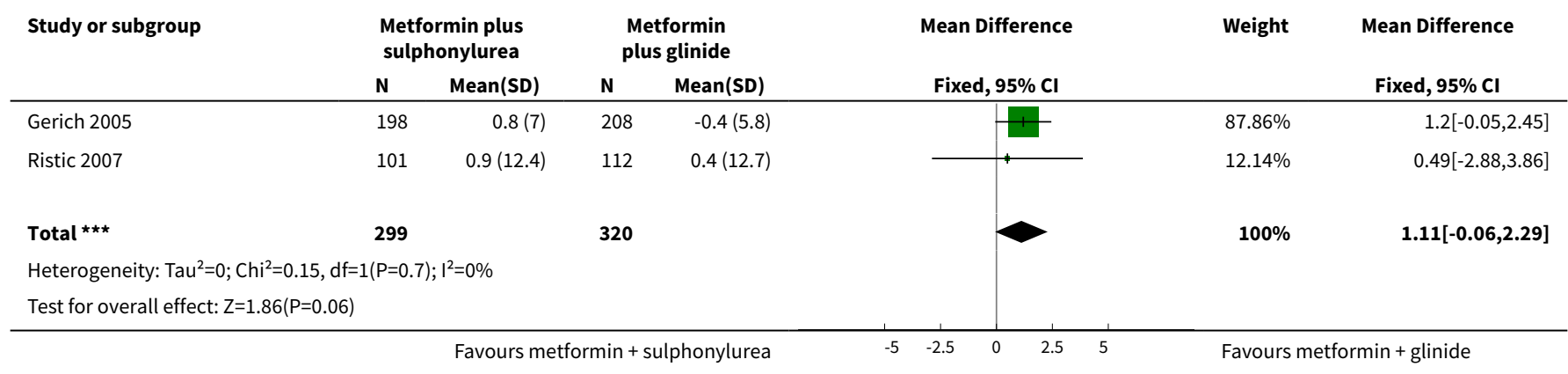

Analysis 7.7. Comparison 7 Metformin plus sulphonylurea vs metformin plus glinide, Outcome 7 Change in HbA1c.

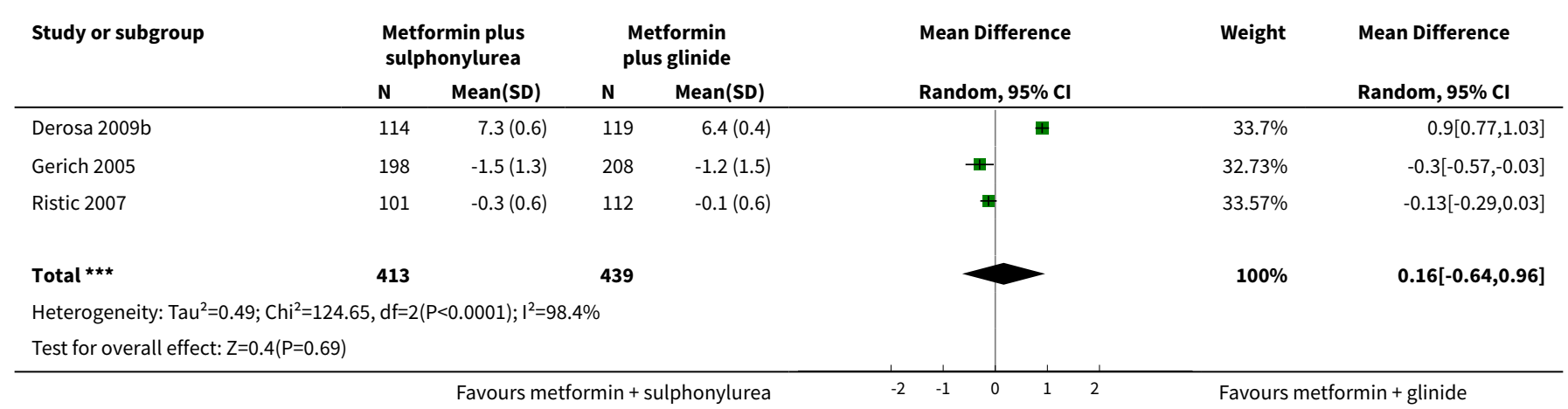

Comparison 8. Metformin plus sulphonylurea vs metformin plus SGLT-2 inhibitor

\begin{tabular}{llllll}
\hline Outcome or subgroup title & No. of studies & $\begin{array}{l}\text { No. of partici- } \\
\text { pants }\end{array}$ & Statistical method & Effect size \\
\hline 1 All-cause mortality & 4 & 5134 & Risk Ratio (M-H, Random, 95\% Cl) & $0.96[0.44,2.09]$ \\
\hline 2 Serious adverse events & 4 & 5134 & Risk Ratio (M-H, Random, 95\% Cl) & $1.02[0.76,1.37]$ \\
\hline 3 Cardiovascular mortality & 3 & 3589 & Risk Ratio (M-H, Random, 95\% Cl) & $1.22[0.33,4.41]$ \\
\hline $\begin{array}{l}\text { 4 Non-fatal myocardial in- } \\
\text { farction }\end{array}$ & 2 & 2264 & Risk Ratio (M-H, Random, 95\% Cl) & $1.43[0.49,4.18]$ \\
\hline 5 Heart failure & 3 & 3809 & $\begin{array}{l}\text { Peto Odds Ratio (Peto, Fixed, 95\% } \\
\text { Cl) }\end{array}$ & $9.21[1.26,67.24]$ \\
\hline \hline
\end{tabular}




\begin{tabular}{|c|c|c|c|c|}
\hline Outcome or subgroup title & No. of studies & $\begin{array}{l}\text { No. of partici- } \\
\text { pants }\end{array}$ & Statistical method & Effect size \\
\hline 6 Non-fatal stroke & 2 & 2775 & Risk Ratio (M-H, Random, 95\% Cl) & $0.87[0.22,3.34]$ \\
\hline $\begin{array}{l}7 \text { Amputation of lower ex- } \\
\text { tremity }\end{array}$ & 1 & & Risk Ratio (M-H, Fixed, 95\% Cl) & Totals not selected \\
\hline $\begin{array}{l}8 \text { Non-serious adverse } \\
\text { events }\end{array}$ & 3 & 3809 & Risk Ratio (M-H, Random, 95\% Cl) & $1.27[1.01,1.59]$ \\
\hline $\begin{array}{l}9 \text { Mild/moderate hypogly- } \\
\text { caemia }\end{array}$ & 3 & 3309 & Risk Ratio (M-H, Random, 95\% Cl) & $5.60[2.38,13.14]$ \\
\hline 10 Serious hypoglycaemia & 4 & 5134 & Risk Ratio (M-H, Random, 95\% Cl) & $6.16[2.92,12.97]$ \\
\hline 11 Weight change & 3 & 3294 & $\begin{array}{l}\text { Mean Difference (IV, Random, 95\% } \\
\mathrm{CI} \text { ) }\end{array}$ & $4.41[4.05,4.77]$ \\
\hline 12 Change in $\mathrm{HbA} 1 \mathrm{c}$ & 4 & 4182 & $\begin{array}{l}\text { Mean Difference (IV, Random, 95\% } \\
\mathrm{CI} \text { ) }\end{array}$ & $0.09[-0.07,0.24]$ \\
\hline
\end{tabular}

Analysis 8.1. Comparison 8 Metformin plus sulphonylurea vs metformin plus SGLT-2 inhibitor, Outcome 1 All-cause mortality.

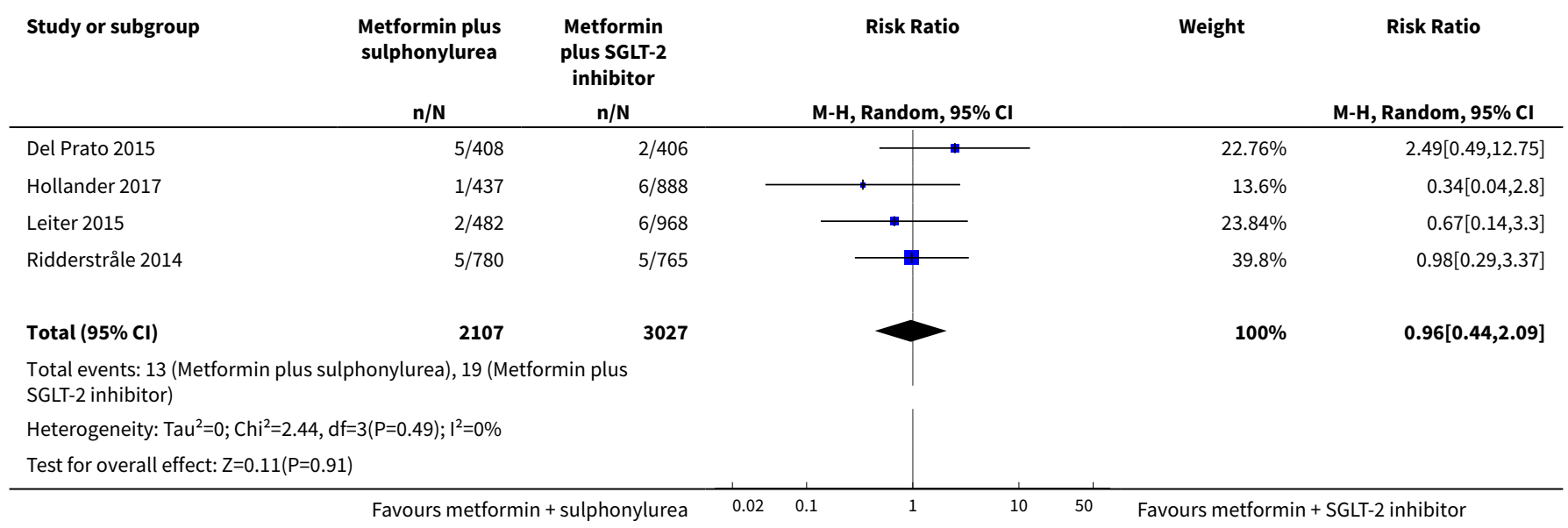

Analysis 8.2. Comparison 8 Metformin plus sulphonylurea vs metformin plus SGLT-2 inhibitor, Outcome 2 Serious adverse events.

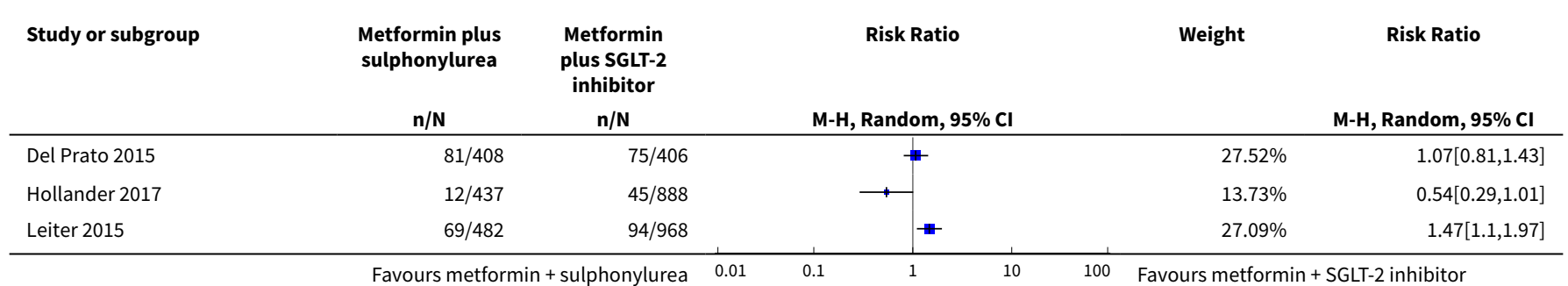




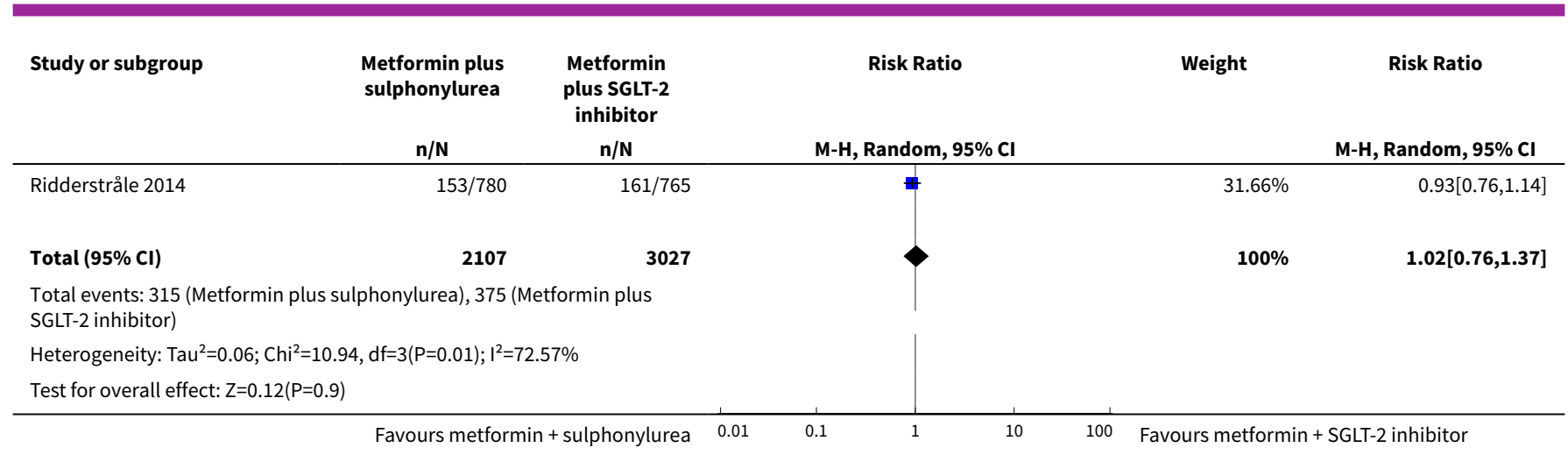

Analysis 8.3. Comparison 8 Metformin plus sulphonylurea vs metformin plus SGLT-2 inhibitor, Outcome 3 Cardiovascular mortality.

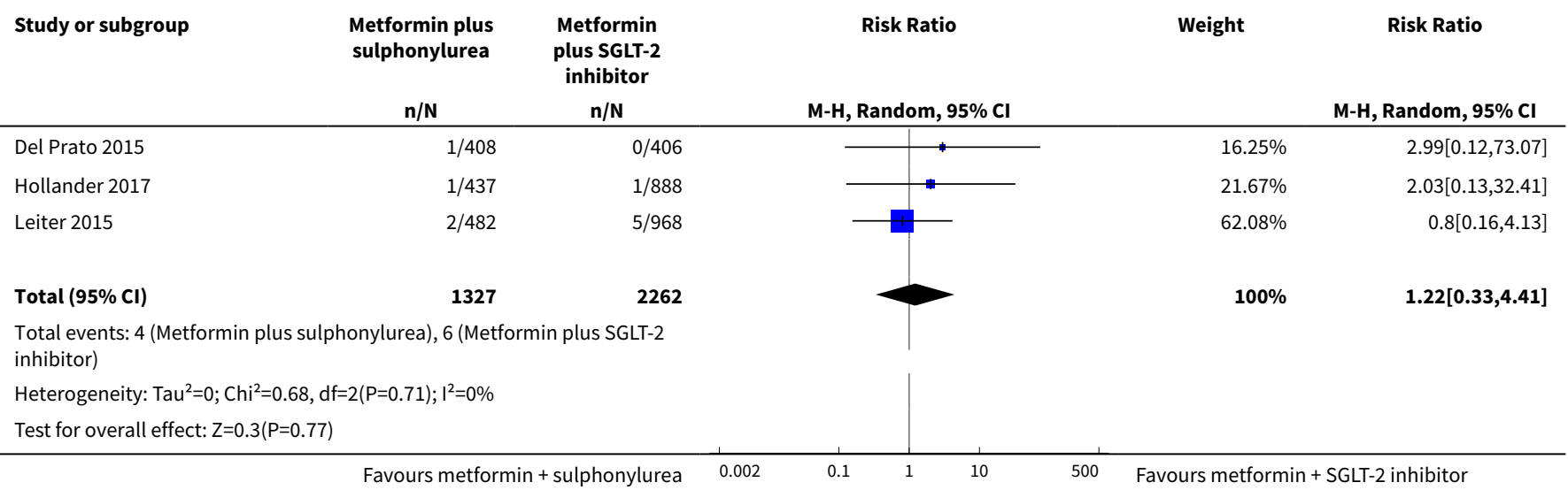

\section{Analysis 8.4. Comparison 8 Metformin plus sulphonylurea vs metformin plus SGLT-2 inhibitor, Outcome 4 Non-fatal myocardial infarction.}

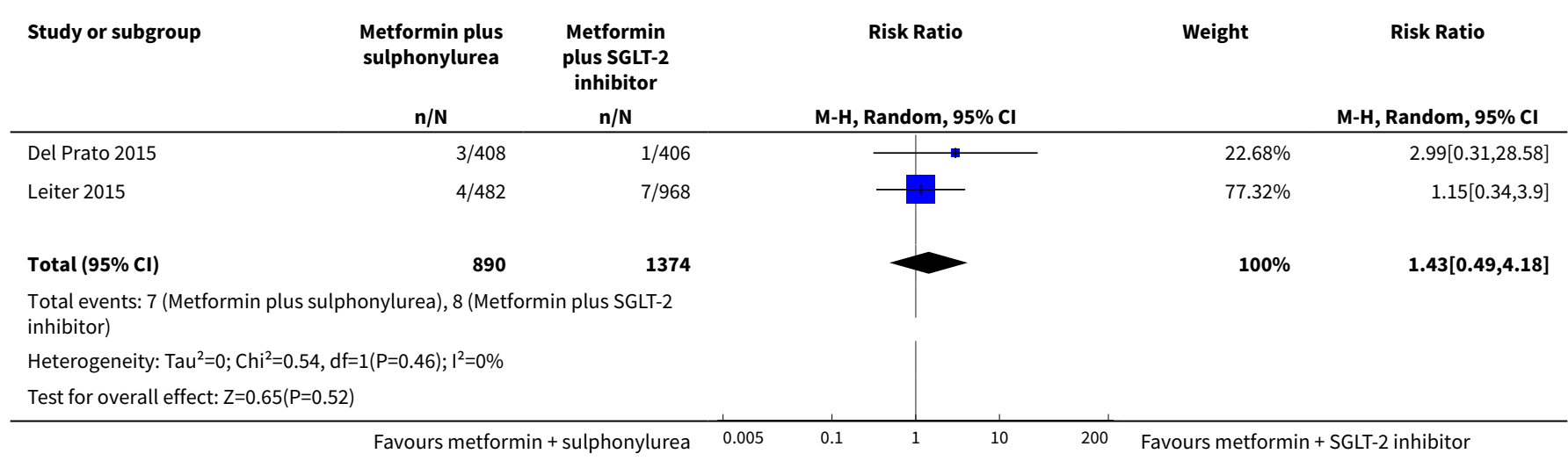


Analysis 8.5. Comparison 8 Metformin plus sulphonylurea vs metformin plus SGLT-2 inhibitor, Outcome 5 Heart failure.

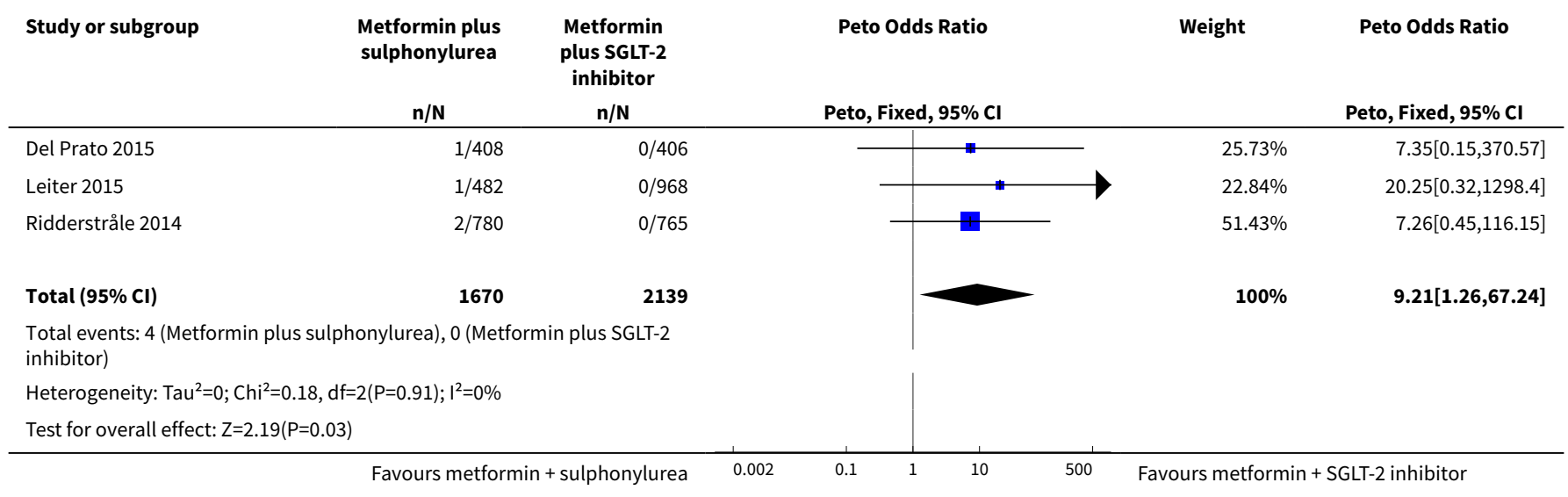

Analysis 8.6. Comparison 8 Metformin plus sulphonylurea vs metformin plus SGLT-2 inhibitor, Outcome 6 Non-fatal stroke.

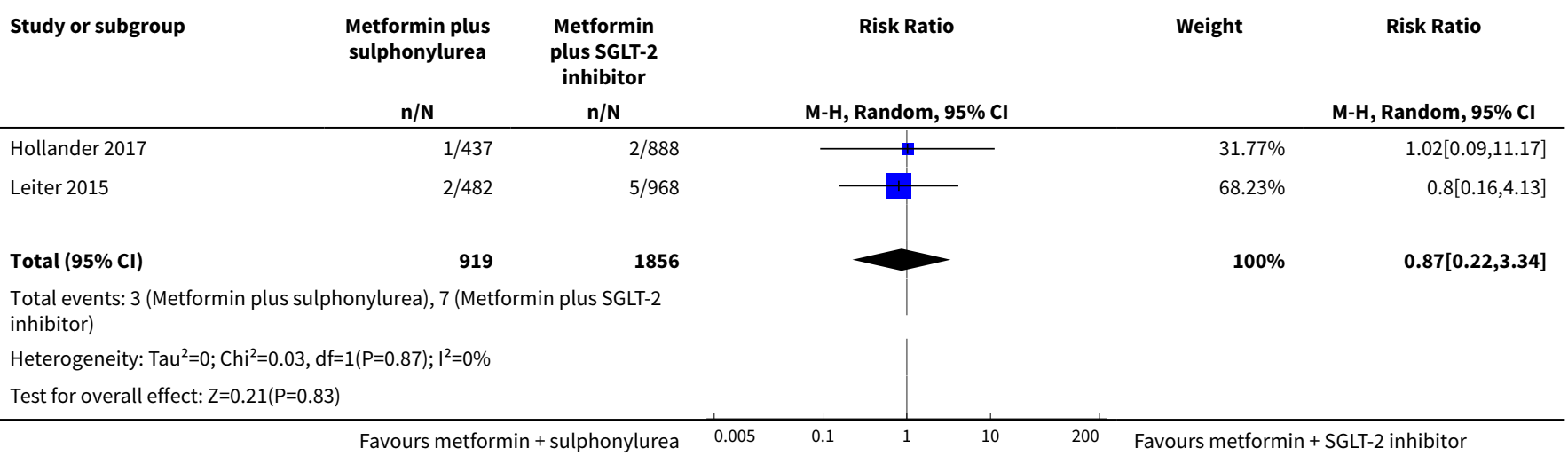

Analysis 8.7. Comparison 8 Metformin plus sulphonylurea vs metformin plus SGLT-2 inhibitor, Outcome 7 Amputation of lower extremity.

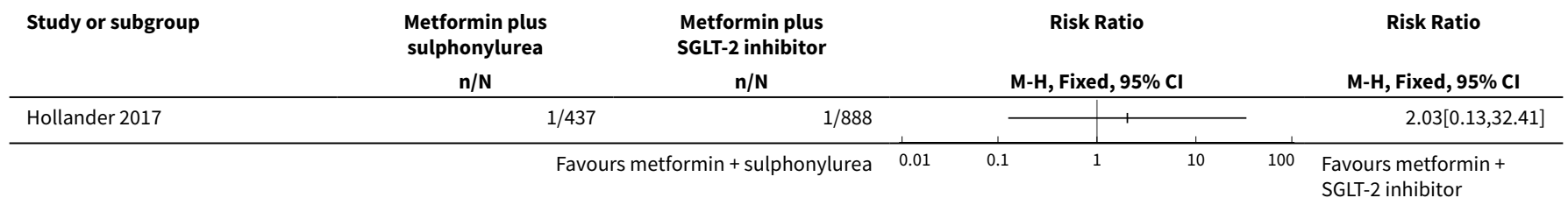


Analysis 8.8. Comparison 8 Metformin plus sulphonylurea vs metformin plus SGLT-2 inhibitor, Outcome 8 Non-serious adverse events.

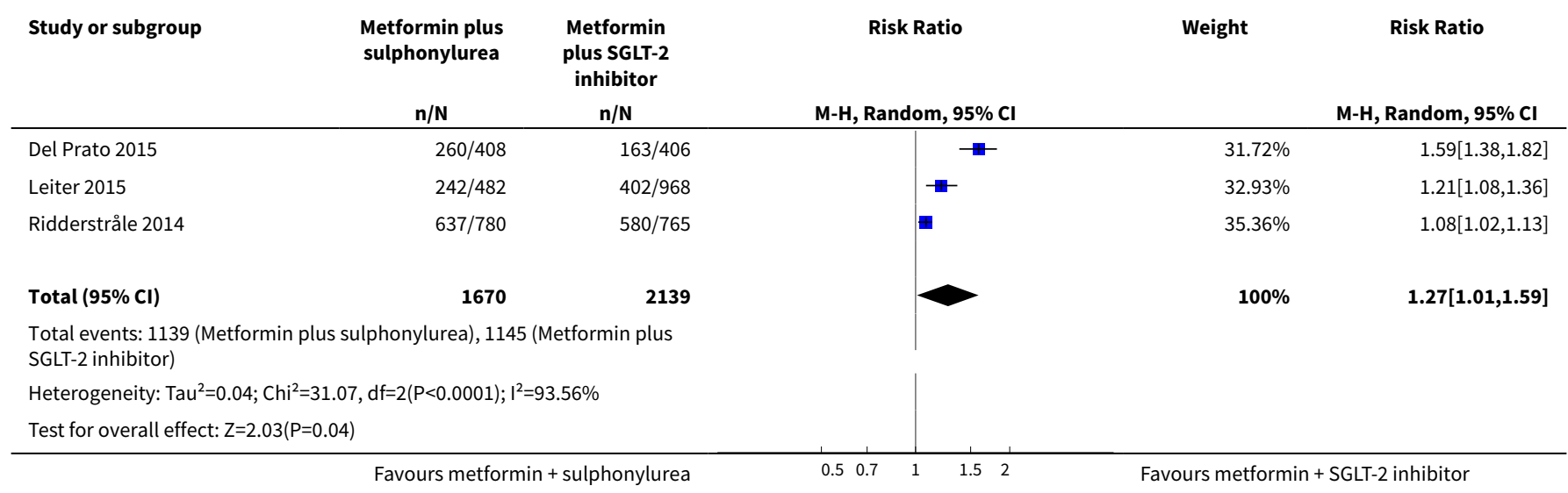

\section{Analysis 8.9. Comparison 8 Metformin plus sulphonylurea vs metformin} plus SGLT-2 inhibitor, Outcome 9 Mild/moderate hypoglycaemia.

\begin{tabular}{|c|c|c|c|c|c|}
\hline \multirow[t]{2}{*}{ Study or subgroup } & \multirow{2}{*}{$\begin{array}{c}\text { Metformin plus } \\
\text { sulphonylurea } \\
n / N\end{array}$} & \multirow{2}{*}{$\begin{array}{c}\text { Metformin } \\
\text { plus SGLT-2 } \\
\text { inhibitor } \\
n / N \\
\end{array}$} & Risk Ratio & \multirow[t]{2}{*}{ Weight } & \multirow{2}{*}{$\begin{array}{c}\text { Risk Ratio } \\
\text { M-H, Random, } 95 \% \mathrm{Cl}\end{array}$} \\
\hline & & & M-H, Random, $95 \% \mathrm{Cl}$ & & \\
\hline Leiter 2015 & $86 / 482$ & $33 / 468$ & $\rightarrow$ & $33.65 \%$ & $2.53[1.73,3.7]$ \\
\hline Ridderstråle 2014 & $228 / 780$ & $41 / 765$ & + & $34.35 \%$ & $5.45[3.97,7.49]$ \\
\hline Total $(95 \% \mathrm{Cl})$ & 1670 & 1639 & & $100 \%$ & $5.6[2.38,13.14]$ \\
\hline \multicolumn{6}{|c|}{$\begin{array}{l}\text { Total events: } 514 \text { (Metformin plus sulphonylurea), } 89 \text { (Metformin plus } \\
\text { SGLT-2 inhibitor) }\end{array}$} \\
\hline \multicolumn{6}{|c|}{ Heterogeneity: $\mathrm{Tau}^{2}=0.53 ; \mathrm{Chi}^{2}=28.15, \mathrm{df}=2(\mathrm{P}<0.0001) ; \mathrm{I}^{2}=92.9 \%$} \\
\hline Test for overall effect & 01) & & & & \\
\hline
\end{tabular}

$\begin{array}{lllllll}\text { Favours metformin + sulphonylurea } & 0.01 & 0.1 & 1 & 10 & 100 & \text { Favours metformin + SGLT-2 inhibitor }\end{array}$

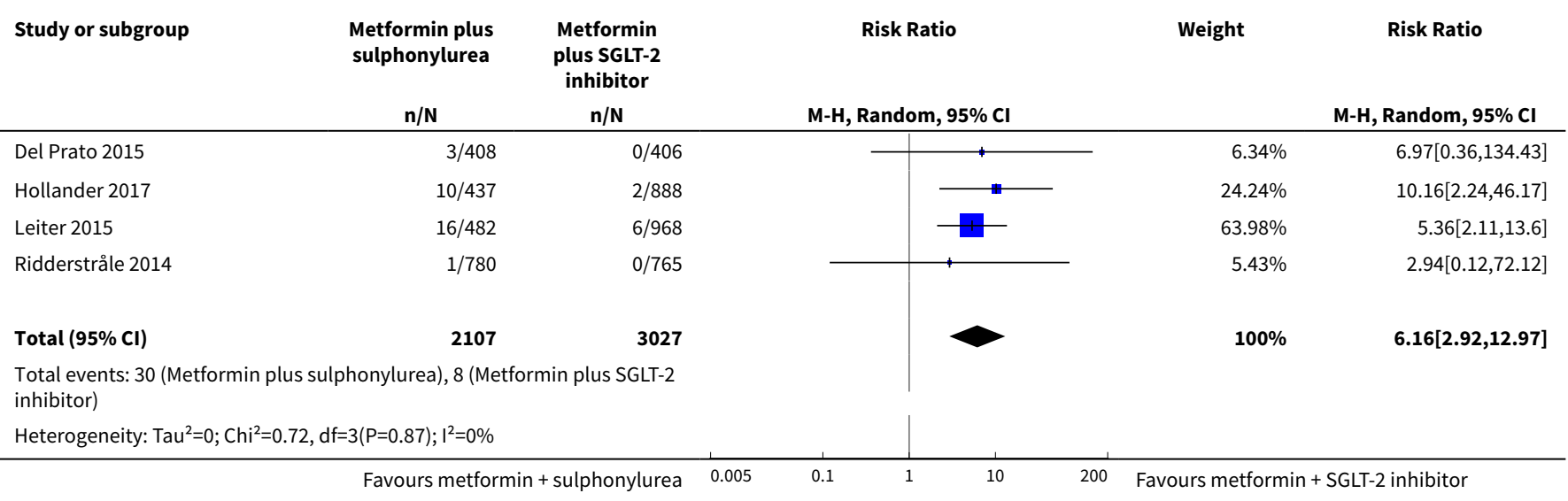




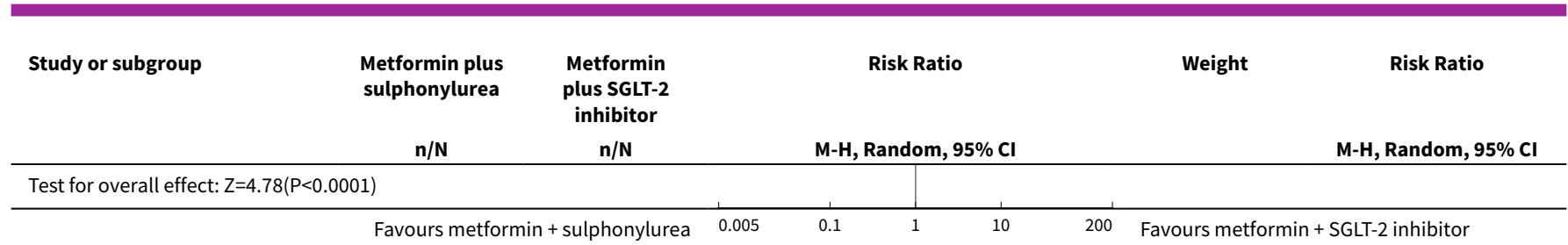

Analysis 8.11. Comparison 8 Metformin plus sulphonylurea vs

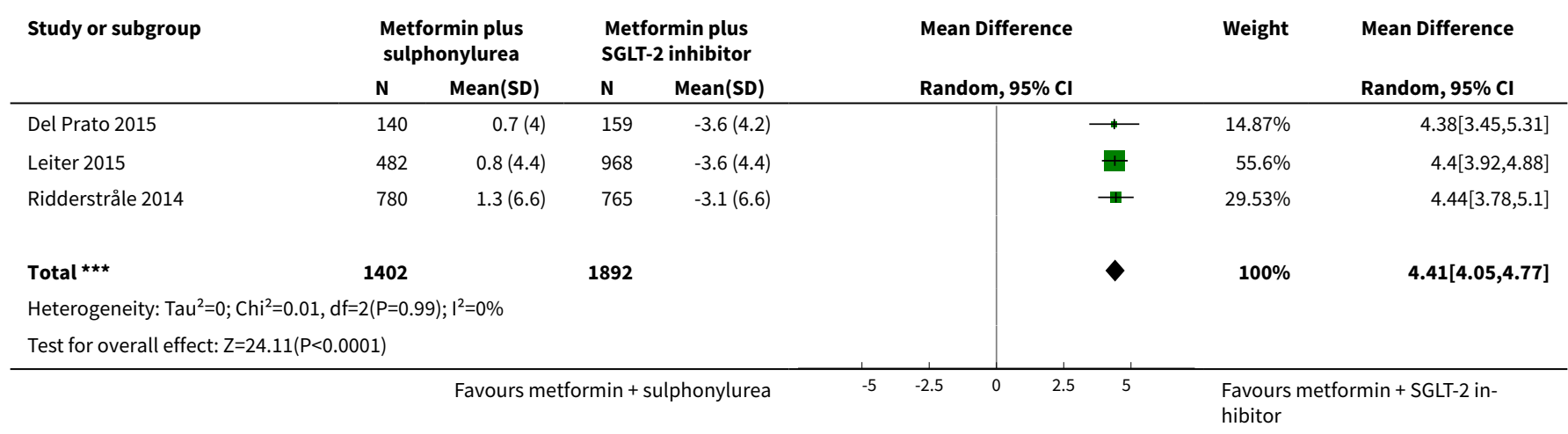

Analysis 8.12. Comparison 8 Metformin plus sulphonylurea vs metformin plus SGLT-2 inhibitor, Outcome 12 Change in HbA1c.

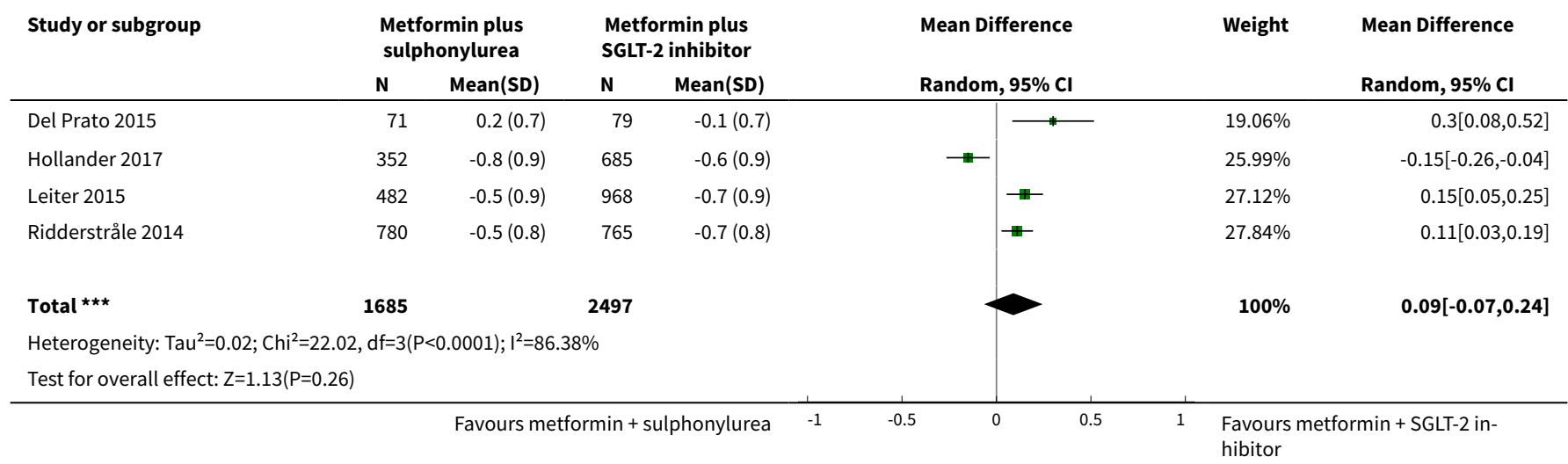




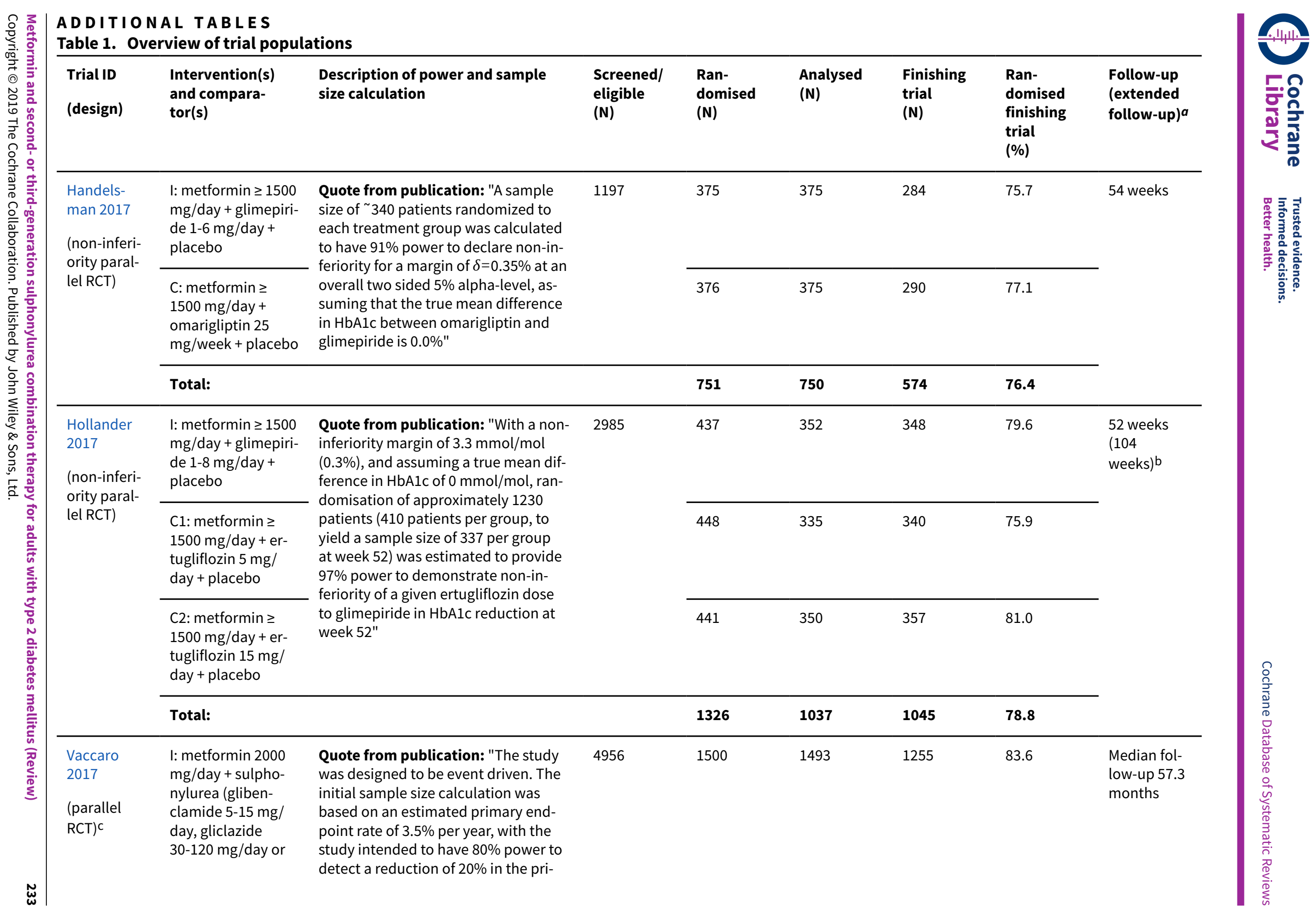




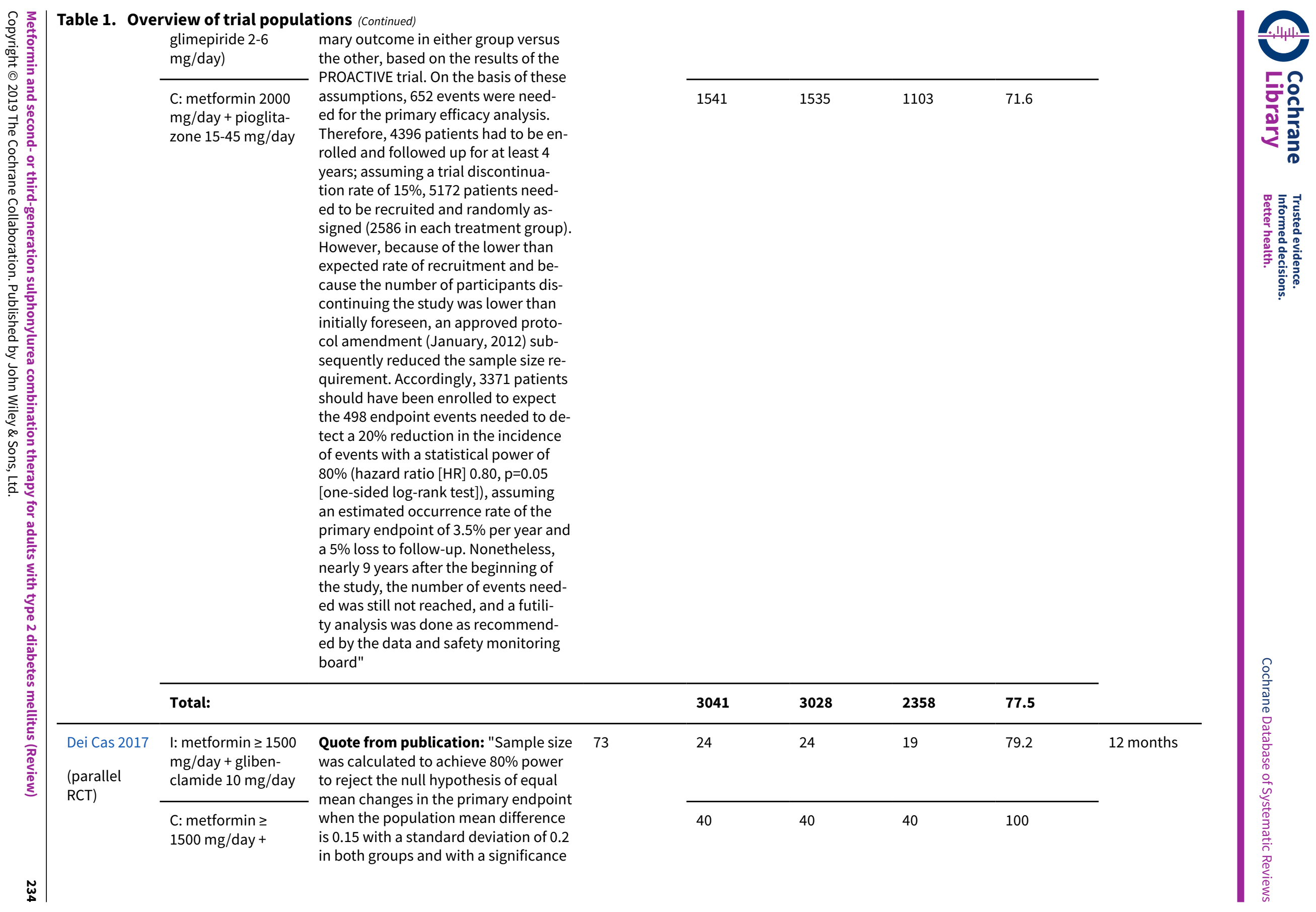




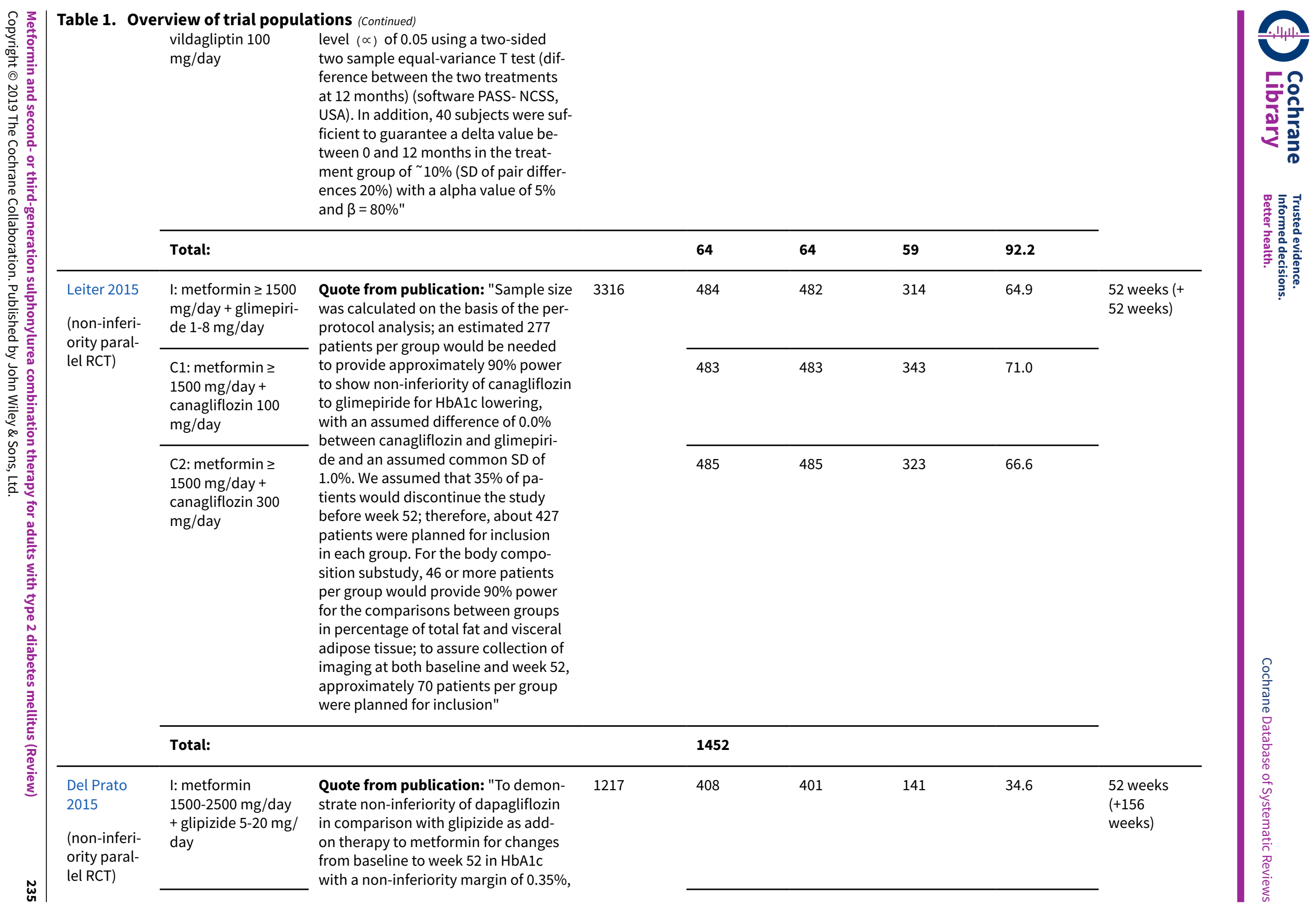


able 1. Overview of trial populations (Continued)

C: metformin assuming a standard deviation (SD)

of $1.25 \%$, and at a one-sided signifi-

406

400

161

39.7

day + dapagliflozin

$2.5-10 \mathrm{mg} /$ day

cance level of $0.025,280$ evaluable pa-

tients were needed in each treatment

group to provide approximately $90 \%$

power (given a true difference of ze-

ro between the 2 treatment groups).

Assuming a $5 \%$ exclusion rate from

the full analysis set, 295 patients per

treatment group are needed for the

full analysis set. Additionally, to have

90\% power for the per-protocol popu-

lation and assuming a $25 \%$ exclusion

rate from the per-protocol population,

373 patients per treatment group (746

patients in total) were planned for ran-

domization"

\begin{tabular}{|c|c|c|c|c|c|c|c|c|}
\hline & Total: & & & $814^{d}$ & 801 & 302 & 37.0 & \\
\hline \multirow[t]{3}{*}{$\begin{array}{l}\text { Schern- } \\
\text { thaner } 2015 \\
\text { (parallel } \\
\text { RCT) }\end{array}$} & $\begin{array}{l}\text { I: metformin at any } \\
\text { dose }+ \text { glimepiri- } \\
\text { de } 1-6 \mathrm{mg} / \text { day }+ \\
\text { placebo }\end{array}$ & \multirow{3}{*}{$\begin{array}{l}\text { Quote from publication: "A sample } \\
\text { size of } 698 \text { patients ( } 349 / \text { treatment } \\
\text { arm) was calculated for detecting su- } \\
\text { periority of saxagliptin in the prima- } \\
\text { ry endpoint, with a two-sided signif- } \\
\text { icance level of } 0.05 \text { and } 80 \% \text { power. } \\
\text { This assumed a } 10 \% \text { dropout rate and } \\
\text { an odds ratio (OR) of } 1.55 \text { for achiev- } \\
\text { ing target HbA1c without hypogly- } \\
\text { caemia with saxagliptin compared } \\
\text { with glimepiride" }\end{array}$} & 957 & 360 & 359 & 285 & 79.2 & \multirow[t]{3}{*}{52 weeks } \\
\hline & $\begin{array}{l}\text { C: metformin } \\
\text { at any dose + } \\
\text { saxagliptin } 5 \mathrm{mg} / \\
\text { day + placebo }\end{array}$ & & & 360 & 359 & 289 & 80.3 & \\
\hline & Total: & & & 720 & 718 & 574 & 79.7 & \\
\hline \multirow[t]{2}{*}{$\begin{array}{l}\text { Del Prato } \\
2014 \mathrm{e} \\
\text { (non-inferi- } \\
\text { ority paral- } \\
\text { lel RCT) }\end{array}$} & $\begin{array}{l}\text { I: metformin } \geq 1500 \\
\text { mg once daily or } \\
\text { maximum tolerat- } \\
\text { ed dose + glipizide } \\
5-20 \text { mg once daily }\end{array}$ & \multirow{2}{*}{$\begin{array}{l}\text { Quote from publication: "The } \\
\text { planned randomization sample size } \\
\text { for the study was between } 815 \text { and } \\
897 \text { patients per treatment arm. This } \\
\text { ensured at least } 95 \% \text { power to de- } \\
\text { clare non-inferiority between either } \\
\text { alogliptin dose ( } 12.5 \text { or } 25 \mathrm{mg} \text { ) and } \\
\text { glipizide at week } 104 \text {, assuming a non- } \\
\text { inferiority margin of } 0.3 \% \text {, no differ- } \\
\text { ence between either alogliptin dose }\end{array}$} & 5789 & 874 & 336 & 427 & 48.9 & \multirow[t]{2}{*}{$\begin{array}{l}104 \text { weeks } \\
\text { (+ } 2 \text { weeks }\end{array}$} \\
\hline & $\begin{array}{l}\mathrm{C} 1: \text { metformin } \geq \\
1500 \text { mg once dai- } \\
\text { ly or maximum } \\
\text { tolerated dose }+\end{array}$ & & & 880 & 371 & 472 & 53.6 & \\
\hline
\end{tabular}


Table 1. Overview of trial populations (Continued)

alogliptin $12.5 \mathrm{mg}$ and glipizide, a standard deviation

once daily

of change from baseline of $1.2 \%$, an

evaluability rate of $60 \%$, and a one-

C2: metformin $\geq$ sided 0.0125 significance level. The

$1500 \mathrm{mg}$ once dai- $\quad 0.0125$ significance level was chosen

ly or maximum

so that, combined with similar analy-

ses conducted at week 52 , the overall

alogliptin $25 \mathrm{mg}$ one-sided type 1 error rate for the trial

once daily was maintained at the 0.025 level"

885

382

493

55.7

Total:

Ahrén 2014 I: metformin $\geq$

$1500 \mathrm{mg}$ daily +

glimepiride 2-4 mg

once daily + place-

ority paral-
lel RCT) bo

C1: metformin $\geq$

$1500 \mathrm{mg}$ daily +

albiglutide $30-50$

mg once weekly +

placebo

C2: metformin $\geq$

$1500 \mathrm{mg}$ daily +

sitagliptin $100 \mathrm{mg}$

once daily + place-

bo

C3: metformin $\geq$

$1500 \mathrm{mg}$ daily +

placebo

Quote from publication: "The

planned sample size provided $>90 \%$

power to demonstrate superiority ver-

sus placebo and noninferiority ver-

sus sitagliptin and glimepiride (non-

inferiority margin $=0.3 \%$ ). Superiori-

ty of albiglutide versus sitagliptin and

glimepiride was tested if noninferiority

was established"

88

190

60.7

60.3

Total:

104 weeks

(+ 52 weeks)

\begin{tabular}{|c|c|c|c|c|c|c|c|c|}
\hline & Total: & & & 1049 & 321 & 628 & 59.9 & \\
\hline \multirow{3}{*}{$\begin{array}{l}\text { Ridderstråle } \\
2014 \\
\text { (non-inferi- } \\
\text { ority paral- } \\
\text { lel RCT) }\end{array}$} & $\begin{array}{l}\text { I: metformin im- } \\
\text { mediate release } \geq\end{array}$ & \multirow{3}{*}{$\begin{array}{l}\text { Quote from publication: "698 pa- } \\
\text { tients per group were needed to pro- } \\
\text { vide a power of at least } 95 \% \text { to show } \\
\text { non-inferiority, based on a margin } \\
\text { of } 0.3 \% \text {, for the primary endpoint at } \\
\text { weeks } 52 \text { and } 104 \text { if the true treatment } \\
\text { effect is } 0.05 \% \text { (in favour of glimepiri- } \\
\text { de) and SD is } 1.2 \% "\end{array}$} & \multirow[t]{3}{*}{2637} & 780 & 780 & $\begin{array}{l}648(2 \\
\text { years) }\end{array}$ & $\begin{array}{l}83.1(2 \\
\text { years })\end{array}$ & \multirow[t]{3}{*}{208 weeks } \\
\hline & $\begin{array}{l}1500 \mathrm{mg} \text { /day plus } \\
\text { glimepiride } 1-4 \\
\text { mg/day }\end{array}$ & & & & & 589 (4 years) & $\begin{array}{l}75.5(4 \\
\text { years })\end{array}$ & \\
\hline & $\begin{array}{l}\text { C: metformin im- } \\
\text { mediate release } \geq\end{array}$ & & & 769 & 765 & $\begin{array}{l}652 \text { ( } 2 \text { years) } \\
610 \text { ( } 4 \text { years) }\end{array}$ & $\begin{array}{l}84.8(2 \\
\text { years })\end{array}$ & \\
\hline
\end{tabular}




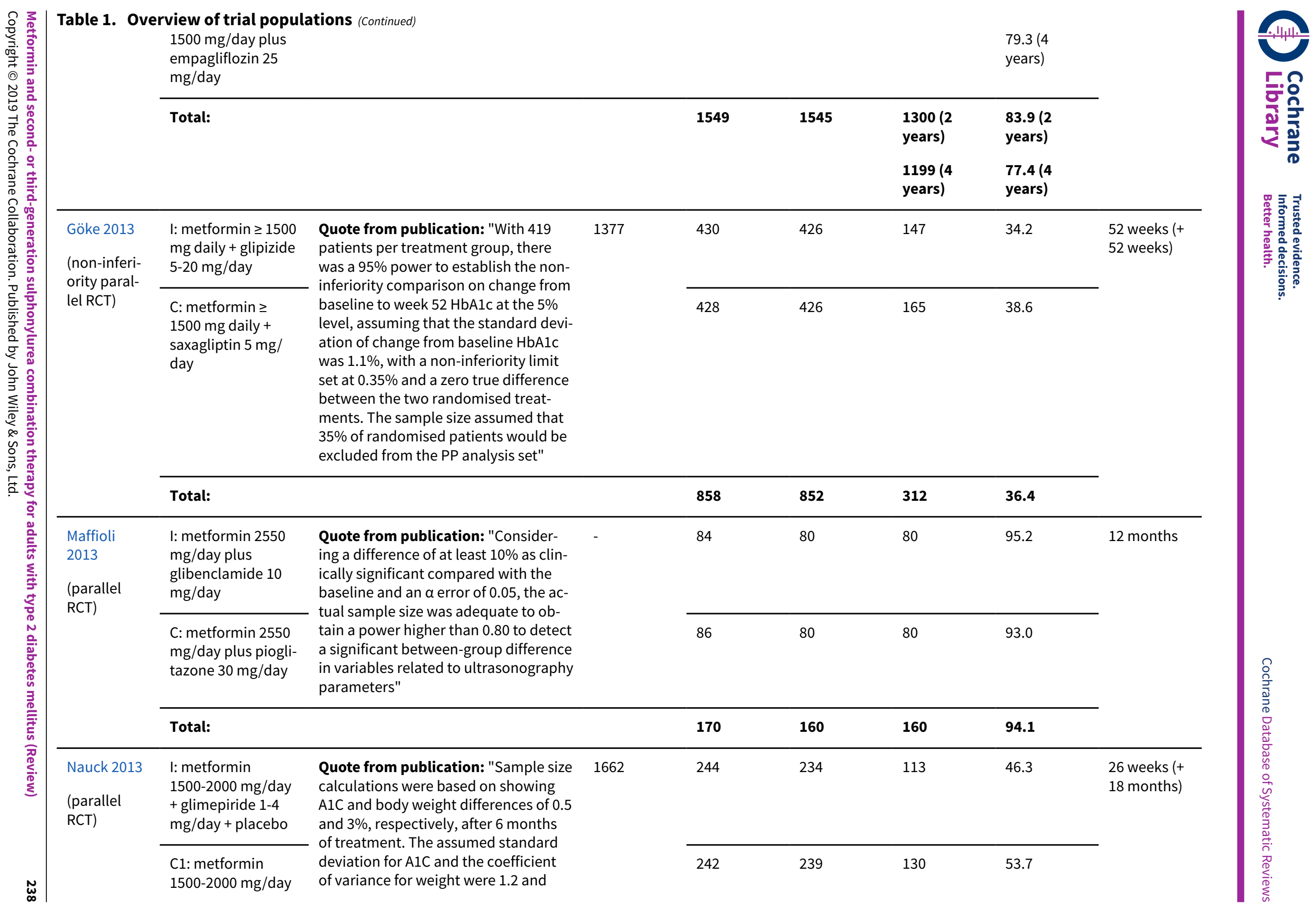




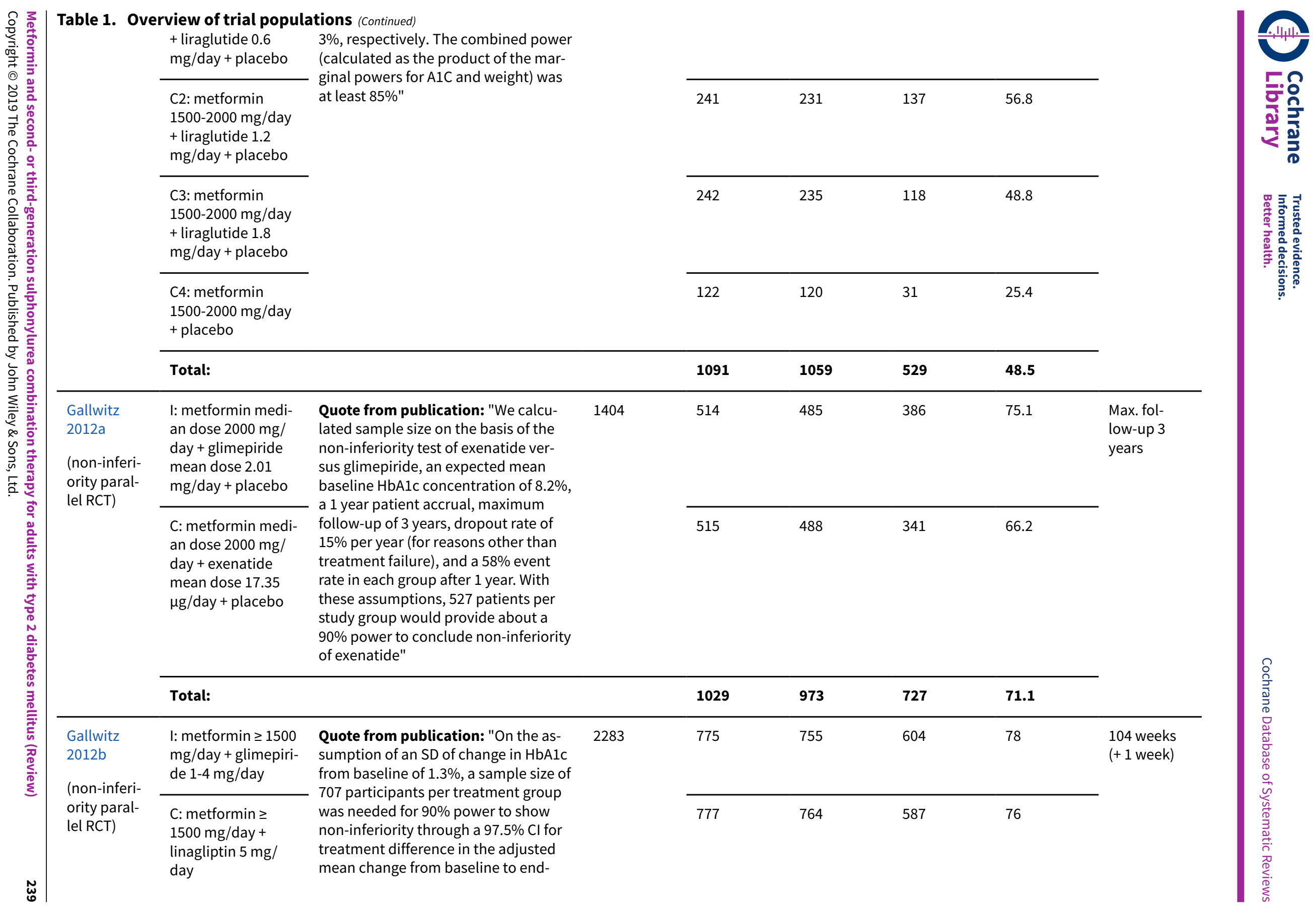




\begin{tabular}{|c|c|c|c|c|c|c|c|c|}
\hline & & $\begin{array}{l}\text { point of }<0.35 \% \mathrm{HbA} 1 \mathrm{c} \text { at the level of } \\
\alpha=0.0125 \text { (one-sided)" }\end{array}$ & & & & & & \\
\hline & Total: & & & 1552 & 1519 & 1191 & 77 & \\
\hline \multirow[t]{3}{*}{$\begin{array}{l}\text { Derosa } \\
2011 a \\
\text { (parallel } \\
\text { RCT) }\end{array}$} & $\begin{array}{l}\text { I: metformin } \\
\text { 1000-2000 mg/day } \\
\text { + glimepiride } 6 \mathrm{mg} / \\
\text { day }\end{array}$ & \multirow{2}{*}{$\begin{array}{l}\text { Quote from publication: "Consider- } \\
\text { ing as clinically significant a difference } \\
\text { of at least the } 10 \% \text { compared to the } \\
\text { baseline and an alpha error of } 0.05 \text {, } \\
\text { the actual sample size was adequate } \\
\text { to obtain a power higher than } 0.80 \text { for } \\
\text { all measured variable" }\end{array}$} & \multirow[t]{2}{*}{-} & 54 & 49 & 49 & 90.7 & \multirow[t]{3}{*}{12 months } \\
\hline & $\begin{array}{l}\text { C: metformin } \\
1000-2000 \mathrm{mg} / \mathrm{day} \\
\text { + exenatide } 20 \mu \mathrm{g} / \\
\text { day }\end{array}$ & & & 57 & 52 & 52 & 91.2 & \\
\hline & Total: & & & 111 & 101 & 101 & 91.0 & \\
\hline \multirow[t]{3}{*}{$\begin{array}{l}\text { Derosa } \\
\text { 2011b } \\
\text { (parallel } \\
\text { RCT) }\end{array}$} & $\begin{array}{l}\text { I: metformin } 1700 \\
\pm 850 \text { mg/day }+ \\
\text { glibenclamide } 5-15 \\
\text { mg/day }\end{array}$ & \multirow{2}{*}{$\begin{array}{l}\text { Quote from publication: "Consider- } \\
\text { ing as clinically significant a difference } \\
\text { of at least the } 10 \% \text { compared to the } \\
\text { baseline and an alpha error of } 0.05, \\
\text { the actual sample size was adequate } \\
\text { to obtain a power higher than } 0.80 \text { for } \\
\text { all measured variables" }\end{array}$} & \multirow[t]{2}{*}{-} & 99 & 95 & 95 & 96.0 & \multirow[t]{3}{*}{12 months } \\
\hline & $\begin{array}{l}\text { C: metformin } 1700 \\
\pm 850 \mathrm{mg} / \text { day }+ \text { pi- } \\
\text { oglitazone } 15-45 \\
\mathrm{mg} / \text { day }\end{array}$ & & & 102 & 99 & 99 & 97.1 & \\
\hline & Total: & & & 201 & 194 & 194 & 96.5 & \\
\hline \multirow[t]{3}{*}{$\begin{array}{l}\text { Petrica } 2011 \\
\text { (parallel } \\
\text { RCT) }\end{array}$} & $\begin{array}{l}\text { I: metformin } 1700 \\
\text { mg/day plus } \\
\text { glimepiride } 4 \text { mg/ } \\
\text { day }\end{array}$ & - & 124 & 39 & 34 & 34 & 87.2 & \multirow[t]{3}{*}{1 year } \\
\hline & $\begin{array}{l}\text { C: metformin } 1700 \\
\mathrm{mg} / \text { day plus piogli- } \\
\text { tazone } 30 \mathrm{mg} / \text { day }\end{array}$ & & & 39 & 34 & 34 & 87.2 & \\
\hline & Total: & & & 78 & 68 & 68 & 87.2 & \\
\hline $\begin{array}{l}\text { Derosa } 2010 \\
\text { (parallel } \\
\text { RCT) }\end{array}$ & $\begin{array}{l}\text { I: metformin } 1500 \\
\pm 500 \mathrm{mg} / \text { day }+ \\
\text { glibenclamide } 15 \\
\mathrm{mg} / \text { day }\end{array}$ & - & - & 65 & 57 & 57 & 87.7 & 12 months \\
\hline
\end{tabular}




\begin{tabular}{|c|c|c|c|c|c|c|c|c|}
\hline & $\begin{array}{l}\text { C: metformin } 1500 \\
\pm 500 \mathrm{mg} / \mathrm{day}+\mathrm{ex}- \\
\text { enatide } 20 \mu \mathrm{g} / \mathrm{day}\end{array}$ & & & 63 & 59 & 59 & 93.7 & \\
\hline & Total: & & & 128 & 116 & 116 & 90.6 & \\
\hline \multirow{3}{*}{$\begin{array}{l}\text { Matthews } \\
2010 \\
\text { (non-inferi- } \\
\text { ority paral- } \\
\text { lel RCT) }\end{array}$} & $\begin{array}{l}\text { I: } \text { metformin } \geq 1500 \\
\text { mg twice a day + } \\
\text { glimepiride 2-6 } \\
\text { mg/day }\end{array}$ & \multirow{2}{*}{$\begin{array}{l}\text { Quote from publication: "With } 3120 \\
\text { patients randomized (i.e. } 1560 \text { pa- } \\
\text { tients per treatment arm) and an } \\
\text { overall } 20 \% \text { discontinuation rate, the } \\
\text { study had } 96 \% \text { power to show non- } \\
\text { inferiority of vildagliptin compared } \\
\text { with glimepiride (one-sided a level } \\
\text { of } 0.0125 \text {, assuming a non-inferiority } \\
\text { margin of } 0.3 \% \mathrm{HbA} 1 \mathrm{c} \text { and a standard } \\
\text { deviation of } 1.25 \%) "\end{array}$} & \multirow[t]{2}{*}{$\begin{array}{l}\text { approx. } \\
6000\end{array}$} & 1556 & 1518 & 953 & 61.2 & \multirow[t]{3}{*}{2 years } \\
\hline & $\begin{array}{l}\text { C: metformin } \geq \\
1500 \text { mg twice a } \\
\text { day + vildagliptin } \\
50 \text { mg twice a day }\end{array}$ & & & 1562 & 1476 & 994 & 63.6 & \\
\hline & Total: & & & 3118 & 2994 & 1947 & 62.4 & \\
\hline \multirow[t]{3}{*}{$\begin{array}{l}\text { Filozof } 2010 \\
\text { (non-inferi- } \\
\text { ority paral- } \\
\text { lel RCT) }\end{array}$} & $\begin{array}{l}\text { I: metformin } 1500 \\
\text { mg/day plus gli- } \\
\text { clazide } 80-320 \text { mg/ } \\
\text { day }\end{array}$ & \multirow{2}{*}{\multicolumn{2}{|c|}{$\begin{array}{l}\text { Quote from publication: "Eight hun- } \\
\text { dred patients ( } 400 \text { per group) were re- } \\
\text { quired to demonstrate non-inferiori- } \\
\text { ty of vildagliptin to gliclazide in HbAlc } \\
\text { reduction, with a one-sided a level of } \\
0.025 \text { at the end of the study with } 92 \% \\
\text { power (assuming a true difference of } \\
0.1 \% \text { in favour of gliclazide, standard } \\
\text { deviation of HbA1c reduction at week } \\
52 \text { of } 1.25 \text { units and discontinuation } \\
\text { rate of } 20 \% \text { over the } 52 \text {-week period)" }\end{array}$}} & 494 & 393 & 412 & 83.4 & \multirow[t]{3}{*}{52 weeks } \\
\hline & $\begin{array}{l}\text { C: metformin } \\
1500 \text { mg/day plus } \\
\text { vildagliptin } 100 \\
\text { mg/day }\end{array}$ & & & 513 & 386 & 407 & 79.3 & \\
\hline & Total: & & & 1007 & 779 & 819 & 81.3 & \\
\hline \multirow{3}{*}{$\begin{array}{l}\text { Seck } 2010 \\
\text { (non-inferi- } \\
\text { ority paral- } \\
\text { lel RCT) }\end{array}$} & $\begin{array}{l}\text { I: metformin } \geq 1500 \\
\mathrm{mg} / \text { day plus glip- } \\
\text { izide } 5-20 \mathrm{mg} / \text { day }\end{array}$ & - & 2141 & 584 & 559 & 264 & 45.2 & \multirow[t]{3}{*}{2 years } \\
\hline & $\begin{array}{l}\text { C: metformin } \geq \\
1500 \mathrm{mg} / \text { day plus } \\
\text { sitagliptin } 100 \mathrm{mg} / \\
\text { day }\end{array}$ & & & 588 & 576 & 255 & 43.4 & \\
\hline & Total: & & & 1172 & 1135 & 519 & 44.3 & \\
\hline
\end{tabular}




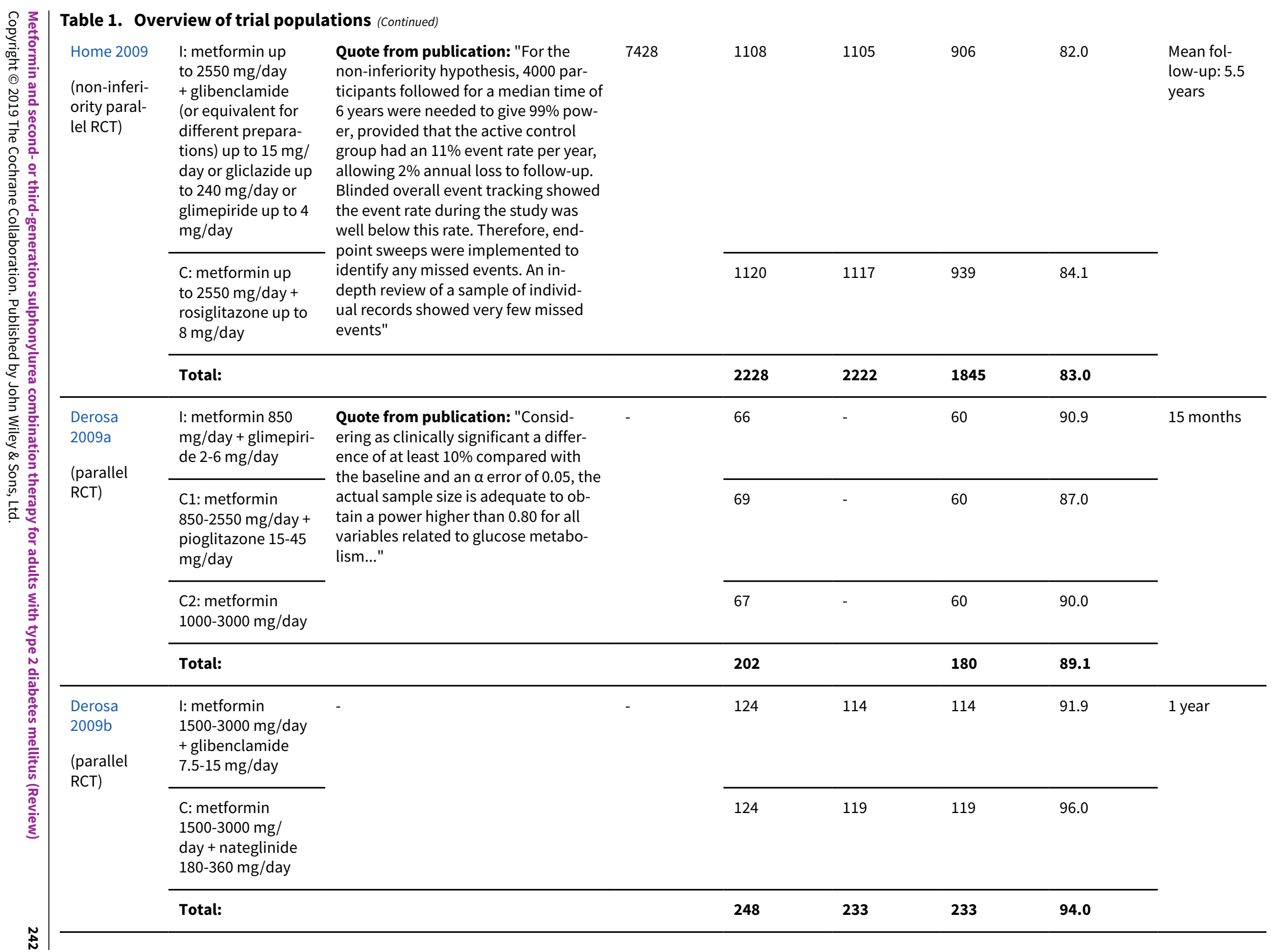




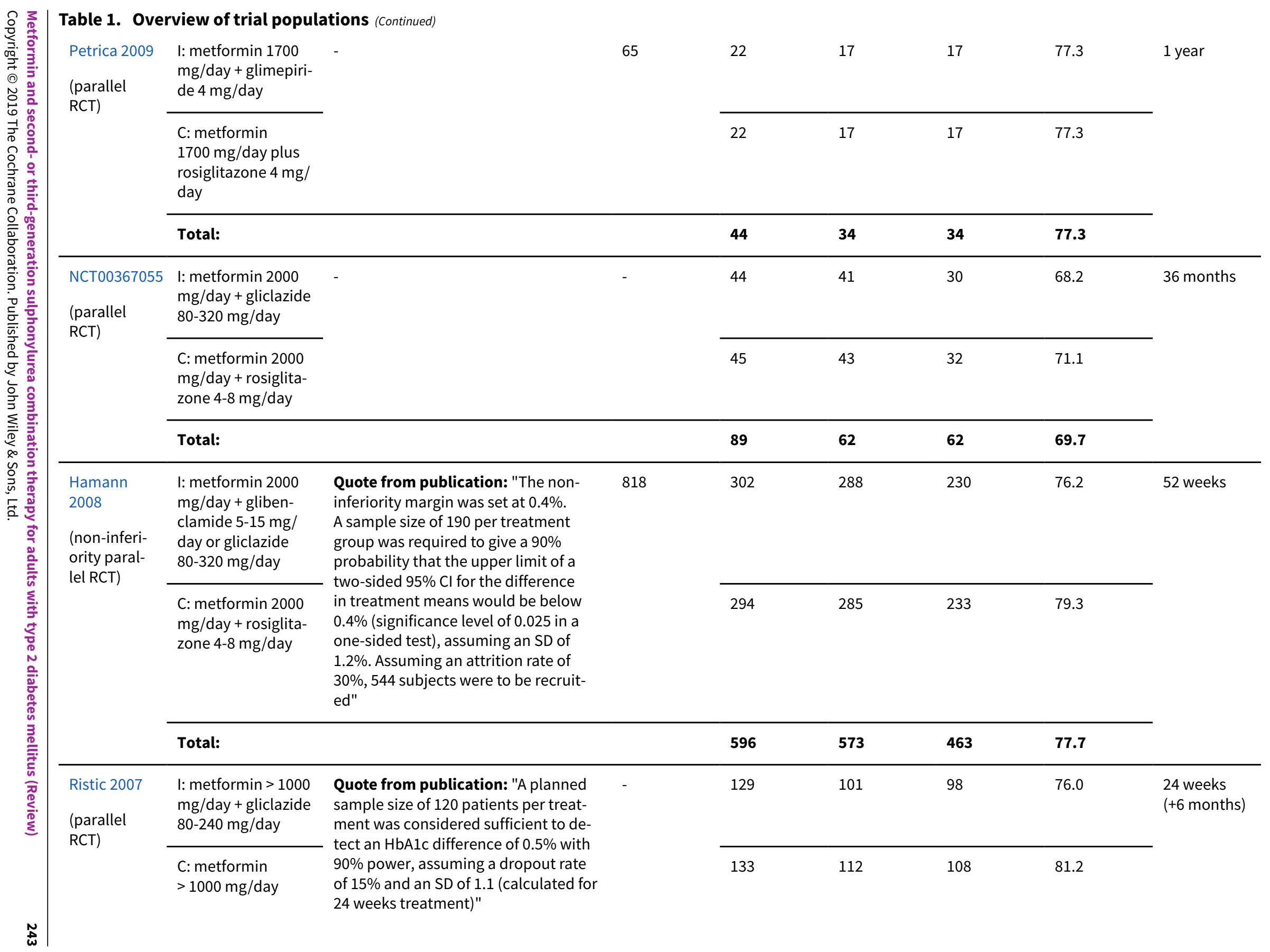




\begin{tabular}{|c|c|c|c|c|c|c|c|c|}
\hline & $\begin{array}{l}+ \text { nateglinide } \\
180-540 \mathrm{mg} / \text { day }\end{array}$ & & & & & & & \\
\hline & Total: & & & 262 & 213 & 206 & 78.6 & \\
\hline \multirow[t]{3}{*}{$\begin{array}{l}\text { Charbonnel } \\
2005 \\
\text { (parallel } \\
\text { RCT) }\end{array}$} & $\begin{array}{l}\text { I: metformin at pre- } \\
\text { study dose }+ \text { gli- } \\
\text { clazide } 80-320 \mathrm{mg} / \\
\text { day }\end{array}$ & \multirow{2}{*}{$\begin{array}{l}\text { Quote from publication: "Sample } \\
\text { size was based on demonstrating a be- } \\
\text { tween-group difference of } 0.35 \% \text { in } \\
\text { the change in HbA1c from baseline to } \\
\text { week } 52 \text { (the primary efficacy variable) } \\
\text { using a two-sided t-test. A total of } 225 \\
\text { patients/group completing at least } 24 \\
\text { weeks of the study was required, on } \\
\text { the basis of a level of } 95 \% \text { power at } 5 \% \\
\text { significance" }\end{array}$} & \multirow[t]{2}{*}{1071} & 313 & - & 238 & 76.0 & \multirow[t]{3}{*}{104 weeks } \\
\hline & $\begin{array}{l}\text { C: metformin at } \\
\text { pre-study dose }+ \\
\text { pioglitazone } 15-45 \\
\mathrm{mg} / \text { day }\end{array}$ & & & 317 & - & 233 & 73.5 & \\
\hline & Total: & & & 630 & - & 471 & 74.8 & \\
\hline \multirow[t]{3}{*}{$\begin{array}{l}\text { Derosa } 2005 \\
\text { (parallel } \\
\text { RCT) }\end{array}$} & $\begin{array}{l}\text { I: metformin } 1500 \\
\text { mg/day plus } \\
\text { glimepiride } 2 \text { mg/ } \\
\text { day }\end{array}$ & \multirow{2}{*}{$\begin{array}{l}\text { Quote from publication: "The study } \\
\text { power was a priori calculated by using } \\
\text { the World Wide Web-available power } \\
\text { calculator of the university of Califor- } \\
\text { nia, Los Angeles, Department of statis- } \\
\text { tics (Los Angeles, CA)" }\end{array}$} & - & 49 & 47 & 47 & 95.9 & \multirow[t]{3}{*}{12 months } \\
\hline & $\begin{array}{l}\text { C: metformin } \\
1500 \mathrm{mg} / \text { day plus } \\
\text { rosiglitazone } 4 \mathrm{mg} / \\
\text { day }\end{array}$ & & & 50 & 48 & 48 & 96.0 & \\
\hline & Total: & & & 99 & 95 & 95 & 96.0 & \\
\hline \multirow[t]{3}{*}{$\begin{array}{l}\text { Gerich } 2005 \\
\text { (parallel } \\
\text { RCT) }\end{array}$} & $\begin{array}{l}\text { I: metformin } \\
500-2000 \mathrm{mg} / \text { day } \\
+ \text { glyburide } 1.25-15 \\
\mathrm{mg} / \text { day + placebo }\end{array}$ & - & 908 & 209 & 198 & 122 & 58.4 & \multirow[t]{3}{*}{104 weeks } \\
\hline & $\begin{array}{l}\text { C: metformin } \\
500-2000 \mathrm{mg} / \\
\text { day + nateglinide } \\
180-540 \mathrm{mg} / \text { day + } \\
\text { placebo }\end{array}$ & & & 219 & 208 & 141 & 64.4 & \\
\hline & Total: & & & 428 & 406 & 263 & 61.4 & \\
\hline Grand total & All interventions & & & 12,863 & & & & \\
\hline
\end{tabular}




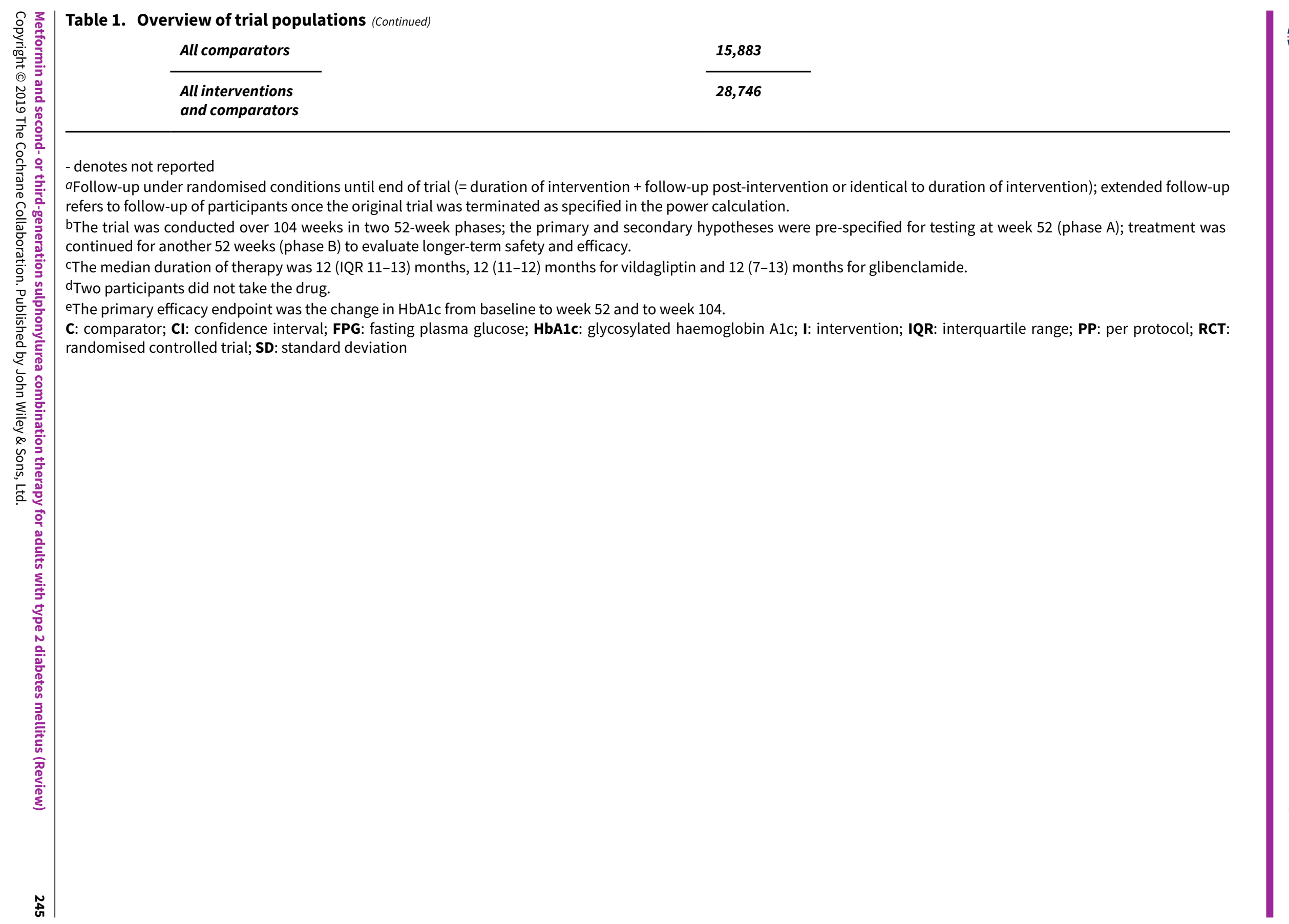


Table 2. Overview of trials (trial arms), comparators, intervention and number of randomised participants

\begin{tabular}{|c|c|c|c|}
\hline Drug class & $\begin{array}{l}\text { Trials (trial arms) } \\
\text { (N)a }\end{array}$ & $\begin{array}{l}\text { Metformin + comparator: randomised par- } \\
\text { ticipants }(\mathrm{N})\end{array}$ & $\begin{array}{l}\text { Metformin + sulphonylurea: ran- } \\
\text { domised participants }(\mathrm{N})^{\mathrm{b}}\end{array}$ \\
\hline \multirow[t]{12}{*}{$\begin{array}{l}\text { Thiazolidine- } \\
\text { diones }\end{array}$} & 1 & Pioglitazone 15-45 mg: 1541 & $\begin{array}{l}\text { Glimepiride/glibenclamide/gli- } \\
\text { clazide: } 1500\end{array}$ \\
\hline & 1 & Pioglitazone 15-45 mg: 102 & \\
\hline & 1 & Pioglitazone 15-45 mg: 69 & Glibenclamide: 99 \\
\hline & 1 & Pioglitazone: 15-45 mg: 317 & Glimepiride: 66 \\
\hline & 1 & Pioglitazone 30 mg: 86 & Gliclazide: 313 \\
\hline & 1 & Pioglitazone 30 mg: 39 & Glibenclamide: 84 \\
\hline & 1 & Rosiglitazone 4 mg: 22 & Glimepiride: 39 \\
\hline & 1 & Rosiglitazone 4 mg: 50 & Glimepiride: 22 \\
\hline & 1 & Rosiglitazone 4-8 mg: 45 & Glimepiride: 49 \\
\hline & 1 & Rosiglitazone 4-8 mg: 294 & Gliclazide: 44 \\
\hline & 1 & Rosiglitazone up to $8 \mathrm{mg}: 1120$ & Glibenclamide/gliclazide: 302 \\
\hline & Total:11 (11) & Total:3685 & $\begin{array}{l}\text { Glibenclamide/gliclazide/glimepiri- } \\
\text { de: } 1108\end{array}$ \\
\hline
\end{tabular}

Total:3626

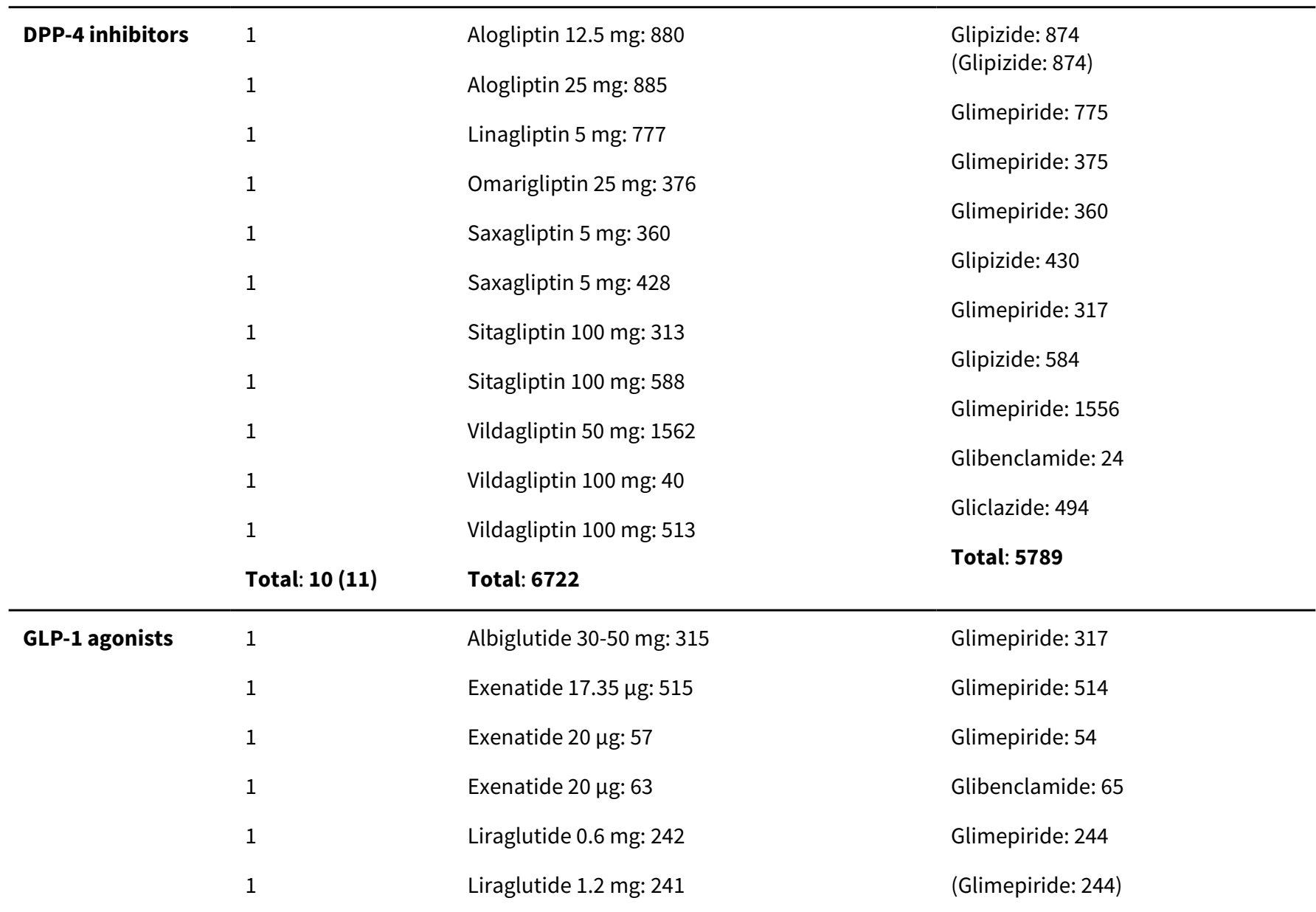


Table 2. Overview of trials (trial arms), comparators, intervention and number of randomised participants (Continued)

1

Total: 5 (7)

\section{SGLT-2 inhibitors}

1

1

1

1

1

1

Total: 4 (6)

\begin{tabular}{|c|c|c|c|}
\hline \multirow[t]{4}{*}{ Glinides } & 1 & Nateglinide $180-360$ mg: 124 & Glibenclamide: 124 \\
\hline & 1 & Nateglinide 180-540 mg: 133 & Gliclazide: 129 \\
\hline & 1 & Nateglinide 180-540 mg: 219 & Glyburide: 209 \\
\hline & Total: 3 (3) & Total: 476 & Total: 462 \\
\hline \multirow{4}{*}{$\begin{array}{l}\text { Metformin } \\
\text { monotherapy }\end{array}$} & 1 & Metformin $\geq 1500 \mathrm{mg:} 104$ & (Glimepiride: 317 ) \\
\hline & 1 & Metformin 1500-2000 mg: 122 & (Glimepiride: 244) \\
\hline & 1 & Metformin 1000-3000 mg: 67 & (Glimepiride: 66) \\
\hline & Total:3 (3) & Total: 293 & (Total: 627) \\
\hline
\end{tabular}

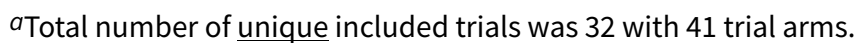

bNumbers of randomised participants for metformin combination therapy do not add up correctly because several trials had one intervention with several comparator groups, the intervention group may therefore appear in several drug classes and is characterised by parentheses; combination therapy data for metformin monotherapy are shown for illustrative purposes.

DPP4-I: dipeptidyl-peptidase 4; GLP-1: glucagon-like peptide-1 agonist; SGLT-2: sodium-glucose transport 2

Table 3. Overview of HbA1c and hypoglycaemia across comparisons

\begin{tabular}{|c|c|c|c|c|}
\hline Comparison & $\begin{array}{l}\text { Better HbA1c } \\
\text { for } M+S\end{array}$ & $\begin{array}{l}\text { Better HbA1c } \\
\text { for comparator }\end{array}$ & $\begin{array}{l}\text { Less hypogly- } \\
\text { caemia for } M+S\end{array}$ & $\begin{array}{l}\text { Less hypoglycaemia for compara- } \\
\text { tor }\end{array}$ \\
\hline$M+S$ vs metformin + placebo & Yes & No & No & Yes: mild or moderate episodes \\
\hline $\begin{array}{l}\text { M+S vs metformin + GLP-1 ago- } \\
\text { nist }\end{array}$ & No & No & No & Yes: mild or moderate episodes \\
\hline $\begin{array}{l}\text { M+S vs metformin + DPP-4 in- } \\
\text { hibitor }\end{array}$ & Yes & No & No & $\begin{array}{l}\text { Yes: mild or moderate and serious } \\
\text { episodes }\end{array}$ \\
\hline $\begin{array}{l}\text { M+S vs metformin + thiazo- } \\
\text { lidinedione }\end{array}$ & No & Yes & No & $\begin{array}{l}\text { Yes: mild or moderate and serious } \\
\text { episodes }\end{array}$ \\
\hline$M+S$ vs metformin + glinide & No & Yes & No & Yes: mild or moderate episodes \\
\hline $\begin{array}{l}\text { M+S vs metformin + SGLT-2 in- } \\
\text { hibitor }\end{array}$ & No & Yes & No & $\begin{array}{l}\text { Yes: mild or moderate and serious } \\
\text { episodes }\end{array}$ \\
\hline
\end{tabular}

Metformin and second- or third-generation sulphonylurea combination therapy for adults with type 2 diabetes mellitus (Review) 
DPP4-I: dipeptidyl-peptidase 4; GLP-1: glucagon-like peptide-1 agonist; HbA1c: glycosylated haemoglobin A1c; M+S: metformin + sulphonylurea; SGLT-2: sodium-glucose transport 2

\section{AP PE N DICES}

\section{Appendix 1. Search strategies}

\section{MEDLINE (Ovid SP)}

1. exp Diabetes Mellitus, Type 2/

2. (MODY or NIDDM or T2D*).tw.

3. (non insulin* depend ${ }^{\star}$ or noninsulin* depend ${ }^{\star}$ or noninsulin?depend ${ }^{\star}$ or non insulin?depend ${ }^{\star}$ ).tw.

4. ((typ? 2 or typ? II or typ?2 or typ?II) adj3 diabet*).tw.

5. (((late or adult* or matur ${ }^{\star}$ or slow or stabl $\left.{ }^{\star}\right)$ adj3 onset) and diabet $\left.{ }^{\star}\right)$.tw.

6. or/1-5

7. Metformin/

8. metformin ${ }^{\star}$. tw.

9. $\mathrm{or} / 7-8$

10. exp Sulfonylurea Compounds/

11. (sulfon?lurea* or sulphon?lurea*).tw.

12. (gl?benclamid* ${ }^{\star}$ glyburid ${ }^{\star}$ or HB 419 OR HB419 or HB 420 OR HB420).tw.

13. (gl?bornurid* or Ro 64563 or Ro 4563 or gluborid $\left.{ }^{\star}\right)$.tw.

14. (glipizid* or gl?diazinamide or glypidizine or K 4024 or K4024 or melizide or napizide).tw.

15. (gliquidon* or AR DF 26 or ARDF 26 or ARDF26).tw.

16. (glisoxepid* or RP 22410 or BS 4231).tw.

17. gl?clopyramid*.tw.

18. (glimepirid* or HOE 490).tw.

19. (gl?clazid* or gl?cazid* or S 1702 or S1702 or S 852 OR S852).tw.

20. or $/ 10-19$

21. 6 and 9 and 20

[22-32: Cochrane Handbook 2008 RCT filter - sensitivity max. version]

22. randomized controlled trial.pt.

23. controlled clinical trial.pt.

24. randomi?ed.ab.

25. placebo.ab.

26. drug therapy.fs. 
(Continued)

27. randomly.ab.

28. trial.ab.

29. groups.ab.

30. or/22-29

31. exp animals/ not humans/

32. 30 not 31

33. 21 and 32

[34: Wong 2006a-systematic reviews filter - SensSpec version]

34. meta analysis.mp,pt. or review.pt. or search*.tw.

35. 21 and 34

36.33 or 35

37. $\left(2015^{\star}\right.$ or $\left.2016^{\star}\right) \cdot d c$.

38. 36 and 37

39. ..dedup 38

Cochrane Central Register of Controlled Trials (CENTRAL) via Cochrane Register of Studies Online (CRSO)
1. MESH DESCRIPTOR Diabetes Mellitus, Type 2 EXPLODE ALL TREES
2. (MODY OR NIDDM OR T2D*):TI,AB,KY
3. (non insulin* depend* OR noninsulin* depend ${ }^{\star}$ OR noninsulin?depend ${ }^{\star}$ OR non insulin?depend ${ }^{\star}$ ):TI,AB,KY
4. ((typ? 2 OR typ? II OR typ?2 OR typ?II) ADJ3 diabet):TI,AB,KY
5. (((late OR adult* OR matur* OR slow OR stabl $\left.{ }^{\star}\right)$ ADJ3 onset) AND diabet $\left.{ }^{\star}\right): T I, A B, K Y$
6. \#1 OR \#2 OR \#3 OR \#4 OR \#5
7. MESH DESCRIPTOR Metformin
8. metformin*:TI,AB,KY

9. \#7 OR \#8

10. MESH DESCRIPTOR Sulfonylurea Compounds EXPLODE ALL TREES

11. (sulfon?lurea* OR sulphon?lurea*):TI,AB,KY

12. (gl?benclamid* OR glyburid* OR HB 419 OR HB419 OR HB 420 OR HB420):TI,AB,KY

13. (gl?bornurid* OR Ro 64563 OR Ro 4563 OR gluborid*):TI,AB,KY

14. (glipizid* OR gl?diazinamide OR glypidizine OR K 4024 OR K4024 OR melizide OR napizide):TI,AB,KY

15. (gliquidon* OR AR DF 26 OR ARDF 26 OR ARDF26):TI,AB,KY

16. (glisoxepid* OR RP 22410 OR BS 4231):TI,AB,KY

17. gl?clopyramid*:TI,AB,KY

18. (glimepirid* OR HOE 490):TI,AB,KY

19. (gl?clazid* OR gl?cazid* OR S 1702 OR S1702 OR S 852 OR S852):TI,AB,KY 
(Continued)

20. \#10 OR \#11 OR \#12 OR \#13 OR \#14 OR \#15 OR \#16 OR \#17 OR \#18 OR \#19

21. \#6 AND \#9 AND \#20

22. 2015 TO 2016:YR

23. \#21 AND \#22

\section{Embase (Ovid SP)}

1. non insulin dependent diabetes mellitus/

2. (MODY or NIDDM or T2D*).tw.

3. (non insulin* depend ${ }^{\star}$ or noninsulin* depend ${ }^{\star}$ or noninsulin?depend ${ }^{\star}$ or non insulin?depend $\left.{ }^{\star}\right)$.tw.

4. ((typ? 2 or typ? II or typ?2 or typ?II) adj3 diabet*).tw.

5. (((late or adult ${ }^{\star}$ or matur ${ }^{\star}$ or slow or stabl $\left.{ }^{\star}\right)$ adj3 onset $)$ and diabet $\left.{ }^{\star}\right)$. tw.

6. or/1-5

7. Metformin/

8. metformin ${ }^{\star}$. tw.

9. $\mathrm{or} / 7-8$

10. gliamilide/ or glibenclamide/ or glibornuride/ or glicaramide/ or gliclazide/ or glicondamide/ or gliflumide/ or glimepiride/ or glipalamide/ or glipentide/ or glipizide/ or gliquidone/ or glisamuride/ or glisolamide/ or glisoxepide/ or glucosulfa/or glybuthiazol/or glybuzole/ or glycyclamide/ or glyhexamide/ or glyoctamide/ or glyparamide/ or glypinamide/ or glyprothiazol/ or glysobuzole/

11. (sulfon?lurea* or sulphon?lurea*).tw.

12. (gl?benclamid* or glyburid* or HB 419 OR HB419 or HB 420 OR HB420).tw.

13. (gl?bornurid ${ }^{\star}$ or Ro 64563 or Ro 4563 or gluborid $\left.{ }^{\star}\right)$.tw.

14. (glipizid* or gl?diazinamide or glypidizine or K 4024 or K4024 or melizide or napizide).tw.

15. (gliquidon* or AR DF 26 or ARDF 26 or ARDF26).tw.

16. (glisoxepid* or RP 22410 or BS 4231).tw.

17. gl?clopyramid*.tw.

18. (glimepirid* or HOE 490).tw.

19. (gl?clazid* or gl?cazid* or S 1702 or S1702 or S 852 OR S852).tw.

20. or/10-19

21.6 and 9 and 20

[22: Wong 2006b "sound treatment studies" filter - BS version]

22. random*.tw. or clinical trial ${ }^{\star}$.mp. or exp health care quality/

23. 21 and 22

24. $\left(2015^{\star}\right.$ or $\left.2016^{\star}\right) \cdot d d$.

25. 23 and 24

26. conference ${ }^{\star}$.pt.

27.25 not 26

Metformin and second- or third-generation sulphonylurea combination therapy for adults with type 2 diabetes mellitus (Review) Copyright @ 2019 The Cochrane Collaboration. Published by John Wiley \& Sons, Ltd. 


\section{ClinicalTrials.gov (Expert search)}

INFLECT EXACT "Interventional" [STUDY-TYPES] AND ( diabetes OR diabetic OR "type 2" OR "type II" OR T2D OR T2DM ) [DISEASE] AND ( (gliamilide OR glibenclamide OR glybenclamide OR glibornuride OR glybornuride OR glicaramide OR gliclazide OR glyclazide OR glicondamide OR gliflumide OR glimepiride OR glipalamide OR glipentide OR glipizide OR glydiazinamide OR glidiazinamide OR glypidizine OR melizide OR napidizide OR gliquidone OR glisamuride OR glisolamide OR glisoxepide OR glucosulfa OR glyburide OR glybuthiazol OR gluboride OR glybuzole OR glycyclamide OR glyhexamide OR glyoctamide OR glyparamide OR glypinamide OR glyprothiazol OR glysobuzole OR gliclopyramide OR glyclopyramide OR "HB 419" OR HB419 OR "HB 420" OR HB420 OR "Ro 64563 " OR "Ro 4563" OR "K 4024" OR K4024 OR "AR DF 26" OR "ARDF 26" OR ARDF26 OR "RP 22410" OR "BS 4231" OR "HOE 490" OR "S 1702" OR S1702 OR "S 852" OR S852 OR sulfonylurea OR sulfonilurea OR sulfonylureas OR sulfonilureas OR sulphonylurea OR sulphonilurea OR sulphonylureas OR sulphonilureas) AND metformin ) [TREATMENT]

\section{WHO International Clinical Trials Registry Platform (ICTRP) Search Portal (Standard search)}

diabet $^{\star}$ AND metformin ${ }^{*}$ AND sulfon* OR

diabet $^{\star}$ AND metformin* AND sulphon* OR

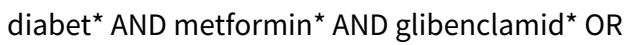

diabet $^{\star}$ AND metformin * AND glybenclamid ${ }^{\star}$ OR

diabet $^{*}$ AND metformin * AND glyburid* OR

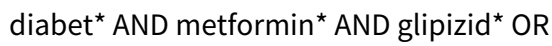

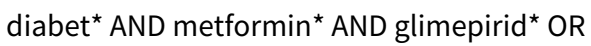

diabet $^{\star}$ AND metformin * AND gliclazid* OR

T2D* AND metformin* AND sulfon* OR

T2D* AND metformin * AND sulphon* OR

T2D* AND metformin * AND glibenclamid ${ }^{\star}$ OR

T2D* AND metformin* AND glybenclamid* OR

T2D* AND metformin* AND glyburid* OR

T2D* AND metformin $^{\star}$ AND glipizid* OR

T2D* AND metformin * AND glimepirid* OR

T2D* AND metformin* AND gliclazid*

\section{Appendix 2. 'Risk of bias' assessment}

\section{'Risk of bias' domains}

\section{Random sequence generation (selection bias due to inadequate generation of a randomised sequence)}

For each included trial, we will describe the method used to generate the allocation sequence in sufficient detail to allow an assessment of whether it should produce comparable groups.

- Low risk of bias: trial authors achieved sequence generation using computer-generated random numbers or a random numbers table. Drawing of lots, tossing a coin, shuffling cards or envelopes, and throwing dice are adequate if an independent person per- 
(Continued)

formed this who was not otherwise involved in the trial. We will consider the use of the minimisation technique as equivalent to being random.

- Unclear risk of bias: insufficient information about the sequence generation process.

- High risk of bias: the sequence generation method was non-random or quasi-random (e.g. sequence generated by odd or even date of birth; sequence generated by some rule based on date (or day) of admission; sequence generated by some rule based on hospital or clinic record number; allocation by judgment of the clinician; allocation by preference of the participant; allocation based on the results of a laboratory test or a series of tests; or allocation by availability of the intervention).

\section{Allocation concealment (selection bias due to inadequate concealment of allocation prior to assignment)}

We will describe for each included trial the method used to conceal allocation to interventions prior to assignment and we will assess whether intervention allocation could have been foreseen in advance of or during recruitment or changed after assignment.

- Low risk of bias: central allocation (including telephone, interactive voice-recorder, internet-based and pharmacy-controlled randomisation); sequentially numbered drug containers of identical appearance; sequentially numbered, opaque, sealed envelopes.

- Unclear risk of bias: insufficient information about the allocation concealment.

- High risk of bias: used an open random allocation schedule (e.g. a list of random numbers); assignment envelopes used without appropriate safeguards; alternation or rotation; date of birth; case record number; any other explicitly unconcealed procedure.

We will also evaluate trial baseline data to incorporate assessment of baseline imbalance into the 'Risk of bias' judgement for selection bias (Corbett 2014).

Chance imbalances may also affect judgements on the risk of attrition bias. In the case of unadjusted analyses, we will distinguish between trials that we rate as being at low risk of bias on the basis of both randomisation methods and baseline similarity, and trials that we judge as being at low risk of bias on the basis of baseline similarity alone (Corbett 2014). We will reclassify judgements of unclear, low, or high risk of selection bias as specified in Appendix 3.

\section{Blinding of participants and study personnel (performance bias due to knowledge of the allocated interventions by partici- pants and personnel during the trial)}

We will evaluate the risk of detection bias separately for each outcome (Hróbjartsson 2013). We will note whether endpoints were self-reported, investigator-assessed, or adjudicated outcome measures (see below).

- Low risk of bias: blinding of participants and key study personnel was ensured, and it was unlikely that the blinding could have been broken; no blinding or incomplete blinding, but we judge that the outcome is unlikely to have been influenced by lack of blinding.

- Unclear risk of bias: insufficient information about the blinding of participants and study personnel; the trial does not address this outcome.

- High risk of bias: no blinding or incomplete blinding, and the outcome is likely to have been influenced by lack of blinding; blinding of trial participants and key personnel attempted, but likely that the blinding could have been broken, and the outcome is likely to be influenced by lack of blinding.

\section{Blinding of outcome assessment (detection bias due to knowledge of the allocated interventions by outcome assessment)}

We will evaluate the risk of detection bias separately for each outcome (Hróbjartsson 2013). We will note whether endpoints were self-reported, investigator-assessed, or adjudicated outcome measures (see below).

- Low risk of bias: blinding of outcome assessment is ensured, and it is unlikely that the blinding could have been broken; no blinding of outcome assessment, but we judge that the outcome measurement is unlikely to have been influenced by lack of blinding.

- Unclear risk of bias: insufficient information about the blinding of outcome assessors; the trial did not address this outcome.

- High risk of bias: no blinding of outcome assessment, and the outcome measurement was likely to have been influenced by lack of blinding; blinding of outcome assessment, but likely that the blinding could have been broken, and the outcome measurement was likely to be influenced by lack of blinding.

\section{Incomplete outcome data (attrition bias due to quantity, nature or handling of incomplete outcome data)}

For each included trial or each outcome, or both, we will describe the completeness of data, including attrition and exclusions from the analyses. We will state whether the trial reported attrition and exclusions, and we will report the number of participants included in the analysis at each stage (compared with the number of randomised participants per intervention/comparator groups). We will also note if the trial reported the reasons for attrition or exclusion, and whether missing data were balanced across groups or were related to outcomes. We will consider the implications of missing outcome data per outcome such as high dropout rates (e.g. above $15 \%$ ) or disparate attrition rates (e.g. difference of $10 \%$ or more between trial arms). 
(Continued)

- Low risk of bias: no missing outcome data; reasons for missing outcome data unlikely to be related to true outcome (for survival data, censoring unlikely to introduce bias); missing outcome data balanced in numbers across intervention groups, with similar reasons for missing data across groups; for dichotomous outcome data, the proportion of missing outcomes compared with observed event risk was not enough to have a clinically relevant impact on the intervention effect estimate; for continuous outcome data, plausible effect size (mean difference or standardised mean difference) among missing outcomes was not enough to have a clinically relevant impact on observed effect size; appropriate methods, such as multiple imputation, were used to handle missing data.

- Unclear risk of bias: insufficient information to assess whether missing data in combination with the method used to handle missing data were likely to induce bias; the trial did not address this outcome.

- High risk of bias: reason for missing outcome data was likely to be related to true outcome, with either imbalance in numbers or reasons for missing data across intervention groups; for dichotomous outcome data, the proportion of missing outcomes compared with observed event risk was enough to induce clinically relevant bias in the intervention effect estimate; for continuous outcome data, plausible effect size (mean difference or standardised mean difference) among missing outcomes enough to induce clinically relevant bias in observed effect size; 'as-treated' or similar analysis done with substantial departure of the intervention received from that assigned at randomisation; potentially inappropriate application of simple imputation.

\section{Selective reporting (reporting bias due to selective outcome reporting)}

We will assess outcome reporting bias by integrating the results of the appendix 'Matrix of trial endpoints (publications and trial documents)' (Boutron 2014; Jones 2015; Mathieu 2009), with those of the appendix 'High risk of outcome reporting bias according to the Outcome Reporting Bias In Trials (ORBIT) classification' (Kirkham 2010). This analysis will form the basis for the judgement of selective reporting.

- Low risk of bias: the trial protocol was available and all the trial's prespecified (primary and secondary) outcomes that were of interest to this review were reported in the prespecified way; the study protocol was unavailable, but it was clear that the published reports included all expected outcomes (ORBIT classification).

- Unclear risk of bias: insufficient information about selective reporting.

- High risk of bias: not all the trial's prespecified primary outcomes were reported; one or more primary outcomes were reported using measurements, analysis methods, or subsets of the data (e.g. subscales) that were not prespecified; one or more reported primary outcomes were not prespecified (unless clear justification for their reporting was provided, such as an unexpected adverse effect); one or more outcomes of interest in the Cochrane Review were reported incompletely so that we cannot enter them into a meta-analysis; the trial report failed to include results for a key outcome that we would expect to have been reported for such a trial (ORBIT classification).

\section{Other bias}

- Low risk of bias: the trial appears to be free from other sources of bias.

- Unclear risk of bias: information was insufficient to assess whether an important risk of bias existed; insufficient rationale or evidence that an identified problem introduced bias.

- High risk of bias: the trial had a potential source of bias related to the specific trial design used; the trial was claimed to be fraudulent; or the trial had some other serious problem.

\section{Appendix 3. Selection bias decisions}

\section{Selection bias decisions for trials that reported unadjusted analyses: comparison of results obtained using method details alone versus results obtained using method details and trial baseline informationa}

\begin{tabular}{|c|c|c|c|}
\hline $\begin{array}{l}\text { Reported randomi- } \\
\text { sation and alloca- } \\
\text { tion concealment } \\
\text { methods }\end{array}$ & $\begin{array}{l}\text { 'Risk of bias' } \\
\text { judgement using } \\
\text { methods reporting }\end{array}$ & Information gained from study characteristics data & $\begin{array}{l}\text { Risk of bias using } \\
\text { baseline informa- } \\
\text { tion and methods } \\
\text { reporting }\end{array}$ \\
\hline
\end{tabular}

\begin{tabular}{ll}
\hline Unclear methods Unclear risk & $\begin{array}{l}\text { Baseline imbalances present for important prognostic vari- } \\
\text { able(s) }\end{array}$
\end{tabular}


Groups appear similar at baseline for all important prognostic variables

Limited or no baseline details

Baseline imbalances present for important prognostic variable(s)

Low risk

truly random sam ple, with robust allocation concealment

\section{Low risk}

Unclear risk

Unclear risk ${ }^{b}$

Groups appear similar at baseline for all important prognostic Low risk variables

Limited baseline details, showing balance in some important Low risk prognostic variablesc

No baseline details Unclear risk

\section{Sequence is not truly High risk} randomised or allocation concealment is inadequate
Baseline imbalances present for important prognostic vari- High risk able(s)
Groups appear similar at baseline for all important prognostic Low risk variables
Limited baseline details, showing balance in some important prognostic variablesc

Unclear risk

No baseline details
High risk

aTaken from Corbett 2014; judgements highlighted in bold indicate situations in which the addition of baseline assessments would change the judgement about risk of selection bias compared with using methods reporting alone.

bImbalance was identified that appears likely to be due to chance.

cDetails for the remaining important prognostic variables are not reported.

\section{Appendix 4. Description of interventions}

\begin{tabular}{|c|c|c|}
\hline Trial ID & Intervention(s) & Comparator(s) \\
\hline Handelsman 2017 & $\begin{array}{l}\text { Metformin + glimepiride + omarigliptin placebo } \\
\text { Metformin dose } \geq 1500 \mathrm{mg} / \text { day + glimepiride titrated } \\
\text { from } 1-6 \mathrm{mg} / \text { day based upon participants self-mon- } \\
\text { itoring blood-glucose results. Omarigliptin placebo } \\
\text { once weekly. } \\
\text { "Patients who were on a maximum tolerated dose of } \\
\text { glimepiride who did not meet progressively stricter } \\
\text { pre-specified glycaemic control criteria post-randomi- } \\
\text { sation (from day } 1 \text { through week } 6 \text {, FPG > } 14.99 \text { mmol/ } \\
\text { L ( } 270 \mathrm{mg} / \mathrm{dL}) \text {; after week } 6 \text { through week } 12 \text {, FPG > } \\
13.32 \mathrm{mmol} / \mathrm{L}(240 \mathrm{mg} / \mathrm{dL}) \text {; after week } 12 \text { through } \\
\text { week } 24, \mathrm{FPG}>11.1 \mathrm{mmol} / \mathrm{L}(200 \mathrm{mg} / \mathrm{dL} \text { ); after week } \\
24 \text { through week } 54 \text {, (HbAlc }>8.0 \% \text { ) were rescued } \\
\text { with open-label insulin glargine, initiated as per the } \\
\text { local country insulin glargine label" }\end{array}$ & $\begin{array}{l}\text { Metformin + omarigliptin + glimepiride placebo: } \\
\text { metformin dose } \geq 1500 \mathrm{mg} / \text { day + omarigliptin } 25 \\
\text { mg once weekly. Glimepiride placebo }\end{array}$ \\
\hline
\end{tabular}


(Continued)

Hollander 2017

\section{Metformin + glimepiride}

Metformin dose $\geq 1500 \mathrm{mg} /$ day + glimepiride titrated from 1-8 mg/day. "To manage both hyperglycaemia and hypoglycaemia, the dose of glimepiride/matching placebo was to be up- and/or down-titrated throughout the study on the basis of glucose levels, and by the investigator's clinical assessment of the glycaemic status."

"Glycaemic rescue therapy with open-label sitagliptin was prescribed for participants who met progressively more stringent glycaemic rescue criteria (FPG values consistently (repeat measurement performed within 7 days) $>15.0 \mathrm{mmol} / \mathrm{L}(270 \mathrm{mg} / \mathrm{dL})$ after randomisation through week 6 ; > $13.3 \mathrm{mmol} / \mathrm{L}(240 \mathrm{mg} / \mathrm{dL})$ after week 6 through week 12 ; > $11.1 \mathrm{mmol} / \mathrm{L}(200 \mathrm{mg} / \mathrm{dL})$ after week 12 through week 26 ; $>11.1 \mathrm{mmol} / \mathrm{L}(200$ $\mathrm{mg} / \mathrm{dL}$ ) or $\mathrm{HbAlc}>64 \mathrm{mmol} / \mathrm{mol}(8.0 \%)$ after week 26). Rescued participants continued on their study medication and background metformin"

\section{Vaccaro 2017}

\section{Metformin + sulphonylurea (glibenclamide, gliclazide or glimepiride)}

"The metformin dose remains constant ( $2 \mathrm{~g} /$ day) throughout the study. The add-on drugs will be uptitrated at any follow-up visit, if necessary, based on home glucose monitoring (i.e. fasting glucose $>120$ $\mathrm{mg} / \mathrm{dL}$ or post prandial glucose $>160 \mathrm{mg} / \mathrm{dL}$ in $>50 \%$ of the home glucose readings performed over the last 8 weeks period). Doses of $5-15 \mathrm{mg}$ for glibenclamide, 30-120 mg for gliclazide and 2-6 mg for glimepiride were used. If, despite the maximal daily dose of the drugs has been reached, blood glucose control is still unsatisfactory, adherence to treatments is assessed, lifestyle recommendations are reinforced and $\mathrm{HbAlC}$ is re-evaluated after three months. A confirmed $\mathrm{HbA} 1 \mathrm{C}>8.0 \%$, will lead to add on a bed-time injection of basal insulin (glargine) and prandial rapid acting insulin boluses, if glucose control is still unsatisfactory. Insulin titration is performed according to a pre-defined algorithm based on self-monitored fasting capillary glucose"
Metformin + ertugliflozin

C1: metformin dose $\geq 1500 \mathrm{mg} /$ day + ertugliflozin $5 \mathrm{mg} /$ day

C2: metformin dose $\geq 1500 \mathrm{mg} /$ day + ertugliflozin $15 \mathrm{mg} /$ day

"Glycemic rescue therapy with open-label sitagliptin was prescribed for participants who met progressively more stringent glycaemic rescue criteria (FPG values consistently (repeat measurement performed within 7 days) $>15.0 \mathrm{mmol} / \mathrm{L}(270 \mathrm{mg} / \mathrm{dL})$ after randomisation through week 6 ; > $13.3 \mathrm{mmol} / \mathrm{L}(240 \mathrm{mg} /$ $\mathrm{dL}$ ) after week 6 through week 12; >11.1 mmol/ $\mathrm{L}(200 \mathrm{mg} / \mathrm{dL})$ after week 12 through week 26; > $11.1 \mathrm{mmol} / \mathrm{L}(200 \mathrm{mg} / \mathrm{dL})$ or HbAlc $>64 \mathrm{mmol} /$ mol (8.0\%) after week 26). Rescued participants continued on their study medication and background metformin"

\section{Metformin + pioglitazone}

"The metformin dose remains constant $(2 \mathrm{~g} /$ day) throughout the study. The add-on drugs will be up-titrated at any follow-up visit, if necessary, based on home glucose monitoring (i.e. fasting glucose $>120 \mathrm{mg} / \mathrm{dL}$ or post prandial glucose $>160 \mathrm{mg} / \mathrm{dl}$ in $>50 \%$ of the home glucose readings performed over the last 8-week period). Doses of $15-45 \mathrm{mg}$ for pioglitazone were used. If, despite the maximal daily dose of the drugs has been reached, blood glucose control is still unsatisfactory, adherence to treatments is assessed, lifestyle recommendations are reinforced and $\mathrm{HbA1 \textrm {C }}$ is re-evaluated after three months. A confirmed $\mathrm{HbA} 1 \mathrm{c}>8.0 \%$, will lead to add on a bed-time injection of basal insulin (glargine) and prandial rapid acting insulin boluses, if glucose control is still unsatisfactory. Insulin titration is performed according to a predefined algorithm based on self-monitored fasting capillary glucose"

Metformin + vildagliptin

Maximum dose of metformin was $2500 \mathrm{mg} /$ day, oral administration Vildaglitptin dose was 100 $\mathrm{mg}$. No dose titration. Oral administration administration. Glibenclamide was $2.5 \mathrm{mg}$ daily, but increased to $5 \mathrm{mg} /$ day administered as $2.5 \mathrm{mg}$ twice daily before breakfast and dinner. After 1 month glibenclamide was increased to maximum of $7.5 \mathrm{mg} /$ day and at 4 months up to $5 \mathrm{mg}$ twice daily in order to achieve preprandial glucose values between 4.4-7.8 $\mathrm{mmol} / \mathrm{L}$ and post-prandial glucose $\leq 11.1 \mathrm{mmol} / \mathrm{L}$. Oral administration

\begin{tabular}{lll}
\hline Leiter 2015 & Metformin + glimepiride (tablet) & Metformin + canagliflozin (tablet) \\
& $\begin{array}{l}\text { Metformin } \geq 1500 \mathrm{mg} / \text { day }+ \text { glimepiride } 1 \mathrm{mg} / \mathrm{day} \\
\text { titrated to a maximum dose of } 6 \text { or } 8 \mathrm{mg} / \mathrm{day} \text { (on the }\end{array}$ & $\begin{array}{l}\mathrm{C} 1 \text { : } \text { metformin } \geq 1500 \mathrm{mg} / \text { day }+ \text { canagliflozin } 100 \\
\mathrm{mg} / \text { day }\end{array}$ \\
\hline
\end{tabular}


(Continued)

basis of maximum approved dose in the country of the investigational site) after 2 or more weeks at the current dose if participants met protocol-specified glycaemic criteria (i.e., $\geq 50 \%$ of fasting self monitored blood glucose readings $>6.0 \mathrm{mmol} / \mathrm{L}$, with no hypoglycaemic events during the 2 weeks preceding clinic visit or telephone contact)

\section{Del Prato 2015 \\ Metformin + glipizide (capsule)}

Metformin 1500-2500 mg/day split/twice daily + glipizide $5 \mathrm{mg} /$ day (dosage level 1) once or split/twice daily. "During an 18-week period and at 21-day intervals, participants were up-titrated to the next dosage level if FPG was $\geq 6.1 \mathrm{mmol} / \mathrm{L}$. Level 2 was glipizide 10 $\mathrm{mg} /$ day and level 3 was glipizide $20 \mathrm{mg} /$ day. Up-titration continued until the maximum tolerable dose level was reached." "After the 18-week titration period, participants entered a 34-week maintenance period, during which no further up-titration was allowed."

"For the 52-week, double-blind extension period, one single attempt at up-titration was allowed if $\mathrm{HbAlc}$ was $>7 \%$, but only if the participant had not already reached the maximum dose."

"For the 104-week, double-blind extension period, one single attempt at up-titration was allowed if HbAlc was > $7 \%$."

"After the 18-week titration period, participants entered a 34-week maintenance period, during which no further up-titration was allowed. Particpants could be down-titrated to the preceding level or potentially down to level 0 (placebo for both arms) in the event of recurrent hypoglycaemia"
C2: metformin $\geq 1500 \mathrm{mg} /$ day + canagliflozin $300 \mathrm{mg} /$ day

Participants assigned to the canagliflozin groups were mock up-titrated
Metformin 1500-2500 mg/day split/twice daily + dapagliflozin $2.5 \mathrm{mg} /$ day (dosage level 1) once daily. "During an 18-week period and at 21day intervals, participants were up-titrated to the next dosage level if FPG was $\geq 6.1 \mathrm{mmol} / \mathrm{L}$. Level 2 was dapagliflozin $5 \mathrm{mg} /$ day and level 3 was dapagliflozin $10 \mathrm{mg} /$ day. Up-titration continued until the maximum tolerable dose level was reached." "After the 18-week titration period, participants entered a 34-week maintenance period, during which no further up-titration was allowed."

"For the 52-week double-blind extension period one single attempt at up-titration was allowed if HbAlc was $>7 \%$, but only if the participant had not already reached the maximum dose."

"For the 104 week double-blind extension period one single attempt at up-titration was allowed if HbA1c was $>7 \%$."

"After the 18-week titration period, participants entered a 34-week maintenance period, during which no further up-titration was allowed. Particpants could be down-titrated to the preceding level or potentially down to level 0 (placebo for both arms) in the event of recurrent hypoglycaemia"

\section{Schernthaner 2015}

Metformin + saxagliptin (tablet) + placebo

Metformin at any dose (baseline mean dose $1647 \mathrm{mg} /$ day) + saxagliptin $5 \mathrm{mg}$ once daily + placebo 1-6 mg/day. "During the titration period (12 weeks), placebo was up-titrated every 3 weeks in 1 or $2 \mathrm{mg} /$ day increments to the optimum dose (fasting plasma glucose $\leq 6.1 \mathrm{mmol} /$ L), up to $6 \mathrm{mg}$ once daily. During maintenance (40 weeks), no up-titration was performed."

"Placebo could be down-titrated if recurrent hypoglycaemia occurred"

Glimepiride could be down-titrated if recurrent hypoglycaemia occurred"

Metformin + alogliptin (tablet)

C1: metformin + alogliptin $12.5 \mathrm{mg}$ (tablet): "Alogliptin $12.5 \mathrm{mg}$, tablets, orally, once daily and the maximum tolerated dose of metformin (1500 mg to $3300 \mathrm{mg}$ daily) for up to 104 weeks" week 20 , participants with persistent hyperglycaem (fasting plasma glucose $\geq 250 \mathrm{mg} / \mathrm{dL}$ ) underwent a dose titration of glipizide up to $20 \mathrm{mg}$ in 5-mg increments in 4-week intervals" 
Metformin + glimepiride (tablet): "Participants received glimepiride $2 \mathrm{mg}$ daily (with masked up-titration to $4 \mathrm{mg}$ daily if required) plus metformin $\geq 1500$ $\mathrm{mg}$ daily plus matching albiglutide placebo as a subcutaneous injection weekly via a fully disposable pen injector system plus matching sitagliptin placebo from week 1 to week 156"
C1: metformin + albiglutide (injection): "Participants received albiglutide $30 \mathrm{mg}$ weekly (with masked up-titration to $50 \mathrm{mg}$ weekly if required) as a subcutaneous injection via a fully disposable pen injector system plus metformin $\geq 1500$ $\mathrm{mg}$ daily plus matching sitagliptin placebo plus matching glimepiride placebo from week 1 to week 156"

C2: metformin + sitagliptin (tablet): "Participants received sitagliptin $100 \mathrm{mg}$ daily plus metformin $\geq 1500 \mathrm{mg}$ daily plus matching albiglutide placebo as a subcutaneous injection weekly via a fully disposable pen injector system plus matching glimepiride placebo from week 1 to week 156"

C3: metformin + placebo: "Participants received metformin $\geq 1500$ milligrams (mg) daily plus matching albiglutide placebo as a subcutaneous injection weekly via a fully disposable pen injector system plus matching sitagliptin placebo plus matching glimepiride placebo from week 1 to week 156"

Metformin + empagliflozin: metformin immediate release $\geq 1500 \mathrm{mg} /$ day was continued at the participant's usual dose. Empagliflozin $25 \mathrm{mg}$ / day

Metformin immediate release $\geq 1500 \mathrm{mg} /$ day was continued at the participant's usual dose.

"Glimepiride was initiated at a dose of $1 \mathrm{mg} /$ day, with a recommendation for up-titration if fasting plasma glucose (assessed with home monitoring) was $>6.1$ $\mathrm{mmol} / \mathrm{L}$ to $2 \mathrm{mg} /$ day at week $4,3 \mathrm{mg} /$ day at week 8 , and $4 \mathrm{mg} /$ day at week 12 . Uptitration was to be withheld if it would place the participant at risk of hypoglycaemia and was not to be done after week 12 . Glimepiride dose could be down-titrated at any time to prevent recurrent hypoglycaemia."

"Rescue treatment could be initiated if, after an overnight fast, a participant had confirmed blood glucose concentrations of $>13.3 \mathrm{mmol} / \mathrm{L}$ during weeks $1-12,>11.1 \mathrm{mmol} / \mathrm{L}$ during weeks $12-28$, or $>10.0$ $\mathrm{mmol} / \mathrm{L}$ (or HbAlc concentration >8\%) after week 28 . The choice and dose of rescue medication were at the discretion of the investigator, but could not include a sulphonylurea drug or SGLT-2 inhibitor"

Göke 2013 Metformin + glipizide (tablet)

Open-label metformin at 1500, 2000, 2500 or $3000 \mathrm{mg}$ daily based on individual metformin dose at enrolment for the duration of the study; the dose remained stable throughout the study.

Glipizide was titrated to an optimal effect (FPG $\leq 110$ $\mathrm{mg} / \mathrm{dL}(\leq 6.1 \mathrm{mmol} / \mathrm{L})$ ) or the highest tolerated dose
"Rescue treatment could be initiated if, after an overnight fast, a participant had confirmed blood glucose concentrations of $>13.3 \mathrm{mmol} / \mathrm{L}$ during weeks $1-12,>11.1 \mathrm{mmol} / \mathrm{L}$ during weeks $12-28$, or $>10.0 \mathrm{mmol} / \mathrm{L}$ (or HbA1c concentration $>8 \%$ ) after week 28 . The choice and dose of rescue medication were at the discretion of the investigator, but could not include a sulphonylurea drug or SGLT-2 inhibitor"
Metformin + saxagliptin (tablet): open-label metformin at 1500, 2000, 2500 or 3000 mg daily based on individual metformin dose at enrolment for the duration of the study; the dose remained stable throughout the study. Saxagliptin $5 \mathrm{mg}$ daily throughout the study. Study medication was taken orally, immediately before or with a meal 
during an 18-week titration period. "Glipizide was initiated at $5 \mathrm{mg} /$ day (morning dose) and titrated in 3week intervals to a maximum of $20 \mathrm{mg} /$ day using a double-dummy technique to ensure blinding. Titration steps were $10 \mathrm{mg} /$ day (morning dose), followed by $15 \mathrm{mg} /$ day (10-mg morning dose, 5-mg evening dose) and $20 \mathrm{mg} /$ day (10-mg morning dose, 10-mg evening dose). Initial titration assessment was at week 3 ; subsequent reassessment for titration occurred at weeks 6, 9, 12, 15 and 18. During the titration period, glipizide could be down-titrated once if hypoglycaemic events occurred and could thereafter be up-titrated once. Evaluation at each titration visit and final decision by the investigator on dose increase or decrease took into account participant glucose measurements made before visits, hypoglycaemic events recorded in the participant diary and investigator's measurements at titration visits. Following the titration period, medication doses remained stable except for instances of glipizide downtitration to mitigate recurrent hypoglycaemia at the discretion of the study investigator; no up-titration was allowed." Study medication was taken orally, immediately before or with a meal

\section{Maffioli 2013}

\section{Metfmormin + glibenclamide (tablet)}

3-month run-in period with metformin $850 \mathrm{mg} 3 \mathrm{x} /$ day followed by 12 months of metformin $850 \mathrm{mg} 3 \times$ /day + glibenclamide $5 \mathrm{mg}$ twice a day. For the last 6 months rosuvastatin $5 \mathrm{mg}$ /day was added.

"Patients were already following a controlled-energy diet (almost $600 \mathrm{kcal}$ daily deficit) on the basis of American Heart Association recommendations that included $50 \%$ of calories from carbohydrates, 30\% from fat ( $6 \%$ saturated), and $20 \%$ from proteins, with a maximum cholesterol content of $300 \mathrm{mg} /$ day and $35 \mathrm{~g} /$ day of fibre. Patients were not treated with vitamins or mineral preparations during the study. Standard diet advice was provided by a dietitian and/or a specialist doctor. The dietitian and/or specialist doctor periodically provided instructions on dietary intake recording procedures as part of a behavior modification program and then later used the patient's food diaries for counselling. Individuals were also encouraged to increase their physical activity by walking briskly for 20-30 min, three to five times per week, or by cycling. The recommended changes in physical activity throughout the study were not assessed."
Metfmormin + pioglitazone (tablet): 3-month run-in period with metformin $850 \mathrm{mg} 3 \mathrm{x} /$ day followed by 12 months of metformin $850 \mathrm{mg} 3 \mathrm{x} /$ day + pioglitazone $15 \mathrm{mg}$ twice a day. For the last 6 months rosuvastatin $5 \mathrm{mg} /$ day was added.

"Patients were already following a controlled-energy diet (almost 600 kcal daily deficit) on the basis of American Heart Association recommendations that included $50 \%$ of calories from carbohydrates, $30 \%$ from fat $(6 \%$ saturated), and $20 \%$ from proteins, with a maximum cholesterol content of $300 \mathrm{mg} /$ day and $35 \mathrm{~g} /$ day of fibre. Patients were not treated with vitamins or mineral preparations during the study. Standard diet advice was provided by a dietitian and/or a specialist doctor. The dietitian and/or specialist doctor periodically provided instructions on dietary intake recording procedures as part of a behavior modification program and then later used the patient's food diaries for counselling. Individuals were also encouraged to increase their physical activity by walking briskly for 20-30 min, three to five times per week, or by cycling. The recommended changes in physical activity throughout the study were not assessed."

\section{Nauck 2013}

\section{Metformin + glimepiride (oral)}

Metformin 1500-2000 mg/day (taken in the morning and in the evening) + glimepiride up to $4 \mathrm{mg}$ (once daily in the morning) + liraglutide placebo (injected subcutaneously once daily at any time of the day in the upper arm, abdomen, or thigh using a pen injector
C1: metformin 1500-2000 mg/day + liraglutide (injection) $0.6 \mathrm{mg} /$ day + glimepiride placebo

C2: metformin 1500-2000 mg/day + liraglutide (injection) $1.2 \mathrm{mg} /$ day + glimepiride placebo

C3: metformin 1500-2000 mg/day + liraglutide (injection) $1.8 \mathrm{mg} /$ day + glimepiride placebo 
device. Participants were encouraged to inject liraglutide placebo at the same time each day.

26-week double-blind + 78-week open-label
Metformin taken in the morning and in the evening. Liraglutide injected subcutaneously once daily at any time of the day in the upper arm, abdomen, or thigh using a pen injector device. Glimepiride placebo taken once daily in the morning. Participants were encouraged to inject liraglutide at the same time each day. 26-week double-blind + 78-week open-label

C4: metformin + placebo (oral or injection): metformin $1500-2000 \mathrm{mg} /$ day (taken in the morning and in the evening) + glimepiride placebo (once daily in the morning) and liraglutide placebo (injected subcutaneously once daily at any time of the day in the upper arm, abdomen, or thigh using a pen injector device. Participants were encouraged to inject liraglutide placebo at the same time each day. 26-week double-blind + 78week open-label
Gallwitz 2012a
Metformin + glimepiride (tablet)

Maximally tolerated dose of metformin (either immediate or extended-release) + glimepiride at starting dose at $1 \mathrm{mg} /$ day, given once daily immediately before breakfast. Glimepiride dose adjusted every 4 weeks, according to tolerability, up to the maximum tolerated dose in accordance with the country specific summary of product characteristics

\section{Gallwitz 2012b}

Metformin + glimepiride (capsule)

Metformin at $1500 \mathrm{mg} /$ day or more. Dose unchanged throughout the study.

Glimepiride at starting dose of $1 \mathrm{mg}$ once daily, uptitrated stepwise in $1 \mathrm{mg}$ increments up to a maximum of $4 \mathrm{mg}$ once daily, at 4-week intervals during the first 12 weeks of treatment. Glimepiride was uptitrated by investigators if the participants self-monitored fasting plasma glucose values were $>6.1 \mathrm{mmol} /$ L. At any time, glimepiride could be down-titrated to prevent recurrent hypoglycaemic events. Placebo identical to linagliptin
Metformin + exenatide (subcutaneous injection): maximally tolerated dose of metformin (either immediate or extended-release) + exenatide at starting dose at $5 \mu \mathrm{g}$ twice daily injected within 60 min before breakfast and evening meal. After 4 weeks exenatide dose adjusted to $10 \mu \mathrm{g}$ twice daily for the remaining study period. If participants had daily episodes of nausea for $>1$ week, the $10 \mu \mathrm{g}$ dose was reduced to $5 \mu \mathrm{g}$ twice daily and could be increased again after nausea subsided

Metformin + linagliptin (tablet): metformin at $1500 \mathrm{mg} /$ day or more. Dose unchanged throughout the study. Linagliptin $5 \mathrm{mg}$ once daily. Placebo identical to glimepiride

\section{Derosa 2011a}

\section{Metformin + glimepiride (tablet)}

Metformin 1000-2000 mg/day + glimepiride $1 \mathrm{mg} 3$ x/ day and titrated after 1 month to $2 \mathrm{mg} 3 \mathrm{x} /$ day.

"Subjects began a controlled-energy diet (near 600 Kcal daily deficit) based on American Heart Association recommendations that included $50 \%$ of calories from carbohydrates, $30 \%$ from fat ( $6 \%$ saturated), and $20 \%$ from proteins, with a maximum cholesterol content of $300 \mathrm{mg} /$ day and $35 \mathrm{~g} /$ day of fibre. Patients were not treated with vitamins or mineral preparations during the study.
Metformin + exenatide (injection): metformin $1000-2000 \mathrm{mg} /$ day + exenatide $5 \mu \mathrm{g}$ twice a day titrated after one month to $10 \mu \mathrm{g}$ twice a day.

"Subjects began a controlled-energy diet (near 600 Kcal daily deficit) based on American Heart Association recommendations that included 50 $\%$ of calories from carbohydrates, $30 \%$ from fat (6\% saturated), and $20 \%$ from proteins, with a maximum cholesterol content of $300 \mathrm{mg} /$ day and $35 \mathrm{~g} /$ day of fibre. Patients were not treated with vitamins or mineral preparations during the study. Standard diet advice was given by a dietitian and/or specialist doctor. Dietit- 
(Continued)

Standard diet advice was given by a dietitian and/ or specialist doctor. Dietitian and/or specialist doctor periodically provided instruction on dietary intake recording procedures as part of a behavior modification program and then later used the subject's food diaries for counselling. Individuals were also encouraged to increase their physical activity by walking briskly for 20 to $30 \mathrm{~min}, 3$ to 5 times per week, or by cycling. The recommended changes in physical activity throughout the study were not assessed."

\section{Derosa 2011b}

Metformin + glibenclamide (tablet)

Metformin (mean dosage: $1700 \pm 850 \mathrm{mg} /$ day) + glibenclamide titrated till $15 \mathrm{mg} /$ day with forced titration every 3 months (independently from their glycaemic control, unless the developed side effects also due to the drug dosage) for 12 months.

"Subjects began a controlled-energy diet (near 600 kcal daily deficit) based on American Heart Association recommendations that included $50 \%$ of calories from carbohydrates, $30 \%$ from fat ( $6 \%$ saturated), and $20 \%$ from proteins, with a maximum cholesterol content of $300 \mathrm{mg} /$ day and $35 \mathrm{~g} /$ day of fibre. Patients were not treated with vitamins or mineral preparations during the study. Standard diet advice was given by a dietitian and / or specialist doctor. Dietitian and / or specialist doctor periodically provided instruction on dietary intake recording procedures as part of a behavior modification program and then later used the subject's food diaries for counselling. Individuals were also encouraged to increase their physical activity by walking briskly for $20-30 \mathrm{~min}, 3-5$ times per week, or by cycle. The recommended changes in physical activity throughout the study were assessed at each visit using the subject's activity diary." ian and/or specialist doctor periodically provided instruction on dietary intake recording procedures as part of a behavior modification program and then later used the subject's food diaries for counselling. Individuals were also encouraged to increase their physical activity by walking briskly for 20 to $30 \mathrm{~min}, 3$ to 5 times per week, or by cycling. The recommended changes in physical activity throughout the study were not assessed."

Metformin + pioglitazone (tablet): metformin (mean dosage: $1700 \pm 850 \mathrm{mg} /$ day) + pioglitazone titrated till $45 \mathrm{mg} /$ day with forced titration every 3 months (independently from their glycaemic control, unless the developed side effects also due to the drug dosage) for 12 months.

"Subjects began a controlled-energy diet (near 600 kcal daily deficit) based on American Heart Association recommendations that included 50 $\%$ of calories from carbohydrates, $30 \%$ from fat (6\% saturated), and $20 \%$ from proteins, with a maximum cholesterol content of $300 \mathrm{mg} /$ day and $35 \mathrm{~g} /$ day of fibre. Patients were not treated with vitamins or mineral preparations during the study. Standard diet advice was given by a dietitian and/or specialist doctor. Dietitian and/or specialist doctor periodically provided instruction on dietary intake recording procedures as part of a behavior modification program and then later used the subject's food diaries for counselling. Individuals were also encouraged to increase their physical activity by walking briskly for 20 - 30 min, 3 - 5 times per week, or by cycle. The recommended changes in physical activity throughout the study were assessed at each visit using the subject's activity diary."

Metformin + pioglitazone: metformin $1700 \mathrm{mg} /$ day + pioglitazone $30 \mathrm{mg} /$ day

$\begin{array}{ll}\text { Petrica } 2011 & \text { Metformin + glimepiride } \\ \text { Metformin } 1700 \mathrm{mg} / \text { day + glimepiride } 4 \mathrm{mg} / \text { day }\end{array}$

Metformin + glibenclamide (tablet)

Metformin $1500 \pm 500 \mathrm{mg} /$ day + glibenclamide $2.5 \mathrm{mg}$ $3 \mathrm{x} /$ day titrated after 1 month to $5 \mathrm{mg} 3 \mathrm{x} /$ day.

"Subjects began a controlled-energy diet (near 600 kcal daily deficit) based on American Heart Association recommendations 17 that included $50 \%$ of calories from carbohydrates, $30 \%$ from fat (6\% saturated), and $20 \%$ from proteins, with a maximum cholesterol content of $300 \mathrm{mg} /$ day and $35 \mathrm{~g} /$ day of fibre. Patients were not treated with vitamins or mineral preparations during the study. Standard diet advice was given by a dietitian and/or specialist doctor. Dietitian and/ or a specialist doctor periodically provided instruction on dietary intake recording procedures as part of a behavior modification program and then later used
Metformin + exenatide (injection): metformin $1500 \pm 500 \mathrm{mg} /$ day + exenatide $5 \mu \mathrm{g}$ twice a day titrated after 1 month to $10 \mu \mathrm{g}$ twice a day.

"Subjects began a controlled-energy diet (near 600 kcal daily deficit) based on American Heart Association recommendations 17 that included $50 \%$ of calories from carbohydrates, 30\% from fat (6\% saturated), and $20 \%$ from proteins, with a maximum cholesterol content of $300 \mathrm{mg} /$ day and $35 \mathrm{~g} /$ day of fibre. Patients were not treated with vitamins or mineral preparations during the study. Standard diet advice was given by a dietitian and/or specialist doctor. Dietitian and/ or a specialist doctor periodically provided instruction on dietary intake recording procedures as part of a behavior modification program and 
(Continued)

the subject's food diaries for counselling. Individuals were also encouraged to increase their physical activity by walking briskly for 20-30 min, three to five times per week, or bicycle. The recommended changes in physical activity throughout the study were not assessed."

Metformin + glimepiride (tablet)

Metformin $\geq 1500 \mathrm{mg}$ twice a day + glimepiride $2 \mathrm{mg}$ / day. Glimepiride could be up-titrated (to a maximum of $6 \mathrm{mg} /$ day) at weeks 4,8 or any later visit if FPG exceeded $6.2 \mathrm{mmol} / \mathrm{L}$ or down-titrated in cases of recurrent hypoglycaemia

Filozof $2010 \quad$ Metformin + gliclazide (tablet)
Metformin $1500 \mathrm{mg} /$ day + gliclazide $80 \mathrm{mg} /$ day up
titrated to a maximum of $320 \mathrm{mg} /$ day if FPG was $>$
$7.0 \mathrm{mmol} / \mathrm{L}$ or fasting blood glucose was $>6.3 \mathrm{mmol} /$
L based on the fasting finger-stick capillary glucose
measurement performed at the study centre. Partici-
pants were up-titrated to the next dose level at week 4
(160 mg), week $8(240 \mathrm{mg})$ and week $12(320 \mathrm{mg})$

then later used the subject's food diaries for counselling. Individuals were also encouraged to increase their physical activity by walking briskly for 20-30 min, three to five times per week, or bicycle. The recommended changes in physical activity throughout the study were not assessed."

Metformin + vildagliptin (tablet): metformin $\geq$ $1500 \mathrm{mg}$ twice a day + vildagliptin $50 \mathrm{mg}$ twice a day

Metformin + vildagliptin (tablet): metformin $1500 \mathrm{mg} /$ day + vildagliptin $50 \mathrm{mg}$ twice daily

Seck $2010 \quad$ Metformin + glipizide
Metformin $\geq 1500 \mathrm{mg} /$ day + glipizide $5 \mathrm{mg} /$ day up-
titrated to a potential maximum dose of $20 \mathrm{mg} /$ day.
In 3-week intervals during the first 18 weeks of treat-
ment, glipizide was up-titrated if pre-meal fingerstick
glucose values were $>6.1 \mathrm{mmol} / \mathrm{L}(110 \mathrm{mg} / \mathrm{dL})$. At the
investigator's discretion, up-titration of glipizide was
withheld if the investigator considered that up-titra-
tion would place the participant at risk for hypogly-
caemia. At any time during the study, glipizide could
be down-titrated to prevent recurrent hypoglycaemic
events

Home 2009 Metformin + SU (tablet)

Metformin, starting dose varied by local practice. Dose increase permitted after 8 weeks of treatment. Maximum daily dose $2550 \mathrm{mg}$.

Suphonylurea, starting dose varied by local practice. Dose increase permitted after 8 weeks of treatment. Maximum daily dose glibenclamide (or equivalent for different preparations) $15 \mathrm{mg}$, gliclazide $240 \mathrm{mg}$ and glimepiride $4 \mathrm{mg}$.

Rescue therapy by transfer to insulin if $\mathrm{HbAlc}>8.5 \%$ confirmed

Derosa 2009a Metformin + glimepiride (tablet):

Metformin $850 \mathrm{mg} /$ day + glimepiride $2 \mathrm{mg} /$ day, both once a day after lunch for 1 month. Forced titration of glimepiride (independently from their glycaemic control, unless they developed adverse effects also due to the drug dosage) to $4 \mathrm{mg} /$ day in the second

\author{
Metformin + sitagliptin \\ Metformin $\geq 1500 \mathrm{mg} /$ day + sitagliptin $100 \mathrm{mg} /$ \\ day
}

Metformin + rosiglitazone (tablet)

Metformin, starting dose varied by local practice. Dose increase permitted after 8 weeks of treatment. Maximum daily dose $2550 \mathrm{mg}$.

Rosiglitazone, starting dose $4 \mathrm{mg} /$ day. Dose increase permitted after 8 weeks of treatment to 8 $\mathrm{mg} /$ day.

Rescue therapy by addition of a third oral agent if $\mathrm{HbA} 1 \mathrm{c}>8.5 \%$ confirmed. In case of HbAlc > $8.5 \%$ confirmed on triple therapy, rosiglitazone was stopped and insulin therapy substituted.

C1: metformin + pioglitazone (tablet)

Metformin $850 \mathrm{mg} /$ day + pioglitazone $15 \mathrm{mg} /$ day, both once a day after lunch for 1 month. Forced titration of metformin and pioglitazone (independently from their glycaemic control, unless they developed adverse effects also due to the drug dosage) to metformin $1700 \mathrm{mg} /$ day 
month and hereafter $6 \mathrm{mg} /$ day till the end of study. Metformin kept constant at $850 \mathrm{mg} /$ day till study end.

"At baseline, patients began a controlled-energy diet ( $600 \mathrm{kcal}$ daily deficit) based on American Diabetes Association recommendations that contained $50 \%$ of calories from carbohydrates, $30 \%$ from fat $(6 \%$ saturated), and $20 \%$ from proteins, with a maximum cholesterol content of $300 \mathrm{mg} / \mathrm{d}$, and $35 \mathrm{~g} / \mathrm{d}$ of fibre. Each centre's standard diet advice was given by a dietitian and/or specialist physician. Dietitians and/or specialists each month for the first 3 months provided instruction on dietary intake, recording procedures as part of a behavior-modification program, and then from month 3 used the patients' food diaries for counselling. During the study, behavior-modification sessions on weight loss strategies were given to individual patients at baseline and then every 3 months until the end of the trial. Individuals were also encouraged to increase their physical activity by walking briskly or riding a stationary bicycle for 20 to 30 minutes, 3 to 5 times per week. The recommended changes in physical activity throughout the study were not assessed."

Derosa 2009b

Metformin + glibenclamide (tablet)

6 months of run-in. Glibenclamide starting dose 2.5 $\mathrm{mg} 3 \mathrm{x} /$ day, titrated to $5 \mathrm{mg} 3 \mathrm{x} /$ day. After 1 month of run-in metformin was added. Metformin starting dose $500 \mathrm{mg} 3 \mathrm{x} /$ day titrated to $1000 \mathrm{mg} 3 \mathrm{x} /$ day.

"At baseline, patients began a controlled-energy diet (600 kcal daily deficit), based on ADA recommendations, that contained $50 \%$ of calories from carbohydrates, $30 \%$ from fat ( $6 \%$ saturated), and 20 $\%$ from proteins, with a maximum cholesterol content of $300 \mathrm{mg} / \mathrm{d}$, and $35 \mathrm{~g} / \mathrm{d}$ of fibre. Each centre's standard diet advice was given by a dietitian and/ or specialist physician. Dietitians and/or specialists each two weeks provided instruction on dietary intake-recording procedures as part of a behavior-modification program and then from month 1 used the patients' food diaries for counselling. During the study, behavior-modification sessions on weight-loss strategies were given to individual patients at baseline, one at 6 months, and four with all patients at 3, 6, 9 and 12 months. Individuals were also encouraged to increase their physical activity by walking briskly or rid-
+ pioglitazone $30 \mathrm{mg} /$ day in the second month and hereafter metformin $2550 \mathrm{mg} /$ day + pioglitazone $45 \mathrm{mg} /$ day till the end of study.

\section{C2: metformin monotherapy (tablet)}

Metformin $1000 \mathrm{mg} /$ day, $500 \mathrm{mg}$, twice a day, after lunch and diner for 1 month. Forced titration (independently from their glycaemic control, unless they developed adverse effects also due to the drug dosage) to $2000 \mathrm{mg} /$ day in the second month and hereafter $3000 \mathrm{mg} /$ day till the end of study.

"At baseline, patients began a controlled-energy diet ( 600 kcal daily deficit) based on American Diabetes Association recommendations that contained $50 \%$ of calories from carbohydrates, $30 \%$ from fat (6\% saturated), and $20 \%$ from proteins, with a maximum cholesterol content of $300 \mathrm{mg} / \mathrm{d}$, and $35 \mathrm{~g} / \mathrm{d}$ of fibre. Each centre's standard diet advice was given by a dietitian and/or specialist physician. Dietitians and/ or specialists each month for the first 3 months provided instruction on dietary intake, recording procedures as part of a behavior-modification program, and then from month 3 used the patients' food diaries for counselling. During the study, behavior-modification sessions on weight loss strategies were given to individual patients at baseline and then every 3 months until the end of the trial. Individuals were also encouraged to increase their physical activity by walking briskly or riding a stationary bicycle for 20 to 30 minutes, 3 to 5 times per week. The recommended changes in physical activity throughout the study were not assessed."

Metformin + nateglinide (tablet)

6 months of run-in. Nateglinide starting dose $60 \mathrm{mg} 3 \mathrm{x} /$ day, titrated to $120 \mathrm{mg} 3 \mathrm{x} /$ day. After 1 month of run-in metformin was added. Metformin starting dose $500 \mathrm{mg} 3 \mathrm{x} /$ day titrated to $1000 \mathrm{mg} 3$ x/day.

"At baseline, patients began a controlled-energy diet (600 kcal daily deficit), based on ADA recommendations, that contained $50 \%$ of calories from carbohydrates, $30 \%$ from fat (6\% saturated), and $20 \%$ from proteins, with a maximum cholesterol content of $300 \mathrm{mg} / \mathrm{d}$, and $35 \mathrm{~g} / \mathrm{d}$ of fibre. Each centre's standard diet advice was given by a dietitian and/or specialist physician. Dietitians and/or specialists each two weeks provided instruction on dietary intake-recording procedures as part of a behavior-modification program and then from month 1 used the patients' food diaries for counselling. During the study, behavior-modification sessions on weight-loss strategies were given to individual patients at baseline, one at 6 months, and four 
(Continued)

ing a stationary bicycle for 20 to $30 \mathrm{~min}, 3-5$ times per week. The recommended changes in physical activity throughout the study were not assessed." with all patients at 3, 6, 9 and 12 months. Individuals were also encouraged to increase their physical activity by walking briskly or riding a stationary bicycle for 20 to $30 \mathrm{~min}, 3-5$ times per week. The recommended changes in physical activity throughout the study were not assessed."

\begin{tabular}{lll}
\hline Petrica 2009 & Metformin + glimepiride & Metmin + rosiglitazone \\
Metformin $1700 \mathrm{mg} /$ day + glimepiride $4 \mathrm{mg} /$ day & $\begin{array}{l}\text { Metformin } 1700 \mathrm{mg} / \text { day + rosiglitazone } 4 \mathrm{mg} / \\
\text { day }\end{array}$
\end{tabular}

NCT00367055 Metformin + Gliclazide (tablet)

Observation period: metformin $2 \mathrm{~g} /$ day

Treatment period: initial dosage gliclazide $80 \mathrm{mg} /$ day + metformin $2 \mathrm{~g} /$ day with the opportunity, in case of FBG $>1.26 \mathrm{~g} / \mathrm{L}$ and after a 4-week treatment period, to adjust progressively their treatment up to $320 \mathrm{mg} /$ day and $2 \mathrm{~g}$ /day of gliclazide/metformin

Metformin + rosiglitazone (tablet)

Observation period: metformin $2 \mathrm{~g} /$ day

Treatment period: initial dosage rosiglitazone 4 $\mathrm{mg} /$ day + metformin $2 \mathrm{~g} /$ day with the opportunity, in case of FBG $>1.26 \mathrm{~g} / \mathrm{L}$ and after a 8-week treatment period, to adjust their treatment up to $8 \mathrm{mg} /$ day and $2 \mathrm{~g} /$ day of rosiglitazone/metformin

\section{Hamann 2008}

\section{Metformin + glibenclamide or gliclazide}

Metformin $2000 \mathrm{mg} /$ day + glibenclamide $5 \mathrm{mg} /$ day or gliclazide $80 \mathrm{mg} /$ day up-titrated to maximum tolerated dose (glibenclamide $15 \mathrm{mg} /$ day, gliclazide $320 \mathrm{mg}$ / day).

"All subjects had their study medications progressively up-titrated at 4,8 and 12 weeks to achieve optimal glycaemic control, unless either their mean daily glucose (determined for the prior 3 days from diary cards) was $<6.1 \mathrm{mmol} / \mathrm{L}$ or they reported frequent or severe hypoglycaemia. Patients who were not uptitrated at one visit because their mean daily glucose was $<6.1 \mathrm{mmol} / \mathrm{L}$ could have the dose up-titrated at the next visit if mean daily glucose was then $\geq 6.1$ $\mathrm{mmol} / \mathrm{L}$. Patients with multiple or severe episodes of hypoglycaemia could, at the investigators discretion, undergo blinded reduction to the previous dose level. Investigators were required to make every effort to confirm hypoglycaemia biochemically and to identify potential causes of hypoglycaemia other than study medication (e.g. a missed meal) before down-titrating the dose level. Individuals with insufficient therapeutic effect (fasting blood glucose $\geq 12 \mathrm{mmol} / \mathrm{L}$ ) after having been on the maximum dose level of study medication for at least 8 weeks were withdrawn from the study"

\section{Metformin + rosiglitazone}

Metformin $2000 \mathrm{mg} /$ day + rosiglitazone $4 \mathrm{mg} /$ day up-titrated to maximum tolerated dose ( 8 $\mathrm{mg} /$ day).

"All subjects had their study medications progressively up-titrated at 4,8 and 12 weeks to achieve optimal glycaemic control, unless either their mean daily glucose (determined for the prior 3 days from diary cards) was $<6.1 \mathrm{mmol} /$ $\mathrm{L}$ or they reported frequent or severe hypoglycaemia. Patients who were not up-titrated at one visit because their mean daily glucose was $<6.1 \mathrm{mmol} / \mathrm{L}$ could have the dose up-titrated at the next visit if mean daily glucose was then $\geq 6.1 \mathrm{mmol} / \mathrm{L}$. Patients with multiple or severe episodes of hypoglycaemia could, at the investigators discretion, undergo blinded reduction to the previous dose level. Investigators were required to make every effort to confirm hypoglycaemia biochemically and to identify potential causes of hypoglycaemia other than study medication (e.g. a missed meal) before downtitrating the dose level. Individuals with insufficient therapeutic effect (fasting blood glucose $\geq 12 \mathrm{mmol} / \mathrm{L}$ ) after having been on the maximum dose level of study medication for at least 8 weeks were withdrawn from the study"
Ristic 2007

\section{Metformin + gliclazide}

Metformin: "participants were kept on their individual maximally tolerated dose (the dose at which the participant was inadequately controlled, based on $\mathrm{HbAlc}$ assessment, which, however, could not be increased because of side-effects) from 8 weeks before entering
Metformin + nateglinide

Metformin: "participants were kept on their individual maximally tolerated dose (the dose at which the participant was inadequately controlled, based on HbA1c assessment, which, however, could not be increased because of 
(Continued)

the study and during the entire course of the study." Mean dose $1834 \mathrm{mg} /$ day.

Gliclazide: "treatment regimen were started at the lowest level ( $80 \mathrm{mg} /$ day) and were titrated to the next dose level on a monthly basis up to a maximum of 240 $\mathrm{mg} /$ day during the first 3 months. Dose levels of study medication were increased if the FPG level was $>7$ $\mathrm{mmol} / \mathrm{L}$, if the participant had not experienced any confirmed hypoglycaemic events (symptomatic and/ or asymptomatic events with plasma glucose concentration $\leq 4.0 \mathrm{mmol} / \mathrm{L}$ ) and if the participant had not experienced $>3$ hypoglycaemic events in the past month." side-effects) from 8 weeks before entering the study and during the entire course of the study." Mean dose $1931 \mathrm{mg} /$ day.

Nateglinide: "treatment regimen were started at the lowest level (60 mg 3 x/day) and were titrated to the next dose level on a monthly basis up to a maximum of $180 \mathrm{mg} 3 \mathrm{x}$ /day during the first 3 months. Dose levels of study medication were increased if the FPG level was $>7 \mathrm{mmol} / \mathrm{L}$, if the participant had not experienced any confirmed hypoglycaemic events (symptomatic and/or asymptomatic events with plasma glucose concentration $\leq 4.0 \mathrm{mmol} / \mathrm{L}$ ) and if the participant had not experienced $>3$ hypoglycaemic events in the past month."

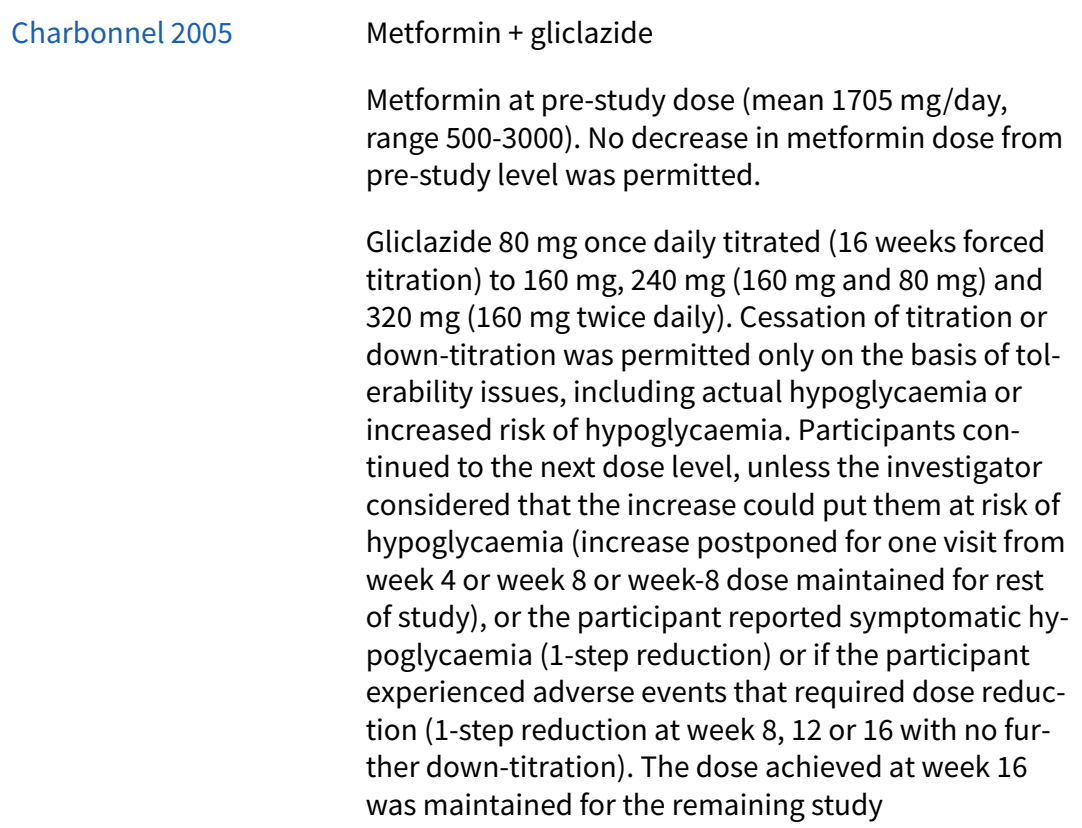

\section{Metformin + pioglitazone}

Metformin at pre-study dose (mean $1705 \mathrm{mg}$ / day, range 500-3000). No decrease in metformin dose from pre-study level was permitted.

Pioglitazone $15 \mathrm{mg}$ once daily titrated (16 weeks forced titration) to 30 and $45 \mathrm{mg}$. Cessation of titration or down-titration was permitted only on the basis of tolerability issues, including actual hypoglycaemia or increased risk of hypoglycaemia. Participants continued to the next dose level, unless the investigator considered that the increase could put them at risk of hypoglycaemia (increase postponed for one visit from week 4 or week 8 or week- 8 dose maintained for rest of study), or the participant reported symptomatic hypoglycaemia (1-step reduction) or if the participant experienced adverse events that required dose reduction (1-step reduction at week 8, 12 or 16 with no further down-titration). The dose achieved at week 16 was maintained for the remaining study

Metformin + rosiglitazone (tablet)

Metformin $1500 \mathrm{mg} /$ day orally (3 x /day) + rosiglitazone $4 \mathrm{mg} /$ day orally (once a day, before lunch).

"Subjects began a controlled-energy diet (approximately $600 \mathrm{kcal}$ daily deficit) based on American Diabetes Association recommendations containing $52 \%$ as carbohydrates, $22 \%$ proteins, $26 \%$ of calories as lipids ( $6 \%$ saturated), with a maximum cholesterol content of 300 $\mathrm{mg} /$ day, and $35 \mathrm{~g}$ fibre. Each centre's standard diet advice was given by a dietitian and/or specialist physician. Dietitians and/or diabetologists periodically provided instruction on dietary intake, recording procedures as part of a behavior modification program, and then later used the subject's food diaries for counselling. During the study, one individual behavior-modification session on weight-loss strategies took place at 
(Continued)

to increase their physical activity by walking briskly for 20-30 min, 3-5 times per week, or bicycle. The recommended changes in physical activity throughout the study were not assessed."

baseline, and four collective educational seminars with all patients were held at 3, 6, 9, and 12 months. Individuals were also encouraged to increase their physical activity by walking briskly for 20-30 min, 3-5 times per week, or bicycle. The recommended changes in physical activity throughout the study were not assessed."

Gerich 2005
Metformin + glyburide (capsules orally) + placebo to match nateglinide

\begin{abstract}
Maintenance period, 4 weeks: 500 mg metformin open-label before the evening meal + $1.25 \mathrm{mg}$ glyburide before breakfast.
\end{abstract}

Titration period 12 weeks: Titration was performed at biweekly visits if FPG $\geq 6.7 \mathrm{mmol} / \mathrm{L}$. Open-label metformin was titrated in $500 \mathrm{mg}$ increments to a maximum of $2000 \mathrm{mg}$ daily. Glyburide was titrated in 1.25 $\mathrm{mg}$ increments to a maximum of $10 \mathrm{mg}$ daily, before breakfast and before dinner.

Monitoring period 88 weeks: study medication remained constant, unless protocol-specified criteria for rescue therapy were met. The dose level was increased to the next highest level or to the "rescue dose level 9" if FPG $\geq 13.3 \mathrm{mmol} / \mathrm{L}, \mathrm{HbA} 1 \mathrm{c} \geq 9.0 \%$, or the participant had symptomatic hyperglycaemia.

Rescue dose: $15 \mathrm{mg}$ of glyburide in divided daily doses
Metformin + nateglinide (tablets orally) + placebo to match glyburide

Maintenance period, 4 weeks: 500 mg metformin open-label before the evening meal $+120 \mathrm{mg}$ nateglinide, before breakfast, lunch, dinner. The dose level was not adjusted during this 4-week maintenance period, unless hypoglycaemia required downward titration to dose level 0 (60 mg nateglinide).

Titration period 12 weeks: titration was performed at biweekly visits if $F P G \geq 6.7 \mathrm{mmol} / \mathrm{L}$. Open-label metformin was titrated in $500 \mathrm{mg}$ increments to a maximum of $2000 \mathrm{mg}$ daily. Nateglinide remained constant at $120 \mathrm{mg}$

Monitoring period 88 weeks: study medication remained constant, unless protocol-specified criteria for rescue therapy were met. The dose level was increased to the next highest level or to the "rescue dose level 9" if FPG $\geq 13.3 \mathrm{mmol} /$ $\mathrm{L}, \mathrm{A} 1 \mathrm{C} \geq 9.0 \%$, or the participant had symptomatic hyperglycaemia.

Rescue dose: $180 \mathrm{mg}$ of nateglinide

ADA: American Diabetes Association; C: comparator; FBG: fasting blood glucose; FPG: fasting plasma glucose; HbA1c: glycosylated haemoglobin A1C; I: intervention; SGLT2: sodium-glucose transport 2 


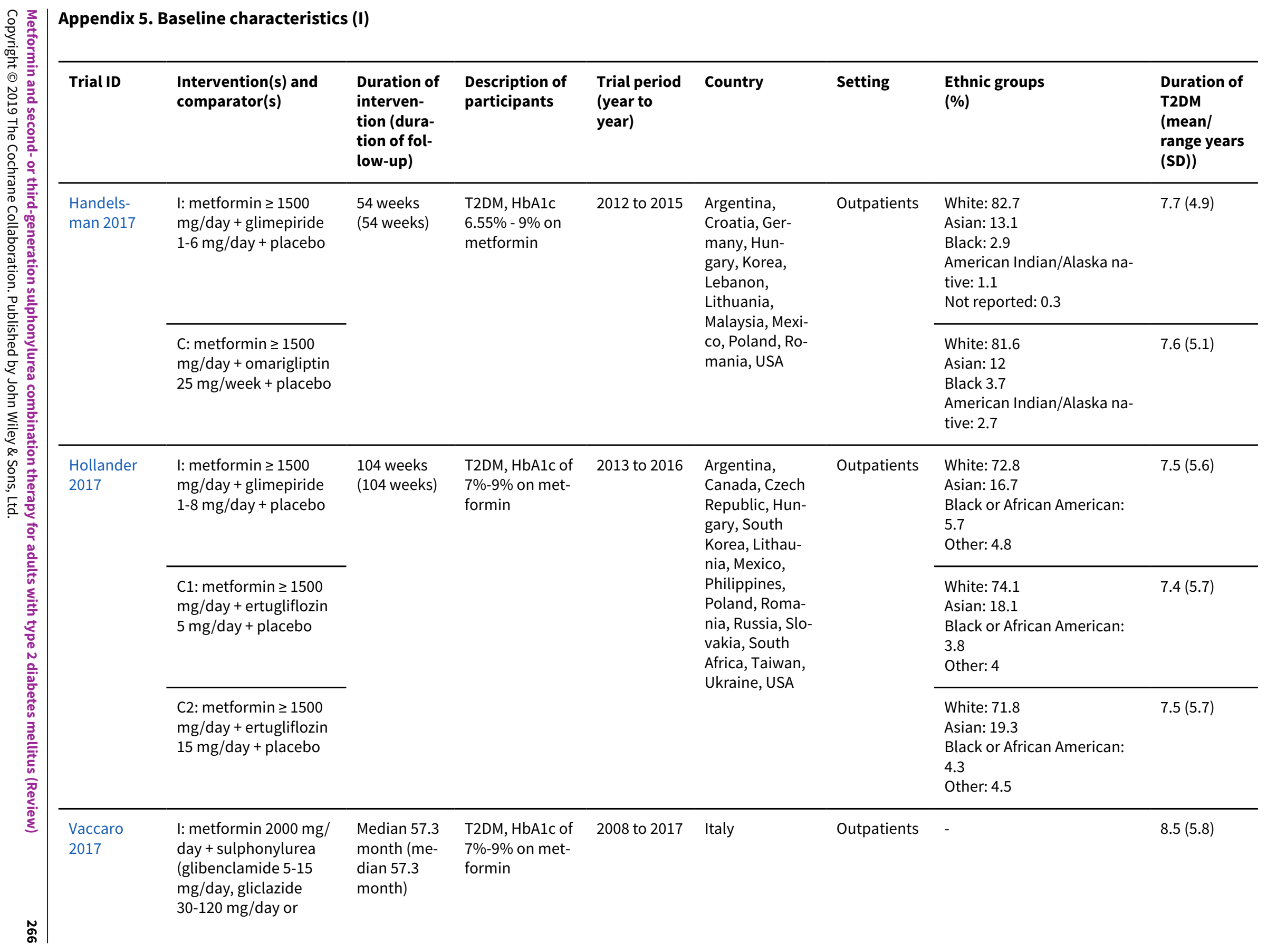




\begin{tabular}{|c|c|c|c|c|c|c|c|c|c|c|}
\hline 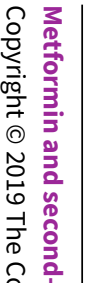 & (Continued) & $\begin{array}{l}\text { glimepiride } 2-6 \mathrm{mg} / \\
\text { day) } \\
\text { C: metformin } 2000 \\
\mathrm{mg} / \text { day + pioglitazone } \\
15-45 \mathrm{mg} / \text { day) }\end{array}$ & & & & & & - & $8.4(5.6)$ & 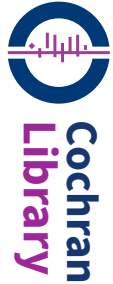 \\
\hline 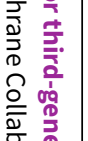 & Dei Cas 2017 & $\begin{array}{l}\text { I: metformin } \geq 1500 \\
\text { mg/day + gliben- } \\
\text { clamide } 10 \text { mg/day }\end{array}$ & $\begin{array}{l}52 \text { weeks } \\
\text { (52 weeks) }\end{array}$ & $\begin{array}{l}\text { T2DM, HbAlc of } \\
7 \%-9 \% \text { on met- } \\
\text { formin }\end{array}$ & 2007 to 2009 & Italy & Outpatients & - & $5(1-10)^{a}$ & 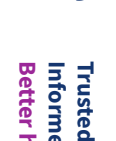 \\
\hline 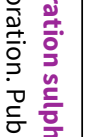 & & $\begin{array}{l}\text { C: metformin } \geq 1500 \\
\text { mg/day + vildagliptin } \\
100 \text { mg/day }\end{array}$ & & & & & & - & $7(4-11)$ & 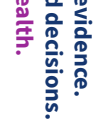 \\
\hline 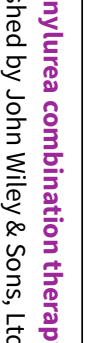 & Leiter 2015 & $\begin{array}{l}\text { I: metformin } \geq 1500 \\
\text { mg/day + glimepiride } \\
\text { 1-8 mg/day }\end{array}$ & $\begin{array}{l}104 \text { week } \\
\text { (104 week) }\end{array}$ & $\begin{array}{l}\text { T2DM, HbA1c } \\
\text { of } 7 \%-9.5 \% \text { on } \\
\text { metformin }\end{array}$ & 2009 to 2013 & $\begin{array}{l}\text { Argentina, Bul- } \\
\text { garia, Cana- } \\
\text { da, Costa Ri- } \\
\text { ca, Denmark, } \\
\text { Finland, Ger- } \\
\text { many, India, Is- } \\
\text { rael, Mexico, } \\
\text { Norway, Philip- } \\
\text { pines, Poland, }\end{array}$ & Outpatients & $\begin{array}{l}\text { White: } 67 \\
\text { Black or African American: } 5 \\
\text { Asian: } 19 \\
\text { Other (includes American } \\
\text { Indian or Alaska Native, Na- } \\
\text { tive Hawaiian or other Pacif- } \\
\text { ic Islander, multiple origin, } \\
\text { and other): } 9\end{array}$ & $6.6(5.0)$ & \\
\hline 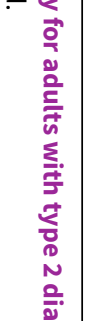 & & $\begin{array}{l}\mathrm{C} 1: \text { metformin } \geq 1500 \\
\mathrm{mg} / \text { day + canagliflozin } \\
100 \mathrm{mg} / \text { day }\end{array}$ & & & & $\begin{array}{l}\text { Romania, Russ- } \\
\text { ian Federation, } \\
\text { Slovakia, South } \\
\text { Korea, Ukraine, } \\
\text { USA }\end{array}$ & & $\begin{array}{l}\text { White: } 67 \\
\text { Black or African American: } 4 \\
\text { Asian: } 21 \\
\text { Other (includes American } \\
\text { Indian or Alaska Native, Na- } \\
\text { tive Hawaiian or other Pacif- } \\
\text { ic Islander, multiple origin, } \\
\text { and other): } 9\end{array}$ & $6.5(5.5)$ & \\
\hline 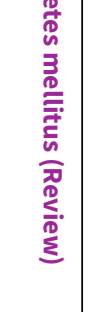 & & $\begin{array}{l}\text { C2: metformin } \geq 1500 \\
\mathrm{mg} / \text { day + canagliflozin } \\
300 \mathrm{mg} / \text { day }\end{array}$ & & & & & & $\begin{array}{l}\text { White: } 69 \\
\text { Black or African American: } 4 \\
\text { Asian: } 19 \\
\text { Other (includes American } \\
\text { Indian or Alaska Native, Na- } \\
\text { tive Hawaiian or other Pacif- } \\
\text { ic Islander, multiple origin, } \\
\text { and other): } 9\end{array}$ & $6.7(5.5)$ & 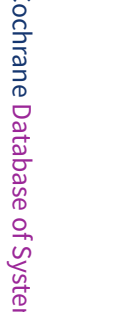 \\
\hline ูู & $\begin{array}{l}\text { Del Prato } \\
2015\end{array}$ & $\begin{array}{l}\text { I: metformin } \\
1500-2500 \mathrm{mg} / \text { day + } \\
\text { glipizide } 5-20 \mathrm{mg} / \text { day }\end{array}$ & $\begin{array}{l}208 \text { weeks } \\
\text { ( } 208 \text { weeks) }\end{array}$ & $\begin{array}{l}\text { People with } \\
\text { inadequate- } \\
\text { ly controlled }\end{array}$ & 2008 to 2013 & $\begin{array}{l}\text { Argentina, } \\
\text { France, Ger- } \\
\text { many, UK, Italy, }\end{array}$ & Outpatients & $\begin{array}{l}\text { American Indian or Alaska } \\
\text { Native: } 0 \\
\text { Asian: } 8.5\end{array}$ & $7(6)$ & 离. \\
\hline
\end{tabular}




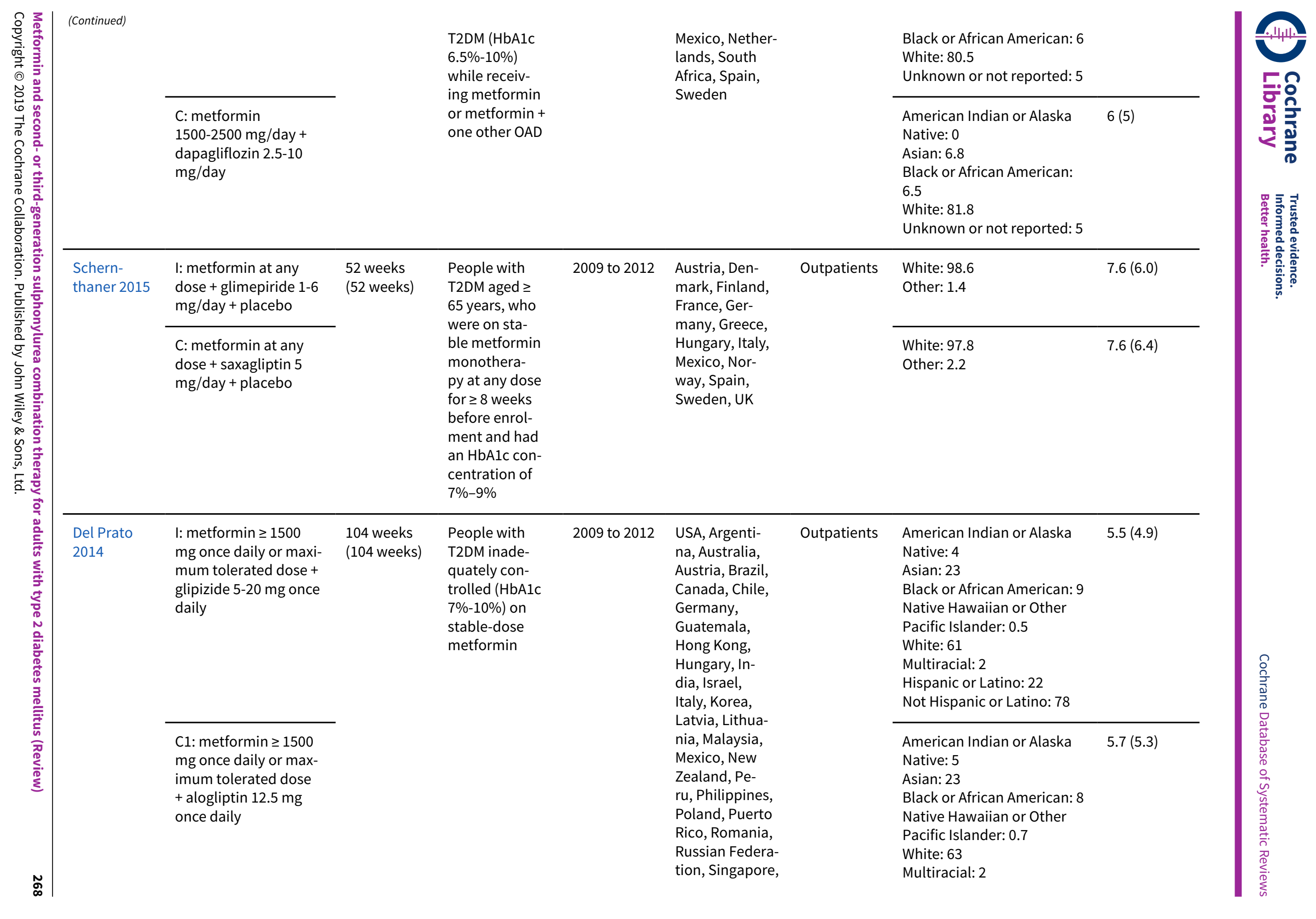




\begin{tabular}{|c|c|c|c|c|c|c|c|c|c|}
\hline 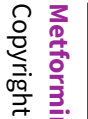 & (Continued) & & & & & $\begin{array}{l}\text { South Africa, } \\
\text { Spain, Thai- }\end{array}$ & & $\begin{array}{l}\text { Hispanic or Latino: } 23 \\
\text { Not Hispanic or Latino: } 77\end{array}$ & \\
\hline 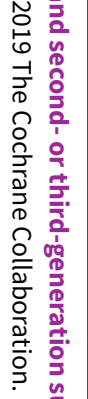 & & $\begin{array}{l}\text { C2: metformin } \geq 1500 \\
\text { mg once daily or maxi- } \\
\text { mum tolerated dose }+ \\
\text { alogliptin } 25 \text { mg once } \\
\text { daily }\end{array}$ & & & & UK & & $\begin{array}{l}\text { American Indian or Alaska } \\
\text { Native: } 5 \\
\text { Asian: } 22 \\
\text { Black or African American: } 8 \\
\text { Native Hawaiian or Other } \\
\text { Pacific Islander: } 0.1 \\
\text { White: } 63 \\
\text { Multiracial: } 1 \\
\text { Hispanic or Latino: } 22 \\
\text { Not Hispanic or Latino: } 78\end{array}$ & $5.4(4.7)$ \\
\hline 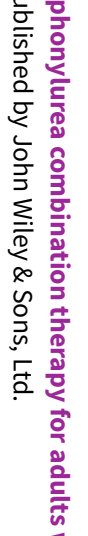 & Ahrén 2014 & $\begin{array}{l}\text { I: metformin } \geq 1500 \mathrm{mg} \\
\text { daily + glimepiride } 2-4 \\
\text { mg once daily + place- } \\
\text { bo + placebo }\end{array}$ & $\begin{array}{l}156 \text { weeks } \\
\text { (156 weeks) }\end{array}$ & $\begin{array}{l}\text { People with } \\
\text { T2DM with in- } \\
\text { adequate gly- } \\
\text { caemic con- } \\
\text { trol (HbA1c } \\
\text { 7\%-10\%) while } \\
\text { taking back- } \\
\text { ground met- } \\
\text { formin }\end{array}$ & 2009 to 2013 & $\begin{array}{l}\text { USA, Albania, } \\
\text { Germany, Hong } \\
\text { Kong, Mexico, } \\
\text { Peru, Philip- } \\
\text { pines, Russ- } \\
\text { ian, Federation, } \\
\text { South Africa, } \\
\text { Spain, UK }\end{array}$ & Outpatients & $\begin{array}{l}\text { African American/African } \\
\text { heritage: } 13 \\
\text { American Indian or Alaskan } \\
\text { native: } 8 \\
\text { Asian - Central/South Asian } \\
\text { heritage: } 1 \\
\text { Asian - East Asian heritage: } \\
1 \\
\text { Asian - South East Asian } \\
\text { heritage: } 3 \\
\text { White Arabic/North African } \\
\text { heritage: } 3 \\
\text { White/European heritage: } \\
72\end{array}$ & $6.0(4.8)$ \\
\hline 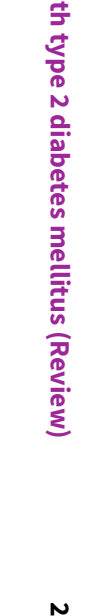 & & $\begin{array}{l}\text { C1: metformin } \geq 1500 \\
\text { mg daily + albiglutide } \\
30-50 \text { mg once weekly } \\
\text { + placebo }\end{array}$ & & & & & & $\begin{array}{l}\text { African American/African } \\
\text { heritage: } 18 \\
\text { American Indian or Alaskan } \\
\text { Native: } 6 \\
\text { Asian - Central/South Asian } \\
\text { heritage: } 1 \\
\text { Asian - East Asian heritage: } \\
2 \\
\text { Asian - South East Asian } \\
\text { heritage: } 4 \\
\text { Native Hawaiian or other } \\
\text { Pacific Islander: } 0 \\
\text { White Arabic/North African } \\
\text { heritage: } 1 \\
\text { White/European heritage: } \\
71\end{array}$ & $6.0(4.3)$ \\
\hline
\end{tabular}

1: metformin $\geq 1500$ mg daily + albiglutid 30-50 mg once weekly placebo

Asian - Central/South Asian

heritage: 4

heritage:

71 


\begin{tabular}{|c|c|c|c|c|c|c|c|c|}
\hline 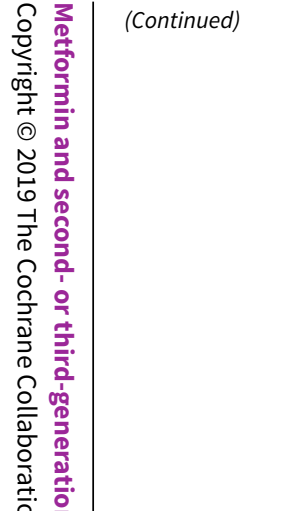 & $\begin{array}{l}\text { C2: } \text { metformin } \geq 1500 \\
\text { mg daily + sitagliptin } \\
100 \text { mg once daily }+ \\
\text { placebo }\end{array}$ & & & & & & $\begin{array}{l}\text { African American/African } \\
\text { heritage: } 12 \\
\text { American Indian or Alaskan } \\
\text { native: } 7 \\
\text { Asian - Central/South Asian } \\
\text { heritage: } 2 \\
\text { Asian - East Asian heritage: } \\
1 \\
\text { Asian - South East Asian } \\
\text { heritage: } 4 \\
\text { White/Caucasian/European } \\
\text { heritage: } 75\end{array}$ & $5.8(4.8)$ \\
\hline 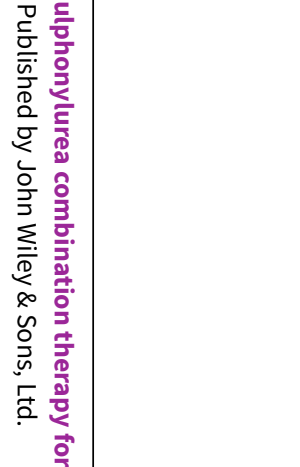 & $\begin{array}{l}\text { C3: } \text { metformin } \geq 1500 \\
\text { mg daily + placebo }\end{array}$ & & & & & & $\begin{array}{l}\text { African American/African: } 23 \\
\text { American Indian or Alaskan } \\
\text { native: } 9 \\
\text { Asian - Central/South Asian } \\
\text { heritage: } 1 \\
\text { Asian - Japanese heritage: } 1 \\
\text { Asian - South East Asian } \\
\text { heritage: } 3 \\
\text { Native Hawaiian or other } \\
\text { Pacific Islander: } 1 \\
\text { White/European heritage: } \\
63\end{array}$ & $6.7(6.6)$ \\
\hline $\begin{array}{l}\text { Ridderstråle } \\
2014\end{array}$ & $\begin{array}{l}\text { I: metformin immedi- } \\
\text { ate release } \geq 1500 \mathrm{mg} / \\
\text { day + glimepiride } 1-4 \\
\text { mg/day }\end{array}$ & $\begin{array}{l}208 \text { weeks } \\
\text { (208 weeks) }\end{array}$ & $\begin{array}{l}\text { People with } \\
\text { T2DM with poor } \\
\text { glycaemic con- } \\
\text { trol (HbA1c } \\
7 \%-10 \%) \text { BMI }\end{array}$ & 2010 to 2015 & $\begin{array}{l}\text { Argentina, } \\
\text { Austria, Cana- } \\
\text { da, Colombia, } \\
\text { Czech Republic, }\end{array}$ & Outpatients & $\begin{array}{l}\text { White: } 67 \\
\text { Asian: } 32 \\
\text { Balck or Afican-American: } 1\end{array}$ & $\begin{array}{l}\leq 1: 12 \\
>1-5: 43 \\
>5-10: 27 \\
>10: 18\end{array}$ \\
\hline 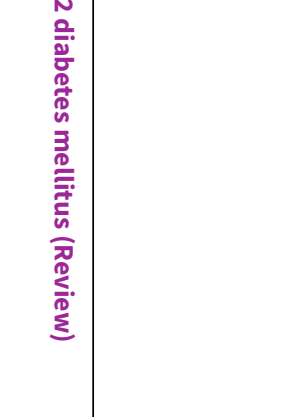 & $\begin{array}{l}\text { C: } \text { metformin immedi- } \\
\text { ate release } \geq 1500 \mathrm{mg} / \\
\text { day }+ \text { empagliflozin } 25 \\
\mathrm{mg} / \text { day }\end{array}$ & & $\begin{array}{l}\leq 45 \mathrm{~kg} / \mathrm{m}^{2} \\
\text { on stable met- } \\
\text { formin } \geq 1500 \\
\mathrm{mg} / \text { day }\end{array}$ & & $\begin{array}{l}\text { Kong, India, } \\
\text { Italy, Malaysia, } \\
\text { Mexico, Nether- } \\
\text { lands, Nor- } \\
\text { way, Philip- } \\
\text { pines, Portugal, } \\
\text { South Africa, } \\
\text { Spain, Sweden, } \\
\text { Switzerland, } \\
\text { Taiwan, Thai- } \\
\text { land, UK, USA }\end{array}$ & & $\begin{array}{l}\text { White: } 65 \\
\text { Asian: } 33 \\
\text { Balck or Afican-American: } 2 \\
\text { Hawaiian or Pacific Islan- } \\
\text { der: }<1\end{array}$ & $\begin{array}{l}\leq 1: 10 \\
>1-5: 45 \\
>5-10: 28 \\
>10: 17\end{array}$ \\
\hline
\end{tabular}




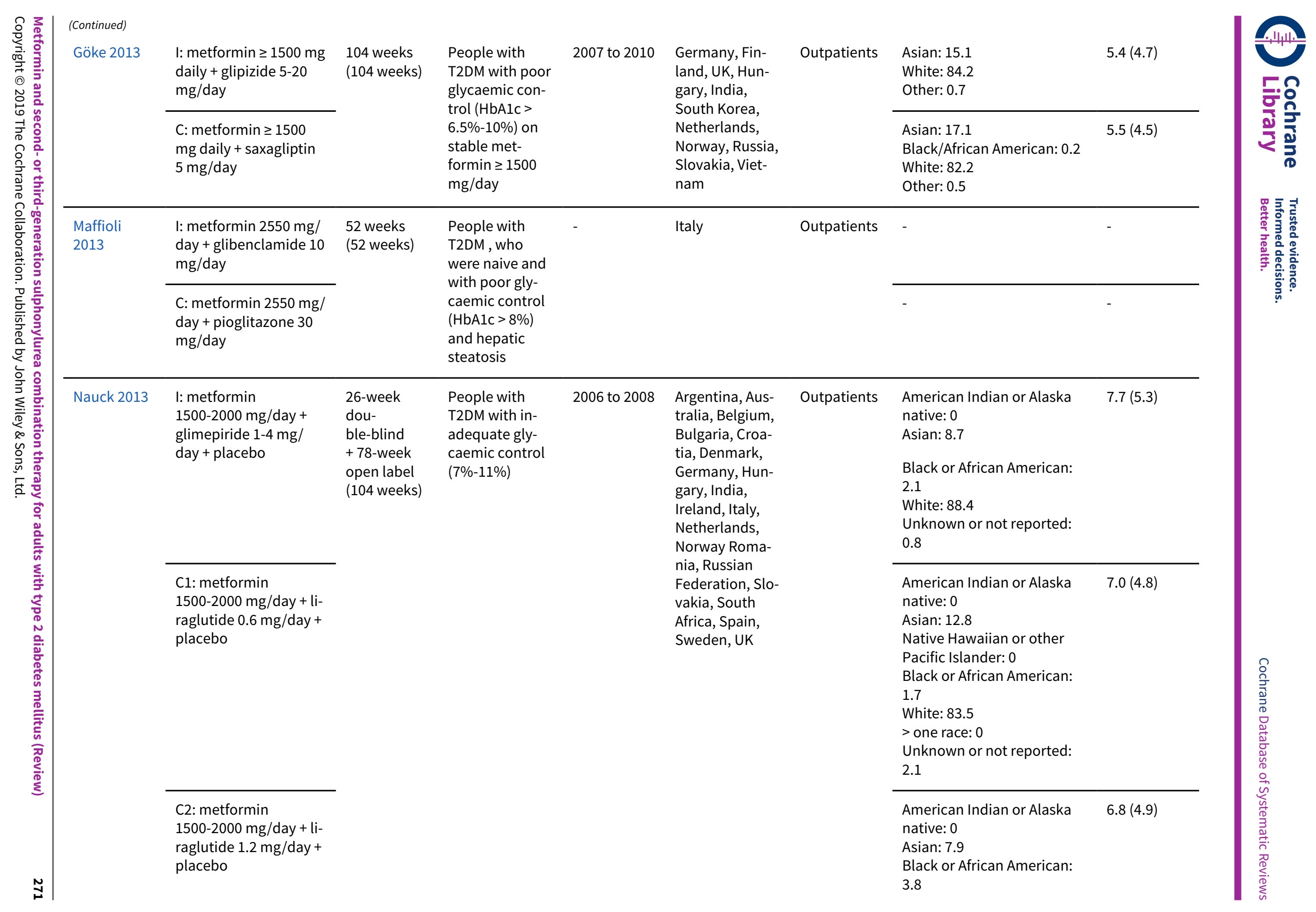




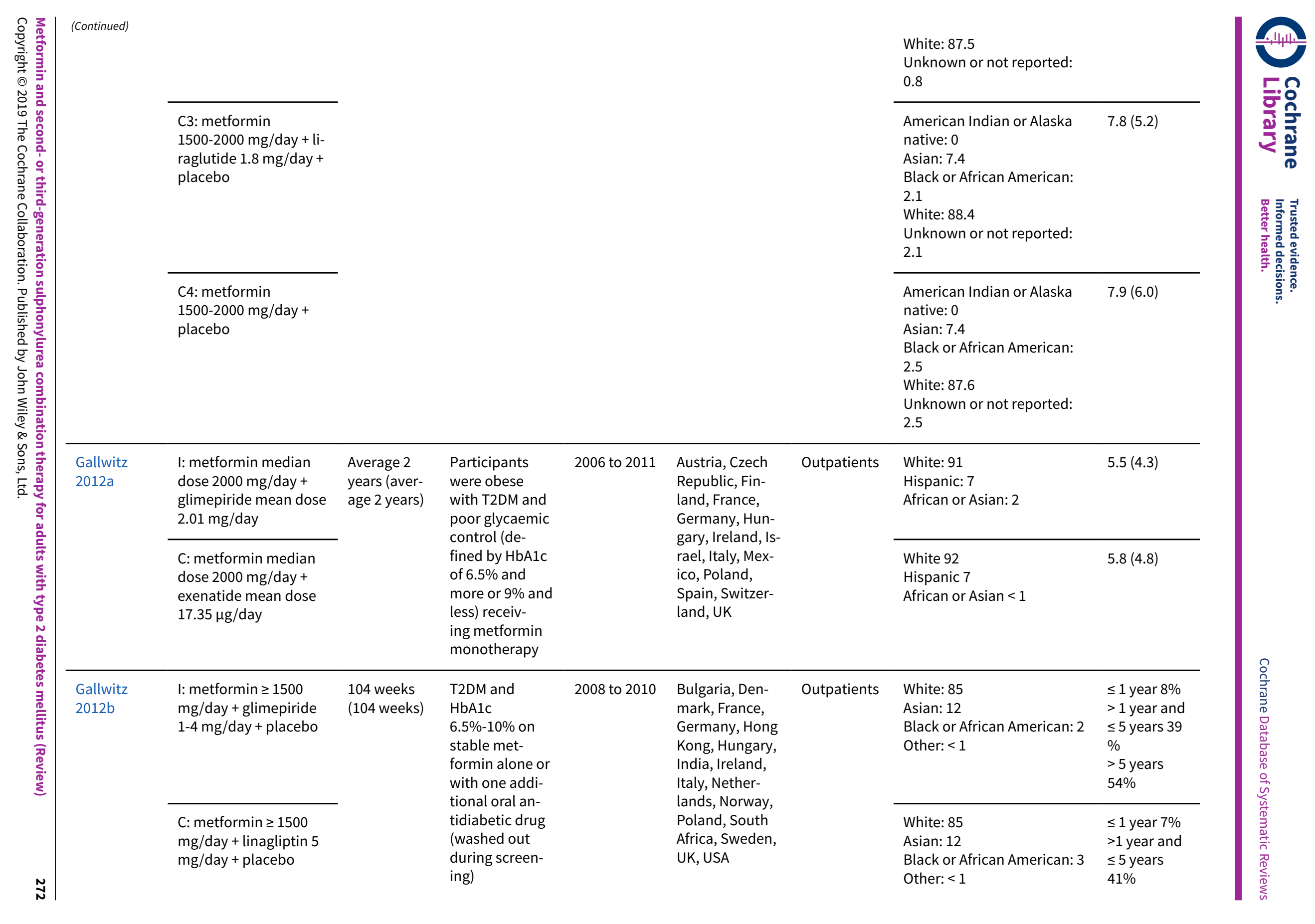




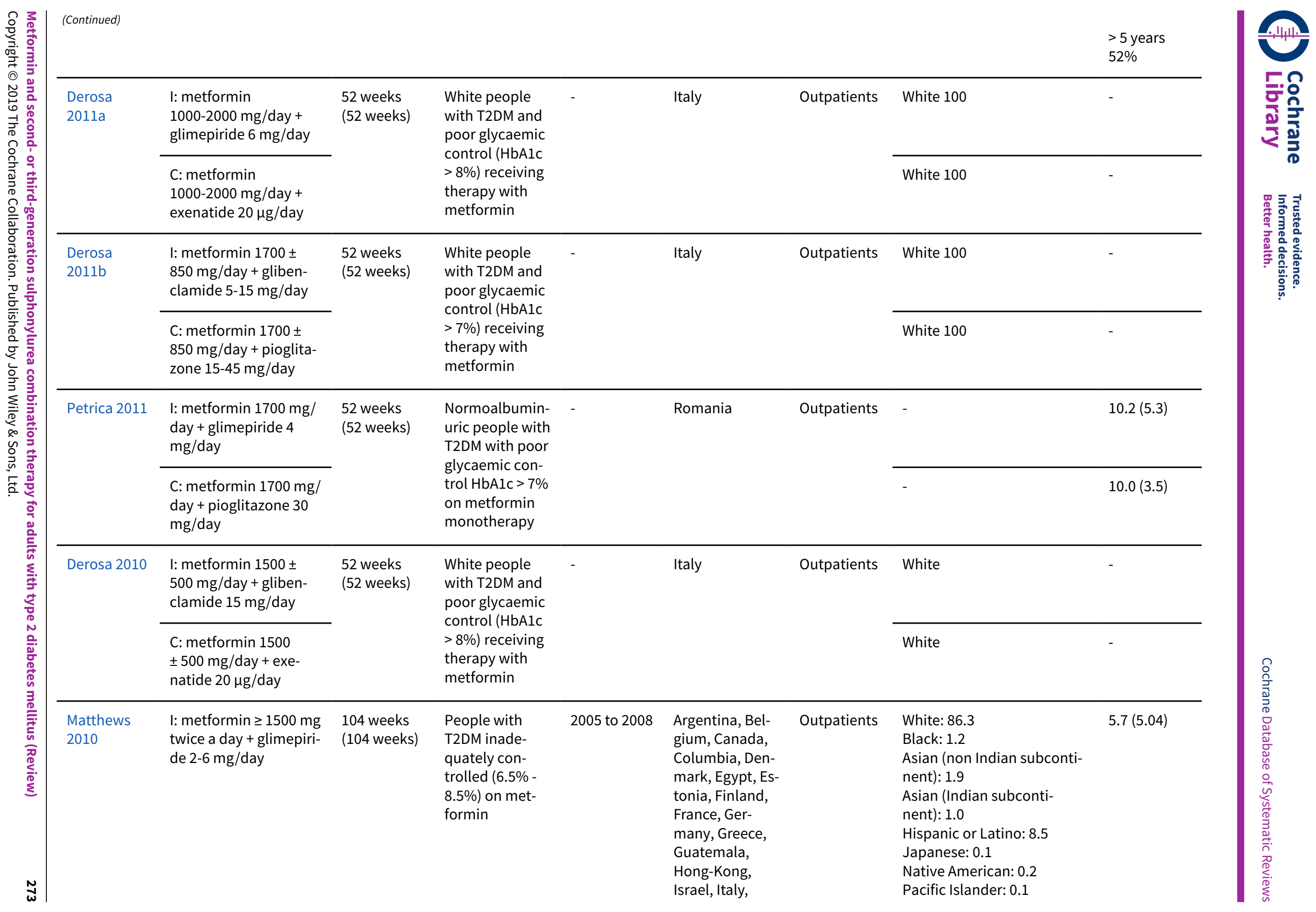




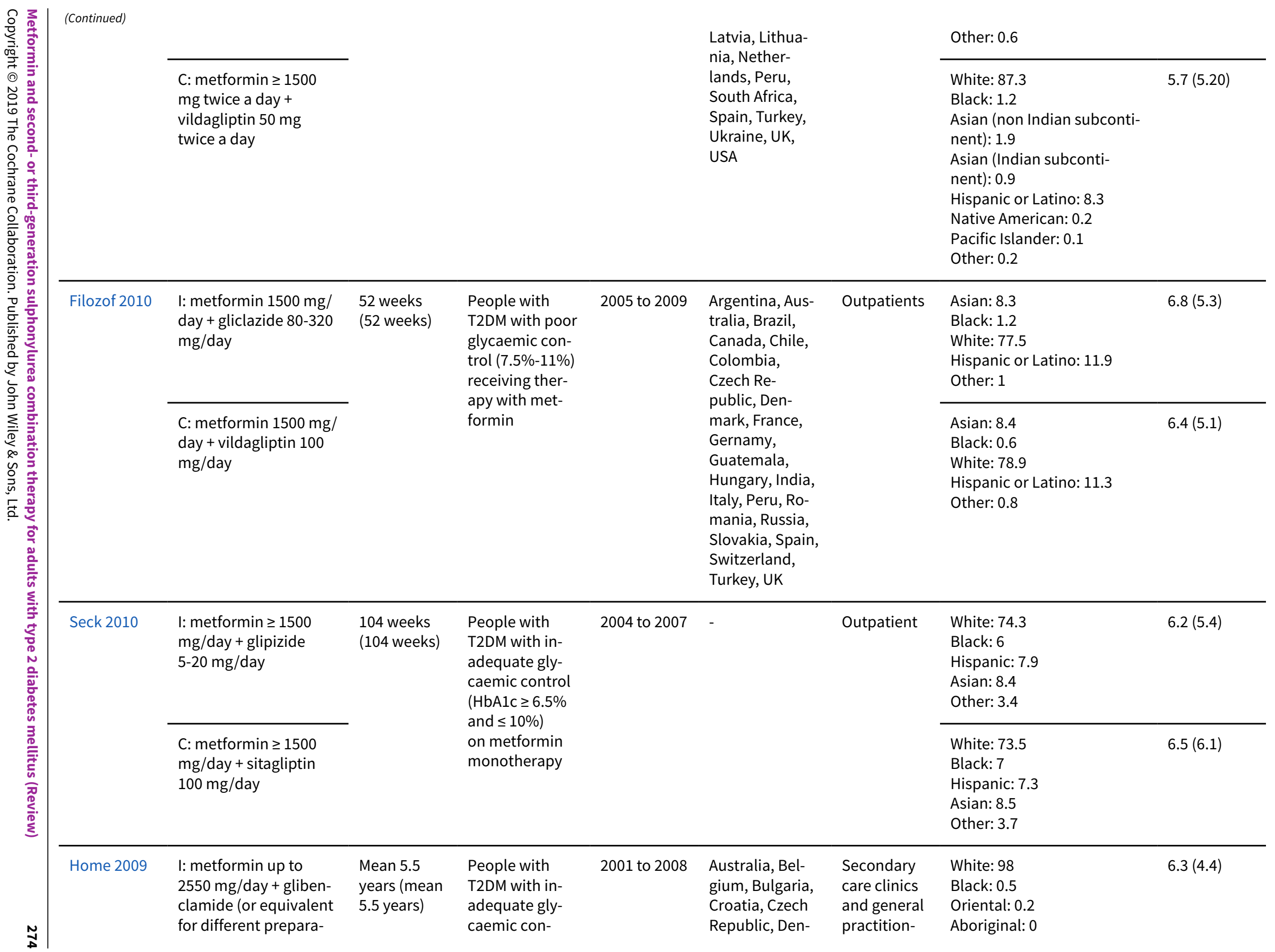




\begin{tabular}{|c|c|c|c|c|c|c|c|c|c|}
\hline 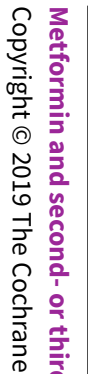 & (Continued) & $\begin{array}{l}\text { tions) up to } 15 \mathrm{mg} / \text { day } \\
\text { or gliclazide up to } 240 \\
\mathrm{mg} / \text { day or glimepiride } \\
\text { up to } 4 \mathrm{mg} / \text { day }\end{array}$ & & $\begin{array}{l}\text { trol (HbA1c } 7 \% \\
-9 \%) \text { on met- } \\
\text { formin }\end{array}$ & & $\begin{array}{l}\text { mark, Esto- } \\
\text { nia, Finland, } \\
\text { France, Ger- } \\
\text { many, Greece, } \\
\text { Hungary, } \\
\text { Italy, Latvia, } \\
\text { Lithuania, } \\
\text { Netherlands, } \\
\text { New Zealand, }\end{array}$ & $\begin{array}{l}\text { er surgeries, } \\
\text { including } \\
\text { site man- } \\
\text { agement or- } \\
\text { ganisations } \\
\text { and private } \\
\text { diabetes } \\
\text { clinics }\end{array}$ & $\begin{array}{l}\text { Asian: } 0.2 \\
\text { Indian: } 0.09 \\
\text { Maori: } 0.09 \\
\text { Middle East Hible: } 0.09 \\
\text { Pacific Islander: } 0.09 \\
\text { Polynesian: } 0.09 \\
\text { Sri Lankan: } 0.2 \\
\text { Tahitian: } 0.09\end{array}$ & \\
\hline 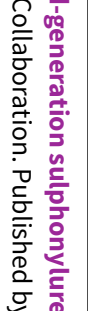 & & $\begin{array}{l}\text { C: metformin up to } \\
2550 \mathrm{mg} / \text { day + rosigli- } \\
\text { tazone up to } 8 \mathrm{mg} / \text { day }\end{array}$ & & & & $\begin{array}{l}\text { Poland, Ro- } \\
\text { mania, Russ- } \\
\text { ian Federa- } \\
\text { tion, Slovakia, } \\
\text { Spain, Sweden, } \\
\text { Ukraine, UK }\end{array}$ & & $\begin{array}{l}\text { White: } 99 \\
\text { Black: } 0.3 \\
\text { Oriental: } 0.4 \\
\text { Aboriginal: } 0.09 \\
\text { African: } 0.09 \\
\text { Asian: } 0.09 \\
\text { Egyptian: } 0.09 \\
\text { Gipsy: } 0.09\end{array}$ & $6.1(4.2)$ \\
\hline 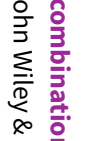 & $\begin{array}{l}\text { Derosa } \\
\text { 2009a }\end{array}$ & $\begin{array}{l}\text { I: metformin } 850 \mathrm{mg} / \\
\text { day + glimepiride 2-6 } \\
\text { mg/day }\end{array}$ & $\begin{array}{l}65 \text { weeks } \\
\text { ( } 65 \text { weeks) }\end{array}$ & $\begin{array}{l}\text { White people } \\
\text { with T2DM, who } \\
\text { were naive and }\end{array}$ & - & Italy & Outpatients & White 100 & \\
\hline 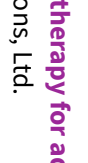 & & $\begin{array}{l}\text { C1: metformin } \\
850-2550 \mathrm{mg} / \text { day + pi- } \\
\text { oglitazone } 15-45 \mathrm{mg} / \\
\text { day }\end{array}$ & & $\begin{array}{l}\text { caemic control } \\
(\mathrm{HbA} 1 \mathrm{c}>6.5 \%)\end{array}$ & & & & White 100 & \\
\hline$\underset{\substack{F \\
E}}{\stackrel{F}{E}}$ & & $\begin{array}{l}\text { C2: metformin } \\
1000-3000 \mathrm{mg} / \text { day }\end{array}$ & & & & & & White 100 & \\
\hline 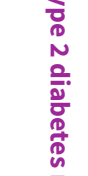 & $\begin{array}{l}\text { Derosa } \\
2009 b\end{array}$ & $\begin{array}{l}\text { I: metformin } \\
1500-3000 \mathrm{mg} / \text { day + } \\
\text { glibenclamide } 7.5-15 \\
\mathrm{mg} / \text { day }\end{array}$ & $\begin{array}{l}52 \text { weeks } \\
\text { (52 weeks) }\end{array}$ & $\begin{array}{l}\text { White people } \\
\text { with T2DM, who } \\
\text { were naive and } \\
\text { with poor gly- }\end{array}$ & - & Italy & Outpatients & White 100 & $4(2)$ \\
\hline 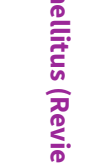 & & $\begin{array}{l}\text { C: metformin } \\
\text { 1500-3000 mg/day + } \\
\text { nateglinide 180-360 } \\
\text { mg/day }\end{array}$ & & $(\mathrm{HbA} 1 \mathrm{c}>7 \%)$ & & & & White 100 & $5(2)$ \\
\hline 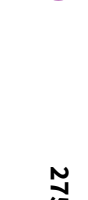 & Petrica 2009 & $\begin{array}{l}\text { I: metformin } 1700 \mathrm{mg} / \\
\text { day + glimepiride } 4 \\
\text { mg/day }\end{array}$ & $\begin{array}{l}52 \text { weeks } \\
\text { ( } 52 \text { weeks) }\end{array}$ & $\begin{array}{l}\text { Normoalbumin- } \\
\text { uric people with } \\
\text { T2DM with poor } \\
\text { glycaemic con- } \\
\text { trol HbA1c > } 7 \%\end{array}$ & - & Romania & Outpatients & - & $10.4(1.84)$ \\
\hline
\end{tabular}




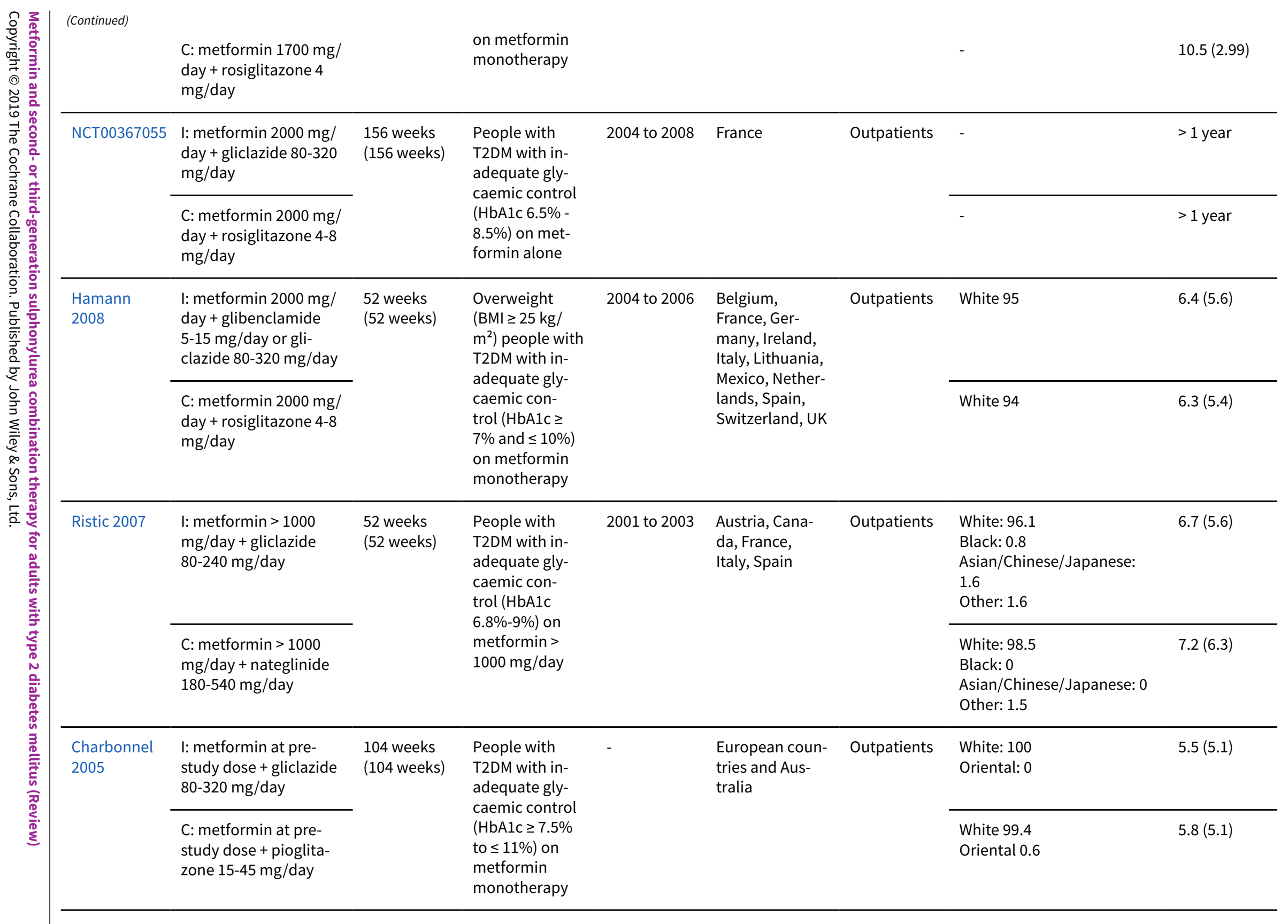




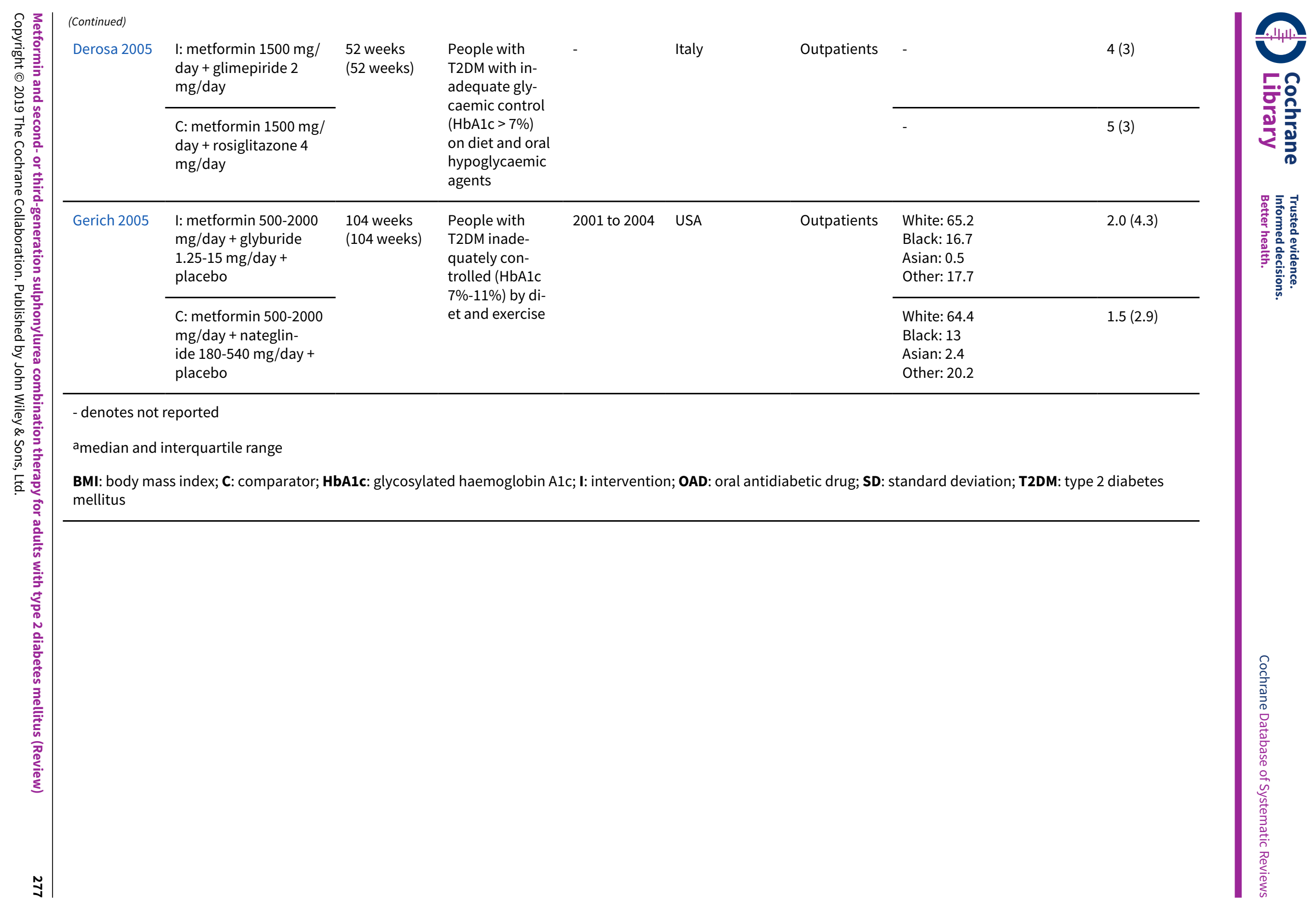




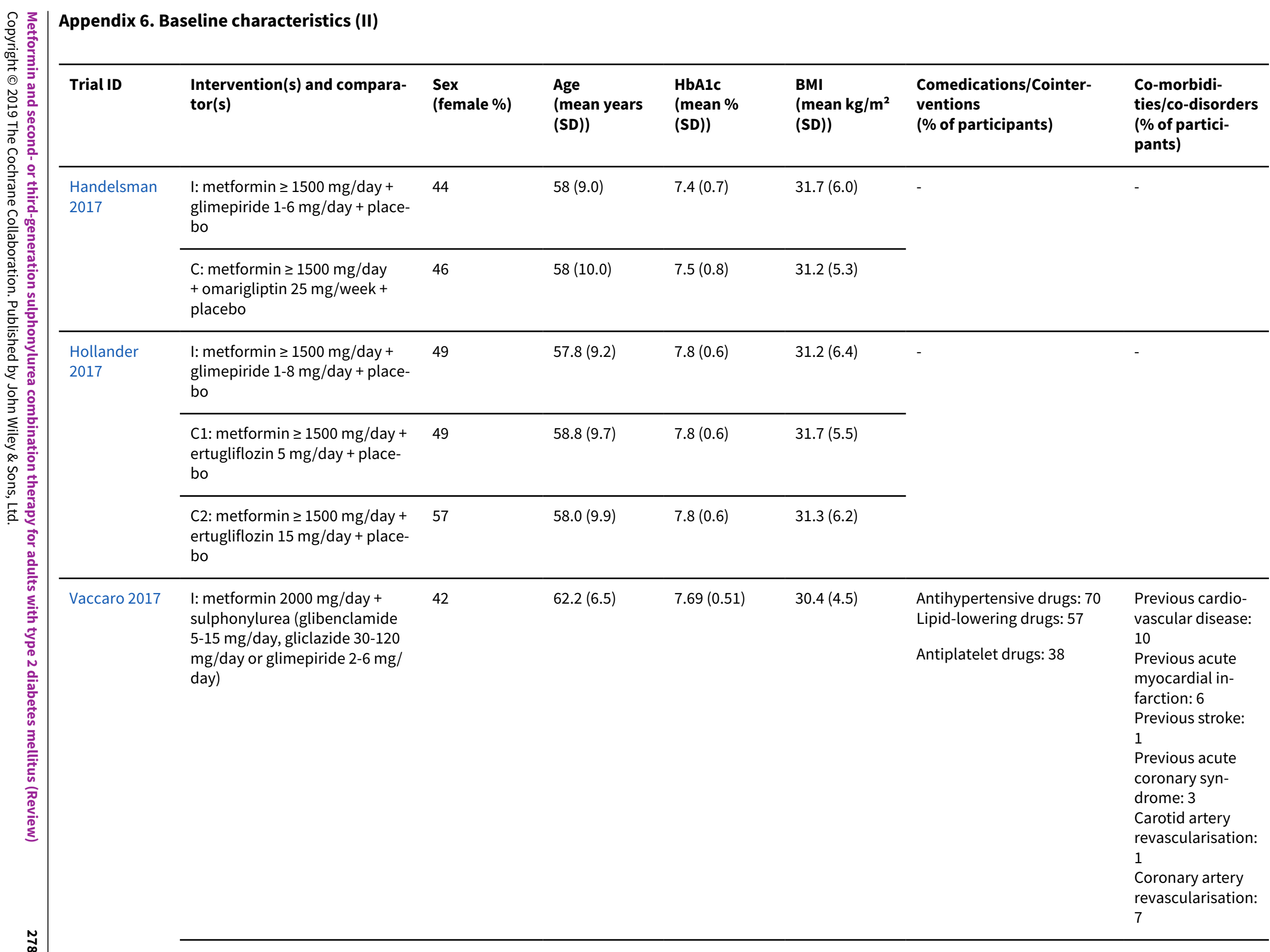




\begin{tabular}{|c|c|c|c|c|c|c|c|}
\hline 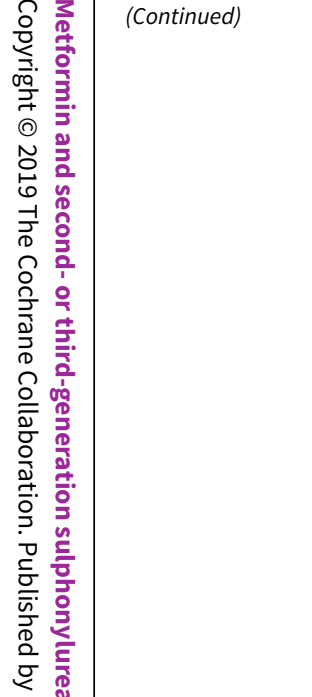 & $\begin{array}{l}\text { C: metformin } 2000 \mathrm{mg} / \text { day + pi- } \\
\text { oglitazone } 15-45 \mathrm{mg} / \text { day) }\end{array}$ & 41 & $62.4(6.4)$ & $7.67(0.50)$ & $30.2(4.4)$ & $\begin{array}{l}\text { Antihypertensive drugs: } 70 \\
\text { Lipid-lowering drugs: } 58 \\
\text { Antiplatelet drugs: } 42\end{array}$ & $\begin{array}{l}\text { Previous cardio- } \\
\text { vascular disease: } \\
12 \\
\text { Previous acute } \\
\text { myocardial in- } \\
\text { farction: } 7 \\
\text { Previous stroke: } \\
2 \\
\text { Previous acute } \\
\text { coronary syn- } \\
\text { drome: } 3 \\
\text { Carotid artery } \\
\text { revascularisation: } \\
1 \\
\text { Coronary artery } \\
\text { revascularisation: } \\
7\end{array}$ \\
\hline 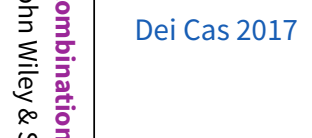 & $\begin{array}{l}\text { I: metformin } \geq 1500 \mathrm{mg} / \text { day + } \\
\text { glibenclamide } 10 \mathrm{mg} / \text { day }\end{array}$ & 29 & $63(10)$ & $7.7(7.5-8.1)^{a}$ & $\begin{array}{l}28.9 \\
(25.4-34.1)^{a}\end{array}$ & $\begin{array}{l}\text { Antihypertensive: } 75 \\
\text { Lipid-lowering: } 75 \\
\text { Anti-platelet: } 42\end{array}$ & - \\
\hline 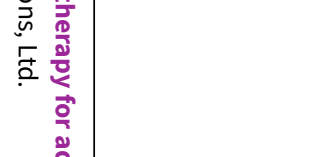 & $\begin{array}{l}\text { C: metformin } \geq 1500 \mathrm{mg} / \text { day + } \\
\text { vildagliptin } 100 \mathrm{mg} / \text { day }\end{array}$ & 35 & $61(9)$ & $7.7(7.4-7.9)^{a}$ & $\begin{array}{l}29.1 \\
(26.8-32.9)^{a}\end{array}$ & $\begin{array}{l}\text { Antihypertensive: } 64 \\
\text { Lipid-lowering: } 67 \\
\text { Anti-platelet: } 51\end{array}$ & - \\
\hline 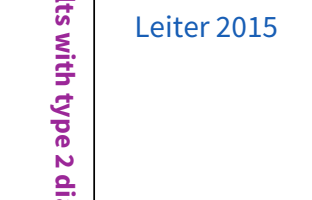 & $\begin{array}{l}\text { I: metformin } \geq 1500 \mathrm{mg} / \text { day + } \\
\text { glimepiride } 1-8 \mathrm{mg} / \text { day }\end{array}$ & 45 & $56.3(9.0)$ & $7.8(0.8)$ & $30.9(5.5)$ & $\begin{array}{l}\text { ACE-inhibitor or ARB use: } \\
62.9 \\
\text { Participants who started or } \\
\text { modified therapy with lipid- } \\
\text { modifying agents: } 13.3\end{array}$ & $\begin{array}{l}\text { Obese: } 51.5 \\
\text { Neuropathy: } 13.9 \\
\text { Retinopathy: } 5.6 \\
\text { Nephropathy: } 3.3\end{array}$ \\
\hline 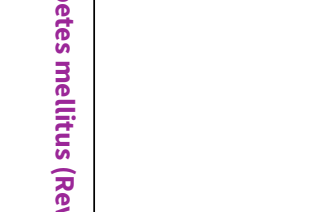 & $\begin{array}{l}\mathrm{C} 1 \text { : } \text { metformin } \geq 1500 \mathrm{mg} / \text { day }+ \\
\text { canagliflozin } 100 \mathrm{mg} / \text { day }\end{array}$ & 48 & $56.4(9.5)$ & $7.8(0.8)$ & $31.0(5.3)$ & $\begin{array}{l}\text { ACE-inhibitor or ARB use: } \\
59.4 \\
\text { Participants who started or } \\
\text { modified therapy with lipid- } \\
\text { modifying agents: } 13.0\end{array}$ & $\begin{array}{l}\text { Obese: } 55.5 \\
\text { Neuropathy: } 15.5 \\
\text { Retinopathy: } 5.4 \\
\text { Nephropathy: } 3.7\end{array}$ \\
\hline$\leqslant$ & $\begin{array}{l}\text { C2: } \text { metformin } \geq 1500 \mathrm{mg} / \text { day }+ \\
\text { canagliflozin } 300 \mathrm{mg} / \text { day }\end{array}$ & 50 & $55.8(9.2)$ & $7.8(0.8)$ & $31.2(5.4)$ & $\begin{array}{l}\text { ACE-inhibitor or ARB use: } 60 \\
\text { Participants who started or } \\
\text { modified therapy with lipid- } \\
\text { modifying agents: } 11.5\end{array}$ & $\begin{array}{l}\text { Obese: } 53.8 \\
\text { Neuropathy: } 12.8 \\
\text { Retinopathy: } 7.6 \\
\text { Nephropathy: } 3.1\end{array}$ \\
\hline
\end{tabular}




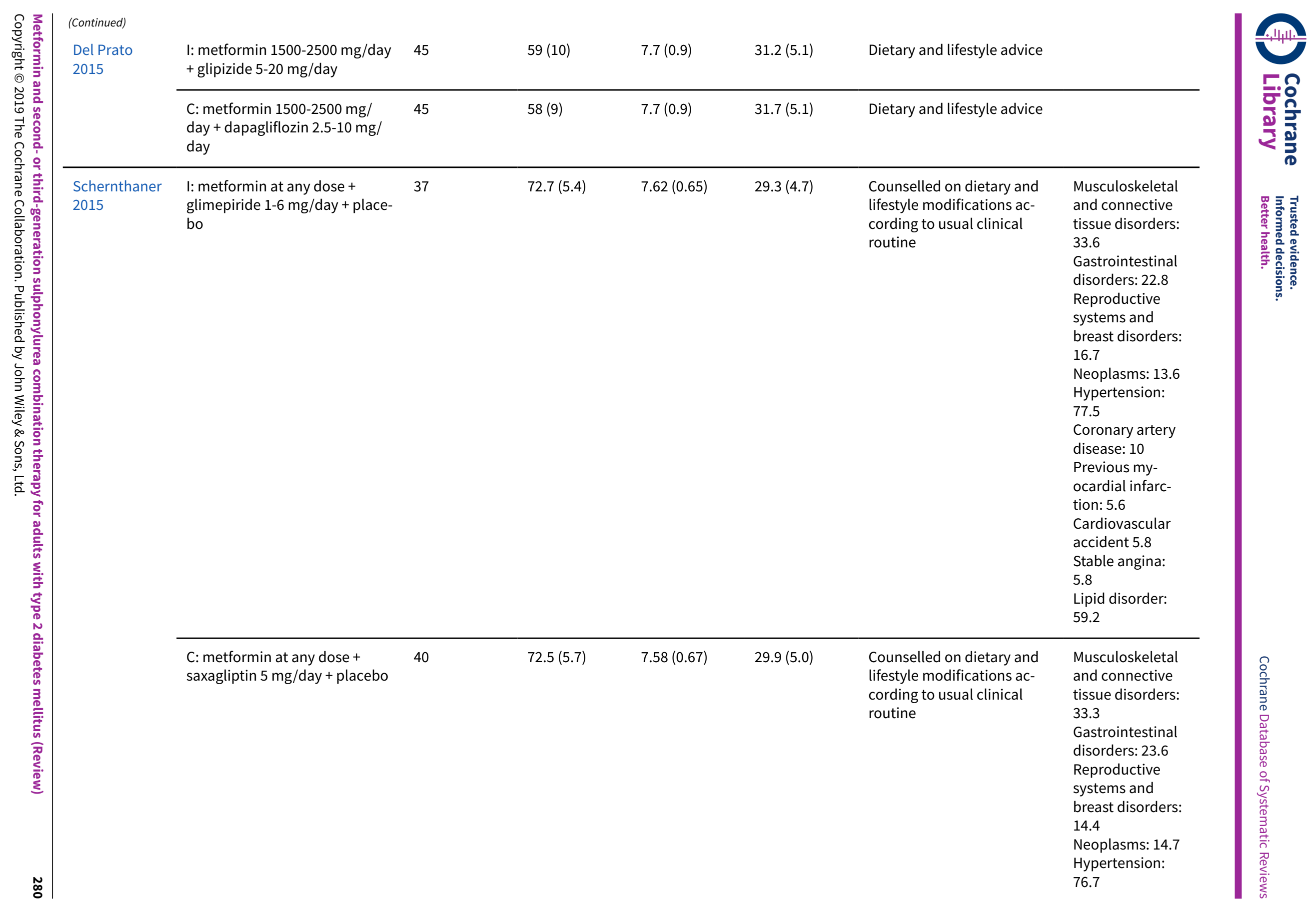




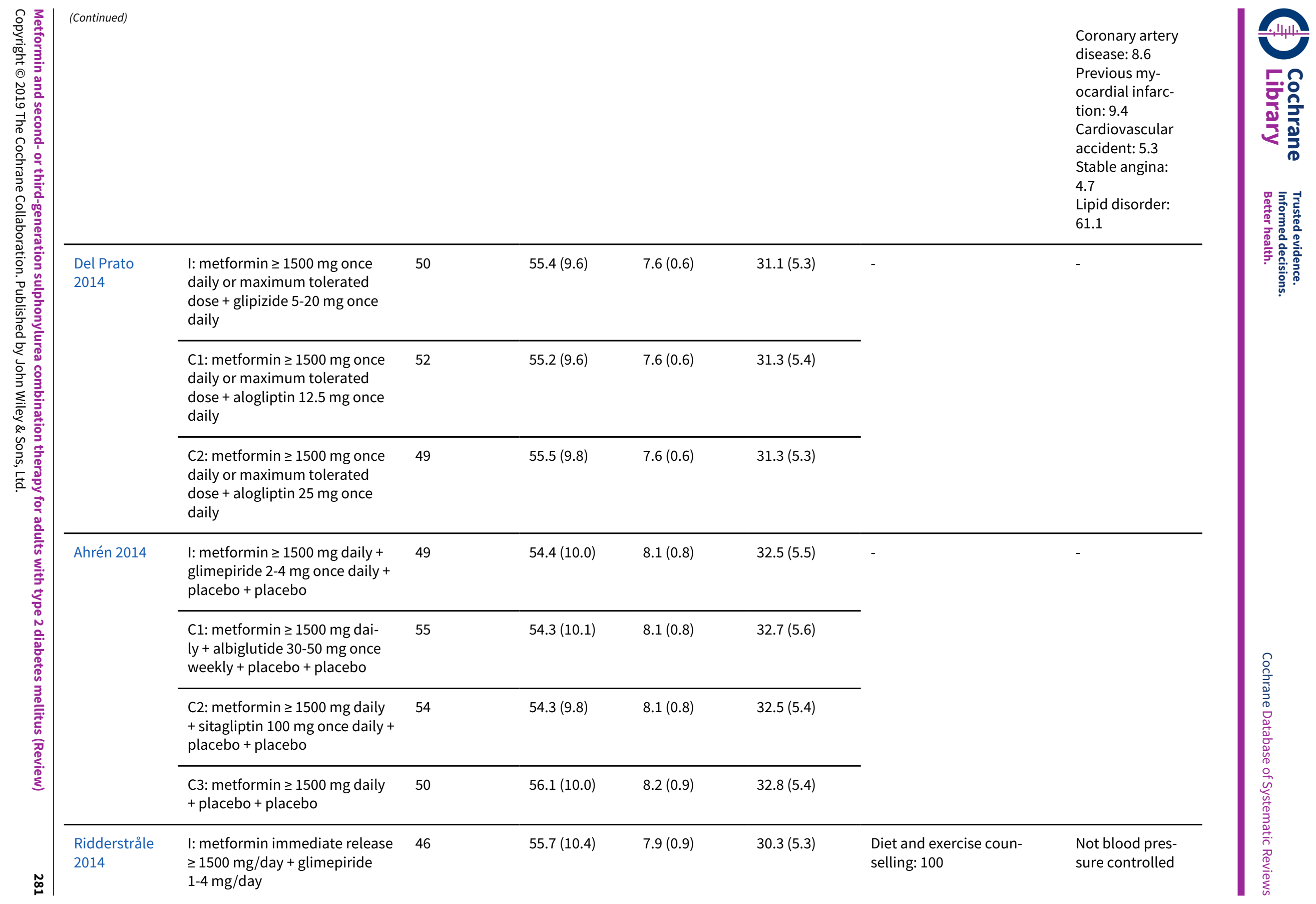


Cardiovascular medications: 74
$<130 \mathrm{mmHg} / 80$

$\mathrm{mmHg}): 69$

Estimated

glomerular filtra-

tion rate 30 to <

60: 3

Macroalbumin-

uria: 2

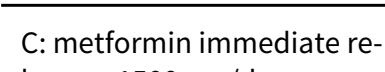

44

$56.2(10.3)$

$7.9(0.8)$

$29.9(5.3)$

Diet and exercise coun-

selling: 100

pagliflozin $25 \mathrm{mg} /$ day

Not blood pres-
Cardiovascular medications: 78 sure controlled

$<130 \mathrm{mmHg} / 80$

$\mathrm{mmHg}$ ): 68

Estimated

glomerular filtra-

tion rate 30 to <

60: 2

Macroalbumin-

uria: 2

\begin{tabular}{|c|c|c|c|c|c|c|c|}
\hline \multirow[t]{2}{*}{ Göke 2013} & $\begin{array}{l}\text { I: metformin } \geq 1500 \mathrm{mg} \text { daily + } \\
\text { glipizide } 5-20 \mathrm{mg} / \text { day }\end{array}$ & 46 & $57.6(10.37)$ & $7.7(0.9)$ & $31.3(6.17)$ & Advice on diet and exercise & \multirow[t]{2}{*}{-} \\
\hline & $\begin{array}{l}\text { C: } \text { metformin } \geq 1500 \mathrm{mg} \text { daily + } \\
\text { saxagliptin } 5 \mathrm{mg} / \text { day }\end{array}$ & 51 & $57.5(10.26)$ & $7.7(0.9)$ & $31.5(5.70)$ & Advice on diet and exercise & \\
\hline \multirow[t]{2}{*}{ Maffioli 2013} & $\begin{array}{l}\text { I: metformin } 2550 \mathrm{mg} / \text { day + } \\
\text { glibenclamide } 10 \mathrm{mg} / \text { day }\end{array}$ & 50 & $61.4(5.6)$ & $8.2(3.6)^{b}$ & $30.2(2.9)$ & $\begin{array}{l}\text { Comedication: } 6 \text { months of } \\
\text { rosuvastatin } 5 \text { mg/day: } 100 \\
\text { Cointervention: con- } \\
\text { trolled-energy diet, be- } \\
\text { haviour-modification pro- } \\
\text { gramme and physical activi- } \\
\text { ty: } 100\end{array}$ & $\begin{array}{l}\text { Overweight: } 100 \\
\text { Hepatic steato- } \\
\text { sis: } 100)\end{array}$ \\
\hline & $\begin{array}{l}\text { C: metformin } 2550 \mathrm{mg} / \text { day + pi- } \\
\text { oglitazone } 30 \mathrm{mg} / \text { day }\end{array}$ & 52 & $62.8(6.3)$ & $8.4(3.4)$ & $30.0(3.0)$ & $\begin{array}{l}\text { Comedication: } 6 \text { months of } \\
\text { rosuvastatin } 5 \text { mg/day: } 100 \\
\text { Cointervention: con- } \\
\text { trolled-energy diet, be- } \\
\text { haviour modification pro- } \\
\text { gramme and physical activi- } \\
\text { ty: } 100\end{array}$ & $\begin{array}{l}\text { Overweight: } 100 \\
\text { Hepatic steato- } \\
\text { sis: } 100\end{array}$ \\
\hline
\end{tabular}


38

$56.0(10.5)$

$8.4(0.9)$

$30.5(4.8)$

day + liraglutide $0.6 \mathrm{mg} /$ day +

placebo

\begin{tabular}{|c|}
\hline $\begin{array}{l}\text { C2: metformin } 1500-2000 \mathrm{mg} / \\
\text { dav + liraglutide } 1.2 \mathrm{mg} / \text { dav + }\end{array}$ \\
\hline
\end{tabular}

placebo

\begin{tabular}{lllll}
\hline C3: metformin $1500-2000 \mathrm{mg} /$ & 41 & $56.8(9.4)$ & $8.3(0.9)$ & $30.9(4.6)$ \\
day + liraglutide $1.8 \mathrm{mg} / \mathrm{day}+$
\end{tabular}

placebo

\begin{tabular}{lllll}
\hline C4: metformin $1500-2000 \mathrm{mg} /$ & 41 & $56.0(9.4)$ & $8.4(1.0)$ & $31.6(4.4)$
\end{tabular}

day + placebo + placebo

\begin{tabular}{|c|c|c|c|c|c|c|c|}
\hline $\begin{array}{l}\text { Gallwitz } \\
2012 a\end{array}$ & $\begin{array}{l}\text { I: metformin median dose } 2000 \\
\mathrm{mg} / \text { day + glimepiride mean } \\
\text { dose } 2.01 \mathrm{mg} / \text { day }\end{array}$ & 48 & $56(9.1)$ & $7.4(0.7)$ & $32.3(3.9)$ & Antihypertensive drugs: 75 & Overweight: 100 \\
\hline & $\begin{array}{l}\text { C: metformin median dose } 2000 \\
\mathrm{mg} / \text { day + exenatide mean dose } \\
17.35 \mu \mathrm{g} / \text { day }\end{array}$ & 44 & $56(1.0)$ & $7.5(0.7)$ & $32.6(4.2)$ & Antihypertensive drugs: 69 & Overweight: 100 \\
\hline \multirow[t]{2}{*}{$\begin{array}{l}\text { Gallwitz } \\
\text { 2012b }\end{array}$} & $\begin{array}{l}\text { I: } \text { metformin } \geq 1500 \mathrm{mg} / \text { day + } \\
\text { glimepiride } 1-4 \mathrm{mg} / \text { day + place- } \\
\text { bo }\end{array}$ & 39 & $59.8(9.4)$ & $7.69(0.9)$ & $30.31(4.6)$ & $\begin{array}{l}\text { Most participants received } \\
\text { other treatments, most } \\
\text { commonly antihypertensive } \\
\text { drugs }\end{array}$ & \\
\hline & $\begin{array}{l}\text { C: } \text { metformin } \geq 1500 \mathrm{mg} / \text { day + } \\
\text { linagliptin } 5 \mathrm{mg} / \text { day + placebo }\end{array}$ & 40 & $59.8(9.4)$ & $7.69(0.9)$ & $30.21(4.8)$ & $\begin{array}{l}\text { Most participants received } \\
\text { other treatments, most } \\
\text { commonly antihypertensive } \\
\text { drugs }\end{array}$ & \\
\hline Derosa 2011a & $\begin{array}{l}\text { I: metformin } 1000-2000 \mathrm{mg} / \text { day } \\
+ \text { glimepiride } 6 \mathrm{mg} / \text { day }\end{array}$ & 52 & $55(6)$ & $8.8(0.8)$ & $28.5(1.4)$ & $\begin{array}{l}\text { Cointervention: con- } \\
\text { trolled-energy diet, behav- } \\
\text { ior modification program } \\
\text { and physical activity: } 100\end{array}$ & Overweight: 100 \\
\hline
\end{tabular}

C: metformin 1000-2000 mg/

51

$56(7)$

$28.4(1.3)$

Cointervention: con-

Overweight: 100 
Antihypertensives:

ACE-inhibitor: 13.1

ARBs: 12.1

Calcium antagonists: 11.1

$\beta$-Blockers: 3.0

Diuretics: 7.1

Dyslipidemia:

15.1

Hypertension +

dyslipidaemia:

a-Blockers: 8.1

Antidyslipidemics:

Statins: 10.1

Fibrates: 4.0

\section{Antiaggregants:}

Acetylsalicylic acid: 40.4

Ticlopidine: 2.0

Cointervention: con-

trolled-energy diet, be-

haviour-modification pro-

gramme and physical activi-

ty: 100

C: metformin $1700 \pm 850 \mathrm{mg} /$

day + pioglitazone 15-45 mg/

day
Concomitant medication

Antihypertensives:

ACE-inhibitor: 14.7

ARBs: 10.8

Calcium antagonists: 9.8

B-Blockers: 3.9

Diuretics: 7.8

a-Blockers: 5.9

Antidyslipidemics:

Statins: 8.8

Fibrates: 5.9

Antiaggregants:

Acetylsalicylic acid: 43.1

Ticlopidine: 1.0
Concomitant disease: 44

Hypertension:

26.5

Dyslipidemia:

11.8

Hypertension + dyslipidaemia: 5.9 


\begin{tabular}{|c|c|c|c|c|c|c|c|}
\hline 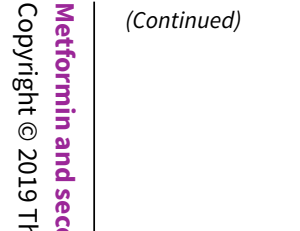 & & & & & & $\begin{array}{l}\text { Cointervention: con- } \\
\text { trolled-energy diet, be- } \\
\text { haviour-modification pro- } \\
\text { gramme and physical activi- } \\
\text { ty: } 100\end{array}$ & \\
\hline Petrica 2011 & $\begin{array}{l}\text { I: metformin } 1700 \mathrm{mg} / \text { day + } \\
\text { glimepiride } 4 \mathrm{mg} / \text { day }\end{array}$ & 62 & $58.8(7.8)$ & $7.5(1.0)$ & $32.1(6.0)$ & $\begin{array}{l}\text { ACE-inhibitor or ARB } \\
\text { Statins }\end{array}$ & - \\
\hline 吾 & $\begin{array}{l}\text { C: metformin } 1700 \mathrm{mg} / \text { day + pi- } \\
\text { oglitazone } 30 \mathrm{mg} / \text { day }\end{array}$ & 65 & $56.9(6.4)$ & $7.7(0.8)$ & $33.7(6.4)$ & $\begin{array}{l}\text { ACE-inhibitor or ARB } \\
\text { Statins }\end{array}$ & \\
\hline 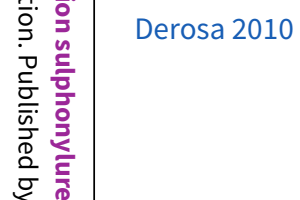 & $\begin{array}{l}\text { I: metformin } 1500 \pm 500 \mathrm{mg} / \text { day } \\
\text { + glibenclamide } 15 \mathrm{mg} / \text { day }\end{array}$ & 49 & $56(7)$ & $8.9(0.8)$ & $28.5(1.4)$ & $\begin{array}{l}\text { Cointervention: con- } \\
\text { trolled-energy diet, be- } \\
\text { haviour-modification pro- } \\
\text { gramme and physical activi- } \\
\text { ty: } 100\end{array}$ & Overweight: 100 \\
\hline 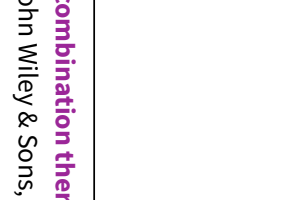 & $\begin{array}{l}\text { C: metformin } 1500 \pm 500 \mathrm{mg} / \\
\text { day + exenatide } 20 \mu \mathrm{g} / \text { day }\end{array}$ & 52 & $57(8)$ & $8.8(0.7)$ & $28.7(1.5)$ & $\begin{array}{l}\text { Cointervention: con- } \\
\text { trolled-energy diet, be- } \\
\text { haviour-modification pro- } \\
\text { gramme and physical activi- } \\
\text { ty: } 100\end{array}$ & Overweight: 100 \\
\hline 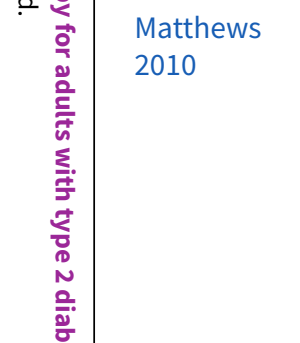 & $\begin{array}{l}\text { I: metformin } \geq 1500 \mathrm{mg} \text { twice a } \\
\text { day + glimepiride } 2-6 \mathrm{mg} / \text { day }\end{array}$ & 46 & 57.5 (9.19) & $7.3(0.66)$ & $31.7(5.26)$ & $\begin{array}{l}\text { Concomitant medications: } \\
\text { ACE-inhibitors } \\
\text { ARBs } \\
\text { Diuretics } \\
\beta \text {-blockers } \\
\text { Lipid-lowering agents } \\
\text { Platelet aggregations in- } \\
\text { hibitors }\end{array}$ & - \\
\hline 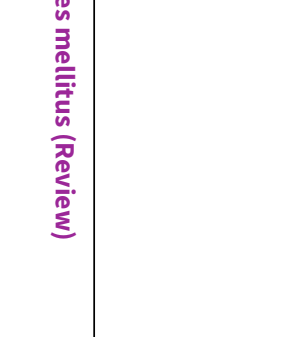 & $\begin{array}{l}\text { C: metformin } \geq 1500 \mathrm{mg} \text { twice a } \\
\text { day + vildagliptin } 50 \mathrm{mg} \text { twice a } \\
\text { day }\end{array}$ & 47 & $57.5(9.07)$ & $7.3(0.65)$ & $31.9(5.33)$ & $\begin{array}{l}\text { Concomitant medications: } \\
\text { ACE-inhibitors } \\
\text { ARBs } \\
\text { Diuretics } \\
\beta \text {-blockers } \\
\text { Lipid-lowering agents } \\
\text { Platelet aggregations in- } \\
\text { hibitors }\end{array}$ & - \\
\hline
\end{tabular}




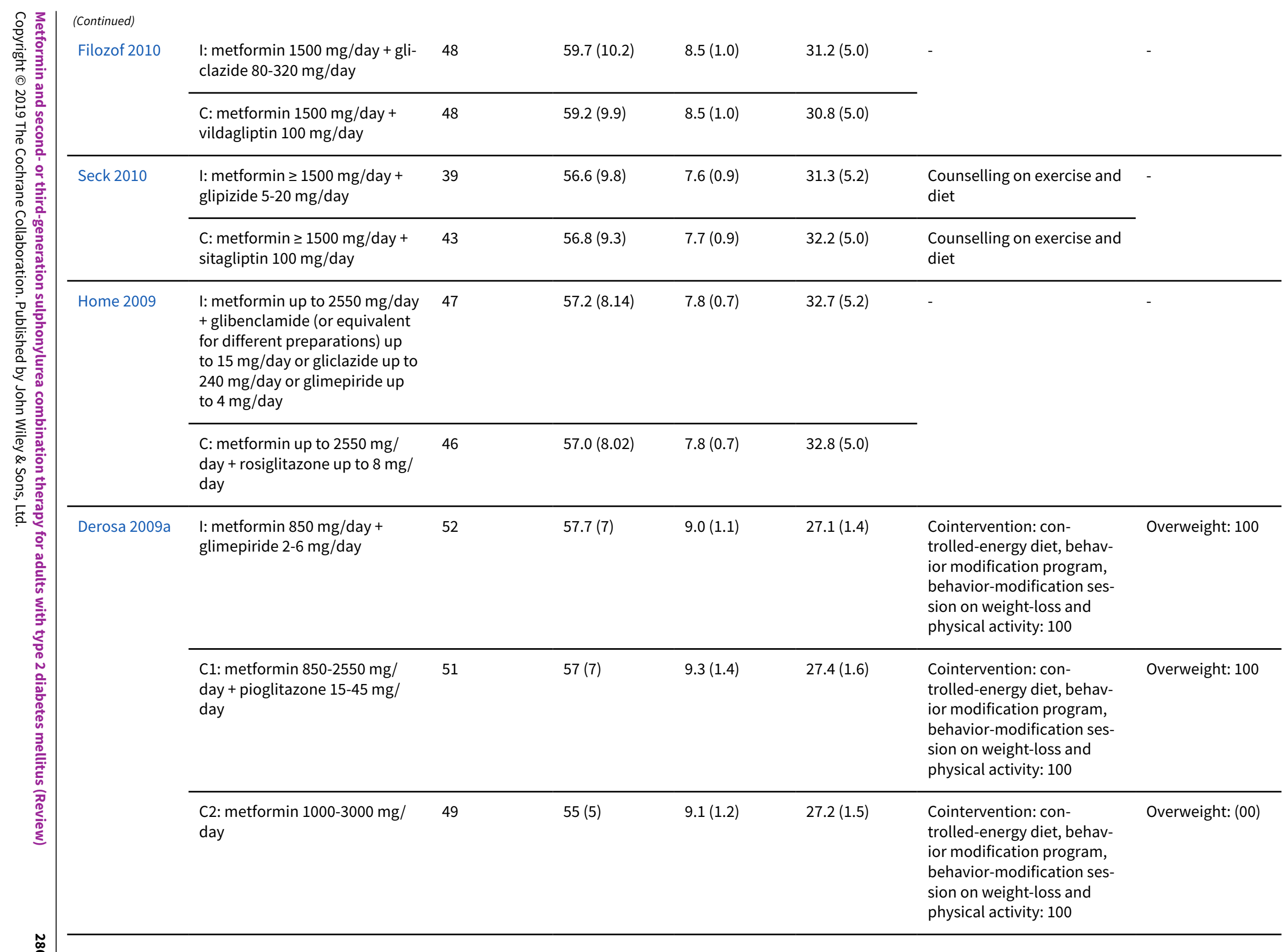


eight-loss and

physical activity: 100

\begin{tabular}{|c|c|c|c|c|c|}
\hline $\begin{array}{l}\text { C: metformin } 1500-3000 \mathrm{mg} / \\
\text { day + nateglinide } 180-360 \mathrm{mg} / \\
\text { day }\end{array}$ & 52 & $55(5)$ & $8.1(1.0)$ & $26.4(1.4)$ & $\begin{array}{l}\text { Cointervention: con- } \\
\text { trolled-energy diet, behav- } \\
\text { ior modification program, } \\
\text { behavior-modification ses- } \\
\text { sion on weight-loss and } \\
\text { physical activity: } 100\end{array}$ \\
\hline
\end{tabular}

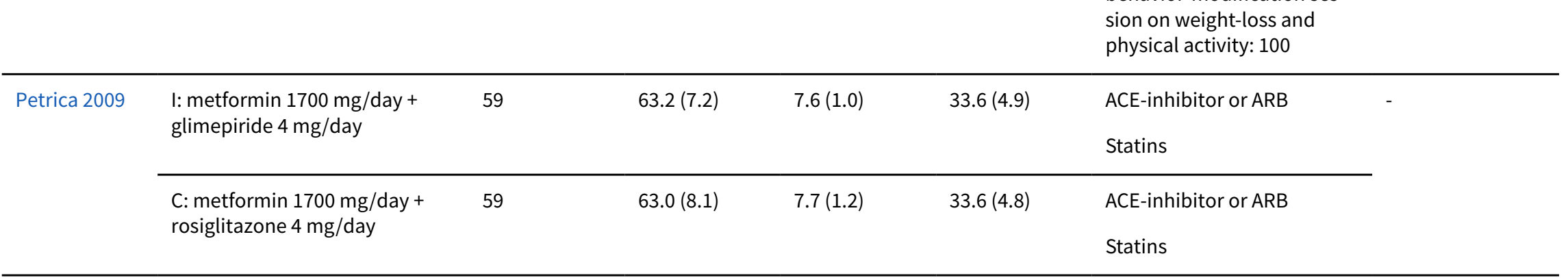

\begin{tabular}{|c|c|c|c|c|}
\hline NCT00367055 & $\begin{array}{l}\text { I: metformin } 2000 \mathrm{mg} / \text { day + gli- } \\
\text { clazide } 80-320 \mathrm{mg} / \text { day }\end{array}$ & 39 & $58.1(8.0)$ & $7.3(0.55)$ \\
\hline
\end{tabular}

clazide $80-320 \mathrm{mg} /$ day

25-30: $49 \%$

\begin{tabular}{lllll}
\multicolumn{1}{l}{} & & $>30: 51 \%$ \\
\hline $\begin{array}{l}\text { C: metformin } 2000 \mathrm{mg} / \text { day }+ \\
\text { rosiglitazone 4-8 mg/day }\end{array}$ & 26 & $58.3(8.4)$ & $7.5(0.55)$ & $<25: 7 \%$ \\
\end{tabular}

> $30: 44 \%$

Hamann 2008 I: metformin $2000 \mathrm{mg} /$ day +

48

$59.3(9.2)$

$8.0(1.0)$

$32.2(4.9)$

gliclazide $80-320 \mathrm{mg} /$ day

\begin{tabular}{lllll}
\hline $\begin{array}{l}\text { C: } \text { metformin } 2000 \mathrm{mg} / \text { day }+ \\
\text { rosiglitazone } 4-8 \mathrm{mg} / \text { day }\end{array}$ & 47 & $58.5(9.6)$ & $8.0(0.9)$ & $33.0(5.9)$
\end{tabular}

Vverweight: 100 Dyslipidaemia:

51

Current smoker:

20

Former smoker: 26 


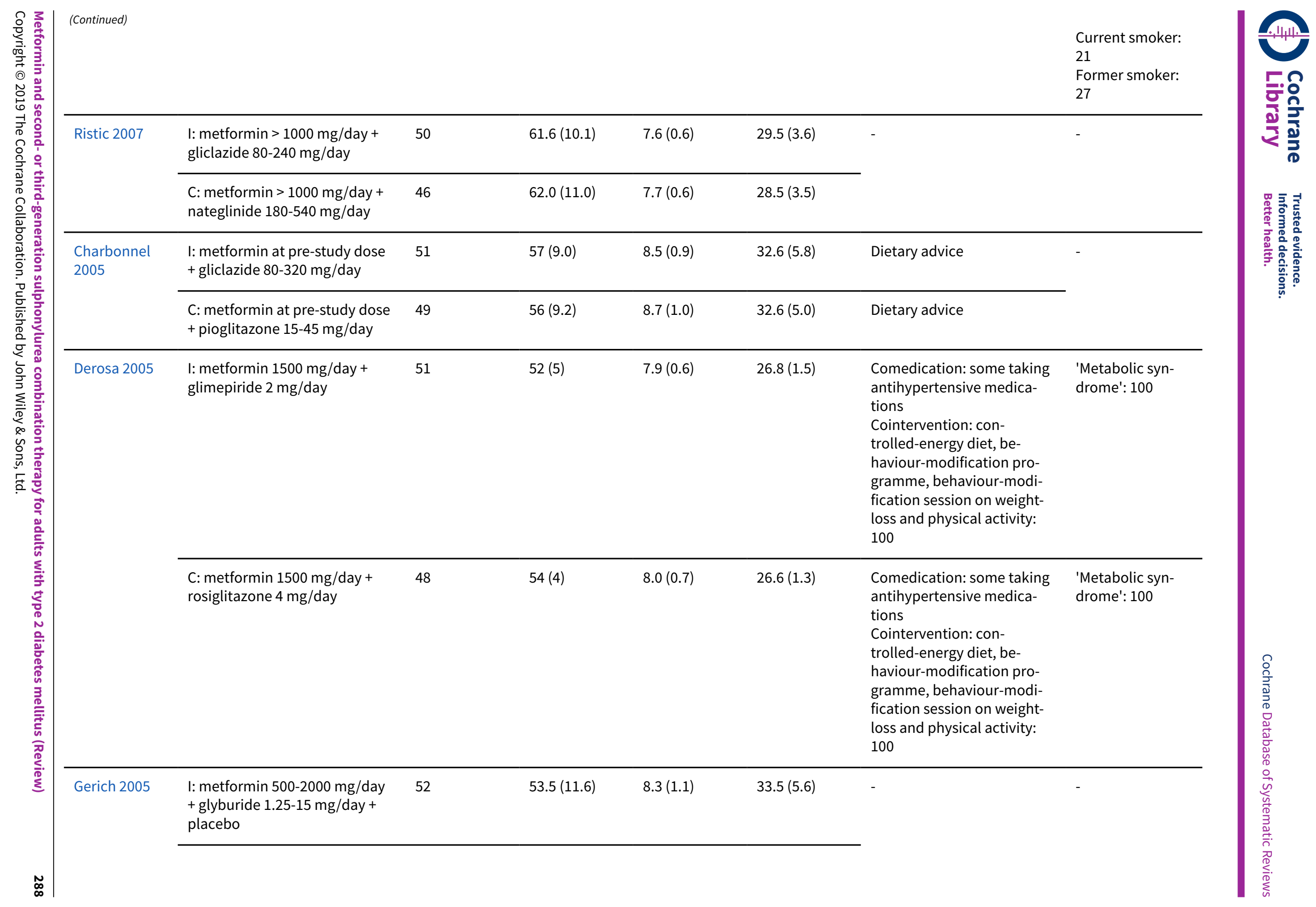




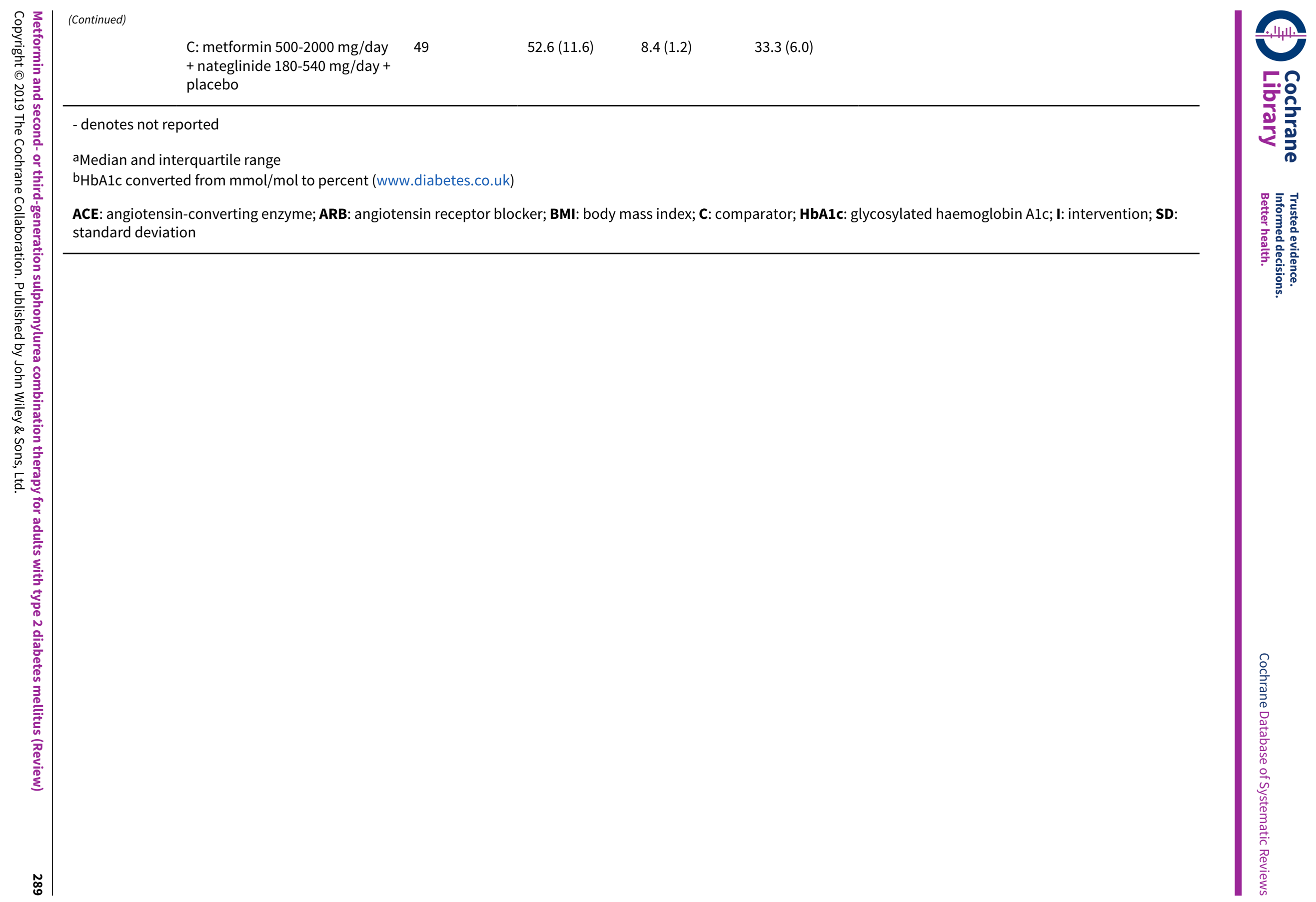




\section{Appendix 7. Matrix of trial endpoints (publications and trial documents)} er's website, published design paper) ${ }^{a, c}$

Source: NCT01682759

Primary outcome measure(s): change from baseline in $\mathrm{HbA1c}$ at week 54, percentage of participants who experienced at least one adverse event excluding data after glycaemic rescue, percentage of participants who discontinued from the study due to an adverse event excluding data after glycaemic rescue

Secondary outcome measure(s): change from baseline in fasting plasma glucose at week 54, percentage of participants achieving a HbAlc of $<6.5 \%$ at week 54, percentage of participants with an adverse event of symptomatic hypoglycaemia excluding data after glycaemic rescue, change from baseline in body weight at week 54 excluding data after glycaemic rescue, percentage of participants achieving a HbA1c of $<7.0 \%$ at week 54

Other outcome measure(s): -

Trial results available in trial register: yes

\section{Endpoints quoted in publication $(\mathbf{s})^{b, c}$}

Primary outcome measure(s): change from baseline in $\mathrm{HbA1c}$ at week 54

Secondary outcome measure(s): change from baseline in FPG at week 54, proportion of participants achieving HbA1c goal of $<6.5 \%(48 \mathrm{mmol} / \mathrm{mol}),<7 \%(53 \mathrm{mmol} / \mathrm{mol})$ at week 54

Other outcome measure(s): any adverse events of symptomatic hypoglycaemia, change from baseline in body weight at week 54, adverse events summary measures, specific adverse events, system organ classes, and predefined limits of change, any adverse event of hypoglycaemia

Endpoints quoted in abstract of publication(s) $)^{b, c}$

Primary outcome measure(s): -

Secondary outcome measure(s): -

Other outcome measure(s): change from baseline in HbA1c week 54, symptomatic hypoglycaemia, adverse events, weight change er's website, published design paper) $)^{a, c}$

\section{Source: NCT01999218}

Primary outcome measure(s): change from baseline in $\mathrm{HbA} 1 \mathrm{C}$ at week 52, number of participants experiencing an adverse event, time frame: up to week 106, number of participants discontinuing study treatment due to an $\mathrm{AE}$, time frame: up to week 104

Secondary outcome measure(s): number of participants with an adverse event of symptomatic hypoglycaemia, time frame: up to week 52, change from baseline in body weight at week 52, change from baseline in systolic blood pressure at week 52

\section{Other outcome measure(s): -}

Trial results available in trial register: no

Endpoints quoted in publication(s) $)^{b, c}$ 
Primary outcome measure(s): change from baseline in HbA1c at week 52

Secondary outcome measure(s): changes from baseline in body weight and systolic blood pressure at week 52

Other outcome measure(s): other efficacy endpoints evaluated at week 52 included the percentage of participants with $\mathrm{HbA} 1 \mathrm{c}<53 \mathrm{mmol} / \mathrm{mol}(7.0 \%)$; changes from baseline in diastolic blood pressure, FPG, homeostasis model assessment of b-cell function, and proinsulin/C-peptide ratio; the percentage of participants requiring rescue medication; and the percentage of participants meeting the composite endpoints of HbAlc

\section{Endpoints quoted in abstract of publication $(s)^{b, c}$}

Primary outcome measure(s): -

Secondary outcome measure(s): -

Other outcome measure(s): change from baseline in $\mathrm{HbA1c}$, body weight and systolic pressure, adverse events, hypoglycaemia, genital mycotic infection, urinary tract infection, hypovolaemia er's website, published design paper)a,c

\section{Source: NCT00700856}

Primary outcome measure(s): a composite endpoint including: all-cause mortality, non fatal myocardial infarction (MI) (including silent MI), non fatal stroke, unplanned coronary revascularisation

Secondary outcome measure(s): a composite ischemic end point of: sudden death, fatal and non fatal MI (including silent MI), fatal and non fatal stroke, major leg amputation (above the ankle), endovascular or surgical interventions on the coronary, leg or carotid arteries. A composite CV endpoint including the primary endpoint plus heart failure, endovascular or surgical intervention on the coronary, leg or carotid arteries, angina, intermittent claudication with an ankle/brachial index $<0.85$. Glycaemic control (changes from baseline in $\mathrm{HbA1c}$, time to failure of oral hypoglycaemic therapy, i.e., HBA1c $>8.0 \%$ on two consecutive occasions three months apart). Major cardiovascular risk factors (lipids, blood pressure, microalbuminuria, inflammation markers, waist circumference). Development of nephropathy: plasma creatinine increase of 2 times above the baseline value or creatinine clearance reduction of $20 \mathrm{ml} / \mathrm{min} / 1.73 \mathrm{~m}^{2}$ or development of microalbuminuria or overt nephropathy (dialysis of plasma creatinine $>3.3 \mathrm{mg} / \mathrm{dL}$ ). Events of heart failure evaluated according to the American Heart Association and the American Diabetes Association consensus on glitazones and heart failure

\section{Other outcome measure(s): -}

Trial results available in trial register: no

\section{Endpoints quoted in publication $(s)^{b, c}$}

Primary outcome measure(s): the primary outcome was a composite of first occurrence of allcause death, non-fatal myocardial infarction (including silent myocardial infarction), non-fatal stroke, or urgent coronary revascularisation

Secondary outcome measure(s): the key secondary outcome was a composite of ischaemic cardiovascular disease, which included first occurrence of sudden death, fatal and non-fatal myocardial infarction (including silent myocardial infarction), fatal and non-fatal stroke, leg amputation above the ankle, and any revascularisation of the coronary, leg, or carotid arteries. An expanded composite cardiovascular outcome was among the remaining secondary outcomes-this included the primary outcome plus heart failure; any revascularisation of the coronary, leg, or carotid arteries; angina confirmed by new ECG abnormalities; and intermittent claudication with an ankle-brachial index less than 0.90 . The other secondary outcomes were new or worsening nephropathy (i.e. new-onset macroalbuminuria, twice the baseline levels of serum creatinine, creatinine 
clearance reduction of $\geq 20 \mathrm{~mL} / \mathrm{min}$ per $1.73 \mathrm{~m}^{2}$, plasma creatinine $>290 \mu \mathrm{mol} / \mathrm{L}$, or need for permanent dialysis), time to failure of hypoglycaemic treatment (defined as $\mathrm{HbAlc} \geq 8 \%$ ( $\geq 64 \mathrm{mmol} /$ mol) on two consecutive visits 3 months apart), and changes in $\mathrm{HbAlc}$ and major cardiovascular risk factors (BMI, waist circumference, plasma lipids, blood pressure, microalbuminuria, C-reactive protein, estimated glomerular filtration rate (eGFR), and heart rate) over time

Other outcome measure(s): adverse events

\section{Endpoints quoted in abstract of publication $(s)^{b, c}$}

Primary outcome measure(s): the primary efficacy outcome is a composite endpoint of all-cause mortality, nonfatal myocardial infarction, nonfatal stroke, and unplanned coronary revascularization

Secondary outcome measure(s): principal secondary outcome is a composite ischemic endpoint of sudden death, fatal and non-fatal myocardial infarction and stroke, endovascular or surgical intervention on the coronary, leg or carotid arteries, major amputations

Other outcome measure(s): adverse effects, quality of life and economic costs will also be evaluated

\section{Dei Cas 2017}

Endpoints quoted in trial document(s) (ClinicalTrials.gov, FDA/EMA document, manufacturer's website, published design paper)a,c

Source: NCT00968812 and NCT01822548

Primary outcome measure(s): absolute and relative change in the circulating endothelial progenitor cells number

Secondary outcome measure(s): absolute and relative change in HbA1c compared to baseline

Other outcome measure(s): adverse events, hypoglycaemia, hyperglycaemia, abnormal findings in physical exam and laboratory workup

Trial results available in trial register: no

Endpoints quoted in publication $(\mathbf{s})^{b, c}$

Primary outcome measure(s): circulating endothelial progenitor cells number

Secondary outcome measure(s): change in HbAlc

Other outcome measure(s): adverse events, hypoglycaemia, hyperglycaemia, BMI, fasting plasma glucose

Endpoints quoted in abstract of publication(s) $)^{b, c}$

Primary outcome measure(s): endothelial progenitor cells number

Secondary outcome measure(s): glycaemic control

Other outcome measure(s): inflammatory biomarkers er's website, published design paper)a,c

Source: NCT00968812

Primary outcome measure(s): change in $\mathrm{HbA1c}$ from baseline to week 52 
Secondary outcome measure(s): "percentage of participants experiencing at least 1 hypoglycemic event from baseline to week 52", "Percent change in body weight from baseline to week 52", "Change in HbA1c from baseline to week 104"

Other outcome measure(s): -

Trial results available in trial register: yes

Endpoints quoted in publication(s) ${ }^{b, c}$

Primary outcome measure(s): change in $\mathrm{HbA1c}$ from baseline to week 52

Secondary outcome measure(s): (Cefalu 2013) "... percentage change from baseline in bodyweight, and proportion of patients with documented hypoglycaemic episodes"; (Leiter 2015) "Secondary end points assessed at week 104 included change in A1C, FPG, and systolic and diastolic BP; percentage change in body weight and fasting plasma lipids (including triglycerides, HDL cholesterol [HDL-C], LDL cholesterol [LDL-C], LDL-C/HDL-C ratio, and non-HDL-C); and the proportion of patients achieving $\mathrm{A} 1 \mathrm{C}, 7.0 \%(53 \mathrm{mmol} / \mathrm{mol}) "$

Other outcome measure(s): (Cefalu 2013)

"Additional endpoints included the proportion of patients achieving HbAlc less than either $7.0 \%$ or 6.5\%; change in fasting plasma glucose and systolic and diastolic blood pressure; and percentage change in fasting plasma lipids, including HDL cholesterol, triglycerides, LDL cholesterol, nonHDL cholesterol, and ratio of LDL cholesterol to HDL cholesterol." "We assessed body composition endpoints for a subset of patients at week 52. Changes from baseline in total fat mass, total lean mass, and percentage of total fat (total fat measurement as a percentage of the sum of total fat measurement, total lean measurement, and bone mineral content)..." "Percentage changes in subcutaneous adipose tissue and visceral adipose tissue, and the change in the ratio of subcutaneous to visceral adipose tissue ..." "We assessed safety with adverse events reports ..." "Additional data collection was prespecified for adverse events of genital mycotic infections and urinary tract infections ..."

Endpoints quoted in abstract of publication $(\mathbf{s})^{b, c}$

Primary outcome measure(s): (Cefalu 2013) "The primary endpoint was change in Hba1c from baseline to week 52..."

Secondary outcome measure(s): -

Other outcome measure(s): -

Del Prato 2015
Endpoints quoted in trial document(s) (ClinicalTrials.gov, FDA/EMA document, manufacturer's website, published design paper)a,c

\section{Source: NCT00660907}

Primary outcome measure(s): adjusted mean change in HbA1c levels from baseline to week 52

Secondary outcome measure(s): adjusted mean change in body weight from baseline to week 52, proportion of participants with at least one episode of hypoglycaemia from baseline to week 52, proportion of participants with body weight reduction of at least $5 \%$

Other outcome measure(s): -

Trial results available in trial register: yes

Endpoints quoted in publication(s)

Primary outcome measure(s): (Nauck 2011, Nauck 2014 in Del Prato 2015, and Del Prato 2015) absolute change in Hba1c from baseline to week 52 
Secondary outcome measure(s): "absolute change in total body weight from baseline to week 52, proportion of participants reporting at least one episode of hypoglycaemia (major, minor or other episode) during the 52 week treatment period, the proportion of participants achieving a total body weight decrease $\geq 5 \%$ from baseline to week 52 "

Other outcome measure(s): (Nauck 2011 in Del Prato 2015) "change from baseline to week 52 for body weight in participants with a baseline $\mathrm{BMI} \geq 30 \mathrm{~kg} / \mathrm{m}^{2}$ and in those with baseline $\mathrm{BMI} \geq 27$ $\mathrm{kg} / \mathrm{m}^{2}$, waist circumference, change in $\mathrm{HbAlc}$ in participants with an $\mathrm{HbAlc}$ of $\geq 7 \%$ at baseline, and FPG. The proportions of participants with $\mathrm{HbAlc}<7 \%$ at week 52 in participants with baseline $\mathrm{HbAlc} \geq 7 \%$ and proportions of participants with $\mathrm{HbA} 1 \mathrm{c} \leq 6.5 \%$ at week 52 were also assessed. Absolute changes from baseline to week 52 for seated systolic and diastolic blood pressure, and percent changes from baseline to week 52 for total cholesterol, LDL cholesterol, HDL cholesterol, triglycerides, and free fatty acids were assessed. Safety and tolerability was assessed by collating data on AEs using the Medical Dictionary for Regulatory Activities (MedDRA version 12.1), hypoglycaemic events, laboratory tests, calculated creatinine clearance, urinary glucose/creatinine ratio, electrocardiographic and physical examinations, and vital signs. In addition, participants were actively questioned at each study visit to assess signs, symptoms, and reports suggestive of genital infections and UTIs. These responses, and those obtained spontaneously, were categorised in the database using a predefined list of MedDRA terms suggestive of genital infections and UTIs."

(Nauck 2014 in Del Prato 2015) "The durability of glycaemic control was evaluated by examining the change from baseline in HbA1c and FPG over 104 weeks and the proportion of participants discontinuing treatment because of a lack of glycaemic control or hypoglycaemia over 104 weeks, and by calculating the CoF for HbAlc and FPG from 18 weeks (end of titration period) to 104 weeks. CoF analyses were not protocol pre-specified. The maintenance of weight loss was evaluated by examining the change from baseline in total body weight over 104 weeks, and the proportion of participants achieving $\geq 5 \%$ reduction in body weight at week 104 . The change from baseline in seated systolic blood pressure over 104 weeks was also evaluated. AEs of special interest included the proportion of participants reporting at least one hypoglycaemic event by weeks 18,52 and week 104, and events suggestive of vulvovaginitis, balanitis and related genital infections (excluding sexually transmitted diseases) and of UTI by 52 and 104 weeks."

(Del Prato 2015) "All variables analysed for the 52-week period were re-examined during the study extensions. All analyses during the extension periods were considered exploratory. All endpoints were reported as changes from a specific time point to weeks 52, 104 and 208. Glycaemic response was analysed in all participants based on baseline HbAlc: $>6.5, \geq 7.0,<8.0, \geq 8.0,<9.0$ and $\geq 9.0 \%$. The additional endpoints assessed included changes from baseline to week 208 in FPG, systolic blood pressure and diastolic blood pressure, total body weight and waist circumference, and rates of, and time to, study discontinuation and glycaemic rescue before or at week 208.

Rates of AEs of interest (including hypoglycaemia, genital and urinary tract infections and renal impairment) were assessed at each study extension to account for participant attrition over time. In addition to the variables of hypoglycaemia and urinary glucose/creatinine ratio, participants were actively questioned on, or self-reported, symptoms suggestive of genital infection or urinary tract infection at each study visit"

\section{Endpoints quoted in abstract of publication $(s)^{b, c}$}

Primary outcome measure(s): (Nauck 2011 in Del Prato 2015) adjusted mean Hba1c change at 52 weeks

Secondary outcome measure(s): adjusted mean weight change at 52 weeks, proportion of participants achieving $\geq 5 \%$ body weight reduction, proportion experiencing hypoglycaemia

Other outcome measure(s): -

Schernthaner 2015 
Primary outcome measure(s): proportion of participants reaching $\mathrm{HbA} 1 \mathrm{c}<7 \%$ after 52 weeks of treatment without confirmed or severe hypoglycaemia

Secondary outcome measure(s): proportion of participants having experienced at least one hypoglycaemic event (confirmed or severe) over the 52-week double-blind treatment period, change from baseline to week 52 in $\mathrm{HbAlc}$, proportion of participants achieving a therapeutic glycaemic response at week 52 defined as $\mathrm{HbA} 1 \mathrm{c}<7.0 \%$, change from baseline to week 52 in FPG, change from baseline to week 52 in insulin, change from baseline to week 52 in $\beta$-cell function

Other outcome measure(s): -

Trial results available in trial register: yes

Endpoints quoted in publication(s) ${ }^{b, c}$

Primary outcome measure(s): proportion of participants achieving an $\mathrm{HbA1c}$ level of $<7.0 \%$ at week 52 without confirmed/severe hypoglycaemia

Secondary outcome measure(s): the key secondary endpoint was the proportion of participants with $\geq 1$ confirmed/severe hypoglycaemic event over the treatment period. Other secondary endpoints included the proportion of participants achievingHbA1c $<7.0$ or $\leq 6.5 \%$ at week 52 , and the change from baseline to week 52 in mean $\mathrm{HbAlc}$

Other outcome measure(s): post hoc analyses were conducted to determine the number of confirmed/severe hypoglycaemic events over time, and the incidence of confirmed/severe hypoglycaemia by HbA1c category at week $52(<7.0 \%$ or $\geq 7.0 \%)$ stratified by age group, and by HbAlc category at week $52(<6.5 \%$; $\geq 6.5$ to $<7.0 \% ; \geq 7.0$ to $<7.5$, and $\geq 7.5 \%)$. Safety and tolerability assessments included adverse events and body weight

Endpoints quoted in abstract of publication $(s)^{b, c}$

Primary outcome measure(s): achievement of $\mathrm{HbAlc}<7.0 \%$ at week 52 without confirmed/severe hypoglycaemia

Secondary outcome measure(s): the key secondary endpoint was incidence of confirmed/severe hypoglycaemia

Other outcome measure(s): safety and tolerability

Del Prato 2014

Endpoints quoted in trial document(s) (ClinicalTrials.gov, FDA/EMA document, manufacturer's website, published design paper)a,c

\section{Source: NCT00856284}

Primary outcome measure(s): "change from baseline in HbA1c at week 52", "change from baseline in HbA1c at week 104"

Secondary outcome measure(s): "change from baseline in HbA1c at other time points", "change from baseline in FPG over time", "percentage of participants with HbA1c less than or equal to $6.5 \%$ ", "percentage of participants with $\mathrm{HbA1c}$ less than or equal to $7.0 \%$ ", "change from baseline in body weight over time"

Other outcome measure(s): -

Trial results available in trial register: yes

Endpoints quoted in publication(s) $)^{b, c}$

Primary outcome measure(s): the primary efficacy endpoint was the change in $\mathrm{HbAlc}$ from baseline to week 52 and to week 104 
Secondary outcome measure(s): secondary and exploratory efficacy endpoints included (i) changes over time in $\mathrm{HbAlC}$ and FPG, (ii) incidence of clinical response ( $\mathrm{HbAlc} \leq 6.5 \%$ and $\leq 7.0 \%$ ) at week 104, (iii) changes in body weight over time, (iv) incidence of hyperglycaemic rescue, and (v) changes in 2-h PPG over time

Other outcome measure(s): safety endpoints included incidence of hypoglycaemia, MACE or pancreatitis, as well as standard general safety endpoints

\section{Endpoints quoted in abstract of publication(s)}

Primary outcome measure(s): the primary endpoint was least square mean change from baseline in $\mathrm{HbA1c}$ level at 104 weeks

Secondary outcome measure(s): -

Other outcome measure(s): FPG, mean weight change, hypoglycaemia, pancreatitis er's website, published design paper) $)^{a, c}$

Source: NCT00838903

Primary outcome measure(s): change from baseline in HbA1c at week 104

Secondary outcome measure(s): change from baseline in $\mathrm{HbA1c}$ at week 156; change from baseline in fasting plasma glucose at week 104 and week 156; number of participants who achieved clinically meaningful $\mathrm{HbA} 1 \mathrm{c}$ response levels of $<6.5 \%,<7 \%$, and $<7.5 \%$ at week 104 and week 156; time to hyperglycaemia rescue until week 156; change from baseline in body weight at week 104 and week 156

Other outcome measure(s): serious adverse events, other adverse events

Trial results available in trial register: yes

Endpoints quoted in publication(s) ${ }^{b, c}$

Primary outcome measure(s): the primary end point was the change in model-adjusted $\mathrm{HbA} 1 \mathrm{c}$ from baseline to week 104 between albiglutide and the comparators

Secondary outcome measure(s): secondary end points included changes in $\mathrm{HbA1c}$, fasting plasma glucose, and weight from baseline over time; the proportion of participants who achieved $\mathrm{HbAlc}$ treatment goals (i.e. $6.5 \%(47.5 \mathrm{mmol} / \mathrm{mol}), 7.0 \%(53.0 \mathrm{mmol} / \mathrm{mol})$ and $7.5 \%(58.5 \mathrm{mmol} /$ $\mathrm{mol})$ ); and time to hyperglycaemic rescue

Other outcome measure(s): safety and tolerability were assessed, including adverse events and serious adverse events; safety events of special interest (i.e. gastrointestinal or hypoglycaemic events, injection-site reactions, pancreatitis, thyroid tumours, potential systematic allergic reactions and cardiovascular events; clinical laboratory evaluations; physical examinations; 12-lead electrocardiograms; vital sign measurements; and immunogenicity

\section{Endpoints quoted in abstract of publication $(s)^{b, c}$}

Primary outcome measure(s): the primary end point was change in $\mathrm{HbAlc}$ from baseline at week 104

Secondary outcome measure(s): secondary end points included fasting plasma glucose, weight, and time to hyperglycaemic rescue

Other outcome measure(s): serious adverse events, diarrhoea, nausea 
(Continued)

Ridderstråle 2014

\section{Endpoints quoted in trial document(s) (ClinicalTrials.gov, FDA/EMA document, manufactur- er's website, published design paper) ${ }^{a, c}$}

Primary outcome measure(s): change from baseline in $\mathrm{HbAlc}$ after 52 and 104 weeks of treatment

Secondary outcome measure(s): change from baseline in body weight after 52 and 104 weeks of treatment, occurrence of confirmed hypoglycaemic AEs during 52 and 104 weeks of treatment

Other outcome measure(s): $\mathrm{HbA1c}<7.0 \%$ or $<6.5 \%$ after 52,104 and 208 weeks of treatment, $\mathrm{HbA} 1 \mathrm{c}$ lowering by $\geq 0.5 \%$ after 52,104 and 208 weeks of treatment, change from baseline in $\mathrm{HbA} 1 \mathrm{c}$ after 208 weeks of treatment, coefficient of durability for $\mathrm{HbA1c}$ response, change from baseline in FPG after 52, 104 and 208 weeks of treatment, change from baseline in mean daily glucose (8point) after 52, 104 and 208 weeks of treatment (substudy), change from baseline in 2-h PPG after 52, 104 and 208 weeks of treatment and follow-up (4 weeks after treatment discontinuation) (substudy), biomarkers of insulin secretion and resistance after 104 and 208 weeks of treatment and (in the mean tolerance test substudy) follow-up (4 weeks after treatment discontinuation), confirmed hypoglycaemic AEs during 208 weeks of treatment

Change from baseline in body weight of $>2 \%$ and $>5 \%$ after 52,104 and 208 weeks of treatment, change from baseline in body weight after 208 weeks of treatment and follow-up (4 weeks after treatment discontinuation), change from baseline in waist circumference after 52, 104 and 208 weeks of treatment and follow-up (4 weeks after treatment discontinuation), changes from baseline in trunk fat, limb fat, fat-free mass and total fat mass (using DXA scan) after 52, 104 and 208 weeks of treatment (substudy), changes from baseline in bone mineral density and T-scores (using DXA scan) after 52, 104 and 208 weeks of treatment (substudy), changes from baseline in abdominal VAT, abdominal SAT and VAT/SAT ratio (using MRI) after 52, 104 and 208 weeks of treatment (substudy), proportion of participants with BP $<130 / 80 \mathrm{mmHg}$ after 52, 104 and 208 weeks of treatment, change from baseline in SBP and DBP after 208 weeks of treatment and follow-up (4 weeks after treatment discontinuation), $\mathrm{HbAlc}<7.0 \%$ or $\mathrm{HbA} 1 \mathrm{c}$ reduction $\geq 1.0 \%$, no confirmed hypoglycaemic AEs, and weight loss $>2 \%$ after 52,104 and 208 weeks of treatment $\mathrm{HbA1c}<6.5 \%$ or $\mathrm{HbA1c}$ reduction $\geq 1.0 \%$, no confirmed hypoglycaemic AEs, and weight loss $>2 \%$ after 52,104 and 208 weeks of treatment, change from baseline in lipid profile after 52, 104 and 208 weeks of treatment and follow-up (4 weeks after treatment discontinuation)

Trial results available in trial register: yes

Endpoints quoted in publication(s) ${ }^{b, c}$

Primary outcome measure(s): change from baseline in $\mathrm{HbA1c}$ concentrations at weeks 52 and 104

Secondary outcome measure(s): occurrence of confirmed hypoglycaemic adverse events (plasma glucose $\leq 3.9 \mathrm{mmol} / \mathrm{L}$ or requiring assistance) up to weeks 52 and 104, changes from baseline in bodyweight, systolic blood, pressure, and diastolic blood pressure at weeks 52 and 104

Other outcome measure(s): percentage of participants who received rescue therapy (increases in the dose of metformin or additional antidiabetes treatment) over 104 weeks, percentage of participants with $\mathrm{HbA1c}$ concentrations of at least $7 \%$ who achieved a level of less than $7 \%$, percentage of participants with bodyweight reductions of $>5 \%$, changes from baseline in fasting plasma glucose at weeks 52 and 104

Safety endpoints were adverse events, vital signs, and clinical laboratory findings. Events consistent with urinary tract infection, genital infection, and volume depletion were identified from adverse events reported spontaneously by the investigator using prospectively defined search categories based on 73,89 , and eight preferred terms, respectively, according to the Medical Dictionary for Regulatory Activities (version 15.0)

\section{Endpoints quoted in abstract of publication $(s)^{b, c}$}

Primary outcome measure(s): change from baseline in HbA1c levels at week 52 and 104

Secondary outcome measure(s): - 
Other outcome measure(s): adverse events, severe adverse events, confirmed hypoglycaemic adverse events

Göke 2013

Endpoints quoted in trial document(s) (ClinicalTrials.gov, FDA/EMA document, manufacturer's website, published design paper) $)^{a, c}$

Source: NCT00575588

Primary outcome measure(s): HbA1c change from baseline to week 52

Secondary outcome measure(s): proportion of participants reporting at least one episode of any hypoglycaemic event over 52 weeks, body weight change from baseline to week 52, mean slope of the regressions of change from week 24 to week 52 in $\mathrm{HbAlc}$

Other outcome measure(s): -

Trial results available in trial register: yes

Endpoints quoted in publication(s) ${ }^{b, c}$

Primary outcome measure(s): -

Secondary outcome measure(s): -

Other outcome measure(s): adverse events, serious adverse events, change from baseline in $\mathrm{HbA1c}$, proportion of participants reporting at least one hypoglycaemic event during 104 weeks of treatment, change from baseline in body weight, durability of change from baseline $\mathrm{HbA} 1 \mathrm{c}$ from week 24 to week 104, change from baseline FPG

Endpoints quoted in abstract of publication(s) $)^{b, c}$

Primary outcome measure(s): -

Secondary outcome measure(s): -

Other outcome measure(s): adverse events, hypoglycaemia, weight change, change from baseline in $\mathrm{HbA} 1 \mathrm{c}$ er's website, published design paper) $)^{a, c}$

$\mathrm{N} / \mathrm{T}$

Endpoints quoted in publication(s) $)^{b, c}$

Primary outcome measure(s): -

Secondary outcome measure(s): -

Other outcome measure(s): "We evaluated the following parameters at baseline, and after 6 and 12 months: body weight, BMI, HbA1c, fasting plasma glucose, postprandial glucose (PPG), fasting plasma insulin (FPI), total cholesterol (TC), low-density lipoprotein-cholesterol (LDL-C), highdensity lipoprotein- cholesterol (HDL-C), triglycerides (Tg), adiponectin (ADN), leptin, tumor necrosis factor-a (TNF-a), interleukin-6 (IL-6), and high-sensitivity C-reactive protein (Hs-CRP). An euglycemic hyperinsulinemic clamp was performed at the baseline, and after 6 and 12 months to determine the glucose infusion rate (GIR); the participants also underwent an ultrasound examination at baseline, and after 6 and 12 months, performed by the same operator, to evaluate steatosis degree, defined as ultrasound grading (0-3), subcutaneous adipose tissue (SAT), and visceral adipose tissue diameter (VAT)"; "... all adverse events were recorded" 
Primary outcome measure(s): -

Secondary outcome measure(s): -

Other outcome measure(s): "Patients underwent an ultrasound examination for evaluation of steatosis degree, subcutaneous adipose tissue, and visceral adipose tissue diameter, an euglycemic hyperinsulinemic clamp, and blood sample collection for evaluation of glycemic control, fasting plasma insulin, lipid profile, adipocytokines at randomization, and after 6 and 12 months" er's website, published design paper) $)^{a, c}$

\section{Source: NCT00318461}

Primary outcome measure(s): change in HbA1c at week 26 and week 104

Secondary outcome measure(s): change in body weight at week 26 and week 104, change in FPG at week 26 and week 104, change in mean prandial increments of plasma glucose based on selfmeasured 7-point plasma glucose profiles at week 26 and week 104, change in mean post prandial plasma glucose based on self-measured 7-point plasma glucose profiles at week 26 and week 104, change in beta-cell function at week 26 and week 104, hypoglycaemic episodes at week 26 and week 104

Other outcome measure(s): -

Trial results available in trial register: yes

Endpoints quoted in publication(s) ${ }^{b, c}$

Primary outcome measure(s): (Nauck 2009 in Nauck 2013, and Nauck 2013) change in Hba1c at week 26 and week 104

Secondary outcome measure(s): change in body weight, FPG, 7-point plasma glucose profiles (before each meal, $90 \mathrm{~min}$ after breakfast, lunch, and dinner, and at bedtime), and beta-cell function based on fasting insulin, fasting C-peptide, fasting proinsulin-to-insulin ratio, and the HOMA-B

Other outcome measure(s): safety variables included adverse events, physical examination, vital signs, ophthalmoscopy, electrocardiogram, biochemical and hematology measures, urinalysis and subject-reported hypoglycemic episodes (based on symptoms and plasma glucose $<3.1$ $\mathrm{mmol} / \mathrm{L}$ ). Analyses of fasting glucagon, lipids (cholesterol, triglycerides (TG), free fatty acids and apolipoprotein B (ApoB)), blood pressure and cardiovascular markers (high-sensitivity C-reactive protein (hsCRP), plasminogen activator inhibitor-1 (PAl-1) and N-terminal B-type natriuretic peptide (NT-proBNP)) were undertaken. (Hermansen 2010 in Nauck 2013) impact of weight on quality of life

Endpoints quoted in abstract of publication(s) $)^{b, c}$

Primary outcome measure(s): -

Secondary outcome measure(s): -

Other outcome measure(s): HbAlc, body weight, hypoglycaemia, nausea 
Primary outcome measure(s): number of participants with treatment failure, time to treatment failure

Secondary outcome measure(s): homeostasis model assessment of beta-cell function, change in HOMA-B from baseline to endpoint, fasting proinsulin/insulin ratio at year 3 , change in fasting proinsulin/insulin ratio from baseline to endpoint, ratio of the 30-min increment in plasma insulin concentration and the 30-min increment in plasma glucose during the oral glucose tolerance test (DI30/DG30 Ratio) at year 3, change in DI30/DG30 ratio from baseline to endpoint, disposition index at year 3, change in disposition index from baseline to endpoint, change in $\mathrm{HbA1c}$ from baseline to year 3, change in $\mathrm{HbA1c}$ from baseline to endpoint, fasting plasma glucose at year 3, change in fasting plasma glucose from baseline to endpoint, postprandial ( 2 hours) plasma glucose at year 3 , change in postprandial ( 2 hours) plasma glucose from baseline to endpoint, change in body weight from baseline to year 3, systolic blood pressure at year 3, diastolic blood pressure at year 3 , heart rate at year 3 , triglycerides at year 3 , total cholesterol at year 3, high-density lipoprotein cholesterol at year 3, hypoglycaemia rate per year, change in $\mathrm{HbA1c}$ from baseline to year 2 for participants randomised at entry in period III, change in $\mathrm{HbAlc}$ from baseline to year 2 for participants not randomised at entry in period III, hypoglycaemia rate per year in period III

Other outcome measure(s): -

Trial results available in trial register: yes

Endpoints quoted in publication(s)

Primary outcome measure(s): the primary outcome was time to inadequate glycaemic control, defined as an $\mathrm{HbAlc}$ concentration of $>9 \%$ after the first 3 months of treatment, or $>7 \%$ at two consecutive visits 3 months apart after the first 6 months

Secondary outcome measure(s): secondary outcomes were markers of $\beta$-cell function, bodyweight, hypoglycaemia, and surrogate markers of cardiovascular risk (blood pressure and heart rate)

Other outcome measure(s): adverse events

\section{Endpoints quoted in abstract of publication $(s)^{b, c}$}

Primary outcome measure(s): time to inadequate glycaemic control and need for alternative treatment, defined as an $\mathrm{HbAlc}$ concentration of $>9 \%$ after the first 3 months of treatment, or $>7 \%$ at two consecutive visits after the first 6 months

Secondary outcome measure(s): -

Other outcome measure(s): adverse events er's website, published design paper) ${ }^{a, c}$

Source: NCT00622284

Primary outcome measure(s): HbA1c change from baseline at week 52, $\mathrm{HbA} 1 \mathrm{c}$ change from baseline at week 104

Secondary outcome measure(s): body weight change from baseline at week 52 , body weight change from baseline at week 104, incidence of hypoglycaemic events up to 52 weeks, incidence of hypoglycaemic events up to 104 weeks, FPG change from baseline at week 52, FPG change from baseline at week 104, percentage of participants with $\mathrm{HbA1c}<7.0 \%$ at week 52, percentage of participants with $\mathrm{HbA} 1 \mathrm{c}<7.0 \%$ at week 104 , percentage of participants with $\mathrm{HbA} 1 \mathrm{c}<6.5 \%$ at week 52 , percentage of participants with $\mathrm{HbA} 1 \mathrm{c}<6.5 \%$ at week 104 , percentage of participants with $\mathrm{HbA} 1 \mathrm{c}$ lowering by $0.5 \%$ at week 104, $2 \mathrm{hr}$ PPG change from baseline at week 104, HbA1c change at week 4, HbAlc change at week $8, \mathrm{HbA1c}$ change at week 12, HbA1c change at week 16, HbA1c change at week $28, \mathrm{HbA1c}$ change at week $40, \mathrm{HbA} 1 \mathrm{c}$ change at week 52, HbA1c change at week $65, \mathrm{HbA} 1 \mathrm{c}$ change at week $78, \mathrm{HbA1c}$ change at week 91 , HbA1c change at week 104, change in baseline lipid 
parameter cholesterol at week 104, change in HDL at week 104, change in LDL at week 104, change in baseline lipid parameter triglyceride at week 104

Other outcome measure(s): -

Trial results available in trial register: yes

\section{Endpoints quoted in publication(s) ${ }^{b, c}$}

Primary outcome measure(s): the primary efficacy endpoint was change in $\mathrm{HbAlc}$ from baseline to week 104

Secondary outcome measure(s): the two key secondary endpoints were occurrence of hypoglycaemic episodes up to 104 weeks and change in bodyweight from baseline to week 104. Other secondary endpoints were change in $\mathrm{HbA1c}$ from baseline to week 52 , $\mathrm{HbA} 1 \mathrm{c}$ reduction over time, occurrence of $\mathrm{HbA} 1 \mathrm{c}$ on treatment of $<7.0 \%$ or $<6.5 \%$ at week 104 , occurrence of $\mathrm{HbAlc}$ reduction of $\geq 0.5 \%$ at week 104, change in FPG from baseline to week 52 and 104, change in 2-h postprandial glucose from baseline to week 104 during a meal tolerance test in a subset of participants who received a standardised breakfast of two nutrition bars and a drink (Ensure Plus, Abbott Nutrition, Columbus, $\mathrm{OH}, \mathrm{USA}$ ), and changes from baseline in total cholesterol, LDL cholesterol, HDL cholesterol, and triglycerides. The changes in plasma proinsulin/insulin ratio and in HOMA-IR from baseline to week 104 were used to indirectly assess pancreatic $\beta$-cell function and insulin resistance

Other outcome measure(s): safety and tolerability endpoints included the incidence and intensity of adverse events, withdrawals because of adverse events, physical examination, 12-lead electrocardiogram, vital signs, and clinical laboratory measures. Hypoglycaemic events and severe hypoglycaemic episodes were also recorded. Treatment-emergent fatal events, suspected events of stroke, myocardial ischaemia (including myocardial infarction), admission to hospital for heart failure, stent thrombosis, and revascularisation procedures

Endpoints quoted in abstract of publication(s) $)^{b, c}$

Primary outcome measure(s): change in HbA1c from baseline to week 104

Secondary outcome measure(s): -

Other outcome measure(s): hypoglycaemia, severe hypoglycaemia, cardiovascular events

Derosa $2011 a$

Endpoints quoted in trial document(s) (ClinicalTrials.gov, FDA/EMA document, manufacturer's website, published design paper) $)^{a, c}$

$\mathrm{N} / \mathrm{T}$

Endpoints quoted in publication(s)b,c

Primary outcome measure(s): the primary endpoint of this study was to evaluate the effect of these two different drugs on body weight, glycemic control, insulin resistance related-parameters and inflammatory biomarkers

Secondary outcome measure(s): -

Other outcome measure(s): "In order to evaluate the tolerability assessments, all adverse events were recorded"

\section{Endpoints quoted in abstract of publication(s)}

Primary outcome measure(s): -

Secondary outcome measure(s): - 
Primary outcome measure(s): the primary endpoint of this study is to evaluate the effect of pioglitazone or glibenclamide when added to metformin therapy on metabolic and inflammatory parameters after an OFL

Secondary outcome measure(s): the effects of pioglitazone and glibenclamide on metabolic and inflammatory parameters after 12 months of treatment in a baseline situation

Other outcome measure(s): -

Endpoints quoted in abstract of publication $(s)^{b, c}$

Primary outcome measure(s): -

Secondary outcome measure(s): -

Other outcome measure(s): "We evaluated glycemic-metabolic parameters [glycated hemoglobin ( $\mathrm{HbA} 1 \mathrm{C}$ ), fasting plasma glucose (FPG), fasting plasma insulin (FPI), homeostasis model assessment (Homa) index], total cholesterol (TC), low density lipoprotein-cholesterol (LDL-C), high density lipoprotein- cholesterol (HDL-C), triglycerides (Tgs), interleukin-6 (IL-6), high sensitivity C-reactive protein (Hs-CRP), tumor necrosis factor- $\alpha$ (TNF- $\alpha$ ), and adiponectin (ADN)" er's website, published design paper)a,c

Endpoints quoted in publication(s) ${ }^{\mathbf{b}, c}$

Primary outcome measure(s): -

Secondary outcome measure(s): -

Other outcome measure(s): plasma asymmetric dimethyl-arginine (ADMA), serum creatinine, GFR, high sensitive C-reactive protein (hSCRP), fibrinogen, glycaemia, glycated haemoglobin (HbA1c), cholesterol, triglycerides, haemoglobin, urine albumin: creatinine ratio (UACR), urinary beta2-microglobulin, and urinary alpha1-microglobulin

Endpoints quoted in abstract of publication $(s)^{b, c}$

\section{Primary outcome measure(s): -}

\section{Secondary outcome measure(s): -}

Other outcome measure(s): urinary albumin:creatinine ratio (UACR), urinary alpha1-microglobulin, urinary beta2-microglobulin, plasma asymmetric dimethyl-arginine (ADMA), GFR, hsC-reactive protein, fibrinogen, $\mathrm{HbA1c}$; pulsatility index, resistance index in the internal carotid artery and middle cerebral artery, intima media thickness in the common carotid artery; cerebrovascular reactivity 
(Continued)

Derosa 2010
Endpoints quoted in trial document(s) (ClinicalTrials.gov, FDA/EMA document, manufacturer's website, published design paper) $)^{a, c}$

$\mathrm{N} / \mathrm{T}$

Endpoints quoted in publication(s) ${ }^{b, c}$

Primary outcome measure(s): -

Secondary outcome measure(s): -

Other outcome measure(s): "The aim of this study was to evaluate the effects of a 1-year treatment with exenatide compared to glibenclamide in type 2 diabetes patients on body weight, glycemic control, and $\beta$-cell function but also on insulin resistance and inflammatory state parameters like resistin, retinol binding protein-4 (RBP-4), and high-sensitivity C-reactive protein (HsCRP)"; "In order to evaluate the tolerability assessments, all adverse events were recorded"

Endpoints quoted in abstract of publication $(s)^{b, c}$

Primary outcome measure(s): -

Secondary outcome measure(s): -

Other outcome measure(s): body weight, glycemic control, $\beta$-cell function, insulin resistance, and inflammatory state

Endpoints quoted in trial document(s) (ClinicalTrials.gov, FDA/EMA document, manufacturer's website, published design paper) $)^{a, c}$

\section{Source: NCT00106340}

Primary outcome measure(s): time to $\mathrm{HbAlc}>8 \%$ at week 104

Source: Novartis Summary report

Primary outcome measure(s): change in Hba1c from baseline to week 104 endpoint

Secondary outcome measure(s): change from baseline in HbA1c at 5 years (2 years, amended), adverse event profile after 5 years of treatment ( 2 years, amended), coefficient of failure for $\mathrm{HbA1c}$ from week $24-5$ years ( 2 years, amended), change from baseline in fasting plasma glucose at 5 years ( 2 years amended), change from baseline in body weight at 5 years ( 2 years amended)

Other outcome measure(s): (Novartis Summary report) risk of failure of glycaemic control over time

Trial results available in trial register: no

\section{Endpoints quoted in publication(s) ${ }^{b, c}$}

Primary outcome measure(s): change from baseline in $\mathrm{Hbalc}$ at study endpoint

Secondary outcome measure(s): $\mathrm{HbA} 1 \mathrm{c}$ responder rates $(\mathrm{HbA} 1 \mathrm{c} \geq 7 \%$ at baseline and $<7 \%$ at week 104 endpoint or $\mathrm{HbA} 1 \mathrm{c}>6.5 \%$ at baseline and $\leq 6.5 \%$ at week 104 endpoint), predefined subgroup analysis according to age ( $\geq 65$ or $<65$ years) at baseline, CoF, FPG, body weight, fasting lipids, $\beta$-cell function and insulin resistance parameters

Other outcome measure(s): AE, SAE, hypoglycaemic events, severe hypoglycaemia

Endpoints quoted in abstract of publication(s) ${ }^{b, c}$

\section{Primary outcome measure(s): -}




\section{Secondary outcome measure(s): -}

Other outcome measure(s): change in Hbalc at 2 years, proportion of participants reaching Hba1c $<7 \%$, hypoglycaemia, body weight er's website, published design paper) $)^{a, c}$

\section{Source: NCT00102466}

Primary outcome measure(s): change from baseline in $\mathrm{HbAlc}$ at 52 weeks

Secondary outcome measure(s): adverse event profile after 52 weeks of treatment, change from baseline in fasting plasma glucose at 52 weeks, participants with endpoint $\mathrm{HbA1c}<7 \%$ at 52 weeks, participants with reduction in $\mathrm{HbAlc} \geq 0.7 \%$ after 52 weeks, participants with reduction in $\mathrm{HbA} 1 \mathrm{c} \geq 0.5 \%$ after 52 weeks

\section{Other outcome measure(s): -}

Trial results available in trial register: no

Endpoints quoted in publication(s)

Primary outcome measure(s): change in $\mathrm{HbA1c}$ from baseline to week 52

Secondary outcome measure(s): proportion of participants achieving $\mathrm{HbAlc}$ targets of $<7.0 \%$ and $\leq 6.5 \%$, changes in FPG, body weight, B-cell function (fasting proinsulin, fasting proinsulin/insulin ratio, HOMA-B) and insulin resistance (fasting insulin, HOMA-IR)

Other outcome measure(s): "Subgroup analyses of change in $\mathrm{HbA1c}$ from baseline at the endpoint were performed according to baseline $\mathrm{HbAlc}$ ( $\mathrm{HbAlc} \leq 8.0 \%$ and $>8.0 \% ; \leq 9.0 \%$ and $>9.0 \%$ ), age ( $\leq 65$ years and $>65$ years), gender and baseline body mass index (BMI) $(<30.0, \geq 30.0$ and $\geq 35.0$ $\left.\mathrm{kg} / \mathrm{m}^{2}\right)$ "; "Safety assessments consisted of monitoring and recording all adverse events (AEs) and serious adverse events (SAEs), regular monitoring of haematology, blood chemistry and urine, and regular assessments of vital signs, electrocardiogram (ECG), physical condition and body weight"

Endpoints quoted in abstract of publication $(\mathbf{s})^{\mathbf{b}, c}$

Primary outcome measure(s): -

Secondary outcome measure(s): -

Other outcome measure(s): change from baseline glycated haemoglobin ( $\mathrm{HbA1c}$ ), proportion of participants reaching $\mathrm{HbAlc}<7 \%$, hypoglycaemic events, fasting plasma glucose, adverse events, serious adverse events, weight change er's website, published design paper) $)^{a, c}$

Source: NCT00094770

Primary outcome measure(s): change from baseline in HbA1c at week 52

Secondary outcome measure(s): change from baseline in HbA1c at week 104, change from baseline in body weight at week 52 , change from baseline in body weight at week 104 , hypoglycemic events at week 52, hypoglycemic events at week 104, number of participants with clinical adverse experiences at week 104, number of participants with serious clinical adverse experiences at week 104 , number of participants with drug-related clinical adverse experiences at week 104, number of participants with laboratory adverse experiences at week 104, number of participants with serious laboratory adverse experiences at week 104, number of participants with drug-related laboratory adverse experiences at week 104 
Other outcome measure(s): -

Trial results available in trial register: yes

Endpoints quoted in publication $(s)^{b, c}$

Primary outcome measure(s): -

Secondary outcome measure(s): -

Other outcome measure(s): HbA1c, FPG, serum insulin and proinsulin, plasma lipid parameters, adverse experiences, body weight, hypoglycaemia

Endpoints quoted in abstract of publication $(s)^{b, c}$

Primary outcome measure(s): -

Secondary outcome measure(s): -

Other outcome measure(s): change in HbA1c from baseline, beta-cell responsiveness, hypoglycaemia, weight change er's website, published design paper) $)^{a, c}$

\section{Source: NCT00379769}

Primary outcome measure(s):

- Number of participants with cardiovascular death/cardiovascular hospitalisation events, from baseline through end of study, up to 7.5 years

- IR outcome: number of participants who died due to any cause, from baseline through end of study, up to 7.5 years

- IR outcome: number of participants with a first occurrence of a MACE defined as CV (or unknown) death, non-fatal MI, and non-fatal stroke based on original RECORD and on contemporary endpoint definitions, from baseline through end of study, up to 7.5 years

- IR outcome: number of participants with a CV (or unknown) death, based on original RECORD and on contemporary endpoint definitions, from baseline through end of study, up to 7.5 years

- IR outcome: number of participants with an event of myocardial infarction (fatal and non-fatal), based on original RECORD and on contemporary endpoint definitions, from baseline through end of study, up to 7.5 years

- IR outcome: number of participants with an event of stroke (fatal and non-fatal), based on original RECORD and on contemporary endpoint definitions, from baseline through end of study, up to 7.5 years

\section{Secondary outcome measure(s):}

- Number of participants with cardiovascular events and all-cause deaths from baseline through end of study, up to 7.5 years

- Total number of cardiovascular hospitalisations and cardiovascular deaths, from baseline through end of study, up to 7.5 years

- Number of participants with first cardiovascular hospitalisations/cardiovascular deaths by stratum, from baseline through end of study, up to 7.5 years

- Number of participants with CV/microvascular events, from baseline through end of study, up to 7.5 years

- Number of participants with glycaemic failure events, from baseline through to end of randomised dual therapy

- Number of participants with addition of third oral agent/switch to insulin, from baseline through end of study, up to 7.5 years 
- The number of participants starting insulin at any time during the study, from baseline through end of study, up to 7.5 years

- Model-adjusted change from baseline in HbA1c at month 60

- Model-adjusted change from baseline in fasting plasma glucose at month 60

- Model-adjusted mean change, from baseline in insulin and pro-insulin at month 60

- Number of HbA1c and FPG responders at month 60

- Model-adjusted ratio to baseline (expressed as a percentage) HOMA beta cell function and insulin sensitivity at month 60

- Model-adjusted ratio to baseline (expressed as a percentage) for TC, LDL, HDL, triglycerides, and FFAs at month 60

- Model-adjusted ratio to baseline (expressed as a percentage) for TC:HDL and LDL:HDL cholesterol ratios at month 60

- Model-adjusted ratio to baseline (expressed as a percentage) for Apo-B at month 60

- Model-adjusted ratio to baseline (expressed as a percentage) for urinary albumin creatinine ratio at month 60

- Model-adjusted change from baseline in body weight at month 60

- Model-adjusted change from baseline in alanine aminotransferase at month 60

- Model-adjusted change from baseline in waist circumference at month 60

- Model-adjusted change from baseline in systolic blood pressure and diastolic blood pressure at month 60

- Model-adjusted ratio to baseline (expressed as a percentage) for C-reactive protein at month 60

- Model-adjusted ratio to baseline (expressed as a percentage) for fibrinogen at month 60

- Model-adjusted ratio to baseline (expressed as a percentage) for plasminogen activator inhibitor-1 antigen at month 60

- Number of participants with the indicated type of neoplasm/cancer event reported as a SAE or death: main study + observational follow-up combined, from the beginning of the main study through the end of the observational follow-up, up to 11.4 years

- Number of participants with the indicated type of neoplasm/cancer event reported as a SAE or death: observational follow-up, from the end of the RECORD study through the end of the observational follow-up, up to 4.0 years

- Number of participants with the indicated type of malignant neoplasms/cancer events reported as an SAE or death by location (including location of special interest): main study + observational follow-up combined, from the beginning of the main study through the end of the observational follow-up, up to 11.4 years

- Number of participants with the indicated type of malignant neoplasms/cancer events reported as an SAE or death by location (including location of special interest): observational follow-up, from the end of the RECORD study through the end of the observational follow-up, up to 4.0 years

- Number of participants who died due to the indicated cancer-related event: main study + observational follow-up combined, from the beginning of the main study through the end of the observational follow-up, up to 11.4 years

- Number of participants who died due to the indicated cancer-related event: observational follow-up, from the end of the RECORD study through the end of the observational follow-up, up to 4.0 years

- Number of participants with a bone fracture event - overall and by gender: main study and observational follow-up combined,from the beginning of the main study through the end of the observational follow-up, up to 11.4 years

- Number of participants with a bone fracture event - overall and by gender: observational follow-up, from the end of the RECORD study through the end of the observational follow-up, up to 4.0 years

- Number of participants with a bone fracture event reported as the indicated serious adverse event (by higher-level group term) or death: main study + observational follow-up combined, from the beginning of the main study through the end of the observational follow-up, up to 11.4 years

- Number of participants with a bone fracture event reported as the indicated serious adverse event (by higher-level group term) or death: observational follow-up, from the end of the RECORD study through the end of the observational follow-up, up to 4.0 years 
- Number of participants with an event of death due to a bone fracture-related event: main study + observational follow-up combined, from the beginning of the main study through the end of the observational follow-up, up to 11.4 years

- Number of participants with the indicated bone fracture by fracture site: main study + observational follow-up combined, from the beginning of the main study through the end of the observational follow-up, up to 11.4 years

- Number of participants with the indicated bone fracture by fracture site: observational follow-up, from the end of the RECORD study through the end of the observational follow-up, up to 4.0 years

- Number of participants with potentially high morbidity fractures: main study + observational follow-up combined, from the beginning of the main study through the end of the observational follow-up, up to 11.4 years

- Number of participants with potentially high morbidity fracture events and non-high morbidity fracture events, in participants with prior hand/upper arm/foot fractures: main study + observational follow-up combined, from the beginning of the main study through the end of the observational follow-up, up to 11.4 years

- Number of participants with bone fracture events of the indicated cause: main study + observational follow-up combined (time frame: from the beginning of the main study through the end of the observational follow-up (up to 11.4 years))

- Number of participants with bone fracture events of the indicated cause: observational follow-up (time frame: from the end of the RECORD study through the end of the observational follow-up (up to 4.0 years))

- Number of bone fracture events with the indicated outcome: main study + observational follow-up combined, from the beginning of the main study through the end of the observational follow-up, up to 11.4 years

- Number of bone fracture events with the indicated outcome: observational follow-up, from the end of the RECORD study through the end of the observational follow-up, up to 4.0 years

- Number of participants with the indicated serious adverse event: observational follow-up, from the end of the RECORD study through the end of the observational follow-up, up to 4.0 years

Other outcome measure(s): -

Trial results available in trial register: yes

Endpoints quoted in publication(s)

Primary outcome measure(s): time from the start of randomised treatment to reach the combined endpoint of (adjudicated) CV death or CV hospitalisation

Secondary outcome measure(s): time to all-cause mortality; time to first occurrence of definite $\mathrm{CHF}$; time to first occurrence of all-cause mortality, $\mathrm{MI}$, stroke, definitive $\mathrm{CHF}$ and unstable angina; time to first occurrence of CV death, MI, stroke and unstable angina; combined CV death or CV hospitalisation plus microvascular events (diabetes-related); time to CV death, acute MI, stroke (MACE) and time to each of the individual endpoints CV death, MI, stroke; total number of each of the events in the CV death or CV hospitalisation endpoint; total number of microvascular events (diabetes-related) at study end; changes in glycaemia and related metabolic parameters; time to failure of glycaemic control (defined as the combination of $\mathrm{HbAlc} 8.5 \% \geq 0$ on two consecutive visits, or $\mathrm{HbAlc} \geq 8.5 \%$ at single visit and moved to post-randomised treatment phase/initiated triple therapy); time to addition of a third oral therapy for rosiglitazone combination groups or switch to insulin for metformin and sulphonylurea combination groups; time to initiation of treatment with insulin; and, general safety through the assessment of changes in physical examinations, vital signs, body weight, clinical laboratory tests, AEs and electrocardiograms. An additional composite endpoint was added to the analysis plan at the request of the Steering Committee (CV death, MI, stroke, unstable angina and $\mathrm{CHF}$ )

Other outcome measure(s): -

\section{Endpoints quoted in abstract of publication(s) $)^{b, c}$}

Primary outcome measure(s): cardiovascular hospitalisation or cardiovascular death 
Other outcome measure(s): er's website, published design paper) $)^{a, c}$

$\mathrm{N} / \mathrm{T}$

Endpoints quoted in publication(s) $)^{b, c}$

Primary outcome measure(s): -

Secondary outcome measure(s): -

Other outcome measure(s): "Anthropometric and metabolic measurements were assessed at baseline, after 3 months, and after 12 months"; "To evaluate the tolerability assessments, all adverse events were recorded"

Endpoints quoted in abstract of publication $(s)^{b, c}$

Primary outcome measure(s): -

Secondary outcome measure(s): -

Other outcome measure(s): "Anthropometric and metabolic measurements were assessed at baseline, after 3 months, and after 12 month" er's website, published design paper) $)^{a, c}$

Endpoints quoted in publication(s) ${ }^{b, c}$

Primary outcome measure(s): -

Secondary outcome measure(s): -

Other outcome measure(s): "Body Mass Index, HbA1c, FPG, PPG, FPI, PPI, HOMA index, TC, LDLC, HDL-C, Tg, Apo A-I, Apo-B, SBP, and DBP values were also assessed at 3, 6, 9 and 12 month"; "Treatment tolerability was assessed at each study visit using an accurate interview of patients by the investigators, and comparisons of clinical and laboratory values with baseline levels"

(Derosa 2007 in Derosa 2009b) BMI, HbAlc, FPG, PPG, FPI, PPI, HOMA index, TC, LDL-C, HDL-C, Tg, $\mathrm{Lp}(\mathrm{a}), \mathrm{Fg}, \mathrm{PAI}-1, \mathrm{tP}-\mathrm{A}, \mathrm{Hcy}, \mathrm{SBP}$, and DBP values were also assessed at 3, 6, 9, and 12 months

\section{Endpoints quoted in abstract of publication(s)}

\section{Primary outcome measure(s): -}

\section{Secondary outcome measure(s): -}

Other outcome measure(s): "We assessed body mass index (BMI), fasting (FPG) and post-prandial (PPG) plasma glucose, glycosylated haemoglobin (HbA1c), fasting (FPI) and post-prandial (PPI) plasma insulin, homeostasis model assessment (HOMA) index, and lipid profile [total cholesterol (TC), low density lipoprotein- cholesterol (LDL-C), high density lipoprotein- cholesterol (HDL-C), triglycerides (Tg), apolipoprotein A-I (Apo A-I), and apolipoprotein B (Apo B)], systolic blood pressure (SBP), and diastolic blood pressure (DBP)" 
(Derosa 2007 in Derosa 2009b) "We assessed body mass index (BMI), glycated hemoglobin (HbA1c), fasting plasma glucose (FPG), postprandial plasma glucose (PPG), fasting plasma insulin (FPI), postprandial plasma insulin (PPI), homeostasis model assessment index (HOMA index), lipid profile with lipoprotein (a) [Lp(a)], fibrinogen (Fg), plasminogen activator inhibitor-1 (PAI-1), tissue plasminogen activator (t-PA), homocysteine (Hcy), systolic blood pressure (SBP), diastolic blood pressure (DBP)"

Petrica 2009

Endpoints quoted in trial document(s) (ClinicalTrials.gov, FDA/EMA document, manufacturer's website, published design paper) $)^{a, c}$

$\mathrm{N} / \mathrm{T}$

Endpoints quoted in publication(s) $)^{b, c}$

Primary outcome measure(s): -

Secondary outcome measure(s): -

Other outcome measure(s): serum creatinine, GFR, C-reactive protein, fibrinogen, glycaemia, $\mathrm{HbA1c}$, cholesterol, triglycerides, haemoglobin, urine albumin/creatinine ratio, serum cystatin $\mathrm{C}$, serum and urinary $\beta 2$-microglobulin, urinary a1-microglobulin

Endpoints quoted in abstract of publication $(s)^{b, c}$

Primary outcome measure(s): -

Secondary outcome measure(s): -

Other outcome measure(s): nephro- and neuroprotective effects, biomarkers in the diagnosis of incipient diabetic nephropathy and cerebral microangiopathy er's website, published design paper) ${ }^{a, c}$

\section{Source: NCT00367055}

Primary outcome measure(s): the primary end point for efficacy was the change from baseline in the insulin secretory capacity after a 36-month treatment measured by the assessment of blood insulin concentrations using the hyperglycaemic clamp technique

\section{Secondary outcome measure(s):}

- Measurement of insulin secretion after treatment for 18 months using a hyperglycaemic clamp test

- Measurement of serum C peptide concentrations at the same time as blood insulin measurements during the clamp test (18 and 36 months)

- Arginine test performed between 180 and 210 minutes after the hyperglycaemic clamp

- Test start, with measurement at baseline, 18 and 36 months of blood insulin and $C$ peptide at the following time: $2,4,6,8,10,15,20$, and 30 minutes

- Change in insulin secretion capacity over 3 years which was also to be evaluated using the meal test technique, with measurements performed before treatment (at baseline) and after treatment (at 18 and 36 months) Measurements of blood insulin and serum C peptide were performed at 15 , 30, 60, 90, 120, 180 minutes.

- Changes in fasting blood glucose and glycated haemoglobin ( $\mathrm{HbA1c}$ ) values were also monitored at the time of the tests (baseline, 18 months, 36 months) in order to evaluate the results of different tests as a function of the change in treatment efficacy.

- Tolerability assessment by evaluating the incidence of adverse events recorded at each visit, whether reported spontaneously by the participant or identified by the interviewers

Other outcome measure(s): - 
Trial results available in trial register: yes

Endpoints quoted in publication(s) ${ }^{b, c}$

$\mathrm{N} / \mathrm{A}$

Endpoints quoted in abstract of publication(s) $)^{b, c}$

N/A

Hamann 2008

Endpoints quoted in trial document(s) (ClinicalTrials.gov, FDA/EMA document, manufacturer's website, published design paper)a,c

Source: NCT00359112

Primary outcome measure(s): change in $\mathrm{HbA1c}$ level from baseline following 52 weeks of treatment

Secondary outcome measure(s): change in FPG, insulin sensitivity, beta cell function, change in PAI-1, CRP, number of hypoglycaemic event, change in 24-h ambulatory blood pressure monitoring, diabetes treatment satisfaction

Other outcome measure(s): -

Trial results available in trial register: no

Endpoints quoted in publication(s)

Primary outcome measure(s): change in HbA1c from baseline following 52 weeks of treatment

Secondary outcome measure(s): -

Other outcome measure(s): FPG, C-peptide, insulin, proinsulin, cardiovascular biomarkers, health outcome, safety

Endpoints quoted in abstract of publication(s) $)^{b, c}$

Primary outcome measure(s): change in $\mathrm{HbA1c}$ from baseline after 52 weeks of treatment

Secondary outcome measure(s): -

Other outcome measure(s): -

Endpoints quoted in trial document(s) (ClinicalTrials.gov, FDA/EMA document, manufacturer's website, published design paper) $)^{a, c}$

\section{Source: CDJN608A 2308E1}

Primary outcome measure(s): HbA1c after 24 weeks, HbA1c after 52 weeks, safety and tolerability during 6-month extension phase

Secondary outcome measure(s): percentage of participants reaching treatment target (endpoint $\mathrm{HbAlc}<7 \%$ and/or a decrease $\geq 0.5 \% \mathrm{HbAlc}$, percentage of participants reaching treatment target (endpoint $\mathrm{HbA1c}<6.5 \%$ and/or a decrease $\geq 1.0 \% \mathrm{HbA1c}$, FPG and bodyweight after 24 weeks and after 52 weeks, prandial effect on glucose and insulin after a standardised meal challenge following 24 and 52 weeks of treatment

Other outcome measure(s): -

Trial results available in trial register: no 
Endpoints quoted in publication(s)

Primary outcome measure(s): -

Secondary outcome measure(s): -

Other outcome measure(s): HbA1c change from baseline to end point, percentage of participants achieving $\mathrm{HbA} 1 \mathrm{c}<7 \%$ and/or a decrease $\geq 0.5 \%$, FPG change from baseline to week 24, prandial plasma glucose, hypoglycaemia, weight

\section{Endpoints quoted in abstract of publication(s) $)^{b, c}$}

Primary outcome measure(s): -

Secondary outcome measure(s): -

Other outcome measure(s): HbA1c change from baseline to week 52, proportion of participants achieving $\mathrm{HbAlc}<7 \%$, FPG change from baseline to week 52, prandial plasma glucose, hypoglycaemia, weight

\section{Charbonnel 2005}

Endpoints quoted in trial document(s) (ClinicalTrials.gov, FDA/EMA document, manufacturer's website, published design paper) $)^{a, c}$

$\mathrm{N} / \mathrm{T}$

Endpoints quoted in publication(s)

Primary outcome measure(s): change in $\mathrm{HbAlc}$

Secondary outcome measure(s): change in fasting plasma glucose, insulin, insulin precursors and lipids

Other outcome measure(s): -

Endpoints quoted in abstract of publication $(s)^{b, c}$

Primary outcome measure(s): -

Secondary outcome measure(s): -

Other outcome measure(s): HbA1c, fasting plasma glucose, insulin and lipids

Endpoints quoted in trial document(s) (ClinicalTrials.gov, FDA/EMA document, manufacturer's website, published design paper) $)^{a, c}$

$\mathrm{N} / \mathrm{T}$

Endpoints quoted in publication(s) ${ }^{b, c}$

Primary outcome measure(s): changes in BMI, $\mathrm{HbAlc}$, lipid profile and lipoprotein parameters were the primary efficacy variables

Secondary outcome measure(s): -

Other outcome measure(s): "FPG, PPG and homeostasis model assessment (HOMA) index were also used to assess efficacy"; "In order to evaluate the tolerability assessments, all adverse events were recorded"; "AEs were recorded at each study visit, using spontaneous reporting, patient interview, and laboratory analysis (including liver enzymes)" 


\section{Endpoints quoted in abstract of publication $(s)^{b, c}$}

Primary outcome measure(s): changes in BMI, glycosylated haemoglobin (HbA1c), Lp(a) and HCT were primary efficacy variables

\section{Secondary outcome measure(s): -}

Other outcome measure(s): FPG, post-prandial plasma glucose (PPG) and homeostasis model assessment index were also used to assess efficacy er's website, published design paper) $)^{a, c}$

Source: CDJN608A US07

Primary outcome measure(s): change from baseline in $\mathrm{HbAlc}$ after 104 weeks of treatment

Secondary outcome measure(s): tolerability and safety as assessed by the incidence of confirmed hypoglycaemia and change in body weight after 104 weeks of treatment; effects of 104 weeks of treatment on markers of beta-cell function (fasting proinsulin/insulin ratio and HOMA-b, calculated from fasting insulin and glucose); effects on serum insulin, glucose, and C-peptide excursions (adjusted $A \cup C_{0-120 m i n}$ ) during an OGTT; incremental changes in glucose and insulin within the first 30 minutes during the OGTT (insulinogenic index); effects on the AIR to glucose ( $A U C_{0-10 ~ m i n}$ ) during an IVGTT in a subset of participants (approximately 40 participants per arm, 80 participants total)

Other outcome measure(s): safety assessments consisted of monitoring and recording all AEs, SSAEs, MACE, and hypoglycemic and hyperglycaemic events: monitoring haematology, blood chemistry, and urine values; and measurements of vital signs and the performance of physical examinations

\section{Trial results available in trial register: no}

\section{Endpoints quoted in publication(s) $)^{b, c}$}

Primary outcome measure(s): the primary efficacy variable was the change from baseline (average of weeks 2 and 0) to week 104 in A1C

Secondary outcome measure(s): secondary efficacy variables included the change from baseline to week 104 in FPG, body weight, and the incremental $A_{U C} C_{0-120 m i n}$ of glucose during oral glucose tolerance tests

Other outcome measure(s): all adverse events

\section{Endpoints quoted in abstract of publication(s) $)^{b, c}$}

Primary outcome measure(s): -

Secondary outcome measure(s): -

Other outcome measure(s): HbA1c, FPG, PPG during an OGTT

\footnotetext{
- denotes not reported

aTrial document(s) refers to all available information from published design papers and sources other than regular publications (e.g. FDA/EMA documents, manufacturer's web sites, trial registers).

bPublication(s) refers to trial information published in scientific journals (primary reference, duplicate publications, companion documents or multiple reports of a primary trial).

cPrimary and secondary outcomes refer to verbatim specifications in publication/records. Other outcome measures refer to all outcomes not specified as primary or secondary outcome measures.
} 
(Continued)

AE: adverse event; AIR: acute insulin response; Apo-B: apolipoprotein B; AUC: area under curve: BMI: body mass index; BP: blood pressure; CHF: congestive heart failure; CoF: coefficient of failure; CV: cardiovascular; DBP: diastolic blood pressure; ECG: electrocardiogram; EMA: European Medicines Agency; FDA: Food and Drug Administration (US); FFA: free fatty acids: FPG: fasting plasma glucose; GFR: glomerular filtration rate; HbA1c: glycosylated haemoglobin A1c; HDL: high-density lipoprotein cholesterol; LDL: low-density lipoprotein cholesterol; HOMA-b: homeostatic model assessment beta-cell function; HOMA-IR: homeostatic model assessment insulin resistance; IR: independent re-adjudication; IVGTT: intravenous glucose tolerance test; MACE: major adverse cardiac event; MI: myocardial infarction; N/A: not applicable; N/T: no trial document available; OGTT: oral glucose tolerance test; PPG: post-prandial plasma glucose; PPGE: postprandial glucose excursions; SAE: serious adverse events; SBP: systolic blood pressure; TC: total cholesterol; UTI: urinary tract infection

\section{Appendix 8. High risk of outcome reporting bias according to ORBIT classification}

\begin{tabular}{|c|c|c|c|c|c|}
\hline Trial ID & Outcome & $\begin{array}{l}\text { High risk of } \\
\text { bias } \\
\text { (category A)a }\end{array}$ & $\begin{array}{l}\text { High risk of } \\
\text { bias } \\
\text { (category } \\
\text { D)b }\end{array}$ & $\begin{array}{l}\text { High risk of } \\
\text { bias } \\
\text { (category E)c }\end{array}$ & $\begin{array}{l}\text { High risk of } \\
\text { bias } \\
\text { (category } \\
\text { G)d }\end{array}$ \\
\hline \multirow[t]{2}{*}{ Maffioli 2013} & All-cause mortality & No & No & No & Yes \\
\hline & Serious adverse events & No & No & Yes & No \\
\hline \multirow[t]{2}{*}{ Derosa $2011 a$} & All-cause mortality & No & No & No & Yes \\
\hline & Serious adverse events & No & No & Yes & No \\
\hline \multirow[t]{2}{*}{ Derosa 2011b } & All-cause mortality & No & No & No & Yes \\
\hline & Serious adverse events & No & No & No & Yes \\
\hline \multirow[t]{5}{*}{ Petrica 2011} & All-cause mortality & No & No & No & Yes \\
\hline & Serious adverse events & No & No & Yes & No \\
\hline & Non-serious adverse events & No & No & Yes & No \\
\hline & Mild/moderate hypoglycaemia & No & No & No & Yes \\
\hline & Serious hypoglycaemia & No & No & No & Yes \\
\hline \multirow[t]{2}{*}{ Derosa 2010} & All-cause mortality & No & No & No & Yes \\
\hline & Serious adverse events & No & No & Yes & No \\
\hline \multirow[t]{2}{*}{ Derosa 2009a } & All-cause mortality & No & No & No & Yes \\
\hline & Serious adverse events & No & No & Yes & No \\
\hline \multirow[t]{3}{*}{ Derosa 2009b } & All-cause mortality & No & No & No & Yes \\
\hline & Serious adverse events & No & No & Yes & No \\
\hline & Non-serious adverse events & No & No & Yes & No \\
\hline
\end{tabular}


(Continued)

\begin{tabular}{|c|c|c|c|c|c|}
\hline & Mild/moderate hypoglycaemia & No & No & No & Yes \\
\hline & Serious hypoglycaemia & No & No & No & Yes \\
\hline \multirow[t]{5}{*}{ Petrica 2009} & All-cause mortality & No & No & No & Yes \\
\hline & Serious adverse events & No & No & Yes & No \\
\hline & Non-serious adverse events & No & No & Yes & No \\
\hline & Mild/moderate hypoglycaemia & No & No & No & Yes \\
\hline & Serious hypoglycaemia & No & No & No & Yes \\
\hline \multirow[t]{4}{*}{ Derosa 2005} & All-cause mortality & No & No & No & Yes \\
\hline & Serious adverse events & No & No & Yes & No \\
\hline & Mild/moderate hypoglycaemia & No & No & No & Yes \\
\hline & Serious hypoglycaemia & No & No & No & Yes \\
\hline \multirow[t]{4}{*}{ Gerich 2005} & Cardiovascular mortality & No & No & Yes & No \\
\hline & Non-fatal myocardial infarction & No & No & Yes & No \\
\hline & Heart failure & No & No & Yes & No \\
\hline & Non-fatal stroke & No & No & Yes & No \\
\hline
\end{tabular}

aClear that outcome was measured and analysed; trial report states that outcome was analysed but reports only that result was not significant (Classification 'A', table 2; Kirkham 2010).

bClear that outcome was measured and analysed; trial report states that outcome was analysed but report no results (Classification 'D', table 2; Kirkham 2010).

'Clear that outcome was measured but was not necessarily analysed; judgement says likely to have been analysed but not reported because of non-significant results (Classification 'E', table 2; Kirkham 2010).

dUnclear whether outcome was measured; not mentioned, but clinical judgement says likely to have been measured and analysed but not reported on the basis of non-significant results (Classification 'G', table 2, Kirkham 2010).

ORBIT: Outcome Reporting Bias In Trials 


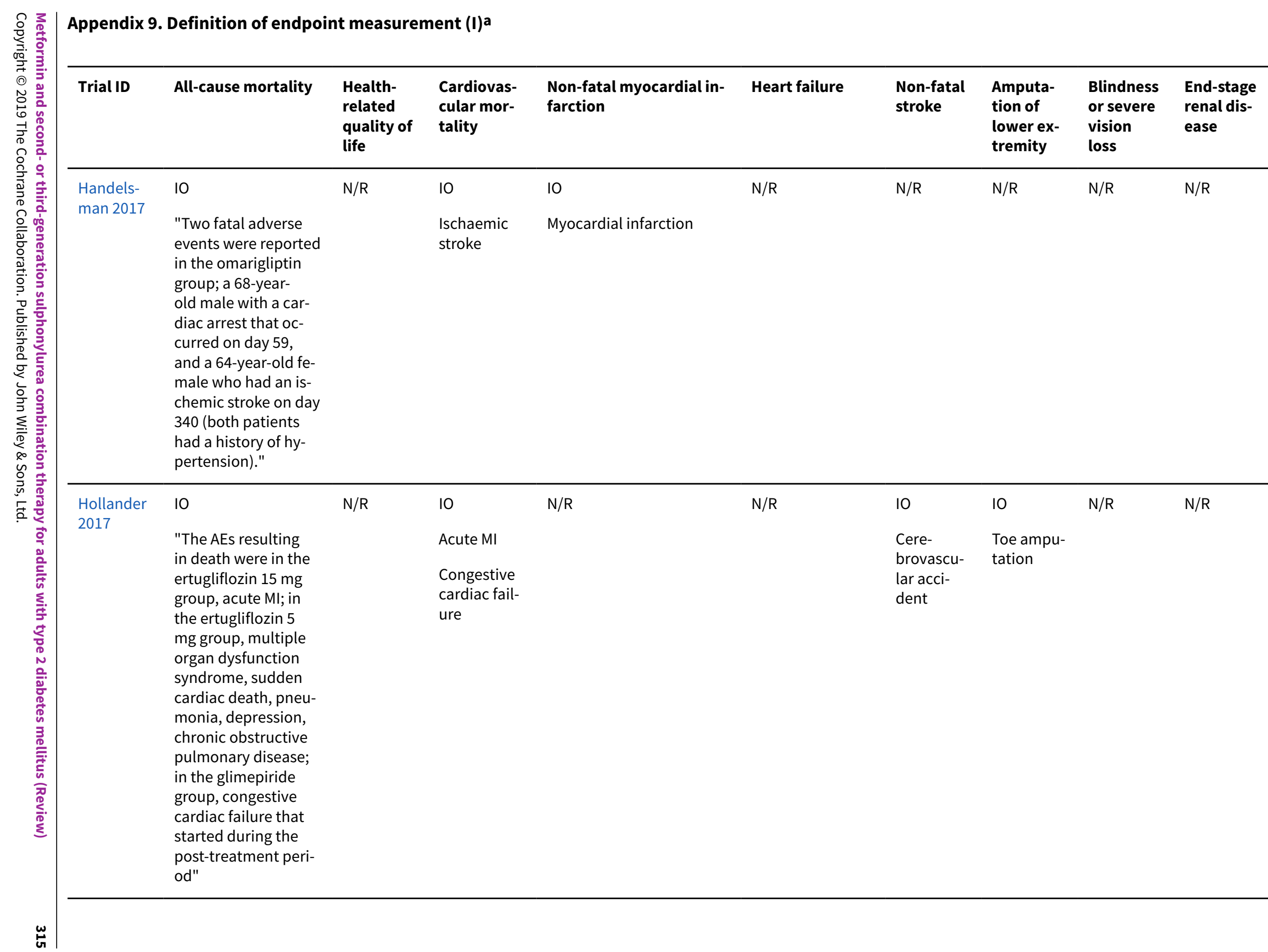


"All deaths, including those from cardiovascular, non-cardiovascular or unknown reason will contribute to the primary end-point"
"Sudden death, unwitnessed unexpected death, fatal MI, fatal stroke, death from heart failure, death related to invasive diagnostic or therapeutic procedures, death due to other cardiovascular causes"
"Non periprocedural MI. Myocardial infarction will be diagnosed if the subject has: Detection of rise of cardiac biomarkers

(preferably troponin) with at least one value above the 99th percentile of the upper reference lim it (URL) together with evidence of myocardial ischemia with at least one of the followings:

- Symptoms of ischaemia - ECG changes indicative of new ischaemia (see ST elevation/ST depression and T wave changes) - Development of pathological Q waves in the ECG - Imaging evidence of new loss of viable myocardium or new regional wall motion abnormality

${ }^{*}$ [ST elevation $=$ new $S T$ elevation at the J point in two contiguous leads with the cut off points: $\geq 0.2 \mathrm{mV}$ in men or $\geq 0.15$ $\mathrm{mV}$ in women in leads $\mathrm{V} 2$ - V3 and/or $0.1 \mathrm{mV}$ in other leads. ST depression and $\mathrm{T}$-wave changes = new horizontal or down sloping ST depression $\geq$ $0.05 \mathrm{mV}$ in two contiguous leads; and/or T inversion $\geq 0.1 \mathrm{mV}$ in two contiguous leads with prominent $\mathrm{R}$ wave or $\mathrm{R}-\mathrm{S}$ ratio $>1$ ].

Silent MI. Documentation of new significant $Q$ waves in at least two consecu-
"Patients with PRE-EXISTING heart failure

Patients presenting with at least two of the following signs or symptoms and requiring intravenous medications (diuretics

or vasodilators or inotropes) or an increase in dosing of oral diuretics:

- increased dyspnea on exertion - orthopnea

- nocturnal dyspnea

- increasing peripheral edema

- pulmonary ede-

- increasing fa-

tigue/decreas-

ing exercise tole ance

renal hypoperfusion (worsening renal function)

- elevated jugular venous pressure

radiological

sign of $\mathrm{CHF}$

new evidence

of left ventricular

systolic dysfunction

Participants with NEW ONSET heart failure (no
"Stroke is "Leg am defined as putation the pres- above the nce of ancle" acute neurological deficit thought to be of vascular origin with symptoms lasting > 24 hours. Subarachnoid hemorrhage may not cause focal deficit. On the basis of clin-

cal symptoms, autopsy and/ or CT/MRI,

trokes

will be

classified

a) definite or probable is-

stroke,

b) definite hemorrhagic stroke, (for both, $a$ \& b, confirmed by 
tive leads or new $Q$ waves in $\mathrm{V} 1$ and $\mathrm{V} 2$ or loss of $\mathrm{R}$ waves on one ECG in com-

parison to the previous

ECG tracings. The new $Q$

waves or $\mathrm{R}$ waves should

not be considered in the

presence of pacemake

rhythm, left ventricular

hypertrophy or pre-ex-

citation syndrome. The

date of MI will be consid-

ered the midway point be-

tween the diagnostic ECG

and the most recent non

diagnostic ECG.

Periprocedural MI:

For percutaneous coro-

nary interventions $(\mathrm{PCl})$

in participants with nor-

mal baseline troponine

values, elevations of car-

diac biomarkers above the

99th percentile URL are

indicative of peri-proce-

dural myocardial necrosis.

y convention, increas-

es of biomarkers $>3 \times 99$ th

percentile URL have been

designated as defining

$\mathrm{PCl}$-related myocardial in -

farction

For coronary artery bypass grafting (CABG) in

participants with normal

baseline troponin values,

elevation of cardiac bio-

markers above the 99th

percentile URL are indica-

tive of peri-procedural

myocardial necrosis. By

convention, increases of

biomarkers $>5 \times$ 99th per-

centile URL plus either previously known

HF)

nitiation or in-

rease in dosage

(if previously

prescribed for

other cause

ie. hyperten-

sion) of loop

diuretic, ACE-

inhibitor/ARB

therapy, or evi-

dence-based be-

ta-blocker ther-

py because of

new heart failure

signs and symp-

toms (see above)

AND AT LEAST

OF THE FOL-

OWING:

$B N P \geq 400 \mathrm{pg} /$

$\mathrm{mL}, \mathrm{NT}$-proBNP

$1500 \mathrm{pg} / \mathrm{ml}$

$\mathrm{OR}$

Structural heart

sease with doc-

umentation of

systolic dysfunc-

tion (LVEF < 45\%)

or diastolic dys-

function not pre-

viously known.

Heart failure re-

quiring at least

an overnight stay

( 2 calender days)

in hospital or in

n acute care set-

ting and day-care

admissions for

the management

of heart failure

are considered

as a hospitaliza-

tion. For sched-
T, MR

scan)

sub-

hage, (a

CT scan

or cere-

brospinal

fluid re-

be avail-

able show-

ing evi-

ence of

bleeding

in the sub-

aracnoid

d) uncer-

tain or un-

known

stroke.

Hem-

orrhag-

stroke

does not

include

hor-

infarct,

into a tu-

mor, in-

to a vas-

cular mal-

traumat-

ic hemor-

rhage." 


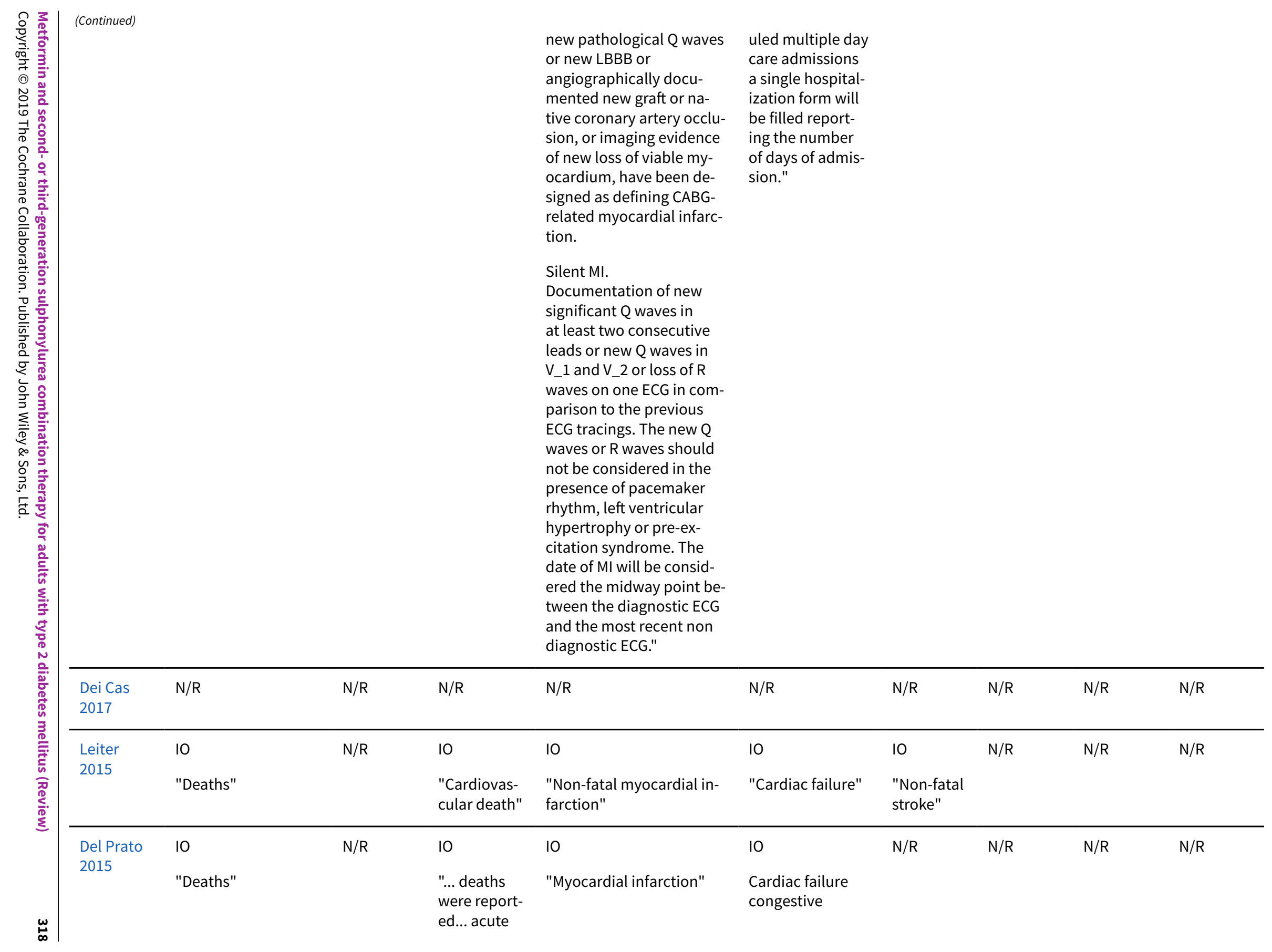




\begin{tabular}{|c|c|c|c|c|c|c|c|c|c|c|c|}
\hline 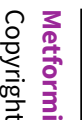 & (Continued) & & & $\begin{array}{l}\text { myocardial } \\
\text { infarction..." }\end{array}$ & & & & & & & , \\
\hline 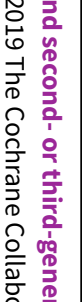 & $\begin{array}{l}\text { Schern- } \\
\text { thaner } \\
2015\end{array}$ & $\begin{array}{l}\text { IO } \\
\text { "There were two } \\
\text { deaths in the study } \\
\text { (saxagliptin: } \\
\text { myocardial infarc- } \\
\text { tion; glimepiride: un- } \\
\text { known cause)..." }\end{array}$ & $N / R$ & $N / R$ & $\mathrm{~N} / \mathrm{R}$ & $\begin{array}{l}\text { IO } \\
\text { Heart failure }\end{array}$ & $N / R$ & $N / R$ & $N / R$ & $N / R$ & 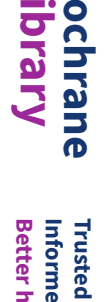 \\
\hline 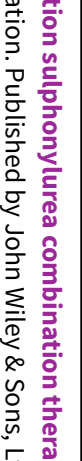 & $\begin{array}{l}\text { Del Prato } \\
2014\end{array}$ & $\begin{array}{l}\text { AO } \\
\text { "Deaths" }\end{array}$ & $N / R$ & $\begin{array}{l}\text { AO } \\
\text { "Cofirmed } \\
\text { MACE (car- } \\
\text { diovascu- } \\
\text { lar death, } \\
\text { non-fatal } \\
\text { myocardial } \\
\text { infarction } \\
\text { or non-fatal } \\
\text { stroke)..." }\end{array}$ & $\begin{array}{l}\text { AO } \\
\text { "Cofirmed MACE (cardio- } \\
\text { vascular death, non-fatal } \\
\text { myocardial infarction or } \\
\text { non-fatal stroke)..." }\end{array}$ & $\begin{array}{l}\text { AO } \\
\text { "Congestive car- } \\
\text { diac failure" }\end{array}$ & $\begin{array}{l}\text { AO } \\
\text { "Cofirmed } \\
\text { MACE (car- } \\
\text { diovascu- } \\
\text { lar death, } \\
\text { non-fatal } \\
\text { myocar- } \\
\text { dial in- } \\
\text { farction or } \\
\text { non-fatal } \\
\text { stroke)..." }\end{array}$ & $N / R$ & $N / R$ & $N / R$ & 旁: \\
\hline 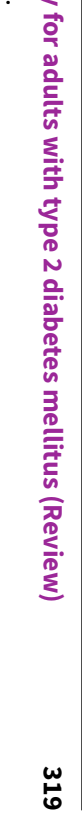 & $\begin{array}{l}\text { Ahrén } \\
2014\end{array}$ & $\begin{array}{l}\text { IO } \\
\text { "Five of the death } \\
\text { were cardiac in na- } \\
\text { ture, } 5 \text { were death } \\
\text { due to cancer, } 2 \\
\text { deaths were from un- } \\
\text { known causes, and } 1 \\
\text { death were due to a } \\
\text { cerebrovascular acci- } \\
\text { dent." }\end{array}$ & $N / R$ & $\begin{array}{l}\text { AO } \\
\text { "Cardio- } \\
\text { vascular } \\
\text { death was } \\
\text { defined as } \\
\text { sudden car- } \\
\text { diac death, } \\
\text { death } \\
\text { caused by } \\
\text { myocar- } \\
\text { dial infarc- } \\
\text { tion, death } \\
\text { caused by } \\
\text { heart fail- } \\
\text { ure, death } \\
\text { caused } \\
\text { by stroke, } \\
\text { death } \\
\text { caused by } \\
\text { other car- }\end{array}$ & $\begin{array}{l}\text { AO } \\
\text { "Acute myocardial infarc- } \\
\text { tion was defined as high } \\
\text { cardiac } \\
\text { enzymes or biomarkers } \\
\text { plus one of the following: } \\
\text { symptoms of ischaemia, } \\
\text { electrocardiographic } \\
\text { changes indicative of new } \\
\text { ischaemia, development } \\
\text { of pathological Q waves, } \\
\text { or imaging evidence of } \\
\text { new loss of viable my- } \\
\text { ocardium or a new region- } \\
\text { al wall motion abnormali- } \\
\text { ty" } \\
\text { "Silent myocardial infarc- } \\
\text { tion was defined as the } \\
\text { development of new elec- }\end{array}$ & $\begin{array}{l}\text { AO } \\
\text { "Hospital admis- } \\
\text { sion for heart fail- } \\
\text { ure was defined } \\
\text { as an emergency } \\
\text { or unplanned ad- } \\
\text { mission to hos- } \\
\text { pital that result- } \\
\text { ed in at least one } \\
\text { overnight } \\
\text { stay with clinical } \\
\text { manifestations } \\
\text { of new or wors- } \\
\text { ening heart fail- } \\
\text { ure, radiological } \\
\text { evidence of pul- } \\
\text { monary oedema } \\
\text { or congestion or } \\
\text { cardiomegaly, } \\
\text { or other imaging }\end{array}$ & $\mathrm{N} / \mathrm{R}$ & $N / R$ & $N / R$ & $N / R$ & 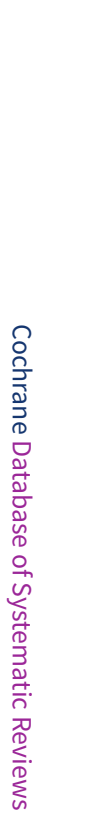 \\
\hline
\end{tabular}




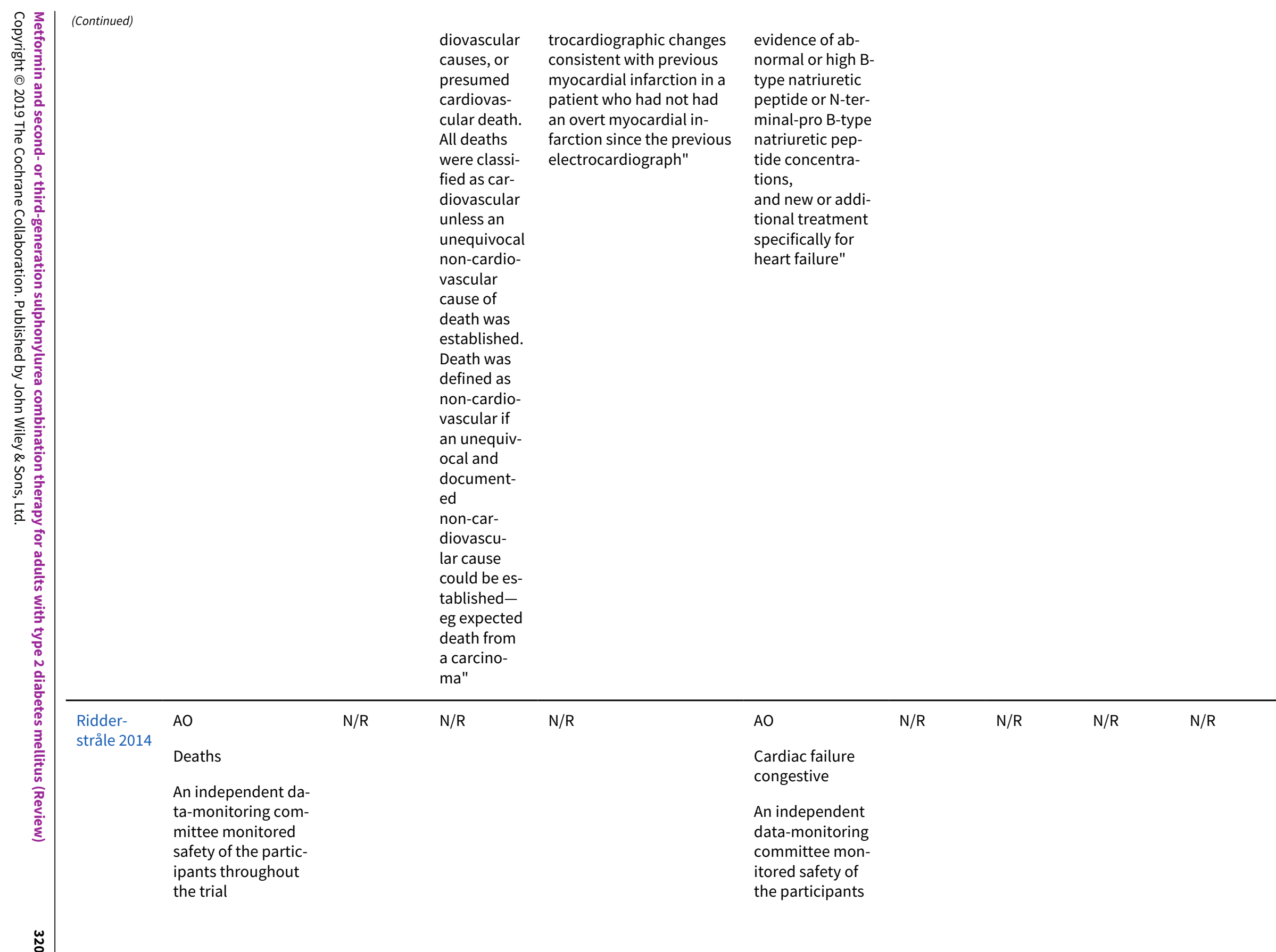




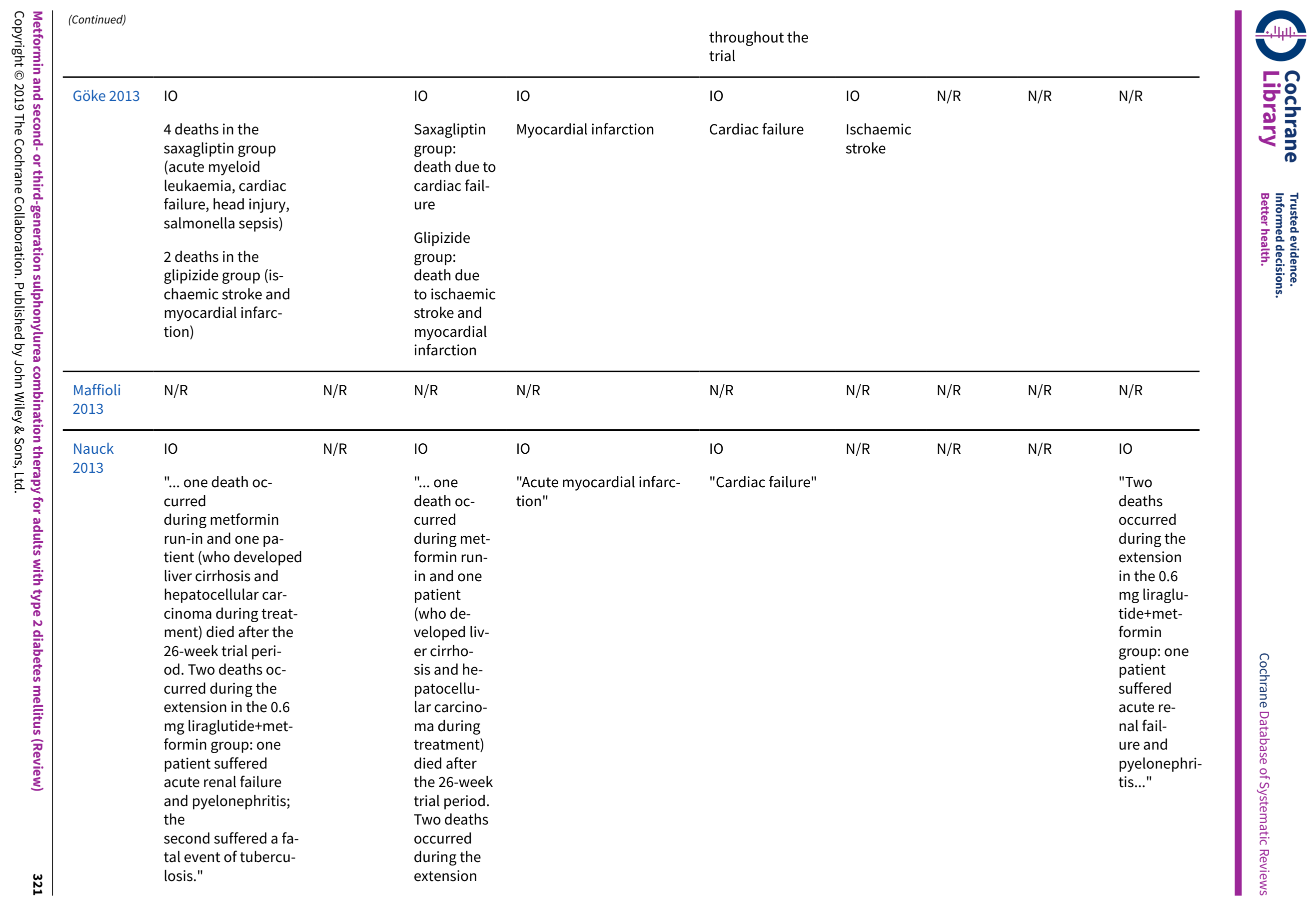




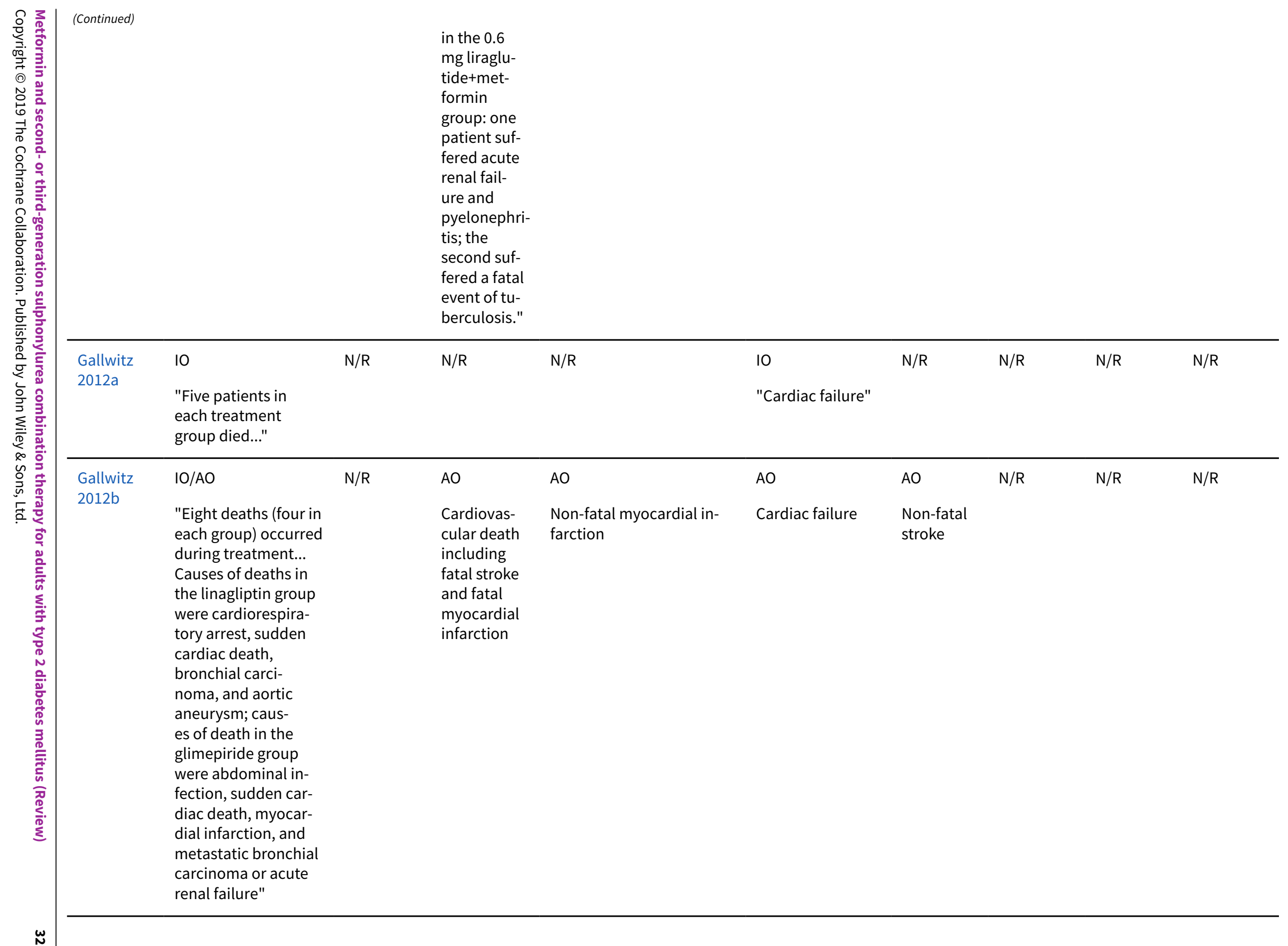




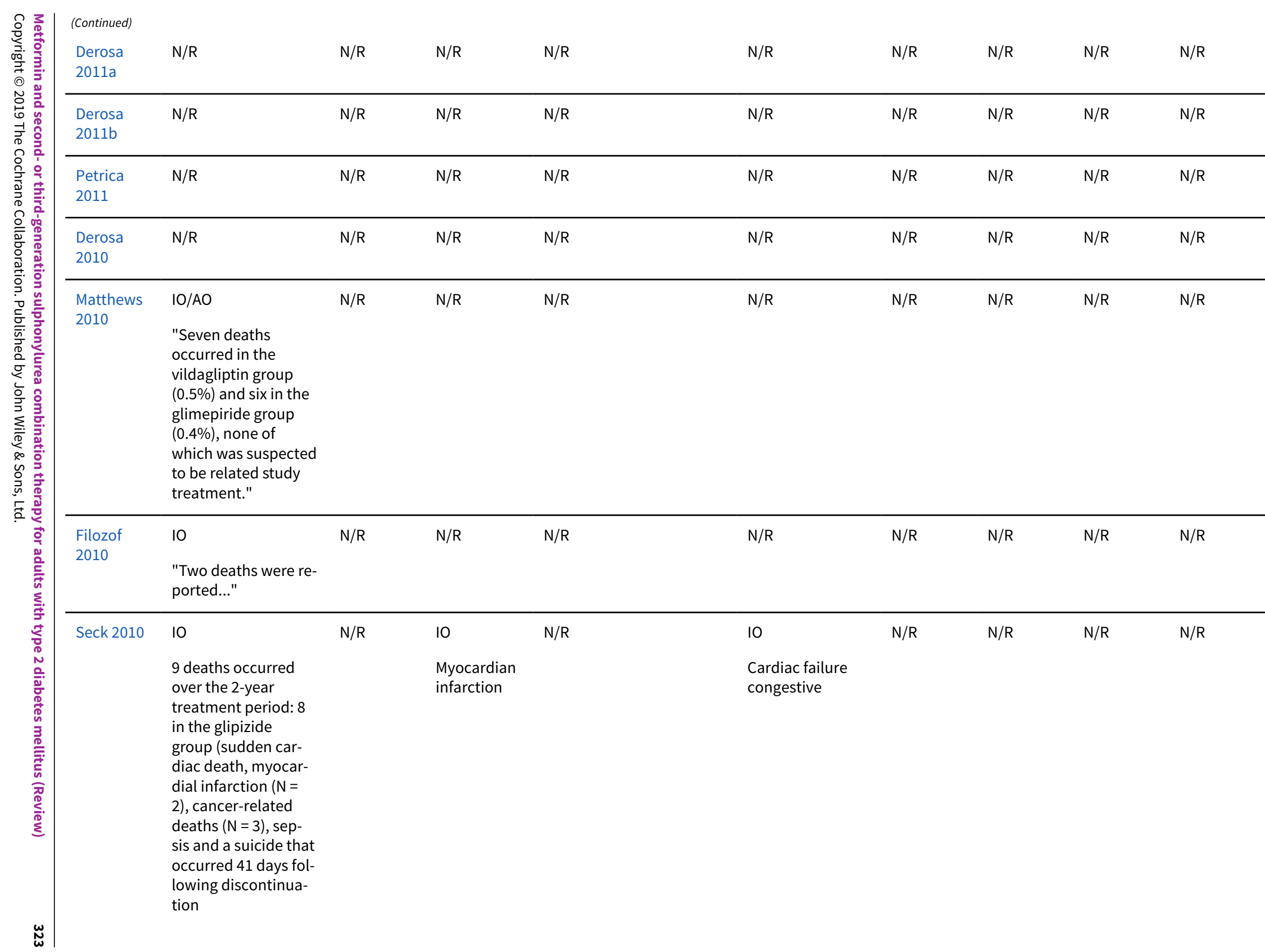




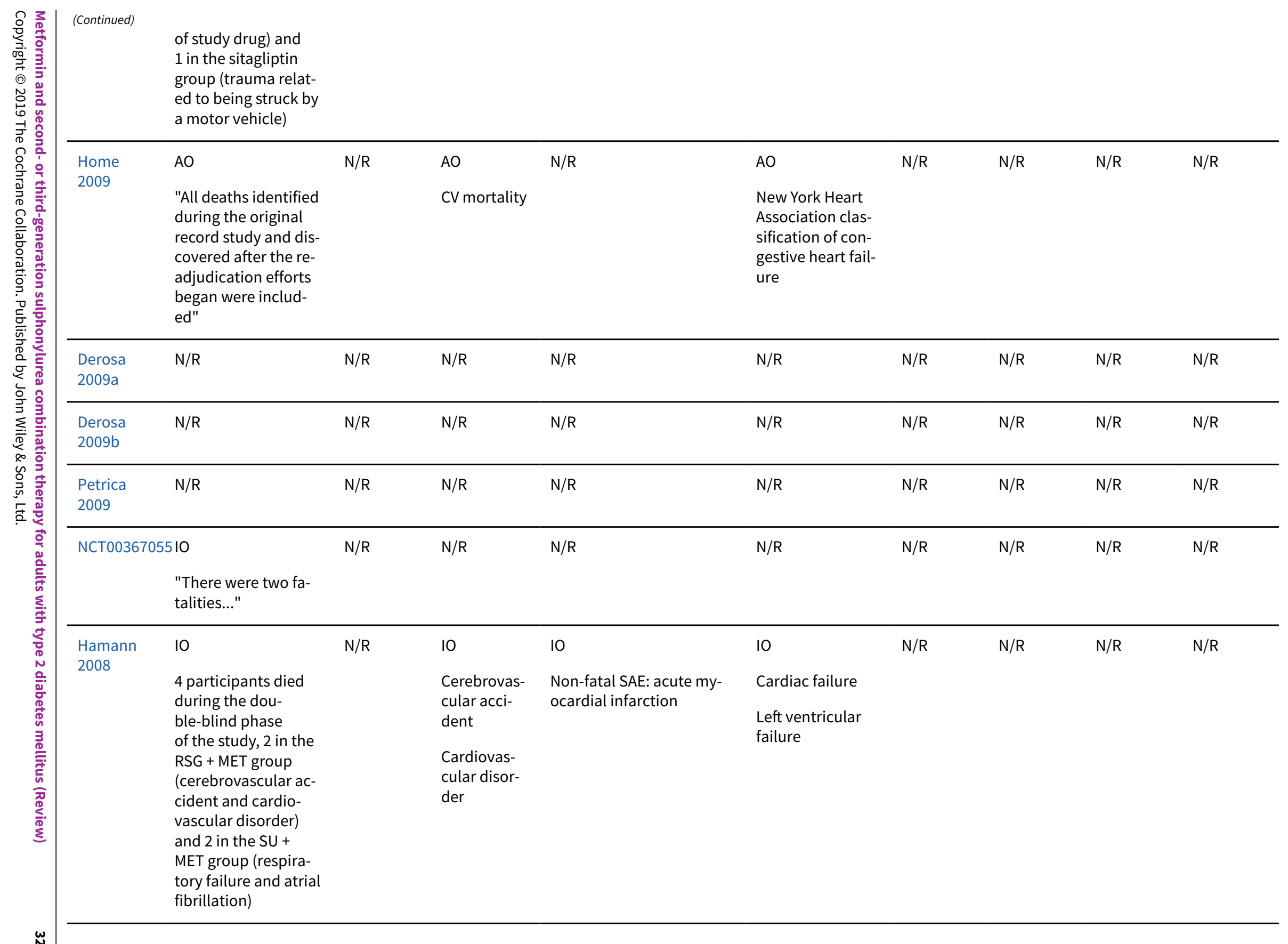




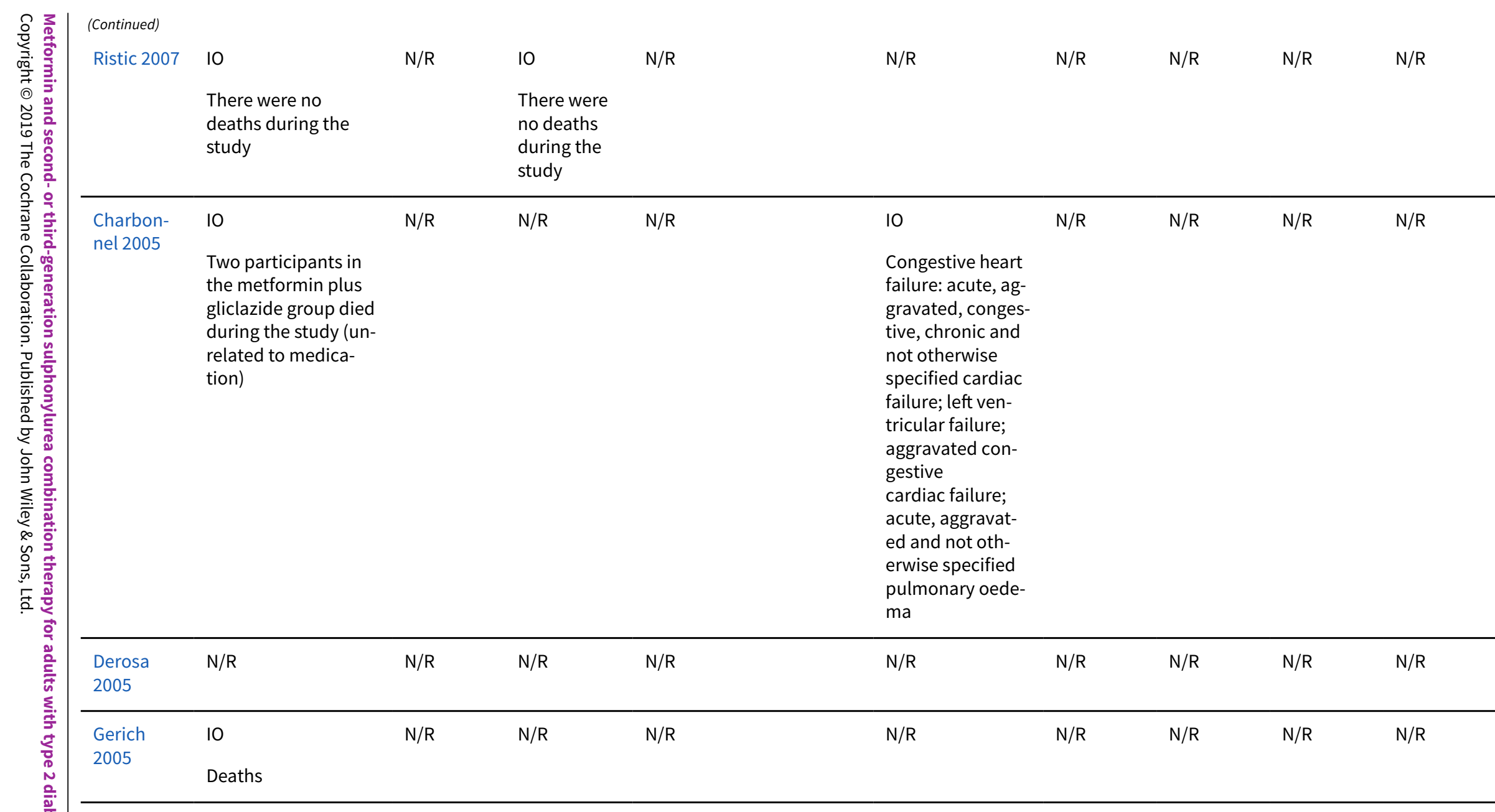

aln addition to definition of endpoint measurement, description who measured the outcome (AO: adjudicated outcome measurement; IO: investigator-assessed outcome measurement; SO: self-reported outcome measurement)

ACE: angiotensin converting enzyme; AE: adverse event; ARB: angiotension receptor blocker; BNP: brain natriuretic peptide; CHF: chronic heart failure; CT: computed tomography; CV: cardiovascular; ECG: electrocardiography; HbA1c: glycosylated haemoglobin A1c; HF: heart failure; LVEF: left ventricular ejection fraction; MACE: major adverse cardiac event; MET: metformin; MI: myocardial infarction; MRI: magnetic resonance imaging; N/D: not defined; N/R: not reported; RSG: rosiglitazone; SU: sulphonylurea 


\section{Appendix 10. Definition of endpoint measurement (II)a}

\begin{tabular}{|c|c|c|c|c|c|}
\hline Trial ID & $\begin{array}{l}\text { Socioeco- } \\
\text { nomic effects }\end{array}$ & $\begin{array}{l}\text { All hypoglycaemic } \\
\text { events }\end{array}$ & $\begin{array}{l}\text { Severe/seri- } \\
\text { ous hypogly- } \\
\text { caemia }\end{array}$ & $\begin{array}{l}\text { Non-seri- } \\
\text { ous adverse } \\
\text { events }\end{array}$ & $\begin{array}{l}\text { Severe/serious } \\
\text { adverse events }\end{array}$ \\
\hline $\begin{array}{l}\text { Handelsman } \\
2017\end{array}$ & $\mathrm{~N} / \mathrm{R}$ & $\begin{array}{l}\text { SO/IO } \\
\text { "Symptomatic hypo- } \\
\text { glycemia: episode with } \\
\text { clinical symptoms } \\
\text { attributed to hypo- } \\
\text { glycemia, without re- } \\
\text { gard to glucose level. } \\
\text { Severe hypoglycemia: } \\
\text { episode that required } \\
\text { assistance, either } \\
\text { medical or non-med- } \\
\text { ical. Episodes with a } \\
\text { markedly depressed } \\
\text { level of consciousness, } \\
\text { a loss of consciousness, } \\
\text { or seizure were classi- } \\
\text { fied as having required } \\
\text { medical } \\
\text { assistance, whether or } \\
\text { not medical assistance } \\
\text { was obtained. } \\
\text { Asymptomatic hypo- } \\
\text { glycemia: finger-stick } \\
\text { glucose values } \leq 3.9 \\
\text { mmol/L ( } 70 \text { mg/dL) } \\
\text { without symptoms." }\end{array}$ & $\begin{array}{l}\text { IO } \\
\text { "Severe hy- } \\
\text { poglycemia: } \\
\text { episode that } \\
\text { required assis- } \\
\text { tance, either } \\
\text { medical or } \\
\text { non-medical. } \\
\text { Episodes with } \\
\text { a markedly } \\
\text { depressed } \\
\text { level of con- } \\
\text { sciousness, } \\
\text { a loss of con- } \\
\text { sciousness, } \\
\text { or seizure } \\
\text { were classi- } \\
\text { fied as hav- } \\
\text { ing required } \\
\text { medical as- } \\
\text { sistance, } \\
\text { whether or } \\
\text { not med- } \\
\text { ical assis- } \\
\text { tance was ob- } \\
\text { tained." }\end{array}$ & $\begin{array}{l}\text { SO } \\
\text { "An adverse } \\
\text { event that is } \\
\text { not a serious } \\
\text { adverse event, } \\
\text { meaning that } \\
\text { it does not re- } \\
\text { sult in death, } \\
\text { is not life- } \\
\text { threatening, } \\
\text { does not re- } \\
\text { quire inpa- } \\
\text { tient hospi- } \\
\text { talization or } \\
\text { extend a cur- } \\
\text { rent hospi- } \\
\text { tal stay, does } \\
\text { not result in } \\
\text { an ongoing } \\
\text { or significant } \\
\text { incapacity } \\
\text { or interfere } \\
\text { substantially } \\
\text { with normal } \\
\text { life functions, } \\
\text { and does not } \\
\text { cause a con- } \\
\text { genital anom- } \\
\text { aly or birth } \\
\text { defect; it also } \\
\text { does not put } \\
\text { the partici- } \\
\text { pant in danger } \\
\text { and does not } \\
\text { require med- } \\
\text { ical or surgical } \\
\text { intervention } \\
\text { to prevent one } \\
\text { of the results } \\
\text { listed above." }\end{array}$ & $\begin{array}{l}\text { IO } \\
\text { "An adverse event that results in } \\
\text { death, is life-threatening, requires } \\
\text { inpatient hospitalization or ex- } \\
\text { tends a current hospital stay, re- } \\
\text { sults in an ongoing or significant } \\
\text { incapacity or interferes substan- } \\
\text { tially with normal life functions, } \\
\text { or causes a congenital anomaly or } \\
\text { birth defect. Medical events that } \\
\text { do not result in death, are not life- } \\
\text { threatening, or do not require hos- } \\
\text { pitalization may be considered se- } \\
\text { rious adverse events if they put the } \\
\text { participant in danger or require } \\
\text { medical or surgical intervention } \\
\text { to prevent one of the results listed } \\
\text { above." }\end{array}$ \\
\hline
\end{tabular}

\begin{tabular}{|c|c|c|c|c|c|}
\hline \multirow{2}{*}{$\begin{array}{l}\text { Hollander } \\
2017\end{array}$} & $\mathrm{~N} / \mathrm{R}$ & SO/IO & 10 & $\mathrm{~N} / \mathrm{R}$ & 10 \\
\hline & & $\begin{array}{l}\text { "Safety assessments in- } \\
\text { cluded the incidence of } \\
\text { adverse events (AEs), } \\
\text { including AEs of spe- } \\
\text { cial interest (sympto- } \\
\text { matic hypoglycemia } \\
\text { [episodes with clinical } \\
\text { symptoms reported by } \\
\text { the investigator as hy- } \\
\text { poglycemia; biochemi- }\end{array}$ & $\begin{array}{l}\text { "...severe hy- } \\
\text { poglycemia } \\
\text { (episodes that } \\
\text { required } \\
\text { medical or } \\
\text { non-medical } \\
\text { assistance) } \\
\text { were record- } \\
\text { ed." }\end{array}$ & & $\mathrm{N} / \mathrm{D}$ \\
\hline
\end{tabular}


cal documentation not required],..."

"Documented hypoglycemia (episodes with a glucose level $\leq$ $3.9 \mathrm{mmol} / \mathrm{L}[70 \mathrm{mg} / \mathrm{dL}]$ with or without symptoms) and severe hypoglycemia (episodes that required medical or non-medical assistance) were recorded."

\begin{tabular}{|c|c|c|c|c|c|}
\hline Vaccaro 2017 & $N / R$ & $\begin{array}{l}\text { SO/IO } \\
\text { "...hypoglycaemic } \\
\text { episodes defined } \\
\text { as a documented glu- } \\
\text { cose value of less than } \\
\text { 3.3 mmol/L and grad- } \\
\text { ed as moderate (not re- } \\
\text { quiring help for treat- } \\
\text { ment) or severe (requir- } \\
\text { ing assistance for treat- } \\
\text { ment)." }\end{array}$ & $\begin{array}{l}\text { SO/IO } \\
\text { "...hypo- } \\
\text { glycaemic } \\
\text { episodes de- } \\
\text { fined as a doc- } \\
\text { umented glu- } \\
\text { cose value } \\
\text { of less than } \\
3.3 \text { mmol/L } \\
\text { and graded as } \\
\text { moderate (not } \\
\text { requiring help } \\
\text { for treatment) } \\
\text { or severe (re- } \\
\text { quiring as- } \\
\text { sistance for } \\
\text { treatment)." }\end{array}$ & $\begin{array}{l}\text { SO/IO } \\
\text { Non-serious } \\
\text { adverse event }\end{array}$ & $\begin{array}{l}\text { IO/AO } \\
\text { "A serious adverse event was de- } \\
\text { fined as death, a life-threatening } \\
\text { episode, hospital admission or } \\
\text { prolongation of existing hospital } \\
\text { admission, or a persistent or sub- } \\
\text { stantial disability." }\end{array}$ \\
\hline Dei Cas 2017 & $N / R$ & $\begin{array}{l}\text { Mild hypoglycaemic } \\
\text { events } \\
\text { so/IO }\end{array}$ & $\begin{array}{l}\text { Severe hy- } \\
\text { poglycaemic } \\
\text { events } \\
\text { so/lo }\end{array}$ & $\begin{array}{l}\text { Adverse } \\
\text { events } \\
\text { so/10 }\end{array}$ & $\begin{array}{l}\text { Adverse events } \\
\text { SO/IO }\end{array}$ \\
\hline Leiter 2015 & $N / R$ & $\begin{array}{l}\text { SO } \\
\text { "... biochemically docu- } \\
\text { mented episodes (con- } \\
\text { current fingerstick glu- } \\
\text { cose or plasma glucose } \\
\leq 3.9 \text { mmol/L with or } \\
\text { without symptoms ..." }\end{array}$ & $\begin{array}{l}\text { SO/IO } \\
\text { "severe } \\
\text { episodes } \\
\text { (those need- } \\
\text { ing assistance } \\
\text { of another in- } \\
\text { dividual or } \\
\text { resulting in } \\
\text { seizure or loss } \\
\text { of conscious- } \\
\text { ness)." }\end{array}$ & $\begin{array}{l}\text { SO/IO } \\
\text { "Other Ad- } \\
\text { verse Events } \\
\text { are adverse } \\
\text { events that } \\
\text { are not Seri- } \\
\text { ous Adverse } \\
\text { Events but } \\
\text { that exceed } \\
\text { the indicat- } \\
\text { ed frequency } \\
\text { threshold." }\end{array}$ & $\begin{array}{l}\text { IO } \\
\text { "Serious Adverse Events include } \\
\text { adverse events that result in } \\
\text { death, require either inpatient hos- } \\
\text { pitalization or the prolongation of } \\
\text { hospitalization, are life-threaten- } \\
\text { ing, result in a persistent or signif- } \\
\text { icant disability/incapacity or re- } \\
\text { sult in a congenital anomaly/birth } \\
\text { defect. Other important medical } \\
\text { events, based upon appropriate } \\
\text { medical judgment, may also be } \\
\text { considered Serious Adverse Events } \\
\text { if a trial participant's health is at } \\
\text { risk and intervention is required to } \\
\text { prevent an outcome mentioned." }\end{array}$ \\
\hline
\end{tabular}

Del Prato $2015 \quad$ IO

$\mathrm{SO} / \mathrm{IO}$

$\mathrm{SO} / \mathrm{IO}$

$\mathrm{SO} / \mathrm{IO}$

10 
(Continued)

Incremental cost-effectiveness ratio, total costs
"Major hypoglycemia was defined as a symptomatic episode requiring external assistance due to severely impaired conscious ness or behavior, with capillary or plasma glucose levels of 54 $\mathrm{mg} / \mathrm{dL}$ (<3.0 mmol/ $\mathrm{L})$ and recovery after glucose or glucagon administration. Minor hypoglycemia was defined as a symptomatic episode with capillary or plasma glucose levels of $63 \mathrm{mg} / \mathrm{dL}$ (<3.5mmol/L), irrespective of the need for external assistance, or an asymptomatic episode with capillary or plasma glucose levels of 63 $\mathrm{mg} / \mathrm{dL}$ ( $<3.5 \mathrm{mmol} / \mathrm{L}$ ) that did not qualify as a major episode. Other hypoglycemia was defined as an episode with symptoms suggestive of hypoglycemia but without measurement confirmation."
"Major hypoglycemia was defined as a symptomatic episode requiring external assistance due to severely impaired consciousness or behavior, with capillary or plasma glucose levels of $54 \mathrm{mg} / \mathrm{dL}$ ( $<3.0 \mathrm{mmol} / \mathrm{L}$ ) and recovery after glucose or glucagon administration."
"Other Adverse Events are adverse events that are not Serious Adverse Events but that exceed the indicated frequency threshold."
"Serious Adverse Events include adverse events that result in death, require either inpatient hospitalization or the prolongation of hospitalization, are life-threatening, result in a persistent or significant disability/incapacity or result in a congenital anomaly/birth defect. Other important medical events, based upon appropriate medical judgment, may also be considered Serious Adverse Events if a trial participant's health is at risk and intervention is required to prevent an outcome mentioned."
Schernthaner N/R

2015

SO/IO
Confirmed hypogly-
caemia was defined
as a symptomatic or
asymptomatic event
with plasma glucose <
3.0 mmol/L, requiring
no external assistance.
Severe hypoglycaemia
was defined as a symp-
tomatic event requiring
external assistance be-
cause of severe impair-
ment in consciousness
or behaviour, with or
without plasma glucose
$<3.0$ mmol/L, but with
prompt recovery after
glucose/glucagon ad-
ministration.

Confirmed hypogly caemia was defined asymptomatic even with plasma glucose $<$ $3.0 \mathrm{mmol} / \mathrm{L}$, requiring Severe hypoglycaemia was defined as a symptomatic event requiring external assistance because of severe impairor behaviour, with or without plasma glucose prompt recovery after ministration.

so/IO
Severe hypo-
glycaemia was
defined as a
symptomatic
event requir-
ing external
assistance be-
cause of se-
vere impair-
ment in
conscious-
ness or behav-
iour, with or
without plas-
ma glucose
$<3.0$ mmol/
L, but with
prompt recov-
ery after glu-
cose/glucagon
administra-
tion.

So

"Other Adverse Events are adverse events that are not Serious Adverse Events but that exceed the indicated frequency threshold." 10

"Serious Adverse Events include adverse events that result in death, require either inpatient hospitalization or the prolongation of hospitalization, are life-threatening, result in a persistent or significant disability/incapacity or result in a congenital anomaly/birth defect. Other important medical events, based upon appropriate medical judgment, may also be considered Serious Adverse Events if a trial participant's health is at risk and intervention is required to prevent an outcome mentioned." 
(Continued)

$\begin{array}{ll}\begin{array}{ll}\text { Incremental } \\ \text { cost-effective- }\end{array} & \text { poglycaemia was de- } \\ \text { ness ratio } & \text { fined as blood glucose } \\ & <3.33 \mathrm{mmol} / \mathrm{L} \text { in the } \\ & \text { presence of symptoms, } \\ & \text { or blood glucose }<2.78 \\ & \text { mmol/L without symp- } \\ & \text { toms. Severe hypogly- } \\ & \text { caemia was defined as } \\ & \text { requiring assistance } \\ & \text { to administer carbo- } \\ & \text { hydrate, glucagon or } \\ & \text { other resuscitative ac- } \\ & \text { tions, with a document- } \\ & \text { ed blood glucose con- } \\ & \text { centration <3.33mmol/ } \\ & \text { lif the clinical situation } \\ & \text { allowed measurement } \\ & \text { of blood glucose." }\end{array}$

"Severe hypoglycaemia was defined as requiring assistance to administer carbohydrate, glucagon or other resuscitative actions, with a documented blood glucose concentration $<3.33$ $\mathrm{mmol} / \mathrm{L}$ if the clinical situation allowed measurement of blood glucose."
"Other Adverse Events are adverse events that are not Serious Adverse Events but that exceed the indicated frequency threshold."

\section{SO}

"Other adverse events are adverse events that are not serious adverse events but that exceed the indicated frequency threshold" = required assistance of another person..."
"Serious Adverse Events include adverse events that result in death, require either inpatient hospitalization or the prolongation of hospitalization, are life-threatening, result in a persistent or significant disability/incapacity or result in a congenital anomaly/birth defect. Other important medical events, based upon appropriate medical judgment, may also be considered Serious Adverse Events if a trial participant's health is at risk and intervention is required to prevent an outcome mentioned."

\section{0}

"Serious adverse events include adverse events that result in death, require either inpatient hospitalization or the prolongation of hospitalization, are life-threatening, result in a persistent or significant disability/incapacity or result in a congenital anomaly/birth defect. Other important medical events, based upon appropriate medical judgment, may also be considered serious adverse events if a trial participant's health is at risk and intervention is required to prevent an outcome mentioned" and asymptomatic $=$ no symptoms but plasma glucose concentration $\leq 3.9 \mathrm{mmol} / \mathrm{L}$."

\begin{tabular}{|c|c|c|c|c|c|}
\hline \multirow{2}{*}{$\begin{array}{l}\text { Ridderstråle } \\
2014\end{array}$} & $\mathrm{~N} / \mathrm{R}$ & $\mathrm{AO}$ & $\mathrm{AO}$ & AO & $\mathrm{AO}$ \\
\hline & & $\begin{array}{l}\text { Hypoglycaemia (not in- } \\
\text { cluding serious) } \\
\text { Hypoglycaemia (serious } \\
\text { AE) } \\
\text { An independent da- } \\
\text { ta-monitoring commit- } \\
\text { tee monitored safe- } \\
\text { ty of the participants } \\
\text { throughout the trial }\end{array}$ & $\begin{array}{l}\text { Hypogly- } \\
\text { caemia (seri- } \\
\text { ous AE) } \\
\text { An indepen- } \\
\text { dent da- } \\
\text { ta-monitor- } \\
\text { ing commit- } \\
\text { tee monitored } \\
\text { safety of the } \\
\text { participants } \\
\text { throughout } \\
\text { the trial }\end{array}$ & $\begin{array}{l}\text { "Other ad- } \\
\text { verse events } \\
\text { are adverse } \\
\text { events that } \\
\text { are not seri- } \\
\text { ous adverse } \\
\text { events but } \\
\text { that exceed } \\
\text { the indicat- } \\
\text { ed frequency } \\
\text { threshold" } \\
\text { An indepen- } \\
\text { dent da- } \\
\text { ta-monitor- } \\
\text { ing commit- }\end{array}$ & $\begin{array}{l}\text { "Serious adverse events include } \\
\text { adverse events that result in } \\
\text { death, require either inpatient hos- } \\
\text { pitalization or the prolongation of } \\
\text { hospitalization, are life-threaten- } \\
\text { ing, result in a persistent or signif- } \\
\text { icant disability/incapacity or re- } \\
\text { sult in a congenital anomaly/birth } \\
\text { defect. Other important medical } \\
\text { events, based upon appropriate } \\
\text { medical judgment, may also be } \\
\text { considered serious adverse events } \\
\text { if a trial participant's health is at } \\
\text { risk and intervention is required to } \\
\text { prevent an outcome mentioned" }\end{array}$ \\
\hline
\end{tabular}


tee monitored An independent data-monitoring safety of the participants throughout the trial.

\begin{tabular}{|c|c|c|c|c|c|}
\hline \multirow[t]{2}{*}{ Göke 2013} & 10 & SO & $\mathrm{SO} / \mathrm{IO}$ & SO & 10 \\
\hline & $\begin{array}{l}\text { Cost per QALY, } \\
\text { incremental } \\
\text { cost }\end{array}$ & $\begin{array}{l}\text { Reported hypogly- } \\
\text { caemia events were a } \\
\text { combination of reports } \\
\text { of either signs or symp- } \\
\text { toms consistent with } \\
\text { hypoglycaemia with } \\
\text { or without document- } \\
\text { ed glucose levels or re- } \\
\text { ported low glucose lev- } \\
\text { els without any symp- } \\
\text { toms. Confirmed hypo- } \\
\text { glycaemia was defined } \\
\text { as a finger-stick glucose } \\
\text { value } \leq 50 \text { mg/dL with } \\
\text { associated symptoms }\end{array}$ & $\begin{array}{l}\text { Severe hypo- } \\
\text { glycaemia }\end{array}$ & $\begin{array}{l}\text { "Other ad- } \\
\text { verse events } \\
\text { are adverse } \\
\text { events that } \\
\text { are not seri- } \\
\text { ous adverse } \\
\text { events but } \\
\text { that exceed } \\
\text { the indicat- } \\
\text { ed frequency } \\
\text { threshold" }\end{array}$ & $\begin{array}{l}\text { "Serious adverse events include } \\
\text { adverse events that result in } \\
\text { death, require either inpatient hos- } \\
\text { pitalization or the prolongation of } \\
\text { hospitalization, are life-threaten- } \\
\text { ing, result in a persistent or signif- } \\
\text { icant disability/incapacity or re- } \\
\text { sult in a congenital anomaly/birth } \\
\text { defect. Other important medical } \\
\text { events, based upon appropriate } \\
\text { medical judgment, may also be } \\
\text { considered serious adverse events } \\
\text { if a trial participant's health is at } \\
\text { risk and intervention is required to } \\
\text { prevent an outcome mentioned" }\end{array}$ \\
\hline Maffioli 2013 & $\mathrm{~N} / \mathrm{R}$ & $\mathrm{N} / \mathrm{R}$ & $\mathrm{N} / \mathrm{R}$ & $\mathrm{N} / \mathrm{R}$ & $N / R$ \\
\hline \multirow[t]{2}{*}{ Nauck 2013} & $\mathrm{~N} / \mathrm{R}$ & $\mathrm{SO}$ & $\mathrm{SO}$ & $\mathrm{sO} / 10$ & 10 \\
\hline & & $\begin{array}{l}\text { "Hypoglycaemic } \\
\text { episodes were defined } \\
\text { as major, minor, or } \\
\text { symptoms only. Major if } \\
\text { the subject was unable } \\
\text { to treat her/himself. Mi- } \\
\text { nor if subject was able } \\
\text { to treat her/himself and } \\
\text { plasma glucose was be- } \\
\text { low } 3.1 \text { mmol/L. Symp- } \\
\text { toms only if subject was } \\
\text { able to treat her/him- } \\
\text { self and with no plasma } \\
\text { glucose measurement } \\
\text { or plasma glucose high- } \\
\text { er than or equal to } 3.1 \\
\text { mmol/L." }\end{array}$ & $\begin{array}{l}\text { "Hypogly- } \\
\text { caemic } \\
\text { episodes were } \\
\text { defined as... } \\
\text { Major if the } \\
\text { subject was } \\
\text { unable to } \\
\text { treat her/him- } \\
\text { self." }\end{array}$ & $\begin{array}{l}\text { "Other ad- } \\
\text { verse events } \\
\text { are adverse } \\
\text { events that } \\
\text { are not seri- } \\
\text { ous adverse } \\
\text { events but } \\
\text { that exceed } \\
\text { the indicat- } \\
\text { ed frequency } \\
\text { threshold." }\end{array}$ & $\begin{array}{l}\text { "Serious adverse events include } \\
\text { adverse events that result in } \\
\text { death, require either inpatient hos- } \\
\text { pitalization or the prolongation of } \\
\text { hospitalization, are life-threaten- } \\
\text { ing, result in a persistent or signif- } \\
\text { icant disability/incapacity or re- } \\
\text { sult in a congenital anomaly/birth } \\
\text { defect. Other important medical } \\
\text { events, based upon appropriate } \\
\text { medical judgment, may also be } \\
\text { considered serious adverse events } \\
\text { if a trial participant's health is at } \\
\text { risk and intervention is required to } \\
\text { prevent an outcome mentioned." }\end{array}$ \\
\hline \multirow[t]{2}{*}{ Gallwitz 2012a } & $\mathrm{N} / \mathrm{R}$ & $\mathrm{SO} / 10$ & $\mathrm{sO} / 10$ & $\mathrm{sO} / 10$ & 10 \\
\hline & & $\begin{array}{l}\text { A hypoglycaemic } \\
\text { episode was defined } \\
\text { as any time a partici- } \\
\text { pant experienced a sign } \\
\text { or symptom associat- } \\
\text { ed with hypoglycaemia } \\
\text { or had a blood glucose } \\
\text { measurement of } \leq 3.9 \\
\text { mmol/L ( } \leq 70 \text { mg/dL), } \\
\text { even if not associated } \\
\text { with a sign or symptom } \\
\text { of hypoglycaemia; se- } \\
\text { vere hypoglycaemia }\end{array}$ & $\begin{array}{l}\text { Severe hypo- } \\
\text { glycaemia was } \\
\text { defined as an } \\
\text { event requir- } \\
\text { ing assistance } \\
\text { of another } \\
\text { person. }\end{array}$ & $\begin{array}{l}\text { "Other ad- } \\
\text { verse events } \\
\text { are adverse } \\
\text { events that } \\
\text { are not seri- } \\
\text { ous adverse } \\
\text { events but } \\
\text { that exceed } \\
\text { the indicat- } \\
\text { ed frequency } \\
\text { threshold." }\end{array}$ & $\begin{array}{l}\text { "Serious adverse events include } \\
\text { adverse events that result in } \\
\text { death, require either inpatient hos- } \\
\text { pitalization or the prolongation of } \\
\text { hospitalization, are life-threaten- } \\
\text { ing, result in a persistent or signif- } \\
\text { icant disability/incapacity or re- } \\
\text { sult in a congenital anomaly/birth } \\
\text { defect. Other important medical } \\
\text { events, based upon appropriate } \\
\text { medical judgment, may also be } \\
\text { considered serious adverse events } \\
\text { if a trial participant's health is at }\end{array}$ \\
\hline
\end{tabular}


was defined as an event requiring assistance of another

person. risk and intervention is required to prevent an outcome mentioned."

\begin{tabular}{|c|c|c|c|c|c|}
\hline \multirow[t]{2}{*}{ Gallwitz 2012b } & $N / R$ & $\mathrm{SO} / 10$ & so/10 & SO/IO & IO/AO \\
\hline & & $\begin{array}{l}\text { Hypoglycaemia (not in- } \\
\text { cluding severe) } \\
\text { Severe hypoglycaemia }\end{array}$ & $\begin{array}{l}\text { Severe hypo- } \\
\text { glycaemia }\end{array}$ & $\begin{array}{l}\text { "Other ad- } \\
\text { verse events } \\
\text { are adverse } \\
\text { events that } \\
\text { are not seri- } \\
\text { ous adverse } \\
\text { events but } \\
\text { that exceed } \\
\text { the indicat- } \\
\text { ed frequency } \\
\text { threshold." }\end{array}$ & $\begin{array}{l}\text { "Serious adverse events include } \\
\text { adverse events that result in } \\
\text { death, require either inpatient hos- } \\
\text { pitalization or the prolongation of } \\
\text { hospitalization, are life-threaten- } \\
\text { ing, result in a persistent or signif- } \\
\text { icant disability/incapacity or re- } \\
\text { sult in a congenital anomaly/birth } \\
\text { defect. Other important medical } \\
\text { events, based upon appropriate } \\
\text { medical judgment, may also be } \\
\text { considered serious adverse events } \\
\text { if a trial participant's health is at } \\
\text { risk and intervention is required to } \\
\text { prevent an outcome mentioned." }\end{array}$ \\
\hline
\end{tabular}

\begin{tabular}{llllll}
\hline Derosa 2011a & $\mathrm{N} / \mathrm{R}$ & $\mathrm{N} / \mathrm{R}$ & $\mathrm{N} / \mathrm{R}$ & $\mathrm{N} / \mathrm{R}$ & $\mathrm{N} / \mathrm{R}$ \\
\hline Derosa 2011b & $\mathrm{N} / \mathrm{R}$ & $\mathrm{N} / \mathrm{R}$ & $\mathrm{N} / \mathrm{R}$ & $\mathrm{N} / \mathrm{R}$ & $\mathrm{N} / \mathrm{R}$ \\
\hline Petrica 2011 & $\mathrm{N} / \mathrm{R}$ & $\mathrm{N} / \mathrm{R}$ & $\mathrm{N} / \mathrm{R}$ & $\mathrm{N} / \mathrm{R}$ & $\mathrm{N} / \mathrm{R}$ \\
\hline Derosa 2010 & $\mathrm{N} / \mathrm{R}$ & $\mathrm{N} / \mathrm{R}$ & $\mathrm{N} / \mathrm{R}$ & $\mathrm{N} / \mathrm{R}$ & $\mathrm{N} / \mathrm{R}$ \\
\hline Matthews & $\mathrm{N} / \mathrm{R}$ & $\mathrm{SO}$ & $\mathrm{IO} / \mathrm{O}$ & $\mathrm{IO}$ \\
2010 & & $\begin{array}{l}\text { "... symptoms sugges- } \\
\text { tive of hypoglycaemia } \\
\text { and confirmed by self- } \\
\text { monitored plasma } \\
\text { glucose }<3.1 \text { mmol/L..." }\end{array}$ & $\begin{array}{l}\text { "... any } \\
\text { episode re- } \\
\text { quiring the as- } \\
\text { sistance of an- } \\
\text { other party..." }\end{array}$ & "SAE's were experienced by..." \\
\hline
\end{tabular}

\begin{tabular}{llllll}
\hline Filozof 2010 & $N / R$ & $N / R$ & $N / R$ & $N / R$ & AO/IO
\end{tabular}

Drug-related SAE

\begin{tabular}{|c|c|c|c|c|c|}
\hline \multirow[t]{2}{*}{ Seck 2010} & $\mathrm{~N} / \mathrm{R}$ & SO & $\mathrm{SO} / 10$ & SO & 10 \\
\hline & & $\begin{array}{l}\text { Hypoglycaemia other } \\
\text { (not including serious) } \\
\text { adverse events } \\
\text { Hypoglycaemia re- } \\
\text { quiring medical inter- } \\
\text { vention or exhibiting } \\
\text { markedly depressed } \\
\text { level of consciousness, } \\
\text { including loss of con- } \\
\text { sciousness or seizure }\end{array}$ & $\begin{array}{l}\text { Hypogly- } \\
\text { caemia requir- } \\
\text { ing medical } \\
\text { intervention } \\
\text { or exhibit- } \\
\text { ing marked- } \\
\text { ly depressed } \\
\text { level of con- } \\
\text { sciousness, in- } \\
\text { cluding loss of } \\
\text { consciousness } \\
\text { or seizure }\end{array}$ & $\begin{array}{l}\text { "Other Ad- } \\
\text { verse Events } \\
\text { are adverse } \\
\text { events that } \\
\text { are not Seri- } \\
\text { ous Adverse } \\
\text { Events but } \\
\text { that exceed } \\
\text { the indicat- } \\
\text { ed frequency } \\
\text { threshold." }\end{array}$ & Serious adverse events \\
\hline Home 2009 & $\mathrm{~N} / \mathrm{R}$ & SO & $\mathrm{SO} / 10$ & SO & IO/AO \\
\hline
\end{tabular}


Hypoglycaemia other (not including serious) adverse events

Hypoglycaemia serious adverse events
Hypogly-

caemia seri-

ous adverse

events
"Other Adverse Events are adverse events that are not Serious Adverse Events but that exceed the indicated frequency threshold."
"Serious Adverse Events include adverse events that result in death, require either inpatient hospitalization or the prolongation of hospitalization, are life-threatening, result in a persistent or significant disability/incapacity or result in a congenital anomaly/birth defect. Other important medical events, based upon appropriate medical judgment, may also be considered Serious Adverse Events if a trial participant's health is at risk and intervention is required to prevent an outcome mentioned."

\begin{tabular}{llllll}
\hline Derosa 2009a & N/R & N/R & N/R & N/R & $N / R$ \\
\hline Derosa 2009b & N/R & N/R & $N / R$ & $N / R$ & $N / R$ \\
\hline Petrica 2009 & N/R & N/R & $N / R$ & $N / R$ & $N / R$ \\
\hline
\end{tabular}

\begin{tabular}{llllll}
\hline NCT00367055 & N/R & SO/IO & N/R & SO/IO & 10
\end{tabular}

Hypoglycaemia
"Other Adverse Events are adverse events that are not Serious Adverse Events but that exceed the indicated frequency threshold."
"Serious Adverse Events include adverse events that result in death, require either inpatient hospitalization or the prolongation of hospitalization, are life-threatening, result in a persistent or significant disability/incapacity or result in a congenital anomaly/birth defect. Other important medical events, based upon appropriate medical judgment, may also be considered Serious Adverse Events if a trial participant's health is at risk and intervention is required to prevent an outcome mentioned."

10

\begin{tabular}{llll}
\hline Hamann $2008 \mathrm{~N} / \mathrm{R}$ & SO & SO & SO \\
& & & \\
& Participants were al- & Non-fatal & Non-seri- \\
so asked to check their & SAE: hypogly- & ous adverse \\
blood glucose levels at & caemia & events, ex- \\
any time they experi- & & cluding hy- \\
enced symptoms of hy- & & poglycaemic \\
poglycaemia and record & & events, that \\
the reading in their di- & & led to with- \\
& ary cards. & drawal
\end{tabular}

\begin{tabular}{lllll}
\hline Ristic 2007 & N/R & N/R & N/R & N/R \\
\hline $\begin{array}{llll}\text { Charbonnel } \\
2005\end{array}$ & N/R & SO/IO & SO/IO & N/R \\
Hypoglycaemia & $\begin{array}{l}\text { Severe or se- } \\
\text { rious hypogly- } \\
\text { caemia }\end{array}$ & Serious adverse events \\
\hline
\end{tabular}

\begin{tabular}{|c|c|c|c|}
\hline Derosa 2005 & $N / R$ & $\mathrm{SO} / 10$ & $\mathrm{SO} / \mathrm{IO}$ \\
\hline
\end{tabular}


(Continued)

$\begin{array}{lll}\text { Mild/moderate hypo- } & \begin{array}{l}\text { Serious hypo- } \\ \text { glycaemia }\end{array} & \text { "...side ef- } \\ & \text { fects... } \\ & \text { transient } \\ & \text { headache... } \\ & \text { transient } \\ & \text { flatulence... } \\ & \text { aspartate } \\ & \text { aminotrans- } \\ & \text { ferase... ala- } \\ & \text { nine amino- } \\ & \text { transferase } \\ & \text { values... in- } \\ & \text { creases..." }\end{array}$

\begin{tabular}{|c|c|c|c|c|c|}
\hline \multirow[t]{2}{*}{ Gerich 2005} & $\mathrm{~N} / \mathrm{R}$ & so & $\mathrm{so} / 10$ & $N / R$ & 10 \\
\hline & & $\begin{array}{l}\text { "Hypoglycemia was de- } \\
\text { fined as symptoms con- } \\
\text { sistent with low blood } \\
\text { glucose confirmed by } \\
\text { a self-monitored blood } \\
\text { glucose determination } \\
\text { of } 3.3 \text { mmol/l plasma } \\
\text { glucose equivalents." }\end{array}$ & $\begin{array}{l}\text { "Severe hy- } \\
\text { poglycemia } \\
\text { (grade 2, re- } \\
\text { quiring assis- } \\
\text { tance from an } \\
\text { outside par- } \\
\text { ty..." }\end{array}$ & & $\begin{array}{l}\text { "Includes deaths, grade } 2 \text { hypo- } \\
\text { glycemic events, and } 1 \text { subject } \\
\text { with a laboratory abnormality } \\
\text { leading to discontinuation" }\end{array}$ \\
\hline
\end{tabular}

aln addition to definition of endpoint measurement, description who measured the outcome (AO: adjudicated outcome measurement; IO: investigator-assessed outcome measurement; SO: self-reported outcome measurement)

AE: adverse event; N/D: not defined; N/R: not reported; QALY: quality-adjusted life year 


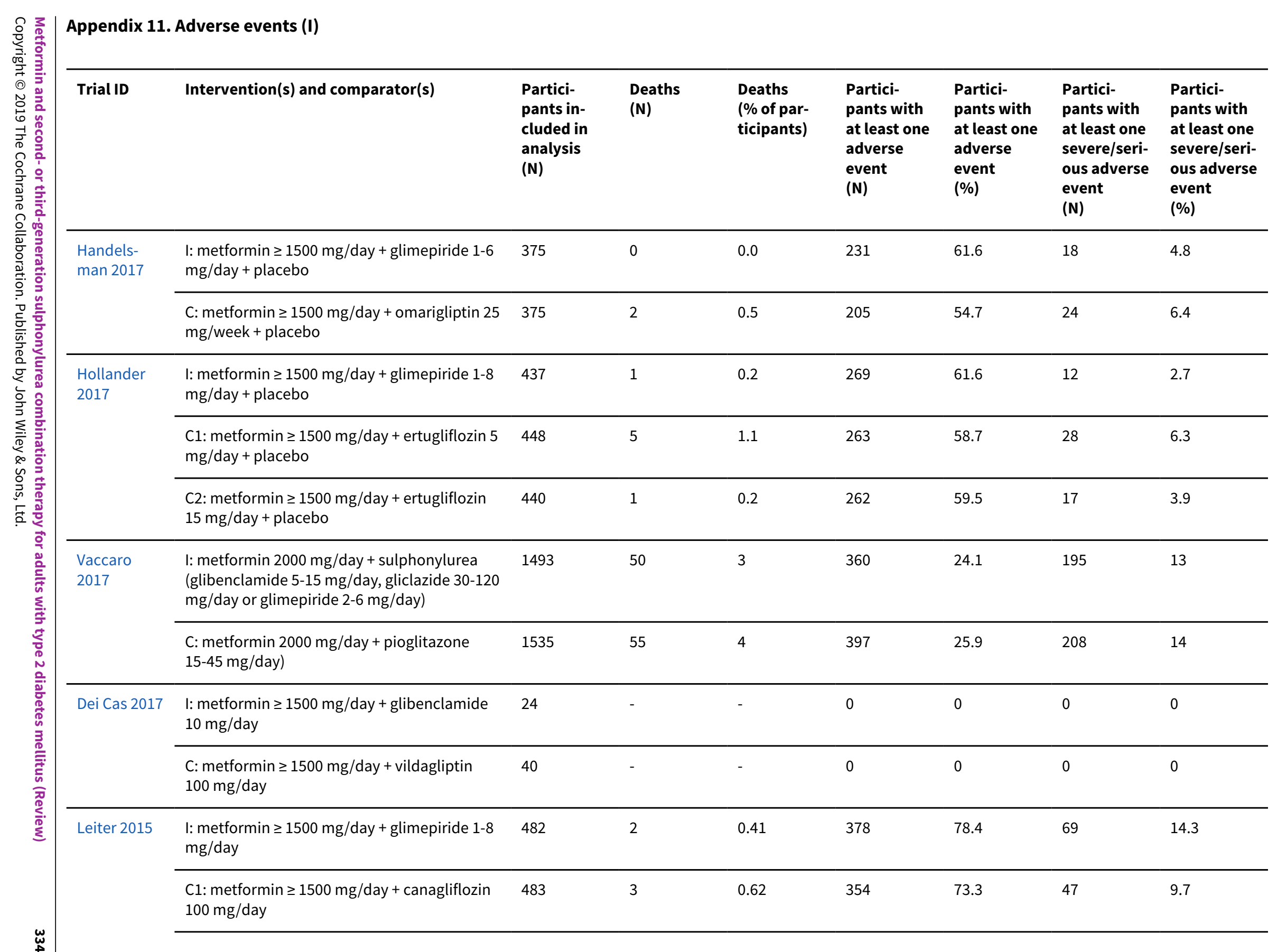




\begin{tabular}{|c|c|c|c|c|c|c|c|c|}
\hline 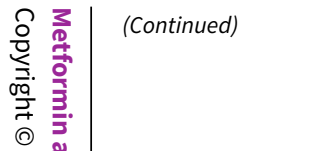 & $\begin{array}{l}\text { C2: } \text { metformin } \geq 1500 \mathrm{mg} / \text { day + canagliflozin } \\
300 \mathrm{mg} / \text { day }\end{array}$ & 485 & 3 & 0.62 & 378 & 77.9 & 47 & 9.7 \\
\hline $\begin{array}{l}\text { Del Prato } \\
2015\end{array}$ & $\begin{array}{l}\text { I: metformin } 1500-2500 \mathrm{mg} / \text { day + glipizide } \\
\text { 5-20 mg/day }\end{array}$ & 408 & 5 & 1.2 & 355 & 87.0 & 81 & 19.9 \\
\hline 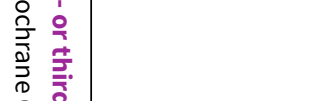 & $\begin{array}{l}\text { C: metformin } 1500-2500 \mathrm{mg} / \text { day + da- } \\
\text { pagliflozin } 2.5-10 \mathrm{mg} / \text { day }\end{array}$ & 406 & 2 & 0.5 & 356 & 87.7 & 75 & 18.5 \\
\hline $\begin{array}{l}\text { Schern- } \\
\text { thaner } 2015\end{array}$ & $\begin{array}{l}\text { I: metformin at any dose + glimepiride } 1-6 \\
\mathrm{mg} / \text { day + placebo }\end{array}$ & 359 & 1 & 0.3 & 213 & 59.3 & 32 & 8.9 \\
\hline 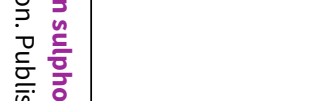 & $\begin{array}{l}\text { C: metformin at any dose + saxagliptin } 5 \mathrm{mg} / \\
\text { day + placebo }\end{array}$ & 359 & 1 & 0.3 & 213 & 59.3 & 41 & 11.4 \\
\hline $\begin{array}{l}\text { Del Prato } \\
2014\end{array}$ & $\begin{array}{l}\text { I: metformin } \geq 1500 \text { mg once daily or maxi- } \\
\text { mum tolerated dose + glipizide 5-20 mg once } \\
\text { daily }\end{array}$ & 869 & 5 & 0.6 & 668 & 76.9 & 81 & 9.3 \\
\hline 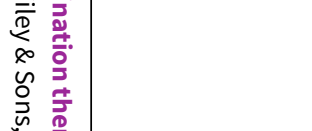 & $\begin{array}{l}\mathrm{C} 1 \text { : metformin } \geq 1500 \mathrm{mg} \text { once daily or maxi- } \\
\text { mum tolerated dose }+ \text { alogliptin } 12.5 \text { mg once } \\
\text { daily }\end{array}$ & 873 & 3 & 0.3 & 688 & 78.8 & 86 & 9.9 \\
\hline 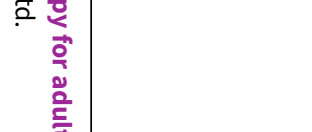 & $\begin{array}{l}\mathrm{C} 2 \text { : metformin } \geq 1500 \mathrm{mg} \text { once daily or maxi- } \\
\text { mum tolerated dose }+ \text { alogliptin } 25 \mathrm{mg} \text { once } \\
\text { daily }\end{array}$ & 878 & 3 & 0.3 & 687 & 78.3 & 97 & 11.1 \\
\hline Ahrén 2014 & $\begin{array}{l}\text { I: } \text { metformin } \geq 1500 \text { mg daily + glimepiride } 2-4 \\
\text { mg once daily + placebo }\end{array}$ & 307 & 6 & 2.0 & 261 & 85 & 36 & 11.7 \\
\hline$\frac{0^{\circ}}{20}$ & $\begin{array}{l}\mathrm{C} 1: \text { metformin } \geq 1500 \mathrm{mg} \text { daily + albiglutide } \\
30-50 \mathrm{mg} \text { once weekly + placebo }\end{array}$ & 302 & 4 & 1.3 & 263 & 87.1 & 44 & 14.6 \\
\hline 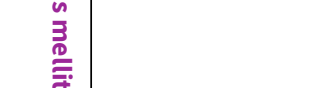 & $\begin{array}{l}\text { C2: metformin } \geq 1500 \text { mg daily + sitagliptin } \\
100 \text { mg once daily + placebo }\end{array}$ & 302 & 2 & 0.7 & 251 & 83.1 & 32 & 10.6 \\
\hline 总. & $\begin{array}{l}\text { C3: } \text { metformin } \geq 1500 \mathrm{mg} \text { daily }+ \text { placebo }+ \\
\text { placebo }\end{array}$ & 101 & 1 & 1.0 & 83 & 82.2 & 15 & 14.9 \\
\hline $\begin{array}{l}\text { Ridderstråle } \\
2014\end{array}$ & $\begin{array}{l}\text { I: metformin immediate release } \geq 1500 \mathrm{mg} / \\
\text { day + glimepiride } 1-4 \mathrm{mg} / \text { day }\end{array}$ & 780 & 5 & 0.6 & 673 & 86 & 153 & 19.6 \\
\hline
\end{tabular}




\begin{tabular}{|c|c|c|c|c|c|c|c|c|c|}
\hline 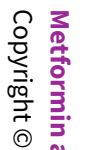 & (Continued) & $\begin{array}{l}\text { C: metformin immediate release } \geq 1500 \mathrm{mg} / \\
\text { day + empagliflozin } 25 \mathrm{mg} / \text { day }\end{array}$ & 765 & 5 & 0.7 & 661 & 86 & 161 & 21.1 \\
\hline 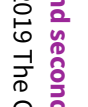 & \multirow[t]{2}{*}{ Göke 2013} & $\begin{array}{l}\text { I: metformin } \geq 1500 \text { mg daily + glipizide 5-20 } \\
\mathrm{mg} / \text { day }\end{array}$ & 430 & 2 & 0.5 & 312 & 72.6 & 55 & 12.8 \\
\hline 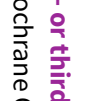 & & $\begin{array}{l}\text { C: metformin } \geq 1500 \text { mg daily + saxagliptin } 5 \\
\text { mg/day }\end{array}$ & 428 & 4 & 0.9 & 287 & 67.1 & 54 & 12.6 \\
\hline 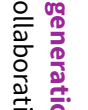 & \multirow[t]{2}{*}{$\begin{array}{l}\text { Maffioli } \\
2013\end{array}$} & $\begin{array}{l}\text { I: metformin } 2550 \mathrm{mg} / \text { day + glibenclamide } 10 \\
\mathrm{mg} / \text { day }\end{array}$ & - & - & - & - & - & - & - \\
\hline 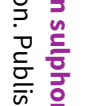 & & $\begin{array}{l}\text { C: metformin } 2550 \mathrm{mg} / \text { day + pioglitazone } 30 \\
\mathrm{mg} / \text { day }\end{array}$ & - & - & - & - & - & - & - \\
\hline 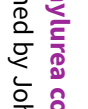 & \multirow[t]{5}{*}{ Nauck 2013} & $\begin{array}{l}\text { I: metformin } 1500-2000 \mathrm{mg} / \text { day + glimepiride } \\
\text { 1-4 mg/day + placebo }\end{array}$ & 242 & 0 & 0 & 128 & 52.9 & 24 & 9.9 \\
\hline 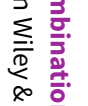 & & $\begin{array}{l}\mathrm{C} 1: \text { metformin } 1500-2000 \mathrm{mg} / \text { day + liraglu- } \\
\text { tide } 0.6 \mathrm{mg} / \text { day + placebo }\end{array}$ & 242 & 2 & 0.8 & 132 & 54.6 & 36 & 14.9 \\
\hline 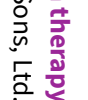 & & $\begin{array}{l}\text { C2: metformin } 1500-2000 \mathrm{mg} / \text { day + liraglu- } \\
\text { tide } 1.2 \mathrm{mg} / \text { day + placebo }\end{array}$ & 240 & 0 & 0 & 144 & 60.0 & 25 & 10.4 \\
\hline$\stackrel{\overrightarrow{0}}{\frac{0}{2}}$ & & $\begin{array}{l}\text { C3: metformin } 1500-2000 \mathrm{mg} / \text { day + liraglu- } \\
\text { tide } 1.8 \mathrm{mg} / \text { day + placebo }\end{array}$ & 242 & 0 & 0 & 158 & 65.3 & 16 & 6.6 \\
\hline 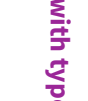 & & $\begin{array}{l}\text { C4: metformin } 1500-2000 \mathrm{mg} / \text { day + placebo + } \\
\text { placebo }\end{array}$ & 121 & 0 & 0 & 44 & 36.4 & 9 & 7.4 \\
\hline 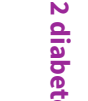 & \multirow[t]{2}{*}{$\begin{array}{l}\text { Gallwitz } \\
2012 a\end{array}$} & $\begin{array}{l}\text { I: metformin median dose } 2000 \mathrm{mg} / \text { day + } \\
\text { glimepiride mean dose } 2.01 \mathrm{mg} / \text { day }\end{array}$ & 508 & 5 & 1.0 & 248 & 48.8 & 68 & 13.4 \\
\hline 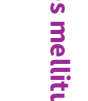 & & $\begin{array}{l}\text { C: metformin median dose } 2000 \mathrm{mg} / \text { day }+ \text { ex- } \\
\text { enatide mean dose } 17.35 \mu \mathrm{g} / \mathrm{day}\end{array}$ & 511 & 5 & 1.0 & 311 & 60.9 & 73 & 14.3 \\
\hline 勇 & \multirow[t]{2}{*}{$\begin{array}{l}\text { Gallwitz } \\
\text { 2012b }\end{array}$} & $\begin{array}{l}\text { I: metformin } \geq 1500 \mathrm{mg} / \text { day + glimepiride } 1-4 \\
\mathrm{mg} / \text { day + placebo }\end{array}$ & 775 & 4 & 1 & 706 & 91 & 162 & 21 \\
\hline & & $\begin{array}{l}\text { C: } \text { metformin } \geq 1500 \mathrm{mg} / \text { day + linagliptin } 5 \\
\mathrm{mg} / \text { day + placebo }\end{array}$ & 776 & 4 & 1 & 663 & 85 & 135 & 17 \\
\hline
\end{tabular}


C: metformin 1000-2000 mg/day + exenatide

$20 \mu \mathrm{g} /$ day

Derosa I: metformin $1700 \pm 850 \mathrm{mg} /$ day + gliben-

2011b clamide $5-15 \mathrm{mg} /$ day

C: metformin $1700 \pm 850 \mathrm{mg} /$ day + pioglita-

zone $15-45 \mathrm{mg} /$ day

Petrica 2011 I: metformin $1700 \mathrm{mg} /$ day + glimepiride 4

$\mathrm{mg} /$ day

C: metformin $1700 \mathrm{mg} /$ day + pioglitazone $30 \quad 34$

$\mathrm{mg} /$ day

Derosa 2010 I: metformin $1500 \pm 500 \mathrm{mg} /$ day + gliben-

clamide $15 \mathrm{mg} /$ day

C: metformin $1500 \pm 500 \mathrm{mg} /$ day + exenatide

$20 \mu \mathrm{g} /$ day

\begin{tabular}{|c|c|c|c|c|c|c|c|c|}
\hline \multirow[t]{2}{*}{$\begin{array}{l}\text { Matthews } \\
2010\end{array}$} & $\begin{array}{l}\text { I: metformin } \geq 1500 \mathrm{mg} \text { twice a day + } \\
\text { glimepiride } 2-6 \mathrm{mg} / \text { day }\end{array}$ & 1546 & 6 & 0.4 & 1335 & 86.4 & 253 & 16.4 \\
\hline & $\begin{array}{l}\text { C: metformin } \geq 1500 \mathrm{mg} \text { twice a day + } \\
\text { vildagliptin } 50 \mathrm{mg} \text { twice a day }\end{array}$ & 1553 & 7 & 0.5 & 1291 & 83.1 & 236 & 15.2 \\
\hline Filozof 2010 & $\begin{array}{l}\text { I: metformin } 1500 \text { mg/day + gliclazide 80-320 } \\
\mathrm{mg} / \text { day }\end{array}$ & 493 & 1 & 0.2 & 302 & 61.3 & 43 & 8.7 \\
\hline \multirow[t]{2}{*}{ Seck 2010} & $\begin{array}{l}\text { I: metformin } \geq 1500 \text { mg/day + glipizide 5-20 } \\
\mathrm{mg} / \text { day }\end{array}$ & 584 & 8 & 1.4 & 480 & 82.2 & 73 & 12.5 \\
\hline & $\begin{array}{l}\text { C: } \text { metformin } \geq 1500 \mathrm{mg} / \text { day + sitagliptin } 100 \\
\mathrm{mg} / \text { day }\end{array}$ & 588 & 1 & 0.2 & 452 & 76.9 & 64 & 10.9 \\
\hline
\end{tabular}

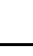




\begin{tabular}{|c|c|c|c|c|c|c|c|c|c|}
\hline 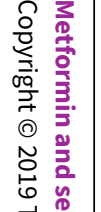 & $\begin{array}{l}\text { (Continued) } \\
\text { Home } 2009\end{array}$ & $\begin{array}{l}\text { I: metformin up to } 2550 \mathrm{mg} / \text { day + gliben- } \\
\text { clamide (or equivalent for different prepara- } \\
\text { tions) up to } 15 \mathrm{mg} / \text { day or gliclazide up to } 240 \\
\mathrm{mg} / \text { day or glimepiride up to } 4 \mathrm{mg} / \text { day }\end{array}$ & 1105 & 67 & 6.1 & 1105 & 100 & 428 & 38.7 \\
\hline 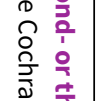 & & $\begin{array}{l}\text { C: metformin up to } 2550 \mathrm{mg} / \text { day + rosiglita- } \\
\text { zone up to } 8 \mathrm{mg} / \text { day }\end{array}$ & 1117 & 57 & 5.1 & 1117 & 100 & 424 & 38.0 \\
\hline 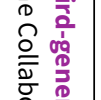 & $\begin{array}{l}\text { Derosa } \\
2009 a\end{array}$ & $\begin{array}{l}\text { I: metformin } 850 \text { mg/day + glimepiride 2-6 } \\
\mathrm{mg} / \text { day }\end{array}$ & - & - & - & - & - & - & - \\
\hline 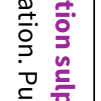 & & $\begin{array}{l}\text { C1: metformin } 850-2550 \mathrm{mg} / \text { day + pioglita- } \\
\text { zone } 15-45 \mathrm{mg} / \text { day }\end{array}$ & - & - & - & - & - & - & - \\
\hline$\frac{\overline{\overline{\vec{m}}}}{\overline{0}} \stackrel{0}{\underline{0}}$ & & C2: metformin $1000-3000 \mathrm{mg} /$ day & - & - & - & - & - & - & - \\
\hline 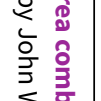 & $\begin{array}{l}\text { Derosa } \\
\text { 2009b }\end{array}$ & $\begin{array}{l}\text { I: metformin } 1500-3000 \mathrm{mg} / \text { day + gliben- } \\
\text { clamide } 7.5-15 \mathrm{mg} / \text { day }\end{array}$ & - & - & - & - & - & - & - \\
\hline 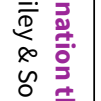 & & $\begin{array}{l}\text { C: metformin } 1500-3000 \mathrm{mg} / \text { day + nateglinide } \\
180-360 \mathrm{mg} / \text { day }\end{array}$ & - & - & - & - & - & - & - \\
\hline 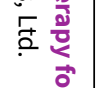 & Petrica 2009 & $\begin{array}{l}\text { I: metformin } 1700 \text { mg/day + glimepiride } 4 \\
\mathrm{mg} / \text { day }\end{array}$ & 22 & - & - & - & - & - & - \\
\hline 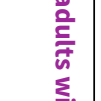 & & $\begin{array}{l}\text { C: metformin } 1700 \mathrm{mg} / \text { day + rosiglitazone } 4 \\
\mathrm{mg} / \text { day }\end{array}$ & 22 & - & - & - & - & - & - \\
\hline 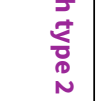 & NCT00367055 & $\begin{array}{l}\text { I: metformin } 2000 \text { mg/day + gliclazide 80-320 } \\
\mathrm{mg} / \text { day }\end{array}$ & 41 & 2 & 4.9 & 33 & 80 & 12 & 29 \\
\hline 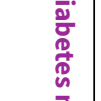 & & $\begin{array}{l}\text { C: metformin } 2000 \mathrm{mg} / \text { day + rosiglitazone 4-8 } \\
\mathrm{mg} / \text { day }\end{array}$ & 43 & 0 & 0 & 35 & 81 & 8 & 19 \\
\hline 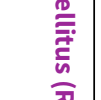 & $\begin{array}{l}\text { Hamann } \\
2008\end{array}$ & $\begin{array}{l}\text { I: metformin } 2000 \mathrm{mg} / \text { day + glibenclamide } \\
5-15 \mathrm{mg} / \text { day or gliclazide } 80-320 \mathrm{mg} / \text { day }\end{array}$ & 301 & 2 & 0.7 & 175 & 58 & 11 & 4 \\
\hline 旁. & & $\begin{array}{l}\text { C: metformin } 2000 \text { mg/day + rosiglitazone 4-8 } \\
\mathrm{mg} / \text { day }\end{array}$ & 294 & 2 & 0.7 & 165 & 56 & 16 & 5 \\
\hline & Ristic 2007 & $\begin{array}{l}\text { I: metformin > } 1000 \mathrm{mg} / \text { day + gliclazide } \\
80-240 \mathrm{mg} / \text { day }\end{array}$ & 101 & 0 & 0 & - & - & - & - \\
\hline
\end{tabular}




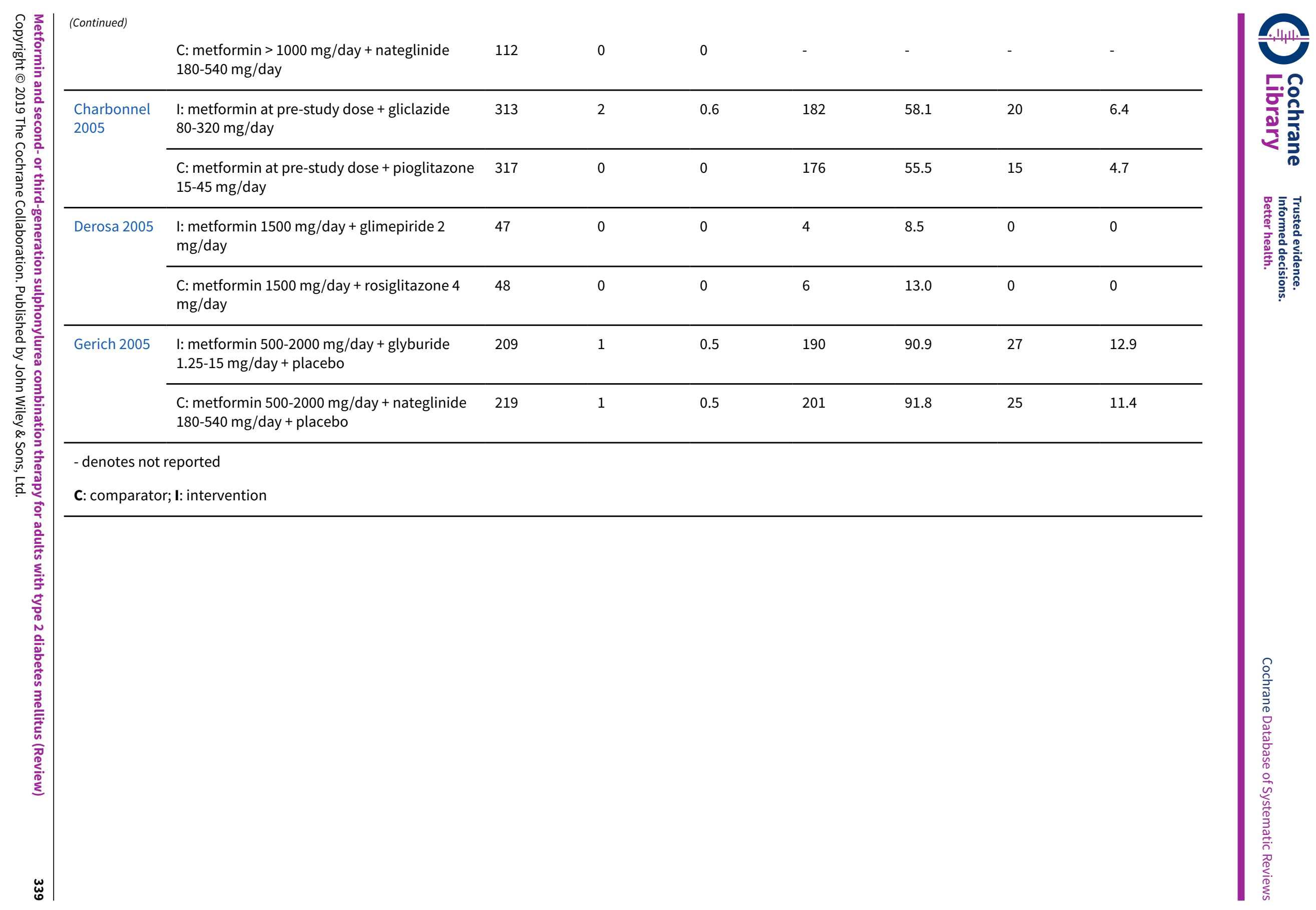




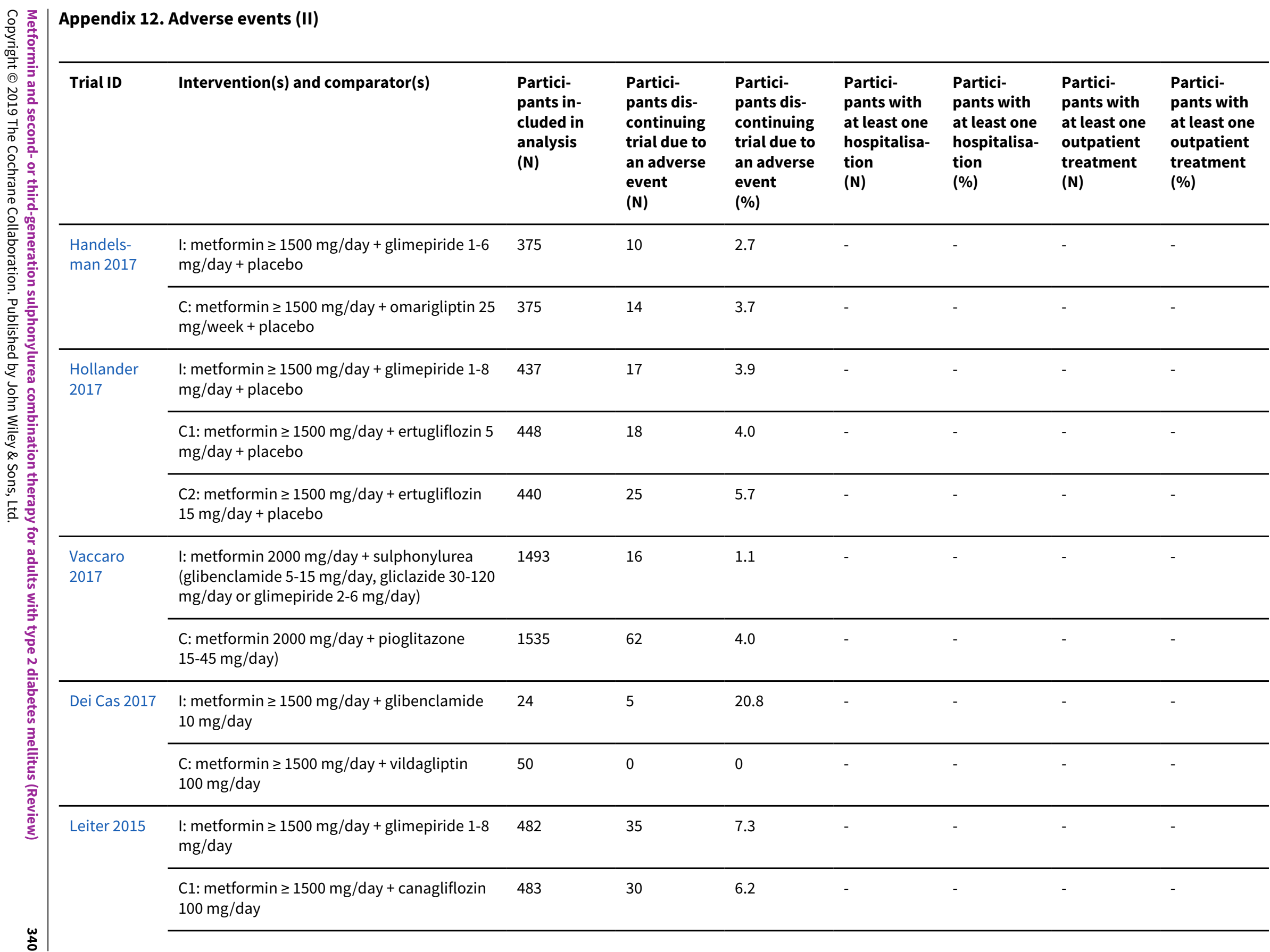




\begin{tabular}{|c|c|c|c|c|c|c|c|c|}
\hline 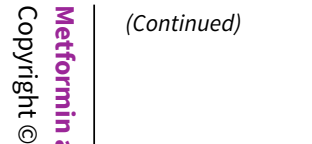 & $\begin{array}{l}\text { C2: metformin } \geq 1500 \mathrm{mg} / \text { day + canagliflozin } \\
300 \mathrm{mg} / \text { day }\end{array}$ & 485 & 46 & 9.5 & - & - & - & - \\
\hline $\begin{array}{l}\text { Del Prato } \\
2015\end{array}$ & $\begin{array}{l}\text { I: metformin } 1500-2500 \mathrm{mg} / \text { day + glipizide } \\
5-20 \mathrm{mg} / \text { day }\end{array}$ & 408 & 46 & 11.3 & - & - & - & - \\
\hline 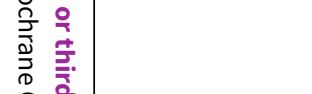 & $\begin{array}{l}\text { C: metformin } 1500-2500 \mathrm{mg} / \text { day + da- } \\
\text { pagliflozin } 2.5-10 \mathrm{mg} / \text { day }\end{array}$ & 406 & 54 & 13.3 & - & - & - & - \\
\hline $\begin{array}{l}\text { Schern- } \\
\text { thaner } 2015\end{array}$ & $\begin{array}{l}\text { I: metformin at any dose + glimepiride 1-6 } \\
\mathrm{mg} / \text { day + placebo }\end{array}$ & 359 & 11 & 3.1 & - & - & - & - \\
\hline 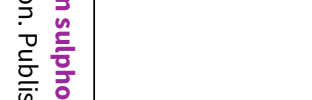 & $\begin{array}{l}\text { C: metformin at any dose + saxagliptin } 5 \mathrm{mg} / \\
\text { day + placebo }\end{array}$ & 359 & 16 & 4.5 & - & - & - & - \\
\hline $\begin{array}{l}\text { Del Prato } \\
2014\end{array}$ & $\begin{array}{l}\text { I: metformin } \geq 1500 \text { mg once daily or maxi- } \\
\text { mum tolerated dose + glipizide 5-20 mg once } \\
\text { daily }\end{array}$ & 869 & 82 & 9.4 & - & - & - & - \\
\hline 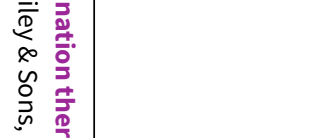 & $\begin{array}{l}\mathrm{C} 1 \text { : metformin } \geq 1500 \mathrm{mg} \text { once daily or maxi- } \\
\text { mum tolerated dose + alogliptin } 12.5 \mathrm{mg} \text { once } \\
\text { daily }\end{array}$ & 873 & 60 & 6.8 & - & - & - & - \\
\hline$\frac{\overrightarrow{0}}{\stackrel{0}{\circ}}$ & $\begin{array}{l}\text { C2: metformin } \geq 1500 \mathrm{mg} \text { once daily or maxi- } \\
\text { mum tolerated dose + alogliptin } 25 \mathrm{mg} \text { once } \\
\text { daily }\end{array}$ & 878 & 74 & 8.4 & - & - & - & - \\
\hline Ahrén 2014 & $\begin{array}{l}\text { I: metformin } \geq 1500 \mathrm{mg} \text { daily + glimepiride } 2-4 \\
\text { mg once daily + placebo + placebo }\end{array}$ & 307 & 17 & 5.5 & - & - & - & - \\
\hline 을 & $\begin{array}{l}\text { C1: metformin } \geq 1500 \mathrm{mg} \text { daily + albiglutide } \\
30-50 \mathrm{mg} \text { once weekly + placebo + placebo }\end{array}$ & 302 & 25 & 8.3 & - & - & - & - \\
\hline 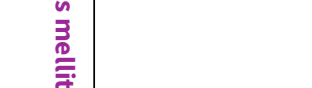 & $\begin{array}{l}\text { C2: metformin } \geq 1500 \text { mg daily + sitagliptin } \\
100 \mathrm{mg} \text { once daily + placebo + placebo }\end{array}$ & 302 & 13 & 4.3 & - & - & - & - \\
\hline 勇. & $\begin{array}{l}\text { C3: metformin } \geq 1500 \mathrm{mg} \text { daily + placebo + } \\
\text { placebo }\end{array}$ & 101 & 5 & 5.0 & - & - & - & - \\
\hline $\begin{array}{l}\text { Ridderstråle } \\
2014\end{array}$ & $\begin{array}{l}\text { I: metformin immediate release } \geq 1500 \mathrm{mg} / \\
\text { day + glimepiride } 1-4 \mathrm{mg} / \text { day }\end{array}$ & 780 & 51 & 6.5 & - & - & - & - \\
\hline
\end{tabular}




\begin{tabular}{|c|c|c|c|c|c|c|c|c|c|}
\hline 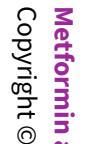 & (Continued) & $\begin{array}{l}\text { C: metformin immediate release } \geq 1500 \mathrm{mg} / \\
\text { day + empagliflozin } 25 \mathrm{mg} / \text { day }\end{array}$ & 765 & 47 & 6.1 & - & - & - & - \\
\hline 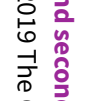 & Göke 2013 & $\begin{array}{l}\text { I: metformin } \geq 1500 \text { mg daily + glipizide 5-20 } \\
\mathrm{mg} / \text { day }\end{array}$ & 430 & 24 & 5.6 & - & - & - & - \\
\hline 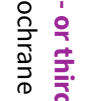 & & $\begin{array}{l}\text { C: metformin } \geq 1500 \text { mg daily + saxagliptin } 5 \\
\text { mg/day }\end{array}$ & 428 & 21 & 4.9 & - & - & - & - \\
\hline 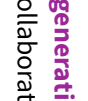 & $\begin{array}{l}\text { Maffioli } \\
2013\end{array}$ & $\begin{array}{l}\text { I: metformin } 2550 \mathrm{mg} / \text { day + glibenclamide } 10 \\
\mathrm{mg} / \text { day }\end{array}$ & 84 & 4 & 4.8 & - & - & - & - \\
\hline 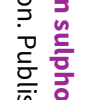 & & $\begin{array}{l}\text { C: metformin } 2550 \mathrm{mg} / \text { day + pioglitazone } 30 \\
\mathrm{mg} / \text { day }\end{array}$ & 86 & 4 & 4.7 & - & - & - & - \\
\hline 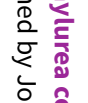 & Nauck 2013 & $\begin{array}{l}\text { I: metformin } 1500-2000 \mathrm{mg} / \text { day + glimepiride } \\
\text { 1-4 mg/day + placebo }\end{array}$ & 244 & 14 & 5.7 & - & - & - & - \\
\hline 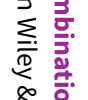 & & $\begin{array}{l}\mathrm{C} 1 \text { : metformin } 1500-2000 \mathrm{mg} / \text { day + liraglu- } \\
\text { tide } 0.6 \mathrm{mg} / \text { day + placebo }\end{array}$ & 242 & 22 & 9.1 & - & - & - & - \\
\hline 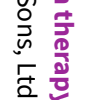 & & $\begin{array}{l}\text { C2: metformin } 1500-2000 \mathrm{mg} / \text { day + liraglu- } \\
\text { tide } 1.2 \mathrm{mg} / \text { day + placebo }\end{array}$ & 241 & 31 & 12.9 & - & - & - & - \\
\hline $\begin{array}{l}\stackrel{0}{\frac{0}{7}} \\
\stackrel{2}{\frac{2}{\sigma}}\end{array}$ & & $\begin{array}{l}\text { C3: metformin } 1500-2000 \mathrm{mg} / \text { day + liraglu- } \\
\text { tide } 1.8 \mathrm{mg} / \text { day + placebo }\end{array}$ & 242 & 35 & 14.5 & - & - & - & - \\
\hline 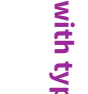 & & $\begin{array}{l}\text { C4: metformin } 1500-2000 \mathrm{mg} / \text { day + placebo + } \\
\text { placebo }\end{array}$ & 122 & 3 & 2.5 & - & - & - & - \\
\hline 高 & $\begin{array}{l}\text { Gallwitz } \\
\text { 2012a }\end{array}$ & $\begin{array}{l}\text { I: metformin median dose } 2000 \mathrm{mg} / \text { day + } \\
\text { glimepiride mean dose } 2.01 \mathrm{mg} / \text { day }\end{array}$ & 508 & 17 & 3.3 & - & - & - & - \\
\hline 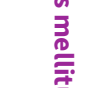 & & $\begin{array}{l}\text { C: metformin median dose } 2000 \mathrm{mg} / \text { day + ex- } \\
\text { enatide mean dose } 17.35 \mu \mathrm{g} / \text { day }\end{array}$ & 511 & 49 & 9.6 & - & - & - & - \\
\hline 盆 & $\begin{array}{l}\text { Gallwitz } \\
\text { 2012b }\end{array}$ & $\begin{array}{l}\text { I: metformin } \geq 1500 \mathrm{mg} / \text { day + glimepiride } 1-4 \\
\mathrm{mg} / \text { day + placebo }\end{array}$ & 775 & 85 & 11 & - & - & - & - \\
\hline & & $\begin{array}{l}\text { C: } \text { metformin } \geq 1500 \mathrm{mg} / \text { day + linagliptin } 5 \\
\mathrm{mg} / \text { day + placebo }\end{array}$ & 776 & 60 & 8 & - & - & - & - \\
\hline
\end{tabular}


C: metformin 1000-2000 mg/day + exenatide

57

4

$20 \mu \mathrm{g} / \mathrm{day}$

Derosa I: metformin $1700 \pm 850 \mathrm{mg} /$ day + gliben- $\quad 99 \quad 3$

2011b

clamide $5-15 \mathrm{mg} /$ day

\begin{tabular}{llll}
\hline C: metformin $1700 \pm 850 \mathrm{mg} /$ day + pioglita- & 102 & 2 & 2.9
\end{tabular}

zone $15-45 \mathrm{mg}$ /day

\begin{tabular}{|c|c|c|c|c|c|c|c|c|}
\hline \multirow[t]{2}{*}{ Petrica 2011} & $\begin{array}{l}\text { I: metformin } 1700 \text { mg/day + glimepiride } 4 \\
\text { mg/day }\end{array}$ & 34 & 4 & 11.8 & - & - & - & - \\
\hline & $\begin{array}{l}\text { C: metformin } 1700 \mathrm{mg} / \text { day + pioglitazone } 30 \\
\mathrm{mg} / \text { day }\end{array}$ & 34 & 3 & 8.8 & - & - & - & - \\
\hline \multirow[t]{2}{*}{ Derosa 2010} & $\begin{array}{l}\text { I: metformin } 1500 \pm 500 \mathrm{mg} / \text { day + gliben- } \\
\text { clamide } 15 \mathrm{mg} / \text { day }\end{array}$ & 65 & 7 & 10.8 & - & - & - & - \\
\hline & $\begin{array}{l}\text { C: metformin } 1500 \pm 500 \mathrm{mg} / \text { day + exenatide } \\
20 \mu \mathrm{g} / \text { day }\end{array}$ & 63 & 4 & 6.3 & - & - & - & - \\
\hline \multirow[t]{2}{*}{$\begin{array}{l}\text { Matthews } \\
2010\end{array}$} & $\begin{array}{l}\text { I: metformin } \geq 1500 \mathrm{mg} \text { twice a day + } \\
\text { glimepiride } 2-6 \mathrm{mg} / \text { day }\end{array}$ & 1546 & 166 & 10.7 & - & - & - & - \\
\hline & $\begin{array}{l}\text { C: metformin } \geq 1500 \mathrm{mg} \text { twice a day + } \\
\text { vildagliptin } 50 \mathrm{mg} \text { twice a day }\end{array}$ & 1553 & 130 & 8.4 & - & - & - & - \\
\hline \multirow[t]{2}{*}{ Filozof 2010} & $\begin{array}{l}\text { I: metformin } 1500 \text { mg/day + gliclazide 80-320 } \\
\mathrm{mg} / \text { day }\end{array}$ & - & 22 & 4.7 & - & - & - & - \\
\hline & $\begin{array}{l}\text { C: metformin } 1500 \text { mg/day + vildagliptin } 100 \\
\text { mg/day }\end{array}$ & - & 33 & 6.7 & - & - & - & - \\
\hline \multirow[t]{2}{*}{ Seck 2010} & $\begin{array}{l}\text { I: metformin } \geq 1500 \mathrm{mg} / \text { day + glipizide } 5-20 \\
\mathrm{mg} / \text { day }\end{array}$ & 584 & 36 & 6.2 & - & - & - & - \\
\hline & $\begin{array}{l}\text { C: metformin } \geq 1500 \mathrm{mg} / \text { day + sitagliptin } 100 \\
\mathrm{mg} / \text { day }\end{array}$ & 588 & 35 & 6.0 & - & - & - & - \\
\hline
\end{tabular}




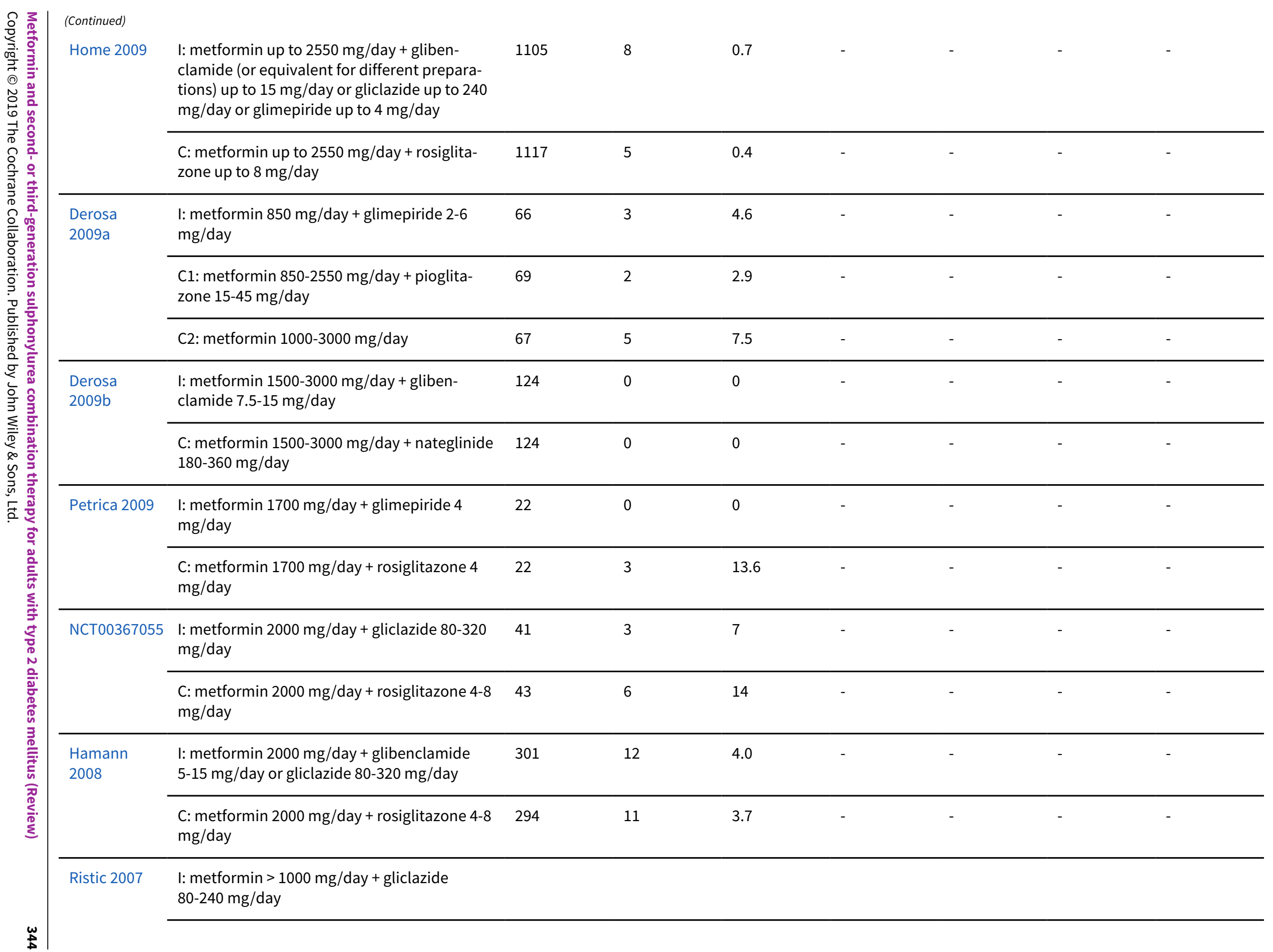




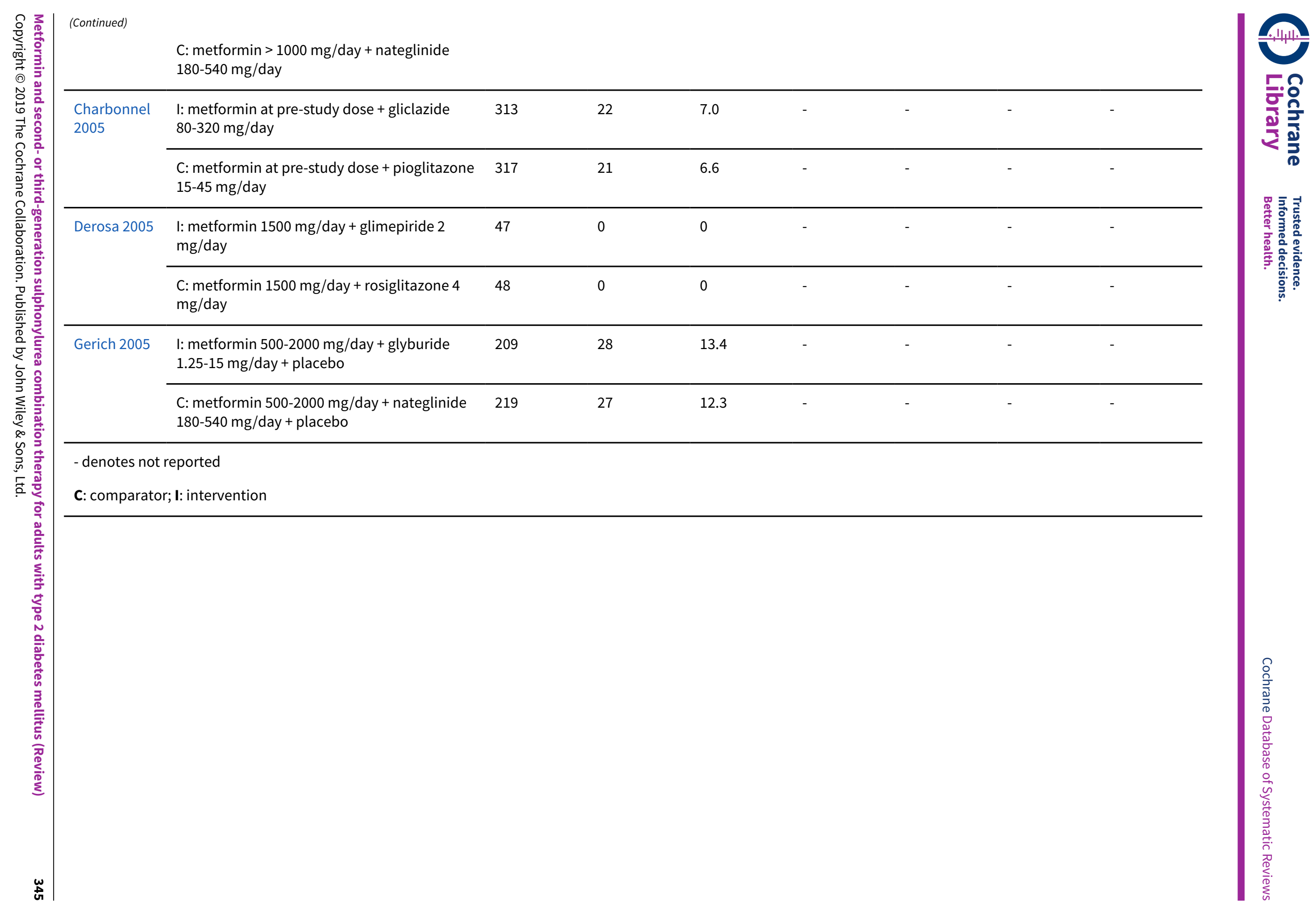




\section{Appendix 13. Adverse events (III)}

\begin{tabular}{|c|c|c|c|c|c|}
\hline Trial ID & Intervention(s) and comparator(s) & $\begin{array}{l}\text { Participants } \\
\text { included in } \\
\text { analysis } \\
\text { (N) }\end{array}$ & $\begin{array}{l}\text { Participants } \\
\text { with a spe- } \\
\text { cific adverse } \\
\text { event } \\
\text { (description) }\end{array}$ & $\begin{array}{l}\text { Participants } \\
\text { with at least } \\
\text { one specif- } \\
\text { ic adverse } \\
\text { event } \\
\text { (N) }\end{array}$ & $\begin{array}{l}\text { Participants } \\
\text { with at least } \\
\text { one specif- } \\
\text { ic adverse } \\
\text { event } \\
(\%)\end{array}$ \\
\hline
\end{tabular}

\begin{tabular}{|c|c|c|c|c|c|}
\hline \multirow[t]{2}{*}{$\begin{array}{l}\text { Handelsman } \\
2017\end{array}$} & $\begin{array}{l}\text { I: } \text { metformin } \geq 1500 \mathrm{mg} / \text { day + glimepiride } \\
1-6 \mathrm{mg} / \text { day + placebo }\end{array}$ & \multicolumn{4}{|l|}{375} \\
\hline & $\begin{array}{l}\text { C: } \text { metformin } \geq 1500 \mathrm{mg} / \text { day + omarigliptin } \\
25 \mathrm{mg} / \text { week + placebo }\end{array}$ & 375 & & & \\
\hline \multirow[t]{3}{*}{$\begin{array}{l}\text { Hollander } \\
2017\end{array}$} & $\begin{array}{l}\text { I: metformin } \geq 1500 \mathrm{mg} / \text { day + glimepiride } \\
1-8 \mathrm{mg} / \text { day + placebo }\end{array}$ & 437 & & & \\
\hline & $\begin{array}{l}\mathrm{C} 1: \text { metformin } \geq 1500 \mathrm{mg} / \text { day + ertugliflozin } \\
5 \mathrm{mg} / \text { day + placebo }\end{array}$ & 448 & & & \\
\hline & $\begin{array}{l}\text { C2: metformin } \geq 1500 \mathrm{mg} / \text { day + ertugliflozin } \\
15 \mathrm{mg} / \text { day + placebo }\end{array}$ & 440 & & & \\
\hline \multirow[t]{2}{*}{ Vaccaro 2017} & $\begin{array}{l}\text { I: metformin } 2000 \mathrm{mg} / \text { day + sulphonylurea } \\
\text { (glibenclamide } 5-15 \mathrm{mg} / \text { day, gliclazide } \\
30-120 \mathrm{mg} / \text { day or glimepiride } 2-6 \mathrm{mg} / \text { day) }\end{array}$ & 1493 & - & - & - \\
\hline & $\begin{array}{l}\text { C: metformin } 2000 \mathrm{mg} / \text { day + pioglitazone } \\
15-45 \mathrm{mg} / \text { day) }\end{array}$ & 1535 & - & - & - \\
\hline \multirow[t]{2}{*}{ Dei Cas 2017} & $\begin{array}{l}\text { I: metformin } \geq 1500 \mathrm{mg} / \text { day + glibenclamide } \\
10 \mathrm{mg} / \text { day }\end{array}$ & 24 & - & - & - \\
\hline & $\begin{array}{l}\text { C: } \text { metformin } \geq 1500 \mathrm{mg} / \text { day + vildagliptin } \\
100 \mathrm{mg} / \text { day }\end{array}$ & 40 & - & - & - \\
\hline \multirow[t]{3}{*}{ Leiter 2015} & $\begin{array}{l}\text { I: metformin } \geq 1500 \mathrm{mg} / \text { day + glimepiride } \\
1-8 \mathrm{mg} / \text { day }\end{array}$ & 482 & & & \\
\hline & $\begin{array}{l}\mathrm{C} 1: \text { metformin } \geq 1500 \mathrm{mg} / \text { day + canagliflozin } \\
100 \mathrm{mg} / \text { day }\end{array}$ & 483 & & & \\
\hline & $\begin{array}{l}\text { C2: metformin } \geq 1500 \mathrm{mg} / \text { day + canagliflozin } \\
300 \mathrm{mg} / \text { day }\end{array}$ & 485 & & & \\
\hline \multirow[t]{2}{*}{ Del Prato 2015} & $\begin{array}{l}\text { I: metformin } 1500-2500 \mathrm{mg} / \text { day + glipizide } \\
\text { 5-20 mg/day }\end{array}$ & 408 & - & - & - \\
\hline & $\begin{array}{l}\text { C: metformin } 1500-2500 \mathrm{mg} / \text { day + da- } \\
\text { pagliflozin } 2.5-10 \mathrm{mg} / \text { day }\end{array}$ & 406 & - & - & - \\
\hline \multirow[t]{2}{*}{$\begin{array}{l}\text { Schernthaner } \\
2015\end{array}$} & $\begin{array}{l}\text { I: metformin at any dose + glimepiride } 1-6 \\
\mathrm{mg} / \text { day + placebo }\end{array}$ & 359 & & & \\
\hline & $\begin{array}{l}\text { C: metformin at any dose + saxagliptin } 5 \mathrm{mg} / \\
\text { day + placebo }\end{array}$ & 359 & & & \\
\hline
\end{tabular}


(Continued)

\begin{tabular}{|c|c|c|c|c|c|}
\hline \multirow[t]{3}{*}{ Del Prato 2014} & $\begin{array}{l}\text { I: metformin } \geq 1500 \mathrm{mg} \text { once daily or max- } \\
\text { imum tolerated dose + glipizide } 5-20 \mathrm{mg} \\
\text { once daily }\end{array}$ & 869 & - & - & - \\
\hline & $\begin{array}{l}\text { C1: metformin } \geq 1500 \text { mg once daily or max- } \\
\text { imum tolerated dose + alogliptin } 12.5 \mathrm{mg} \\
\text { once daily }\end{array}$ & 873 & - & - & - \\
\hline & $\begin{array}{l}\text { C2: metformin } \geq 1500 \text { mg once daily or maxi- } \\
\text { mum tolerated dose + alogliptin } 25 \text { mg once } \\
\text { daily }\end{array}$ & 878 & - & - & - \\
\hline \multirow[t]{3}{*}{ Ahrén 2014} & $\begin{array}{l}\text { I: metformin } \geq 1500 \text { mg daily + glimepiride } \\
2-4 \text { mg once daily + placebo + placebo }\end{array}$ & 307 & $\begin{array}{l}\text { (1) injection } \\
\text { site reaction } \\
\text { (2) upper res- } \\
\text { piratory tract } \\
\text { infection }\end{array}$ & $\begin{array}{l}\text { (1) } 9 \\
\text { (2) } 32\end{array}$ & $\begin{array}{l}\text { (1) } 2.9 \\
\text { (2) } 10.4\end{array}$ \\
\hline & $\begin{array}{l}\text { C1: metformin } \geq 1500 \text { mg daily + albiglutide } \\
30-50 \text { mg once weekly + placebo }+ \text { placebo }\end{array}$ & 302 & $\begin{array}{l}\text { (1) injection } \\
\text { site reaction } \\
\text { (2) upper res- } \\
\text { piratory tract } \\
\text { infection }\end{array}$ & $\begin{array}{l}\text { (1) } 33 \\
\text { (2) } 58\end{array}$ & $\begin{array}{l}\text { (1) } 10.9 \\
\text { (2) } 19.2\end{array}$ \\
\hline & $\begin{array}{l}\text { C2: metformin } \geq 1500 \text { mg daily + sitagliptin } \\
100 \mathrm{mg} \text { once daily + placebo + placebo }\end{array}$ & 302 & $\begin{array}{l}\text { (1) injection } \\
\text { site reaction } \\
\text { (2) upper res- } \\
\text { piratory tract } \\
\text { infection }\end{array}$ & $\begin{array}{l}\text { (1) } 5 \\
\text { (2) } 33\end{array}$ & $\begin{array}{l}\text { (1) } 1.7 \\
\text { (2) } 10.9\end{array}$ \\
\hline
\end{tabular}

Ridderstråle I: metformin immediate release $\geq 1500 \mathrm{mg} / \quad 780$

2014 day + glimepiride $1-4 \mathrm{mg} /$ day

C: metformin immediate release $\geq 1500 \mathrm{mg} / \quad 765$

day + empagliflozin $25 \mathrm{mg} /$ day

\begin{tabular}{|c|c|}
\hline Göke 2013 & $\begin{array}{l}\text { I: metformin } \geq 1500 \text { mg daily + glipizide 5-20 } \\
\mathrm{mg} / \text { day }\end{array}$ \\
\hline
\end{tabular}

C: metformin $\geq 1500 \mathrm{mg}$ daily + saxagliptin $5 \quad 428$

$\mathrm{mg} /$ day

Maffioli $2013 \quad$ I: metformin $2550 \mathrm{mg} /$ day + glibenclamide

$10 \mathrm{mg} /$ day

C: metformin $2550 \mathrm{mg} /$ day + pioglitazone 30

$\mathrm{mg} /$ day

\begin{tabular}{llllll}
\hline Nauck 2013 & I: metformin $1500-2000 \mathrm{mg} /$ day + glimepiri- & 242 & (1) Nausea & (1) 10 & (1) 4.1 \\
& de $1-4 \mathrm{mg} /$ day + placebo & & (2) Diarhea & (2) 14 & (2) 5.8 \\
& & & (3) Vomiting & (3) 1 & (3) 0.4
\end{tabular}


(Continued)

\begin{tabular}{|c|c|c|c|c|}
\hline $\begin{array}{l}\mathrm{C} 1: \text { metformin } 1500-2000 \mathrm{mg} / \text { day + liraglu- } \\
\text { tide } 0.6 \mathrm{mg} / \text { day + placebo }\end{array}$ & 242 & $\begin{array}{l}\text { (1) Nausea } \\
\text { (2) Diarhea } \\
\text { (3) Vomiting }\end{array}$ & $\begin{array}{l}\text { (1) } 30 \\
\text { (2) } 31 \\
\text { (3) } 19\end{array}$ & $\begin{array}{l}\text { (1) } 12.4 \\
\text { (2) } 12.8 \\
\text { (3) } 7.9\end{array}$ \\
\hline
\end{tabular}

\begin{tabular}{lllll}
\hline C2: metformin $1500-2000 \mathrm{mg} /$ day + liraglu- & \multirow{2}{*}{240} & (1) Nausea & (1) 42 & (1) 17.5 \\
tide $1.2 \mathrm{mg} /$ day + placebo & & (2) Diarhea & (2) 27 & (2) 11.3 \\
& (3) Vomiting & (3) 18 & (3) 7.5
\end{tabular}

\begin{tabular}{|c|c|c|c|c|}
\hline $\begin{array}{l}\text { C3: metformin } 1500-2000 \mathrm{mg} / \text { day + liraglu- } \\
\text { tide } 1.8 \mathrm{mg} / \text { day + placebo }\end{array}$ & 242 & $\begin{array}{l}\text { (1) Nausea } \\
\text { (2) Diarhea } \\
\text { (3) Vomiting }\end{array}$ & $\begin{array}{l}\text { (1) } 52 \\
\text { (2) } 40 \\
\text { (3) } 24\end{array}$ & $\begin{array}{l}\text { (1) } 21.5 \\
\text { (2) } 15.5 \\
\text { (3) } 9.9\end{array}$ \\
\hline
\end{tabular}

\begin{tabular}{lllll}
\hline C4: metformin $1500-2000 \mathrm{mg} /$ day + placebo & 121 & (1) Nausea & (1) 5 & (1) 4.1 \\
+ placebo & & (2) Diarhea & (2) 5 & (2) 4.1 \\
& & (3) Vomiting & (3) 0 & (3) 0.0
\end{tabular}

\begin{tabular}{|c|c|c|c|c|c|}
\hline \multirow[t]{2}{*}{ Gallwitz 2012b } & $\begin{array}{l}\text { I: metformin median dose } 2000 \mathrm{mg} / \text { day + } \\
\text { glimepiride mean dose } 2.01 \mathrm{mg} / \mathrm{day}\end{array}$ & 775 & & & \\
\hline & $\begin{array}{l}\text { C: metformin median dose } 2000 \mathrm{mg} / \text { day + } \\
\text { exenatide mean dose } 17.35 \mu \mathrm{g} / \mathrm{day}\end{array}$ & 776 & & & \\
\hline \multirow[t]{2}{*}{ Gallwitz 2012a } & $\begin{array}{l}\text { I: } \text { metformin } \geq 1500 \mathrm{mg} / \text { day + glimepiride } \\
1-4 \mathrm{mg} / \text { day + placebo }\end{array}$ & 508 & Nausea & 11 & 2 \\
\hline & $\begin{array}{l}\text { C: metformin } \geq 1500 \mathrm{mg} / \text { day + linagliptin } 5 \\
\mathrm{mg} / \text { day + placebo }\end{array}$ & 511 & Nausea & 147 & 29 \\
\hline
\end{tabular}

\begin{tabular}{ll}
\hline Derosa 2011a & $\begin{array}{l}\text { I: metformin } 1000-2000 \mathrm{mg} / \text { day + glimepiri- } \\
\text { de } 6 \mathrm{mg} / \text { day }\end{array}$ \\
\cline { 2 - 2 } & $\begin{array}{l}\text { C: } \text { metformin } 1000-2000 \mathrm{mg} / \text { day + exenatide } \\
20 \mu \mathrm{g} / \text { day }\end{array}$ \\
\hline Derosa 2011b & $\begin{array}{l}\text { I: metformin } 1700 \pm 850 \mathrm{mg} / \text { day + gliben- } \\
\text { clamide } 5-15 \mathrm{mg} / \mathrm{day}\end{array}$ \\
\hline
\end{tabular}

C: metformin $1700 \pm 850 \mathrm{mg} /$ day + pioglita-

zone $15-45 \mathrm{mg} /$ day

\begin{tabular}{|c|c|c|c|c|c|}
\hline \multirow[t]{2}{*}{ Petrica 2011} & $\begin{array}{l}\text { I: metformin } 1700 \text { mg/day + glimepiride } 4 \\
\text { mg/day }\end{array}$ & 34 & & & \\
\hline & $\begin{array}{l}\text { C: metformin } 1700 \text { mg/day + pioglitazone } 30 \\
\text { mg/day }\end{array}$ & 34 & & & \\
\hline \multirow[t]{2}{*}{ Derosa 2010} & $\begin{array}{l}\text { I: metformin } 1500 \pm 500 \mathrm{mg} / \text { day + gliben- } \\
\text { clamide } 15 \mathrm{mg} / \text { day }\end{array}$ & - & & & \\
\hline & $\begin{array}{l}\text { C: metformin } 1500 \pm 500 \mathrm{mg} / \text { day + exenatide } \\
20 \mu \mathrm{g} / \text { day }\end{array}$ & - & & & \\
\hline \multirow[t]{2}{*}{$\begin{array}{l}\text { Matthews } \\
2010\end{array}$} & $\begin{array}{l}\text { I: metformin } \geq 1500 \mathrm{mg} \text { twice a day + } \\
\text { glimepiride } 2-6 \mathrm{mg} / \text { day }\end{array}$ & 1546 & Dizziness & 247 & 16.0 \\
\hline & $\begin{array}{l}\text { C: metformin } \geq 1500 \mathrm{mg} \text { twice a day + } \\
\text { vildagliptin } 50 \mathrm{mg} \text { twice a day }\end{array}$ & 1553 & Dizziness & 128 & 8.2 \\
\hline
\end{tabular}


(Continued)

\begin{tabular}{|c|c|c|c|c|c|}
\hline \multirow[t]{2}{*}{ Filozof 2010} & $\begin{array}{l}\text { I: metformin } 1500 \mathrm{mg} / \text { day + gliclazide } \\
80-320 \mathrm{mg} / \text { day }\end{array}$ & \multicolumn{4}{|l|}{493} \\
\hline & $\begin{array}{l}\text { C: metformin } 1500 \text { mg/day + vildagliptin } 100 \\
\text { mg/day }\end{array}$ & 510 & & & \\
\hline \multirow[t]{2}{*}{ Seck 2010} & $\begin{array}{l}\text { I: metformin } \geq 1500 \text { mg/day + glipizide 5-20 } \\
\text { mg/day }\end{array}$ & 584 & & & \\
\hline & $\begin{array}{l}\text { C: metformin } \geq 1500 \mathrm{mg} / \text { day + sitagliptin } 100 \\
\text { mg/day }\end{array}$ & 588 & & & \\
\hline \multirow[t]{2}{*}{ Home 2009} & $\begin{array}{l}\text { I: metformin up to } 2550 \mathrm{mg} / \text { day + gliben- } \\
\text { clamide (or equivalent for different prepa- } \\
\text { rations) up to } 15 \mathrm{mg} / \text { day or gliclazide up to } \\
240 \mathrm{mg} / \text { day or glimepiride up to } 4 \mathrm{mg} / \text { day }\end{array}$ & 1105 & - & - & - \\
\hline & $\begin{array}{l}\text { C: metformin up to } 2550 \mathrm{mg} / \mathrm{day}+\text { rosiglita- } \\
\text { zone up to } 8 \mathrm{mg} / \mathrm{day}\end{array}$ & 1117 & - & - & - \\
\hline Derosa 2009a & $\begin{array}{l}\text { I: metformin } 850 \text { mg/day + glimepiride 2-6 } \\
\text { mg/day }\end{array}$ & - & & & \\
\hline
\end{tabular}

C1: metformin $850-2550 \mathrm{mg} /$ day + pioglita-

zone $15-45 \mathrm{mg} /$ day

C2: metformin 1000-3000 mg/day

Derosa 2009b I: metformin 1500-3000 mg/day + gliben-

clamide $7.5-15 \mathrm{mg} /$ day

C: metformin 1500-3000 mg/day + nateglin-

ide $180-360 \mathrm{mg} /$ day

\begin{tabular}{|c|c|c|c|c|c|}
\hline \multirow[t]{2}{*}{ Petrica 2009} & $\begin{array}{l}\text { I: metformin } 1700 \text { mg/day + glimepiride } 4 \\
\text { mg/day }\end{array}$ & 22 & & & \\
\hline & $\begin{array}{l}\text { C: metformin } 1700 \mathrm{mg} / \text { day + rosiglitazone } 4 \\
\mathrm{mg} / \text { day }\end{array}$ & 22 & & & \\
\hline \multirow[t]{2}{*}{ NCT00367055 } & $\begin{array}{l}\text { I: metformin } 2000 \mathrm{mg} / \text { day + gliclazide } \\
80-320 \mathrm{mg} / \text { day }\end{array}$ & 41 & Bronchitis & 1 & 2 \\
\hline & $\begin{array}{l}\text { C: metformin } 2000 \mathrm{mg} / \text { day + rosiglitazone } \\
4-8 \mathrm{mg} / \text { day }\end{array}$ & 43 & Bronchitis & 8 & 19 \\
\hline \multirow[t]{2}{*}{ Hamann 2008} & $\begin{array}{l}\text { I: metformin } 2000 \mathrm{mg} / \text { day + glibenclamide } \\
\text { 5-15 mg/day or gliclazide } 80-320 \mathrm{mg} / \text { day }\end{array}$ & 301 & & & \\
\hline & $\begin{array}{l}\text { C: metformin } 2000 \mathrm{mg} / \text { day + rosiglitazone } \\
4-8 \mathrm{mg} / \text { day }\end{array}$ & 294 & & & \\
\hline \multirow[t]{2}{*}{ Ristic 2007} & $\begin{array}{l}\text { I: } \text { metformin > } 1000 \mathrm{mg} / \text { day + gliclazide } \\
80-240 \mathrm{mg} / \text { day }\end{array}$ & & & & \\
\hline & $\begin{array}{l}\text { C: } \text { metformin > } 1000 \mathrm{mg} / \text { day + nateglinide } \\
180-540 \mathrm{mg} / \text { day }\end{array}$ & & & & \\
\hline
\end{tabular}




\section{(Continued)}

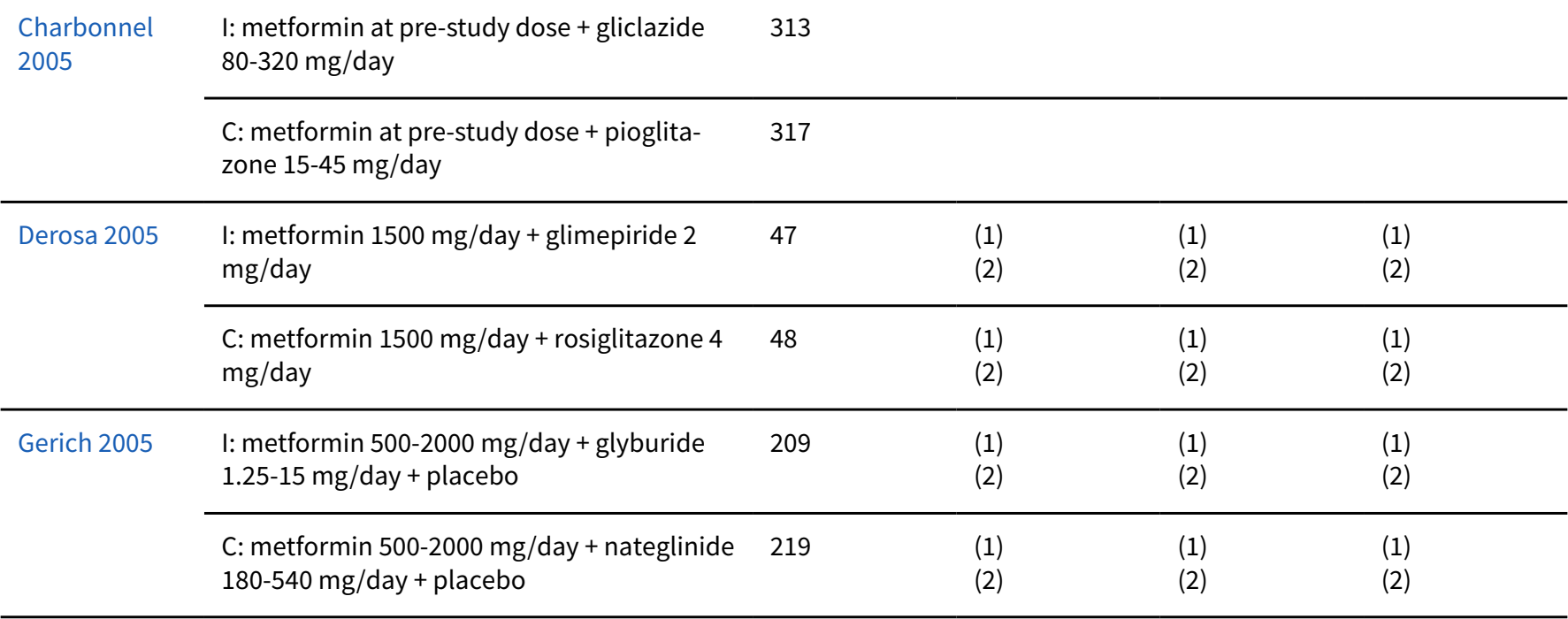

\footnotetext{
- denotes not reported
}

C: comparator; I: intervention 


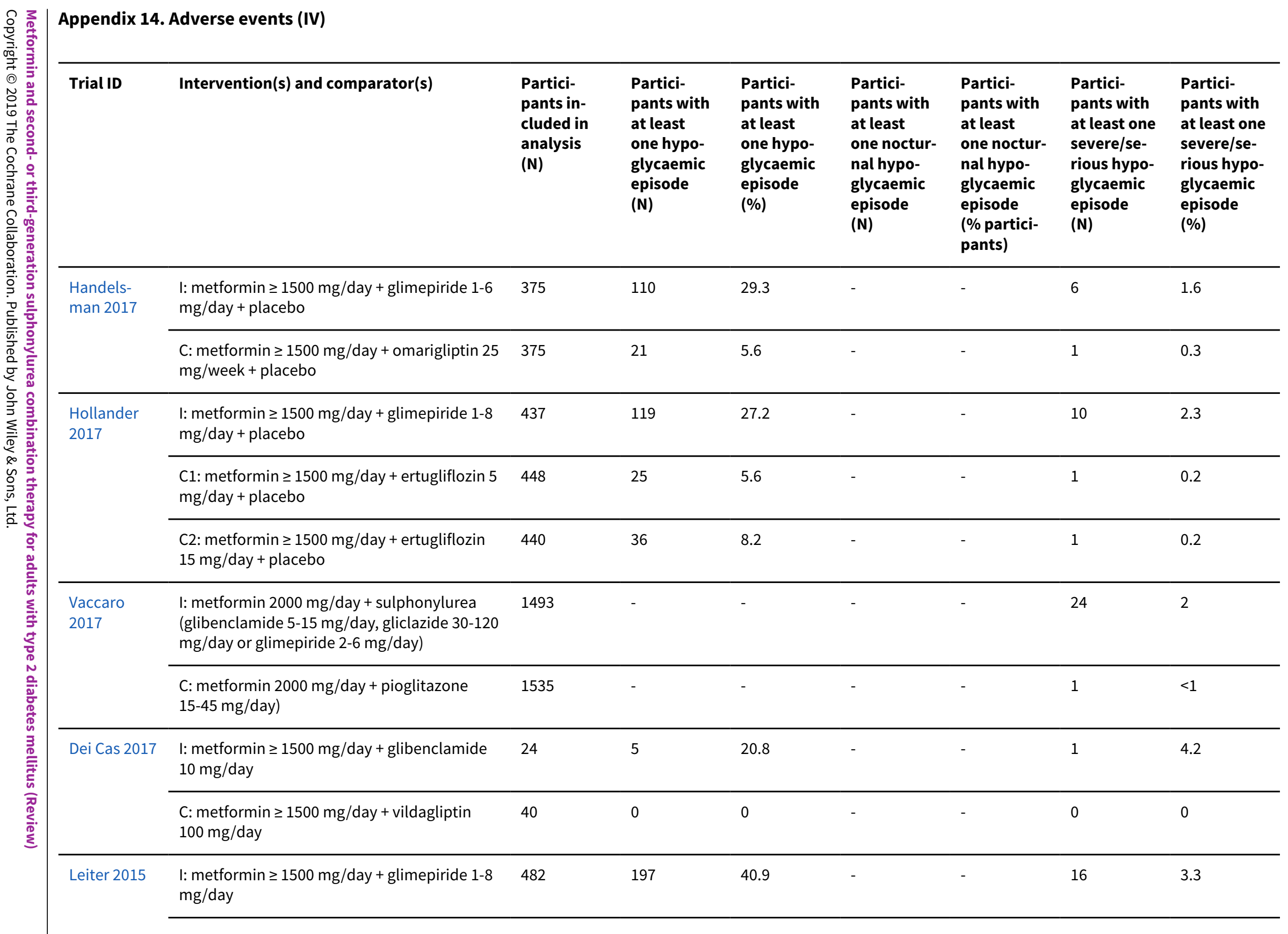


C2: metformin $\geq 1500 \mathrm{mg} /$ day + canagliflozin

485

40

8.2

3

0.6

$300 \mathrm{mg} /$ day

\begin{tabular}{|c|c|c|c|c|c|c|c|c|}
\hline \multirow[t]{2}{*}{$\begin{array}{l}\text { Del Prato } \\
2015\end{array}$} & $\begin{array}{l}\text { I: metformin } 1500-2500 \mathrm{mg} / \text { day + glipizide } \\
\text { 5-20 mg/day }\end{array}$ & 408 & 210 & 51.5 & - & - & 3 & 0.7 \\
\hline & $\begin{array}{l}\text { C: metformin } 1500-2500 \mathrm{mg} / \mathrm{day}+\mathrm{da}- \\
\text { pagliflozin } 2.5-10 \mathrm{mg} / \text { day }\end{array}$ & 406 & 22 & 5.4 & - & - & 0 & 0.0 \\
\hline \multirow[t]{2}{*}{$\begin{array}{l}\text { Schern- } \\
\text { thaner } 2015\end{array}$} & $\begin{array}{l}\text { I: metformin at any dose + glimepiride 1-6 } \\
\mathrm{mg} / \text { day + placebo }\end{array}$ & 359 & 125 & 34.8 & - & - & 1 & 0.3 \\
\hline & $\begin{array}{l}\text { C: metformin at any dose + saxagliptin } 5 \mathrm{mg} / \\
\text { day + placebo }\end{array}$ & 359 & 21 & 5.8 & - & - & 0 & 0 \\
\hline \multirow[t]{3}{*}{$\begin{array}{l}\text { Del Prato } \\
2014\end{array}$} & $\begin{array}{l}\text { I: metformin } \geq 1500 \text { mg once daily or maxi- } \\
\text { mum tolerated dose + glipizide 5-20 mg once } \\
\text { daily }\end{array}$ & 869 & 202 & 23.2 & - & - & 5 & 0.6 \\
\hline & $\begin{array}{l}\mathrm{C} 1 \text { : metformin } \geq 1500 \text { mg once daily or maxi- } \\
\text { mum tolerated dose + alogliptin } 12.5 \text { mg once } \\
\text { daily }\end{array}$ & 873 & 22 & 2.5 & - & - & 1 & 0.1 \\
\hline & $\begin{array}{l}\text { C2: metformin } \geq 1500 \text { mg once daily or maxi- } \\
\text { mum tolerated dose }+ \text { alogliptin } 25 \text { mg once } \\
\text { daily }\end{array}$ & 878 & 12 & 1.4 & - & - & 0 & 0 \\
\hline \multirow[t]{4}{*}{ Ahrén 2014} & $\begin{array}{l}\text { I: metformin } \geq 1500 \mathrm{mg} \text { daily + glimepiride } 2-4 \\
\mathrm{mg} \text { once daily + placebo + placebo }\end{array}$ & 307 & 102 & 33.2 & - & - & 1 & 0.3 \\
\hline & $\begin{array}{l}\mathrm{C} 1: \text { metformin } \geq 1500 \mathrm{mg} \text { daily + albiglutide } \\
30-50 \mathrm{mg} \text { once weekly + placebo + placebo }\end{array}$ & 302 & 35 & 11.6 & - & - & 0 & 0 \\
\hline & $\begin{array}{l}\text { C2: metformin } \geq 1500 \mathrm{mg} \text { daily + sitagliptin } \\
100 \mathrm{mg} \text { once daily + placebo + placebo }\end{array}$ & 302 & 25 & 8.3 & - & - & 1 & 0.3 \\
\hline & $\begin{array}{l}\text { C3: } \text { metformin } \geq 1500 \mathrm{mg} \text { daily + placebo + } \\
\text { placebo }\end{array}$ & 101 & 18 & 17.2 & - & - & 0 & 0 \\
\hline
\end{tabular}




\begin{tabular}{|c|c|c|c|c|c|c|c|c|}
\hline 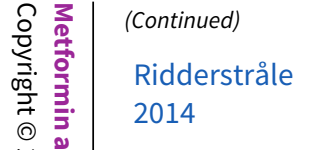 & $\begin{array}{l}\text { I: metformin immediate release } \geq 1500 \mathrm{mg} / \\
\text { day + glimepiride } 1-4 \mathrm{mg} / \text { day }\end{array}$ & 780 & 228 & 29.2 & - & - & 1 & 0.1 \\
\hline 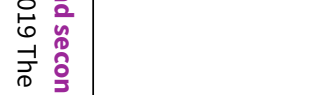 & $\begin{array}{l}\text { C: metformin immediate release } \geq 1500 \mathrm{mg} / \\
\text { day + empagliflozin } 25 \mathrm{mg} / \text { day }\end{array}$ & 765 & 41 & 5.4 & - & - & 0 & 0 \\
\hline Göke 2013 & $\begin{array}{l}\text { I: metformin } \geq 1500 \text { mg daily + glipizide 5-20 } \\
\mathrm{mg} / \text { day }\end{array}$ & 430 & 165 & 38.4 & - & - & 7 & 0 \\
\hline 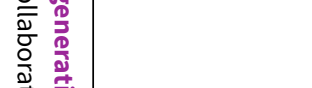 & $\begin{array}{l}\text { C: metformin } \geq 1500 \text { mg daily + saxagliptin } 5 \\
\mathrm{mg} / \text { day }\end{array}$ & 428 & 15 & 3.5 & - & - & 1 & 1.6 \\
\hline $\begin{array}{l}\text { Maffioli } \\
2013\end{array}$ & $\begin{array}{l}\text { I: metformin } 2550 \text { mg/day + glibenclamide } 10 \\
\mathrm{mg} / \text { day }\end{array}$ & - & - & - & - & - & - & - \\
\hline 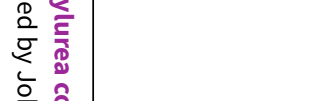 & $\begin{array}{l}\text { C: metformin } 2550 \mathrm{mg} / \text { day + pioglitazone } 30 \\
\mathrm{mg} / \text { day }\end{array}$ & - & - & - & - & - & - & - \\
\hline Nauck 2013 & $\begin{array}{l}\text { I: metformin } 1500-2000 \mathrm{mg} / \text { day + glimepiride } \\
1-4 \mathrm{mg} / \text { day + placebo }\end{array}$ & 242 & 58 & 24.0 & - & - & 0 & 0 \\
\hline 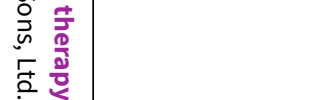 & $\begin{array}{l}\mathrm{C} 1: \text { metformin } 1500-2000 \mathrm{mg} / \text { day + liraglu- } \\
\text { tide } 0.6 \mathrm{mg} / \text { day + placebo }\end{array}$ & 242 & 12 & 5.0 & - & - & 0 & 0 \\
\hline 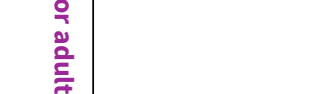 & $\begin{array}{l}\text { C2: metformin } 1500-2000 \mathrm{mg} / \text { day + liraglu- } \\
\text { tide } 1.2 \mathrm{mg} / \text { day + placebo }\end{array}$ & 240 & 10 & 4.2 & - & - & 1 & 0.4 \\
\hline 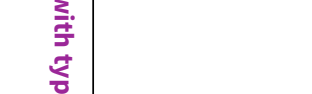 & $\begin{array}{l}\text { C3: metformin } 1500-2000 \mathrm{mg} / \text { day + liraglu- } \\
\text { tide } 1.8 \mathrm{mg} / \text { day + placebo }\end{array}$ & 242 & 10 & 4.1 & - & - & 0 & 0 \\
\hline 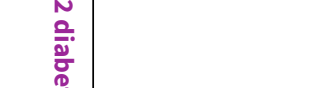 & $\begin{array}{l}\text { C4: metformin } 1500-2000 \mathrm{mg} / \text { day + placebo + } \\
\text { placebo }\end{array}$ & 121 & 3 & 2.5 & - & - & 0 & 0 \\
\hline $\begin{array}{l}\text { Gallwitz } \\
\text { 2012a }\end{array}$ & $\begin{array}{l}\text { I: metformin median dose } 2000 \mathrm{mg} / \text { day + } \\
\text { glimepiride mean dose } 2.01 \mathrm{mg} / \text { day }\end{array}$ & 508 & 338 & 67 & 82 & 16 & 0 & 0 \\
\hline 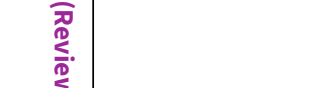 & $\begin{array}{l}\text { C: metformin median dose } 2000 \mathrm{mg} / \text { day + ex- } \\
\text { enatide mean dose } 17.35 \mu \mathrm{g} / \mathrm{day}\end{array}$ & 511 & 186 & 36 & 53 & 10 & 1 & 0.2 \\
\hline $\begin{array}{l}\text { Gallwitz } \\
\text { 2012b }\end{array}$ & $\begin{array}{l}\text { I: metformin } \geq 1500 \mathrm{mg} / \text { day + glimepiride } 1-4 \\
\mathrm{mg} / \text { day + placebo }\end{array}$ & 775 & 280 & 36 & - & - & 12 & 1.5 \\
\hline
\end{tabular}




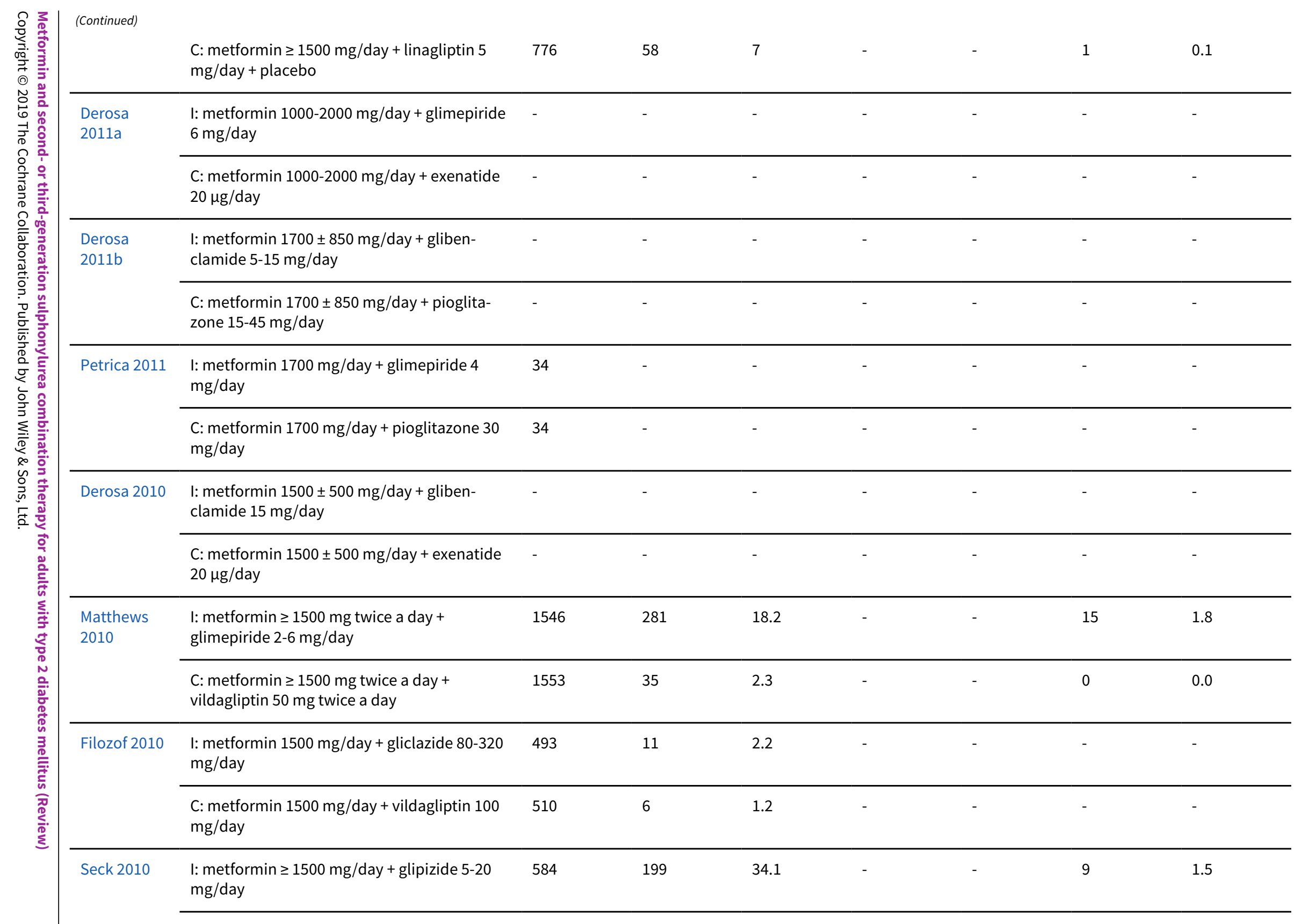




\begin{tabular}{|c|c|c|c|c|c|c|c|c|}
\hline 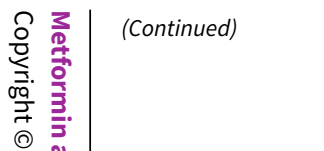 & $\begin{array}{l}\text { C: metformin } \geq 1500 \mathrm{mg} / \text { day + sitagliptin } 100 \\
\mathrm{mg} / \text { day }\end{array}$ & 588 & 31 & 5.3 & - & - & 1 & 0.2 \\
\hline \multirow[t]{2}{*}{ Home 2009} & $\begin{array}{l}\text { I: metformin up to } 2550 \mathrm{mg} / \text { day + gliben- } \\
\text { clamide (or equivalent for different prepara- } \\
\text { tions) up to } 15 \mathrm{mg} / \text { day or gliclazide up to } 240 \\
\mathrm{mg} / \text { day or glimepiride up to } 4 \mathrm{mg} / \text { day }\end{array}$ & 1105 & 190 & 17.2 & - & - & 5 & 0.5 \\
\hline & $\begin{array}{l}\text { C: metformin up to } 2550 \mathrm{mg} / \text { day + rosiglita- } \\
\text { zone up to } 8 \mathrm{mg} / \text { day }\end{array}$ & 1117 & 51 & 4.6 & - & - & 5 & 0.5 \\
\hline \multirow[t]{3}{*}{$\begin{array}{l}\text { Derosa } \\
2009 a\end{array}$} & $\begin{array}{l}\text { I: metformin } 850 \text { mg/day + glimepiride 2-6 } \\
\text { mg/day }\end{array}$ & - & 3 & - & - & - & - & - \\
\hline & $\begin{array}{l}\mathrm{C} 1 \text { : metformin } 850-2550 \mathrm{mg} / \text { day + pioglita- } \\
\text { zone } 15-45 \mathrm{mg} / \text { day }\end{array}$ & - & 2 & - & - & - & - & - \\
\hline & C2: metformin $1000-3000 \mathrm{mg} /$ day & - & 0 & - & - & - & - & - \\
\hline \multirow[t]{2}{*}{$\begin{array}{l}\text { Derosa } \\
2009 b\end{array}$} & $\begin{array}{l}\text { I: metformin } 1500-3000 \mathrm{mg} / \text { day + gliben- } \\
\text { clamide } 7.5-15 \mathrm{mg} / \text { day }\end{array}$ & - & - & - & - & - & - & - \\
\hline & $\begin{array}{l}\text { C: metformin } 1500-3000 \mathrm{mg} / \text { day + nateglinide } \\
180-360 \mathrm{mg} / \text { day }\end{array}$ & - & - & - & - & - & - & - \\
\hline \multirow[t]{2}{*}{ Petrica 2009} & $\begin{array}{l}\text { I: metformin } 1700 \text { mg/day + glimepiride } 4 \\
\text { mg/day }\end{array}$ & 22 & - & - & - & - & - & - \\
\hline & $\begin{array}{l}\text { C: metformin } 1700 \mathrm{mg} / \text { day + rosiglitazone } 4 \\
\mathrm{mg} / \text { day }\end{array}$ & 22 & - & - & - & - & - & - \\
\hline \multirow[t]{2}{*}{ NCT00367055 } & $\begin{array}{l}\text { I: metformin } 2000 \text { mg/day + gliclazide 80-320 } \\
\mathrm{mg} / \text { day }\end{array}$ & 41 & 8 & 20 & - & - & - & - \\
\hline & $\begin{array}{l}\text { C: metformin } 2000 \text { mg/day + rosiglitazone 4-8 } \\
\mathrm{mg} / \text { day }\end{array}$ & 43 & 1 & 2 & - & - & - & - \\
\hline \multirow[t]{2}{*}{$\begin{array}{l}\text { Hamann } \\
2008\end{array}$} & $\begin{array}{l}\text { I: metformin } 2000 \mathrm{mg} / \text { day + glibenclamide } \\
\text { 5-15 mg/day or gliclazide } 80-320 \mathrm{mg} / \text { day }\end{array}$ & 301 & 91 & 30 & - & - & 1 & 0.3 \\
\hline & $\begin{array}{l}\text { C: metformin } 2000 \text { mg/day + rosiglitazone 4-8 } \\
\text { mg/day }\end{array}$ & 294 & 19 & 6 & - & - & 0 & 0 \\
\hline
\end{tabular}




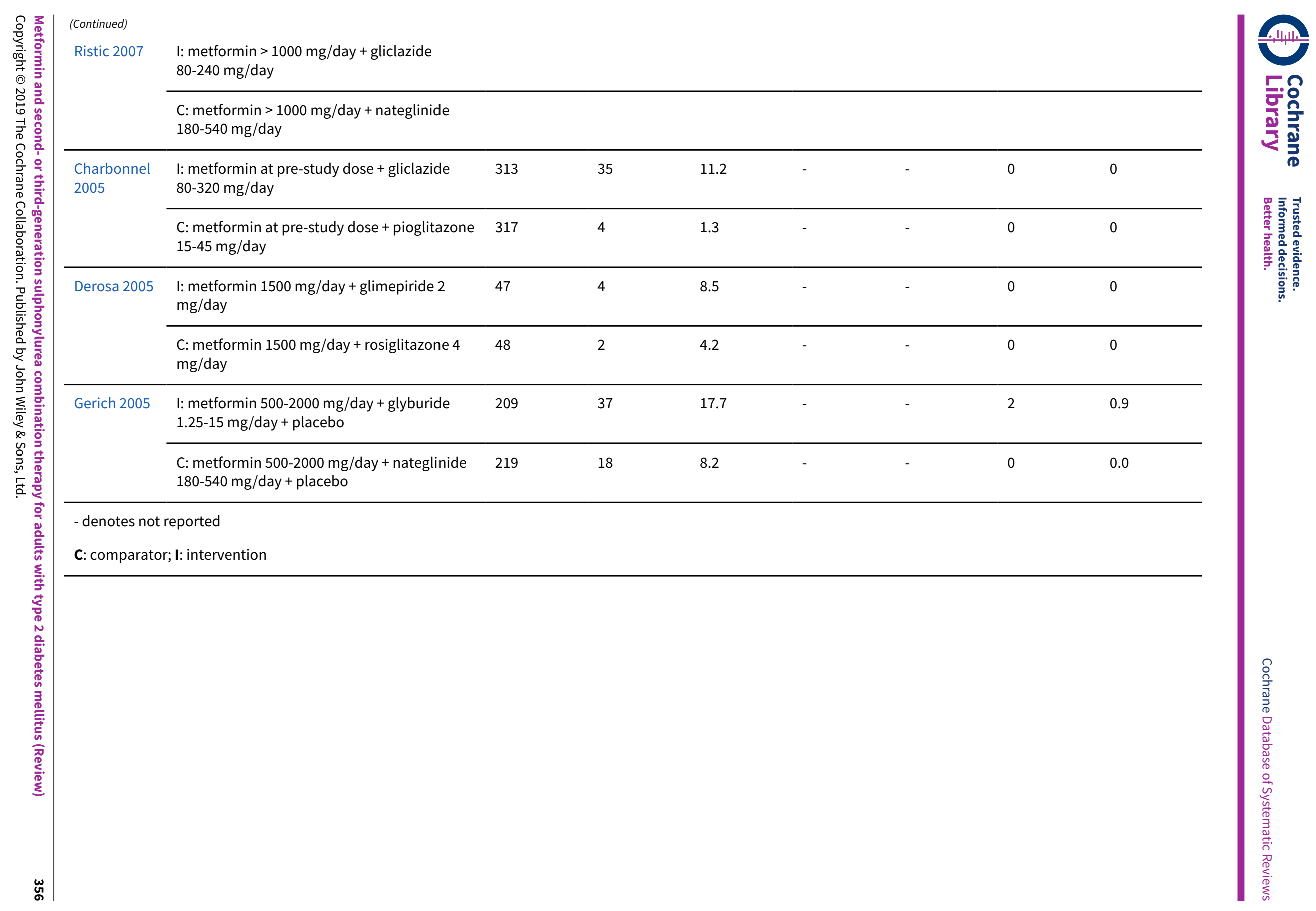




\section{Appendix 15. Survey of trial investigators providing information on studies}

\begin{tabular}{|c|c|c|c|c|}
\hline Trial ID & $\begin{array}{l}\text { Date trial au- } \\
\text { thor contacted }\end{array}$ & $\begin{array}{l}\text { Date trial au- } \\
\text { thor replied }\end{array}$ & $\begin{array}{l}\text { Date trial author was asked for } \\
\text { additional information } \\
\text { (short summary) }\end{array}$ & $\begin{array}{l}\text { Date trial author provided data } \\
\text { (short summary) }\end{array}$ \\
\hline $\begin{array}{l}\text { Handelsman } \\
2017\end{array}$ & 6 March 2018 & No reply & $\mathrm{N} / \mathrm{A}$ & N/A \\
\hline Hollander 2017 & $\begin{array}{l}5 \text { March } 2018 \\
\text { (email sent to } \\
\text { last author Brett } \\
\text { Lauring) }\end{array}$ & No reply & $\mathrm{N} / \mathrm{A}$ & $\mathrm{N} / \mathrm{A}$ \\
\hline Vaccaro 2017 & $\begin{array}{l}15 \text { February } 2018 \\
27 \text { February (au- } \\
\text { thor Sartore was } \\
\text { contacted) }\end{array}$ & $\begin{array}{l}15 \text { February } 2018 \\
1 \text { March } 2018 \\
\text { (author Chilel- } \\
\text { li responded on } \\
\text { behalf of author } \\
\text { Sartore) }\end{array}$ & $\begin{array}{l}16 \text { February } 2018 \\
\text { Additional material on the trial? } \\
\text { Additional RCT to be included? } \\
\text { Data on remaining outcomes? } \\
5 \text { March } 2018 \\
\text { Author Chilelli was asked about } \\
\text { a reference for which no full-text } \\
\text { was found }\end{array}$ & $\begin{array}{l}22 \text { February } 2018 \\
\text { No additional material on the trial } \\
\text { No additional RCT to be included } \\
\text { Provided data on non-serious ad- } \\
\text { verse events } \\
8 \text { March } 2018 \text { (an unpublished sub- } \\
\text { study to the Vaccaro } 2017 \text { trial was } \\
\text { received from author Chilelli) }\end{array}$ \\
\hline Dei Cas 2017 & 5 July 2017 & 5 July 2017 & $\begin{array}{l}6 \text { July } 2017 \\
\text { Additional material on the trial? } \\
\text { Additional RCT to be included? } \\
\text { Data on remaining outcomes? }\end{array}$ & $\begin{array}{l}18 \text { July } 2017 \text { (data on remaining } \\
\text { outcomes) }\end{array}$ \\
\hline Leiter 2015 & 2 February 2017 & No reply & $\mathrm{N} / \mathrm{A}$ & N/A \\
\hline Del Prato 2015 & 3 March 2017 & $\begin{array}{l}10 \text { March (mail } \\
\text { not delivered } \\
\text { due to problem } \\
\text { with permission } \\
\text { or security) }\end{array}$ & $\mathrm{N} / \mathrm{A}$ & $\mathrm{N} / \mathrm{A}$ \\
\hline $\begin{array}{l}\text { Schernthaner } \\
2015\end{array}$ & 2 August 2017 & 2 August 2017 & $\begin{array}{l}3 \text { August } 2017 \\
\text { Additional material on the trial? } \\
\text { Additional RCT to be included? } \\
\text { Data on remaining outcomes? }\end{array}$ & No reply \\
\hline Del Prato 2014 & 5 January 2017 & No reply & $\mathrm{N} / \mathrm{A}$ & $\mathrm{N} / \mathrm{A}$ \\
\hline Ahrén 2014 & 5 December 2016 & 8 December 2016 & 5 December 2016 & $\begin{array}{l}8 \text { December 2016: "I have forward- } \\
\text { ed you to the relevant person at } \\
\text { GSK, who should be able to get the } \\
\text { data you need. You should hear } \\
\text { from him directly" } \\
\text { Since then: no response }\end{array}$ \\
\hline $\begin{array}{l}\text { Ridderstråle } \\
2014\end{array}$ & 18 July 2017 & $\begin{array}{l}18 \text { July } 2017 \text { - } \\
\text { mail not deliv- } \\
\text { ered }\end{array}$ & $\mathrm{N} / \mathrm{A}$ & N/A \\
\hline Göke 2013 & $\begin{array}{l}10 \text { July } 2017 \\
\text { (contact person: }\end{array}$ & 14 July 2017 & $\begin{array}{l}14 \text { July } 2017 \\
\text { Additional material on the trial? } \\
\text { Additional RCT to be included? }\end{array}$ & $\begin{array}{l}9 \text { August } 2017 \text { (will look in to some } \\
\text { of the questions. Advised us to } \\
\text { contact AZ in Denmark as well. }\end{array}$ \\
\hline
\end{tabular}


(Continued)

Ingrid Gause- Imputation method? $\quad$ Email sent to AZ Denmark $10 \mathrm{Au}-$

Nilsson) Data on remaining outcomes?
gust 2017)

28 September (had questions regarding definition of hypoglycaemia)

\begin{tabular}{lllll}
\hline Maffioli 2013 & 25 January 2017 & No reply & N/A & N/A \\
\hline Nauck 2013 & 28 March 2017 & 28 March 2017 & N/A & N/A \\
& $\begin{array}{l}\text { Mail not deliv- } \\
\text { ered }\end{array}$ & \\
& & & \\
& & & \\
& &
\end{tabular}

$\begin{array}{llll}\text { Gallwitz 2012a } & 6 \text { July } 2017 & 10 \text { July } 2017 & 10 \text { July } 2017\end{array}$

Additional material on the trial? Additional RCT to be included? Data on remaining outcomes?

Gallwitz 2012b $\quad 7$ July $2017 \quad 10$ July 2017

10 July 2017

Additional material on the trial? Additional RCT to be included? Data on remaining outcomes?
29 July 2017 (no additional publications on the trial)

29 July 2017 (no additional publications on the trial, attached a review of linagliptin)

Derosa 2011a $\quad 18$ January $2017 \quad 25$ January $2017 \quad 18$ January 2017

14 March 2017 (answer provided by colleague Pamela Maffioli. Provided data on remaining outcomes, but did not mention number of participants included in safety-analysis. No reply after this)

Derosa 2011b 19 January $2017 \quad 25$ January $2017 \quad 19$ January 2017

14 March 2017 - answer provided by colleague Pamela Maffioli. Provided data on remaining outcomes, but did not mention number of participants included in safety-analysis. No reply after this

\begin{tabular}{|c|c|c|c|c|}
\hline Petrica 2011 & 16 July 2017 & 17 July 2017 & $\begin{array}{l}3 \text { August } 2017 \\
\text { Additional material on the trial? } \\
\text { Additional RCT to be included? } \\
\text { Trial protocol available? } \\
\text { Data on remaining outcomes? }\end{array}$ & No reply \\
\hline \multirow[t]{2}{*}{ Derosa 2010} & 12 January 2017 & 12 January 2017 & 12 January 2017 & $\begin{array}{l}12 \text { January 2017: "Thank you very } \\
\text { much for your consideration. } \\
\text { Please, give me some few days and } \\
\text { I will happy to send you an email } \\
\text { with all the information that I can } \\
\text { have" }\end{array}$ \\
\hline & & & & Since then: no response \\
\hline Matthews 2010 & 11 March 2017 & No reply & $\mathrm{N} / \mathrm{A}$ & N/A \\
\hline Filozof 2010 & 4 February 2017 & 9 February 2017 & $\begin{array}{l}21 \text { February } 2017 \text { (the primary } \\
\text { author does not have access to } \\
\text { trial data any more) }\end{array}$ & $\mathrm{N} / \mathrm{A}$ \\
\hline
\end{tabular}


(Continued)

$\begin{array}{llll}\text { Seck } 2010 & 14 \text { July } 2017 & \begin{array}{l}14 \text { July } 2017 \\ \text { (email not found) }\end{array} & \text { N/A N/A } \\ & \end{array}$

\begin{tabular}{|c|c|c|c|c|}
\hline Home 2009 & 1 April 2017 & 1 April 2017 & $\begin{array}{l}7 \text { April } 2017 \\
\text { Additional material on the trial? } \\
\text { Additional RCT to be included? } \\
\text { Imputation method? } \\
\text { Data on missing outcomes? }\end{array}$ & $\begin{array}{l}7 \text { April } 2017 \text { (information on impu } \\
\text { tation method) } \\
10 \text { April } 2017 \text { (provided data for Cl } \\
\text { mortality) }\end{array}$ \\
\hline
\end{tabular}

\begin{tabular}{lll}
\hline Derosa 2009a & 20 January 2017 25 January 2017 & 25 January 2017 \\
& Additional material on the trial? \\
& Additional RCT to be included? \\
& Trial protocol available? \\
& Data on remaining outcomes? \\
\hline
\end{tabular}

\begin{tabular}{|c|c|c|c|c|}
\hline Derosa 2009b & 24 January 2017 & 24 January 2017 & $\begin{array}{l}25 \text { January } 2017 \\
\text { Additional material on the trial? } \\
\text { Additional RCT to be included? } \\
\text { Trial protocol available? } \\
\text { Data on remaining outcomes? }\end{array}$ & $\begin{array}{l}14 \text { March } 2017 \text { (answer provid- } \\
\text { ed by colleague Pamela Maffioli. } \\
\text { Provided data on remaining out- } \\
\text { comes, but did not mention num- } \\
\text { ber of participants included in } \\
\text { safety-analysis. No reply after this) }\end{array}$ \\
\hline
\end{tabular}

\begin{tabular}{|c|c|c|c|c|}
\hline NCT00367055 & $\begin{array}{l}\text { No contact de- } \\
\text { tails available }\end{array}$ & No reply & $\mathrm{N} / \mathrm{A}$ & $\mathrm{N} / \mathrm{A}$ \\
\hline Hamann 2008 & 12 July 2017 & $\begin{array}{l}12 \text { July } 2017 \\
\text { (email not found) }\end{array}$ & $\mathrm{N} / \mathrm{A}$ & $\mathrm{N} / \mathrm{A}$ \\
\hline Ristic 2007 & 17 July 2017 & $\begin{array}{l}17 \text { July } 2017 \\
\text { (email not found) }\end{array}$ & $\mathrm{N} / \mathrm{A}$ & $\mathrm{N} / \mathrm{A}$ \\
\hline Charbonnel 2005 & 13 July 2017 & No reply & $\mathrm{N} / \mathrm{A}$ & $\mathrm{N} / \mathrm{A}$ \\
\hline Derosa 2005 & 10 January 2017 & 25 January 2017 & $\begin{array}{l}25 \text { January } 2017 \\
\text { Additional material on the trial? } \\
\text { Additional RCT to be included? } \\
\text { Trial protocol available? } \\
\text { Data on remaining outcomes? }\end{array}$ & $\begin{array}{l}14 \text { March } 2017 \text { (answer provid- } \\
\text { ed by colleague Pamela Maffioli. } \\
\text { Provided data on remaining out- } \\
\text { comes) }\end{array}$ \\
\hline Gerich 2005 & 16 March 2017 & $\begin{array}{l}16 \text { March } 2017 \\
\text { (email not found) }\end{array}$ & $\mathrm{N} / \mathrm{A}$ & $\mathrm{N} / \mathrm{A}$ \\
\hline Cryer 2005 & $\begin{array}{l}2 \text { August } 2017 \\
\text { (contacted the } \\
\text { author if sep- } \\
\text { arate data are } \\
\text { available) }\end{array}$ & No reply & $\mathrm{N} / \mathrm{A}$ & $\mathrm{N} / \mathrm{A}$ \\
\hline
\end{tabular}

N/A: not applicable; $\mathbf{R C T}$ : randomised controlled trial 


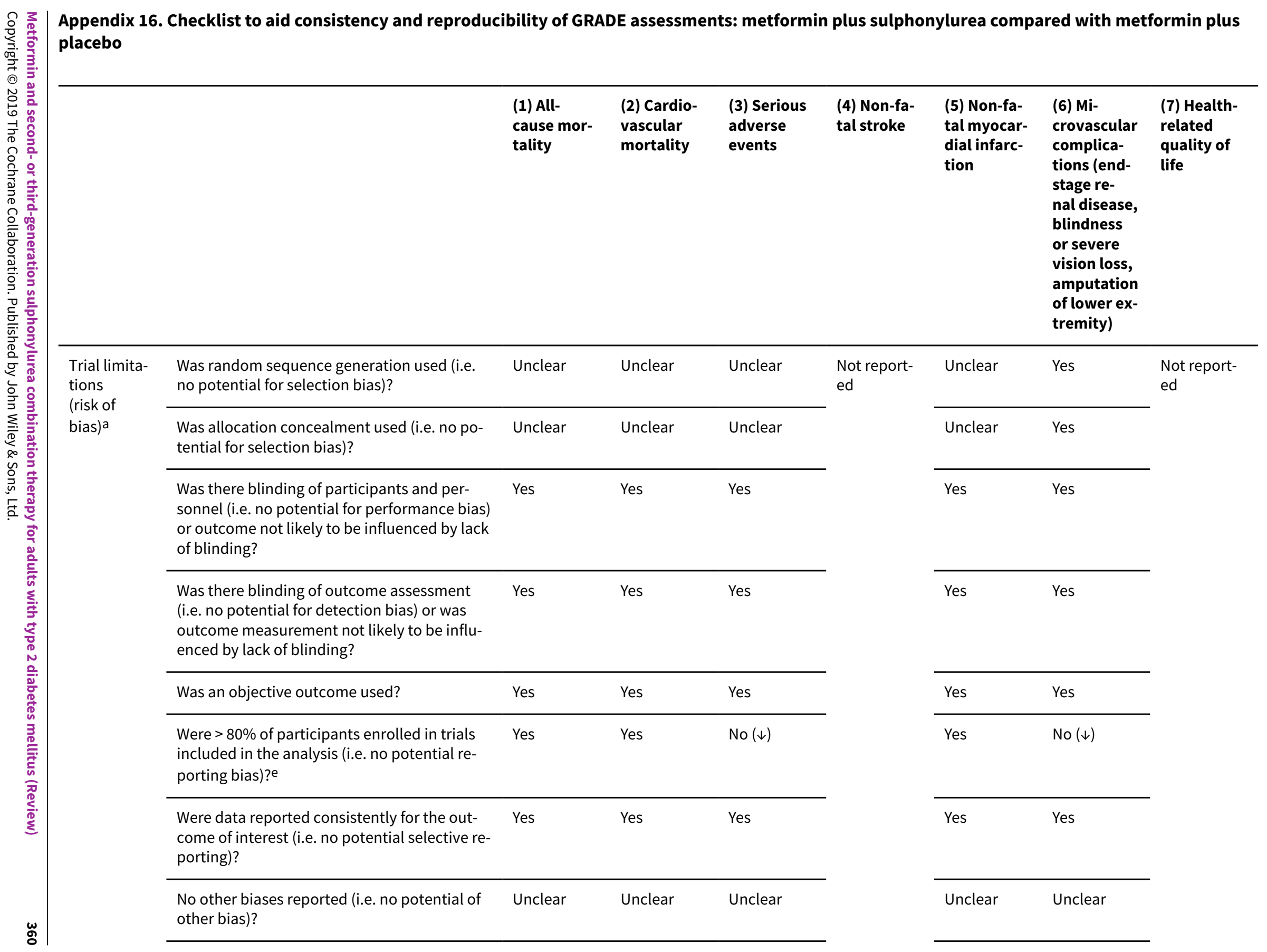




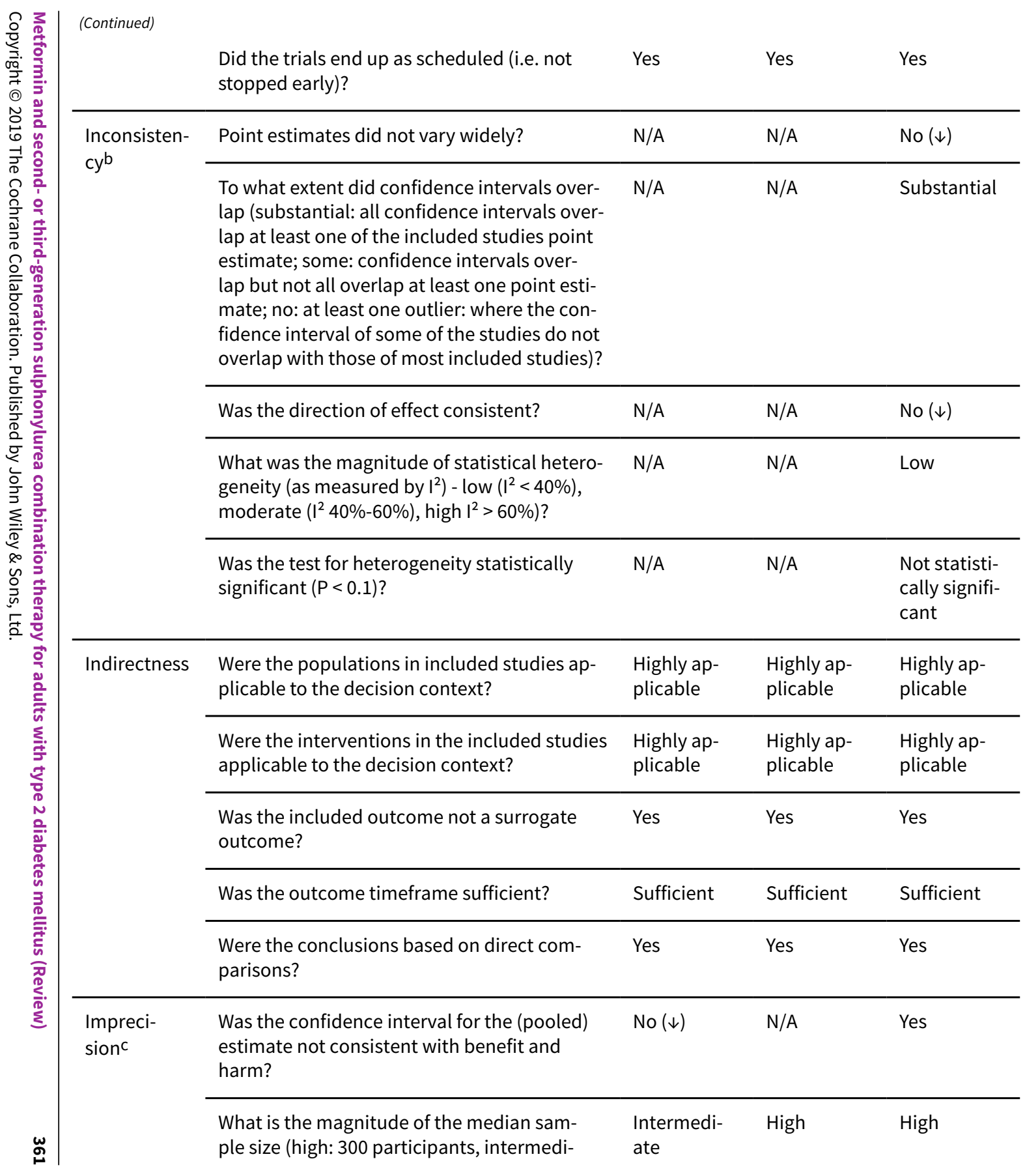

\begin{tabular}{lll} 
Yes & Yes \\
\hline No $(\downarrow)$ & N/A & \\
\hline Substantial & N/A &
\end{tabular}

\begin{tabular}{ll}
\hline No $(\downarrow)$ & N/A \\
\hline Low & N/A
\end{tabular}

\begin{tabular}{ll}
\hline $\begin{array}{l}\text { Not statisti- } \\
\text { cally signifi- } \\
\text { cant }\end{array}$ & N/A \\
\hline $\begin{array}{l}\text { Highly ap- } \\
\text { plicable }\end{array}$ & $\begin{array}{l}\text { Highly ap- } \\
\text { plicable }\end{array}$ \\
\hline $\begin{array}{l}\text { Highly ap- } \\
\text { plicable }\end{array}$ & $\begin{array}{l}\text { Highly ap- } \\
\text { plicable }\end{array}$ \\
\hline Yes & Yes \\
\hline Sufficient & Sufficient \\
\hline Yes & Yes \\
\hline Yes & N/A \\
\hline High & High
\end{tabular}


ate: $100-300$ participants, low: $<100$ participants)?e

What was the magnitude of the number of included studies (large: > 10 studies, moderate:

Small ( $\downarrow)$ Small $(\downarrow)$ Small $(\downarrow)$ 5-10 studies, small: $<5$ studies)?

Was the outcome a common event (e.g. oc-
curs $>1 / 100)$ ?
biasd

\begin{tabular}{llll} 
Was a comprehensive search conducted? & Yes & Yes & Yes \\
\hline Was grey literature searched? & Yes & Yes & Yes \\
\hline $\begin{array}{l}\text { Were no restrictions applied to study selec- } \\
\text { tion on the basis of language? }\end{array}$ & Yes & Yes & Yes \\
\hline $\begin{array}{l}\text { There was no industry influence on studies in- } \\
\text { cluded in the review? }\end{array}$ & Unclear & Unclear & Unclear \\
\hline $\begin{array}{l}\text { There was no evidence of funnel plot asym- } \\
\text { metry? }\end{array}$ & N/A & N/A & N/A
\end{tabular}

\begin{tabular}{llll}
\hline $\begin{array}{l}\text { There was no discrepancy in findings be- } \\
\text { tween published and unpublished trials? }\end{array}$ & N/A & N/A & N/A
\end{tabular}

\section{Publication}

\begin{tabular}{ll}
\hline Small $(\downarrow)$ & Small $(\downarrow)$ \\
\hline No $(\downarrow)$ & No $(\downarrow)$ \\
\hline Yes & Yes \\
\hline Yes & Yes \\
\hline Yes & Yes \\
\hline Unclear & Unclear \\
\hline N/A & N/A \\
\hline N/A & N/A
\end{tabular}

aQuestions on risk of bias are answered in relation to the majority of the aggregated evidence in the meta-analysis rather than to individual trials.

bQuestions on inconsistency are primarily based on visual assessment of forest plots and the statistical quantification of heterogeneity based on ${ }^{2}$.

cWhen judging the width of the confidence interval it is recommended to use a clinical decision threshold to assess whether the imprecision is clinically meaningful. dQuestions address comprehensiveness of the search strategy, industry influence, funnel plot asymmetry and discrepancies between published and unpublished trials. eDepends on the context of the systematic review area.

$(\downarrow)$ : key item for potential downgrading the quality of the evidence (GRADE) as shown in the footnotes of the 'Summary of finding' table(s); GRADE: Grading of Recommendations Assessment, Development and Evaluation; N/A: not applicable 


\begin{tabular}{|c|c|c|c|c|c|c|c|c|c|}
\hline \multirow{4}{*}{\multicolumn{2}{|c|}{ 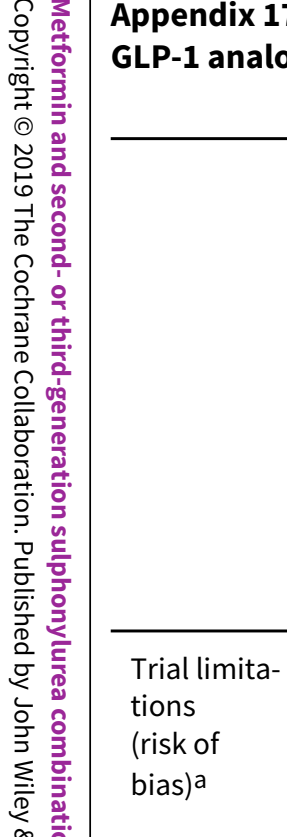 }} & hecklist to ald consistency and reprodu & bility of GR & E assessm & s: mettorn & plus sulph & ylurea com & red with m & rmın plus \\
\hline & & & \multirow[t]{2}{*}{$\begin{array}{l}\text { (1) All- } \\
\text { cause mor- } \\
\text { tality }\end{array}$} & \multirow[t]{2}{*}{$\begin{array}{l}\text { (2) Cardio- } \\
\text { vascular } \\
\text { mortality } \\
\\
\text { Yes }\end{array}$} & \multirow[t]{2}{*}{$\begin{array}{l}\text { (3) Serious } \\
\text { adverse } \\
\text { events } \\
\\
\text { Yes }\end{array}$} & \multirow[t]{3}{*}{$\begin{array}{l}\text { (4) Non-fa- } \\
\text { tal stroke } \\
\\
\text { Not report- } \\
\text { ed }\end{array}$} & \multirow[t]{2}{*}{$\begin{array}{l}\text { (5) Non-fa- } \\
\text { tal myocar- } \\
\text { dial infarc- } \\
\text { tion } \\
\\
\text { Unclear }\end{array}$} & \multirow[t]{2}{*}{$\begin{array}{l}\text { (6) Mi- } \\
\text { crovascular } \\
\text { complica- } \\
\text { tions (end- } \\
\text { stage re- } \\
\text { nal disease, } \\
\text { blindness } \\
\text { or severe } \\
\text { vision loss, } \\
\text { amputation } \\
\text { of lower ex- } \\
\text { tremity) }\end{array}$} & $\begin{array}{l}\text { (7) Health } \\
\text { related } \\
\text { quality of } \\
\text { life }\end{array}$ \\
\hline & & $\begin{array}{l}\text { Was random sequence generation used (i.e. } \\
\text { no potential for selection bias)? }\end{array}$ & & & & & & & $\begin{array}{l}\text { Not report- } \\
\text { ed }\end{array}$ \\
\hline & & $\begin{array}{l}\text { Was allocation concealment used (i.e. no po- } \\
\text { tential for selection bias)? }\end{array}$ & Yes & Yes & Yes & & Unclear & Yes & \\
\hline 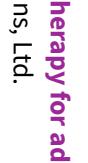 & & $\begin{array}{l}\text { Was there blinding of participants and per- } \\
\text { sonnel (i.e. no potential for performance bias) } \\
\text { or outcome not likely to be influenced by lack } \\
\text { of blinding? }\end{array}$ & Yes & Yes & Yes & & Yes & Yes & \\
\hline 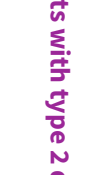 & & $\begin{array}{l}\text { Was there blinding of outcome assessment } \\
\text { (i.e. no potential for detection bias) or was } \\
\text { outcome measurement not likely to be influ- } \\
\text { enced by lack of blinding? }\end{array}$ & Yes & Yes & Yes & & Yes & Yes & \\
\hline$\frac{0}{0}$ & & Was an objective outcome used? & Yes & Yes & Yes & & Yes & Yes & \\
\hline 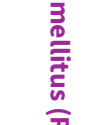 & & $\begin{array}{l}\text { Were }>80 \% \text { of participants enrolled in trials } \\
\text { included in the analysis (i.e. no potential re- } \\
\text { porting bias)? }\end{array}$ & Yes & Yes & Unclear & & Unclear & Unclear & \\
\hline 市. & & $\begin{array}{l}\text { Were data reported consistently for the out- } \\
\text { come of interest (i.e. no potential selective re- } \\
\text { porting)? }\end{array}$ & Yes & Yes & Yes & & Yes & N/A & \\
\hline & & $\begin{array}{l}\text { No other biases reported (i.e. no potential of } \\
\text { other bias)? }\end{array}$ & Unclear & Unclear & Unclear & & Unclear & Unclear & \\
\hline
\end{tabular}


Inconsisten-

cyb

Point estimates did not vary widely?

N/A

$\mathrm{N} / \mathrm{A}$

To what extent did confidence intervals over-

lap (substantial: all confidence intervals over-

Substantial

Substantial

ap at least one of the included studies point

estimate; some: confidence intervals over-

lap but not all overlap at least one point esti-

mate; no: at least one outlier: where the con-

fidence interval of some of the studies do not

overlap with those of most included studies)?

\begin{tabular}{llll}
\hline Was the direction of effect consistent? & Unclear & N/A & Yes \\
\hline
\end{tabular}

What was the magnitude of statistical hetero-

Low

$\mathrm{N} / \mathrm{A}$

Low

geneity (as measured by $\mathrm{I}^{2}$ ) - low $\left(\mathrm{I}^{2}<40 \%\right)$

moderate $\left(I^{2} 40 \%-60 \%\right)$, high $\left.\mathrm{I}^{2}>60 \%\right)$ ?

Was the test for heterogeneity statistically

significant $(P<0.1)$ ?

Not statisti-

N/A

cally signifi-

Not statisti-

cant

cally signifi-

\begin{tabular}{lllll}
\hline Indirectness & $\begin{array}{l}\text { Were the populations in included studies ap- } \\
\text { plicable to the decision context? }\end{array}$ & $\begin{array}{l}\text { Highly ap- } \\
\text { plicable }\end{array}$ & $\begin{array}{l}\text { Highly ap- } \\
\text { plicable }\end{array}$ & $\begin{array}{l}\text { Highly ap- } \\
\text { plicable }\end{array}$ \\
\cline { 2 - 5 } $\begin{array}{l}\text { Were the interventions in the included studies } \\
\text { applicable to the decision context? }\end{array}$ & $\begin{array}{l}\text { Highly ap- } \\
\text { plicable }\end{array}$ & $\begin{array}{l}\text { Highly ap- } \\
\text { plicable }\end{array}$ & $\begin{array}{l}\text { Highly ap- } \\
\text { plicable }\end{array}$ \\
\cline { 2 - 5 } & $\begin{array}{l}\text { Was the included outcome not a surrogate } \\
\text { outcome? }\end{array}$ & Yes & Yes & Yes \\
\cline { 2 - 5 } & \begin{tabular}{l} 
Was the outcome timeframe sufficient? \\
\cline { 2 - 5 }
\end{tabular} & Sufficient & Sufficient & Sufficient \\
Were the conclusions based on direct com- & Yes & Yes & Yes \\
\hline $\begin{array}{l}\text { Impreci- } \\
\text { sionc }\end{array}$ & $\begin{array}{l}\text { Was the confidence interval for the (pooled) } \\
\text { estimate not consistent with benefit and } \\
\text { harm? }\end{array}$ & No $(\downarrow)$ & No $(\downarrow)$ & No $(\downarrow)$ \\
\hline
\end{tabular}

What is the magnitude of the median sam ple size (high: 300 participants, intermedi-
High

High

\begin{tabular}{ll}
\hline N/A & N/A \\
\hline Substantial & $N / A$
\end{tabular}

\begin{tabular}{ll}
\hline N/A & N/A \\
\hline Low & $N / A$
\end{tabular}

Not statisti- N/A

cally signifi-

cant

\begin{tabular}{ll}
\hline $\begin{array}{l}\text { Highly ap- } \\
\text { plicable }\end{array}$ & $\begin{array}{l}\text { Highly ap- } \\
\text { plicable }\end{array}$ \\
\hline $\begin{array}{l}\text { Highly ap- } \\
\text { plicable }\end{array}$ & $\begin{array}{l}\text { Highly ap- } \\
\text { plicable }\end{array}$ \\
\hline Yes & Yes \\
\hline Sufficient & Sufficient \\
\hline Yes & Yes \\
\hline No $(\downarrow)$ & N/A \\
\hline High & High
\end{tabular}


ate: $100-300$ participants, low: $<100$ participants)?e

What was the magnitude of the number of included studies (large: > 10 studies, moderate:

Small ( $\downarrow)$

Small $(\downarrow)$

Small ( $\downarrow)$

5-10 studies, small: $<5$ studies)?

Was the outcome a common event (e.g. oc-

No $(\downarrow)$

No $(\downarrow)$

Yes curs $>1 / 100)$ ?

Publication

biasd

Was a comprehensive search conducted?

Yes

Yes

Yes

Was grey literature searched?

Were no restrictions applied to study selec-

tion on the basis of language?

There was no industry influence on studies in- Unclear
cluded in the review?

\begin{tabular}{|c|c|c|c|}
\hline $\begin{array}{l}\text { There was no evidence of funnel plot asym- } \\
\text { metry? }\end{array}$ & $\mathrm{N} / \mathrm{A}$ & $\mathrm{N} / \mathrm{A}$ & $\mathrm{N} / \mathrm{A}$ \\
\hline
\end{tabular}

There was no discrepancy in findings be-

Yes

Yes

Yes

tween published and unpublished trials?

aQuestions on risk of bias are answered in relation to the majority of the aggregated evidence in the meta-analysis rather than to individual trials.

bQuestions on inconsistency are primarily based on visual assessment of forest plots and the statistical quantification of heterogeneity based on ${ }^{2}$.

cWhen judging the width of the confidence interval it is recommended to use a clinical decision threshold to assess whether the imprecision is clinically meaningful. dQuestions address comprehensiveness of the search strategy, industry influence, funnel plot asymmetry and discrepancies between published and unpublished trials. eDepends on the context of the systematic review area.

$(\downarrow)$ : key item for potential downgrading the quality of the evidence (GRADE) as shown in the footnotes of the 'Summary of finding' table(s); GRADE: Grading of Recommendations Assessment, Development and Evaluation; N/A: not applicable

Small $(\downarrow) \quad$ Small $(\downarrow)$

\begin{tabular}{ll}
\hline No $(\downarrow)$ & No $(\downarrow)$ \\
\hline Yes & Yes \\
\hline Yes & Yes \\
\hline Yes & Yes \\
\hline Unclear & Unclear \\
\hline N/A & N/A \\
\hline
\end{tabular}

Yes

N/A

-




\begin{tabular}{|c|c|c|c|c|c|c|c|c|c|}
\hline \multirow{4}{*}{\multicolumn{2}{|c|}{ 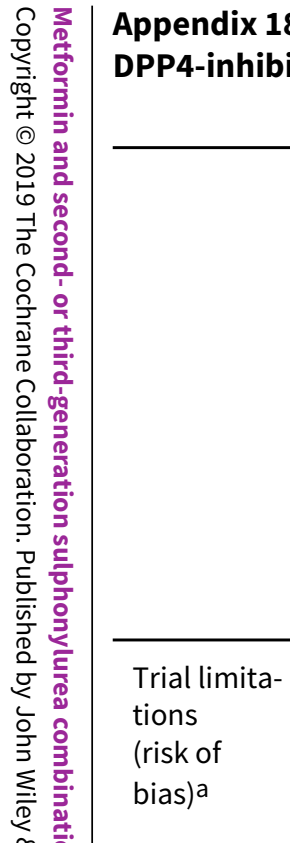 }} & \multirow{2}{*}{$\begin{array}{l}\text { Checklist to aid consistency and reproduc } \\
\text { or } \\
\end{array}$} & \multirow{2}{*}{$\begin{array}{l}\text { bility of GR } \\
\text { (1) All- } \\
\text { cause mor- } \\
\text { tality }\end{array}$} & \multirow[b]{2}{*}{$\begin{array}{l}\text { (2) Cardio- } \\
\text { vascular } \\
\text { mortality }\end{array}$} & \multirow[b]{2}{*}{$\begin{array}{l}\text { (3) Serious } \\
\text { adverse } \\
\text { events }\end{array}$} & \multirow[b]{2}{*}{$\begin{array}{l}\text { (4) Non-fa- } \\
\text { tal stroke }\end{array}$} & \multirow[b]{2}{*}{$\begin{array}{l}\text { (5) Non-fa- } \\
\text { tal myocar- } \\
\text { dial infarc- } \\
\text { tion }\end{array}$} & \multirow[b]{2}{*}{$\begin{array}{l}\text { (6) Mi- } \\
\text { crovascular } \\
\text { complica- } \\
\text { tions (end- } \\
\text { stage re- } \\
\text { nal disease, } \\
\text { blindness } \\
\text { or severe } \\
\text { vision loss, } \\
\text { amputation } \\
\text { of lower ex- } \\
\text { tremity) }\end{array}$} & \multirow[b]{2}{*}{$\begin{array}{l}\text { (7) Health- } \\
\text { related } \\
\text { quality of } \\
\text { life }\end{array}$} \\
\hline & & & & & & & & & \\
\hline & & $\begin{array}{l}\text { Was random sequence generation used (i.e. } \\
\text { no potential for selection bias)? }\end{array}$ & Yes & Unclear & Yes & Yes & Yes & Yes & $\begin{array}{l}\text { Not report- } \\
\text { ed }\end{array}$ \\
\hline & & $\begin{array}{l}\text { Was allocation concealment used (i.e. no po- } \\
\text { tential for selection bias)? }\end{array}$ & Yes & Unclear & Yes & Yes & Yes & Yes & \\
\hline 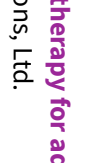 & & $\begin{array}{l}\text { Was there blinding of participants and per- } \\
\text { sonnel (i.e. no potential for performance bias) } \\
\text { or outcome not likely to be influenced by lack } \\
\text { of blinding? }\end{array}$ & Yes & Yes & Yes & Yes & Yes & Yes & \\
\hline 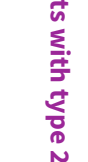 & & $\begin{array}{l}\text { Was there blinding of outcome assessment } \\
\text { (i.e. no potential for detection bias) or was } \\
\text { outcome measurement not likely to be influ- } \\
\text { enced by lack of blinding? }\end{array}$ & Yes & Yes & Yes & Yes & Yes & Yes & \\
\hline$\frac{0}{0}$ & & Was an objective outcome used? & Yes & Yes & Yes & Yes & Yes & Yes & \\
\hline 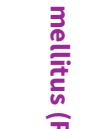 & & $\begin{array}{l}\text { Were }>80 \% \text { of participants enrolled in trials } \\
\text { included in the analysis (i.e. no potential re- } \\
\text { porting bias)? }\end{array}$ & Yes & Yes & Unclear & Unclear & Unclear & Yes & \\
\hline & & $\begin{array}{l}\text { Were data reported consistently for the out- } \\
\text { come of interest (i.e. no potential selective re- } \\
\text { porting)? }\end{array}$ & Yes & Yes & Unclear & Yes & Yes & Yes & \\
\hline & & $\begin{array}{l}\text { No other biases reported (i.e. no potential of } \\
\text { other bias)? }\end{array}$ & Unclear & Unclear & Unclear & Unclear & Unclear & Unclear & \\
\hline
\end{tabular}




\begin{tabular}{|c|c|c|c|c|c|c|c|c|}
\hline 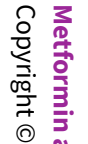 & (Continued) & $\begin{array}{l}\text { Did the trials end up as scheduled (i.e. not } \\
\text { stopped early)? }\end{array}$ & Yes & Yes & Yes & Yes & Yes & Yes \\
\hline & \multirow{5}{*}{$\begin{array}{l}\text { Inconsisten- } \\
\text { cyb }\end{array}$} & Point estimates did not vary widely? & No $(\downarrow)$ & No $(\downarrow)$ & No $(\downarrow)$ & Yes & Yes & N/A \\
\hline 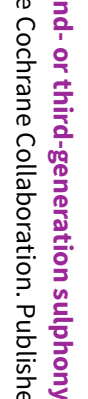 & & $\begin{array}{l}\text { To what extent did confidence intervals over- } \\
\text { lap (substantial: all confidence intervals over- } \\
\text { lap at least one of the included studies point } \\
\text { estimate; } \\
\text { some: confidence intervals overlap but not } \\
\text { all overlap at least one point estimate; no: at } \\
\text { least one outlier: where the confidence inter- } \\
\text { val of some } \\
\text { of the studies do not overlap with those of } \\
\text { most included studies)? }\end{array}$ & Substantial & Substantial & Substantial & Substantial & Substantial & $\mathrm{N} / \mathrm{A}$ \\
\hline t & & Was the direction of effect consistent? & No $(\downarrow)$ & No $(\downarrow)$ & No $(\downarrow)$ & Yes & Yes & $\mathrm{N} / \mathrm{A}$ \\
\hline 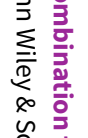 & & $\begin{array}{l}\text { What was the magnitude of statistical hetero- } \\
\left.\text { geneity (as measured by } I^{2}\right)- \text { low }\left(I^{2}<40 \%\right) \text {, } \\
\left.\text { moderate }\left(I^{2} 40 \%-60 \%\right) \text {, high } I^{2}>60 \%\right) \text { ? }\end{array}$ & Low & Low & Low & Low & Low & $\mathrm{N} / \mathrm{A}$ \\
\hline 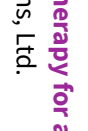 & & $\begin{array}{l}\text { Was the test for heterogeneity statistically } \\
\text { significant }(P<0.1) \text { ? }\end{array}$ & $\begin{array}{l}\text { Not statisti- } \\
\text { cally signifi- } \\
\text { cant }\end{array}$ & $\begin{array}{l}\text { Not statisti- } \\
\text { cally signifi- } \\
\text { cant }\end{array}$ & $\begin{array}{l}\text { Not statisti- } \\
\text { cally signifi- } \\
\text { cant }\end{array}$ & $\begin{array}{l}\text { Not statisti- } \\
\text { cally signifi- } \\
\text { cant }\end{array}$ & $\begin{array}{l}\text { Not statisti- } \\
\text { cally signifi- } \\
\text { cant }\end{array}$ & $\mathrm{N} / \mathrm{A}$ \\
\hline 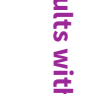 & \multirow[t]{5}{*}{ Indirectness } & $\begin{array}{l}\text { Were the populations in included studies ap- } \\
\text { plicable to the decision context? }\end{array}$ & $\begin{array}{l}\text { Highly ap- } \\
\text { plicable }\end{array}$ & $\begin{array}{l}\text { Highly ap- } \\
\text { plicable }\end{array}$ & $\begin{array}{l}\text { Highly ap- } \\
\text { plicable }\end{array}$ & $\begin{array}{l}\text { Highly ap- } \\
\text { plicable }\end{array}$ & $\begin{array}{l}\text { Highly ap- } \\
\text { plicable }\end{array}$ & $\begin{array}{l}\text { Highly ap- } \\
\text { plicable }\end{array}$ \\
\hline 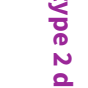 & & $\begin{array}{l}\text { Were the interventions in the included studies } \\
\text { applicable to the decision context? }\end{array}$ & $\begin{array}{l}\text { Highly ap- } \\
\text { plicable }\end{array}$ & $\begin{array}{l}\text { Highly ap- } \\
\text { plicable }\end{array}$ & $\begin{array}{l}\text { Highly ap- } \\
\text { plicable }\end{array}$ & $\begin{array}{l}\text { Highly ap- } \\
\text { plicable }\end{array}$ & $\begin{array}{l}\text { Highly ap- } \\
\text { plicable }\end{array}$ & $\begin{array}{l}\text { Highly ap- } \\
\text { plicable }\end{array}$ \\
\hline 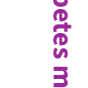 & & $\begin{array}{l}\text { Was the included outcome not a surrogate } \\
\text { outcome? }\end{array}$ & Yes & Yes & Yes & Yes & Yes & Yes \\
\hline$\overline{\overline{\vec{n}}}$ & & Was the outcome timeframe sufficient? & Sufficient & Sufficient & Sufficient & Sufficient & Sufficient & Sufficient \\
\hline 変. & & $\begin{array}{l}\text { Were the conclusions based on direct com- } \\
\text { parisons? }\end{array}$ & Yes & Yes & Yes & Yes & Yes & Yes \\
\hline & $\begin{array}{l}\text { Impreci- } \\
\text { sionc }\end{array}$ & $\begin{array}{l}\text { Was the confidence interval for the (pooled) } \\
\text { estimate not consistent with benefit and } \\
\text { harm? }\end{array}$ & No $(\downarrow)$ & No $(\downarrow)$ & No $(\downarrow)$ & No $(\downarrow)$ & No $(\downarrow)$ & $\mathrm{N} / \mathrm{A}$ \\
\hline
\end{tabular}


What is the magnitude of the median sample size (high: 300 participants, intermedi-

High

High

High

High

High

$\operatorname{Low}(\downarrow)$ ate: 100-300 participants, low: $<100$ participants)?

\begin{tabular}{|c|c|c|c|c|c|c|}
\hline $\begin{array}{l}\text { What was the magnitude of the number of in- } \\
\text { cluded studies (large: }>10 \text { studies, moderate: } \\
5-10 \text { studies, small: }<5 \text { studies)?e }\end{array}$ & Moderate & Moderate & Moderate & Small $(\downarrow)$ & Moderate & Small $(\downarrow)$ \\
\hline $\begin{array}{l}\text { Was the outcome a common event (e.g. oc- } \\
\text { curs }>1 / 100) \text { ? }\end{array}$ & No $(\downarrow)$ & No $(\downarrow)$ & Yes & No $(\downarrow)$ & No $(\downarrow)$ & No $(\downarrow)$ \\
\hline Was a comprehensive search conducted? & Yes & Yes & Yes & Yes & Yes & Yes \\
\hline Was grey literature searched? & Yes & Yes & Yes & Yes & Yes & Yes \\
\hline $\begin{array}{l}\text { Were no restrictions applied to study selec- } \\
\text { tion on the basis of language? }\end{array}$ & Yes & Yes & Yes & Yes & Yes & Yes \\
\hline $\begin{array}{l}\text { There was no industry influence on studies in- } \\
\text { cluded in the review? }\end{array}$ & Unclear & Unclear & Unclear & Unclear & Unclear & Yes \\
\hline $\begin{array}{l}\text { There was no evidence of funnel plot asym- } \\
\text { metry? }\end{array}$ & N/A & N/A & N/A & N/A & $\mathrm{N} / \mathrm{A}$ & $\mathrm{N} / \mathrm{A}$ \\
\hline $\begin{array}{l}\text { There was no discrepancy in findings be- } \\
\text { tween published and unpublished trials? }\end{array}$ & Yes & Yes & Yes & Yes & Yes & $\mathrm{N} / \mathrm{A}$ \\
\hline
\end{tabular}

aQuestions on risk of bias are answered in relation to the majority of the aggregated evidence in the meta-analysis rather than to individual trials.

bQuestions on inconsistency are primarily based on visual assessment of forest plots and the statistical quantification of heterogeneity based on $I^{2}$.

cWhen judging the width of the confidence interval it is recommended to use a clinical decision threshold to assess whether the imprecision is clinically meaningful.

dQuestions address comprehensiveness of the search strategy, industry influence, funnel plot asymmetry and discrepancies between published and unpublished trials.

eDepends on the context of the systematic review area.

$(\downarrow)$ : key item for potential downgrading the quality of the evidence (GRADE) as shown in the footnotes of the 'Summary of finding' table(s); GRADE: Grading of Recommendations Assessment, Development and Evaluation; N/A: not applicable 


\begin{tabular}{|c|c|c|c|c|c|c|c|c|c|c|}
\hline 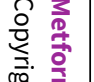 & $\begin{array}{l}\text { Appendix } 19 \\
\text { long acting }\end{array}$ & $\begin{array}{l}\text { Checklist to aid consistency and reproduc } \\
\text { P4-inhibitor }\end{array}$ & bility of GR & assessm & s: metforn & plus sulph & /lurea com & ed with me & ormin plus & \\
\hline 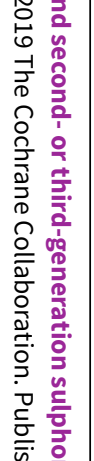 & & & $\begin{array}{l}\text { (1) All- } \\
\text { cause mor- } \\
\text { tality }\end{array}$ & $\begin{array}{l}\text { (2) Cardio- } \\
\text { vascular } \\
\text { mortality }\end{array}$ & $\begin{array}{l}\text { (3) Serious } \\
\text { adverse } \\
\text { events }\end{array}$ & $\begin{array}{l}\text { (4) Non-fa- } \\
\text { tal stroke }\end{array}$ & $\begin{array}{l}\text { (5) Non-fa- } \\
\text { tal myocar- } \\
\text { dial infarc- } \\
\text { tion }\end{array}$ & $\begin{array}{l}\text { (6) Mi- } \\
\text { crovascular } \\
\text { complica- } \\
\text { tions (end- } \\
\text { stage re- } \\
\text { nal disease, } \\
\text { blindness } \\
\text { or severe } \\
\text { vision loss, } \\
\text { amputation } \\
\text { of lower ex- } \\
\text { tremity) }\end{array}$ & $\begin{array}{l}\text { (7) Health- } \\
\text { related } \\
\text { quality of } \\
\text { life }\end{array}$ & 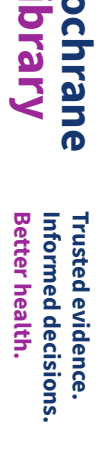 \\
\hline 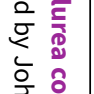 & $\begin{array}{l}\text { Trial limita- } \\
\text { tions }\end{array}$ & $\begin{array}{l}\text { Was random sequence generation used (i.e. } \\
\text { no potential for selection bias)? }\end{array}$ & Yes & Yes & Yes & $\begin{array}{l}\text { Not report- } \\
\text { ed }\end{array}$ & Yes & $\begin{array}{l}\text { Not report- } \\
\text { ed }\end{array}$ & $\begin{array}{l}\text { Not report- } \\
\text { ed }\end{array}$ & \\
\hline 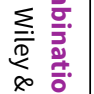 & bias)a & $\begin{array}{l}\text { Was allocation concealment used (i.e. no po- } \\
\text { tential for selection bias)? }\end{array}$ & Yes & Yes & Yes & & Yes & & & \\
\hline 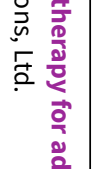 & & $\begin{array}{l}\text { Was there blinding of participants and per- } \\
\text { sonnel (i.e. no potential for performance bias) } \\
\text { or outcome not likely to be influenced by lack } \\
\text { of blinding? }\end{array}$ & Yes & Yes & Yes & & Yes & & & \\
\hline 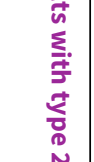 & & $\begin{array}{l}\text { Was there blinding of outcome assessment } \\
\text { (i.e. no potential for detection bias) or was } \\
\text { outcome measurement not likely to be influ- } \\
\text { enced by lack of blinding? }\end{array}$ & Yes & Yes & Yes & & Yes & & & \\
\hline$\frac{10}{0}$ & & Was an objective outcome used? & Yes & Yes & Yes & & Yes & & & $\delta$ \\
\hline 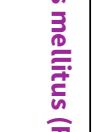 & & $\begin{array}{l}\text { Were }>80 \% \text { of participants enrolled inlmpre } \\
\text { trials included in the analysis (i.e. no poten- } \\
\text { tial reporting bias)? }\end{array}$ & Yes & Yes & Yes & & Yes & & & 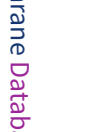 \\
\hline 市. & & $\begin{array}{l}\text { Were data reported consistently for the out- } \\
\text { come of interest (i.e. no potential selective re- } \\
\text { porting)? }\end{array}$ & Yes & Yes & Yes & & Yes & & & 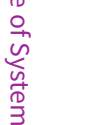 \\
\hline & & $\begin{array}{l}\text { No other biases reported (i.e. no potential of } \\
\text { other bias)? }\end{array}$ & Unclear & Unclear & Unclear & & Unclear & & & 勇. \\
\hline
\end{tabular}




\begin{tabular}{|c|c|c|c|c|c|}
\hline \multicolumn{2}{|r|}{ (Continued) } & $\begin{array}{l}\text { Did the trials end up as scheduled (i.e. not } \\
\text { stopped early)? }\end{array}$ & Yes & Yes & Yes \\
\hline & \multirow{5}{*}{$\begin{array}{l}\text { Inconsisten- } \\
\text { cyb }\end{array}$} & Point estimates did not vary widely? & $\mathrm{N} / \mathrm{A}$ & $\mathrm{N} / \mathrm{A}$ & $\mathrm{N} / \mathrm{A}$ \\
\hline 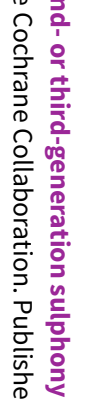 & & $\begin{array}{l}\text { To what extent did confidence intervals over- } \\
\text { lap (substantial: all confidence intervals over- } \\
\text { lap at least one of the included studies point } \\
\text { estimate; } \\
\text { some: confidence intervals overlap but not } \\
\text { all overlap at least one point estimate; no: at } \\
\text { least one outlier: where the confidence inter- } \\
\text { val of some } \\
\text { of the studies do not overlap with those of } \\
\text { most included studies)? }\end{array}$ & $\mathrm{N} / \mathrm{A}$ & N/A & $\mathrm{N} / \mathrm{A}$ \\
\hline & & Was the direction of effect consistent? & $\mathrm{N} / \mathrm{A}$ & $\mathrm{N} / \mathrm{A}$ & $\mathrm{N} / \mathrm{A}$ \\
\hline 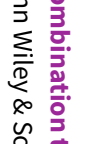 & & $\begin{array}{l}\text { What was the magnitude of statistical hetero- } \\
\left.\text { geneity (as measured by } \mathrm{I}^{2}\right)- \text { low }\left(1^{2}<40 \%\right) \text {, } \\
\left.\text { moderate }\left(1^{2} 40 \%-60 \%\right) \text {, high } 1^{2}>60 \%\right) \text { ? }\end{array}$ & $\mathrm{N} / \mathrm{A}$ & $\mathrm{N} / \mathrm{A}$ & $\mathrm{N} / \mathrm{A}$ \\
\hline$\underbrace{\infty}$ & & $\begin{array}{l}\text { Was the test for heterogeneity statistically } \\
\text { significant }(P<0.1) \text { ? }\end{array}$ & N/A & N/A & $\mathrm{N} / \mathrm{A}$ \\
\hline 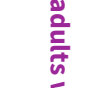 & \multirow[t]{5}{*}{ Indirectness } & $\begin{array}{l}\text { Were the populations in included studies ap- } \\
\text { plicable to the decision context? }\end{array}$ & $\begin{array}{l}\text { Highly ap- } \\
\text { plicable }\end{array}$ & $\begin{array}{l}\text { Highly ap- } \\
\text { plicable }\end{array}$ & $\begin{array}{l}\text { Highly ap- } \\
\text { plicable }\end{array}$ \\
\hline 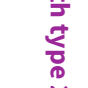 & & $\begin{array}{l}\text { Were the interventions in the included studies } \\
\text { applicable to the decision context? }\end{array}$ & $\begin{array}{l}\text { Highly ap- } \\
\text { plicable }\end{array}$ & $\begin{array}{l}\text { Highly ap- } \\
\text { plicable }\end{array}$ & $\begin{array}{l}\text { Highly ap- } \\
\text { plicable }\end{array}$ \\
\hline$\frac{\overrightarrow{\sigma_{0}}}{\bar{\phi}}$ & & $\begin{array}{l}\text { Was the included outcome not a surrogate } \\
\text { outcome? }\end{array}$ & Yes & Yes & Yes \\
\hline 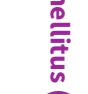 & & Was the outcome timeframe sufficient? & $\begin{array}{l}\text { Insufficient } \\
(\downarrow)\end{array}$ & $\begin{array}{l}\text { Insufficient } \\
(\downarrow)\end{array}$ & Sufficient \\
\hline 尊. & & $\begin{array}{l}\text { Were the conclusions based on direct com- } \\
\text { parisons? }\end{array}$ & Yes & Yes & Yes \\
\hline & $\begin{array}{l}\text { Impreci- } \\
\text { sionc }\end{array}$ & $\begin{array}{l}\text { Was the confidence interval for the (pooled) } \\
\text { estimate not consistent with benefit and } \\
\text { harm? }\end{array}$ & No $(\downarrow)$ & No $(\downarrow)$ & No $(\downarrow)$ \\
\hline
\end{tabular}

Yes

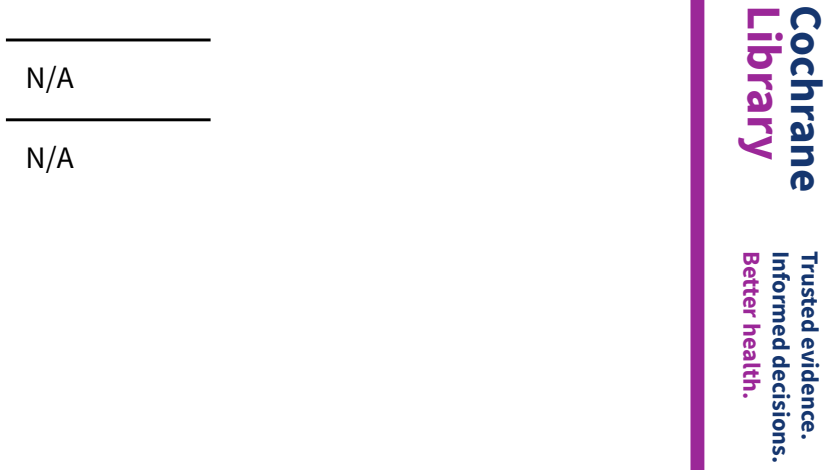

\begin{tabular}{l}
\hline N/A \\
\hline N/A \\
\hline N/A \\
\hline Highly ap- \\
plicable \\
\hline Highly ap- \\
plicable \\
\hline Yes \\
\hline Insufficient \\
$(\downarrow)$ \\
\hline Yes \\
\hline No $(\downarrow)$ \\
\hline
\end{tabular}


What is the magnitude of the median sample size (high: 300 participants, intermediate: 100-300 participants, low: $<100$ participants)?e

What was the magnitude of the number of in-

Small ( $\downarrow)$

Small $(\downarrow)$

Small $(\downarrow)$

Small ( $\downarrow)$

5-10 studies, small: < 5 studies)?e

Was the outcome a common event (e.g. occurs $>1 / 100)$ ?

No $(\downarrow)$

No $(\downarrow)$

No $(\downarrow)$

No $(\downarrow)$

\section{Publication}

Was a comprehensive search conducted?

Yes

Yes

Yes

Was grey literature searched?

Yes

Yes

Were no restrictions applied to study selec-

Yes

Yes

tion on the basis of language?
tion

There was no industry influence on studies in- Yes

Yes

Yes

cluded in the review?

\begin{tabular}{llll}
\hline $\begin{array}{l}\text { There was no evidence of funnel plot asym- } \\
\text { metry? }\end{array}$ & N/A & N/A & N/A \\
\hline $\begin{array}{l}\text { There was no discrepancy in findings be- } \\
\text { tween published and unpublished trials? }\end{array}$ & N/A & N/A & N/A
\end{tabular}

\begin{tabular}{l}
\hline Yes \\
\hline Yes \\
\hline Yes \\
\hline Unclear \\
\hline N/A \\
\hline N/A
\end{tabular}

aQuestions on risk of bias are answered in relation to the majority of the aggregated evidence in the meta-analysis rather than to individual trials.

bQuestions on inconsistency are primarily based on visual assessment of forest plots and the statistical quantification of heterogeneity based on $I^{2}$.

cWhen judging the width of the confidence interval it is recommended to use a clinical decision threshold to assess whether the imprecision is clinically meaningful.

dQuestions address comprehensiveness of the search strategy, industry influence, funnel plot asymmetry and discrepancies between published and unpublished trials.

eDepends on the context of the systematic review area.

$(\downarrow)$ : key item for potential downgrading the quality of the evidence (GRADE) as shown in the footnotes of the 'Summary of finding' table(s); GRADE: Grading of Recommendations Assessment, Development and Evaluation; N/A: not applicable 


\begin{tabular}{|c|c|c|c|c|c|c|c|c|c|}
\hline \multirow{4}{*}{\multicolumn{2}{|c|}{ 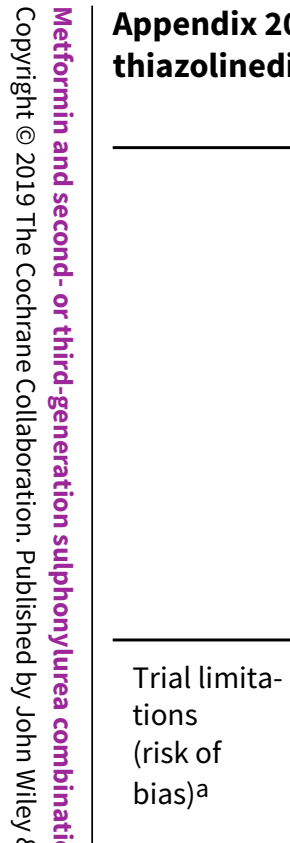 }} & \multirow{2}{*}{$\begin{array}{l}\text { Checklist to aid consistency and reproduc } \\
\text { ne }\end{array}$} & \multirow{2}{*}{$\begin{array}{l}\text { bility of GR } \\
\text { (1) All- } \\
\text { cause mor- } \\
\text { tality }\end{array}$} & \multirow[b]{2}{*}{$\begin{array}{l}\text { (2) Cardio- } \\
\text { vascular } \\
\text { mortality }\end{array}$} & \multirow[b]{2}{*}{$\begin{array}{l}\text { (3) Serious } \\
\text { adverse } \\
\text { events }\end{array}$} & \multirow[b]{2}{*}{$\begin{array}{l}\text { (4) Non-fa- } \\
\text { tal stroke }\end{array}$} & \multirow[b]{2}{*}{$\begin{array}{l}\text { (5) Non-fa- } \\
\text { tal myocar- } \\
\text { dial infarc- } \\
\text { tion }\end{array}$} & \multirow[b]{2}{*}{$\begin{array}{l}\text { (6) Mi- } \\
\text { crovascular } \\
\text { complica- } \\
\text { tions (end- } \\
\text { stage re- } \\
\text { nal disease, } \\
\text { blindness } \\
\text { or severe } \\
\text { vision loss, } \\
\text { amputation } \\
\text { of lower ex- } \\
\text { tremity) }\end{array}$} & \multirow[b]{2}{*}{$\begin{array}{l}\text { (7) Health- } \\
\text { related } \\
\text { quality of } \\
\text { life }\end{array}$} \\
\hline & & & & & & & & & \\
\hline & & $\begin{array}{l}\text { Was random sequence generation used (i.e. } \\
\text { no potential for selection bias)? }\end{array}$ & Yes & Yes & Yes & Yes & Yes & Yes & $\begin{array}{l}\text { Not report- } \\
\text { ed }\end{array}$ \\
\hline & & $\begin{array}{l}\text { Was allocation concealment used (i.e. no po- } \\
\text { tential for selection bias)? }\end{array}$ & Yes & Yes & Yes & Yes & Yes & Yes & \\
\hline 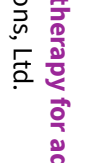 & & $\begin{array}{l}\text { Was there blinding of participants and per- } \\
\text { sonnel (i.e. no potential for performance bias) } \\
\text { or outcome not likely to be influenced by lack } \\
\text { of blinding? }\end{array}$ & Yes & Yes & Yes & Yes & Yes & Yes & \\
\hline 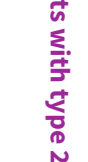 & & $\begin{array}{l}\text { Was there blinding of outcome assessment } \\
\text { (i.e. no potential for detection bias) or was } \\
\text { outcome measurement not likely to be influ- } \\
\text { enced by lack of blinding? }\end{array}$ & Yes & Yes & Yes & Yes & Yes & Yes & \\
\hline$\frac{0}{0}$ & & Was an objective outcome used? & Yes & Yes & Yes & Yes & Yes & Yes & \\
\hline 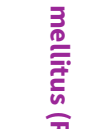 & & $\begin{array}{l}\text { Were }>80 \% \text { of participants enrolled in trials } \\
\text { included in the analysis (i.e. no potential re- } \\
\text { porting bias)? }\end{array}$ & Yes & Yes & No $(\downarrow)$ & Yes & No $(\downarrow)$ & No $(\downarrow)$ & \\
\hline & & $\begin{array}{l}\text { Were data reported consistently for the out- } \\
\text { come of interest (i.e. no potential selective re- } \\
\text { porting)? }\end{array}$ & Yes & Yes & Yes & Yes & Yes & Yes & \\
\hline & & $\begin{array}{l}\text { No other biases reported (i.e. no potential of } \\
\text { other bias)? }\end{array}$ & Unclear & Unclear & Unclear & Unclear & Unclear & Unclear & \\
\hline
\end{tabular}




\begin{tabular}{|c|c|c|c|c|c|c|c|c|}
\hline 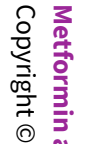 & (Continued) & $\begin{array}{l}\text { Did the trials end up as scheduled (i.e. not } \\
\text { stopped early)? }\end{array}$ & Yes & Yes & Yes & Yes & Yes & Yes \\
\hline 峁 & \multirow{5}{*}{$\begin{array}{l}\text { Inconsisten- } \\
\text { cyb }\end{array}$} & Point estimates did not vary widely? & Yes & Yes & Yes & $\mathrm{N} / \mathrm{A}$ & Yes & N/A \\
\hline 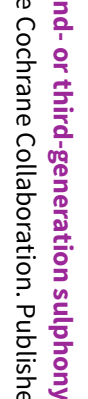 & & $\begin{array}{l}\text { To what extent did confidence intervals over- } \\
\text { lap (substantial: all confidence intervals over- } \\
\text { lap at least one of the included studies point } \\
\text { estimate; } \\
\text { some: confidence intervals overlap but not } \\
\text { all overlap at least one point estimate; no: at } \\
\text { least one outlier: where the confidence inter- } \\
\text { val of some } \\
\text { of the studies do not overlap with those of } \\
\text { most included studies)? }\end{array}$ & Substantial & Substantial & Substantial & $\mathrm{N} / \mathrm{A}$ & Substantial & $\mathrm{N} / \mathrm{A}$ \\
\hline$\$$ & & Was the direction of effect consistent? & Yes & Yes & No $(\downarrow)$ & $\mathrm{N} / \mathrm{A}$ & Yes & $\mathrm{N} / \mathrm{A}$ \\
\hline 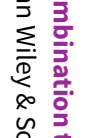 & & $\begin{array}{l}\text { What was the magnitude of statistical hetero- } \\
\left.\text { geneity (as measured by } I^{2}\right)- \text { low }\left(I^{2}<40 \%\right) \text {, } \\
\left.\text { moderate }\left(1^{2} 40 \%-60 \%\right) \text {, high } 1^{2}>60 \%\right) \text { ? }\end{array}$ & Low & Low & Low & $\mathrm{N} / \mathrm{A}$ & Low & $\mathrm{N} / \mathrm{A}$ \\
\hline 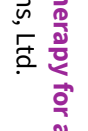 & & $\begin{array}{l}\text { Was the test for heterogeneity statistically } \\
\text { significant }(P<0.1) \text { ? }\end{array}$ & $\begin{array}{l}\text { Not statisti- } \\
\text { cally signifi- } \\
\text { cant }\end{array}$ & $\begin{array}{l}\text { Not statisti- } \\
\text { cally signifi- } \\
\text { cant }\end{array}$ & $\begin{array}{l}\text { Not statisti- } \\
\text { cally signifi- } \\
\text { cant }\end{array}$ & $\mathrm{N} / \mathrm{A}$ & $\begin{array}{l}\text { Not statisti- } \\
\text { cally signifi- } \\
\text { cant }\end{array}$ & $\mathrm{N} / \mathrm{A}$ \\
\hline 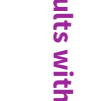 & \multirow[t]{5}{*}{ Indirectness } & $\begin{array}{l}\text { Were the populations in included studies ap- } \\
\text { plicable to the decision context? }\end{array}$ & $\begin{array}{l}\text { Highly ap- } \\
\text { plicable }\end{array}$ & $\begin{array}{l}\text { Highly ap- } \\
\text { plicable }\end{array}$ & $\begin{array}{l}\text { Highly ap- } \\
\text { plicable }\end{array}$ & $\begin{array}{l}\text { Highly ap- } \\
\text { plicable }\end{array}$ & $\begin{array}{l}\text { Highly ap- } \\
\text { plicable }\end{array}$ & $\begin{array}{l}\text { Highly ap- } \\
\text { plicable }\end{array}$ \\
\hline 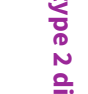 & & $\begin{array}{l}\text { Were the interventions in the included studies } \\
\text { applicable to the decision context? }\end{array}$ & $\begin{array}{l}\text { Highly ap- } \\
\text { plicable }\end{array}$ & $\begin{array}{l}\text { Highly ap- } \\
\text { plicable }\end{array}$ & $\begin{array}{l}\text { Highly ap- } \\
\text { plicable }\end{array}$ & $\begin{array}{l}\text { Highly ap- } \\
\text { plicable }\end{array}$ & $\begin{array}{l}\text { Highly ap- } \\
\text { plicable }\end{array}$ & $\begin{array}{l}\text { Highly ap- } \\
\text { plicable }\end{array}$ \\
\hline$\frac{6}{0}$ & & $\begin{array}{l}\text { Was the included outcome not a surrogate } \\
\text { outcome? }\end{array}$ & Yes & Yes & Yes & Yes & Yes & Yes \\
\hline$\overline{\overline{\vec{n}}}$ & & Was the outcome timeframe sufficient? & Sufficient & Sufficient & Sufficient & Sufficient & Sufficient & Sufficient \\
\hline 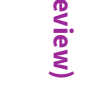 & & $\begin{array}{l}\text { Were the conclusions based on direct com- } \\
\text { parisons? }\end{array}$ & Yes & Yes & Yes & Yes & Yes & Yes \\
\hline & $\begin{array}{l}\text { Impreci- } \\
\text { sionc }\end{array}$ & $\begin{array}{l}\text { Was the confidence interval for the (pooled) } \\
\text { estimate not consistent with benefit and } \\
\text { harm? }\end{array}$ & No $(\downarrow)$ & No $(\downarrow)$ & No $(\downarrow)$ & No $(\downarrow)$ & No $(\downarrow)$ & $\mathrm{N} / \mathrm{A}$ \\
\hline
\end{tabular}


What is the magnitude of the median sample size (high: 300 participants, intermedi-

High

High

High

High

High

$\operatorname{Low}(\downarrow)$ ate: 100-300 participants, low: $<100$ participants)?

\begin{tabular}{|c|c|c|c|c|c|c|}
\hline $\begin{array}{l}\text { What was the magnitude of the number of in- } \\
\text { cluded studies (large: }>10 \text { studies, moderate: } \\
5-10 \text { studies, small: }<5 \text { studies)?e }\end{array}$ & Small $(\downarrow)$ & Small $(\downarrow)$ & Small $(\downarrow)$ & Small $(\downarrow)$ & Small $(\downarrow)$ & Small $(\downarrow)$ \\
\hline $\begin{array}{l}\text { Was the outcome a common event (e.g. oc- } \\
\text { curs }>1 / 100) \text { ? }\end{array}$ & Yes & Yes & Yes & Yes & Yes & No $(\downarrow)$ \\
\hline Was a comprehensive search conducted? & Yes & Yes & Yes & Yes & Yes & Yes \\
\hline Was grey literature searched? & Yes & Yes & Yes & Yes & Yes & Yes \\
\hline $\begin{array}{l}\text { Were no restrictions applied to study selec- } \\
\text { tion on the basis of language? }\end{array}$ & Yes & Yes & Yes & Yes & Yes & Yes \\
\hline $\begin{array}{l}\text { There was no industry influence on studies in- } \\
\text { cluded in the review? }\end{array}$ & Unclear & Unclear & Unclear & Yes & Unclear & Yes \\
\hline $\begin{array}{l}\text { There was no evidence of funnel plot asym- } \\
\text { metry? }\end{array}$ & N/A & N/A & N/A & N/A & $\mathrm{N} / \mathrm{A}$ & $\mathrm{N} / \mathrm{A}$ \\
\hline $\begin{array}{l}\text { There was no discrepancy in findings be- } \\
\text { tween published and unpublished trials? }\end{array}$ & N/A & $\mathrm{N} / \mathrm{A}$ & $\mathrm{N} / \mathrm{A}$ & $\mathrm{N} / \mathrm{A}$ & $\mathrm{N} / \mathrm{A}$ & $\mathrm{N} / \mathrm{A}$ \\
\hline
\end{tabular}

aQuestions on risk of bias are answered in relation to the majority of the aggregated evidence in the meta-analysis rather than to individual trials.

bQuestions on inconsistency are primarily based on visual assessment of forest plots and the statistical quantification of heterogeneity based on $I^{2}$.

cWhen judging the width of the confidence interval it is recommended to use a clinical decision threshold to assess whether the imprecision is clinically meaningful.

dQuestions address comprehensiveness of the search strategy, industry influence, funnel plot asymmetry and discrepancies between published and unpublished trials.

eDepends on the context of the systematic review area.

$(\downarrow)$ : key item for potential downgrading the quality of the evidence (GRADE) as shown in the footnotes of the 'Summary of finding' table(s); GRADE: Grading of Recommendations Assessment, Development and Evaluation; N/A: not applicable 


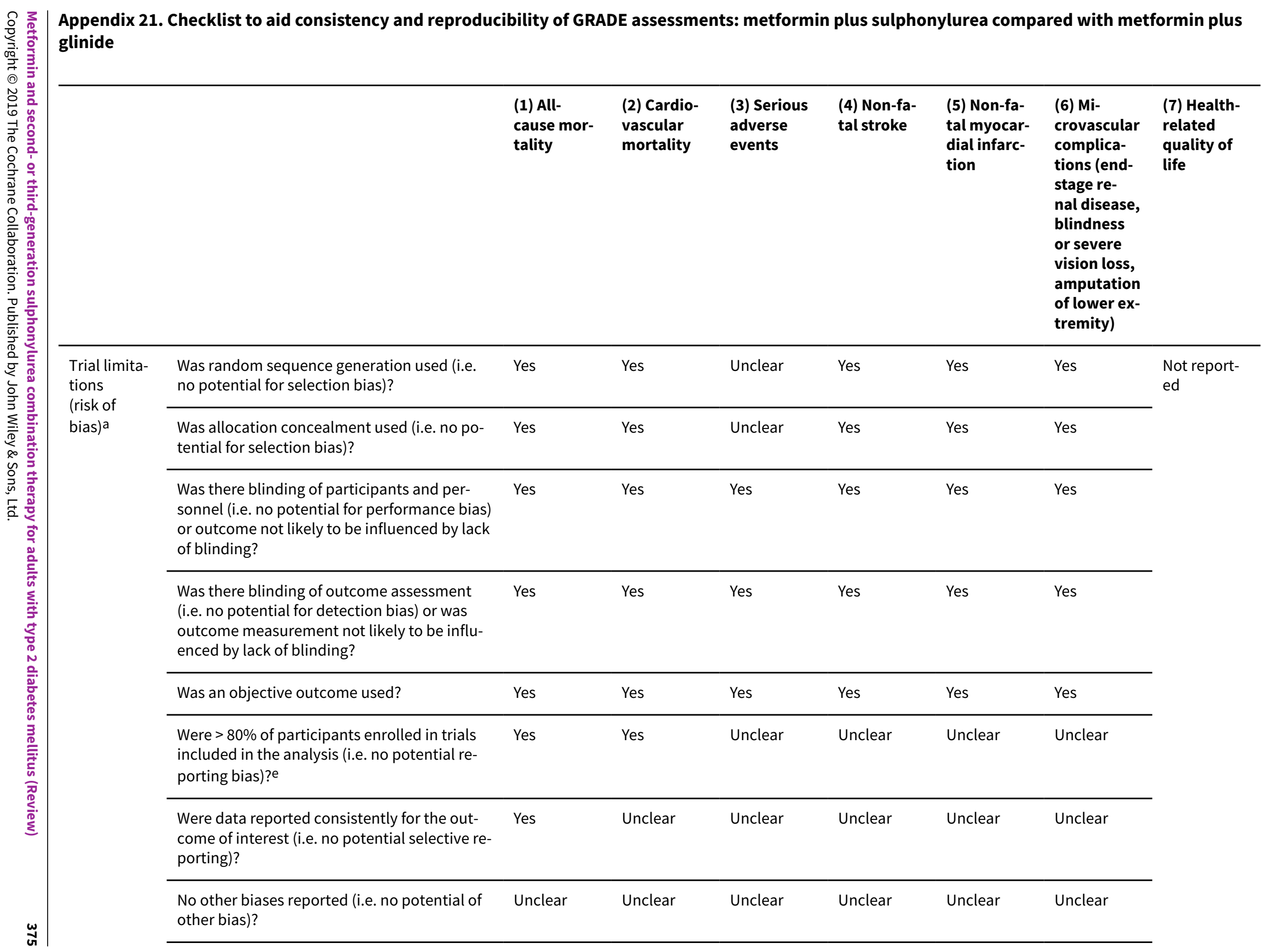




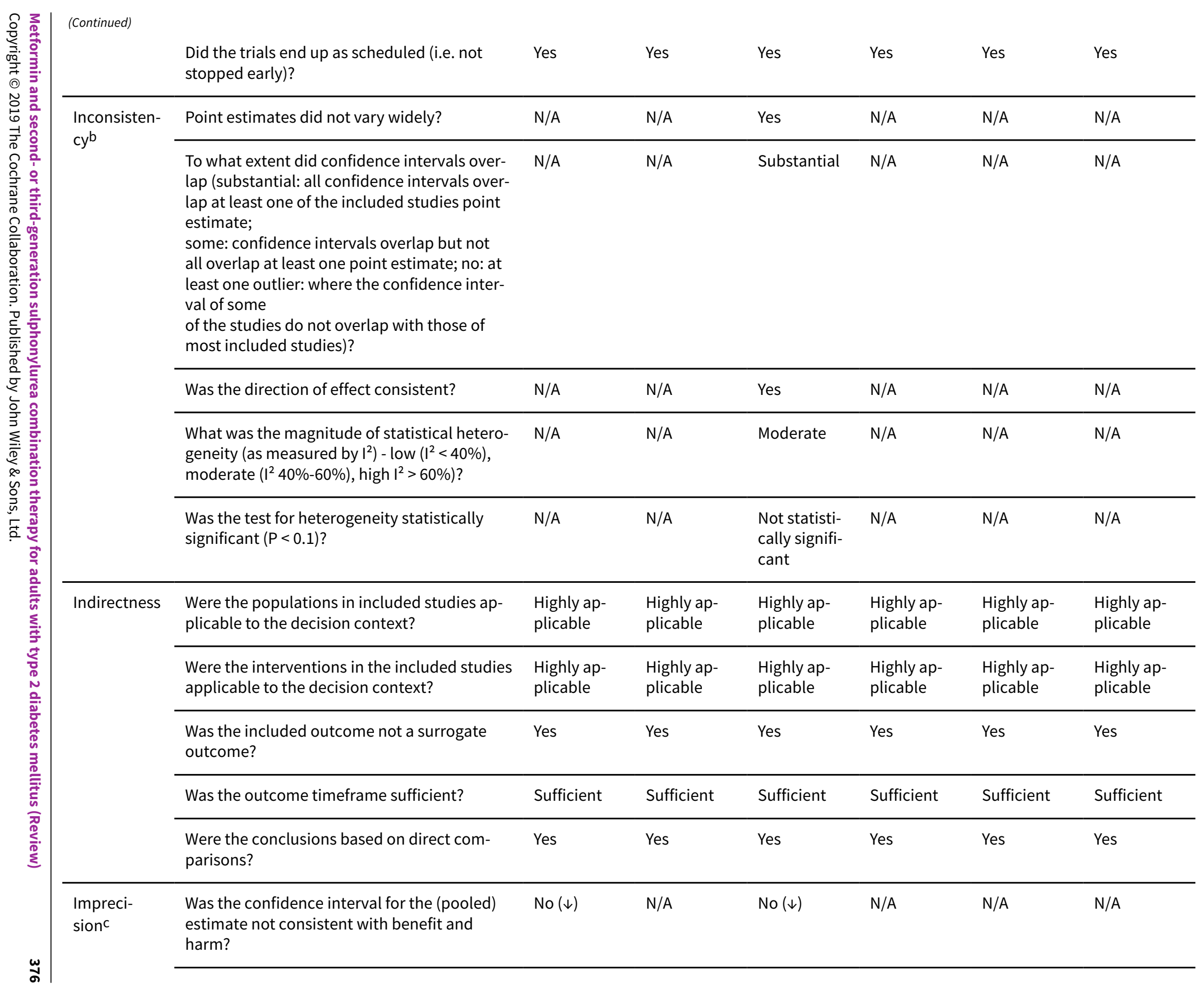




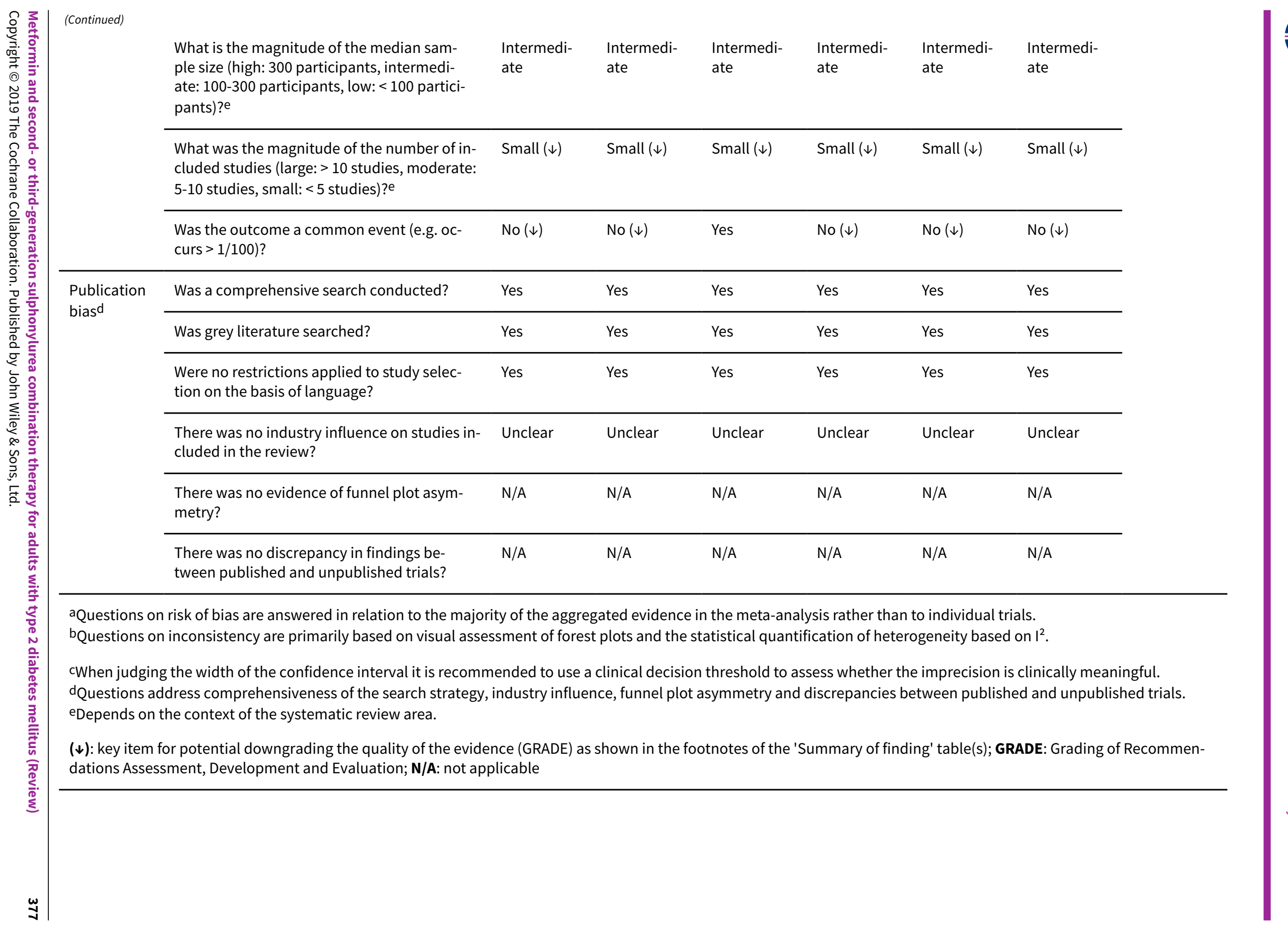




\begin{tabular}{|c|c|c|c|c|c|c|c|c|c|}
\hline \multirow{4}{*}{\multicolumn{2}{|c|}{ 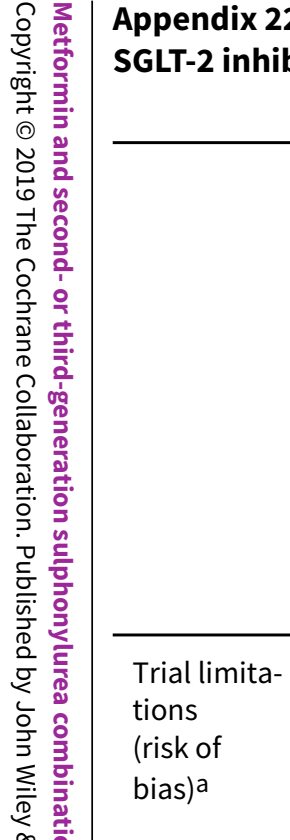 }} & \multirow{2}{*}{$\begin{array}{l}\text { Checklist to aid consistency and reproduci } \\
\text { or }\end{array}$} & \multirow{2}{*}{$\begin{array}{l}\text { oility of GR- } \\
\text { (1) All- } \\
\text { cause mor- } \\
\text { tality }\end{array}$} & \multirow[b]{2}{*}{$\begin{array}{l}\text { (2) Cardio- } \\
\text { vascular } \\
\text { mortality }\end{array}$} & \multirow[b]{2}{*}{$\begin{array}{l}\text { (3) Serious } \\
\text { adverse } \\
\text { events }\end{array}$} & \multirow[b]{2}{*}{$\begin{array}{l}\text { (4) Non-fa- } \\
\text { tal stroke }\end{array}$} & \multirow[b]{2}{*}{$\begin{array}{l}\text { (5) Non-fa- } \\
\text { tal myocar- } \\
\text { dial infarc- } \\
\text { tion }\end{array}$} & \multirow[b]{2}{*}{$\begin{array}{l}\text { (6) Mi- } \\
\text { crovascular } \\
\text { complica- } \\
\text { tions (end- } \\
\text { stage re- } \\
\text { nal disease, } \\
\text { blindness } \\
\text { or severe } \\
\text { vision loss, } \\
\text { amputation } \\
\text { of lower ex- } \\
\text { tremity) }\end{array}$} & \multirow[b]{2}{*}{$\begin{array}{l}\text { (7) Health- } \\
\text { related } \\
\text { quality of } \\
\text { life }\end{array}$} \\
\hline & & & & & & & & & \\
\hline & & $\begin{array}{l}\text { Was random sequence generation used (i.e. } \\
\text { no potential for selection bias)? }\end{array}$ & Yes & Yes & Yes & Yes & Yes & Yes & $\begin{array}{l}\text { Not report- } \\
\text { ed }\end{array}$ \\
\hline & & $\begin{array}{l}\text { Was allocation concealment used (i.e. no po- } \\
\text { tential for selection bias)? }\end{array}$ & Yes & Yes & Yes & Yes & Yes & Yes & \\
\hline 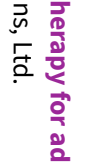 & & $\begin{array}{l}\text { Was there blinding of participants and per- } \\
\text { sonnel (i.e. no potential for performance bias) } \\
\text { or outcome not likely to be influenced by lack } \\
\text { of blinding? }\end{array}$ & Yes & Yes & Yes & Yes & Yes & Yes & \\
\hline 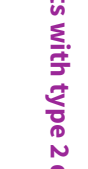 & & $\begin{array}{l}\text { Was there blinding of outcome assessment } \\
\text { (i.e. no potential for detection bias) or was } \\
\text { outcome measurement not likely to be influ- } \\
\text { enced by lack of blinding? }\end{array}$ & Yes & Yes & Yes & Yes & Yes & Yes & \\
\hline$\underset{0}{0}$ & & Was an objective outcome used? & Yes & Yes & Yes & Yes & Yes & Yes & \\
\hline 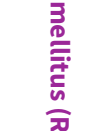 & & $\begin{array}{l}\text { Were }>80 \% \text { of participants enrolled in trials } \\
\text { included in the analysis (i.e. no potential re- } \\
\text { porting bias)?e }\end{array}$ & Yes & Yes & No $(\downarrow)$ & No $(\downarrow)$ & No $(\downarrow)$ & Unclear & \\
\hline हैं & & $\begin{array}{l}\text { Were data reported consistently for the out- } \\
\text { come of interest (i.e. no potential selective re- } \\
\text { porting)? }\end{array}$ & Yes & Yes & Yes & Yes & Yes & Yes & \\
\hline & & $\begin{array}{l}\text { No other biases reported (i.e. no potential of } \\
\text { other bias)? }\end{array}$ & Unclear & Unclear & Unclear & Unclear & Unclear & Unclear & \\
\hline
\end{tabular}




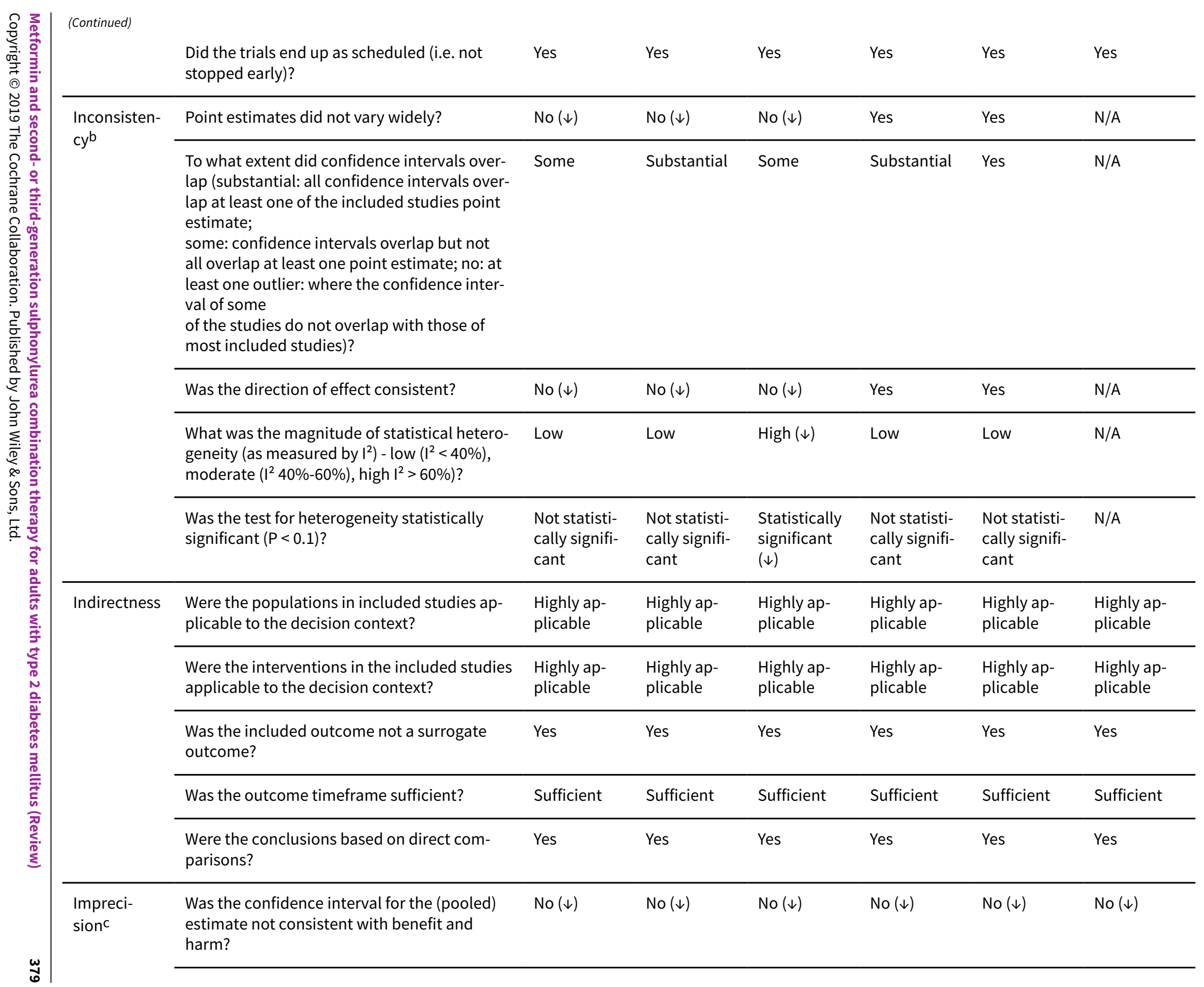


What is the magnitude of the median sample size (high: 300 participants, intermedi-

High

High

High

High

High

High ate: $100-300$ participants, low: $<100$ participants)?e

\begin{tabular}{|c|c|c|c|c|c|c|}
\hline $\begin{array}{l}\text { What was the magnitude of the number of in- } \\
\text { cluded studies (large: }>10 \text { studies, moderate: } \\
5-10 \text { studies, small: }<5 \text { studies)?e }\end{array}$ & Small $(\downarrow)$ & Small $(\downarrow)$ & Small $(\downarrow)$ & Small $(\downarrow)$ & Small $(\downarrow)$ & Small $(\downarrow)$ \\
\hline $\begin{array}{l}\text { Was the outcome a common event (e.g. oc- } \\
\text { curs }>1 / 100) \text { ? }\end{array}$ & No $(\downarrow)$ & No $(\downarrow)$ & Yes & No $(\downarrow)$ & No $(\downarrow)$ & No $(\downarrow)$ \\
\hline Was a comprehensive search conducted? & Yes & Yes & Yes & Yes & Yes & Yes \\
\hline Was grey literature searched? & Yes & Yes & Yes & Yes & Yes & Yes \\
\hline $\begin{array}{l}\text { Were no restrictions applied to study selec- } \\
\text { tion on the basis of language? }\end{array}$ & Yes & Yes & Yes & Yes & Yes & Yes \\
\hline $\begin{array}{l}\text { There was no industry influence on studies in- } \\
\text { cluded in the review? }\end{array}$ & Unclear & Unclear & Unclear & Unclear & Unclear & Unclear \\
\hline $\begin{array}{l}\text { There was no evidence of funnel plot asym- } \\
\text { metry? }\end{array}$ & N/A & N/A & N/A & N/A & $\mathrm{N} / \mathrm{A}$ & $\mathrm{N} / \mathrm{A}$ \\
\hline $\begin{array}{l}\text { There was no discrepancy in findings be- } \\
\text { tween published and unpublished trials? }\end{array}$ & N/A & $\mathrm{N} / \mathrm{A}$ & $\mathrm{N} / \mathrm{A}$ & $\mathrm{N} / \mathrm{A}$ & $\mathrm{N} / \mathrm{A}$ & $\mathrm{N} / \mathrm{A}$ \\
\hline
\end{tabular}

aQuestions on risk of bias are answered in relation to the majority of the aggregated evidence in the meta-analysis rather than to individual trials.

bQuestions on inconsistency are primarily based on visual assessment of forest plots and the statistical quantification of heterogeneity based on $I^{2}$.

cWhen judging the width of the confidence interval it is recommended to use a clinical decision threshold to assess whether the imprecision is clinically meaningful.

dQuestions address comprehensiveness of the search strategy, industry influence, funnel plot asymmetry and discrepancies between published and unpublished trials.

eDepends on the context of the systematic review area.

$(\downarrow)$ : key item for potential downgrading the quality of the evidence (GRADE) as shown in the footnotes of the 'Summary of finding' table(s); GRADE: Grading of Recommendations Assessment, Development and Evaluation; N/A: not applicable 


\section{CONTRIBUTIONS OF AUTHORS}

All review authors read and approved the final review draft.

Kasper S Madsen (KM): protocol draft, acquisition of trial reports, trial selection, data extraction, data analysis, data interpretation, writing draft, and future review updates

Pernille Kähler (PK): trial selection, data extraction, review of drafts and future review updates

Bianca Hemmingsen (BH): protocol draft, acquisition of trial reports, trial selection, data extraction, data analysis, data interpretation, review of drafts and future review updates

Sten Madsbad (SM): protocol draft, review of drafts and future review updates

Bernd Richter (BR): protocol draft, search strategy development, data analysis, data interpretation, review of drafts and future review updates

Maria-Inti Metzendorf (MIM): protocol draft, search strategy development, review of drafts and future review updates

Lise Katrine Kähler (LK): trial selection, data extraction, review of drafts and future review updates

Filip Gnesin (FG): trial selection, data extraction, review of drafts and future review updates

\section{DECLARATIONS OF INTEREST}

KM: had an inadvertent conflict of interest because he had owned a small number of shares with Novo Nordisk A/S before registering the title. Without prompting KM amended the situation by selling the shares, so he no longer has a direct financial benefit as first author. Cochrane Metabolic and Endocrine Disorders contacted the Cochrane Funding Arbiter for guidance, who agreed to allow the review to proceed with KM as a first author, providing that this issue was clearly explained in the declarations of interest. KM received a scholarship from Michaelsen Fonden.

PK: equities in Novo Nordisk A/S.

LK: none known.

SM: Advisory Boards: Novartis Pharma, Novo Nordisk, Merck Sharp \& Dome, Sanofi-Aventis, AstraZeneca, Johnson \& Johnson, BoehringerIngelheim, Eli Lilly, Intarcia Therapeutics, Bristol-Meyer Squibb. Fee for lectures: Novo Nordisk, Merck, Sharp \& Dome, Astra-Zeneca, SanofiAventis, Novartis Pharma, Eli Lilly, Bristol-Meyer Squibb, Boeringer-Ingelheim. Grants for research: Novo Nordisk.

FG: none known.

MIM: none known.

BR: none known.

$\mathrm{BH}$ : none known.

\section{SOURCES OF SUPPORT}

\section{Internal sources}

- No sources of support supplied

\section{External sources}

- Michaelsen Fonden, Denmark.

Financial support: non-pharmaceutical fund, which hands out scholarships to medical students on reaserch leave from the university

\section{DIFFERENCES BETWEEN PROTOCOL AND REVIEW}

We changed data extraction of mild, moderate and serious hypoglycaemia to extraction of mild or moderate and serious hypoglycaemia as this was the more common way of reporting hypoglycaemia in publications.

We performed subgroup analysis dividing thiazolidinediones in rosiglitazone and pioglitazone. 


\section{NOTES}

Portions of the background and methods sections, the appendices, additional tables and figures 1 to 3 of this review are based on a standard template established by Cochrane Metabolic and Endocrine Disorders.

\section{NDEX TERMS}

\section{Medical Subject Headings (MeSH)}

Diabetes Mellitus, Type 2 [ ${ }^{\star}$ drug therapy]; Drug Therapy, Combination; Hypoglycemia [chemically induced]; Hypoglycemic Agents [*therapeutic use]; Metformin [*therapeutic use]; Sulfonylurea Compounds [ ${ }^{\star}$ therapeutic use]; Treatment Outcome

\section{MeSH check words}

Humans 